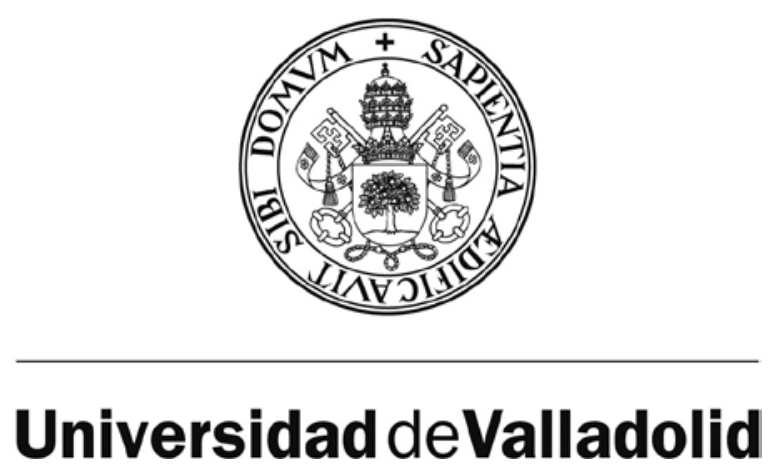

ESCUELA TÉCNICA SUPERIOR DE INGENIEROS DE TELECOMUNICACIÓN DEPARTAMENTO DE TEORÍA DE LA SEÑAL Y COMUNICACIONES E INGENIERIA TELEMÁTICA

TESIS DOCTORAL:

\title{
NOVEL ERBIUM(III) AND YTTERBIUM(III)-BASED MATERIALS FOR OPTOELECTRONIC AND TELECOMMUNICATION APPLICATIONS
}

Presentada por Pablo Martín Ramos para optar al grado de doctor por la Universidad de Valladolid

Dirigida por:

Dr. Pedro Chamorro Posada

Dr. Jesús Martín Gil 
One of the ways of stopping science would be only to do experiments in the region where you know the law. But

experimenters search most diligently, and with the greatest effort, in exactly those places where it seems most likely that we can prove our theories wrong. In other words, we are trying to prove ourselves wrong as quickly as possible, because only in that way can we find progress.

Richard P. Feynman 


\section{ACKNOWLEDGEMENTS}

It is a pleasure to thank the many people who have made this Thesis possible.

It is difficult to overstate my gratitude to my Ph.D. supervisors, Prof. Pedro Chamorro-Posada and Prof. Jesús Martín-Gil. Throughout these years, they have provided encouragement, sound advice, useful feedback and constructive comments. Without their steady and wise guiding hand, I would have been lost.

Besides my advisors, many others have gained my deepest respect and most grateful acknowledgement. This multidisciplinary research Thesis -with components of Chemistry, Physics, Materials Science and Electronics- would not have been feasible without the dedication and willingness of our outstanding collaborators, who have gladly granted me full-access to their research facilities: Prof. Ana María Matos-Beja, Prof. Manuela Ramos-Silva, Prof. José Antonio Paixão, Prof. Ermelinda S. Eusebio, Dr. Pedro S. Pereira da Silva, Joao Pedro Martins and Micael Miranda at the CEMDRX and the Chemistry Department, Universidade de Coimbra (Coimbra, Portugal); Prof. Carmen Coya, Prof. Ángel L. Álvarez and Miguel García at the Organic Optoelectronics group, Universidad Rey Juan Carlos (Madrid, Spain); Prof. Carlos Zaldo at the Photonic Materials group, ICMM-CSIC (Madrid, Spain); Prof. Victor Lavín, Prof. Inocencio R. Martín, Prof. Fernando Lahoz, Prof. Ulises R. Rodríguez and Sergio F. León at the MALTA Consolider Team, Universidad de la Laguna (Canary Islands, Spain); Prof. Laura C.J. Pereira at the Solid State group, Instituto Superior Técnico (Lisboa, Portugal); Dr. Hagen Klauk at the Organic Electronics group, Max Planck Institute for Solid State Research (Stuttgart, Germany); Michael Strecker at the Institute for Large Area Microelectronics, Stuttgart University (Stuttgart, Germany); Prof. Tony Heinz at the Columbia Nanoscale Science and Engineering Center (NSEC) and Prof. Ioannis Kymissis at the Columbia Laboratory for Unconventional Electronics, Columbia University (New York, USA); Prof. Samuel K. Kassegne and Prof. Long C. Lee at the MEMS Research Group and the Photonics Group, respectively, San Diego State University (San Diego, USA); Prof. Humberto Michinel and José A. Nóvoa at the Applied Physics Department, Universidade de Vigo (Orense, Spain); and Dr. Mario Salvalaggio, at Istituto Eni Donegani (Novara, Italy). I apologize in advance to anyone I have overlooked. I owe a great deal to their expertise, their teaching of the advanced techniques involved and their tireless support when I got in a muddle during the actual experiments. They are the ones who have shaped the way I will keep conducting research and approaching problems in the future. I hope this Thesis passes by some of the insights they have taught me.

I am also indebted to the financial support received from the Fulbright Foundation (a twoyear Fulbright Postgraduate Fellowship for the Columbia University's Fu Foundation School of Engineering and Applied Science at New York), Caja Madrid Foundation and Caja España Foundation (a one-year Postgraduate scholarship for the San Diego State University at San Diego, California), the German Academic Exchange Service, DAAD (a five-month scholarship for the Max Planck Institute at Stuttgart), Santander Universities (who has funded my several short research stays at Coimbra University through JPI-2013 program), Junta de Castilla y León through project VA300A12-1, the University of Valladolid (FPI 2011 Ph.D. scholarship and Prometeo research award 2010) and the Spanish Ministry of Education (FPU 2010 Ph.D. scholarship).

Finally, I wish to thank my family for providing a loving environment for me: to my fiancée, Raquel; to my sister, Clara; to my grandmother, Teo; and to my parents, Carmen and Javier. They bore me, supported me, raised me, taught me, and loved me. To them I dedicate this Thesis. 


\begin{abstract}
This Ph.D. Thesis is a contribution within the bounds of the interdisciplinary area constituted by the interrelation between Materials Engineering and Electrical Engineering, insofar as the main results of the conducted research herein referred are related to the characterization of novel materials and devices with applications in these areas.

Key aspects of this work have been the molecular design by semiempirical quantum chemistry methods (Sparkle/PM7), the synthesis, structural elucidation by X-ray diffraction and characterization by DSC, FTIR, Raman, NMR and EPR spectroscopies, UV-Vis-NIR absorption, excitation and photoluminescence of the novel complexes: 44 erbium(III) and ytterbium(III) ternary complexes with an octacoordinated environment which consists of six oxygen atoms from the $\beta$-diketonate ligands and two nitrogen atoms from a Lewis base.

The main properties of the new complexes, on which their applications are based, are: their emission at crucial wavelengths in the infrared region, their nonlinear optical behavior and their molecular magnet characteristics. With regard to the former, the novel materials -either in solid state, in solution or dispersed in matrices (vitreous and non-vitreous)- efficiently emit at wavelengths that make them suitable for the manufacturing of active (lasers and optical amplifiers) and passive (waveguides) optical architectures. On the other hand, their NLO properties are promising for their use in optical switching, optical limiters and other ultrafast applications. Regarding their single-ion magnetic behavior, they could find use in information storage, quantum computation and spintronics.

Of particular engineering interest have been the manufacturing and characterization of solution-processed NIR-OLED devices emitting in the third communication window 1.54 $\mu \mathrm{m})$, in which the excellent film forming properties and ambipolar behavior of the developed materials have enabled their use as active layers without resorting to host matrices. The designed fabrication procedure, together with arc-erosion nano-patterning technology, paves the way to the manufacturing of large area NIR-OLEDs by cost-effective methods.
\end{abstract}




\section{RESUMEN}

La presente Tesis Doctoral constituye una aportación enmarcada en el área multidisciplinar constituida por la interrelación de la Ingeniería de Materiales con las Ingenierías Electrónica y de Telecomunicación en tanto que las principales contribuciones de la investigación realizada son relativas a la caracterización de nuevos materiales y dispositivos con aplicación en estas áreas.

Aspectos fundamentales de este trabajo han sido los de diseño molecular mediante métodos semiempíricos (Sparkle/PM7), síntesis, elucidación estructural por difracción de rayos X y caracterización por DSC, espectroscopías FTIR, Raman, RMN y RPE, absorción óptica UV-VisNIR, excitación y fotoluminescencia de los nuevos compuestos: 44 complejos ternarios de erbio(III) e iterbio(III) en un entorno de octacoordinación asegurado por seis oxígenos procedentes de tres ligandos $\beta$-dicetonato y dos nitrógenos de una base de Lewis.

Las principales propiedades de los nuevos complejos, en las cuales están fundamentadas sus aplicaciones, son: su emisión a longitudes de onda críticas en el infrarrojo, su comportamiento óptico no lineal y su funcionamiento como magnetos moleculares. En relación con la primera de estas propiedades, los nuevos materiales, tanto en estado sólido como en disolución o dispersados en matrices (vítreas y no vítreas), emiten de modo eficiente a longitudes de onda que les hacen útiles para la fabricación de arquitecturas ópticas activas (láseres y amplificadores ópticos) y pasivas (guías de ondas). Por otra parte, sus propiedades en NLO los hacen prometedores para su aplicación en interruptores ópticos, limitadores ópticos y otras aplicaciones ultrarrápidas. En lo que respecta a su comportamiento magnético como imanes mono-moleculares, encuentran aplicación en almacenamiento de información, computación cuántica y espintrónica.

De especial interés ingenieril ha sido la fabricación y caracterización de dispositivos NIROLEDs emisores en tercera ventana $(1.54 \mu \mathrm{m})$ procesados desde solución, en los que las excelentes propiedades de formación de películas y el comportamiento ambipolar de los materiales desarrollados han permitido su utilización como capas activas sin necesidad de recurrir a matrices anfitrionas. El procedimiento desarrollado, conjuntamente con la tecnología de nano-ablación por descarga eléctrica, abre la puerta a la producción de NIROLEDs de área amplia por métodos de bajo coste. 


\section{CONTENTS}

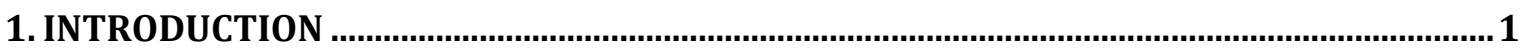

1.1. Luminescence of near-infrared emitting lanthanide complexes and non-radiative loses ........... 2

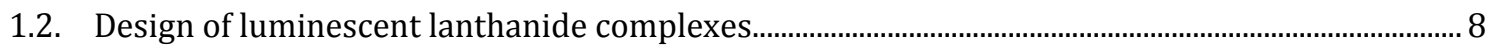

1.3. Lanthanide complexes-based luminescent hybrid materials ..........................................................10

1.4. OLEDs and NIR-OLEDs: device structure and operation principle ................................................15

1.5. Erbium luminescence in the near-infrared telecommunication window. EDFAs y YEDFAs......18

1.5.1. Amplification of telecommunication signals by EDFAs.............................................................18

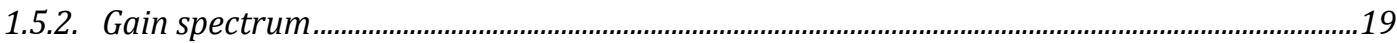

1.5.3. Glass:Er, Yb fibers (ytterbium-sensitized fibers or ytterbium-codoped fibers) .......................20

1.5.4. Design requirements for materials used in amplifiers and waveguides for

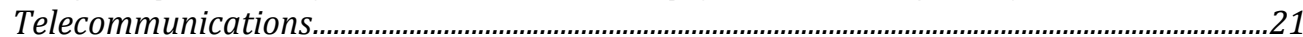

1.6. Erbium/Ytterbium complexes in third generation photovoltaics .....................................................23

1.7. Erbium/Ytterbium complexes as nonlinear optical chromophores...............................................2

1.8. Single Molecular Magnet (SMM) properties of $\operatorname{Ln}(\mathrm{III})$ complexes ..................................................28

\section{SYNTHESIS, STRUCTURAL MODELING AND CHARACTERIZATION OF THE NOVEL ERBIUM(III) AND YTTERBIUM(III) COMPLEXES AS EMITTERS..................................... 29}

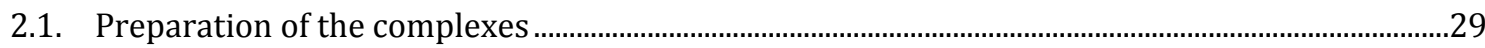

2.2. Crystal structure modeling by semiempirical methods ....................................................................39

2.2.1. Introduction to semiempirical methods...................................................................................39

2.2.2. The Sparkle model, a semiempirical approach to the quantum chemical calculation of

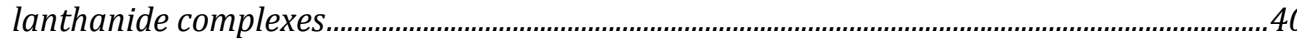

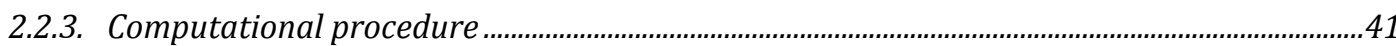

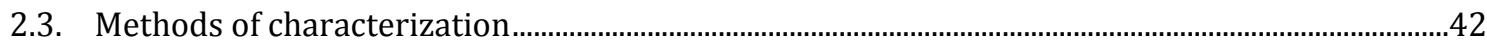

2.3.1. Characterization of crystal structures by $X$-ray diffraction..........................................................42

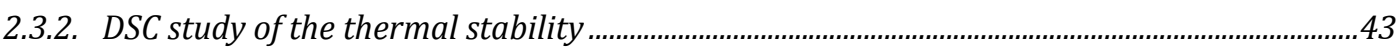

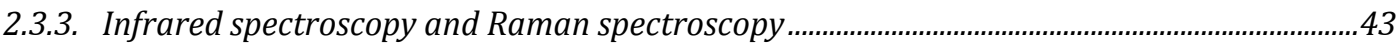

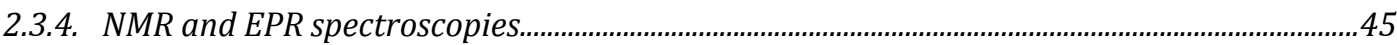

2.3.5. Optical absorption, excitation and photoluminescence studies...................................................46

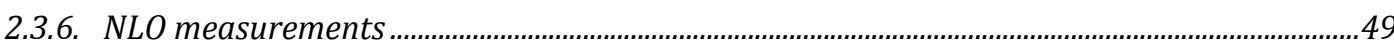

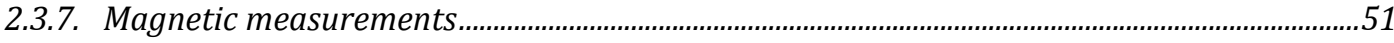

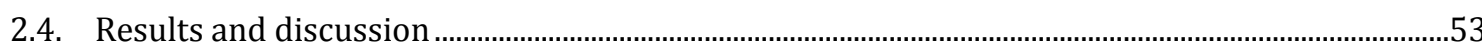

2.4.1. Determination of crystal structures by X-ray diffraction..........................................................53

Preliminary $X$-ray powder diffraction patterns..........................................................................53

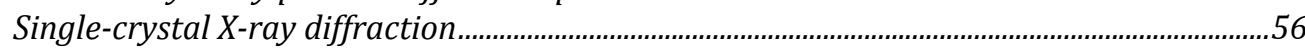

Semiempirical models ........................................................................................................................93

Assessment of the accuracy of semiempirical quantum chemistry methods..........................97

Remarks on structural parameters...................................................................................... 101

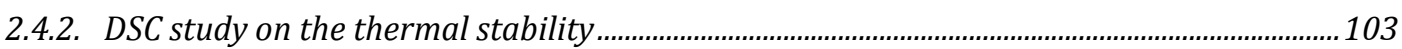

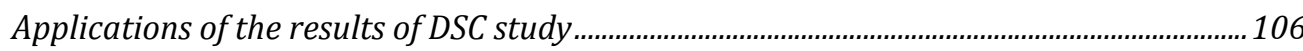


Assessment of the presumable lanthanidomesogen behavior of some of the complexes.. 106 On the special thermal behavior of dibenzoylmethanate complexes in methanol solution and their applications in chemical vapor deposition and sonochemistry..............................108

2.4.3. Infrared and Raman spectroscopies........................................................................................... 108

IR spectra for erbium ternary complexes .................................................................................. 108

IR spectra for selected $\mathrm{Yb}(\mathrm{III})$ complexes with 5-nitro-1,10-phenanthroline........................114

IR spectra for other $\mathrm{Yb}(\mathrm{III})$ complexes.................................................................................. 116

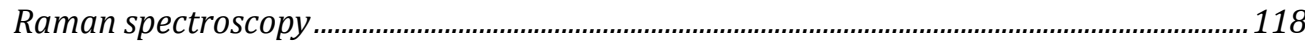

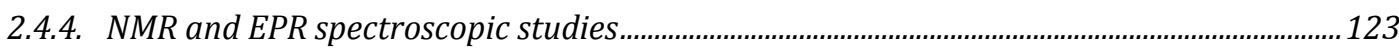

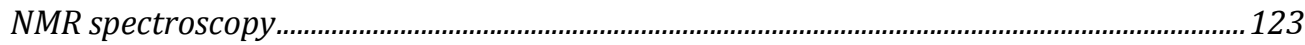

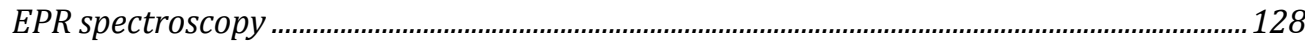

2.4.5. Optical absorption, excitation and photoluminescence studies...............................................130

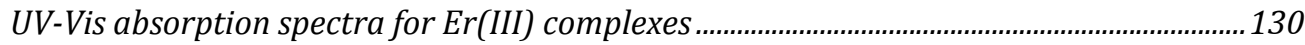

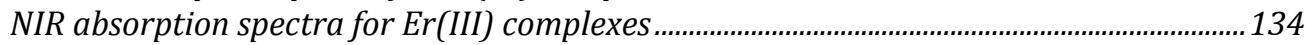

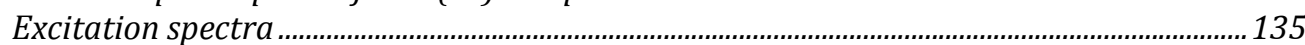

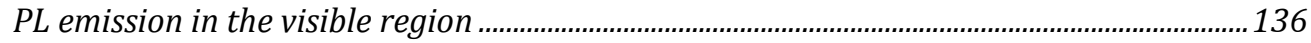

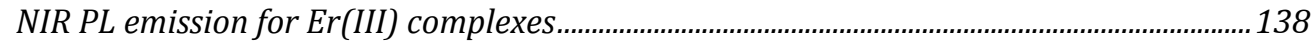

NIR PL emission for selected Yb(III) complexes with 5-nitro-1,10-phenanthroline ........... 143

Visible PL emission lifetimes...............................................................................................145

NIR PL emission lifetimes for Er(III) complexes..................................................................... 148

NIR PL emission lifetimes for selected $\mathrm{Yb}(\mathrm{III})$ complexes with $5 \mathrm{NO}_{2}$ phen.............................150

2.4.6. $\mathrm{NLO}$ properties of $\mathrm{Yb}(\mathrm{III})$ complexes with Vildagliptin ligand..................................................155

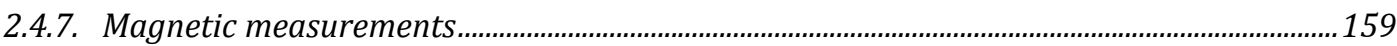

\section{IMPLICATIONS OF THE NEW MATERIALS FOR OPTOELECTRONIC APPLICATIONS.166}

3.1. Correlation between structural data and photoluminescence properties

3.2. Specific role of the organic ligands on the photoluminescence of ternary complexes and their relationship with pumping wavelengths

3.3. Applicability of hybrid materials based on lanthanide complexes to optical networks.

3.4. Approaches for co-doping in YEDFAs: separate $\mathrm{Er}^{3+}$ and $\mathrm{Yb}^{3+}$ complexes vs. ' $\mathrm{Er}^{3+}-\mathrm{Yb}^{3+}$ cocrystalline' complexes

\section{APPLICATIONS OF THE NOVEL COMPLEXES IN OPTOELECTRONICS AND} TELECOMMUNICATIONS

4.1. Assessment of the suitability of the new complexes for the fabrication of waveguides and optical amplifiers

4.1.1. Studies with mesoporous glass and fluorinated polymers........................................................172

Assay on the impregnation of mesoporous glass with solutions of erbium complexes..... 172 Assay on the fabrication of doped fluorinated polymer films ................................................ 174

4.1.2. Studies with doped ormosils and PMMA............................................................................. 175

4.2. Assessment of the suitability of the new complexes for the fabrication of NIR-OLEDs ........... 183

4.2.1. Precedents in the manufacturing of NIR-OLEDs.......................................................................183

4.2.2. Fabrication process and device characterization ….............................................................187

NIR-OLEDs with AZO/PEDOT:PSS/PVK:PBD:Er-complex/Ba/Al structure ..........................187 NIR-OLEDs with ITO/PEDOT:PSS/Er-complex/Ca/Al structure ............................................. 191 


\section{INTRODUCTION}

Photonics has been deemed the $21^{\text {st }}$ Century revolutionary technology that would create as enormous impact as Electronics did in the $20^{\text {th }}$ Century. Innovation in Photonics is often enabled by progress in materials science and technology, exemplified in this case by the novel luminescent ternary lanthanide(III) $\beta$-diketonate complexes for infrared organic light emission diodes (NIR-OLED), erbium/ytterbium doped glass fibre amplifiers (EDFA/YEDFA), erbium-doped polymeric materials, planar waveguides and plenty of other examples.

The attainment of results in aforementioned investigations, conducted at the Department of Signal Theory and Communications and Telematics Engineering at the University of Valladolid since 2007 and which have been the subject of two final year dissertations (entitled "Novel NIR-OLED devices for Telecommunications Electronics" (2008) and "Erbium(III) and ytterbium(III) complexes with fluorinated ligands for photonic applications in Telecommunications: EDFAs and YEDFAs" (2010)), besides the opportunity of expanding and integrating them in a body of common analysis, have encouraged the submission of this $\mathrm{PhD}$ Thesis.

This Thesis covers the design, synthesis and characterization of new mixed-ligand erbium(III) and ytterbium(III) multifunctional materials, together with an assessment of their possible applications. Particular emphasis has been placed on their use as dopants in hybrid materials suitable for passive and active optical architectures and as the active layer for solution-processed OLEDs emitting in the $1.54 \mu \mathrm{m}$ NIR band, but their use as upconverters for third-generation solar cells, non-linear optical chromophores or Single Ion Magnets is also discussed. The problem being addressed (the search for engineering applications to new photoluminescent materials) is within the field of study of this $\mathrm{PhD}$ program in Information Technology and Communications, since it incorporates the concepts, contents and methodological resources specific to Electronics and Telecommunications Engineering.

It should be clarified that this document does not aim to report or discuss the results in a comprehensive and very detailed way, since this goal has been achieved in the articles already published in several of Materials Science and Crystallography journals, namely Journal of Materials Chemistry $C$ ( 2 articles, $\left.\mathrm{SCI}_{2012}=6.101, \mathrm{Q} 1\right)$, Journal of Physical Chemistry $C$ $\left(\mathrm{SCI}_{2012}=4.814, \mathrm{Q} 1\right)$, Dalton Transactions $\left(\mathrm{SCI}_{2012}=3.806, \mathrm{Q} 1\right)$, Polyhedron $\left(\mathrm{SCI}_{2011}=2.057, \mathrm{Q} 2\right)$, Journal of Chemical Crystallography $\left(\mathrm{SCI}_{2012}=0.513, \mathrm{Q} 4\right)$ and Acta Crystallographica Section E $\left(\mathrm{SCI}_{2011}=0.347, \mathrm{Q} 4\right)$, in MRS and CDE proceedings, in peer-reviewed journals without impact factor (e.g., Optica Pura y Aplicada), in several other manuscripts which are still in a submitted status or which will be submitted in the next few months, and in the posters and communications presented to 20 national and international symposiums. All materials herein referred are protected under Spanish patent ES 2388091 A1 and World patent WO/2012/120175.

The Thesis is organized as follows: an overview of the fundamental concepts of lanthanide luminescence, lanthanide-based materials and their technological applications is presented in Section 1; the design, synthesis, experimental methods, equipment and results from the characterization for the novel materials included in this study are presented in Section 2; Section 3 briefly discusses their implications for Optoelectronic applications; these applications are discussed in-depth in Section 4; and in Section 5 the drawn conclusions are presented. Bibliographical references are included in Section 6. 


\subsection{Luminescence of near-infrared emitting lanthanide complexes and non- radiative loses}

\section{Precisions on the luminescence of lanthanide ions}

When the light emission by lanthanide ions is discussed, the term "luminescence" is to be used, rather than the terms "fluorescence" or "phosphorescence". The reason is that the terms fluorescence and phosphorescence are used to describe light emission by organic molecules, and that these terms incorporate information on the emission mechanism: fluorescence is singlet-to-singlet emission and phosphorescence is triplet-to-triplet emissiona. In the case of lanthanides, the emission is due to transitions inside the $4 f$-shell, i.e. these transitions are intraconfigurational $f-f$ transitions. Because the partially filled $4 f$-shell is well shielded from its environment by the closed $5 s^{2} 5 p^{6}$ shell, the ligands in the first and second coordination spheres perturb the electronic configurations of the trivalent lanthanide ions only to a very limited extent. This shielding is responsible for the specific properties of lanthanide luminescence, more particularly for the narrow-band emission and for the long lifetimes of the excited states. Depending on the method of excitation, different types of luminescence are considered, e.g. photoluminescence (emission after excitation by irradiation with electromagnetic radiation), electroluminescence (emission by recombination of electrons and holes under the influence of an electric field), chemiluminescence (non-thermal production of light by a chemical reaction) or triboluminescence (emission observed by applying mechanical stress to crystals or by fracture of crystals).

The optical properties of the lanthanide cations are different from those of other metal cations and molecular species because they absorb and emit light over narrow wavelength ranges. The $f-f$ transitions of the lanthanide cations are Laporte-forbidden and therefore of low intensity. However, Weissman discovered in 1942 that when organic ligands were chelated to $\mathrm{Eu}^{3+}$ and then irradiated at the $\pi \rightarrow \pi^{*}$ transition of the chelated ligands, sensitized $4 f \rightarrow 4 f$ emission from $\mathrm{Eu}^{3+}$ resulted [1]. This effect is described in detail below.

\section{Sensitization processes: "antenna effect"}

Because the molar absorption coefficients $\varepsilon$ of most of the transitions in the absorption spectra of the trivalent lanthanide ions are smaller than $10 \mathrm{~L} \mathrm{~mol}^{-1} \mathrm{~cm}^{-1}$, only a very limited amount of radiation is absorbed by direct excitation in the $4 f$ levels. Since the luminescence intensity is not only proportional to the luminescence quantum yield but also to the amount of light absorbed, weak light absorption results in weak luminescence. However, the problem of weak light absorption can be overcome by the so-called antenna effect (or sensitization) in which energy is transferred from the surroundings of the metal ion (either an inorganic matrix or an organic ligand). In addition to providing an efficient path for the excitation of lanthanide ions, this sensitization process also has the advantage that now the "Stokes' shifts" are very large, which allows an easy spectral separation of the remaining matrix luminescence from the metal ion emission.

In the earlier 1990s, Lehn [2] coined the term "antenna" to denote the absorption, energy transfer, emission sequence involving distinct absorbing (the ligand) and emitting (the lanthanide ion) components in luminescent lanthanide complexes which work as light conversion molecular devices (LMCDs). This two-step excitation process allows one to achieve a large excited-state population using light fluences $\left(\mathrm{J} / \mathrm{cm}^{2}\right)$ four to five orders of magnitude lower than those required for bare ions and allows overcoming the weak absorptivity of

\footnotetext{
a However, it should be noted that when lanthanide complexes are used, the internal efficiency is in theory not limited to $25 \%$ because the excitation energy can be transferred both from an excited singlet or triplet to the lanthanide ion, that is, the internal efficiency could theoretically be as high as $100 \%$, as it happens in phosphorescent OLEDs (see Section 1.4).
} 
lanthanide ions $[3,4]$. The scheme in Figure 1 shows, as an example for the erbium case, a simplified energy level scheme illustrating the energy transfer processes [5,6,7]. After optical excitation in the UV-vis, ligand singlet excited states $S_{1}$ can either decay to the ground state $S_{0}$ (molecular fluorescence), or to triplet states $T_{\mathrm{n}}$ through an intersystem crossing (ISC) mechanism enhanced by heavy atom effect. The triplet state $T_{1}$ can be deactivated radiatively to the ground state, $S_{0}$, by the spin-forbidden transition $T_{1} \rightarrow S_{0}$. This results in molecular phosphorescence. Alternatively, excited triplets can subsequently populate the upper levels of the lanthanide ion via resonance energy transfer (RET) [8] which can occur either via Dexter [9] or Förster [10] mechanisms, depending on total angular momentum variation $(\Delta J)$ undergone by the lanthanide ion [11]. After this indirect excitation by energy transfer, the lanthanide ion may be deactivated by non-radiative processes or it may undergo a radiative transition to a lower $4 f$ state by characteristic line-like photoluminescence (that is, following a fast excited-state ion relaxation, the radiative decay ${ }^{(2 \mathrm{~S}+1)} \Gamma_{\mathrm{J}} \rightarrow{ }^{(2 \mathrm{~S}+1)} \Gamma_{\mathrm{J}}$ yields the emission from the $\left.\operatorname{Ln}^{3+}\right)[12]$.

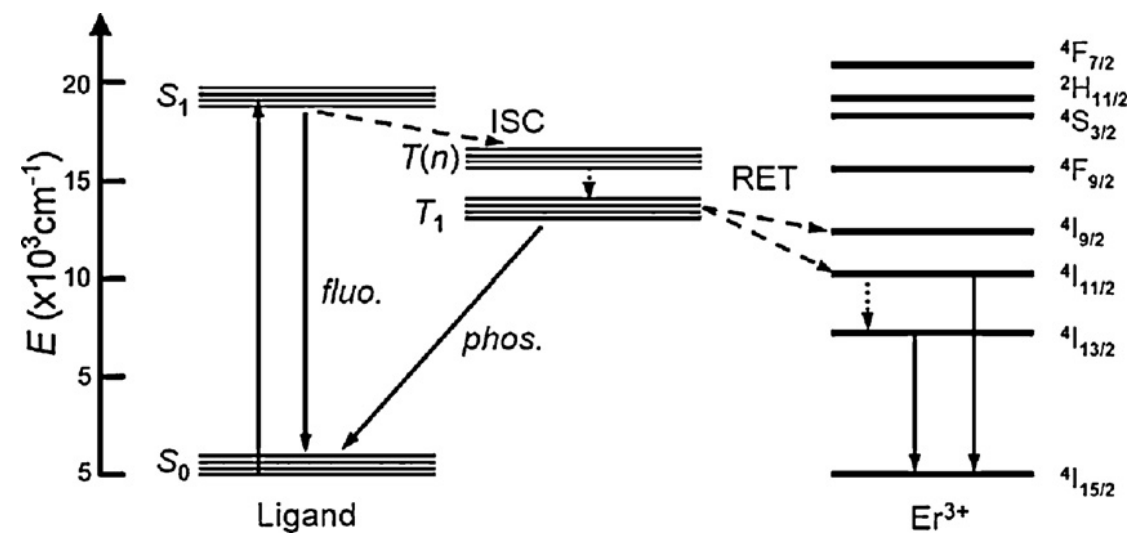

Figure 1. Jablonski diagram illustrating the two-step excitation process for erbium by means of antenna effect. Dashed arrows represent non-radiative relaxation mechanisms.

An analogous mechanism is shown in Figure 2 for $\mathrm{Yb}^{3+}$ :

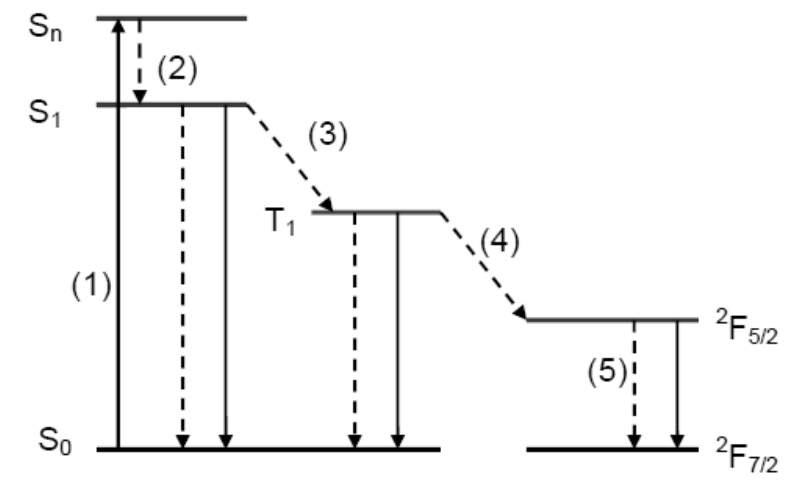

Figure 2. Jablonski diagram showing the mechanism for ligand sensitized $\mathrm{Yb}^{3+}$ luminescence. Solid lines indicate radiative transitions. Dashed lines represent non-radiative transitions. (1) Upon radiation with UV-vis light, the organic ligands of the ytterbium(III) complex are excited from the singlet ground state to a singlet excited state $\left(S_{0} \rightarrow S_{\mathrm{n}}\right)$. The molecule undergoes fast internal conversion to lower vibrational levels of the $S_{1}$ state, for instance through interaction with solvent molecules. (2) The $S_{\mathrm{n}}$ state decays into the lowest energy singlet excited state $\left(S_{\mathrm{n}} \rightarrow S_{1}\right)$ on the ligand. (3) The singlet state intersystem crosses into the ligand-based triplet state $\left(S_{1} \rightarrow T_{1}\right)$. (4) Energy transfer to the lanthanide then occurs. (5) The energy on the lanthanide can then undergo radiative decay resulting in lanthanide-centered luminescence [13]. 
Although Kleinerman proposed a mechanism of direct transfer of energy from the excited singlet state $S_{1}$ to the energy levels of the lanthanide ion, this mechanism is now considered not to be of great importance [14]. Indeed this process is often not efficient due to the short lifetime of the singlet excited state.

In a simplified model, the overall (or absolute) luminescence quantum yield $\Phi_{\text {tot }}$ of sensitized luminescence from a lanthanide complex can be written:

$$
\Phi_{\text {tot }}=\eta_{I S C} \cdot \eta_{E T} \cdot \Phi_{L n}=\eta_{\text {sens }} \cdot \Phi_{L n}=\eta_{\text {sens }} \cdot \tau_{\text {obs }} / \tau_{\text {rad }}
$$

where $\Phi_{\mathrm{Ln}}$ is the intrinsic quantum yield for direct excitation of the lanthanide ion, $\eta_{\mathrm{ISC}}$ represents the efficacy of the intersystem crossing process and $\eta_{\mathrm{ET}}$ is the effectiveness of the $3 \pi \pi^{*}-$ Ln (ligand-to-metal) transfer. These latter terms can be combined in the sensitization efficiency parameter $\eta_{\text {sens. }}$.

Ligand-to-metal sensitization quantum yield, $\eta_{\text {sens, }}$ is a key parameter for the design of luminescent lanthanide complexes since it critically influences the overall emission quantum yield. Several relaxation steps are involved in the sensitization of the lanthanide ion, including the mixing between ligand singlet and triplet states (intersystem crossing, ISC), and resonance energy transfer (RET) from the triplet state to the ion. These processes compete with other external relaxation mechanisms such as triplet oxygen quenching or internal intraligand charge-transfer which may lead to low overall quantum yields. Therefore, for obtaining high lanthanide luminescence efficiencies upon excitation of the antenna, it is then necessary to design and select suitable ligands ensuring high sensitization efficiencies.

Enhancement of spin-orbit coupling due to the heavy-atom effect associated with the lanthanide ions is expected to result in acceleration of the ISC process and to efficient $\eta_{\text {ISC. }}$ Ligand-to-metal energy transfer $\left(\eta_{\mathrm{ET}}\right)$ depends on the distance as well as on the energy overlap between the sensitizer and the lanthanide ion. For efficient lanthanide ion sensitization, the triplet states of the ligand must be closely matched to, or slightly above, the metal ion's emitting resonance levels, but not so close that thermal back energy transfer competes effectively with $\mathrm{Ln}^{3+}$ emission [15]. Typically, an energy gap of $1500 \mathrm{~cm}^{-1}$ is recommended to prevent this detrimental phenomenon. Other references, such as [16], state that triplet excited state should be at least $1850 \mathrm{~cm}^{-1}$ higher than the lowest emitting levels of the $\mathrm{Ln}^{3+}$ ion: when the energy gap is higher, the energy transferred from the triplet flows through non-radiative excited states of the metal until it reaches the emissive levels and the metal centered emission occurs; on the contrary, a lower energy gap strongly limits the emission quantum yield because of thermal deactivation due to aforementioned back energy transfer and $\mathrm{O}_{2}$-quenching towards the chromophore triplet level. When the energy transfer is not very efficient, it is possible to observe some remaining ligand emission in combination with the lanthanidecentered emission.

$\Phi_{\mathrm{Ln}}$ is severely limited by several competitive non-radiative decay processes undergone by the emitting lanthanide ion and depends on the ratio between the rate constants of radiative $\left(\kappa_{\mathrm{rad}}=1 / \tau_{\mathrm{rad}}\right)$ and excited state deactivation ( $\left.\kappa_{\mathrm{obs}}=1 / \tau_{\mathrm{obs}}\right)$ processes. $\tau_{\mathrm{obs}}$ is the observed NIR decay time of a lanthanide compound (which is influenced by non-radiative processes) and $\tau_{\text {rad }}$ is the "natural" radiative lifetime of a given lanthanide ion in the absence of radiation-less deactivation $[17,18]$.

Only the overall quantum yields $\Phi_{\text {tot }}$ and luminescence lifetimes $\tau_{\text {obs }}$ can be directly measured and reliable values are found in the literature, whereas the other terms of the equation above are often difficult to be determined and only rough estimations are reported in most cases. Regarding the radiative lifetime, $\tau_{\text {rad, }}$ many authors consider this quantity to be a constant value for a given lanthanide ion. However, this is an incorrect assumption. Equally wrong is the assumption that $\tau_{\text {rad }}$ can be obtained by measurement of $\tau_{\text {obs }}$ after cooling the sample to a 
sufficiently low temperature ( $77 \mathrm{~K}$ or lower). The best approach to obtain $\tau_{\text {rad }}$ is by calculation of this value with the aid of the experimentally derived Judd-Ofelt intensity parameters, $\Omega_{\lambda}(\lambda=2,4,6)$. These parameters can be derived from optical absorption spectra of the lanthanide complex [19]. The Judd-Ofelt parametrization scheme works remarkably well for $\mathrm{Er}^{3+}$ ions. The two papers of Judd and Ofelt on the theory of intensities of lanthanide spectra are citation classics (Judd-Ofelt theory) [20,21], but the well-known $\Omega_{\lambda}$ intensity parameters were introduced by Axe [22]. The reader who is interested in more theoretical aspects of lanthanide spectroscopy is referred to the specialized literature. The classic works in this field are the books by Dieke [23], Wybourne [24], Judd [25], and Hüfner [26], although it must be admitted that they are not easy to understand for readers without a strong background in mathematics. Several other books are also available $[27,28]$.

From a Chemistry point of view, so as to design highly efficient molecular luminescent edifices, the choice of the antenna ligand involves considering, apart from aforementioned requirements regarding its triplet state, other two important aspects [29]: its excitation wavelength should be above ca. $350 \mathrm{~nm}$ to facilitate the use of inexpensive excitation sources and to avoid the use of expensive quartz optics, and a short distance between the sensitizer and the $\mathrm{Ln}^{3+}$ cation is also required in order to ensure fast energy transfer (therefore, the best results can be obtained when the antenna directly coordinates the metal center). This latter situation has been mainly exploited with chromophores containing binding sites for the $\mathrm{Ln}^{3+}$ cation such as: aza-aromatic compounds (bipyridine, phenanthroline, azatriphenylene, terpyridine) or phenolate aromatics [2-hydroxyisophthalamide (IAM), 1hydroxypyridin-2-one (1,2-HOPO)].

\section{Luminescence of lanthanide complexes embedded in a matrix}

Back in 1997, Li and co-workers [30] studied interactions between poly(N-vinylcarbazole) (PVK) and various $\mathrm{Ln}^{3+}$ complexes and found that efficient energy transfer processes occur between PVK and lanthanide complexes.

They proved that energy transfer processes from a matrix to a compound should be taken into account when rare-earth compounds are used as dopants. It is generally agreed [31] that the energy, which is generated in OLED devices (covered in Section 1.4) containing lanthanide complexes as dopants upon charge recombination, is transferred from the matrix to the singlet excited state of the ligand, from which it is transferred to the triplet level of the ligand as a result of intersystem crossing, and is finally transferred to the metal ion. In this connection, two main problems should be taken into account in the design of devices: (1) the efficiency of energy transfer from the matrix to lanthanide complexes; (2) the efficiency of energy transfer from the triplet level of the ligand to the metal ion (already discussed in previous section).

According to Heil et al. [32], there are three main mechanisms of excitation of lanthanide complexes in a matrix:

1. A complex is excited as a result of energy transfer from the matrix to the complex. In this case, the Frenkel exciton should be formed within the emitter layer, in which the resonance transfer of the electronic excitation energy to the complex molecule occurs. If the energy transfer occurs by the Förster mechanism (a dipole mechanism), spectral overlap between the emission bands of the matrix and the absorption bands of the complex should take place. If the energy transfer is based on exchange interactions according to the Dexter mechanism (an exchange mechanism), the correspondence between the energy levels and interactions of the involved orbitals are of importance.

2. A complex becomes excited as a result of charge transfer from the matrix, i.e. electrons and holes, which are separately transported to the complex molecule, recombine with the result that the energy is released, and the latter is transferred to the metal ion serving as an 
emission center. This requires particular properties of the complex providing simultaneous electron and hole capture.

3. If the emission layer consists only of complex molecules, the latter, as in the previous case, are excited due to recombination of the opposite charges on the complex molecules. Excitation of the complex is most efficient if the charge injection (electron and hole injection) in a layer is balanced and the charges are present at a high concentration and are highly labile. The charge balance can be controlled by introducing an additional charge-blocking layer.

\section{Non-radiative losses}

The near-infrared photoluminescence of trivalent erbium ions is due to the ${ }^{4} \mathrm{I}_{13 / 2} \rightarrow{ }^{4} \mathrm{I}_{15 / 2}$ transition, with the emission maximum located around $1550 \mathrm{~nm}$ (i.e. $6450 \mathrm{~cm}^{-1}$ ). For ytterbium ions, the emission maximum appears at $977 \mathrm{~nm}$ and is due to the ${ }^{2} \mathrm{~F}_{5 / 2} \rightarrow{ }^{2} \mathrm{~F}_{7 / 2}$ transition.

Because of the relatively small energy gap between the excited state ${ }^{4} I_{13 / 2}$ and the ground state, the excited state is efficiently quenched by the vibronic coupling with high energy $\mathrm{O}-\mathrm{H}$, $\mathrm{N}-\mathrm{H}$ or $\mathrm{C}-\mathrm{H}$ stretching vibrations in the neighbourhood of the $\mathrm{Er}^{3+}$ ion (originated from the ligands, polymer matrix or solvents) ${ }^{\mathrm{b}}$. This shows up in the reduction of the emission decay time from the value $\tau \approx 8 \mathrm{~ms}$ in the isolated ion to values in the range of $1-2 \mu$ s in typical lanthanide complexes. Consequently, the overall radiative efficiency is generally as low as $\varphi \approx 10^{-4}$.

Coordination of $\mathrm{O}-\mathrm{H}$ and $\mathrm{C}-\mathrm{H}$ oscillators to the lanthanide ion will increase the non-radiative rates of the lanthanide excited states particularly as the energy of the near-infrared transitions decrease. This is because the non-radiative rate increases exponentially as the energy gap decreases. From Figure 3, it is clear that solvents containing $\mathrm{O}-\mathrm{H}$ bonds will rapidly deactivate the excited states of the lanthanides.

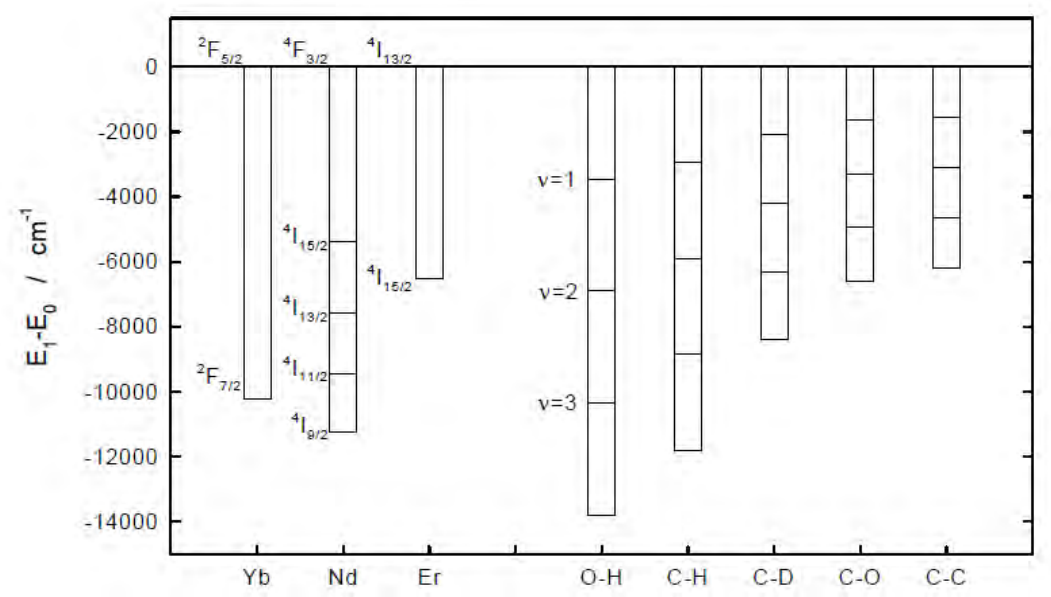

Figure 3. Representation of the radiative transition energies of $\mathrm{Yb}^{3+}, \mathrm{Nd}^{3+}$, and $\mathrm{Er}^{3+}$ and the vibrational energies of common bonds found in organic systems [13].

Consequently, the first objective of our work has been to achieve a reduction of non-radiative losses by designing ligands that minimize quenching by $\mathrm{O}-\mathrm{H}$ and $\mathrm{N}-\mathrm{H}$ oscillators. Deuteration

\footnotetext{
${ }^{b}$ On the other hand, the intramolecular ligand-to-erbium transfer processes seem to be very efficient and fast (some studies [Bertolo, L; et al. Eur. J. Inorg. Chem., 2006, 2370] suggest that the efficiency of these processes is always close to $100 \%$ ) and are not affected by these effects.
} 
and halogenation (i.e., the substitution of $\mathrm{H}$ atoms in the aromatic rings with halogens atoms such as fluorine [33,34,35], chlorine [36] and bromine [37]) both decrease these losses [38]. Absorptions are diminished (C-D) or absent (C-F), because the third overtone of C-D is located at $1.5 \mu \mathrm{m}$ and the third overtone of $\mathrm{C}-\mathrm{F}$ at $2.6 \mu \mathrm{m}$. Halogenation is preferred for the $1.55 \mu \mathrm{m}$ window, because C-D vibrations absorb here (second overtone of the C-D vibration).

It has been well reported that fluorination of hydrogen containing ligands, together with exclusion of coordinated water, can extend the lifetime of infrared luminescence from $\mathrm{Ln}^{3+}$ complexes. In the late 1990s, Yanagida and Hasegawa et al. described in several papers the improved NIR photoluminescence of several deuterated and fluorinated $\mathrm{Nd}^{3+} \beta$-diketonate complexes in solution [39]. With regard to $\mathrm{Er}^{3+}$ complexes, a moderate increase of about $30 \%$ in the emission efficiency has been found in erbium quinolinates with a measured lifetime of $\tau=1.9 \mu$ s [37]. Quite differently, a much more noticeable growth to $\tau=741 \mu$ s has been achieved in an imidodiphosphinate complex [40].

These findings, coincident with previous results obtained by us in the course of two final degree projects defended in 2008 and 2010, have encouraged the continuation of the investigations that are now the subject of this report and which have resulted in the manufacturing of Engineering devices.

The introduction of fluorine atoms into the ligands can greatly improve the quantum yield of the ${ }^{4} \mathrm{I}_{13 / 2}$ to ${ }^{4} \mathrm{I}_{15 / 2}$ transition in erbium-organic framework materials, as it is illustrated in Figure 4, in which the energy level diagrams of the $\mathrm{Er}^{3+}$ ion, $\mathrm{C}-\mathrm{H}\left(2950 \mathrm{~cm}^{-1}\right)$ and C-F $(1220$ $\mathrm{cm}^{-1}$ ) vibrations, are presented. It can be seen that the second-order $\mathrm{C}-\mathrm{H}$ bond vibrational frequencies $\left(5900 \mathrm{~cm}^{-1}\right)$ are almost in resonance with the emission bands of the $\mathrm{Er}^{3+}$ ion at $1.5 \mu \mathrm{m}\left({ }^{4} \mathrm{I}_{13 / 2}\right.$ to $\left.{ }^{4} \mathrm{I}_{15 / 2}\right)$, while only the fifth-order C-F bond vibrational frequencies $\left(6100 \mathrm{~cm}^{-1}\right)$ can approach in resonance with such an emission transition; thus, the effect of the C-F vibration on the fluorescence quenching of the $\mathrm{Er}^{3+}$ ion emission is much lower than that of the $\mathrm{C}-\mathrm{H}$ vibration [41].

It should be noted that fluorination does not significantly modify the energy of the triplet levels, so the resonant transfer to the lanthanide ion is not affected [34].

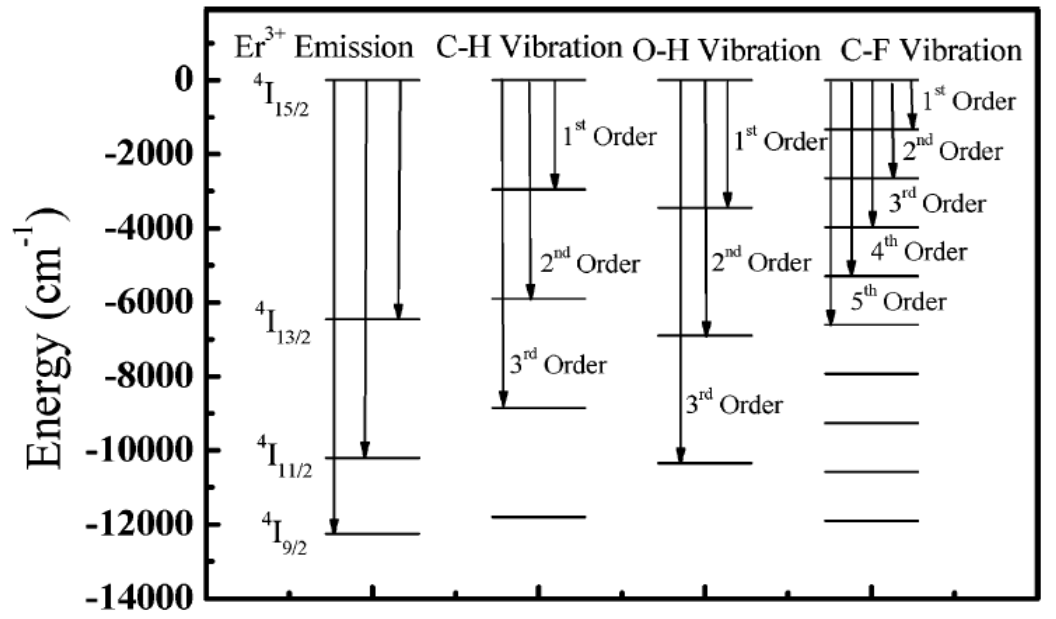

Figure 4. Schematic representation of the vibrational quenching of the ${ }^{4} \mathrm{I}_{13 / 2}$ emission of $\mathrm{Er}^{3+}$ by the vibrational modes of $\mathrm{CH}, \mathrm{OH}$, and $\mathrm{CF}$ functional groups.

In the case of 'host-guest' systems, further minimization of aforementioned loss processes is usually pursued by replacing the hydrogen atoms with heavier atoms via deuteration or fluorination of the polymer host material too, in order to shift the frequencies of the relevant vibrational overtones to less detrimental ranges [42]. 


\subsection{Design of luminescent lanthanide complexes}

The electronic, magnetic and photophysical properties of $\mathrm{Ln}^{3+}$ complexes strongly depend on the control of the coordination sphere of the metal. Accordingly, particular care should be paid to the design of ligands to optimize the property of interest. For instance, a review that describes the basic principles for the design of organic ligands for alkali and alkali-earth cations has been reported by Lehn in 1973 [43]. The parameters to be considered in order to attain control over chemical, structural and thermodynamical properties of the complex, which in the end govern its functionality, are clearly described. Among these parameters the ligand topology (dimensionality, connectivity, shape, size, and chirality), the binding sites (nature, electronic properties, number, shape, arrangement), the layer properties (rigidity/flexibility and the lipophilicity/hydrophilicity ratios, thickness), the environment properties and counter-ions effect are particularly important. These general rules apply to any kind of ligand, independently of the metal cation, and are particularly important in the case of $\mathrm{Ln}^{3+}$, whose coordination is substantially based on Van der Waals electrostatic interactions and is similar to that of alkali-earth cations, in particular $\mathrm{Ca}^{2+}$.

In designing a convenient chemical environment for a luminescent lanthanide ion, chemists and engineers have to take into account four main requirements, two of which have been set out above, in Section 1.1. In brief, the first one is a chemical constraint in that the coordination cavity should provide enough donor atoms to saturate the coordination number requirement of the $\mathrm{Ln}^{3+}$ ion as well as a large bonding strength ensuring a good thermodynamic stability and possibly also kinetic inertness. The second requirement pertains to the efficiency of the sensitization process, i.e. the energy level scheme of the ligands should be such that it maximizes the energy transfer path $\left(\eta_{\text {sens }}\right)$. The third requirement deals with preventing non-radiative de-activation processes. Finally, the fourth requirement would be that the ligands should have the electron- and hole-transport properties to promote charge injection and exciton generation in the complex.

To meet these requirements, several synthetic strategies have been developed during the last decades, which include (1) linear polydentate and multifunctional ligands such as polyaminocarboxylates, $\beta$-diketonates and acyclic Schiff base derivatives, (2) macrocyclic receptors, among which porphyrins, derivatized alixarenes and pendant-arm fitted cyclens are the most investigated, and (3) podates and self-assembled structures.

Most research in luminescent organolanthanide complexes has focused on visible emitters such as $\mathrm{Eu}^{3+}$ (red light), $\mathrm{Tb}^{3+}$ (green light) and $\mathrm{Tm}^{3+}$ (blue light) for their use as inorganic phosphors in display technologies. Another is the case of the complexes of $\mathrm{Er}^{3+}$ and $\mathrm{Yb}^{3+}$ which, due to synthesis problems, lack of proper instrumentation for characterization and low confidence in their applications, have not received adequate attention. However, overcoming these difficulties through the development of a significant number of new $\mathrm{Er}^{3+}$ and $\mathrm{Yb}^{3+}$ complexes, the demonstration of their versatility as emitters and for the fabrication of new devices in Electronics and Telecommunications, should allow us to validate their application in Engineering, as claimed by the present work.

A review that describes a large number of ligands and the emission properties of their $\mathrm{Ln}^{3+}$ (mostly $\mathrm{Eu}^{3+}$ and $\mathrm{Tb}^{3+}$ ) complexes has been recently reported [44]. An extensive review on lanthanide $\beta$-diketonate complexes has been written by Binnemans [13]. For a comprehensive review on all sorts of $\mathrm{Ln}^{3+}$-based NIR-emitting molecular edifices (including macrocyclic ligands, such as porphyrinates, derivatized coronands and cryptands derivatized cyclens, derivatized calixarenes and resorcinarenes; acyclic ligands, including quinolinates, terphenyl-based ligands, polyaminocarboxylates, dyes, podants and dendrimers; heterometallic functional assemblies; inorganic clusters; zeolites and composite mesoporous materials; nanoparticles, etc.), the reader is referred to the review by Comby and Bünzli [45]. 


\section{$\beta$-diketonates and additional ligands}

Amongst the synthetic strategies discussed above aimed at improving lanthanide luminescent quantum efficiencies, in this Thesis $\beta$-diketonates have been chosen as the preferred sensitizing ligands.

It must be clarified that all throughout this document, $\beta$-diketonates are denoted by their abbreviation, and a difference is made between the $\beta$-diketone and the corresponding $\beta$ diketonate ligand that is obtained by deprotonation of the $\beta$-diketone (i.e. the conjugate base of the $\beta$-diketone). For instance, Hacac stands for acetylacetone, and acac is the acetylacetonate ligand.

The simplest $\beta$-diketone is acetylacetone (Hacac), were the substituents on both carbonyl groups are methyl groups. All other $\beta$-diketones can be considered as derived from acetylacetone by substitution of the $\mathrm{CH}_{3}$ groups by other groups. The choice of the substituents influences the properties of the corresponding rare-earth complexes. For instance, branched alkyl chains such as the tert-butyl group increase the solubility in organic solvents and the volatility. Perfluorinated alkyl groups increase the Lewis acidity. The $\beta$ diketones with aromatic substituents have a stronger light absorption than the $\beta$-diketones with only aliphatic substituents. The substituents have also an influence on the position of the energy levels of the ligand (singlet and triplet states), and, as it has already been indicated, the position of the triplet is important, because this is one of the determining factors for the luminescence outputc.

Many $\beta$-diketones are commercially available, so that the synthesis of the rare-earth $\beta$ diketonates can often be restricted to the synthesis of the complexes, without the need to bother about the ligand synthesis. Only in the case that exotic $\beta$-diketones are needed or when new $\beta$-diketones are designed, the worker in the field of rare-earth $\beta$-diketonates has to synthesize the $\beta$-diketones himself/herself. The classic method for the synthesis of $\beta$ diketones is the Claisen condensation between a deprotonated methylketone and an ethyl or a methyl ester.

Ternary rare-earth $\beta$-diketonates contain one or two additional ligands besides the $\beta$-diketonate ligands. These ligands act as Lewis bases, and form adducts with tris $\beta$-diketonate complexes because of the tendency of the rare-earth ion to expand its coordination sphere and to achieve a coordination number higher than six (typically eight or nine). Because the trivalent rare-earth ions are hard Lewis acids, the tris $\beta$-diketonate complexes form preferentially complexes with oxygen-donor or nitrogen-donor Lewis bases. Two very popular Lewis bases are the $\mathrm{N}$-donor ligands 1,10-phenanthroline (phen) and 2,2'-bipyridine (bipy), because the resulting lanthanide complexes show often an intense luminescence. These $\mathrm{N}$-donor ligands can be modified by substituents on the heterocycle ring. According to Yang et al. [46], the presence of a rigid planar structure in the complex causes a higher intensity of the sensitized luminescence, because such structure allows a better energy transfer.

The fact that the introduction of $\mathrm{N}, \mathrm{N}$-donor ligands increases luminescence is attributed to the intramolecular energy transfer from the $\beta$-diketonate ligand to $\mathrm{N}, \mathrm{N}$-donor (the triplet level of the $\beta$-diketonate ligand is much higher than that of $\mathrm{N}, \mathrm{N}$-donor ligand), with the result that $\Delta E$ approaches the optimal value [47].

Further information on synthetic strategies, structural properties and rare-earth $\beta$ diketonate complexes for which crystal structures have been determined by single crystal Xray diffraction, physical and chemical properties, luminescence, etc. can be found in [13].

\footnotetext{
c Nonetheless, it should be noted that McGehee et al. [Adv. Mater., 1999, 11, 1349] found that, in spite of the different nature of the substituents, the triplet levels of the tta, dbm, flta, flpa and flha ligands present in several $\mathrm{Ln}^{3+}$-complexes are located in close proximity. Hence, the corresponding $\beta$-diketonates should have similar efficiency of energy transfer from these ligands to the $\mathrm{Ln}^{3+}$ ion.
} 


\subsection{Lanthanide complexes-based luminescent hybrid materials}

For about one decade, there has been a strong interest in lanthanide-based organic-inorganic hybrid materials. In these materials, a molecular lanthanide complex is embedded in an inorganic host matrix (e.g. sol-gel-derived materials), or alternatively, an inorganic lanthanide compound (such as a lanthanide-doped nanoparticle) is embedded in an organic polymer matrix. Of course, the distinction between these classes is not well-defined, as illustrated by the organically modified xerogels. The study of luminescent lanthanide compounds in hybrid materials is not only of fundamental interest, because these materials have also a high potential for different applications as photonic materials (optical amplifiers, optical waveguides, OLEDs, etc.). In general, these hybrid materials have superior mechanical properties, better processability, thermal stability and luminescence output than the pure molecular lanthanide complexes.

The main chemical routes to designing hybrid materials suitable for optoelectronic applications are schematized in Figure 5. Path A corresponds to conventional sol-gel chemistry, where hybrid networks are obtained through the hydrolysis of metallic alkoxides condensed with or without organically modified metal alkoxides and incorporating lanthanide ions. This strategy is simple and low-cost, and it yields amorphous hybrid materials where lanthanide ions are distributed within the host material according to the chemical nature affinity. These materials can be transparent and easily shaped as films or monoliths, which is important for some advanced technologies.

A better control of the local structures of these hybrid materials and their degrees of organization can be achieved by means of routes $\mathrm{B}$ and $\mathrm{C}$, which respectively provide organofunctional Ln-doped nanoparticles and Ln-doped nanostructures with periodic porosity. The synthesis of discrete luminescent nanoparticles (path B) has especially been explored in the past years because of their interesting properties resulting under quantum confinement effects. The organization and texturing of hybrid networks templated by organic structuredirecting agents through self-assembly approaches (route $\mathrm{C}$ ) has also provided new materials with potential uses in Optics.

Due to the number of scientific publications in this field, this section is far from providing an exhaustive review on the previously performed research activities, only aiming at presenting a broad overview of the different technological alternatives currently available. For a more detailed discussion on these methodologies, the reader can refer to specific pertinent literature. In this framework, exhaustive reviews on different strategies towards lanthanidedoped hybrid materials have been reported $[48,49]$. Particularly interesting is the review by Binnemans [50], which covers the literature until March 2009. Further information can also be found in Section 7 of [13] (specific to lanthanide $\beta$-diketonates) or in Section 3.4 of [45].

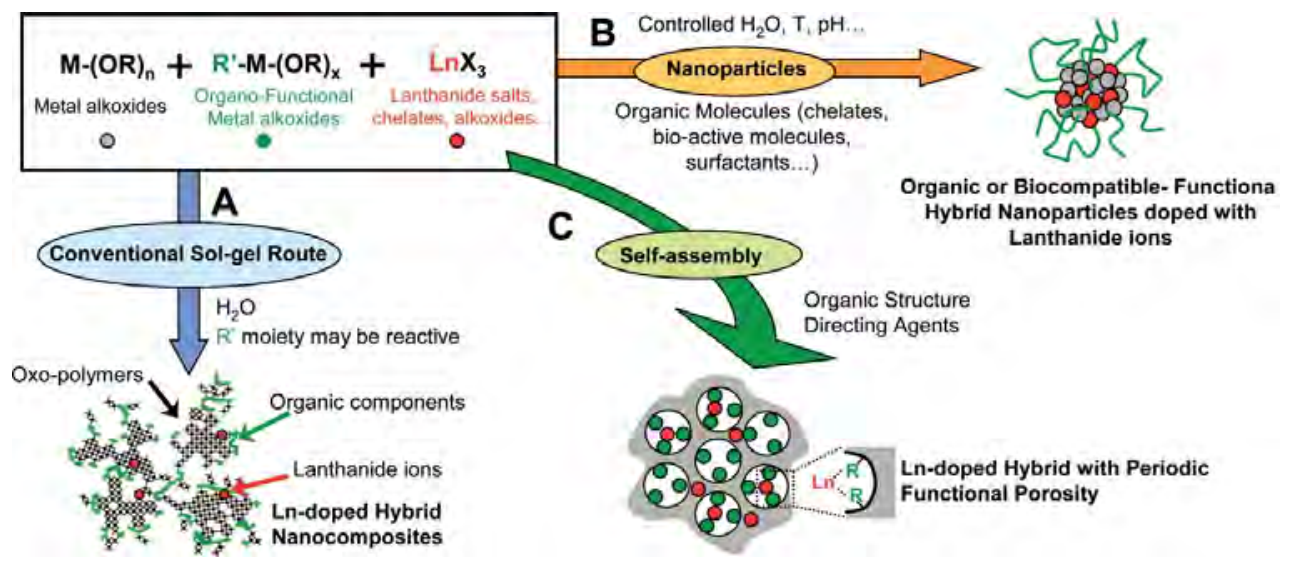

Figure 5. Different pathways to prepare hybrid materials suitable for optical applications [48] 


\section{Sol-gel hybrid materials}

\section{Inorganic sol-gel systems}

Inorganic glasses are excellent transparent host matrices for trivalent lanthanide ions, and lanthanide-doped glasses have been intensively studied in the past. However, it is not easy to sensitize lanthanide luminescence in inorganic glasses, especially not when no co-doping with transition metal ions is made. Because inorganic glasses (for instance silicate, phosphate, fluoride or fluorophosphate glasses) are typically processed at very high temperatures, up to temperatures above $1000^{\circ} \mathrm{C}$, these host matrices are not the most suitable option to trap highly luminescent lanthanide complexes such as lanthanide $\beta$ diketonates. Molecular lanthanide complexes are not thermally stable enough to withstand the high processing temperatures and the organic ligands would be decomposed. In addition, the preparation of silicate glasses with high lanthanide concentrations is problematic due to the low solubility of lanthanide oxides in these glass matrices and to the resulting phase separation.

It is possible to prepare silicate and other glasses by a low-temperature route, namely by the sol-gel route, a chemical synthesis technique that is used for the preparation of gels, glasses, and ceramic powders. Not only does this allow avoidance of the problems of phase separation and crystallization that are often observed for high-temperature melt processes, but it is also possible to encapsulate organic compounds or metal complexes in the sol-gel glass. It is often observed that encapsulation in sol-gel glasses increases the photostability of organic luminophores. The sol-gel process can be used to prepare bulk samples (monoliths) as well as thin films and fibers. The gels can easily be formed in different shapes.

Sol-gel glasses are obtained by controlled hydrolysis of metal alkoxides. For instance, silicon alkoxides react with water and undergo hydrolysis and polycondensation, which leads to the formation of a silicate network. The starting products for the preparation of silicate sol-gel glasses are tetraorthosilicates or tetraalkoxysilanes, $\mathrm{Si}(\mathrm{OR})_{4}$, like tetraethylorthosilicate (TEOS, $\left.\mathrm{Si}\left(\mathrm{OC}_{2} \mathrm{H}_{5}\right)_{4}\right)$. Another often used alkoxide precursor is tetramethylorthosilicate $\left(\mathrm{TMOS}, \mathrm{Si}\left(\mathrm{OCH}_{3}\right)_{4}\right)$.

To get thin films, the silica gel is spin-coated or dip-coated prior to gelation on a suitable substrate. In the spin coating process, an excess amount of a sol solution is placed on the substrate, which is then rotated at high speed to spread the fluid by centrifugal force. The thickness of the film depends on the spinning rate, the concentration and viscosity of the solution, and the solvent. In the dip coating process, a substrate is immersed in the sol and then slowly withdrawn at a constant rate.

Three general methods can be used to incorporate or immobilize luminescent complexes into sol-gel glasses: (1) impregnation; (2) doping; (3) chemical immobilization. To impregnate a complex into the sol-gel glass, the silica matrix is immersed for some time in a solution that contains a fairly high concentration of the luminescent complex. During the period of immersion, the complex will diffuse into the channels or pores of the silica glass. To dope the complex in the sol-gel glass, the complex is added to the silica sol prior to gelation. During gelation, the complex is trapped in the pores and channels of the silica host. It is also possible to dope the complex in the silica matrix by adding a metal salt and ligand to the silica gel, whereby the complex itself is formed in situ in the gel or in the xerogel (often during a heat treatment) ${ }^{\mathrm{d}}$. Chemical immobilization of the complex is achieved by addition of organosilicon compounds with coordinating groups to the sol-gel precursor solution, so that organically

\footnotetext{
d Lanthanide complexes can be directly dissolved in the sol-gel precursor solution. However, the lanthanide complexes may not be stable in the acidic precursor solution. In that case, the ligands and a lanthanide salt can be added to the precursor solution instead of the complex. The complex would be formed in situ during the transformation of the gel into a xerogel.
} 
modified silicates (ormosils, discussed later) are obtained. The most often used method for incorporation of the luminescent complexes into the silica matrix is the doping method.

As it happens in inorganic glasses, the good luminescence performance of lanthanide-doped xerogels and derived glasses is hampered by hydroxyl quenching and clustering of the lanthanide ions, problems which can be solved by replacing the lanthanide salt precursors by molecular lanthanide complexes.

It should be noted that a xerogel doped with molecular lanthanide complexes cannot be transformed into a compact silica glass, because the limited stability of the molecular complexes prevents heating the samples to $800^{\circ} \mathrm{C}$ or higher. However, a heat treatment at $250^{\circ} \mathrm{C}$ (or lower if the complexes have a low thermal stability) is beneficial for the luminescence intensities because at these temperatures water molecules and volatile organic components can be removed by evaporation.

In other cases, lanthanide complexes with organic ligands may only be used to obtain a homogeneous dispersion of the lanthanide ions in the glass matrix rather than to take advantage of the antenna effect. These xerogels would then be heated to high temperatures in order to destroy all organic material and to remove water molecules as efficiently as possible.

\section{Confinement of liquids in silica matrices}

The silica gel formed by the sol-gel process is a two-component system consisting of an interconnected pore network and a liquid phase. The liquid phase is unwanted and is removed during the drying step. The resulting xerogel contains only a small portion of remaining liquid, and this can be further removed by heat treatment, as described in previous section. However, the silica network can also be used to confine liquids within its porous network. Generally, these are nonvolatile liquids in which luminescent lanthanide ions or lanthanide complexes have been dissolved.

Poly(ethylene glycol) (PEG), for example, has been shown to assist and stabilize in situ formation of lanthanide complexes, and transparent monolithic gels can be obtained by mixing the PEG solutions with prehydrolyzed TMOS. The gel formation has no effect on the photophysical properties of the original liquid mixture, which indicates that the silica network just acts to immobilize the PEG solution and does not interact with the lanthanide ions.

Lanthanide-doped ionogels have also been introduced as a new type of luminescent materials. Ionogels are hybrid materials consisting of an ionic liquid (i.e., low-melting organic salts with interesting properties like a negligible vapor pressure, a wide electrochemical window and tunable physicochemical properties) confined inside the nanosized pores of a silica matrix. The ionogels can be obtained as perfect monoliths featuring both the transparency of silica and the outstanding ionic conductivity performances of the ionic liquid, despite the nanometer scale of confinement. The mechanical properties of ionogels are also very similar to those of regular sol-gel hybrid materials.

\section{Organically modified xerogels}

The purely inorganic glasses that are prepared by the controlled hydrolysis of metal alkoxides have some disadvantages if one wants to entrap molecular lanthanide complexes in the glass matrix. First of all, the solubility of the lanthanide complexes in this host is quite low (a few weight percent at maximum) and crystallization of the complex in the glass matrix is often observed. This leads to loss of transparency of the silica gels. Second, these glasses easily crack due to the surface tension of the liquid in the pores. Long drying methods are necessary to reduce this cracking. Third, the mechanical properties of the glasses that have not been heat treated are poor. The resulting glasses are very brittle. It should be remembered that such glasses cannot be heat treated to a high temperature because of the 
limited thermal stability of the lanthanide complexes. These disadvantages can be overcome by incorporation of organic components in the backbone of the xerogel network. Siliconcontaining hybrid materials are known under the specific name organically modified silicates (ormosils). Most of the ormosils are made by co-condensation of TEOS or TMOS with an organosilicon compound that contains two or three alkoxide groups (such as methyltrimethoxysilane (MTMS), triethoxyphenylsilane (TEPS), 3-methacryloxypropyltrimethoxysilane (MEMO) or 3-glycidoxypropyltrimethoxy- silane (GPTMS or GLYMO) ${ }^{\mathrm{e}}$ ).

Again, although in many cases the lanthanide complexes are dissolved in the sol, it is also possible to synthesize the complex in situ in the sol or in the gel.

\section{Silica/polymer nanocomposites}

Organic-inorganic polymer associations can be obtained by polymerization of a monomer like methyl methacrylate in the sol. An organosilicon compound containing a vinyl group like vinyltrimethoxysilane or 3-(trimethoxysilyl)propyl methacrylate (MPTMA) can be present in the precursor solution besides the tetraalkoxysilane. Polymerization can be done by photochemical or by radicalar initiation, obtaining a composite silicate-PMMA matrix, which can be doped with the $\mathrm{Ln}^{3+}$ complexes.

\section{Covalently bonded complexes}

Another approach to avoid clustering of lanthanide ions in a hybrid matrix is grafting the lanthanide complex via a covalent bond to the backbone of the matrix. This type of hybrid material can be considered a Class II hybridf. The covalent bonding of lanthanide complexes to the hybrid matrix also reduces the risk of leaching the complex out from the matrix. Additional advantages of covalent attachment of lanthanide complexes to the matrix are that in general higher doping concentrations can be reached and that the luminescent hybrid materials have a better homogeneity.

Nonetheless, it should be stressed that, according to Armelao et al. [29], antenna complexes simply dispersed via sol-gel and not covalently linked to the $\mathrm{SiO}_{2}$ matrix, can show a uniform distribution inside the glassy layers, similar to that found for the covalently linked analogues. Irrespective of the structural difference of the employed chromophores and their concentration in the sol-gel silica matrices, similar in-depth distribution features were evidenced from secondary ion mass spectrometry (SIMS) profiles. X-ray Photoelectron Spectroscopy (XPS) pointed out, together with SIMS results, the integrity of the complexes and the efficacy of this synthesis strategy for the realization of highly dispersed host-guest systems. Overall luminescence quantum yields of the films were high and only slightly affected by the nature of the host-guest chemical interactions between the matrix and the chromophores.

Consequently, whenever the synthesis of antenna complexes suitably functionalized for the covalent linking to the host medium is very demanding or almost impossible to be achieved, comparable luminescent efficiencies can be obtained by embedding the proper luminescent molecular components into transparent host matrices. Hence, for solid-state lighting purposes, the choice between covalent grafting vs. embedding of the complexes will be driven, once the best sensitizer is identified, by the possibility of an easy functionalization procedure for the antenna unit.

\footnotetext{
e This latter has been found to greatly increase the luminescence output of lanthanide complexes in the hybrid matrix.

${ }^{\mathrm{f}}$ Hybrid materials can be classified according to the bonding between the organic and inorganic part of the network [Sánchez, C; Ribot, F. New J. Chem., 1994, 18, 1007]. In Class I materials, organic molecules are blended into the inorganic network. In Class II materials, the inorganic and organic constituents are linked via covalent bonds.
} 


\section{Porous hybrid materials: mesoporous silicates and other macroporous materials}

The mesoporous molecular sieves of the M41S family (e.g. MCM41, MCM48 and SBA15) were developed by researchers at Mobil Corporation. These materials exhibit narrow pore size distributions, similar to those found for zeolites. Whereas the pore sizes of zeolites are typically less than $10 \AA$, the pores of the M41S materials can be tailored between 15 and more than $100 \AA$. The M41S materials can be obtained in different compositions, either as pure silica or as aluminosilicate. These mesoporous materials are synthesized via a sol-gel process by hydrolysis and condensation of a tetraalkoxysilane or an organosilicon compound in the presence of a surfactant. Therefore, these materials can also be considered as sol-gelderived materials. An advantage is that the pores of these mesoporous materials are large enough to encapsulate lanthanide complexes without the need for the "ship-in-bottle" approach which is necessary in conventional zeolites.

Compared to mesoporous materials, macroporous materials have several advantages, namely that they can be a beneficial host for chemical species too large for mesoporous materials and that 3D-ordered macroporous materials (3DOM) have photonic stop bands with potential applications in optical waveguides, optical circuits, and low-threshold lasers.

\section{Polymer materials: complexes blended with polymers}

Polymers have several advantages over glasses besides their better processability, including a lighter density and higher flexibility. In general the production of polymers is cheaper than that of glasses, and much less energy is required. Lanthanide complexes can be incorporated in many types of optically transparent polymers. Examples are poly(methyl methacrylate) (PMMA), poly(vinyl alcohol) (PVA), polyethylene (PE), polystyrene (PS), polyurethanes, polyesters, polycarbonates, polyimides, and epoxy resins. Fluorinated or deuterated polymers are of particular interest as a host matrix for infrared luminescent lanthanide complexes. Examples of perfluorinated polymers are CYTOP ${ }^{\circledR}$ (Asahi Glass Company) and poly-(hexafluoro isopropyl methacrylate) (P-FiPMA). An example of a deuterated polymer is deuterated poly(methyl methacrylate) (PMMA- $\left.d_{8}\right)$.

There are different methods for incorporating lanthanide complexes into polymers. First of all, one has to distinguish between 'host-guest' systems and systems in which the lanthanide complexes are an integral part of the polymer. In a host-guest system, the lanthanide complex is dissolved in the polymer matrix or blended with the polymer matrix.

To prepare host-guest systems, two techniques can be used. The first technique involves dissolution of the lanthanide complex directly into the monomer or into the monomer solution. After addition of an appropriate initiator, the monomer solution is polymerized by either thermal polymerization of photo-polymerization to form a uniformly doped polymer. In the second technique, the lanthanide complex and the pure polymer are both dissolved in a co-solvent. The solvent is then evaporated, and a uniformly doped polymer is obtained. There is a limit to the amount of dopant that can be incorporated in the polymer host. This limit is determined by the solubility of the lanthanide complex in the host polymer. Beyond this limit, aggregation of the complexes occurs, and this ruins the optical quality of the doped polymer.

Lanthanide complexes that contain polymerizable groups can be copolymerized together with another monomer. This results in a copolymer in which the lanthanide complex is part of the polymer backbone or of the side chain. Alternatively, a polymer with pendant ligands (such as 1,10-phenanthroline) can form adducts with lanthanide complexes.

\section{Lanthanide-doped nanoparticles}

Intense research on Ln-doped nanopartices dispersed in water or organic solvents has been conducted. The older (pre-1998) work on lanthanide-doped nanoparticles has been reviewed by Tissue [51]. Several reviews describe the more recent developments [52,53,54,55,56]. 


\subsection{OLEDs and NIR-OLEDs: device structure and operation principle}

An OLED, an organic light-emitting diode, consists of a sequence of organic layers between two electrodes, an anode for hole and a cathode for electron injection, respectively. These layers can be deposited by various techniques such as plasma deposition, chemical vapor deposition or spin coating from a solution. Schematically, the basic layers are assigned to the following functions:

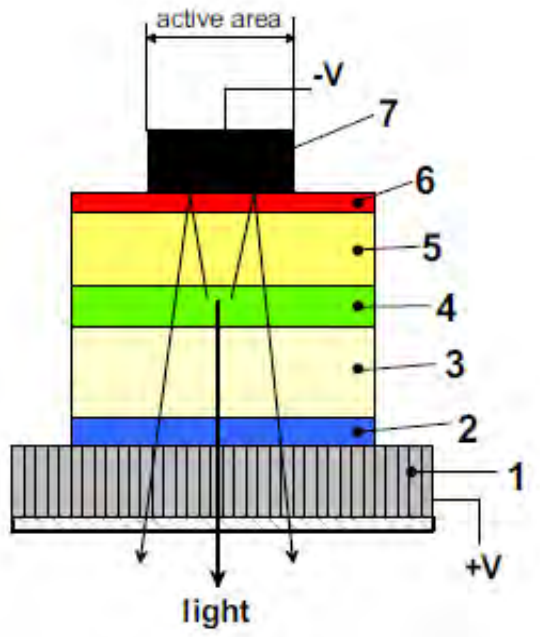

$1 . .$. anode on substrate

2 ... hole injection layer (HIL)

3 ... hole transport layer ( $\mathrm{HTL})$

4 ... emission layer (EML)

5 ... electron transport layer (ETL)

6 ... electron injection layer (EIL)

7 ... cathode

Figure 6. Layer sequence in an organic light-emitting diode

Under positive bias, electrons are injected from the cathode and holes from the anode. The charge carriers drift through the transport layers and meet in the emission layer. There, they form excitons (neutral excited states or bound electron-hole pairs) which show a certain probability to decay radiatively.

In order to achieve a high efficiency (emitted photons per injected electrons), the layers have to fulfill certain requirements [57]. The hole injection layer (HIL) has to ease hole injection from the anode into the hole transport layer (HTL). This might be achieved by choosing the energetic level of the highest occupied molecular orbital (HOMO) to be in between the HOMO of the HTL and the ionization potential of the anode. For the simple picture given in Figure 7, the HOMO and LUMO levels are treated similar to valence and conduction bands in inorganic semiconductors and the vacuum level is assumed to be aligned. The hole transport layer should have a high hole mobility and must hinder electrons coming from the cathode from reaching the anode (this would result in a 'shunt' current). Additionally, the transmission of all organic layers has to be high in the emission wavelength region. The HOMO and LUMO (lowest unoccupied molecular level) positions of the emission layer (EML) have to make hole and electron injection from the neighboring layers possible. Additionally, the photoluminescence efficiency (ratio of radiatively recombining excitons to created excitons) of the emissive material should be high and the emission should be red-shifted to the absorption (Stokes-shift). The properties of the electron transport (ETL) and electron injection (EIL) layers should be complementary to those of the HIL and HTL (i.e suitable LUMO levels, high electron mobility). The anode should have a high ionization potential to inject holes into the HOMO of the HIL. Accordingly, the cathode should be a low workfunction metal like magnesium or calcium [57]. Finally, at least one electrode must be transparent for visible light in order to achieve a high light outcoupling efficiency. For that reason, in most cases, indium-tin-oxide (ITO) is used as transparent anode; ITO is a nonstoichiometric composition consisting of $\mathrm{In}_{2} \mathrm{O}_{3}$ (80-90\%) and $\mathrm{SnO}_{2}$ (10-20\%). Other alternatives include aluminum doped zinc-oxide (AZO), fluorine-tin-oxide (FTO), zinc- 
indium-oxide (ZIO), zinc-indium-tin-oxide (ZITO), gallium-indium-oxide (GIO), titaniumnitride (TiN), polyaniline (PANI), etc.

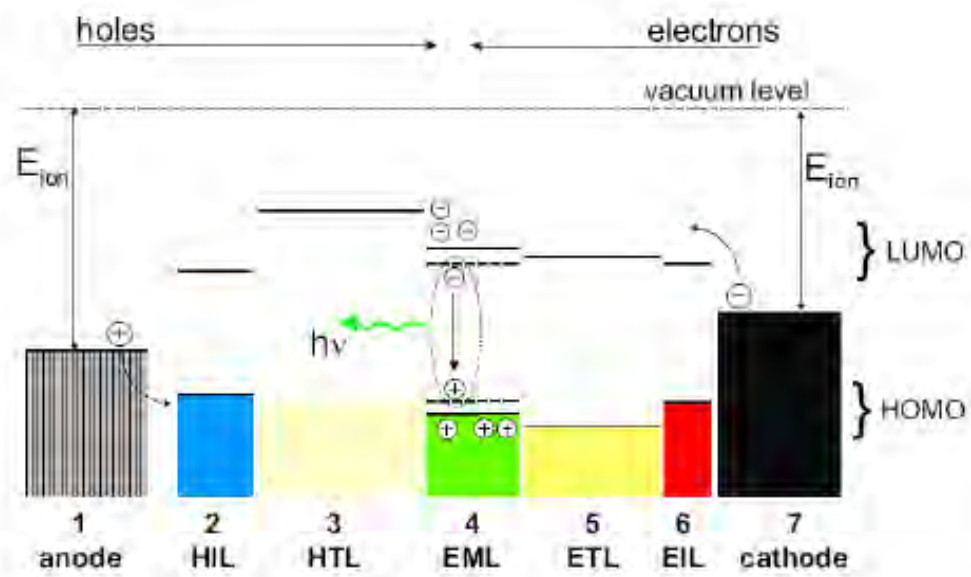

Figure 7. Schematic view on a favorable energetic situation in an OLED [57]

Almost all realized OLEDs consist of fewer layers than those proposed in above figures. This is due to the fact that some organic materials combine several of the properties mentioned above in one layer. e.g., in Tang's device from 1987 (layer sequence: ITOanode/TPD/Alq 3 /Mg:Ag-cathode) [58] TPD serves for hole injection, hole transport and electron blocking, whereas $\mathrm{Alq}_{3}$ features reasonable electron transport, electron injection.

Today, the most used hole transport materials (HTL) for OLEDs are PVK and PEDOT:PSS (which exhibits a conductivity of $460 \pm 20 \mathrm{~S}^{\cdot} \mathrm{cm}^{-1}$ and a work function of $5.35 \pm 0.05 \mathrm{eV}$ ).

The emission layer (EML) is doped with a fluorescent/phosphorescent dyeg, which provides for suitable emission either at visible or near infrared wavelengths. Until recently, OLED research was only focused on visible emission but now NIR-OLED devices are also the focus of some R\&D efforts. The emission wavelength of any OLED depends on the energy gap between the relaxed and the excited state, which depends almost wholly on the structure of the dye molecule. Tweaking the precise structure will allow to tweak the band gap so that an accurate complex with its central atom can emit in the infrared very efficiently.

The goal is to make NIR-OLEDs with high lifetimes and efficiencies which make them suitable for laser technology, optical sensors, telecommunications, night-vision displays, greenhouse illumination, skin cancer and acne treatment and sensing applications. However, only a few materials are available for their use in NIR-OLEDs. These materials include low band-gap polymers, molecules containing rare-earth metals such as $\mathrm{Nd}^{3+}, \mathrm{Er}^{3+}$, small molecule hostguest systems, and small molecules doped in a polymer matrix. Furthermore, most of these materials have proven to be too unstable to be used in practical devices. Thus, there is a need for additional materials for NIR-OLEDs.

The performance of NIR-OLEDs is tested by measuring the current density-voltage and the luminance-voltage characteristics. The turn-on-voltage is defined as the voltage required in

\footnotetext{
$\mathrm{g}$ Theoretically $75 \%$ triplet and $25 \%$ singlet excited states are formed, although in PLEDs this ratio is different, closer to 50-50 [Cao et al. Nature, 1999, 397, 414; Wohlgenannt et al. Nature, 2001, 409, 494]. In fluorescent OLEDs, the decay of triplet excitons is quantum mechanically forbidden by selection rules. Hence only the formation of singlet excitons results in the emission of useful radiation, placing a theoretical limit on the internal quantum efficiency of $25 \%$. On the contrary, phosphorescent OLEDs generate light from both triplet and singlet excitons, allowing the internal quantum efficiency of such devices to reach nearly $100 \%$.
} 
order to achieve a luminance of $1 \mathrm{~cd} / \mathrm{m}^{2}$. Preferably, this value should be as low as possible, but in some lanthanide-based OLEDs the values are between 5 and $15 \mathrm{~V}$. The luminance will increase with increasing voltage up to a maximum value. Increasing the voltage further will then cause a decrease of luminance. In OLEDs one can distinguish the external quantum efficiency $\left(\eta_{\mathrm{ex}}\right)$ and the power efficiency $\left(\eta_{\mathrm{p}}\right)$. The external quantum efficiency is defined as the ratio of the number of emitted quanta to the number of charge carriers. The luminous efficacy of the source, in lumens per Watt $\left(\mathrm{lm}^{\cdot} \mathrm{W}^{-1}\right)$, is the light power of the emitting source as perceived by the human eye divided by the electrical input power. The luminance or brightness in candelas per $\mathrm{m}^{2}\left(\mathrm{~cd} \cdot \mathrm{m}^{-2}\right)$ gives the ratio of the light intensity of the light source as perceived by the human eye emitted in a certain direction and the area of the OLED in that direction, while the current efficiency in $c d \cdot A^{-1}$ relates the same intensity to the current in the device. The power efficiency is the ratio of the luminous flux emitted by the OLED and the consumed electric power.

\section{Additional comments on the anode material for NIR-OLEDs}

Prior to 2009, transparent electrodes for NIR-OLEDs had not been studied systematically. Guan et al. [59] investigated the optical, electrical and electronic properties of indium-tinoxide (100 $\Omega / \mathrm{sq})$, ITO $(12 \Omega / \mathrm{sq})$, polyaniline (PANI), zinc-oxide (ZnO) and aluminum-doped $\mathrm{ZnO}$ (AZO) films for NIR-OLEDs emitting at $1.55 \mu \mathrm{m}$. They concluded that the performance of NIR-OLEDs with an ITO $(100 \Omega / \mathrm{sq})$ anode was significantly better than that of those devices in which ITO $(12 \Omega / \mathrm{sq})$ or PANI were used, and this is due to its much higher NIR transmittance.

The optical transmission spectra of the ITO $(100 \Omega / \mathrm{sq})$ and ITO $(12 \Omega / \mathrm{sq})$ coated glass substrates (shown in Figure 8) are generally consistent with the results obtained by Hamberg et al. [60]. Despite the fact that their behaviour is similar in the visible region, the optical transmittance of ITO $(100 \Omega / \mathrm{sq})$ is much higher $(>85 \%)$ than that of ITO $(12 \Omega / \mathrm{sq})$ in the NIR region $(<35 \%)$. This is due to the higher Sn content in the later (the Sn:In:O molar ratios of ITO $(12 \Omega / \mathrm{sq})$ and ITO (100 $\Omega / \mathrm{sq})$ are 1:11.2:10.1 and 1:13.4:11.8, respectively). That increase in Sn content has little effect on crystal quantities of ITO and the transmittance varys little in the visible range, but Sn ions are very efficient scatterers in the infrared range. Therefore, an increase of the Sn content causes an increase of the infrared reflectance and a concomitant decrease of the infrared transmittance. The thinner films may also contribute to the higher NIR transmittance of ITO $(100 \Omega / \mathrm{sq})$.

Therefore, in NIR-OLED devices, only AZO or ITO (100 $\Omega /$ sq) should be used.

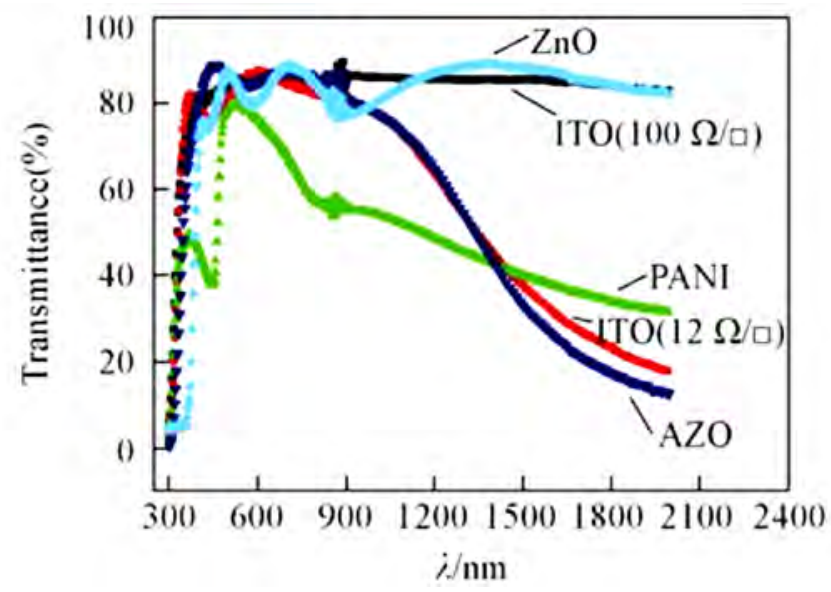

Figure 8. Optical transmission spectra of ZnO ( 400 nm), AZO ( $130 \mathrm{~nm})$, PANI $(\sim 110 \mathrm{~nm})$, ITO (100 $\Omega / \mathrm{sq})$ and ITO $(12 \Omega / \mathrm{sq})$ coated glass substrates [59]. 


\subsection{Erbium luminescence in the near-infrared telecommunication window. EDFAs y YEDFAs}

\subsubsection{Amplification of telecommunication signals by EDFAs}

The increasing demand for high speed information transfer is driving the switch from electrical to optical communications systems. Amplification of telecommunication signals is currently achieved by erbium-doped glass fibre amplifiers (EDFAs), which are based on the infrared photoluminescence of erbium(III) around $1550 \mathrm{~nm}$ (third telecommunication window) [61].

A typical setup of a simple EDFA is shown in Figure 9. Its core is the erbium-doped optical fiber, which is normally a single-mode fiber. In the shown case, the active fiber is pumped with light from two laser diodes (bidirectional pumping). The pump light, which most often has a wavelength $\sim 980 \mathrm{~nm}$ and sometimes $\sim 1450 \mathrm{~nm}$, excites the erbium ions $\left(\mathrm{Er}^{3+}\right.$ ) into the ${ }^{4} \mathrm{I}_{13 / 2}$ state (in the case of $980 \mathrm{~nm}$ pumping via ${ }^{4} \mathrm{I}_{11 / 2}$ ), from where they can amplify light in the $1.5 \mu \mathrm{m}$ wavelength region via stimulated emission back to the ground-state manifold ${ }^{4} \mathbf{I}_{15 / 2}$.

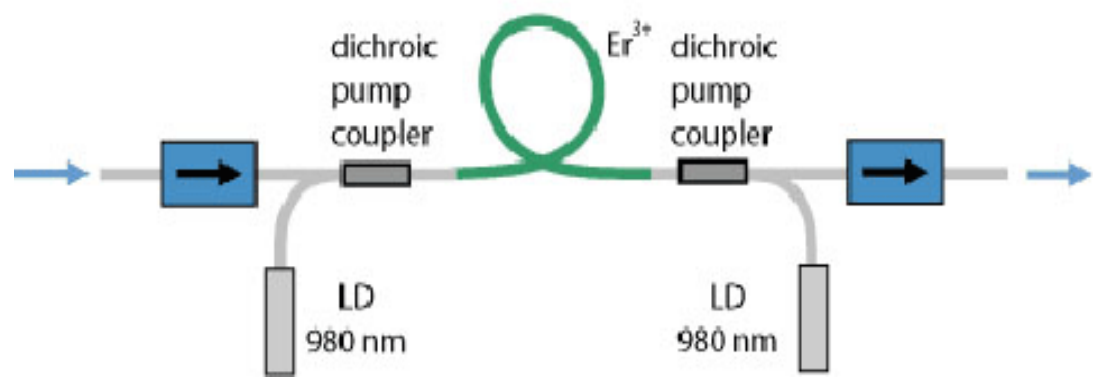

Figure 9. Setup of a simple erbium-doped fiber amplifier (EDFA) [62]

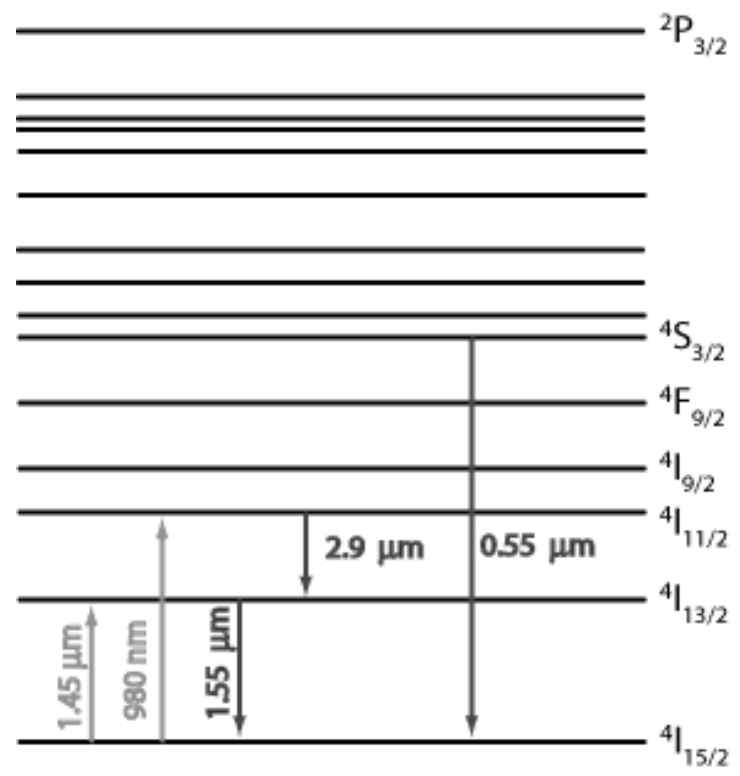

Figure 10. Energy level structure of the trivalent erbium ion, and some common optical transitions 
The upper-state lifetime of ${ }^{4} \mathrm{I}_{13 / 2}$ is around $8-10 \mathrm{~ms}$, whereas all higher-lying levels have lifetimes of at most a few microseconds due to fast multi-phonon decay.

The behavior of $\mathrm{Er}^{3+}$ ions is completely different in fluoride glasses, with a lower phonon energy, which strongly reduces multi-phonon transition rates. In such glasses, a variety of higher-lying energy levels can be populated, and a number of different pumping and lasing schemes are possible. For example, it is possible to realize green-emitting up-conversion fiber lasers [63] or lasers emitting around $2.9 \mu \mathrm{m}$ [64].

In addition to the mentioned optical and multi-phonon transitions, many energy transfer processes can occur, particularly for high doping concentrations. For example, cooperative up-conversion can occur, where one erbium ion in ${ }^{4} \mathrm{I}_{13 / 2}$ transfers energy to a second ion in that level, resulting in one ion in ${ }^{4} I_{9 / 2}$ and another one in the ground-state manifold. Due to the multitude of excited levels, and also because of the different behavior of ions in clusters, it is difficult to obtain reliable spectroscopic data for comprehensive modeling of such processes.

\subsubsection{Gain spectrum}

The shape of the erbium gain spectrum depends both on the host glass and on the excitation level, because the erbium ions have a quasi-three-level transition. Figure 11 shows data for a common type of glass, which is some variant of silica with additional dopants e.g. to avoid clustering of erbium ions.

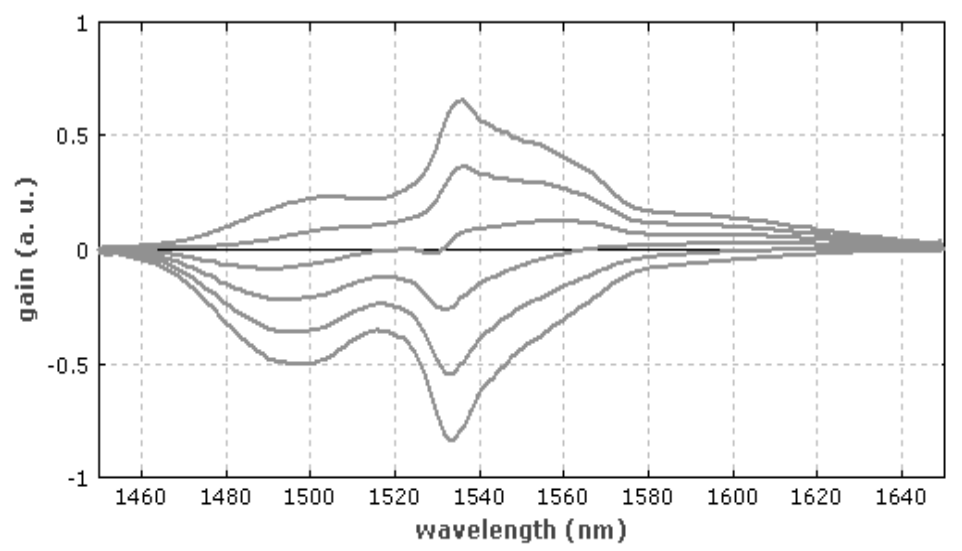

Figure 11. Gain and absorption (negative gain) of erbium $\left(\mathrm{Er}^{3+}\right)$ ions in germanium-aluminum-silicate glass for excitation levels from 0 to $100 \%$ in steps of $20 \%$ [62]

Strong three-level behavior (with transparency reached only for $>50 \%$ excitation) occurs at $1535 \mathrm{~nm}$. In that spectral region, the unpumped fiber exhibits substantial losses, but the high emission cross section allows for a high gain for strong excitation. At longer wavelengths (e.g. $1580 \mathrm{~nm}$ ), a lower excitation level is required for obtaining gain, but the maximum gain is smaller.

The maximum gain usually occurs in the wavelength region around 1530-1560 nm, with the $1530 \mathrm{~nm}$ peak being most pronounced for high excitation levels. The local excitation level depends on the emission and absorption cross sections and on the pump and signal intensity (apart from that of ASE light). The average excitation level over the whole fiber length depends on the pump and signal powers, but also on the fiber length and the erbium concentration. Such parameters are used to optimize EDFAs for a particular wavelength region, such as the telecommunications C or L bands [65,66,67]. 
In high-gain amplifiers, amplified spontaneous emission (ASE) is often a limiting factor for the achievable gain. Due to the quasi-three-level nature of the erbium ions, ASE powers can be different between forward and backward direction, and the maximum ASE can occur at a wavelength which differs from that of maximum gain.

The noise figure of an EDFA is slightly larger than the theoretical limit of $3 \mathrm{~dB}$ for a high-gain amplifier; this is mainly due to the quasi-three-level nature. Relatively low-noise performance can be achieved with suitable amplifier design, taking into account particularly the erbium excitation level near the signal input end, which can be strongly influenced by, e.g., the pump direction.

A particular attraction of EDFAs is their large gain bandwidth, which is typically tens of nanometers and thus actually more than enough to amplify data channels with the highest data rates without introducing any effects of gain narrowing. A single EDFA may be used for simultaneously amplifying many data channels at different wavelengths within the gain region; this technique is called wavelength division multiplexing. Before such fiber amplifiers were available, there was no practical method for amplifying all channels e.g. between long fiber spans of a fiber-optic link: one had to separate all data channels, detect and amplify them electronically, optically resubmit and again combine them. The introduction of fiber amplifiers thus brought an enormous reduction in the complexity, along with a corresponding increase in reliability. Very long lifetimes are possible by using redundant down-rated pump diodes [62].

A good flatness of the gain in a wide wavelength region (as required e.g. for wavelength division multiplexing), can be obtained by using optimized glass hosts (e.g. telluride or fluoride fibers, or some combination of amplifier sections with different glasses) or by combination with appropriate optical filters, such as long-period fiber Bragg gratings.

The only competitors to erbium-doped fiber amplifiers in the $1.5 \mu \mathrm{m}$ region are Raman amplifiers, which profit from the development of higher power pump lasers. Raman amplification can also be done in the transmission fiber. Nevertheless, EDFAs remain very dominant.

\subsubsection{Glass:Er,Yb fibers (ytterbium-sensitized fibers or ytterbium-codoped fibers)}

Particularly in bulk crystals, but also in fibers, efficient pump absorption on the ${ }^{4} \mathrm{I}_{15 / 2} \rightarrow{ }^{4} \mathrm{I}_{11 / 2}$ transition is difficult to achieve, because the absorption cross sections are relatively small, and the doping concentration is limited by the need to avoid excessive quenching processes. A common method to solve this problem (achieving a high gain in a shorter length) is codoping with ytterbium $\left(\mathrm{Yb}^{3+}\right)$ [68] or praseodymium $\left(\mathrm{Pr}^{3+}\right)$ sensitizer ions [69]. The ytterbium ions can efficiently absorb pump radiation e.g. at $980 \mathrm{~nm}$ (or even at longer wavelengths such as $1064 \mathrm{~nm}$ ), and then transfer the energy to erbium ions in the groundstate manifold, bringing them into ${ }^{4} \mathrm{I}_{11 / 2}$. From that level, the ions are quickly transferred into the upper laser level ${ }^{4} I_{13 / 2}$, so that energy transfer back to ytterbium is suppressed.

For a proper choice of the material composition of the fiber core, this energy transfer should be fairly efficient. However, the use of pure erbium-doped fibers is still more common in the telecommunications market.

Erbium-doped double-clad fibers can be used for generating very high output powers of tens of watts or even more. As the pump absorption efficiency can be weak in this case, an ytterbium-sensitized core may again be useful [62].

It is also possible to amplify ultra-short pulses in the $1.5 \mu \mathrm{m}$ region to relatively high energies, using EDFAs in the form of amplifier chains. This exploits the relatively high 
saturation energy of such amplifiers, particularly when using erbium-doped large mode area fibers [62].

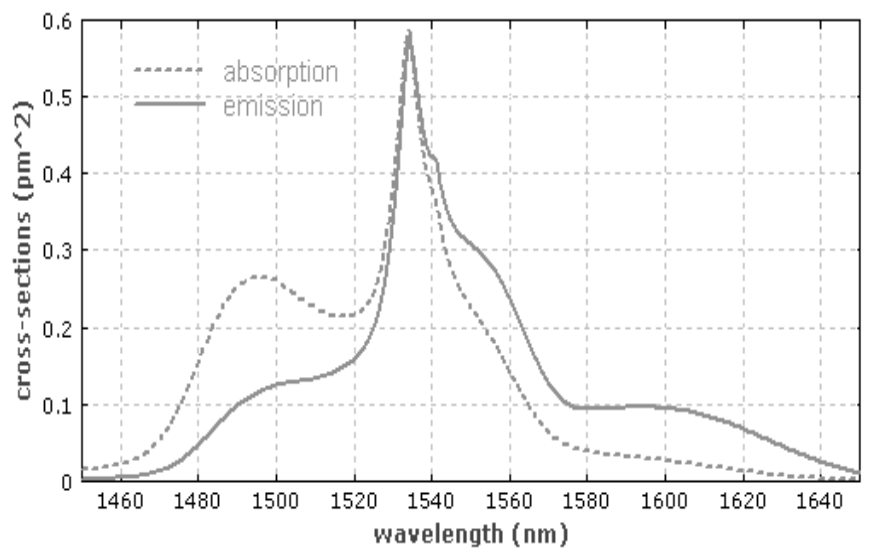

Figure 12. Absorption and emission cross sections for erbium ions in Er:Yb-doped phosphate glass, with data from Ref. $[70,71]$

\subsubsection{Design requirements for materials used in amplifiers and waveguides for Telecommunications}

The main problem for lanthanide-containing optical fibers is the minimization of nonradiative deactivation of the excited states. The literature agrees on the following main strategies for addressing this problem [72]:

(1) Suppression of vibrational deactivation (including the one induced by water molecules). This problem can be dealt with by adding long perfluorinated lateral chains on $\beta$-diketonates, by deuterating the $\mathrm{CH}$ bridge and by working in deuterated solvents. The longer the perfluorinated chain, the less water molecules penetrate into the inner coordination sphere. They are also expelled by coordination of strong donors such as DMSO.

(2) Inhibition of energy migration between luminescent centers and of up-conversion processes. Diffusion of $\mathrm{Ln}^{3+}$ chelates in solution induces collisions between molecules, leading to energy transfer via cross-relaxation and excitation migration. Migration of energy by hopping onto a neighboring ion does not quench luminescence by itself, but it enhances the probability of quenching by permitting the energy to finally migrate onto a site where more rapid nonradiative decay takes place. The only way of avoiding this "concentration quenching" is to keep luminescent centers far apart so that the probability of transfer is reduced. This can be done either by having large complexes which possess long fluorinated alkyl chains, or by dispersing them into polymer matrices.

Up-conversion and excited state absorption (ESA) are the main gain-limiting factors in $\mathrm{Er}^{3+}$ doped planar optical amplifiers, either glass- or polymer-based. Up-conversion increases 510 fold the pump power required to achieve population inversion. The ESA cross section is strongly material dependent and this phenomenon also increases the pump power needed, so that when both gain-limiting factors are present, pump powers of $10-100 \mathrm{~mW}$ are required, which considerably increases the cost of optical amplifiers. It is hoped that the introduction of sensitizers such as $\mathrm{Yb}^{3+}, \mathrm{Ag}^{+}$or silicon nanocrystals into inorganic optical fibers will help solving this problem. Organic chelates in polymer fibers may also be an altemative.

(3) Optimization of the sensitization ability of the ligand and of the emission intensity. $\eta_{\text {sens }}$ for $\beta$-diketonates is often between $20 \%$ and $30 \%$ only, but is larger with unsymmetrical ligands compared to symmetrical ones. Adding a second ligand to form a ternary complex, or a dye to 
transfer energy on the metal ion, improves the sensitization, although it is difficult to go beyond a factor of two, possibly three.

(4) Choice of an adequate host medium and defect-free fabrication of the waveguide. The ideal host should disperse the luminescent centers sufficiently to avoid concentration quenching while simultaneously allowing high enough concentration of these centers and minimizing optical losses. The latter originate from several sources: scattering is a major one and arises from imperfections in the waveguide, such as rough sidewalls or defects in the core material. Another loss mechanism is the mismatch between the device waveguide mode and the incident light source mode. Finally, in a waveguide doped with a chromophoric organic complex, absorption of both the chromophore and the polymer backbone also contributes to the loss. The host material should not modify the complex, or at least not the inner coordination sphere of the luminescent ion, and should not provide non-radiative deactivation paths, henceforth the many fluorinated polymers and functionalized zeolites which have been tested. 


\subsection{Erbium/Ytterbium complexes in third generation photovoltaics}

Photovoltaics (PV) have the potential to make a large contribution to solving the problem of climate change. To make electricity from PV competitive with fossil fuel technologies, the price needs to be reduced by a factor of 2-5. Currently $90 \%$ of the solar cell market is based on crystalline silicon wafers, with thicknesses of 200-300 $\mu \mathrm{m}$. Because of this, over the past ten years, there has been a great deal of research in order (i) to enhance the efficiency limit of silicon solar cells through spectrum modification and (ii) to decrease the thicknesses through thin-film solar cells [73].

\section{Lanthanide-based materials for spectrum modification}

Spectrum modification is one of the Third Generation concepts suggested to overcome the classical efficiency limit of silicon solar cells by M.A. Green in 2003 [74]. These concepts show extreme promise. While the classical efficiency limit is currently estimated to be $29 \%$ [75], detailed-balance calculations show that this could improve to approximately $37 \%$ using spectrum modification at one sun $[76,77]$.

There are three losses in a silicon solar cell that spectrum modification can reduce (Figure 13). The first of these is thermalization, which occurs when an electron-hole pair with energy greater than the band gap of silicon $\left(E_{\mathrm{g}}=1.12 \mathrm{eV}, \lambda_{\mathrm{g}}=1100 \mathrm{~nm}\right)$ is created and the excess energy is lost as heat because the electron (and hole) relax to the conduction (and valence) band edges. Thermalization losses can be reduced by using down-conversion whereby, for example, a photon with twice the energy of the band gap is converted into two photons with exactly the energy of the band gap. The second loss mechanism is imperfect collection due to recombination close to or at the surface. Since high energy photons are absorbed in this region they are more likely to be affected and the result is a reduced spectral response at shorter wavelengths. This loss can be reduced by using photoluminescence, whereby photons are shifted into an energy range where the cell has a higher spectral response. For a typical, industrial solar cell, photoluminescence would be beneficial if wavelengths shorter than approximately $500 \mathrm{~nm}$ could be shifted into the range 500-1000 $\mathrm{nm}$. The third loss mechanism is transmission, which occurs because photons with energy less than the band gap of silicon are not absorbed. Transmission losses can be reduced by using up-conversion whereby two or more low energy photons combine to create one higher energy photon.

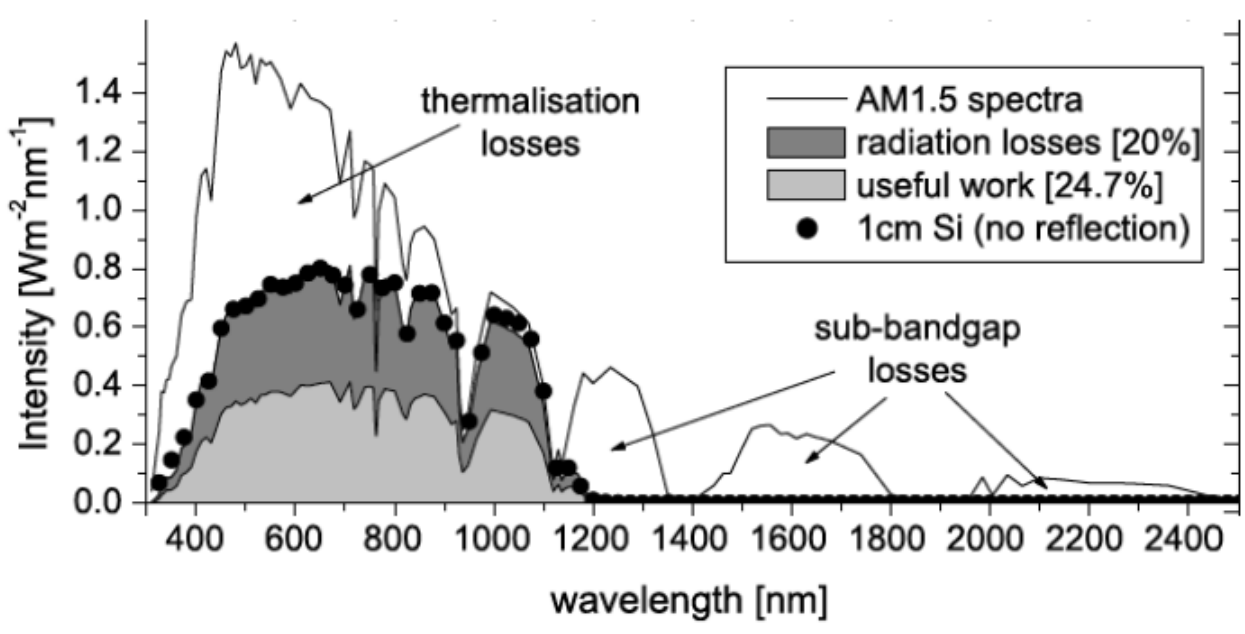

Figure 13. 24.7\% PERL cell spectral losses. Amount of spectral irradiance absorbed by the PERL cell (assuming a cut-off wavelength of $1107 \mathrm{~nm}(1.12 \mathrm{eV})$ ). Ref: Sandia National Laboratories. 
Modifying the incoming spectrum can be realized by incorporating layers above (for downconversion and photoluminescence) or below (for up-conversion) existing solar cells made with established high-efficiency processes. This is illustrated in Figure 14, which shows the down-conversion of a high energy photon $\left(E>2 E_{g}\right)$ and the up-conversion of two low energy photons $\left(0.5 E_{g}<E<E_{g}\right)$. No modification of the active layer is needed, as it is the case with the impurity photovoltaic effect (IPV) and the intermediate band solar cell. Neither a complicated structure such as that used for tandem solar cells or quantum well solar cells is necessary.

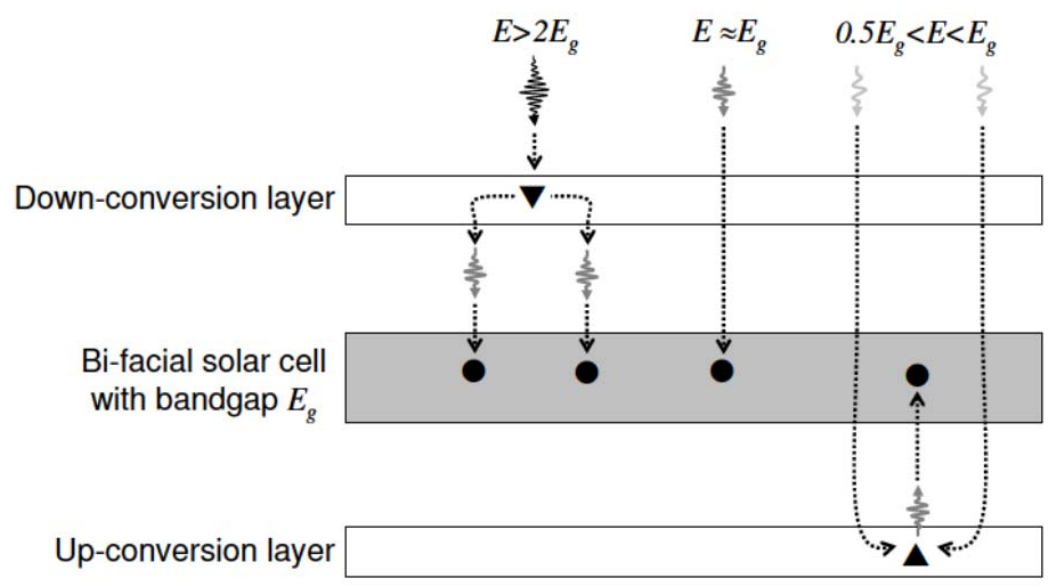

Figure 14. Photon losses and utilization using down- and up-conversion layers on the front and the rear, respectively. $\bullet$ represent $e^{-}-h^{+}$generation. $\boldsymbol{\nabla}$ and $\boldsymbol{\Delta}$ represent down- and up-conversion luminescence, respectively [78]

\section{Conversion to higher energies}

The expected improvement due to the application of an up-converter is a higher number of available photons and therefore additional electron-hole pairs in the cell bulk. This would result in an increase in short circuit current, $J_{s c}$, and therefore in the efficiency of the cell. Theoretical studies based on detailed-balance calculations predict a maximum conversion limit of 37.4\% [77] for an ideal up-converter on the rear side of a cell (without converter $30 \%)$ under non-concentrated sunlight. The efficiency enhancement due to an up-converter can also be increased by using concentrated light up to about $55 \%$ at 100 suns. Nevertheless, up-conversion has the major disadvantage of being a nonlinear process which only becomes really efficient at high incident power (for an up-conversion process of two steps, the intensity of up-conversion light $I$ is related to the incident light intensity $l_{0}$ by $I \propto I_{0}^{2}$, for three step up-conversion, the relation is $I \propto I_{0}^{3}$ ).

Up-conversion schemes relying on lanthanides have been well-studied for decades, since the 1960s. An extended review on up-converter materials with lanthanides, actinides and transition metals was written by Auzel [79]. The interested reader is also referred to the comprehensive reviews by Strümpel et al. [80], by van der Ende et al. [81] and by Wild et al. [82], being the latest the most up-to-date (from 2011).

The main challenges for applying up-conversion are the narrow and weak absorption lines of lanthanides and aforementioned quadratic power dependence of the up-conversion efficiency. High up-conversion efficiency $(\sim 25 \%)$ is only reached for high excitation powers and $100 \%$ efficiency can never be reached.

Reasonably successful proof-of-principle experiments with $\mathrm{Er}^{3+}$ doped up-converters have been reported mostly for $\mathrm{c}-\mathrm{Si}$, while for wider band-gap semiconductor solar cells the 
efficient $\mathrm{Yb}^{3+}-\mathrm{Er}^{3+}$ up-conversion couple is promising. The first experimental proof of the extension of spectral response of silicon solar cells to $1.5 \mu \mathrm{m}$ was obtained by Shalav et al. $[78,83]$ using erbium/ytterbium-doped sodium yttrium fluoride, $\mathrm{NaYF}_{4}: \mathrm{Er}^{3+} / \mathrm{Yb}^{3+}(20 \%)$, the most efficient up-converter under excitation at about $1500 \mathrm{~nm}$ known to date, with an absolute power conversion efficiency of $5.5 \%$ for the conversion of 980 to $540 \mathrm{~nm}$ light, corresponding to an internal quantum efficiency of $23 \%$. $\mathrm{Yb}^{3+}$ has only one energy level around $980 \mathrm{~nm}\left(10200 \mathrm{~cm}^{-1}\right)$ with a lifetime of $\sim 2 \mathrm{~ms}$, making it an ideal sensitizer for near infrared to visible up-conversion. $\mathrm{Er}^{3+}$ has an energy level around $980 \mathrm{~nm}$ and $490 \mathrm{~nm}$ $\left(20400 \mathrm{~cm}^{-1}\right)$, allowing for two close to resonant energy transfer steps from $\mathrm{Yb}^{3+}$ to $\mathrm{Er}^{3+}$.

There have also been attempts to enhance the exchange possibilities by using host lattices, where erbium ions occur in pairs, so-called dimers (discussed in Section 3.4). This has been investigated by Hehlen et al. [84]. The presence of dimers leads to a more complex system of processes since not only the energy transfer for radiative processes is enhanced (higher upconversion efficiency), but also that for non-radiative processes (cross-relaxation, enhanced concentration quenching). No improvement due to dimer-pairs has been seen.

Recently, F. Lahoz et al. [85] (from the MALTA Consolider Team at the University of La Laguna, Tenerife, Spain) have successfully developed a holmium $\left(\mathrm{Ho}^{3+}\right)$-doped transparent nanostructured oxyfluoride glass-ceramic material that achieves an up-conversion efficiency two orders of magnitude better than its precursor glass. Two- and three-photon absorption processes produce two emission bands in this up-conversion material centred at 650 and $910 \mathrm{~nm}$ under infrared excitation at $1170 \mathrm{~nm}$, where solar irradiation is about a factor of two more intense than in the $1550 \mathrm{~nm}$ absorption range of Er-doped phosphors. This material could be used in combination with existing erbium-doped materials to create higherefficiency silicon solar cells.

\section{Conversion to lower energies}

There are two possibilities to use the high energy part of the solar spectrum $(\sim 3.5-2.3 \mathrm{eV}$ or 350-550 nm) more efficiently: photoluminescence and down-conversion. The two techniques are distinguished by their quantum efficiencies. For photoluminescence, the quantum efficiency is always less than or equal to one, whereas for down-conversion the quantum efficiency exceeds one (more than one photon is emitted for each incoming photon) provided non-radiative losses can be prevented. Therefore, the latter is the option that we will focus on. Down-conversion is a linear process and the efficiency is independent of the incident power. The benefit of down-conversion was calculated by Trupke et al. [76]. Under the assumptions of an ideal down-converter on the front side of a silicon solar cell under non-concentrated sunlight, they showed using detailed-balance calculations that the efficiency limit was increased to $36.6 \%$ (without converter $30 \%$ ).

Quantum cutting through down-conversion has only been studied in the last decade. It offers great potential for efficiency enhancement of narrow band-gap solar cells, e.g. crystalline silicon solar cells ${ }^{\mathrm{h}}$. Several couples have proven to serve as potential down-converters (e.g., $\mathrm{Er}^{3+}-\mathrm{Yb}^{3+}, \mathrm{Ho}^{3+}-\mathrm{Yb}^{3+}$ or $\mathrm{Nd}^{3+} \mathrm{Yb}^{3+}$. In these couples $\mathrm{Yb}^{3+}$ serves as an ideal acceptor (the roles are reversed in comparison to those in sensitized up-conversion) with a single excited state, just above the band-gap of c-Si. High internal quantum yields (close to 200\%) have been demonstrated and are independent of the incident power.

Before implementation in solar cell systems can be realized two serious issues need to be addressed: a sensitizer is needed which absorbs strongly in the region 300-500 nm and

\footnotetext{
h The review by Strümpel et al. [80] points out that UV to vis quantum cutting examples in the literature are not useful for improving solar cell efficiency, since the (V)UV excitation wavelengths involved are not (or are minimally) present in the terrestrial solar spectrum. However, vis to near-infrared (NIR) quantum cutting is still of promising benefit to c-Si solar cells.
} 
which transfers efficiently to the donor; and losses arising from concentration quenching due to the high $\mathrm{Yb}^{3+}$-concentrations need to be minimized. Finally, even though the $\mathrm{Yb}^{3+}$ emission wavelength of $980 \mathrm{~nm}$ is above the band-gap of c-Si, absorption by c-Si at this wavelength is relatively weak; therefore, for complete absorption, a thicker c-Si layer is needed.

\section{Applicability of the novel complexes to down-conversion}

Doping a top transparent PMMA layer with the novel $\mathrm{Er}^{3+}$ and $\mathrm{Yb}^{3+}$ complexes (in a similar fashion to that discussed in Section 4.1.2) would be a promising approach, since the energy level structure of $\mathrm{Yb}^{3+}$-as discussed above- is ideally suited for use in the down-conversion for c-Si solar cells. The $\mathrm{Yb}^{3+}$ ion has a single excited state ${ }^{2} \mathrm{~F}_{5 / 2}$ approximately $10000 \mathrm{~cm}^{-1}$ above the ${ }^{2} \mathrm{~F}_{7 / 2}$ ground state, corresponding to an emission around $1000 \mathrm{~nm}$. The absence of other energy levels allows $\mathrm{Yb}^{3+}$ to exclusively 'pick up' energy packages of $10000 \mathrm{~cm}^{-1}$ from other co-doped lanthanide ions and emit photons at $\sim 1000 \mathrm{~nm}$, which can be absorbed by cSi. Efficient down-conversion using $\mathrm{Yb}^{3+}$ via resonant energy transfer requires donor ions with an energy level at about $20000 \mathrm{~cm}^{-1}$ and an intermediate energy level at approximately $10000 \mathrm{~cm}^{-1}$, and -according to the literature- $\mathrm{Er}^{3+}-\mathrm{Yb}^{3+}$ is again a potential ion couple which would fulfill the requisites, despite the fact that in this case their donor-acceptor roles would be reversed.

Finally, regarding the two issues that needed to be addressed so as to achieve efficient downconversion (namely finding a sensitizer which absorbs efficiently light in the UV and visible part of the spectrum up to $\sim 500 \mathrm{~nm}$ and transfers the energy to the down-conversion couple, and solving the concentration quenching issues), the selected $\beta$-diketonate and $\mathrm{N}, \mathrm{N}$-donor ligands would fulfill the first requisite and simultaneously act as spacers. Thus, in spite of the fact that no assays with solar cells have been conducted in the experimental part of this Thesis, and that this possible application of the novel materials will not be discussed in depth, it should be stressed that the complexes presented in Section 2 would be a simple and effective solution for down-conversion purposes (instead of utilizing quantum dots, transition metal ions or plasmonic coupling, which have also been proposed in the literature as feasible approaches). 


\subsection{Erbium/Ytterbium complexes as nonlinear optical chromophores}

Whereas lanthanide complexes have been intensively studied for their luminescent and magnetic properties, there are only a few recent reports on their peculiar nonlinearity of the optical (NLO) properties. NLO properties mean that when a molecule is placed in an intense light beam, there is no linear relationship between the induced electric dipole moment and the applied electric field. The characterization of the nonlinear optical response of materials [86] is a key issue in many areas of photonics such as optical switching [87], optical communications [88], optical limiters [89] and many other ultrafast applications [90]. Depending on the sign and amount of the nonlinearity of the medium, an enormous variety of interesting effects can be experimentally observed, including bright and dark solitons [91], non-diffractive beams and vortices [92], or self-organized structures [93], among other phenomena [94].

\section{Complexes with second order NLO properties in the literature}

Valore et al. [95] showed that intermolecular $\pi$-stacking interactions lead to an enhancement of the solid-state second-order NLO properties of lanthanide-based single-chain magnets [Ln(hfac) $)_{3}\left(4^{\prime}\right.$-phenoxybenzyl-4,4,5,5-tetramethylimidazoline-1-oxyl-3-oxi-de)] (hfac = hexafluoroacetylacetonate). Such enhancement was reported to be linked to the increased number of $f$ electrons but not to the effect of the increased Lewis acidity of the lanthanide according to the decrease of the ionic radius along the lanthanide series.

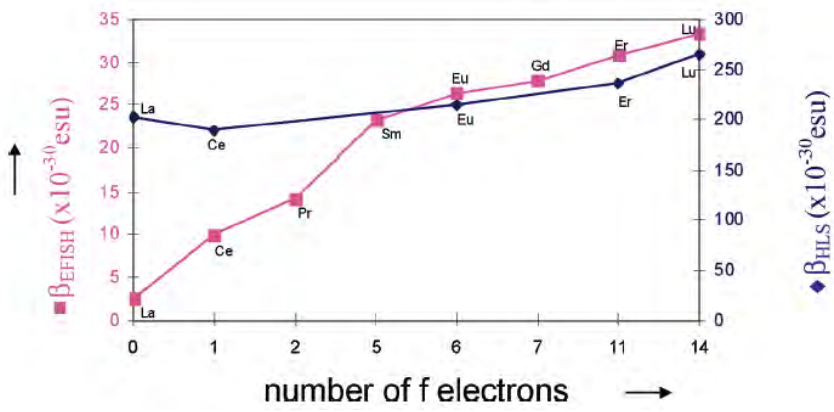

Figure 15. Dependence of $\beta_{\mathrm{EFISH}}$ and $<\beta_{\mathrm{HLS}}>\left(\times 10^{-30} \mathrm{esu}\right)$ upon the number of $f$ electrons [95]

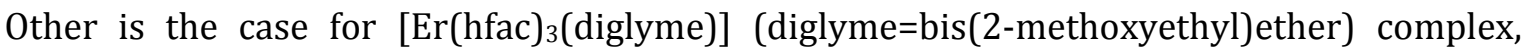
because its NLO properties cannot be considered in terms of classical push-pull systems: the potential donor part (the diglyme moiety) is connected to the potential acceptor part (the hfac moieties) not by a $\pi$-delocalized linker but by the $f$ electrons of a lanthanide ion. This confirmed not only that $f$ electrons may tune the second-order NLO response of lanthanide complexes, but -most importantly- that this tuning can be 10 times higher for the dipolar contribution to the quadratic hyperpolarizability than for the octupolar contribution [95].

\section{Applications of complexes with third order NLO properties}

The development of high-performance all-optical photonic processing devices based on selfor cross-phase modulation effects [96] or optical solitons [94] relies on the buildup of new highly transparent optical materials with a large and fast third-order NLO response. In particular, the use of Ln(III) complexes as NLO media is a highly promising and largely unexplored field [97]. This type of lanthanide complexes has been demonstrated to be compatible with Si technology $[98,99]$ and could also be employed for nonlinear hybridsilicon photonics applications $[100,101]$ in the second and third optical communication windows. One of the objectives of this PhD Thesis will focus on the study of the third-order NLO response of the new highly coordinated Ln(III) complexes. 


\subsection{Single Molecular Magnet (SMM) properties of Ln(III) complexes}

The interest in nanoscale magnetic materials has been driven by the rapid growth in highspeed computers and high-density magnetic storage devices with the promise of a revolution in information technology $[102,103]$. In the early 1990s, great excitement followed the discovery that a molecular transition metal coordination compound could, at liquid-helium temperatures, retain magnetization for long periods of time in the absence of an external magnetic field [104]. The dodecametallic manganese-acetate cage $\left[\mathrm{Mn}_{12} \mathrm{O}_{12}(\mathrm{OAc})_{16}\left(\mathrm{H}_{2} \mathrm{O}\right)_{4}\right]$ $\left(\mathrm{Mn}_{12} \mathrm{Ac}\right)$ became the progenitor of a large family of magnetic materials known as single molecule magnets (SMMs) [105].

Notwithstanding the huge intrinsic interest in SMMs, provided that SMMs can be considered as molecular analogues of classical bulk ferromagnets, it was also realized that they could in principle be developed for new technological applications involving the storage and processing of digital information: these materials open up a popular avenue to nanoscale electronic devices, sensors and high-density data storage media at the molecular level (the ultimate size limit) [106]. In contrast to bulk magnets currently used for this purpose, such as neodymium-iron boride magnets [102], the molecular nature of SMMs offers unique attributes that may allow information to be stored with much higher densities, and to be processed at unprecedented speeds [107]. Completely new applications of SMMs have also been envisaged, including in the development of molecular spintronics [106(c),108]. Further, SMMs provide unique opportunities to observe quantum effects (quantum tunneling of magnetization (QTM) and quantum phase interference) [109], because they straddle the interface between classical and quantum mechanical behavior and all magnetic particles, based on tailor-made molecules, are identical and monodisperse [109(d)].

Ultimately, however, SMM-based technology can only be realized when two major problems have been solved. First, the unique properties of SMMs are currently only accessible using liquid helium cooling; therefore, either the operating temperatures need to rise significantly, or applications so novel and important need to be discovered that temperature ceases to be an issue. Second, depositing and addressing individual molecules of SMMs on surfaces have only been explored with very few examples. One of the grand challenges in this field is still, therefore, to design and to synthesize efficient SMMs that function at temperatures likely to be of practical use, or which show physics that goes beyond what can be achieved with classical magnets.

The initial studies of SMMs were just confined to the realm of coordination complexes based on $3 d$ metals, including SMMs and single chain magnets (SCMs). However, there has been a growing realization that single-ion anisotropy is the crucial property to consider when designing SMMs with large anisotropy barriers [110]. Therefore, particular emphasis has been placed since 2003 -and specially in the last five years- on the design of new SMMs applying $4 f$ metal ions [111], as a result of their significant magnetic anisotropy arising from the large, unquenched orbital angular momentum. SMMs based on coordination compounds of the $f$-elements, particularly those of the lanthanides, have accounted for some of the most eye-catching recent advances in molecular magnetism [111(a),112]. Possibly of even greater significance is that lanthanide SMMs (Ln-SMMs) have already shown considerable potential to be developed for surface deposition and device applications.

The reader interested in the first decade of progress in studies of SMMs based solely on complexes of the lanthanides is referred to the comprehensive review by Woodruff et al. [113]. Hybrid $d-f$ compounds also constitute an important class of SMM, but coverage of this area is beyond the scope of this Thesis (see, for example, Ref. [114]). 


\section{SYNTHESIS, STRUCTURAL MODELING AND CHARACTERIZATION OF THE NOVEL ERBIUM(III) AND YTTERBIUM(III) COMPLEXES AS EMITTERS}

\subsection{Preparation of the complexes}

\section{Materials}

All reagents and solvents employed were commercially available and used as supplied without further purification. All the procedures for complex preparation were carried out under nitrogen and using dry reagents to avoid the presence of water and oxygen, which can quench metal photoluminescence.

\section{General synthesis procedure}

Under stirring, the $\beta$-diketone ( $3 \mathrm{mmol}$ ) methanol solution $(20 \mathrm{ml}$ ) is added to $1 \mathrm{mmol}$ of $\mathrm{Ln}\left(\mathrm{NO}_{3}\right)_{3} \cdot 5 \mathrm{H}_{2} \mathrm{O}$ in methanol, where $\mathrm{Ln}$ is either $\mathrm{Er}(\mathrm{III})$ or $\mathrm{Yb}(\mathrm{III})$. The mixture is neutralized by adding potassium methoxide $(3 \mathrm{mmol})$ dropwise under vigorous stirring until potassium nitrate precipitates. $\mathrm{KNO}_{3}$ is removed by decanting, and the $\mathrm{N}, \mathrm{N}$-donor $(1 \mathrm{mmol})$ is finally added. The mixture is heated to $75^{\circ} \mathrm{C}$ and stirred overnight, then washed with dioxane, and finally dried in vacuum to give product in $90-95 \%$ yield (based on $\mathrm{Er} / \mathrm{Yb}$ ). Crystals suitable for X-ray analysis were obtained by slow evaporation of a methanol-dioxane solution at RT.

\section{List of novel complexes synthesized in this Thesis}

1. $\left[\operatorname{Er}(\mathrm{acac})_{3}(\mathrm{bath})\right]$<smiles>CC(C)OC(C)(C)C1(C)C2=CC=C(c3ccccc3)c3ccc(-c4ccccc4)c2c31</smiles>

2. $\left[\operatorname{Er}(\mathrm{dbm})_{3}(\right.$ bipy) $]$

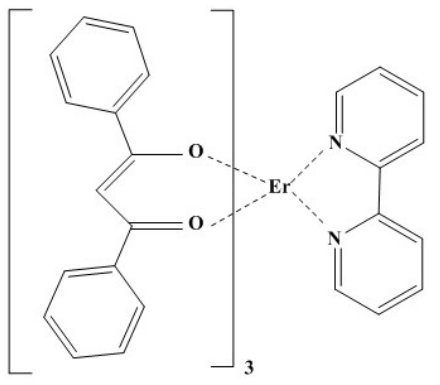

Tris(acetylacetonate)mono(bathophenanthroline)erbium(III)

Chemical formula: $\mathrm{C}_{39} \mathrm{H}_{37} \mathrm{ErN}_{2} \mathrm{O}_{6}$

MW: 796.98

Anal. Calcd. For $\mathrm{C}_{39} \mathrm{H}_{37} \mathrm{ErN}_{2} \mathrm{O}_{6}$ : C, 58.77; H, 4.68; Er, 20.99; N, 3.51; O, 12.05. Found: C, 58.79; H, 4.66; N, 3.51.

Chemical formula: $\mathrm{C}_{55} \mathrm{H}_{41} \operatorname{ErN}_{2} \mathrm{O}_{6}$ MW: 993.18

Anal. Calcd. for $\mathrm{C}_{55} \mathrm{H}_{41} \mathrm{ErN}_{2} \mathrm{O}_{6}$ : C, 66.51; H, 4.16; Er, 16.84; N, 2.82; 0, 9.67. Found: C, $66.42 ; \mathrm{H}, 4.20 ; \mathrm{N}, 2.88$. 
3. $\left[\operatorname{Er}(\mathrm{dbm})_{3}(\mathrm{bath})\right]$

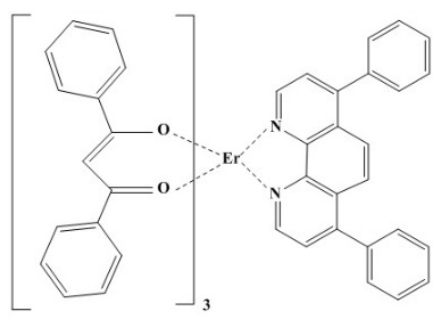

4. $\left[\operatorname{Er}(\mathrm{dbm})_{3}\left(5 \mathrm{NO}_{2}\right.\right.$ phen $\left.)\right]$

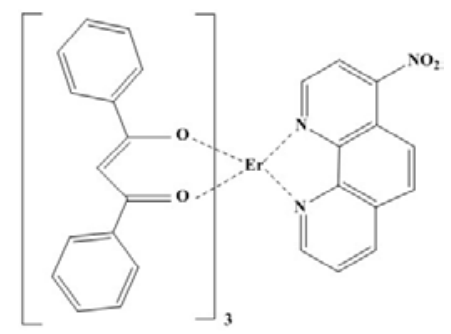

5. $(\mathrm{tba})\left[\operatorname{Er}(\mathrm{dbm})_{4}\right]$

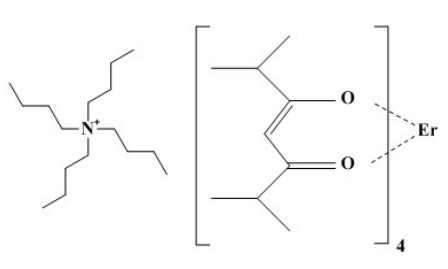

6. $\left[\operatorname{Er}(\mathrm{dnm})_{3}(\right.$ bipy $\left.)\right]$

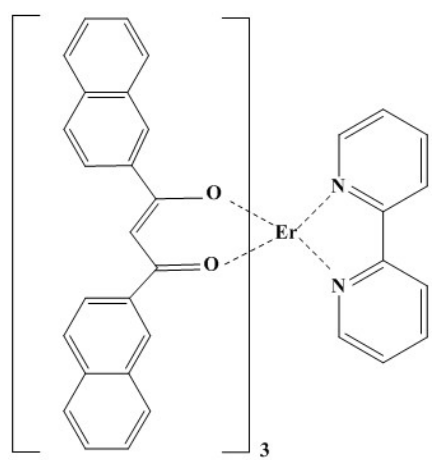

Tris(dibenzoylmethanate)mono(bathophenanthroline)erbium(III)

Chemical formula: $\mathrm{C}_{69} \mathrm{H}_{49} \mathrm{ErN}_{2} \mathrm{O}_{6}$

MW: 1169.39

Anal. Calcd. for $\mathrm{C}_{69} \mathrm{H}_{49} \mathrm{ErN}_{2} \mathrm{O}_{6}$ : C, 70.87; H, 4.22; Er, 14.30; N, 2.40; O, 8.21. Found: C, 71.03; H, 4.12; N, 2.42 .

Tris(dibenzoylmethanate)mono(5-nitro-1,10-

phenanthroline)erbium(III)

Chemical formula: $\mathrm{C}_{57} \mathrm{H}_{40} \mathrm{ErN}_{3} \mathrm{O}_{8}$

MW: 1062.20

Anal. Calcd. for $\mathrm{C}_{57} \mathrm{H}_{40} \mathrm{ErN}_{3} \mathrm{O}_{8}$ : C, 64.45; H, 3.80; Er, 15.75; N, 3.96; O, 12.05;. Found: C, 64.42; H, 4.0; N, 3.88.

(Tetrabutylammonium)tetra(dibenzoylmethanate)erbium(III)

Chemical formula: $\mathrm{C}_{76} \mathrm{H}_{80} \mathrm{ErNO}_{8}$

MW: 1302.71

Anal. Calcd. for $\mathrm{C}_{76} \mathrm{H}_{80} \mathrm{ErNO}_{8}$ : C, 70.07; H, 6.19; Er, 12.84; N, 1.08; O, 9.83. Found: $\mathrm{C}, 69.93 ; \mathrm{H}, 6.21 ; \mathrm{N}, 1.03$

Note: (tba) $\left[\operatorname{Er}(\mathrm{dbm})_{4}\right]$ was obtained as a sub-product of the synthesis of $\mathrm{Er}\left(\mathrm{NO}_{3}\right)_{3}$ with $\mathrm{Hdbm}, \mathrm{CsOH}$ and 2,2'-bipyridine in acetonitrile reaction when the substitution of cesium hydroxide by tetrabutylammonium hydroxide (TBAOH) was assayed.

Tris(1,3-di(2-naphthyl)-1,3-propanedionate)mono(2,2'bipyridine)erbium(III)

Chemical formula: $\mathrm{C}_{79} \mathrm{H}_{53} \mathrm{ErN}_{2} \mathrm{O}_{6}$

MW: 1293.53

Anal. Calcd. for $\mathrm{C}_{79} \mathrm{H}_{53} \mathrm{ErN}_{2} \mathrm{O}_{6}$ : C, 73.35; H, 4.13; $\mathrm{Er}, 12.93 ; \mathrm{N}, 2.17 ; \mathrm{O}$, 7.42. Found: C, 74.01; H, 3.96; N, 2.18. 


\section{7. $\left[\operatorname{Er}(\mathrm{dnm})_{3}(\right.$ bath $\left.)\right]$}

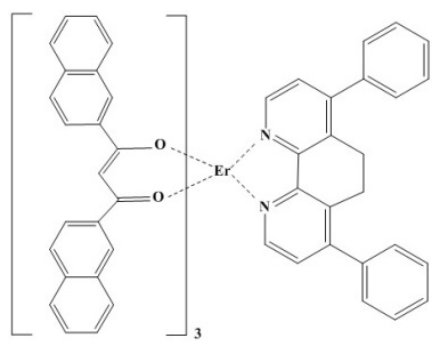

8. $\left[\operatorname{Er}(\mathrm{dnm})_{3}\left(5 \mathrm{NO}_{2}\right.\right.$ phen $\left.)\right]$

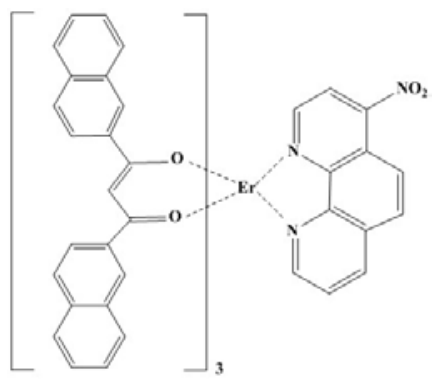

9. $\left[\operatorname{Er}(\mathrm{h})_{3}(\right.$ bipy $\left.)\right]$<smiles>CCC(=O)C1(C)c2ccccc2C1(C)OC(C)=CC(C)C</smiles>

10. $\left[\operatorname{Er}(\mathrm{h})_{3}(\right.$ bath $\left.)\right]$

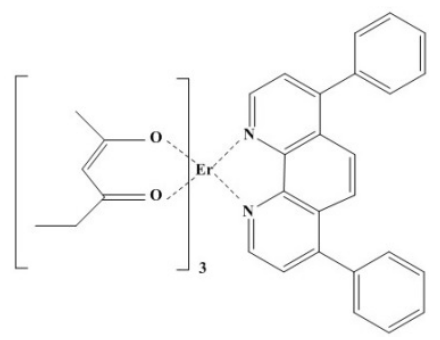

11. $\left[\operatorname{Er}(\mathrm{h})_{3}\left(5 \mathrm{NO}_{2}\right.\right.$ phen $\left.)\right]$

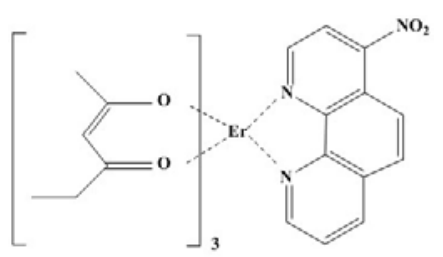

Tris(1,3-di(2-naphthyl)-1,3-

propanedionate)mono(bathophenanthroline)erbium(III)

Chemical formula: $\mathrm{C}_{93} \mathrm{H}_{61} \operatorname{ErN}_{2} \mathrm{O}_{6}$

MW: 1469.75

Anal. Calcd. for $\mathrm{C}_{93} \mathrm{H}_{61} \mathrm{ErN}_{2} \mathrm{O}_{6}$ : C, 76.00; H, 4.18; $\mathrm{Er}, 11.38 ; \mathrm{N}, 1.91 ; \mathrm{O}$ 6.53. Found: C, 76.22; H, 4.12; N, 2.08.

Tris(1,3-di(2-naphthyl)-1,3-propanedionate)mono(5-nitro-1,10phenanthroline)erbium(III)

Chemical formula: $\mathrm{C}_{81} \mathrm{H}_{52} \mathrm{ErN}_{3} \mathrm{O}_{8}$

MW: 1362.55

Anal. Calcd. for $\mathrm{C}_{81} \mathrm{H}_{52} \mathrm{ErN}_{3} \mathrm{O}_{8}$ : C, 71.40; H, 3.85; Er, 12.28; N, 3.08; O, 9.39. Found: C, 71.83; H, 4.05; N, 2.98.

Tris(2,4-hexanedionate)mono(2,2-bipyridine)erbium(III)

Chemical formula: $\mathrm{C}_{28} \mathrm{H}_{35} \mathrm{ErN}_{2} \mathrm{O}_{6}$

MW: 662.84

Anal. Calcd. for $\mathrm{C}_{28} \mathrm{H}_{35} \mathrm{ErN}_{2} \mathrm{O}_{6}$ : C, 50.74; H, 5.32; Er, 25.23; N, 4.23; O, 14.48. Found: C, 50.47; H, 5.26; N, 4.32.

Tris(2,4-hexanedionate)mono(bathophenanthroline)erbium(III)

Chemical formula: $\mathrm{C}_{42} \mathrm{H}_{43} \mathrm{ErN}_{2} \mathrm{O}_{6}$

MW: 838.06

Anal. Calcd. for $\mathrm{C}_{42} \mathrm{H}_{43} \mathrm{ErN}_{2} \mathrm{O}_{6}$ : C, 60.12; H, 5.17; Er, 19.93; N, 3.34; 0 , 11.44. Found: C, 59.93; H, 5.06; N, 3.42 .

Tris(2,4-hexanedionate)mono(5-nitro-1,10-

phenanthroline)erbium(III)

Chemical formula: $\mathrm{C}_{30} \mathrm{H}_{34} \mathrm{ErN}_{3} \mathrm{O}_{8}$

MW: 731.86

Anal. Calcd. for $\mathrm{C}_{30} \mathrm{H}_{34} \mathrm{ErN}_{3} \mathrm{O}_{8}$ : C, 49.23; H, 4.68; Er, 22.85; N, 5.74; O, 17.49. Found: C, 49.47; H, 5.06; N, 5.72 . 
12. $\left[\operatorname{Er}(\mathrm{h})_{2}(\right.$ bipy $\left.)\left(\mathrm{NO}_{3}\right)\right]$

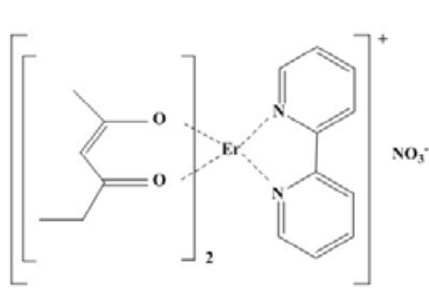

Note: $\left[\operatorname{Er}(\mathrm{h})_{2}(\right.$ bipy $\left.)\left(\mathrm{NO}_{3}\right)\right]$ was obtained as a sub-product of $\left[\operatorname{Er}(\mathrm{h})_{3}(\right.$ bipy $\left.)\right]$ synthesis

13. $\left[\operatorname{Er}(\mathrm{hd})_{3}(\right.$ bipy $\left.)\right]$<smiles>CCC(=O)CC</smiles>

14. $\left[\operatorname{Er}(\mathrm{dmh})_{3}(\right.$ bipy $\left.)\right]$

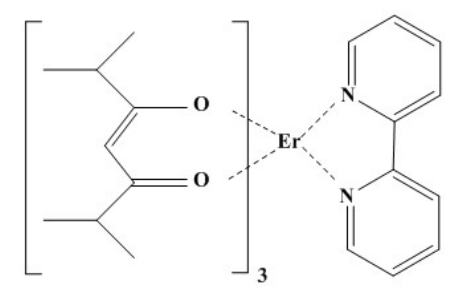

15. $\left[\operatorname{Er}(\mathrm{dmh})_{3}(\right.$ bath $\left.)\right]$

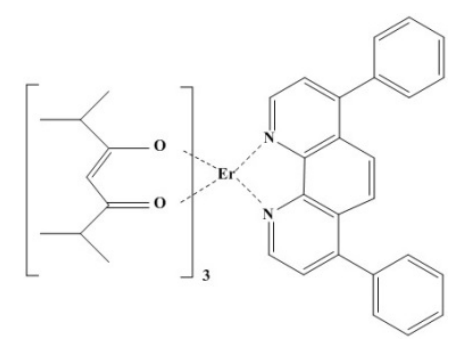

16. $\left[\mathrm{Er}(\mathrm{dmh})_{3}\left(5 \mathrm{NO}_{2}\right.\right.$ phen $\left.)\right]$

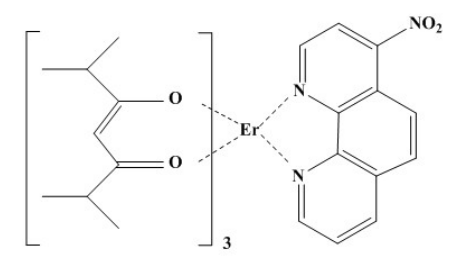

Nitrate-bis(2,4-hexanedionate)mono(2,2-bipyridine)erbium(III)

Chemical formula: $\mathrm{C}_{22} \mathrm{H}_{24} \mathrm{ErN}_{3} \mathrm{O}_{7}$

MW: 609.71

Anal. Calcd. for $\mathrm{C}_{22} \mathrm{H}_{24} \mathrm{ErN}_{3} \mathrm{O}_{7}$ : C, 43.27; H, 4.13; Er, 27.39; N, 6.88; O, 18.34. Found: C, 42.83; $\mathrm{H}, 4.06$; N, 6.82

Tris(3,5-heptanedionate)mono(2,2-bipyridine)erbium(III)

Chemical formula: $\mathrm{C}_{31} \mathrm{H}_{41} \mathrm{ErN}_{2} \mathrm{O}_{6}$

MW: 704.92

Anal. Calcd. for $\mathrm{C}_{31} \mathrm{H}_{41} \mathrm{ErN}_{2} \mathrm{O}_{6}$ : C, 52.82; H, 5.86; Er, 23.73; N, 3.97; 0, 13.62. Found: C, $52.83 ; \mathrm{H}, 5.76$; N, 3.82 .

Tris(2,6-dimethyl-3,5-heptanedionate)mono(2,2bipyridine)erbium(III).

Chemical formula: $\mathrm{C}_{37} \mathrm{H}_{53} \mathrm{ErN}_{2} \mathrm{O}_{6}$

MW: 789.08

Anal. Calcd. for $\mathrm{C}_{37} \mathrm{H}_{53} \mathrm{ErN}_{2} \mathrm{O}_{6}$ : C, 56.32; H, 6.77; Er, 21.20; N, 3.55; 0, 12.17. Found: C, 55.93; H, 6.76; N, 3.57.

Tris(2,6-dimethyl-3,5-

heptanedionate)mono(bathophenanthroline)erbium(III)

Chemical formula: $\mathrm{C}_{51} \mathrm{H}_{61} \operatorname{ErN}_{2} \mathrm{O}_{6}$

MW: 965.30

Anal. Calcd. for $\mathrm{C}_{51} \mathrm{H}_{61} \mathrm{ErN}_{2} \mathrm{O}_{6}$ : C, 63.46; H, 6.37; $\mathrm{Er}, 17.33 ; \mathrm{N}, 2.90$; 0 , 9.94. Found: C, $63.83 ; \mathrm{H}, 6.36$;, 2.82 .

Tris(2,6-dimethyl-3,5-heptanedionate)mono(5-nitro-1,10phenanthroline)erbium(III)

Chemical formula: $\mathrm{C}_{39} \mathrm{H}_{52} \mathrm{ErN}_{3} \mathrm{O}_{8}$

MW: 858.10

Anal. Calcd. for $\mathrm{C}_{39} \mathrm{H}_{52} \mathrm{ErN}_{3} \mathrm{O}_{8}$ : C, 54.59; H, 6.11; Er, 19.49; N, 4.90; O, 14.92. Found: C, 54.85; H, 6.01; N, 4.82 . 
17. [Er(thd $)_{3}($ bath $\left.)\right]$

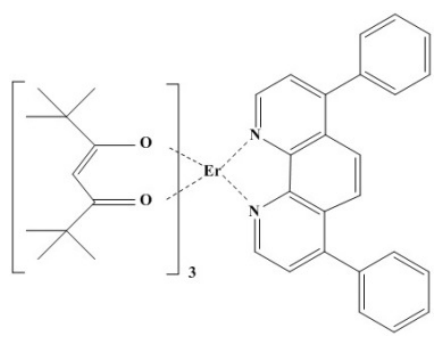

18. $\left[\operatorname{Er}(\mathrm{od})_{3}(\mathrm{bipy})\right]$<smiles></smiles>

19. $\left[\operatorname{Er}(\mathrm{od})_{3}(\right.$ bath $\left.)\right]$

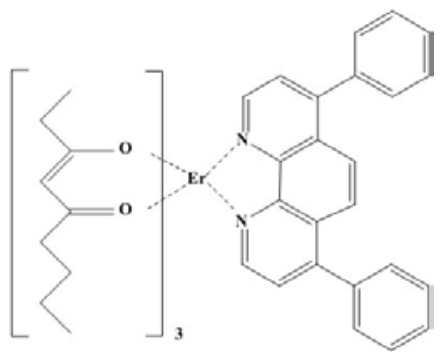

20. $\left[\mathrm{Er}(\mathrm{od})_{3}\left(5 \mathrm{NO}_{2}\right.\right.$ phen $\left.)\right]$

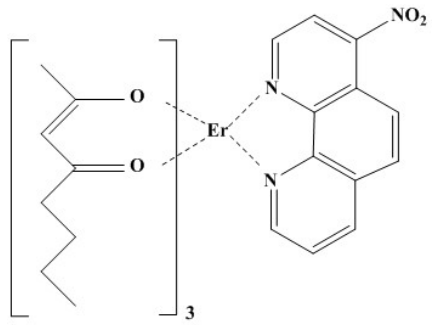

21. [Er(nd) ${ }_{3}($ bipy)]<smiles>CCCCCC(=O)C1(CC)c2ccccc2N2C=CC=C[In]21</smiles>

Tris(2,2,6,6,-tetramethyl-3,5-

heptanedionate)mono(bathophenanthroline)erbium(III)

Chemical formula: $\mathrm{C}_{57} \mathrm{H}_{73} \mathrm{ErN}_{2} \mathrm{O}_{6}$

MW: 1049.46

Anal. Calcd. for $\mathrm{C}_{57} \mathrm{H}_{73} \operatorname{ErN}_{2} \mathrm{O}_{6}$ : C, 65.23; H, 7.01; Er, 15.94; N, 2.67; O 9.15. Found: C, 65.03; H, 6.76; N, 2.62.

Tris(2,4-octanedionate)mono(2,2-bipyridine)erbium(III)

Chemical formula: $\mathrm{C}_{34} \mathrm{H}_{47} \mathrm{ErN}_{2} \mathrm{O}_{6}$

MW: 747.00

Anal. Calcd. for $\mathrm{C}_{34} \mathrm{H}_{47} \mathrm{ErN}_{2} \mathrm{O}_{6}$ : C, 54.67; H, 6.34; Er, 22.39; N, 3.75; O, 12.85. Found: C, 54.61; H, 6.26; N, 3.78.

Tris(2,4-octanedionate)mono(bathophenanthroline)erbium(III)

Chemical formula: $\mathrm{C}_{48} \mathrm{H}_{55} \mathrm{ErN}_{2} \mathrm{O}_{6}$

MW: 923.22

Anal. Calcd. for $\mathrm{C}_{48} \mathrm{H}_{55} \mathrm{ErN}_{2} \mathrm{O}_{6}$ : C, 62.45; H, 6.00; Er, 18.12; N, 3.03; O, 10.40. Found: C, 62.61; H, 6.06; N, 3.08.

Tris(2,4-octanedionate)mono(5-nitro-1,10phenanthroline)erbium(III)

Chemical formula: $\mathrm{C}_{36} \mathrm{H}_{46} \mathrm{ErN}_{3} \mathrm{O}_{8}$

MW: 816.02

Anal. Calcd. for $\mathrm{C}_{36} \mathrm{H}_{46} \mathrm{ErN}_{3} \mathrm{O}_{8}$ : C, 52.99; H, 5.68; Er, 20.50; N, 5.15; O, 15.69. Found: C, 52.61; H, 5.66; N, 5.08.

Tris(2,4-nonanedionate)mono(2,2-bipyridine)erbium(III)

Chemical formula: $\mathrm{C}_{37} \mathrm{H}_{53} \mathrm{ErN}_{2} \mathrm{O}_{6}$

MW: 789.08

Anal. Calcd. for $\mathrm{C}_{37} \mathrm{H}_{53} \mathrm{ErN}_{2} \mathrm{O}_{6}$ : C, 56.32; H, 6.77; Er, 21.20; N, 3.55; O, 12.17. Found: C, 56.23; H, 6.76; N, 3.58 . 
22. $\left[\operatorname{Er}(\mathrm{tfa})_{3}(\mathrm{bipy})\right]$

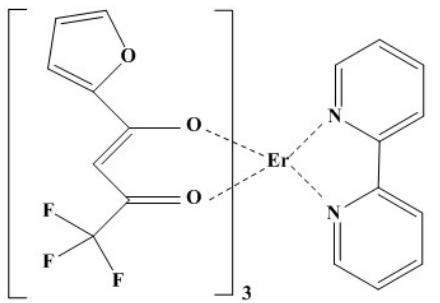

23. $\left[\operatorname{Er}(\mathrm{tfnb})_{3}(\mathrm{bipy})\right]$

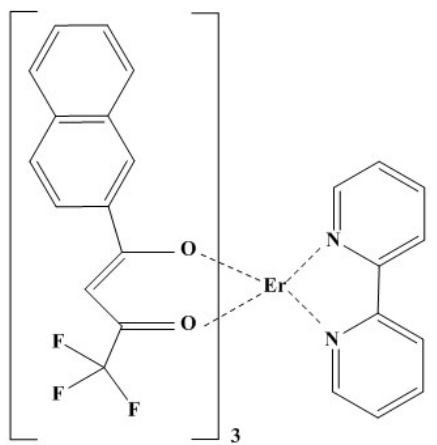

24. $\left[\operatorname{Er}(\mathrm{tfac})_{3}(\mathrm{bipy})\right]$

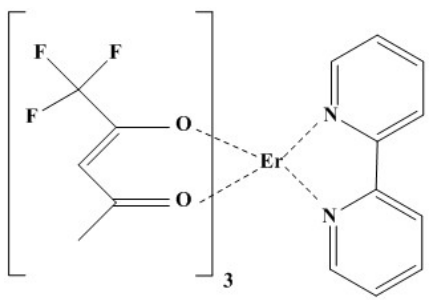

25. $\left[\operatorname{Er}(\mathrm{tfac})_{3}(\mathrm{bath})\right]$

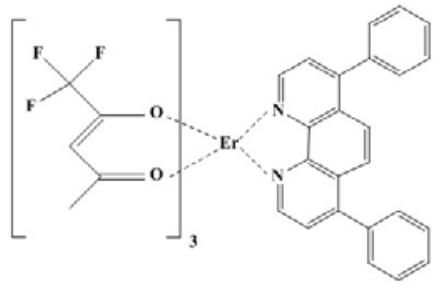

26. $\left[\mathrm{Er}(\mathrm{tfac})_{3}\left(5 \mathrm{NO}_{2}\right.\right.$ phen $\left.)\right]$<smiles>CC(=O)OC(C)(OC(C(C)=O)C(F)(F)F)C1c2cccc3ccc([N+](=O)[O-])c1c23</smiles>

Tris(4,4,4-trifluoro-1-(2-furyl)-1,3-butanedionate)mono(2,2' bipyridine)erbium(III)

Chemical formula: $\mathrm{C}_{34} \mathrm{H}_{20} \mathrm{ErF}_{9} \mathrm{~N}_{2} \mathrm{O}_{9}$

MW: 938.77

Anal. Calcd. for $\mathrm{C}_{34} \mathrm{H}_{20} \mathrm{ErF}_{9} \mathrm{~N}_{2} \mathrm{O}_{9}$ : C, 43.50; H, 2.15; $\mathrm{Er}, 17.82 ; \mathrm{F}, 18.21 ; \mathrm{N}$, 2.98; 0, 15.34. Found: C, 44.01; H, 2.16; N, 2.88.

Tris(4,4,4-trifluoro-1-(2-naphthyl)-1,3-butanedionate)mono(2,2'bipyridine)erbium(III)

Chemical formula: $\mathrm{C}_{52} \mathrm{H}_{32} \mathrm{ErF}_{9} \mathrm{~N}_{2} \mathrm{O}_{6}$

MW: 1119.06

Anal. Calcd. for $\mathrm{C}_{52} \mathrm{H}_{32} \mathrm{ErF}_{9} \mathrm{~N}_{2} \mathrm{O}_{6}$ : C, 55.81; H, 2.88; Er, 14.95; F, 15.28; N, 2.50; 0, 8.58. Found: C, 56.01; H, 2.96; N, 2.48.

Tris(1,1,1-trifluoro-2,4-pentanedionate)mono(2,2'bipyridine)erbium(III)

Chemical formula: $\mathrm{C}_{25} \mathrm{H}_{20} \mathrm{ErF}_{9} \mathrm{~N}_{2} \mathrm{O}_{6}$

MW: 782.68

Anal. Calcd. for $\mathrm{C}_{25} \mathrm{H}_{20} \mathrm{ErF}_{9} \mathrm{~N}_{2} \mathrm{O}_{6}$ : C, 38.36; $\mathrm{H}, 2.58$; $\mathrm{Er}, 21.37 ; \mathrm{F}, 21.85 ; \mathrm{N}$, 3.58; 0, 12.27. Found: C, 38.01; H, 2.56; N, 3.48.

Tris(1,1,1-trifluoro-2,4pentanedionate)mono(bathophenantroline)erbium(III)

Chemical formula: $\mathrm{C}_{39} \mathrm{H}_{28} \mathrm{ErF}_{9} \mathrm{~N}_{2} \mathrm{O}_{6}$

MW: 958.89

Anal. Calcd. for $\mathrm{C}_{39} \mathrm{H}_{28} \mathrm{ErF}_{9} \mathrm{~N}_{2} \mathrm{O}_{6}$ : C, 48.85; H, 2.94; Er, 17.44; F, 17.83; N, 2.92; 0, 10.01. Found: C, 48.81; H, 2.96; N, 2.98 .

Tris(1,1,1-trifluoro-2,4-pentanedionate)mono(5-nitro-1,10phenanthroline)erbium(III)

Chemical formula $\mathrm{C}_{27} \mathrm{H}_{19} \mathrm{ErF}_{9} \mathrm{~N}_{3} \mathrm{O}_{8}$

MW: 851.70

Anal. Calcd. for $\mathrm{C}_{27} \mathrm{H}_{19} \mathrm{ErF}_{9} \mathrm{~N}_{3} \mathrm{O}_{8}$ : C, 38.08; H, 2.25; Er, 19.64; F, 20.08; N, 4.93; 0, 15.03. Found: C, 38.01; H, 2.29; N, 4.98. 
27. $\left[\operatorname{Er}(\mathrm{tpm})_{3}\right.$ (bipy)]

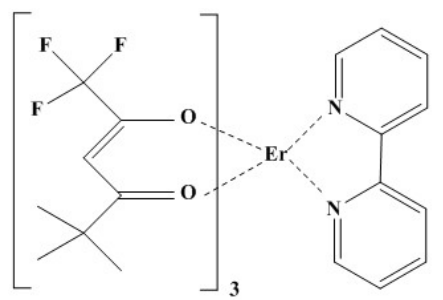

28. $\left[\operatorname{Er}(\mathrm{tpm})_{3}(\mathrm{bath})\right]$

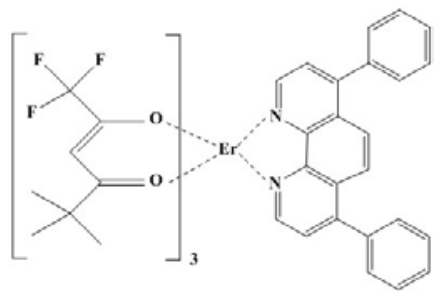

29. $\left[\operatorname{Er}(\operatorname{tpm})_{3}\left(5 \mathrm{NO}_{2}\right.\right.$ phen $\left.)\right]$

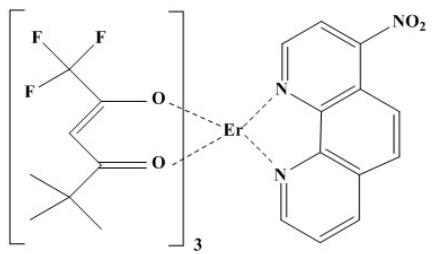

30. [Er(fhd $)_{3}\left(5 \mathrm{NO}_{2}\right.$ phen $\left.)\right]$

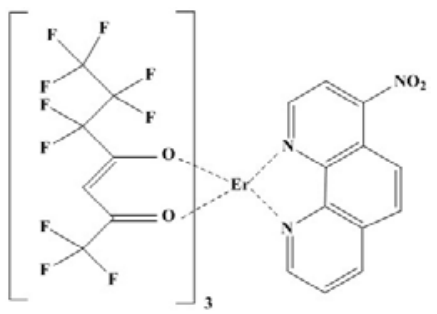

31. [Er(fod) $)_{3}$ (bipy)]

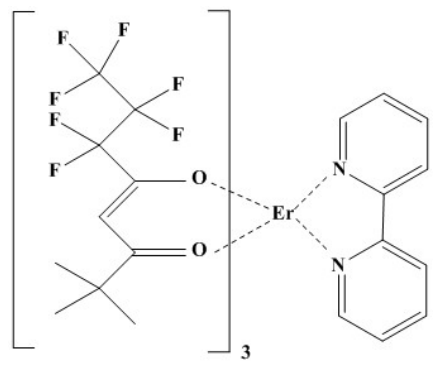

Tris(1,1,1-trifluoro-5,5-dimethyl-2,4-hexanedionate)mono(2,2'bipyridine)erbium(III)

Chemical formula: $\mathrm{C}_{34} \mathrm{H}_{38} \mathrm{ErF}_{9} \mathrm{~N}_{2} \mathrm{O}_{6}$

MW: 908.92

Anal. Calcd. for $\mathrm{C}_{34} \mathrm{H}_{38} \mathrm{ErF}_{9} \mathrm{~N}_{2} \mathrm{O}_{6}$ : C, 44.93; $\mathrm{H}, 4.21$; $\mathrm{Er}, 18.40 ; \mathrm{F}, 18.81 ; \mathrm{N}$, 3.08; 0, 10.56. Found: C, 44.81; H, 4.26; N, 2.98.

Tris(1,1,1-trifluoro-5,5-dimethyl-2,4hexanedionate)mono(bathophenanthroline)erbium(III)

Chemical formula: $\mathrm{C}_{48} \mathrm{H}_{46} \mathrm{ErF}_{9} \mathrm{~N}_{2} \mathrm{O}_{6}$

MW: 1085.13

Anal. Calcd. for $\mathrm{C}_{48} \mathrm{H}_{46} \mathrm{ErF}_{9} \mathrm{~N}_{2} \mathrm{O}_{6}$ : C, 53.13; H, 4.27; Er, 15.41; F, 15.76; N, 2.58; 0, 8.85. Found: C, 52.91; H, 4.30; N, 2.58.

Tris(1,1,1-trifluoro-5,5-dimethyl-2,4-hexanedionate)mono(5-nitro1,10-phenanthroline)erbium(III)

Chemical formula: $\mathrm{C}_{36} \mathrm{H}_{37} \mathrm{ErF}_{9} \mathrm{~N}_{3} \mathrm{O}_{8}$

MW: 976.94

Anal. Calcd. for $\mathrm{C}_{36} \mathrm{H}_{37} \mathrm{ErF}_{9} \mathrm{~N}_{3} \mathrm{O}_{8}$ : C, 44.21; H, 3.81; Er, 17.10; F, 17.48; N 4.30; 0, 13.09;. Found: C, 44.09; H, 3.86; N, 4.18.

Tris(1,1,1,5,5,6,6,7,7,7-decafluoro-2,4-heptanedionate)mono(5-nitro1,10-phenanthroline)erbium(III)

Chemical formula: $\mathrm{C}_{33} \mathrm{H}_{10} \mathrm{ErF}_{30} \mathrm{~N}_{3} \mathrm{O}_{8}$

MW: 1313.66

Anal. Calcd. for $\mathrm{C}_{33} \mathrm{H}_{10} \mathrm{ErF}_{30} \mathrm{~N}_{3} \mathrm{O}_{8}$ : C, 30.17; H, 0.77; Er, 12.73; F, 43.39; N, 3.20; 0, 9.74. Found: C, 29.96; H, 0.76; N, 3.18.

Tris(6,6,7,7,8,8,8-heptafluoro-2,2-dimethyl-3,5octanedionate)mono(2,2'-bipyridine)erbium(III)

Chemical formula: $\mathrm{C}_{40} \mathrm{H}_{38} \mathrm{ErF}_{21} \mathrm{~N}_{2} \mathrm{O}_{6}$

MW: 1208.96

Anal. Calcd. for $\mathrm{C}_{40} \mathrm{H}_{38} \mathrm{ErF}_{21} \mathrm{~N}_{2} \mathrm{O}_{6}$ : C, 39.74; H, 3.17; Er, 13.83; F, 33.00; N, 2.32; 0, 7.94. Found: C, 39.76; H, 3.16; N, 2.38. 
32. $\left[\operatorname{Er}(\text { fod })_{3}\right.$ (bath)]

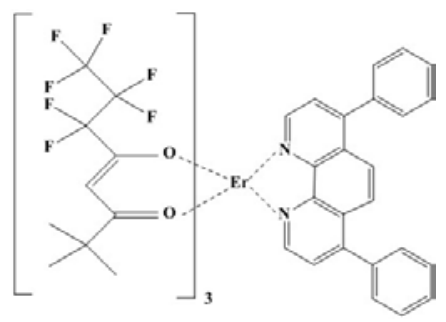

33. $\left[\operatorname{Er}(\text { fod })_{3}\left(5 \mathrm{NO}_{2}\right.\right.$ phen $\left.)\right]$

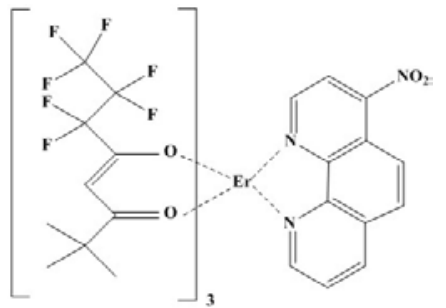

34. $\left[\mathrm{Na}_{5}(\mathrm{OH})_{4}\left(\mathrm{H}_{2} \mathrm{O}\right)_{14} \mathrm{Er}\left(\mathrm{NO}_{3}\right)_{4}\right]$

35. $\left[\mathrm{Yb}(\mathrm{h})_{3}\left(5 \mathrm{NO}_{2}\right.\right.$ phen $\left.)\right]$<smiles></smiles>

36. $\left[\mathrm{Yb}(\mathrm{dmh})_{3}\left(5 \mathrm{NO}_{2}\right.\right.$ phen $\left.)\right]$<smiles></smiles>

Tris(6,6,7,7,8,8,8-heptafluoro-2,2-dimethyl-3,5-

octanedionate)mono(bathophenanthroline)erbium(III)

Chemical formula: $\mathrm{C}_{54} \mathrm{H}_{46} \mathrm{ErF}_{21} \mathrm{~N}_{2} \mathrm{O}_{6}$

MW: 1385.18

Anal. Calcd. for $\mathrm{C}_{54} \mathrm{H}_{46} \mathrm{ErF}_{21} \mathrm{~N}_{2} \mathrm{O}_{6}$ : C, 46.82; H, 3.35; Er, 12.07; F, 28.80; N, 2.02; 0, 6.93. Found: C, 46.71; H, 3.29; N, 2.05.

Tris(6,6,7,7,8,8,8-heptafluoro-2,2-dimethyl-3,5octanedionate)mono(5-nitro-1,1-phenanthroline)erbium(III)

Chemical formula: $\mathrm{C}_{42} \mathrm{H}_{37} \mathrm{ErF}_{21} \mathrm{~N}_{3} \mathrm{O}_{8}$

MW: 1277.98

Anal. Calcd. for $\mathrm{C}_{42} \mathrm{H}_{37} \mathrm{ErF}_{21} \mathrm{~N}_{3} \mathrm{O}_{8}$ : C, 39.47; H, 2.92; Er, 13.09; F, 31.22; N, 3.29; 0, 10.02. Found: C, 39.66; H, 2.66; N, 3.28

tetradecaaqua-tetrahydroxo-pentasodium tetranitrato erbium(III)

Chemical formula: $\mathrm{H}_{32} \mathrm{ErNa}_{5} \mathrm{~N}_{4} \mathrm{O}_{30}$

MW: 850.47

Anal. Calcd. for $\mathrm{H}_{32} \mathrm{ErNa}_{5} \mathrm{~N}_{4} \mathrm{O}_{30}: \mathrm{H}, 3.79 ; \mathrm{Er}, 19.67 ; \mathrm{Na}, 13.52 ; \mathrm{N}, 6.59 ; \mathrm{O}$ 56.44. Found. H, 4.12; N, 6.70.

Tris(2,4-hexanedionate)mono(5-nitro-1,10phenanthroline)ytterbium(III)

Chemical formula: $\mathrm{C}_{30} \mathrm{H}_{34} \mathrm{~N}_{3} \mathrm{O}_{8} \mathrm{Yb}$

MW: 737.64

Anal. Calcd. for $\mathrm{C}_{30} \mathrm{H}_{34} \mathrm{~N}_{3} \mathrm{O}_{8} \mathrm{Yb}$ : C, 48.85; $\mathrm{H}, 4.65 ; \mathrm{N}, 5.70 ; 0,17.35 ; \mathrm{Yb}$, 23.46. Found: C, 49.08; H, 4.62; N, 5.98 .

Tris(2,6-dimethyl-3,5-heptanedionate)mono(5-nitro-1,10phenanthroline)ytterbium(III)

Chemical formula: $\mathrm{C}_{39} \mathrm{H}_{52} \mathrm{~N}_{3} \mathrm{O}_{8} \mathrm{Yb}$

MW: 863.88

Anal. Calcd. for $\mathrm{C}_{39} \mathrm{H}_{52} \mathrm{~N}_{3} \mathrm{O}_{8} \mathrm{Yb}$ : C, 54.22; $\mathrm{H}, 6.07 ; \mathrm{N}, 4.86 ; 0,14.82 ; \mathrm{Yb}$, 20.03. Found: C, 54.28; H, 6.02; N, 4.91. 
37. $\left[\mathrm{Yb}(\mathrm{tfa})_{3}\left(5 \mathrm{NO}_{2}\right.\right.$ phen $\left.)\right]$

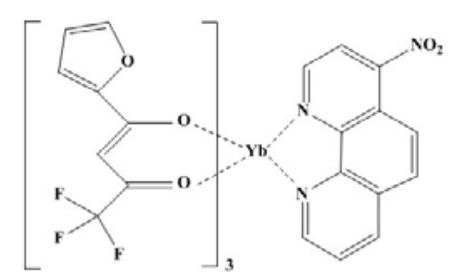

38. $\left[\mathrm{Yb}(\mathrm{tfnb})_{3}\left(5 \mathrm{NO}_{2}\right.\right.$ phen $\left.)\right]$
Tris(4,4,4-trifluoro-1-(2-furyl)-1,3-butanedionate)mono(5-nitro-1,10phenanthroline)ytterbium(III)

Chemical formula: $\mathrm{C}_{36} \mathrm{H}_{19} \mathrm{~F}_{9} \mathrm{~N}_{3} \mathrm{O}_{11} \mathrm{Yb}$ MW: 1013.57

Anal. Calcd. for $\mathrm{C}_{36} \mathrm{H}_{19} \mathrm{~F}_{9} \mathrm{~N}_{3} \mathrm{O}_{11}$ Yb: C, 42.66; H, 1.89; F, 16.87; N, 4.15; O, 17.36; Yb, 17.07. Found: C, 42.68; H, 1.82; N, 3.98.

Tris(4,4,4-trifluoro-1-(2-naphthyl)-1,3-butanedionate)mono(5-nitro1,10-phenanthroline)ytterbium(III)

Chemical formula: $\mathrm{C}_{54} \mathrm{H}_{31} \mathrm{~F}_{9} \mathrm{~N}_{3} \mathrm{O}_{8} \mathrm{Yb}$

MW: 1193.86

Anal. Calcd. for $\mathrm{C}_{54} \mathrm{H}_{31} \mathrm{~F}_{9} \mathrm{~N}_{3} \mathrm{O}_{8} \mathrm{Yb}$ : C, 54.33; H, 2.62; F, 14.32; N, 3.52; O, 10.72; Yb, 14.49. Found: C, 54.68; H, 2.62; N, 3.68.

39. $\left[\mathrm{Yb}(\mathrm{tfac})_{3}\left(5 \mathrm{NO}_{2}\right.\right.$ phen $\left.)\right]$

Tris(1,1,1-trifluoro-2,4-pentanedionate)mono(5-nitro-1,10phenanthroline)ytterbium(III)

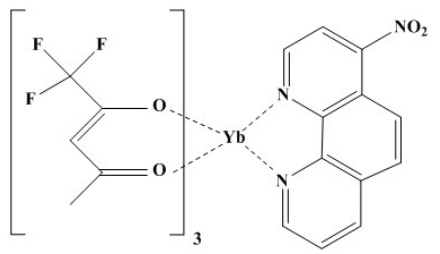

Chemical formula: $\mathrm{C}_{27} \mathrm{H}_{19} \mathrm{~F}_{9} \mathrm{~N}_{3} \mathrm{O}_{8} \mathrm{Yb}$

MW: 857.48

Anal. Calcd. for $\mathrm{C}_{27} \mathrm{H}_{19} \mathrm{~F}_{9} \mathrm{~N}_{3} \mathrm{O}_{8} \mathrm{Yb}$ : C, 37.82; H, 2.23; F, 19.94; N, 4.90; O, 14.93; Yb, 20.18. Found: C, 39.08; H, 2.22; N, 4.98.

40. $\mathrm{Yb}(\mathrm{tpm})_{3}\left(5 \mathrm{NO}_{2}\right.$ phen $\left.)\right]$

Tris(1,1,1-trifluoro-5,5-dimethyl-2,4-hexanedionate)mono(5-nitro1,10-phenanthroline)ytterbium(III)

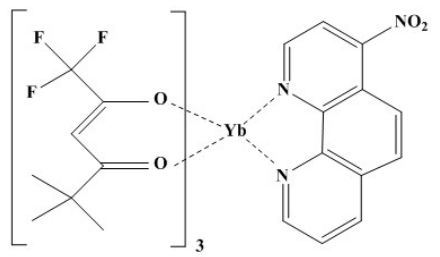

Chemical formula: $\mathrm{C}_{36} \mathrm{H}_{37} \mathrm{~F}_{9} \mathrm{~N}_{3} \mathrm{O}_{8} \mathrm{Yb}$

MW: 983.72

Anal. Calcd. for $\mathrm{C}_{36} \mathrm{H}_{37} \mathrm{~F}_{9} \mathrm{~N}_{3} \mathrm{O}_{8} \mathrm{Yb}$ : C, 43.95; H, 3.79; F, 17.38; N, 4.27; O, 13.01; Yb, 17.59. Found: C, 44.09; H, 3.86; N, 4.28.

41. [Yb(fhd $)_{3}\left(5 \mathrm{NO}_{2}\right.$ phen $\left.)\right]$

Tris(1,1,1,5,5,6,6,7,7,7-decafluoro-2,4-heptanedionate)mono(5-nitro1,10-phenanthroline)ytterbium(III)

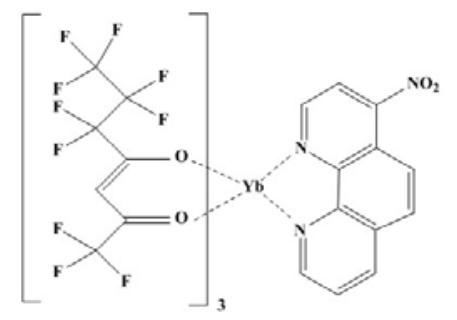

Chemical formula: $\mathrm{C}_{33} \mathrm{H}_{10} \mathrm{~F}_{30} \mathrm{~N}_{3} \mathrm{O}_{8} \mathrm{Yb}$

MW: 1319.44

Anal. Calcd. for $\mathrm{C}_{33} \mathrm{H}_{10} \mathrm{~F}_{30} \mathrm{~N}_{3} \mathrm{O}_{8} \mathrm{Yb}$ : C, 30.04; H, 0.76; F, 43.20; N, 3.18; O, 9.70; Yb, 13.11. Found: C, 29.90; H, 0.78; N, 3.18. 
42. $\left[\mathrm{Na}_{5}(\mathrm{OH})_{4}\left(\mathrm{H}_{2} \mathrm{O}\right)_{14} \mathrm{Yb}\left(\mathrm{NO}_{3}\right)_{4}\right]$ tetradecaaqua-tetrahydroxo-pentasodium tetranitrato ytterbium(III)

Chemical formula: $\mathrm{H}_{32} \mathrm{Na}_{5} \mathrm{~N}_{4} \mathrm{O}_{30} \mathrm{Yb}$

MW: 856.25

Anal. Calcd. for $\mathrm{H}_{32} \mathrm{Na}_{5} \mathrm{~N}_{4} \mathrm{O}_{30} \mathrm{Yb}: \mathrm{H}, 3.77 ; \mathrm{Na}, 13.42 ; \mathrm{N}, 6.54 ; 0,56.06 ; \mathrm{Yb}$, 20.21. Found: H, 3.85; N, 6.61.

43. [Yb(vilda) $)_{3}$ (bipy)]

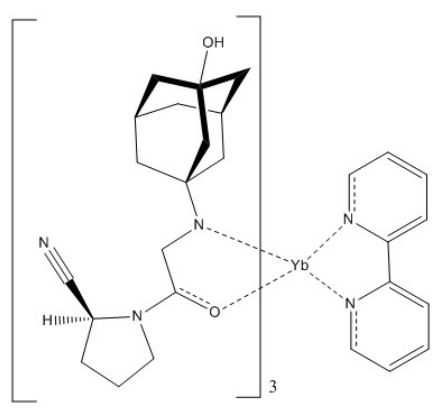

44. $[\text { Yb(vilda) })_{2}($ acac) (bipy)]

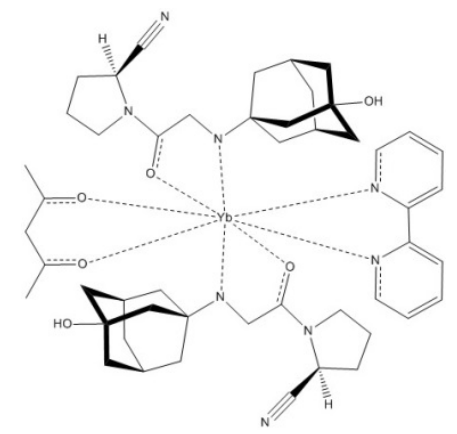

Tris $\{(S)$-1-[N-(3-hydroxy-1-adamantyl)glycylate]pyrrolidine-2carbonitrile)\}mono(2,2'-bipyridine)ytterbium(III)

Chemical formula: $\mathrm{C}_{61} \mathrm{H}_{80} \mathrm{~N}_{11} \mathrm{O}_{6} \mathrm{Yb}$

MW: 1236.39

Anal. Calcd. for $\mathrm{C}_{61} \mathrm{H}_{80} \mathrm{~N}_{11} \mathrm{O}_{6} \mathrm{Yb}: \mathrm{C}, 59.26 ; \mathrm{H}, 6.52 ; \mathrm{N}, 12.46 ; \mathrm{O}, 7.76 ; \mathrm{Yb}$, 14.00. Found: C, 59.86; H, 6.71; N, 12.26.

Chemical formula: $\mathrm{C}_{49} \mathrm{H}_{64} \mathrm{~N}_{8} \mathrm{O}_{6} \mathrm{Yb}$

MW: 1034.12

Anal. Calcd. for $\mathrm{C}_{49} \mathrm{H}_{64} \mathrm{~N}_{8} \mathrm{O}_{6} \mathrm{Yb}$ : C, 56.91; $\mathrm{H}, 6.24 ; \mathrm{N}, 10.84 ; 0,9.28 ; \mathrm{Yb}$, 16.73. Found: C, 57.03; H, 6.30; N, 11.02 . 


\subsection{Crystal structure modeling by semiempirical methods}

Prior to the elucidation of the structure by single crystal X-ray diffraction, it is convenient to conduct a preliminary study aimed at exploring the equilibrium energy configuration for each of the complexes. This step can substantially facilitate the subsequent structural refinement process once SC-XRD data has been collected (for those complexes in which high quality single crystals are available). For those complexes in which the material precipitates as crystalline powder, preventing SC-XRD elucidation, these computational models can replace to a large extent the actual structural elucidation. Furthermore, these methods can calculate the electronic spectra for each of the optimized structures, predicting HOMO and LUMO levels, UV-Vis absorption properties, NMR and FTIR spectra.

\subsubsection{Introduction to semiempirical methods}

In computational chemistry, semiempirical methods occupy a position intermediate between molecular mechanics and ab initio theory. By using approximations to avoid computationally intensive steps, and by using empirically determined parameters to obtain the best fit of predicted results to a training set of reference data, a method that is considerably faster than $a b$ initio methods and considerably more versatile than molecular mechanics methods can be developed. Methods of this type-part quantum theory and part empirical-are known as semiempirical methods. One of the more robust families of such methods are the neglect of diatomic differential overlap (NDDO) methods $[115,116]$ first developed by Pople. Following the pioneering work of Dewar and Thiel in developing the modified neglect of differential overlap (MNDO) method [117,118], several modifications were made to the NDDO formalism in attempts to increase its accuracy and generality, among which the most popular are AM1 [119], PM3 [120,121], PM6 [122] and RM1 [123].

AM1 was made to improve MNDO by adding a stabilizing Gaussian function to the core-core interaction to represent the hydrogen bond. Despite the fact that this was an oversimplification of a very complicated phenomenon, the overall effect was similar, and for the first time NDDO methods gave a good, albeit limited, model of hydrogen bonding.

In the course of the next several years, improvements were made to the method of parameter optimization. The result of this was the PM3 method, which culminated in the parameterization of all the elements in the main group in 2004.

In 2006, RM1 (Recife Model 1) was created as a modern re-parameterization of AM1, that is, as an attempt to maintain the mathematical structure and qualities of AM1, while significantly improving its quantitative accuracy with the help more advanced techniques available for nonlinear optimization. RM1 ended up representing an overall improvement over PM3. Nonetheless, it should be taken into consideration that the training set consisted only of molecules which involved $\mathrm{C}, \mathrm{H}, \mathrm{N}, \mathrm{O}, \mathrm{P}, \mathrm{S}, \mathrm{F}, \mathrm{Cl}, \mathrm{Br}$ and I atoms, representative of organic and biochemistry, so it was mostly focused on pharmaceutical research applications.

As each new method became available, it exhibited some advantages over previous methods; thus, when PM6 (the name was chosen to avoid any confusion with two other unpublished methods, PM4 and PM5) was developed in 2007, the average unsigned error (AUE) in the calculated $\Delta \mathrm{H}_{\mathrm{f}}$ values for simple organic compounds decreased by about $30 \%$ relative to PM3. In PM6 the NDDO method was modified by the adoption of Voityuk's core-core diatomic interaction parameters, which resulted in a significant reduction in errors for compounds of main-group elements, and, together with Thiel's $d$-orbital approximation, allowed extension of the NDDO method to the whole of the transition metal block.

Aforementioned advantages made each new method more attractive than its predecessors for modeling chemical systems. Unfortunately, an inevitable drawback of new methods is 
that, with increased usage, new limitations or faults become apparent. Many of these faults were present in earlier methods but were hidden by more severe limitations in those methods, and only became evident when the masking effects of the earlier faults were eliminated.

In late 2012 - early 2013, PM7 [124] has been presented. A significant increase in accuracy has been achieved after relatively minor changes were made to the approximations and after proxy reference data functions representing non-covalent interactions were introduced. The result has been that the average unsigned error in the heats of formation of organic solids calculated using PM7 have decreased by more than 50\% relative to that for PM6, previously the most accurate of the NDDO methods. At the same time, errors in PM7 geometries have been reduced by over one-third relative to those of PM6.

Such improvements are a result of an examination of the causes of the remaining sources of error, which suggested that further increases in accuracy could be achieved mainly by improving the training and survey reference data sets. The most important of these compendia are the NIST WebBook [125] and the Cambridge Structural Database (CSD) [126], where large amounts of critically reviewed (i.e., accurate) reference data are available.

In this Thesis, PM7 is the preferred option for the simulation of the ground state geometries of the complexes. Nonetheless, it should be noted that PM6 has also been extensively used prior to the publication of the former.

\subsubsection{The Sparkle model, a semiempirical approach to the quantum chemical calculation of lanthanide complexes}

In 1994, the Sparkle model was conceived and a first version was presented [127] within AM1. The Sparkle model recognizes the contracted nature of the $4 f$ orbitals of the lanthanide trications, of electronic configuration [Xe] $4 \mathrm{fn}$, coexisting with a poor overlap with the orbitals of the ligands, which assigns a predominantly ionic character to organo-lanthanide complexes. Accordingly, the angular effects of the $f$ orbitals are shielded from external perturbations by the filled $5 s^{2}$ and $5 p^{6}$ orbitals and are not taken into account. As such, the Sparkle model regards the lanthanide trications as triple positively charged closed shell inert gas electron densities without any angular steric properties. The Sparkle model thus replaces the trivalent rare earth ion by a Coulombic charge of $+3 e$ superimposed to a repulsive exponential potential of the form $\exp (-\alpha r)$, which accounts for the size of the ion. At the same time, the then two variables of the model were parameterized using only one complex, tris(acetylacetonate)mono(1,10-phenanthroline) europium(III). That original Sparkle model was a new concept in lanthanide complex modeling, although its accuracy could only be regarded as qualitative.

The Sparkle Model was improved in a subsequent article [128], in 2004, by the addition of two Gaussian functions to the core-core repulsion energy term, and by including the lanthanide mass, which allowed the calculation of vibrations and thermochemical quantities [129]. Major and significant improvements to the parametrization procedure were then carried out, eventually leading to Sparkle/AM1, the first semiempirical quantum chemical model to be parametrized for the whole lanthanide series [130]. Sparkle/AM1 lanthanides functioned as new elements to the semiempirical molecular orbital model AM1: i.e., when a lanthanide complex was calculated, the lanthanide ion was modeled as a sparkle, whereas the ligands were modeled by AM1. The model was improved to the point that it could be regarded as semiquantitative: Sparkle/AM1 coordination polyhedron geometries were comparable to, if not better than, geometries obtained with the best contemporary ab initio calculations with effective core potentials ( $a b$ initio/ECP). Besides, Sparkle model calculations were hundreds of times faster. 
More recently, in order to allow the user a choice for the modeling of the organic motif of the complexes, Sparkle/PM3 was subsequently introduced [131].

When PM6 was published and released, it already parametrized for 70 elements with the exception of the lanthanides from cerium to ytterbium ( $\mathrm{Z}=58$ and 70 , respectively). Therefore, in order to broaden the range of applicability of PM6, the group at the Universidade Federal de Pernambuco again generalized the Sparkle Model by introducing Sparkle/PM6 parameters for all lanthanide trications, from La(III) to Lu(III) [132].

In December 2012, Sparkle/PM7 elements La-Lu (Z=57-71) have also been added, increasing the total number of elements in PM7 to 83, and thus enabling the application of PM7 to the complexes presented in this Thesis.

\subsubsection{Computational procedure}

The ground state geometries and the corresponding vibrational frequencies were obtained using the Sparkle/AM1 [119,130], Sparkle/PM3 [120,130], Sparkle/PM6 [122,132] and Sparkle/PM7 [124,133] models implemented in the MOPAC2012 [134] software. No imaginary vibrational frequencies were found for any of the optimized geometries. The vibrational spectra obtained were also corrected using the scaling factors for the AM1 [135], PM3 [135] and PM6 [136] models.

The electronic spectra for each of the optimized structures were calculated using the ORCA software [137] via the intermediate neglect of differential overlap/spectroscopic (INDO/S) method and configuration interaction truncated with singles (CIS) $[138,139]$ replacing the $\mathrm{Ln}^{3+}$ ( $\mathrm{Ln}=\mathrm{Er}$ or $\mathrm{Yb}$ ) ion with a point charge as described in Ref. [140].

Semi-empirical calculations were also performed in order to optimize the solid crystalline structures using a treatment of periodic boundary conditions [138] in MOPAC2012 [134] software. In all the calculations, we used the keywords SPARKLE XYZ and GNORM=1: the Sparkle model to treat the $\mathrm{Er}^{3+}$ ions, Cartesian coordinates, and the same exit criteria (when the gradient norm drops below 1.0) as in Ref. [141]. We replicated the crystallographic unit cell $l, m$ and $n$ times along the Cartesian axis $x, y$ and $z$, respectively. In each case, the keyword MERS $=(l, m, n)$ was used, where $l, m$ and $n$ could be either 1 or 2 in each calculation. 


\subsection{Methods of characterization}

\subsubsection{Characterization of crystal structures by X-ray diffraction}

Prior to structural characterisation, powder diffractograms were obtained using an EnrafNonius FR590 powder diffractometer equipped with an INEL120 detector (Debye-Scherrer geometry). The powder was used to fill a glass capillary, which was slowly rotating upon data collection.
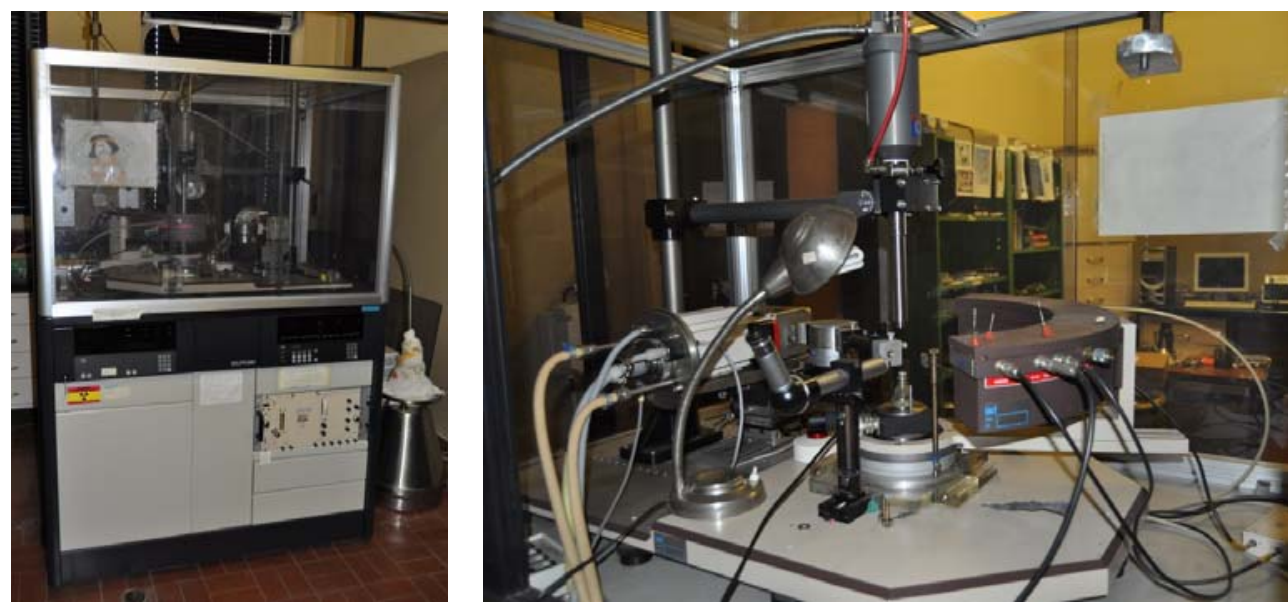

Figure 16. Enraf-Nonius FR590 powder diffractometer

For the determination of the crystal structure by X-ray diffraction, crystals of the complexes were glued to glass fibre and mounted on a Bruker Apex II diffractometer. Diffraction data

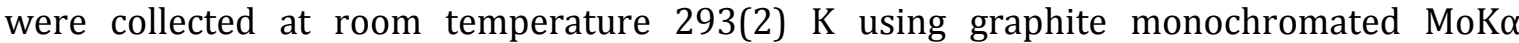
$(\lambda=0.71073 \AA)$ radiation. Absorption corrections were made using SADABS [142]. The structures were solved by direct methods using SHELXS-97 and refined anisotropically (non$\mathrm{H}$ atoms) by full-matrix least-squares on $F^{2}$ using the SHELXL-97 program [143]. All the hydrogen atoms were placed at calculated positions and allowed to ride on their parent atoms using SHELXL-97 defaults. PLATON [144] was generally used to analyse the structure and figure plotting, but Diamond v.3.2 and ChemBioOffice Ultra v.13 have also been used.

In those complexes in which disorder was observed, as shown by elongated anisotropic thermal parameters, the terminal groups of some $\beta$-diketonate ligands or the $\mathrm{N}, \mathrm{N}$-donor had to be refined in two mutually exclusive positions.

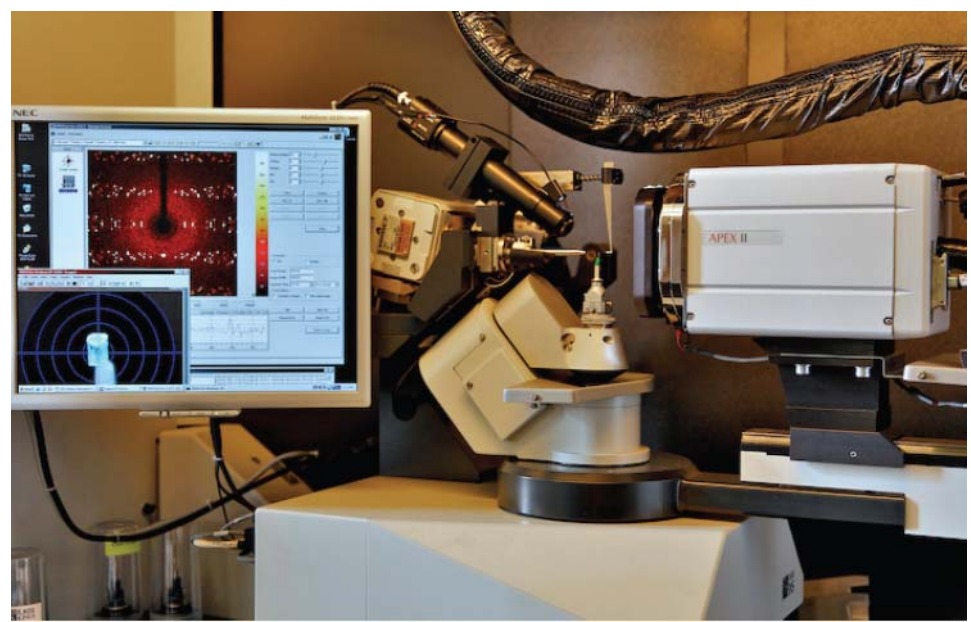

Figure 17. Bruker Apex II diffractometer 


\subsubsection{DSC study of the thermal stability}

Differential scanning calorimetry (DSC) data were obtained on a DSC TA Instruments mod. Q100 v.9.0 or on a DSC Pyris1 Perkin Elmer equipped with an intracooler cooling unit at $-25^{\circ} \mathrm{C}$ (ethylenglycol-water, $1: 1 \mathrm{v} / \mathrm{v}$, cooling mixture), at a heating rate $\beta=10^{\circ} \mathrm{C} / \mathrm{min}$, under a $\mathrm{N}_{2}$ purge $(20 \mathrm{~mL} / \mathrm{min})$. Samples were hermetically sealed in aluminium pans, and an empty pan was used as a reference. Temperature calibration was performed with high-grade standards, biphenyl (CRM LGC 2610) and indium (Perkin-Elmer, $x=99.99 \%$ ), which was also used for enthalpy calibration $[145,146]$.

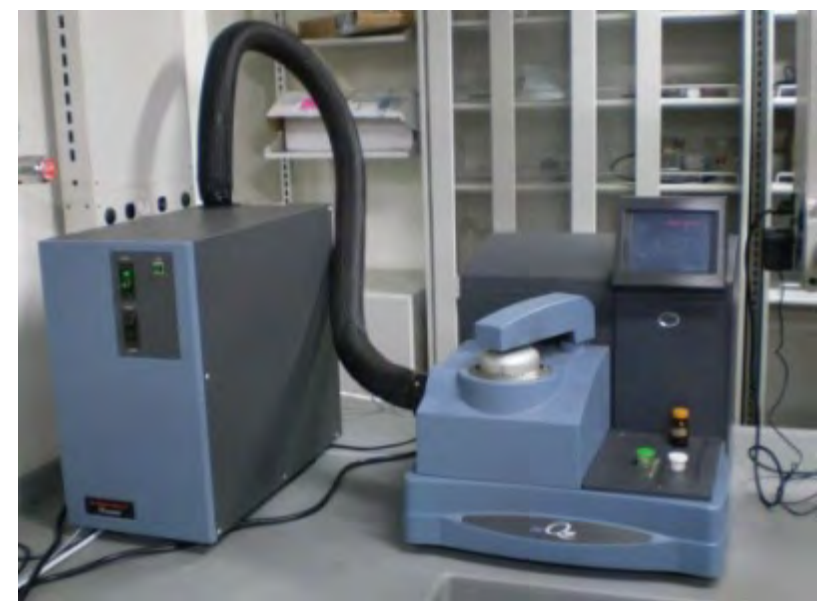

Figure 18. TA Instruments Thermo DSC mod. Q100

\subsubsection{Infrared spectroscopy and Raman spectroscopy}

Infrared spectra for complexes were recorded by a Thermo Nicolet 380 FT-IR apparatus. In most records (carried out in the range 4000-400 $\mathrm{cm}^{-1}$ ) the $\mathrm{KBr}$ pellet method was used, but in some of them a diamond Attenuated Total Reflectance (ATR) module was used instead.

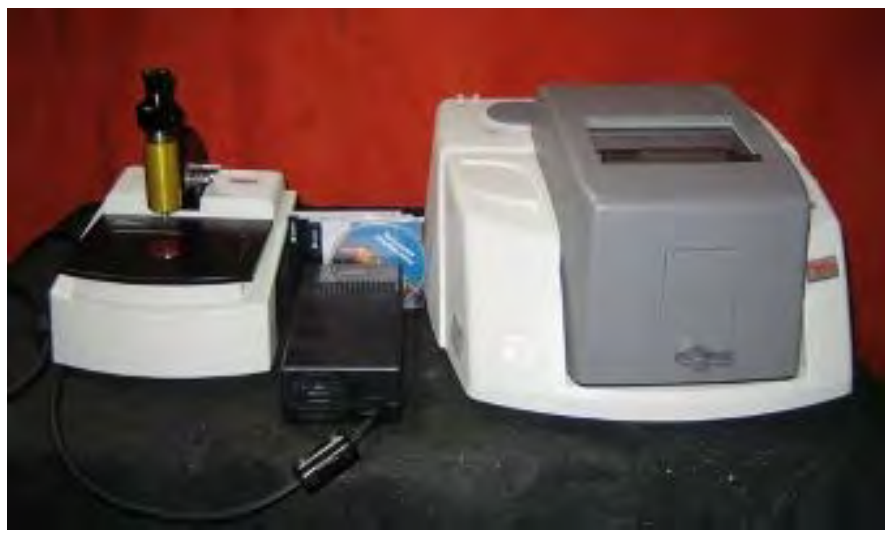

Figure 19. Thermo Nicolet 380 FT-IR spectrometer and its ATR-FTIR module

Usefulness of FT-IR spectroscopy. Though assigning the IR bands does not 'identify' the molecule (i.e., the inherent complexity of the novel compounds makes it impossible to assign all the possible frequencies observed in the IR spectrum), it does provide quite useful information about its nature. In particular, this technique proves useful for verifying the "authenticity" of a compound in fast and reliable way (analogously to the fingerprint of an 
individual). Therefore, a convenient application of IR spectra is the comparative analysis of the spectra of an "unknown" compound (e.g. a new batch of one of the novel complexes, synthetized using different reagents) with that obtained for the initial batch for which the crystal structure by SC-XRD was obtained, so as to confirm that it is the same product prior to manufacturing the devices.

Raman spectra have been recorded with two apparatus, at different excitation laser lines:

One has been a LabRam (Jobin-Ivon) Raman dispersive spectrometer, with a confocal microscope, three excitation laser lines (wavelengths of 514, 633 and $785 \mathrm{~nm}$ ) and a CCD Peltier-cooled detector. This equipment has the accessories needed to obtain Spectral Imaging and Depth Profile.

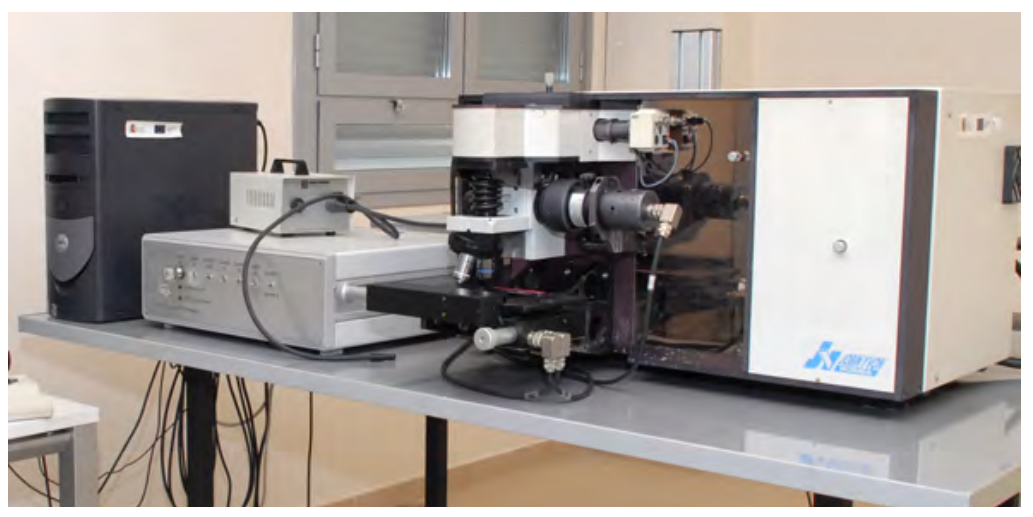

Figure 20. LabRam (Jobin-Ivon) Raman dispersive spectrometer

The other was a FT-Raman (Bruker RFS/100) spectrometer with coupled microscope, that allows to work in worksurface ( $90^{\circ}$ and $180^{\circ}$ geometries) or in the microscope $\left(180^{\circ}\right)$. This equipment has a near infrared excitation laser source (1064 nm, Nd-YAG), particularly suitable for the analysis of fluorescent samples with problems in the visible. The detector is a liquid nitrogen cooled germanium detector, allowing a spectral range 3500 to 50 (stokes) and -100 to $-2000 \mathrm{~cm}^{-1}$ (anti-stokes). Additionally, a controlled environment chamber LINKAM FTIR600, that allows to work in a temperature range from -196 to $250^{\circ} \mathrm{C}$, and to control the spectrum acquisition atmosphere is available.

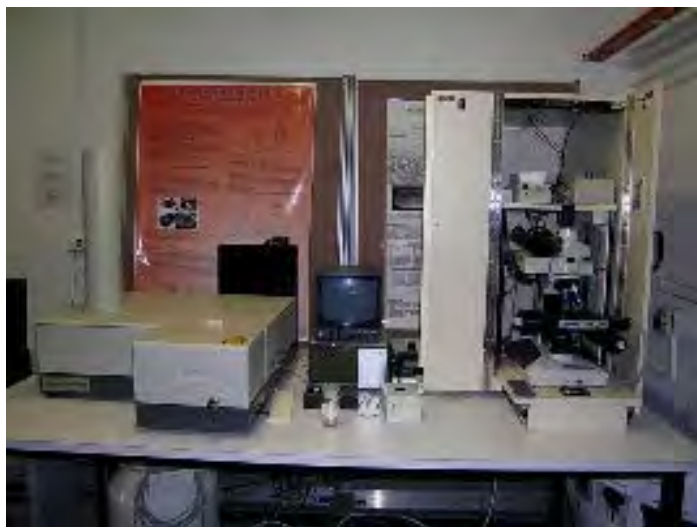

Figure 21. FT-Raman Spectrometer RFS100 with fibre-optically coupled microscope 
Usefulness of Raman spectroscopy. Like in the case of IR spectra, since a Raman spectrum typically contains a number of well-resolved spectral bands, the information available as the band positions, intensities and shapes (which, in fact, is complimentary to the one available from FT-IR spectroscopy) constitute a unique fingerprint of the molecule that can also be exploited for qualitative analysis.

The organic compounds contain functional groups with highly deformable electron clouds and are good scatterers of the radiation. The stretching vibrations of certain functional groups give rise to weak and undetectable IR signals whereas in Raman spectra these are quite strong and can provide useful information about the environment of the group in the molecule. In addition, Raman spectroscopy is very useful for measuring symmetric vibrations from the $\mathrm{C}-\mathrm{C}$ bonds. Further, since in Raman spectrum we measure the shifts in the frequencies rather than the frequencies themselves it makes regions below $600 \mathrm{~cm}^{-1}$ accessible, which otherwise cannot be explored in IR spectroscopy. This feature is quite significant for analyzing coordination compounds because the metal-ligand bonds generally absorb in this region.

\subsubsection{NMR and EPR spectroscopies}

NMR spectroscopy is one of the principal techniques used to obtain physical, chemical, electronic and structural information about molecules due to either the chemical shift, Zeeman effect, or the Knight shift effect, or a combination of both, on the resonant frequencies of the nuclei present in the sample. It can provide information on the topology, dynamics and three-dimensional structure of molecules in solution and the solid state.

${ }^{1} \mathrm{H}$-NMR spectra were registered from deuterated chloroform solution $\left(\mathrm{CDCl}_{3}\right)$ using a 400 MHz NMR spectrometer from Varian Mercury 400 (9.4 T) at $400.123 \mathrm{MHz} .{ }^{13} \mathrm{C}-\mathrm{NMR}$ and ${ }^{19} \mathrm{~F}$ NMR spectra were recorded at 100.6 and $376.5 \mathrm{MHz}$, respectively.

Electron paramagnetic resonance (EPR) is a technique for studying materials with unpaired electrons. The basic concepts of EPR are analogous to those of nuclear magnetic resonance but it is electron spins that are excited instead of the spins of atomic nuclei. By application of a strong magnetic field $B$ to material containing paramagnetic species, the individual magnetic moment arising via the electron "spin" of the unpaired electron can be oriented either parallel or anti-parallel to the applied field. This creates distinct energy levels for the unpaired electrons, making it possible for net absorption of electromagnetic radiation (in the form of microwaves) to occur. Detailed knowledge of the type and strength of pair interactions between high-spin metal ions is paramount to the understanding and design of molecular magnetic materials. Available EPR/ESR properties in ADF are: an $A$ tensor, nucleus-electron hyperfine interaction; a $g$-tensor, the $g$-factor deviation from $g_{\mathrm{e}}$ through spin-orbit coupling effects; and a $D$-tensor, zero-field splitting (ZFS) of electronic states with $S \geq 1$ (spin-orbit coupling part only). The $g$-factors and ZFS parameters are studied using high-order perturbation formulae. Contributions to $g$-factors and ZFS parameters from the charge-transfer mechanism can be calculated by different procedures.

EPR spectra were recorded with a Bruker EMX apparatus in the X band at a temperature range of 300-340 K, at the Unidad de Resonancia Magnética at Universidade de Santiago de Compostela, Spain. 

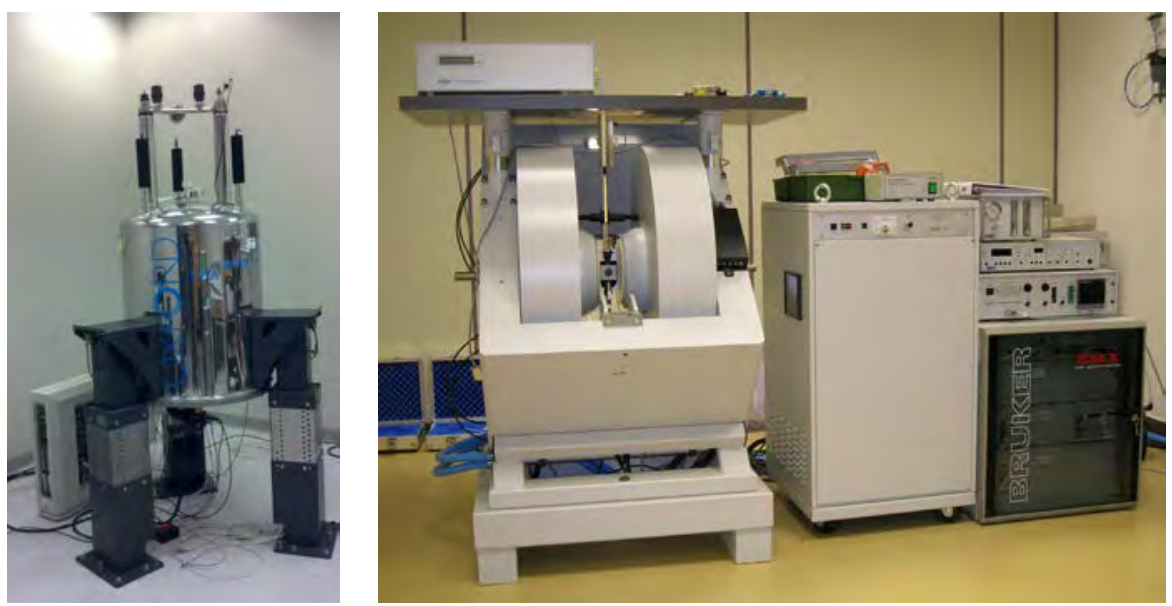

Figure 22. Varian Mercury 400 NMR spectrometer (left) and Bruker EMX EPR spectrometer (right)

\subsubsection{Optical absorption, excitation and photoluminescence studies}

\section{Absorption spectra}

The 200-800 $\mathrm{nm}$ range absorption spectra for the methanol-diluted solutions $\left(10^{-5}\right.$ and $\left.10^{-3} \mathrm{M}\right)$ were recorded with either a Cary 4000 Varian spectrophotometer or a Varian 5E spectrophotometeri.

For the samples in powder form, a Shimazdu UV-2450 UV-Vis spectrophotometer was used instead.
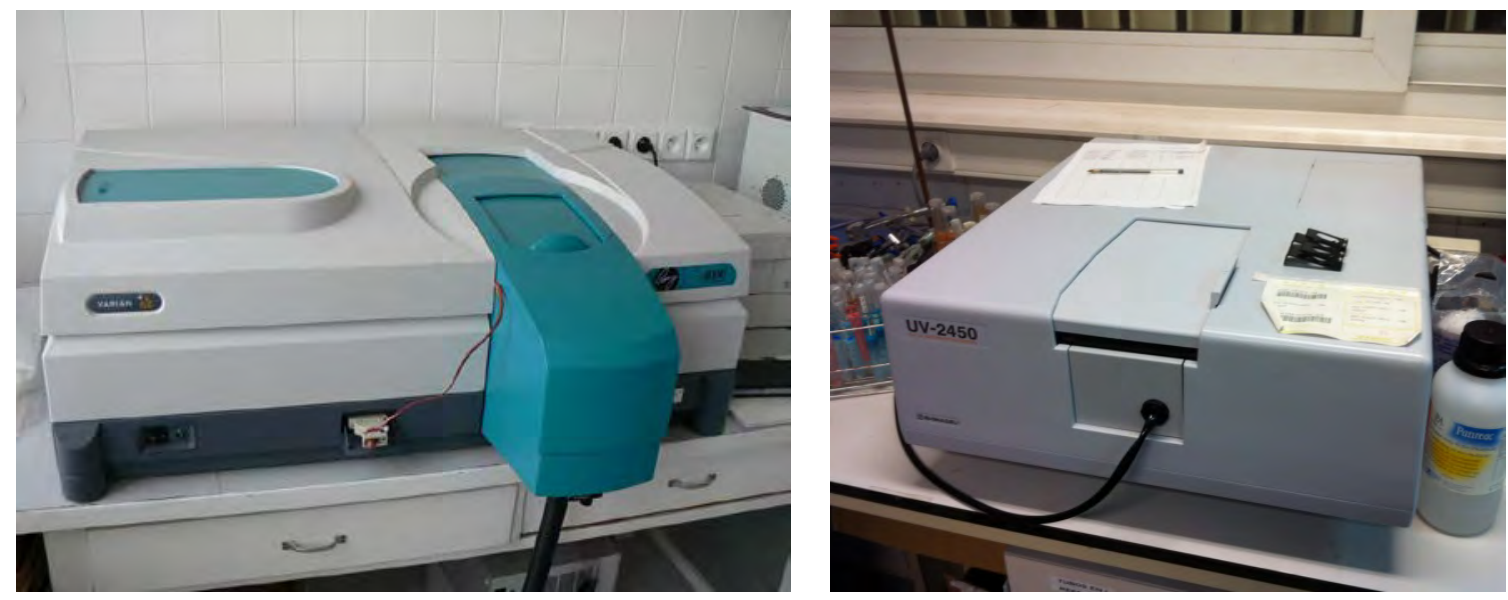

Figure 23. Cary 4000 Varian (left) and Shimazdu UV-2450 UV-Vis spectrophotometers (right)

The absorbance in the 900-3500 nm NIR range was recorded using a Varian 660-IR FT-IR spectrometer in $10 \mathrm{wt} \% \mathrm{KBr}$ pellets. All the spectra have been measured at room temperature and have been corrected by the spectral response of the experimental setups.

\footnotetext{
${ }^{i}$ In the particular case of [Er(acac) $)_{3}($ bath $\left.)\right]$, the excitation and emission spectra in chloroform were recorded with a modular spectrofluorimeter PTI Quantamaster QM-2000-6 at Eni Donegani Institute (Novara, Italy).
} 


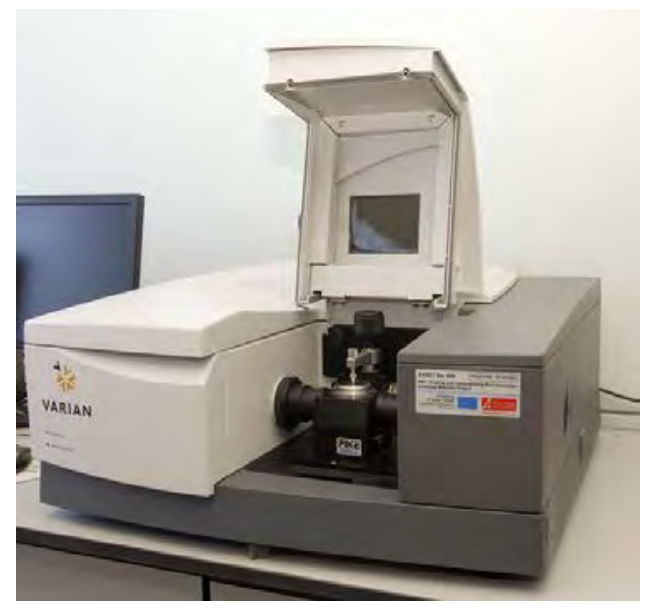

Figure 24. Varian 660-IR FT-IR spectrometer

\section{Photoluminescent emission}

For the samples in powder form, the visible photoluminescence spectrum was excited with a $405 \mathrm{~nm}$ laser diode, and collected with a Shamrock monochromator ( 0.303 focal length) with Andor Newton cooled CCD camera. For the samples in methanol solution, a Jobin-Yvon HR 460 monochromator coupled to a $77 \mathrm{~K}$ cooled CCD was used instead.
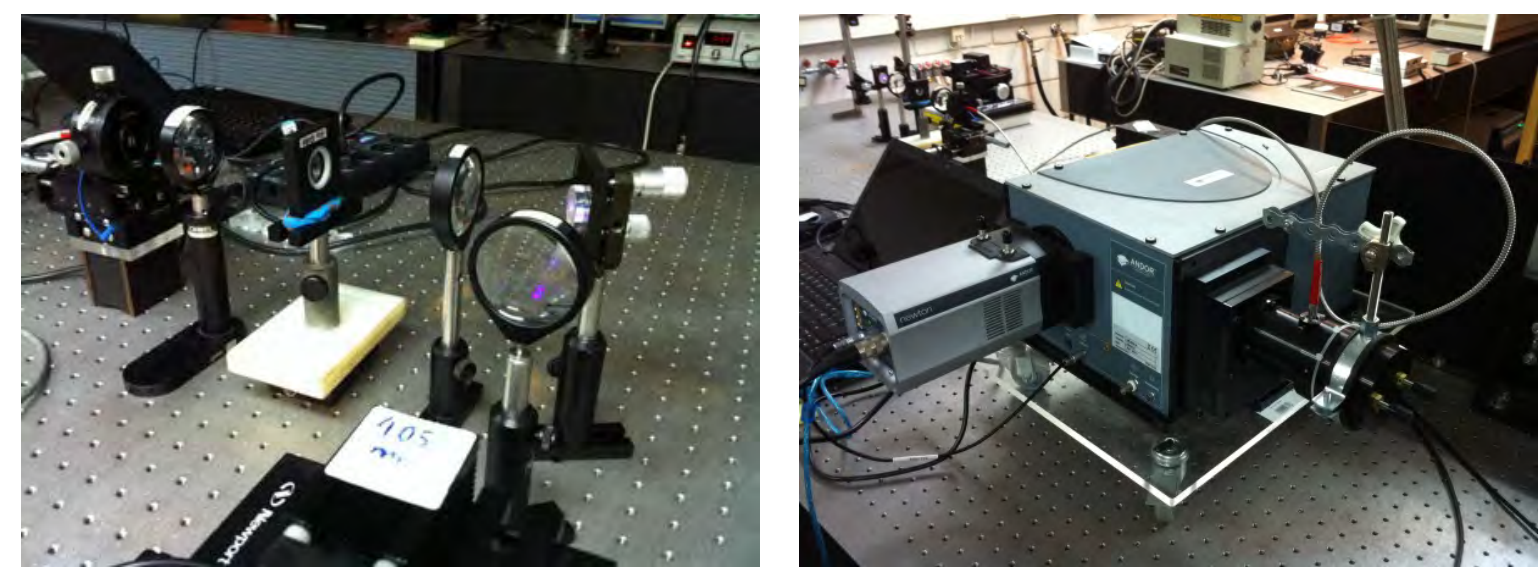

Figure 25. Setup for visible PL measurements and Shamrock monochromator with Andor Newton cooled CCD camera

The ligand lifetimes have been measured using an Edinburgh Instruments LifeSpec II fluorescence spectrometer, exciting the complex at $\lambda=405 \mathrm{~nm}$ with an Edinburgh Instruments EPL-405 picosecond pulsed diode laser (temporal pulse width at half maximum about 80 ps), and using Edinburg Instruments F900 acquisition software. 


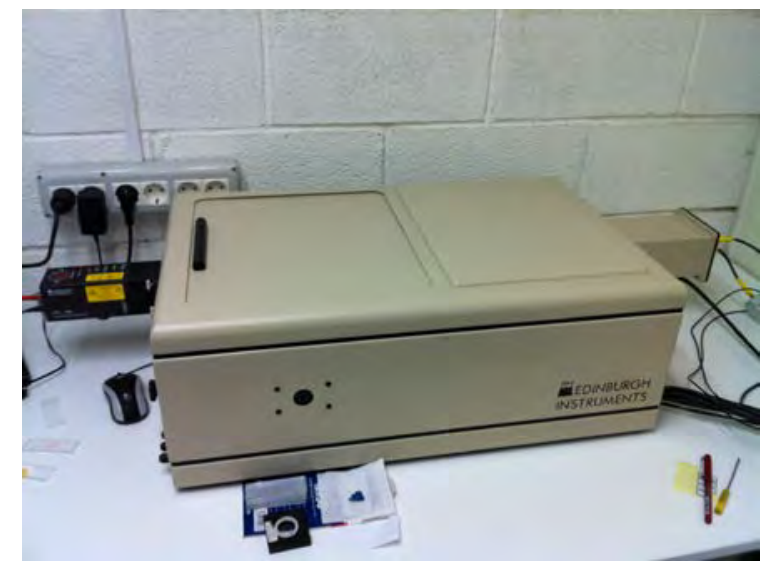

Figure 26. Edinburgh Instruments LifeSpec II fluorescence spectrometer

The NIR photoluminescence spectrum were excited at the ligand absorption at around 337 nm with a $\mathrm{N}_{2}$ laser, and at $532 \mathrm{~nm}$ resonantly with the ${ }^{4} \mathrm{I}_{15 / 2} \rightarrow{ }^{2} \mathrm{H}_{11 / 2}$ transition of the $\mathrm{Er}^{3+}$ absorption with a $500 \mathrm{~mW} \mathrm{cW}$ laser diode. NIR photoluminescence time decay measurements were excited at $980 \mathrm{~nm}$ resonantly with the ${ }^{4} \mathrm{I}_{15 / 2} \rightarrow^{4} \mathrm{I}_{11 / 2}$ transition of the Er ${ }^{3+}$ ions with an OPO (EKSPLA NT 342/3/UVE). The NIR luminescence was analyzed with a Peltier-cooled InGaAs Hamamatsu pin photodiode G5851-21 at $-25^{\circ} \mathrm{C}$ and a Horiba Jobin Yvon Triax 180 monochromator. Lifetimes were measured by using a Tektronix (model 3840 ) oscilloscope. All the spectra have been measured at room temperature and have been corrected by the spectral response of the experimental setups.
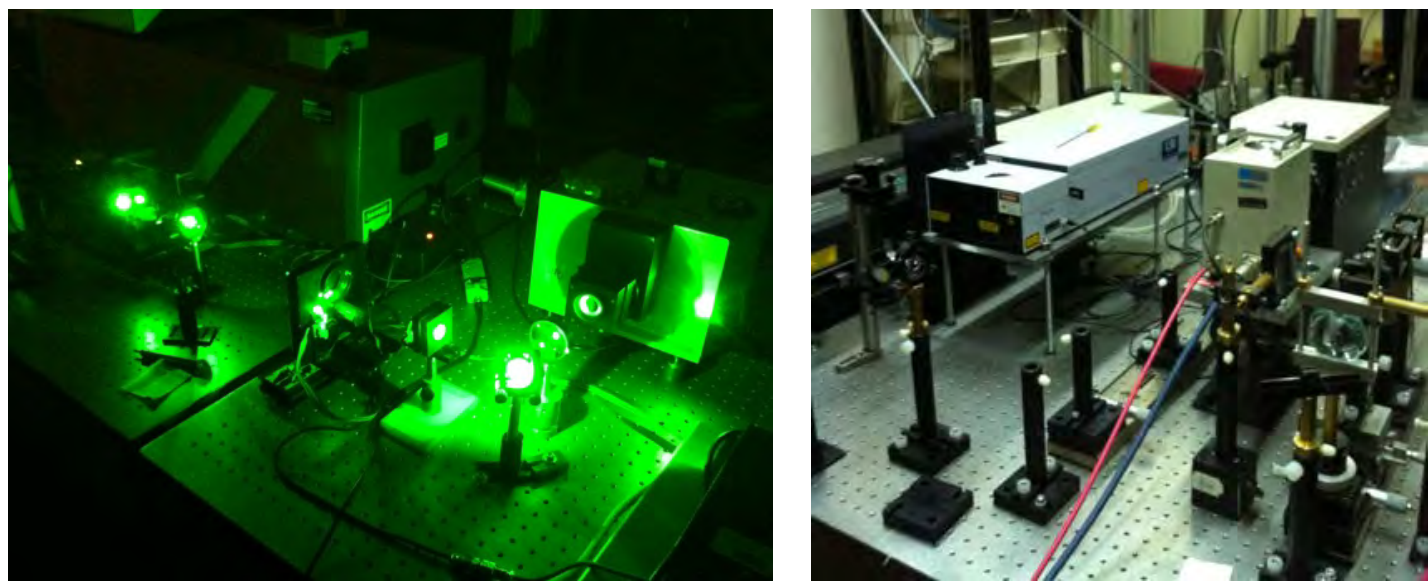

Figure 27. Setup for NIR PL spectra (left) and for Er-decay lifetime measurements (right)

For the quantitative PL emission intensity comparison in the NIR region, a Jobin Yvon FluoroLog-3 spectrofluorometer equipped with an InGaAs detector (liquid nitrogen cooled, range $0.8-1.6 \mu \mathrm{m})$ and a $450 \mathrm{~W}$ Xenon lamp $\left(\lambda_{\text {exc }}=350 \mathrm{~nm}\right)$ were usedi. The spectra were acquired with $1 \mathrm{~s}$ integration time at $1 \mathrm{~nm}$ step. Samples were analysed directly as powderk. Due to the low emission intensities of some samples, a $14 \mathrm{~nm}$ bandwidth was used in both excitation and emission.

In order to evidence the ligand sensitization effect, emission spectra upon direct excitation of the $\mathrm{Er}^{3+}$ hypersensitive transition at $522 \mathrm{~nm}$ were also collected using this equipment.

j These measurements were conducted by Prof. M. Salvalaggio at Eni Donegani Institute facilities.

k Additional spectra were collected for some samples dissolved in $\mathrm{CCl}_{3}$ and $\mathrm{D}_{2} \mathrm{O}$, which were analyzed in a $1 \mathrm{~cm}^{3}$ QS cuvette. 


\subsubsection{NLO measurements}

\section{Z-scan and P-scan techniques}

The Z-scan technique, proposed by Sheik-Bahae and coworkers in the late 80's [147], and its variation called P-scan [148], are particularly useful for the characterization of both the nonlinear refractive index $\left(n_{2}\right)$ and nonlinear absorption $(\beta)$ of optical materials, due to their optimal combination of simplicity and accuracy.

The Z-scan transmittance curve is a function of the sample position along the $z$-axis, perpendicular to the surface of the material. The nonlinear refractive transmittance curve shows a maximum and a minimum around the focus of the beam, located at $z=0$. If $n_{2}$ is positive (i.e., a focusing material), the nonlinearity presents a minimum for $z<0$ and a maximum for $z>0$. Conversely, if the sign is negative (defocusing case), the curve exhibits a maximum in the negative part of the $z$ axis and a minimum for positive $z[149,150]$. A more general theoretical study of the technique including samples of arbitrary thickness and higher-order effects is presented in ref. [151].

To measure the nonlinear absorptive response, the same Z-scan setup can be used in openaperture configuration [152], where the transmittance curve has to be symmetrical with a minimum at $z=0$.

In the case of the P-scan technique, the measurements are made by varying the incident power keeping the sample placed at the maximum of the Z-scan transmittance curve. A simple linear fit gives the result [153].

These techniques make it possible to know, for a given material, the magnitude and sign of both the real and imaginary parts of the third order susceptibility $\chi^{3}$.

\section{Theoretical background of Z-scan technique}

In a material that exhibits a third-order optical susceptibility $\chi^{3}$, the real and imaginary parts of the nonlinear refractive index can be written as [86]:

$$
\begin{aligned}
& n=n_{0}+n_{2} \cdot I \\
& \alpha=\alpha_{0}+\beta \cdot I
\end{aligned}
$$

where $n_{0}$ is the linear refractive index, $n_{2}$ is the nonlinear refractive index (Kerr coefficient), $\alpha_{0}$ is the linear absorbance, $\beta$ is the nonlinear absorbance and $I$ is the irradiance on focus.

May we consider a Gaussian laser beam propagating along the $z$-axis, and focused at $z=0$. A planar layer of the sample material is placed perpendicular to the $z$-axis and can be displaced around the focus. As the refractive index depends on the irradiance, the lensing effect will vary depending on the position. Thus, the variation with $z$ of the beam flux incident on a far circular aperture will display a characteristic peak-valley shape.

For a cubic nonlinearity, as in the previous formulae, a small phase change of the form $\Delta \phi_{0}(t)=\kappa \cdot \Delta n_{0}(t) \cdot L_{e f f}$ is obtained. Therefore, the transmittance curve through a small enough aperture can be written as [151]:

$$
T(z)=\frac{\int_{-\infty}^{\infty} P_{T}\left(\Delta \phi_{0}(t)\right) d t}{S \int_{-\infty}^{\infty} P_{i}(t) d t}
$$

where $P_{T}\left(\Delta \phi_{0}(t)\right)=c \epsilon_{0} n_{0} \pi \int_{o}^{r_{a}}\left|E_{a}(r, t)\right|^{2} r d r, P_{i}(t)=\pi \omega^{2} I_{0}(t) / 2$ and $S=1-\exp \left(-2 r_{a}^{2} /\right.$ $\left.\omega_{a}^{2}\right) . E_{a}(r, t)$ is the electric field pattern at the aperture. For ultrafast or instantaneous nonlinearity, the change in the nonlinear refractive index must be considered: $\left\langle\Delta n_{0}(t)\right\rangle=$ $\Delta n_{0}(t) / \sqrt{2}$. For cumulative effects, such as the cumulative thermal effect, $\left\langle\Delta n_{0}(t)\right\rangle=(A$. $F) / 2$ is used, where $A$ is a constant which depends on the nature of the nonlinearity and $F$ is the fluence [153]. 
If we consider the far-field condition in Eq. (3), the normalized transmittance for Z-scan can be obtained as follows [153]:

$$
T\left(z, \Delta \phi_{0}\right) \cong 1-\frac{4 \cdot \Delta \phi_{0} \cdot x}{\left(x^{2}+9\right)\left(x^{2}+1\right)}
$$

where $x=z / z_{0}$ and $\Delta \phi_{0}=\kappa \cdot n_{2} \cdot I_{0} \cdot L_{e f f}$, being $\kappa$ the wavenumber.

The nonlinear absorbance curve is obtained with open-aperture Z-scan, so that the system is insensitive to the nonlinear refraction. Under these conditions, the transmittance curve is expected to have a minimum centered at $z=0$. Thus, let us consider a medium with the nonlinearity described in Eq. (2). Then, the normalized transmittance curve for the nonlinear absorbance can be written as [153]:

$$
T(z, S=1)=\frac{\sum_{m=0}^{\infty}\left[-q_{0}(z, 0)\right]}{(m+1)^{3 / 2}}
$$

where $q_{0}=\beta \cdot I_{0}(t) \cdot L_{e f f} /\left(1+z^{2} / z_{0}^{2}\right)$.

\section{Equipment}

The experimental setup (Figure 28) includes a titanium-sapphire laser source (Tsunami, Spectra Physics) operating at a wavelength of $810 \mathrm{~nm}$. The temporal duration of the pulses is $80 \mathrm{fs}$ and the repetition rate is $80.75 \mathrm{MHz}$. The maximum average power of this laser is $2 \mathrm{~W}$. The system was calibrated using carbon disulfide $\left(\mathrm{CS}_{2}\right)$, a very well characterized material known to be a good reference. The $\mathrm{CS}_{2}$ measurements are in agreement with those published by other groups, such as Ganeev et al. [154].

The first part of the system consists of two lenses, labeled $L_{1}$ and $L_{2}$. These lenses are used to collimate and select the beam size incident on $L_{3}$ with focal length $f=15 \mathrm{~cm}$, which focuses the laser on the sample $(S)$. The waveplate $\left(W_{1}\right)$ and the polarizing-cube $\left(P C_{1}\right)$ are used to change the incident power at the sample. The beam waist in the focal plane of $L_{3}$ has a radius of 6 $\mu \mathrm{m}$. The sample is placed on a moving track that enables the displacement of the sample along the $z$-axis around the focal point.

With the second pair of polarizing-cube $\left(P C_{2}\right)$ and waveplate $\left(W_{2}\right)$, the beam leaving the sample is split between the two detectors which are at the end of each path. The first signal comes from a fast photodiode $(P H)$ with a response time of nanoseconds, located behind a diaphragm $\left(A P_{1}\right)$, which is used to obtain the experimental curve for the calculation of the nonlinear refractive index. The second measurement is performed with a powermeter $(P W)$ placed in front of a lens $\left(L_{5}\right)$ that focuses the beam in order to measure the nonlinear absorbance.

For the preparation of the samples solutions, the complexes were dissolved in methanol. The solution was then placed inside a quartz cuvette (which transmits $88 \%$ of incident light) with $1 \mathrm{~mm}$ thickness. This thickness is large enough so that the medium does not recover the initial conditions between each laser pulse, and thus the material displays cumulative thermal effects leading to a defocusing response.

So as to characterize the focusing regime of the material, a small amount of each sample was placed between two glass slides and kept under pressure for several days in order to achieve a homogeneous thin layer. Subsequently, the sample thickness was determined with a profilometer. The thin samples prepared for this study have a thickness of $5 \mu \mathrm{m}$. 

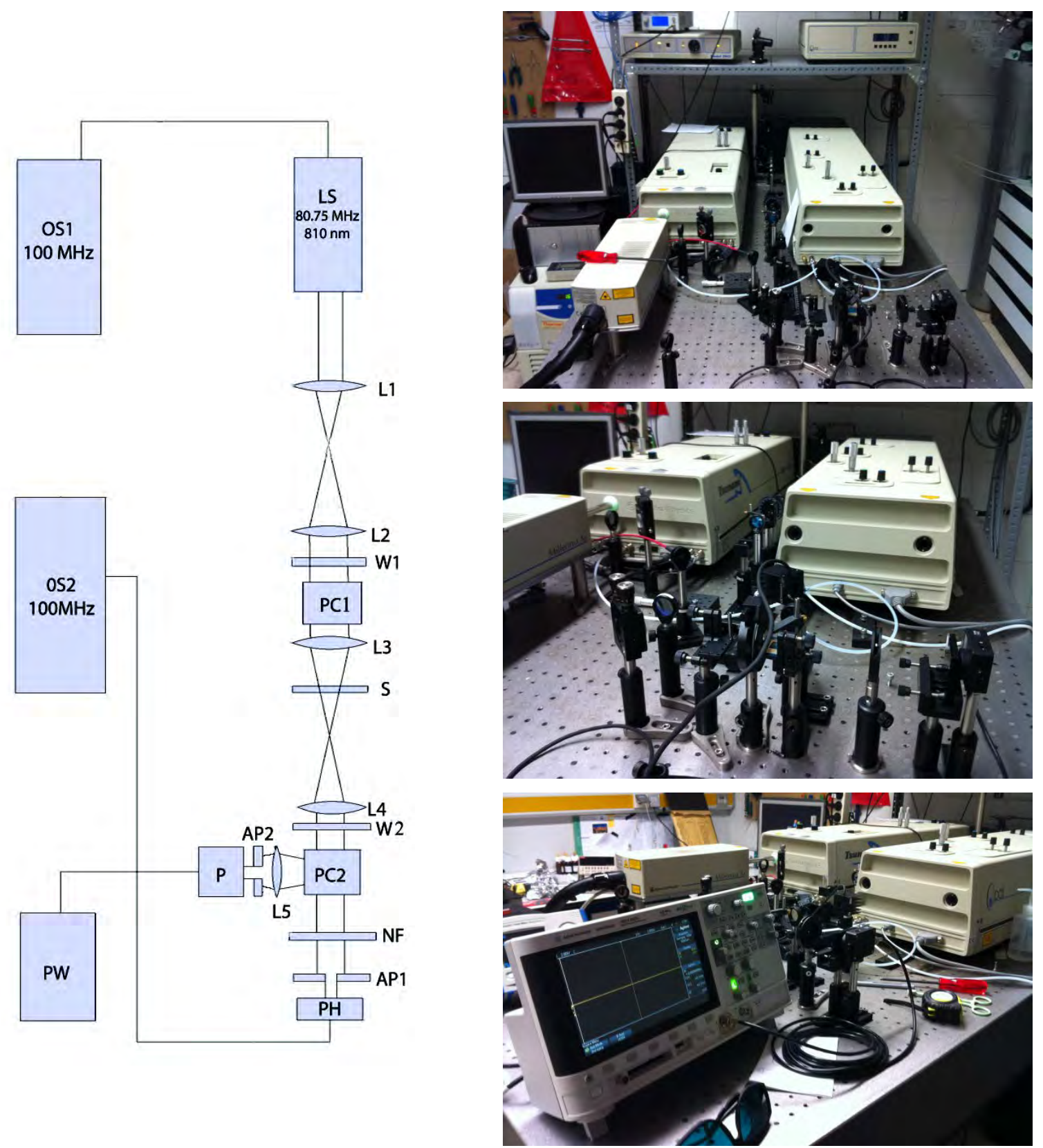

Figure 28. Sketch of the experimental setup (left), general view (top right), closer view of the second part of the system (center right) and oscilloscope connected to the photodiode (bottom right).

\subsubsection{Magnetic measurements}

The magnetic measurements aimed at studying the SMM/SIM behaviour have been conducted at the Solid State Group, UCQR, IST/CTN at the Instituto Superior Técnico in Bobadela, Portugal.

Magnetic measurements were performed in polycrystalline powder samples weighing about 20-40 mg. Measurements were taken using a 6.5 T S700X SQUID magnetometer (Cryogenic Ltd.) in the temperature range 1.8-300 $\mathrm{K}$ under several applied magnetic fields from $100 \mathrm{G}$ to $1 \mathrm{kG}$. This unit (S700X from Cryogenic Ltd) is one of the most sensitive instruments for DC and AC measurements of magnetic properties as a function of magnetic field and temperature, with a resolution down to $10^{-8} \mathrm{emu}$. The S700X comprises a standard variable 
temperature insert operating in the range $1.5-400 \mathrm{~K}$ inside a $7 \mathrm{~T}$ magnet. The temperature range of operation can be extended down to $0.3 \mathrm{~K}$ with a ${ }^{3} \mathrm{He}$ insert. In addition to static magnetization, the AC magnetic susceptibility can be measured from 0.01 to $500 \mathrm{~Hz}$. The presence of a secondary pick-up coil in the main magnet assembly allows the simultaneous measurement of both axial and transverse components of the magnetization. This transverse axis facility and a motor to rotate the sample, in steps of 0.1 degree, enable the determination of sample anisotropic effects. This equipment is mainly dedicated to study samples with relatively low moments such as molecular materials, small single crystals and films.

Above $1.8 \mathrm{~K}$ field dependent magnetization up to $5 \mathrm{~T}$ at different temperatures and $\mathrm{AC}$ susceptibility measurements were taken using a 12 Tesla multicharacterization system MagLab 2000 (Oxford Instruments) by using a a 5 Oe oscillating field in the $30-10000 \mathrm{~Hz}$ frequency range under zero and 500 Oe static fields. Additional isothermal AC susceptibility measurements, $\chi_{A C}=f(\omega)$, were taken in the $10-10000 \mathrm{~Hz}$ frequency range, within 1.8 and $6 \mathrm{~K}$, the temperature range in which the relaxation time reaches a maximum. The Oxford Inst. magnetic properties probe is designed to make AC susceptibility measurements in conjunction with a lock-in amplifier and AC current source and DDC moment measurements in conjunction with a storage voltmeter. The probe consists of 2 main parts, the coil set and the extraction head which contains a reciprocating mechanical assembly. The probe is suitable to be used in a variable temperature insert sample space. Samples are top loaded on a flexible sample rod to minimize the overall system height and detachable tips are supplied to allow a range of different shapes to be attached with varnish, vacuum grease or PTFE tape. The tips are made of a thermal plastic in order to reduce the background signal from them. Samples mounted in a transparent gelatin capsule placed at the end of a Kapton straw. A Motor Drive Unit is used to control the extraction head to position the sample accurately at the center of both pick up coils for ACS or to move the sample at a selectable speed trough the coils for DC extraction. The sense coil set carries a calibrated ceramic oxy-nitride temperature sensor to monitor the stability of the sample space region.

All the data were corrected for diamagnetic contributions from the core diamagnetism estimated using Pascal's constants to give $\chi_{D}=-811.4 \times 10^{-6} \mathrm{emu} / \mathrm{mol}$.
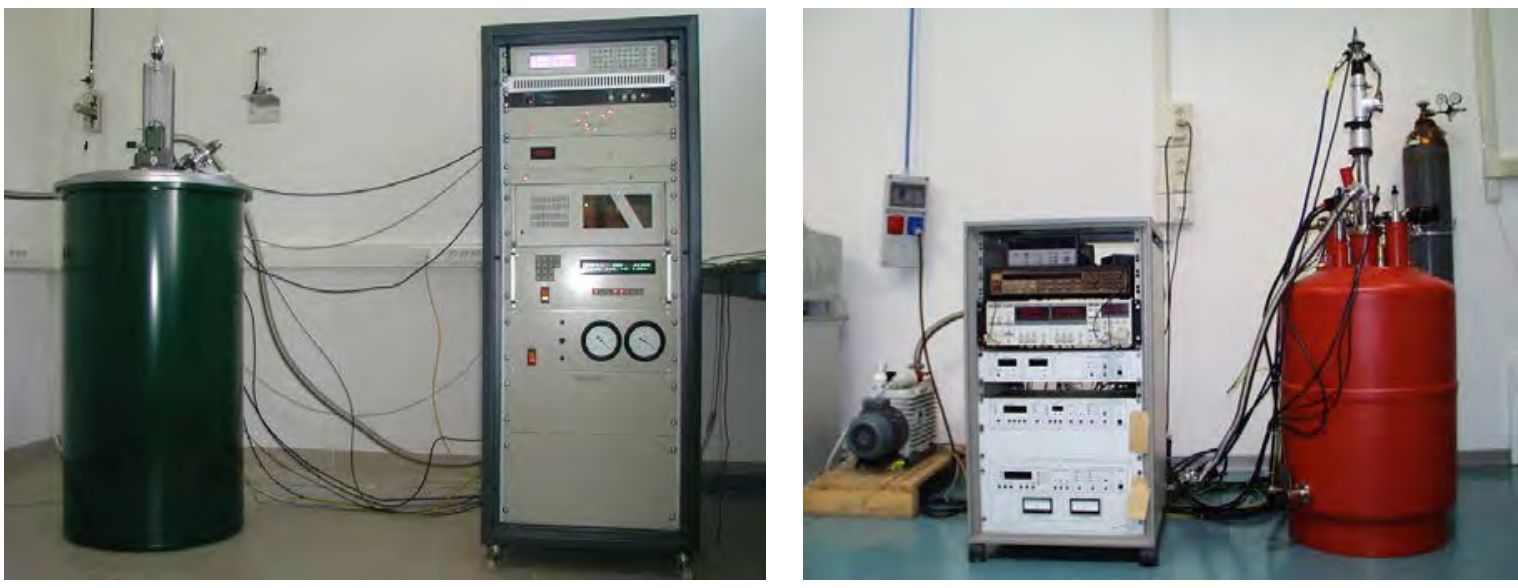

Figure 29. S700X SQUID magnetometer (left) and multicharacterization system MagLab 2000 (right) 


\subsection{Results and discussion}

\subsubsection{Determination of crystal structures by X-ray diffraction}

\section{Preliminary X-ray powder diffraction patterns}

The experimental diffraction pattern for those $\mathrm{Er}^{3+}$ complexes which have been elucidated by single crystal X-ray diffraction are shown in Figure 31, together with the simulated powder patterns obtained from the single crystal structure using PLATON [144]. The experimental diffractograms show a background higher for low theta angles as expected from the diffuse scattering of X-rays by glass and air, a common characteristic when using rotating capillaries in a Debye-Scherrer geometry.

In general, there is a good match between simulated and experimental diffractograms: the peaks appear at the predicted theta angles at the same relative intensities, but some bathophenanthroline complexes show broader lines. There are many factors that determine the width of a diffraction peak: instrumental broadening, disorder, small crystallite size, the presence of defects to the perfect lattice or differences in strain in different grains, but the crystallite size is usually the sole factor of interest. The broadening could be analysed using old mathematics (the Debye function) or more rigorously using new approaches (such as cubic harmonic function method or the Warren-Averbach method), but we believe that the broadening exhibited by the samples may be due to poor crystallinity. With regard to the intensities, it is stated that non-random distributions of crystallites, such as those resulting from platy or needle-like crystals, can change the relative intensities of the diffraction peaks. In fact, the diffracted intensity for a polycrystalline sample is given by the incoherent sum of scattered intensities over the ensemble of crystallites.

Powder diffraction patterns below show that, regardless of the higher or lower crystallinity, all the materials synthesized contain the same structure as the small single crystals used for single-crystal X-ray diffraction.

In the particular case of [ $\operatorname{Er}(\mathrm{acac})_{3}$ (bath)], for illustrative purposes, a quantitative analysis has been conducted so as to measure the goodness-of-fit: a Rietveld refinement [155] was performed with Fullprof software [156] using the powder-diffraction data measured at room temperature. The overall parameters such as cell parameters, $2 \theta$ zero, scale factor, full-width at half-maximum and asymmetry parameter were allowed to refine in the range of 5 to $50^{\circ}$, with a final Bragg reliability factor of $14.4 \%$. As expected, the good agreement between experimental and calculated patterns (Figure 30) confirms the high purity of the sample.

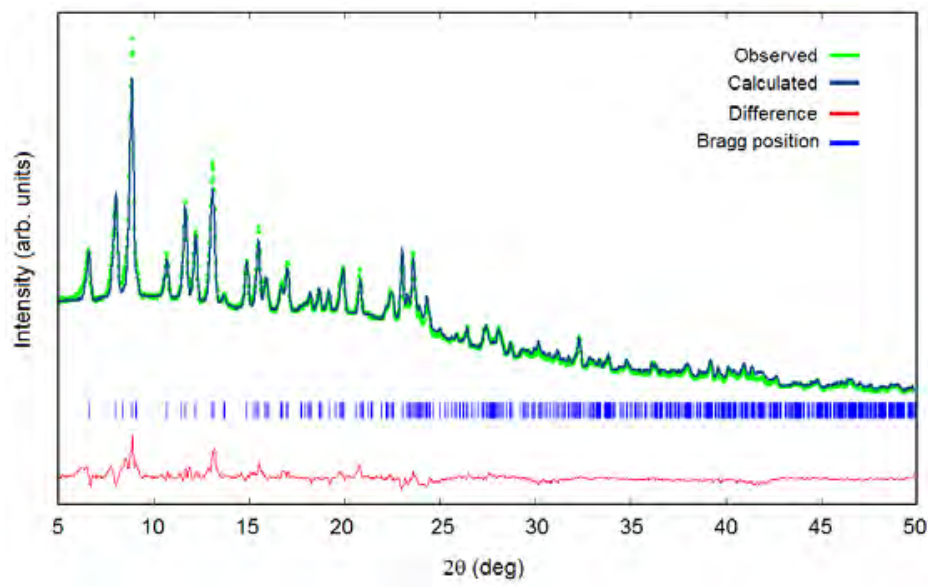

Figure 30. [ $\operatorname{Er}(\mathrm{acac})_{3}($ bath)]. Results from a least-squares fit using the Rietveld method: experimental powder diffraction pattern (green); simulated diffraction pattern using the Rietveld method (darkblue); difference between the observed and calculated intensities (red), and corresponding Bragg positions (blue). 


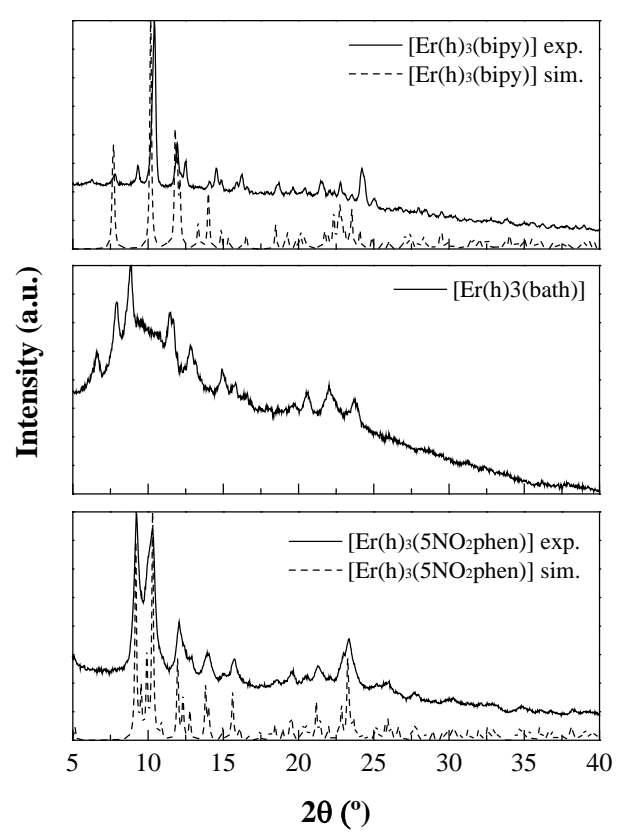

[Er(h) $)_{3}(\mathrm{~N}, \mathrm{~N}-$ donor $\left.)\right]$

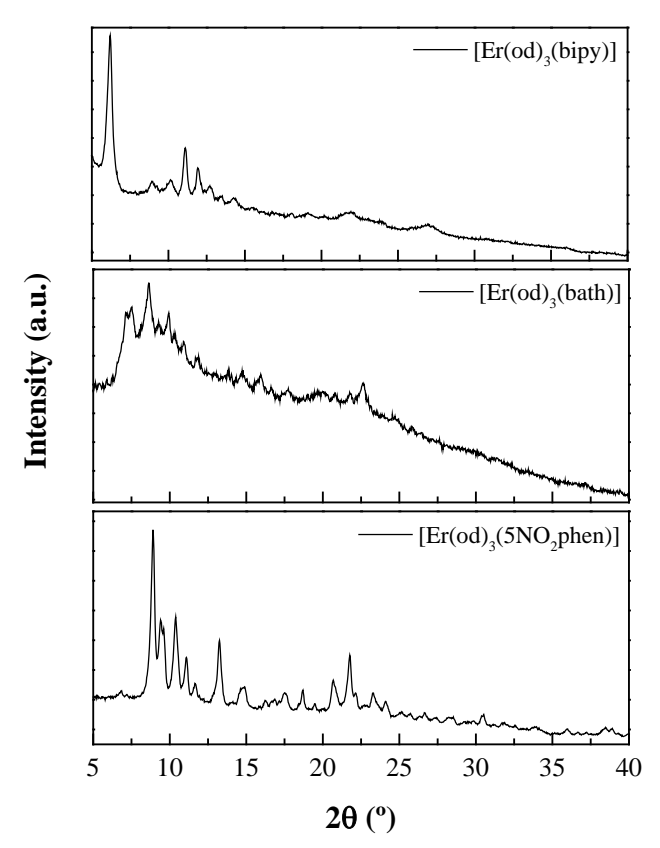

$\left[\operatorname{Er}(\mathrm{od})_{3}(\mathrm{~N}, \mathrm{~N}-\right.$ donor $\left.)\right]$

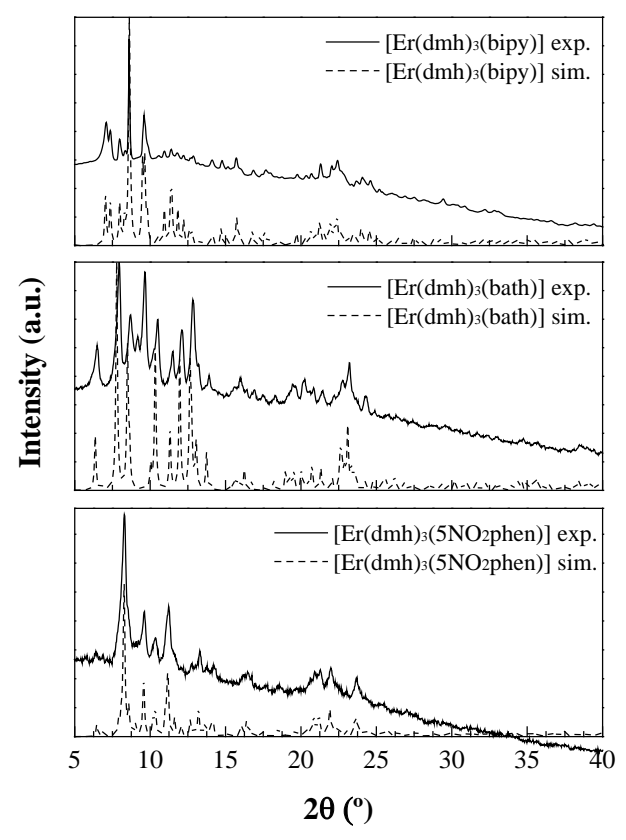

[Er(dmh) $)_{3}(\mathrm{~N}, \mathrm{~N}-$ donor $\left.)\right]$

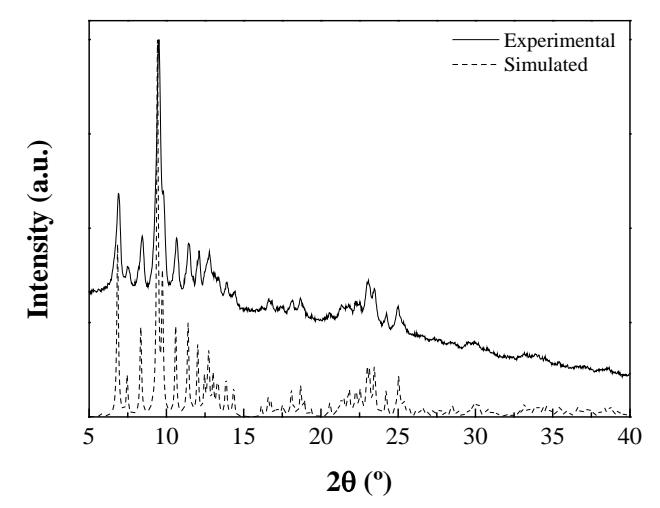

[Er(hd) $)_{3}$ (bipy)]

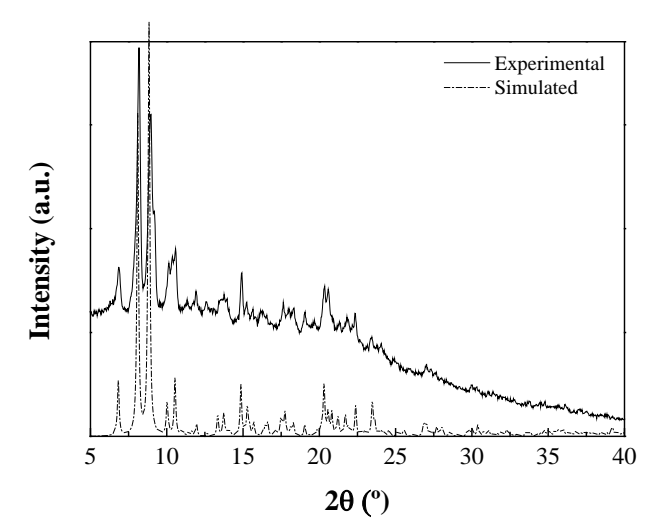

[Er(thd) $)_{3}$ (bath)] 


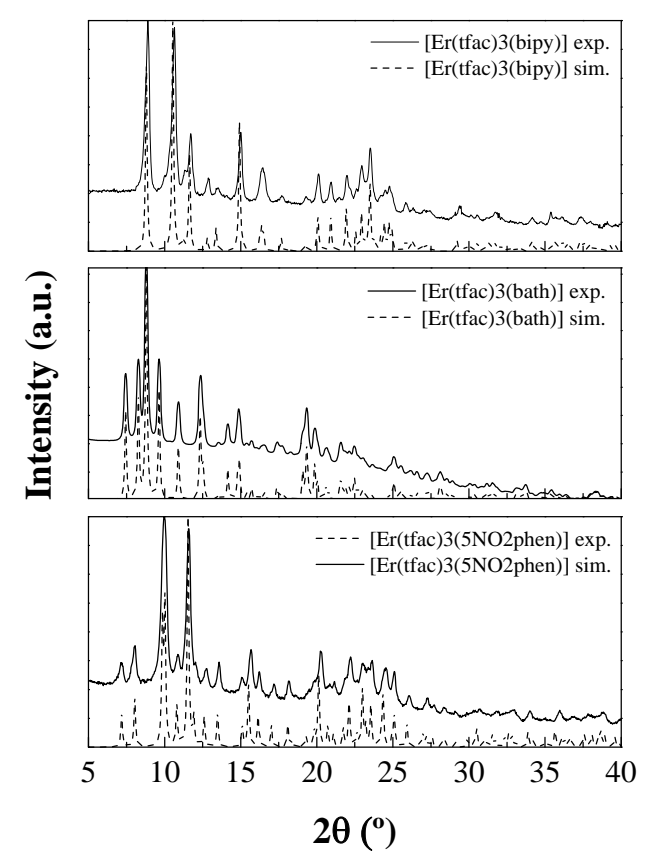

[Er(tfac) $3(\mathrm{~N}, \mathrm{~N}-$ donor $)]$

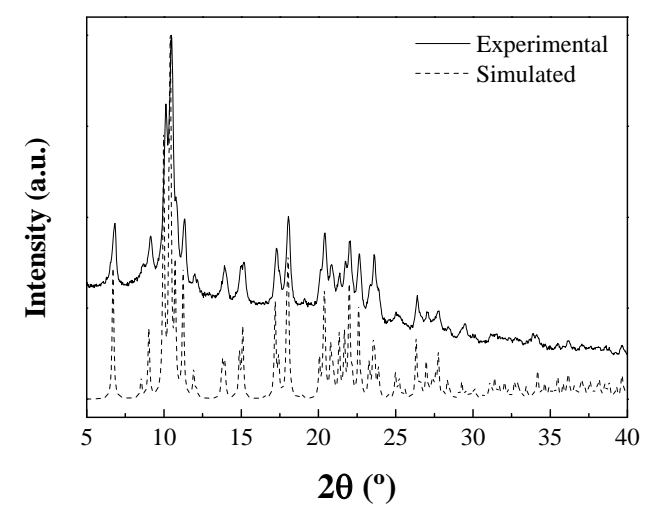

[Er(tfa)3(bipy)]

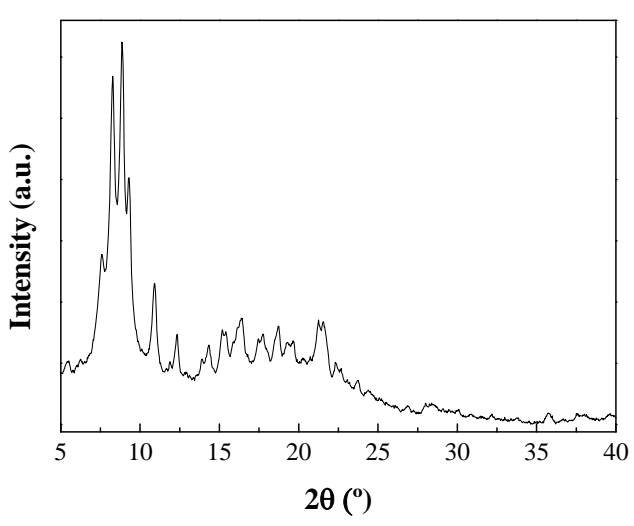

[Er(fod) $)_{3}$ (bath)]

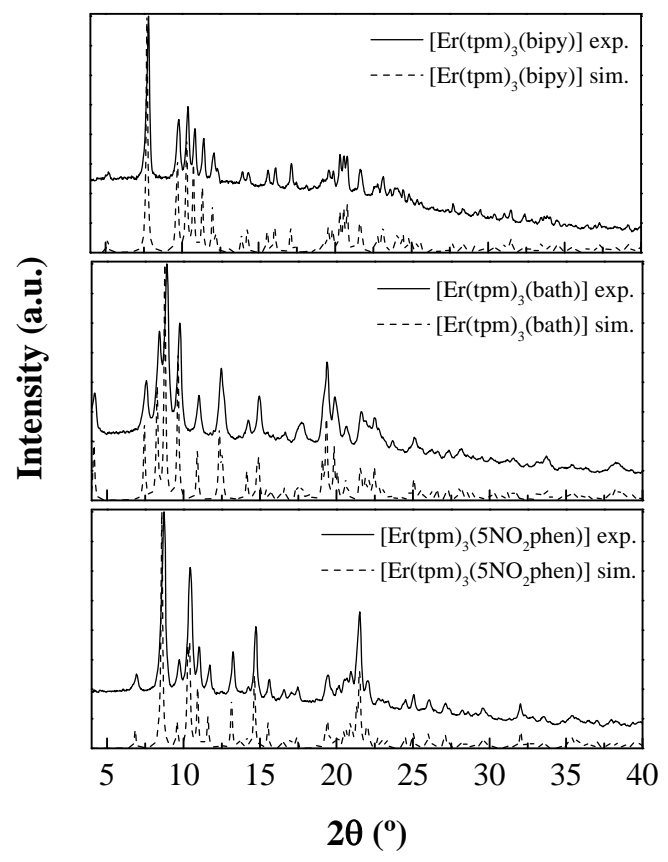

[Er(tpm) $)_{3}(\mathrm{~N}, \mathrm{~N}-$ donor $\left.)\right]$

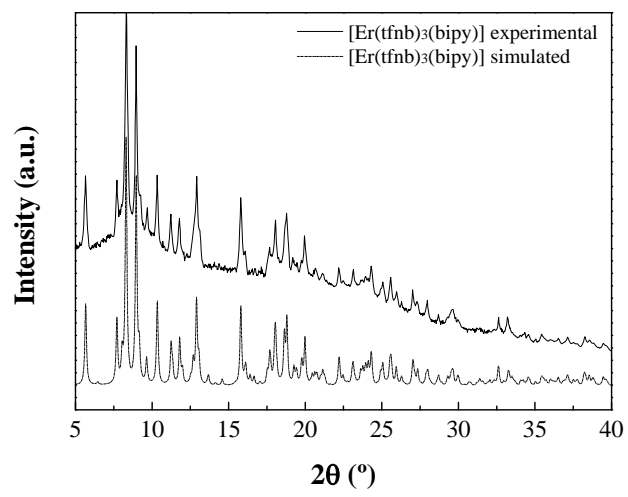

[Er(tfnb) $)_{3}$ (bipy)]

Figure 31. Experimental (solid line) vs. simulated (dashed line) X-ray powder diffraction patterns for selected Er(III) complexes 


\section{Single-crystal X-ray diffraction}

A brief summary of the unit cell and refinement parameters, together with selected bond lengths and angles, is reported below for most complexes. Atomic coordinates, thermal parameters, and bond lengths and angles have been deposited at the Cambridge Crystallographic Data Centre (CCDC).

\section{Structures}

1. [Er(acac) $)_{3}$ (bath)] crystallizes in the triclinic space group $P-1$, with cell parameters

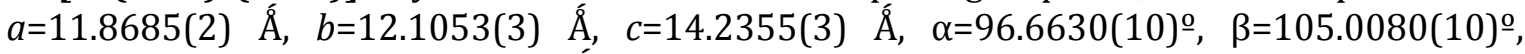
$\gamma=110.1770(10)^{\circ}, V=1806.05(7) \AA^{3}$ (Figure 32 , Table 1). The lanthanide ion is eightcoordinated by six $\mathrm{O}$ atoms from the acetylacetonate anions and two $\mathrm{N}$ atoms from the bathophenanthroline molecule, forming a distorted square-antiprismatic structure in which the set $\mathrm{N} 1, \mathrm{~N} 2,03,04$ and the set $01,02,05,06$ form two approximate squares, respectively (Figure 32). The trivalent erbium ion lies approximately in the middle of the antiprism with a distance of $1.39 \AA$ to the face containing the $\mathrm{N}$ atoms and $1.19 \AA$ to the opposite face containing exclusively oxygens. The angle between the least-squares planes of the square faces is just $2^{\circ}$. The Er-N distances are 2.545(2) and 2.550(2) Å and the Er-O distances are in the range 2.289(2)-2.313(2) Å. The N-Er-N bite angle is 63.6․ Selected geometric parameters are given in Table 2.

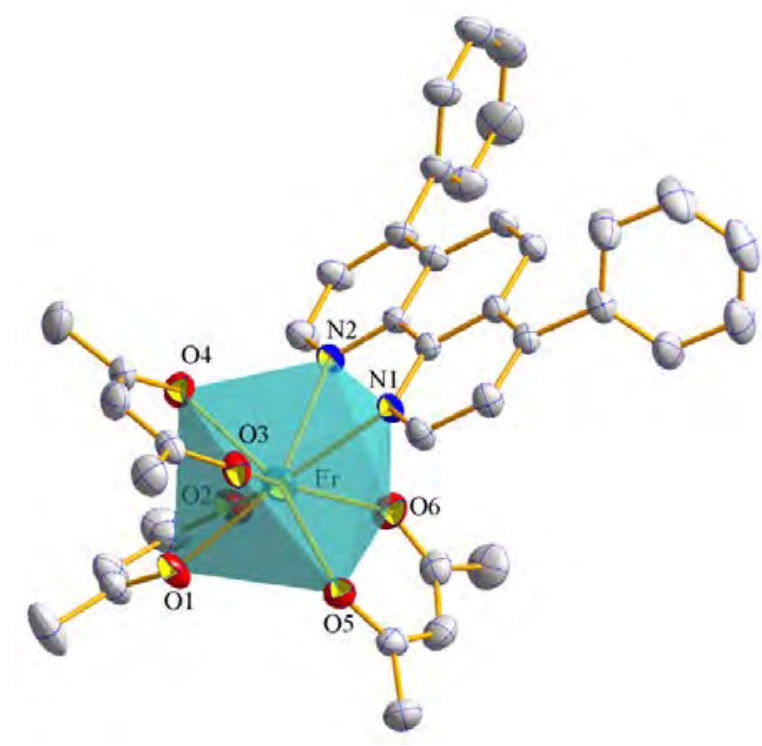

Figure 32. A perspective view of the eightfold coordination polyhedron of [Er(acac) $\left.)_{3}(\mathrm{bath})\right]$, possessing a distorted square-antiprismatic geometry. Displacement ellipsoids are drawn at the $30 \%$ probability level. Hydrogen atoms were omitted for clarity.

The six-membered chelate rings formed by Er(III) and the acetylacetonate ligands possess nearly planar geometries. The chelating ring associated with bathophenanthroline also possesses nearly planar geometry. The slight distortion of the $\beta$-diketonate rings occurs along the axis connecting the two oxygen atoms of the ligand. The bathophenanthroline molecules are not planar as the terminal phenyl rings are rotated 61.36(15) and 70.29(17)o with respect to the central phenantrhroline unit.

The comparison of Er-O bond lengths in $\left[\operatorname{Er}(\mathrm{acac})_{3}(\mathrm{bath})\right]$ with the corresponding bonds lengths in $\left[\operatorname{Er}(\mathrm{acac})_{3}\right]$ is directly related to the interactions of this mono ligand complex with the additional ligand, bathophenathroline, whose introduction in the coordination sphere of 
erbium stimulates redistribution of electronic density in the ternary complex. The Er-bath bonding is not purely electrostatic and its formation is accompanied by a decrease in the Eracac bond energy. Consequently, the Er-O bond lengths in $\left[\operatorname{Er}(\mathrm{acac})_{3}(\mathrm{bath})\right]$ are larger than that in $\left[\operatorname{Er}(\mathrm{acac})_{3}\right]$.

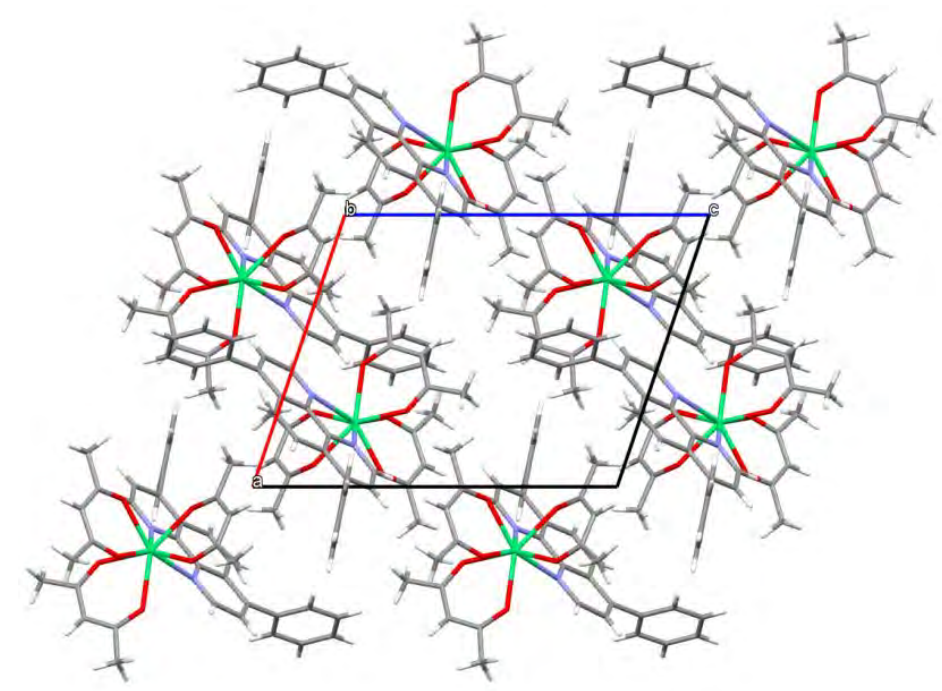

Figure 33. Packing diagram of [Er(acac) $)_{3}$ (bath)]

Table 1. Crystal data and structure refinement for $\left[\operatorname{Er}(\text { acac })_{3}(\right.$ bath $\left.)\right]$

\begin{tabular}{|c|c|}
\hline Complex & {$\left[\operatorname{Er}(\mathrm{acac})_{3}(\mathrm{bath})\right]$} \\
\hline Empirical formula & $\mathrm{C}_{39} \mathrm{H}_{37} \mathrm{ErN}_{2} \mathrm{O}_{6}$ \\
\hline Formula weight & 796.97 \\
\hline Temperature $(\mathrm{K})$ & 293(3) \\
\hline Wavelength $(\AA)$ & 0.71073 \\
\hline Crystal system & Triclinic \\
\hline Space group & $P-1$ \\
\hline$a(\AA)$ & $11.8685(2)$ \\
\hline$b(\AA)$ & $12.1053(3)$ \\
\hline$c(\AA)$ & $14.2355(3)$ \\
\hline$\alpha(\underline{\mathrm{q}})$ & $96.6630(10)$ \\
\hline$\beta(\stackrel{\circ}{)}$ & $105.0080(10)$ \\
\hline$\gamma(\underline{0})$ & $110.1770(10)$ \\
\hline Volume $\left(\AA^{3}\right)$ & $1806.05(7)$ \\
\hline$Z$ & 2 \\
\hline Calculated density $\left(\mathrm{g} \mathrm{cm}^{-3}\right)$ & 1.466 \\
\hline Absorption coefficient $\left(\mathrm{mm}^{-1}\right)$ & 2.370 \\
\hline$F(000)$ & 802 \\
\hline Crystal size $\left(\mathrm{mm}^{3}\right)$ & $0.26 \times 0.18 \times 0.10$ \\
\hline$\theta$ range for data collection $(\underline{o})$ & $1.93-30.64$ \\
\hline Index ranges & $-17<h<17 ;-17<k<17 ;-20<l<20$ \\
\hline Reflections collected & 46456 \\
\hline Independent reflections & 11070 \\
\hline Completeness to $2 \theta=55^{\circ}$ & $99.9 \%$ \\
\hline Refinement method & Full matrix LS on $\mathrm{F}^{2}$ \\
\hline Data/restrains/parameters & $9229 / 0 / 439$ \\
\hline Goodness-of-fit on $\mathrm{F}^{2}$ & 1.047 \\
\hline Final $R$ indices $[I>2 \sigma(I)]$ & $R=0.0276 ; \mathrm{w} R=0.0651$ \\
\hline$R$ indices (all data) & $R=0.0401 ; \mathrm{w} R=0.0724$ \\
\hline Largest diff. peak and hole & $-0.549 / 1.132$ \\
\hline
\end{tabular}


Table 2. Selected distances and angles $\left(\AA \stackrel{\mathrm{A}}{{ }^{\circ}}\right)$ for $\left[\operatorname{Er}(\text { acac })_{3}(\right.$ bath $\left.)\right]$

\begin{tabular}{cccc} 
Bond & Distance & Bonds & Angle \\
\hline Er1-N1 & $2.550(2)$ & 01-Er1-05 & $78.27(7)$ \\
Er1-N2 & $2.5454(19)$ & 01-Er1-04 & $82.78(8)$ \\
Er1-01 & $2.3038(18)$ & 05-Er1-03 & $81.08(7)$ \\
Er1-02 & $2.2897(19)$ & 04-Er1-02 & $80.29(8)$ \\
Er1-03 & $2.3114(18)$ & 03-Er1-02 & $141.78(7)$ \\
Er1-04 & $2.306(2)$ & 01-Er1-N1 & $146.72(6)$ \\
Er1-05 & $2.2890(19)$ & 06-Er1-N1 & $77.17(8)$ \\
Er1-06 & $2.313(2)$ & 06-Er1-N2 & $68.90(7)$ \\
Er1-N avg. & 2.548 & 05-Er1-N2 & $134.02(7)$ \\
Er1-0 avg. & 2.302 & & \\
\hline
\end{tabular}

2. [Er(dbm) $)_{3}$ (bipy)] crystallizes in a monoclinic unit cell with the $P 2_{1} / c$ space group. The unit cell contains four complexes (Figure 34, Table 3). Within each complex, the Er(III) ion is coordinated in a distorted square-antiprismatic geometry by six 0 atoms from the dibenzoylmetanate anions and two $\mathrm{N}$ atoms from the 2,2'-bipyridine molecule.The trivalent erbium ion lies approximately in the middle of the antiprism with a distance of $1.37 \AA$ to the face containing the $\mathrm{N}$ atoms and $1.17 \AA$ to the opposite face containing exclusively oxygens. The angle between the least-squares planes of the square faces is 2.07(9)‥ The Er-N distances are 2.545(2) and 2.566(2) $\AA$ and the Er-O distances lie in the range 2.2402(18)$2.3079(18) \AA$. The N-Er-N bite angle is 62.86(9)‥ Selected geometric parameters are given in Table 2 . The chelating rings are approximately planar. The crystal structure is stabilized by $\mathrm{C}-\mathrm{H} . . . \pi$ and $\pi \ldots \pi$ interactions.

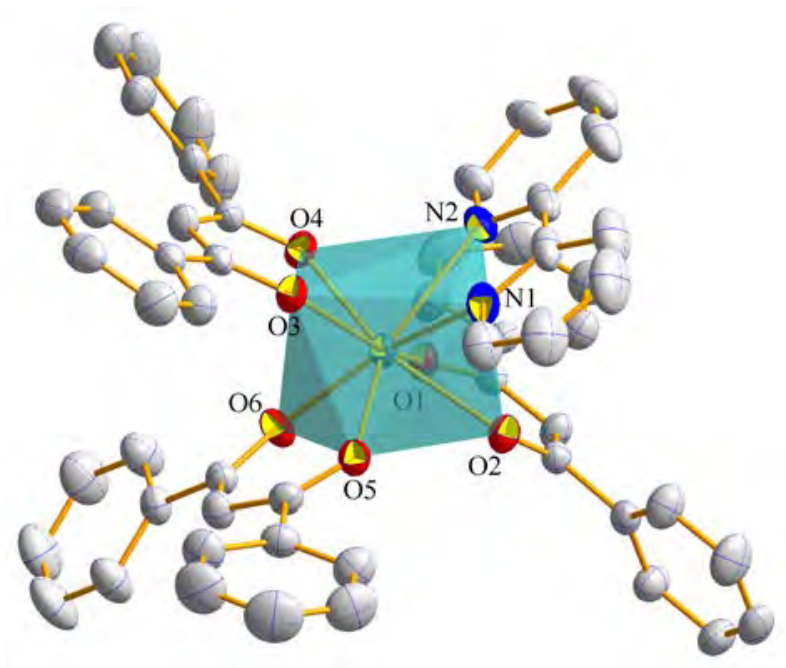

Figure 34. A perspective view of the eightfold coordination square antiprism of [ $\left.\operatorname{Er}(\mathrm{dbm})_{3}(\mathrm{bipy})\right]$. Displacement ellipsoids are drawn at the $30 \%$ probability level. Hydrogen atoms were omitted for clarity. 


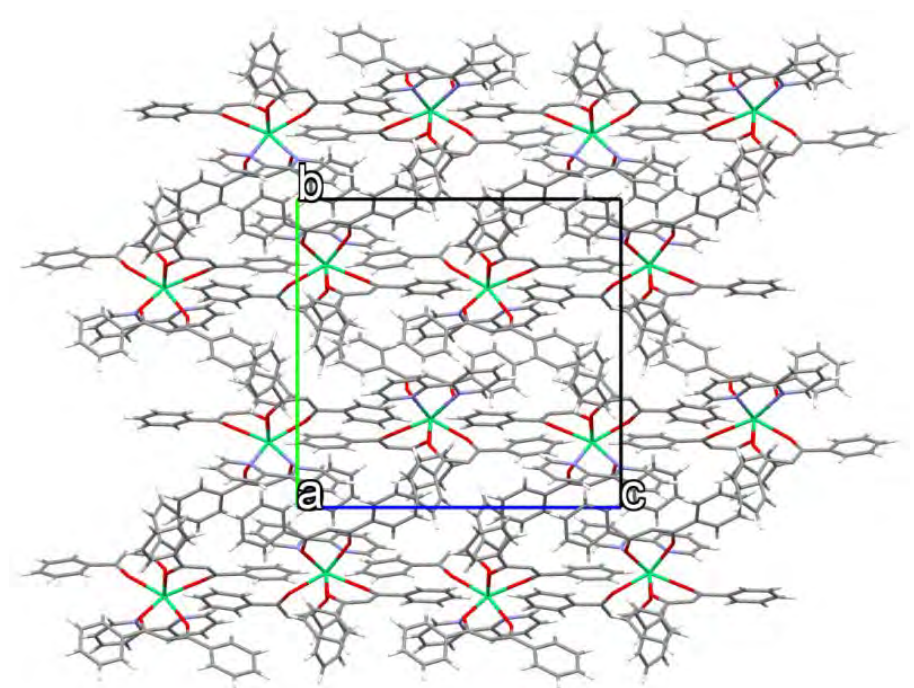

Figure 35. Packing diagram of $\left[\operatorname{Er}(\mathrm{dbm})_{3}(\right.$ bipy $\left.)\right]$

A solvated form of $\left[\operatorname{Er}(\mathrm{dbm})_{3}(\right.$ bipy)] crystallizes in a triclinic unit cell with the $P-1$ space group. The unit cell contains four complexes. Within each complex, the Er(III) ion is coordinated in a distorted square-antiprismatic geometry by six 0 atoms from the dibenzoylmethanate anions and two $\mathrm{N}$ atoms from the 2,2'-bipyridine molecule.

The solvent molecules (probably 1,4-dioxane) are disordered in the crystal and the resulting electron density was found to be uninterpretable. The solvent contribution to the structure factors was taken into account by back-Fourier transformation of all density found in the disordered solvent area using the SQUEEZE routine in PLATON [157]. The formula mass and density do not take the solvent into account.

Table 3. Crystal data and structure refinement for $\left[\operatorname{Er}(\mathrm{dbm})_{3}(\mathrm{bipy})\right]$ and its solvated form

\begin{tabular}{|c|c|c|}
\hline Complex & {$\left[\operatorname{Er}(\mathrm{dbm})_{3}(\mathrm{bipy})\right]$} & Solvated $\left[\operatorname{Er}(\mathrm{dbm})_{3}(\mathrm{bipy})\right]$ form \\
\hline Empirical formula & $\mathrm{C}_{55} \mathrm{H}_{38} \mathrm{ErN}_{2} \mathrm{O}_{6}$ & $\mathrm{C}_{110} \mathrm{H}_{75} \mathrm{Er}_{2} \mathrm{~N}_{4} \mathrm{O}_{12}$ \\
\hline Formula weight & 990.13 & 1979.26 \\
\hline Temperature $(\mathrm{K})$ & $293(3)$ & $293(2)$ \\
\hline Wavelength $(\AA)$ & 0.71073 & 0.71073 \\
\hline Crystal system & Monoclinic & Triclinic \\
\hline Space group & $P 2_{1} / c$ & $P-1$ \\
\hline$a(\AA)$ & $14.9946(6)$ & $15.2886(6)$ \\
\hline$b(\AA)$ & $17.0429(7)$ & $17.7424(7)$ \\
\hline$c(\AA)$ & $22.7907(8)$ & $20.7735(7)$ \\
\hline$\alpha(\underline{0})$ & 90 & $73.910(3)$ \\
\hline$\beta(\underline{0})$ & $128.291(2)$ & $70.046(2)$ \\
\hline$\gamma(\underline{\mathrm{o}})$ & 90 & $68.647(3)$ \\
\hline Volume $\left(\AA^{3}\right)$ & $4571.3(3)$ & $4857.3(3)$ \\
\hline$Z$ & 4 & 2 \\
\hline Calculated density $\left(\mathrm{g} \mathrm{cm}^{-3}\right)$ & 1.439 & 1.353 \\
\hline Absorption coefficient $\left(\mathrm{mm}^{-1}\right)$ & 1.899 & 1.778 \\
\hline$F(000)$ & 1992 & 1990 \\
\hline Crystal size $\left(\mathrm{mm}^{3}\right)$ & $0.33 \times 0.18 \times 0.18$ & - \\
\hline$\theta$ range for data collection & $2.10-25.66^{\circ}$ & $1.78-25.82^{\mathrm{o}}$ \\
\hline Index ranges & $-18<h<18 ;-20<k<20 ;-27<l<27$ & $-18<h<18 ;-21<k<21 ;-25<l<25$ \\
\hline Reflections collected & 82332 & 93233 \\
\hline Independent reflections & 8649 & 7648 \\
\hline Completeness to $2 \theta=51^{\circ}$ & $99.9 \%$ & $99.4 \%$ \\
\hline
\end{tabular}


Refinement method

Data/restrains/parameters Goodness-of-fit on $\mathrm{F}^{2}$

Final $R$ indices $[I>2 \sigma(I)]$ $R$ indices (all data)

Largest diff. peak and hole
Full matrix LS on $\mathrm{F}^{2}$ 8649/0/577

1.058

$R=0.0227 ; \mathrm{w} R=0.0537$

$R=0.0353 ; \mathrm{w} R=0.0599$ $-0.283 / 0.662$
Full matrix LS on $\mathrm{F}^{2}$ $18621 / 0 / 513$ 1.006

$R=0.1013 ; \mathrm{w} R=0.2479$

$R=0.1992 ; \mathrm{w} R=0.2893$ $-4.084 / 5.496$

Table 4. Selected distances and angles $\left(\AA \AA^{,},-\right.$for $\left[\operatorname{Er}(\mathrm{dbm})_{3}(\mathrm{bipy})\right]$

\begin{tabular}{cccc} 
Bond & Distance & Bonds & Angle \\
\hline Er1-N1 & $2.566(2)$ & 01-Er1-02 & $72.91(6)$ \\
Er1-N2 & $2.545(2)$ & 02-Er1-05 & $76.50(6)$ \\
Er1-01 & $2.2975(18)$ & 05-Er1-06 & $73.58(7)$ \\
Er1-02 & $2.3058(18)$ & 06-Er1-01 & $77.26(7)$ \\
Er1-03 & $2.2971(18)$ & 01-Er1-05 & $128.72(7)$ \\
Er1-04 & $2.3079(18)$ & 02-Er1-06 & $106.90(7)$ \\
Er1-05 & $2.3065(19)$ & 03-Er1-04 & $72.13(7)$ \\
Er1-06 & $2.2402(18)$ & 03-Er1-N1 & $76.80(7)$ \\
Er1-N avg. & 2.556 & 04-Er1-N2 & $73.60(8)$ \\
Er1-0 avg. & 2.2925 & & \\
\hline
\end{tabular}

6. [Er(dnm) $)_{3}$ (bipy)] crystallizes in the orthorhombic $P 2_{1} / c$ space group (Figure 36 , Table 5 ). The unit cell contains eight complexes (Figure 37). Further refinement is still required prior to its submission to CCDC.

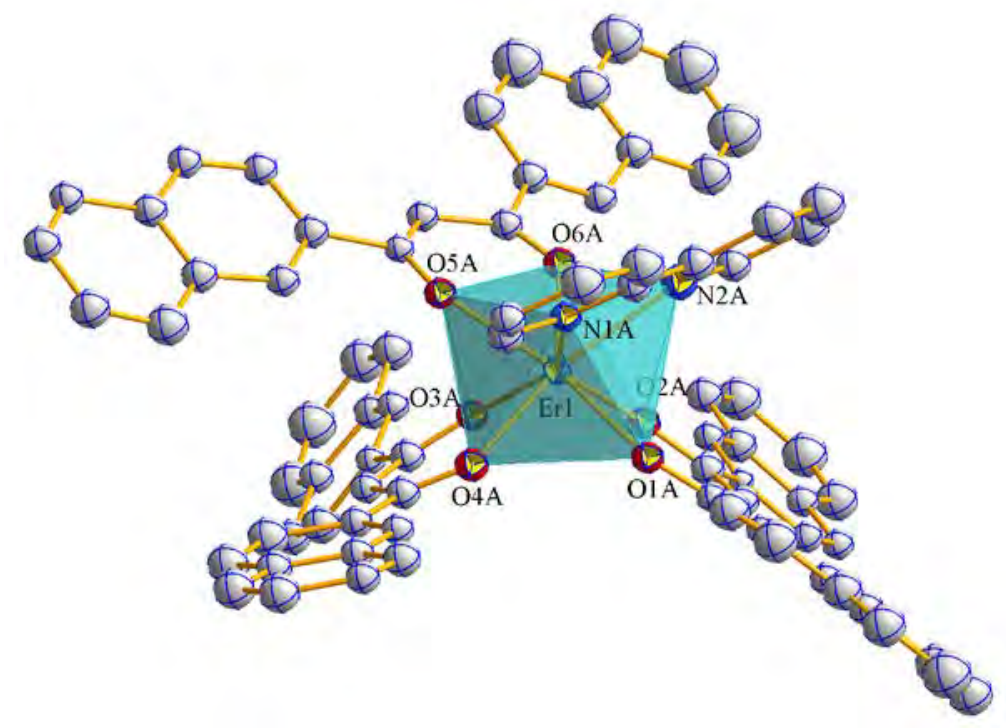

Figure 36. A perspective view of the eightfold coordination square antiprism of [ $\left.\operatorname{Er}(\mathrm{dnm})_{3}(\mathrm{bipy})\right]$. Hydrogen atoms were omitted for clarity. 


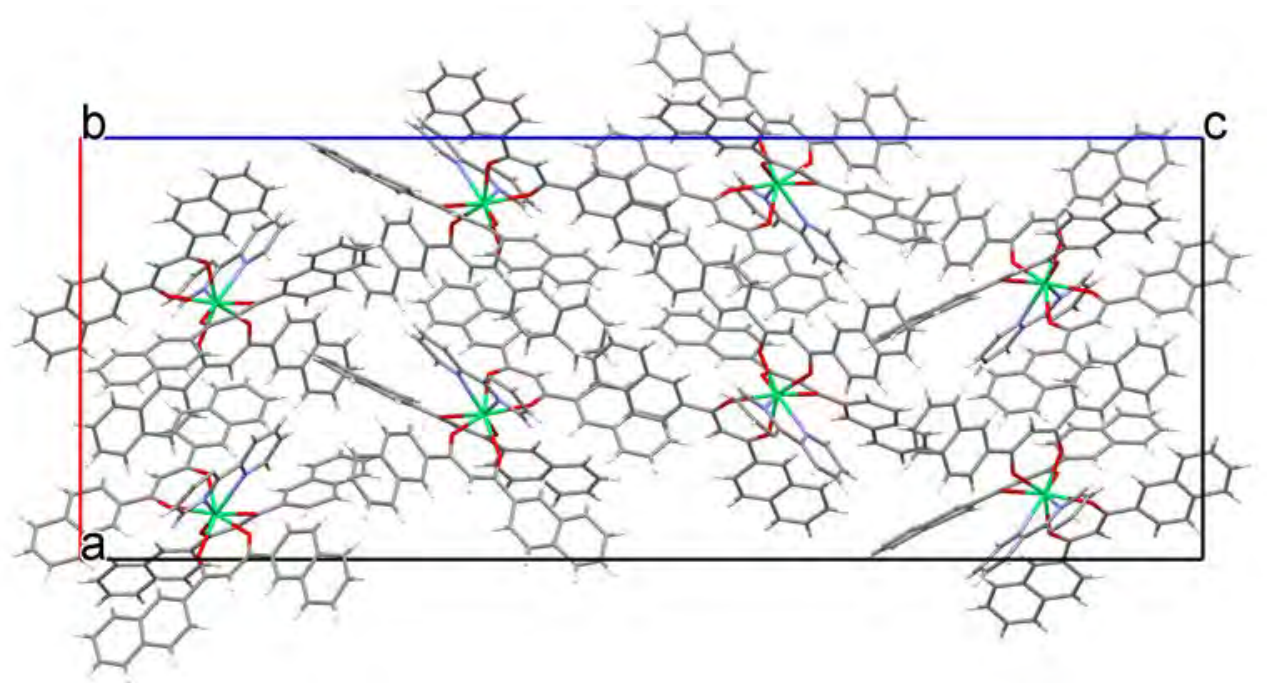

Figure 37. Packing diagram of $\left[\operatorname{Er}(\mathrm{dnm})_{3}\right.$ (bipy)]

8. [Er(dnm $)_{3}\left(\mathbf{5 N O}_{2}\right.$ phen)] crystallizes in the triclinic $P$-1 space group, with cell parameters $a=10.2326(3) \quad \AA \quad, \quad b=13.5753(4) \quad \AA \quad, \quad c=23.9980(7) \quad \AA \quad, \quad \alpha=93.855(2)^{\circ}, \quad \beta=96.4920(10)^{\circ}$, $\gamma=110.2500(10)^{\circ}, \mathrm{V}=3087.18(16) \AA^{3}, \mathrm{Z}=2$ (Figure 38, Table 5).

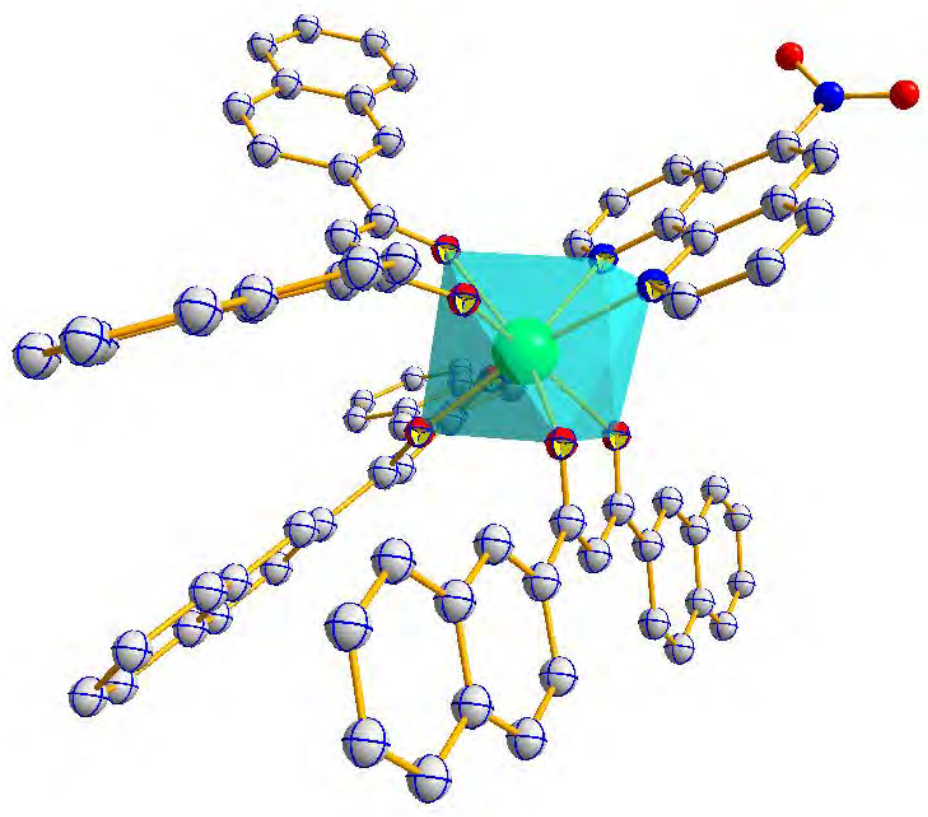

Figure 38. A perspective view of the eightfold coordination square antiprism of $\left[\operatorname{Er}(\mathrm{dnm})_{3}\left(5 \mathrm{NO}_{2}\right.\right.$ phen $\left.)\right]$. Hydrogen atoms were omitted for clarity. 
Table 5. Crystal data and structure refinement for the complexes with 1,3-di(2-naphthyl)-1,3propanedione

\begin{tabular}{|c|c|c|}
\hline Complex & {$\left[\operatorname{Er}(\mathrm{dnm})_{3}(\mathrm{bipy})\right]$} & {$\left[\mathrm{Er}(\mathrm{dnm})_{3}\left(5 \mathrm{NO}_{2}\right.\right.$ phen $\left.)\right]$} \\
\hline Empirical formula & $\mathrm{C}_{79} \mathrm{H}_{53} \mathrm{ErN}_{2} \mathrm{O}_{6}$ & $\mathrm{C}_{81} \mathrm{H}_{52} \mathrm{ErN}_{3} \mathrm{O}_{8}$ \\
\hline Formula weight & 1293.53 & 1362.55 \\
\hline Temperature (K) & $293(3)$ & $293(3)$ \\
\hline Wavelength $(\AA)$ & 0.71073 & 0.71073 \\
\hline Crystal system & Orthorhombic & Triclinic \\
\hline Space group & $P 2_{1} / c$ & $P-1$ \\
\hline$a(\AA)$ & $20.0598(15)$ & $10.2326(3)$ \\
\hline$b(\AA)$ & $11.4092(11)$ & $13.5753(4)$ \\
\hline$c(\AA)$ & $53.335(4)$ & $23.9980(7)$ \\
\hline$\alpha(\underline{0})$ & 90.00 & $93.855(2)$ \\
\hline$\beta(\underline{0})$ & 90.00 & $96.4920(10)$ \\
\hline$\gamma(\underline{\mathrm{o}})$ & 90.00 & $110.2500(10)$ \\
\hline Volume $\left(\AA^{3}\right)$ & $12206.6(17)$ & $3087.18(16)$ \\
\hline$Z$ & 8 & 2 \\
\hline Calculated density $\left(\mathrm{g} \mathrm{cm}^{-3}\right)$ & 1.407 & 1.466 \\
\hline Absorption coefficient $\left(\mathrm{mm}^{-1}\right)$ & 1.434 & 1.424 \\
\hline$F(000)$ & 5248 & 1382 \\
\hline$\theta$ range for data collection & $1.53-25.84^{\circ} \mathrm{o}$ & $1.91-25.72^{\circ}$ \\
\hline Index ranges & $-15<h<24 ;-13<k<13 ;-65<l<52$ & $-12<h<12 ;-16<k<16 ;-29<l<29$ \\
\hline Reflections collected & 97776 & 63914 \\
\hline Independent reflections & 6114 & 9875 \\
\hline Completeness to $2 \theta=51^{\circ}$ & $99.5 \%$ & $99.6 \%$ \\
\hline Refinement method & Full matrix LS on $\mathrm{F}^{2}$ & Full matrix LS on $\mathrm{F}^{2}$ \\
\hline Data/restrains/parameters & $21242 / 1 / 796$ & $11739 / 0 / 838$ \\
\hline Goodness-of-fit on $\mathrm{F}^{2}$ & 0.888 & 1.179 \\
\hline Final $R$ indices $[I>2 \sigma(I)]$ & $R=0.0854 ; \mathrm{w} R=0.1091$ & $R=0.0330 ; \mathrm{w} R=0.0904$ \\
\hline$R$ indices (all data) & $R=0.3195 ; \mathrm{w} R=0.1677$ & $R=0.0488 ; \mathrm{w} R=0.1256$ \\
\hline Largest diff. peak and hole & $-1.092 / 0.837$ & $-1.026 / 1.148$ \\
\hline
\end{tabular}

Table 6. Selected distances and angles $\left(\AA ̊, \frac{o}{}\right)$ for $\left[\operatorname{Er}(\mathrm{dnm})_{3}(\mathrm{bipy})\right]$

\begin{tabular}{cccc} 
Bond & Distance & Bonds & Angle \\
\hline Er1-N1 & $2.533(18)$ & 01-Er1-02 & $71.6(6)$ \\
Er1-N2 & $2.52(2)$ & 02-Er1-05 & $148.1(6)$ \\
Er1-01 & $2.303(18)$ & 05-Er1-06 & $73.8(6)$ \\
Er1-02 & $2.266(15)$ & 06-Er1-01 & $139.5(7)$ \\
Er1-03 & $2.285(14)$ & 01-Er1-05 & $139.9(6)$ \\
Er1-04 & $2.227(17)$ & 02-Er1-06 & $81.0(5)$ \\
Er1-05 & $2.312(13)$ & 03-Er1-04 & $71.9(6)$ \\
Er1-06 & $2.281(15)$ & 03-Er1-N1 & $145.9(6)$ \\
Er1-N avg. & 2.527 & 04-Er1-N2 & $141.3(7)$ \\
Er1-0 avg. & 2.279 & N1-Er1-N2 & $62.3(7)$ \\
\hline
\end{tabular}

Table 7. Selected distances and angles $(\AA ̊ \stackrel{\mathrm{o}}{-})$ for $\left[\operatorname{Er}(\mathrm{dnm})_{3}\left(5 \mathrm{NO}_{2}\right.\right.$ phen $\left.)\right]$

\begin{tabular}{cccc} 
Bond & Distance & Bonds & Angle \\
\hline Er1-N1 & $2.568(4)$ & 01-Er1-02 & $71.68(11)$ \\
Er1-N2 & $2.555(4)$ & O2-Er1-05 & $143.47(11)$ \\
Er1-01 & $2.287(3)$ & 05-Er1-06 & $72.13(11)$ \\
Er1-02 & $2.305(3)$ & 06-Er1-01 & $144.29(11)$ \\
Er1-03 & $2.253(3)$ & 01-Er1-05 & $73.00(11)$ \\
Er1-04 & $2.308(3)$ & 02-Er1-06 & $143.94(11)$ \\
Er1-05 & $2.300(3)$ & 03-Er1-04 & $72.39(11)$ \\
Er1-06 & $2.295(3)$ & 03-Er1-N1 & $147.07(13)$ \\
Er1-N avg. & 2.562 & 04-Er1-N2 & $135.79(11)$ \\
Er1-0 avg. & 2.291 & N1-Er1-N2 & $63.28(13)$ \\
\hline
\end{tabular}


9. [Er(h) $)_{3}$ (bipy)] crystallizes in a monoclinic system, space group $P 2_{1} / c$, with cell parameters $a=9.4068(2) \bar{A}, b=23.0764(4) \bar{A}, c=13.1493(3) \bar{A}, \alpha=90.000, \beta=92.5718(12)^{\circ}$, $\gamma=90.00^{\circ}, \mathrm{V}=2851.51(10) \AA^{3}, \mathrm{Z}=1$ (Figure 39, Table 8).

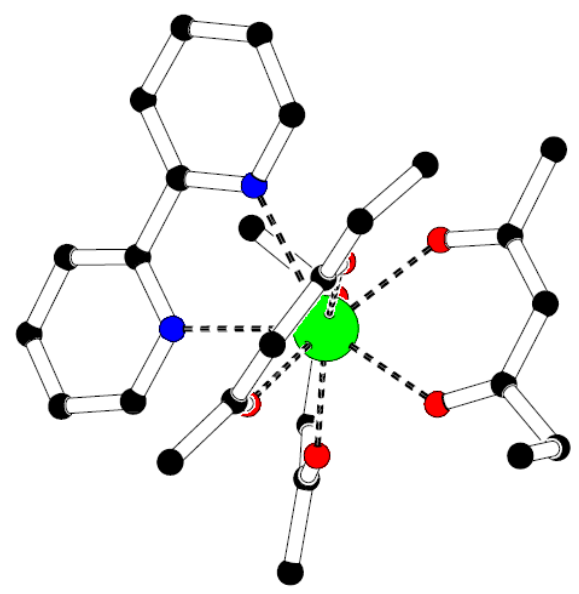

Figure 39. Structural diagram of [Er(h) $)_{3}($ bipy) $]$

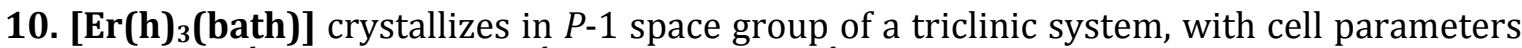

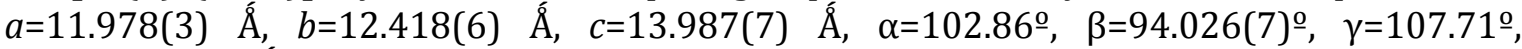
$\mathrm{V}=1910.6(14) \AA^{3}, \mathrm{Z}=2$ (Figure 40, Table 8). Further refinement is required prior to submission to CCDC.

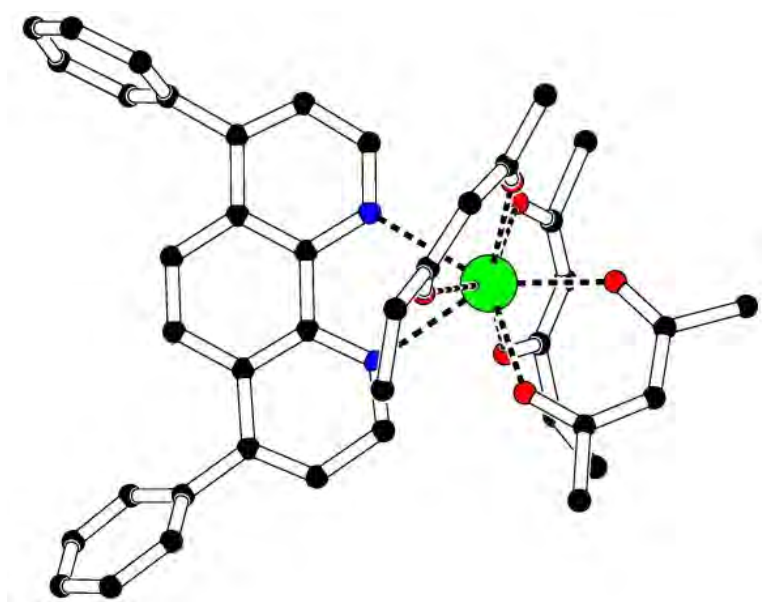

Figure 40. Structural diagram of $\left[\operatorname{Er}(\mathrm{h})_{3}(\right.$ bath $\left.)\right]$

11. [Er(h) ${ }_{3}\left(\mathbf{5 N O}_{2}\right.$ phen)] crystallizes in $P 2_{1} / c$ space group of a monoclinic system, with cell

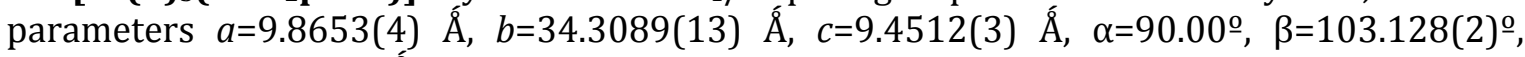
$\gamma=90.00^{\circ}, V=3115.3(2) \AA^{3}, Z=4$ (Figure 41, Table 8). 


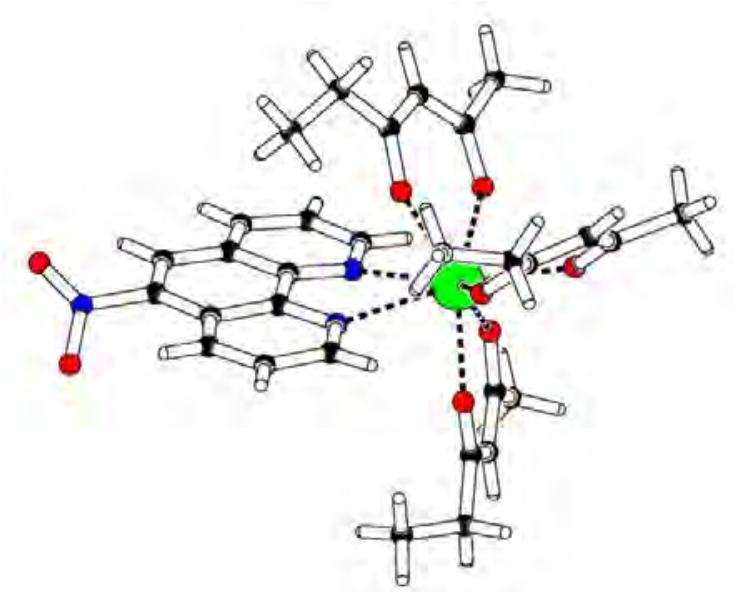

Figure 41. Structural diagram of $\left[\operatorname{Er}(\mathrm{h})_{3}\left(5 \mathrm{NO}_{2}\right.\right.$ phen $\left.)\right]$

12. The Er(III) quaternary chelate complex with bipyridine (bipy), 2,4-hexanedione (h) and nitrate, [Er(h) 2 (bipy)(NO $\left.\mathbf{N}_{3}\right)$ ], is monoclinic and crystallizes in $P 2_{1} / c$ space group with cell parameters $a=9.6753(2) \AA \bar{A}, b=33.8796(9) \AA \bar{\alpha}, c=16.6589(5) \AA \bar{\alpha}, \alpha=90.00^{\circ}, \beta=117.647(2)^{\circ}$, $\gamma=90.00^{\circ}, V=4837.2(2) \AA^{3}, Z=8$ (Figure 42 , Table 8). This is a rare complex whose only antecedent is the also $\operatorname{Er}(\mathrm{III})$ quaternary chelate with terpyridine (terpy), acetylacetonate (acac) and nitrate, Er(terpy)(acac) $\left(\mathrm{NO}_{3}\right)_{2}$, reported by Y. Fukuda, A. Nakao and K. Hayashi [158] (Figure 43).

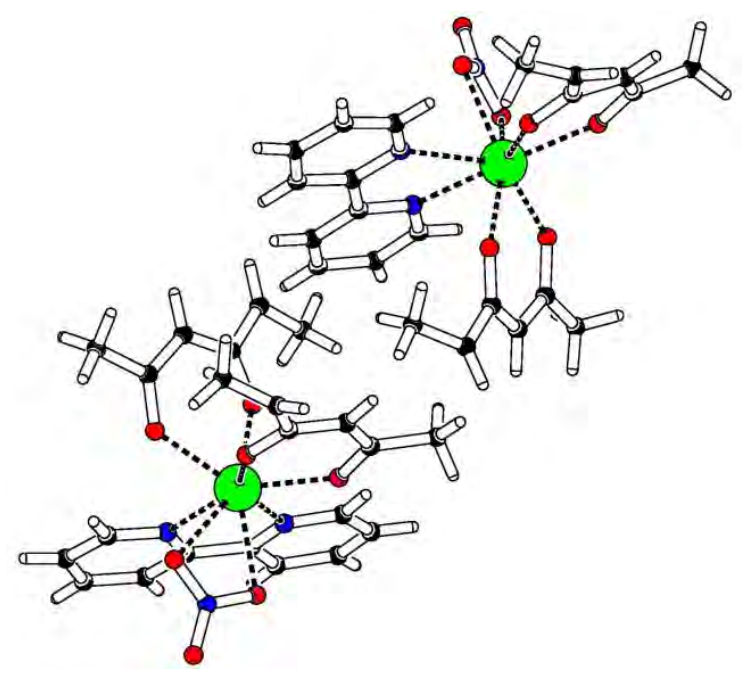

Figure 42. Structural diagram of two independent $\left[\operatorname{Er}(\mathrm{h})_{2}(\right.$ bipy $\left.)\left(\mathrm{NO}_{3}\right)\right]$ complexes

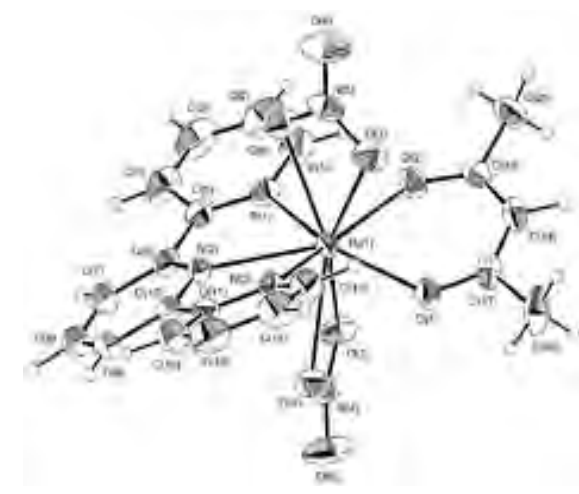

Figure 43. Structure of $\left[\operatorname{Er}(\right.$ terpy $\left.)(\mathrm{acac})\left(\mathrm{NO}_{3}\right)_{2}\right][158]$ 
Table 8. Crystal data and structure refinement for the complexes with 2,4-hexanedione

\begin{tabular}{|c|c|c|c|c|}
\hline Complex & {$\left[\operatorname{Er}(\mathrm{h})_{3}(\right.$ bipy $\left.)\right]$} & {$\left[\operatorname{Er}(\mathrm{h})_{2}(\mathrm{bipy})\left(\mathrm{NO}_{3}\right)\right]$} & {$\left[\operatorname{Er}(\mathrm{h})_{3}(\mathrm{bath})\right]$} & {$\left[\operatorname{Er}(\mathrm{h})_{3}\left(5 \mathrm{NO}_{2}\right.\right.$ phen $\left.)\right]$} \\
\hline Empirical formula & $\mathrm{C}_{28} \mathrm{H}_{35} \mathrm{ErN}_{2} \mathrm{O}_{6}$ & $\mathrm{C}_{22} \mathrm{H}_{24} \mathrm{ErN}_{3} \mathrm{O}_{7}$ & $\mathrm{C}_{42} \mathrm{H}_{43} \mathrm{ErN}_{2} \mathrm{O}_{6}$ & $\mathrm{C}_{30} \mathrm{H}_{34} \mathrm{ErN}_{3} \mathrm{O}_{8}$ \\
\hline Formula weight & 662.84 & 609.71 & 838.06 & 731.86 \\
\hline Temperature (K) & $293(2)$ & $293(2)$ & $293(2)$ & $293(2)$ \\
\hline Wavelength $(\AA)$ & 0.71073 & 0.71073 & 0.71073 & 0.71073 \\
\hline Crystal system & Monoclinic & Monoclinic & Triclinic & Monoclinic \\
\hline Space group & $P 2_{1} / c$ & $P 2_{1} / c$ & $P-1$ & $P 2_{1} / c$ \\
\hline$a(\AA)$ & $9.4068(2)$ & $9.6753(2)$ & $11.978(3)$ & $9.8653(4)$ \\
\hline$b(\AA)$ & $23.0764(4)$ & $33.8796(9)$ & $12.418(6)$ & $34.3089(13)$ \\
\hline$c(\AA)$ & $13.1493(3)$ & $16.6589(5)$ & $13.987(7)$ & $9.4512(3)$ \\
\hline$\alpha(\underline{\mathrm{o}})$ & 90.00 & 90.00 & 102.86 & 90.00 \\
\hline$\beta(\underline{\mathrm{o}})$ & $92.5718(12)$ & $117.647(2)$ & $94.026(7)$ & $103.128(2)$ \\
\hline$\gamma(\underline{\mathrm{o}})$ & 90.00 & 90.00 & 107.71 & 90.00 \\
\hline Volume $\left(\AA^{3}\right)$ & 2851.51(10) & $4837.2(2)$ & $1910.6(14)$ & $3115.3(2)$ \\
\hline$Z$ & 4 & 8 & 2 & 4 \\
\hline $\begin{array}{l}\text { Calculated density } \\
\qquad\left(\mathrm{g} \mathrm{cm}^{-3}\right)\end{array}$ & 1.544 & 1.674 & 2.934 & 1.560 \\
\hline $\begin{array}{l}\text { Absorption coefficient } \\
\qquad\left(\mathrm{mm}^{-1}\right)\end{array}$ & 2.985 & 3.515 & 4.490 & 2.746 \\
\hline$F(000)$ & 1332 & 2408 & 1720 & 1468 \\
\hline Crystal size $\left(\mathrm{mm}^{3}\right)$ & $0.22 \times 0.04 \times 0.04$ & $0.21 \times 0.05 \times 0.04$ & - & $0.16 \times 0.13 \times 0.13$ \\
\hline$\theta$ range for data collection & $1.76-25.78^{\circ}$ & $1.51-19.32^{\mathrm{o}}$ & $1.78-31.53^{\circ}$ & $2.20-25.77^{\circ} \stackrel{0}{0}$ \\
\hline & $-11-<h<11$ & $-8<h<8$ & $-17-<h<16$ & $-12<h<12$ \\
\hline \multirow[t]{2}{*}{ Index ranges } & $-28<k<28$ & $-31<k<31$ & $-17<k<17$ & $-41<k<41$ \\
\hline & $-16<l<15$ & $-15<l<15$ & $-18<l<19$ & $-11<l<11$ \\
\hline Reflections collected & 42461 & 70035 & 30627 & 64553 \\
\hline Independent reflections & 4164 & 3026 & 4693 & 4634 \\
\hline Completeness to $2 \theta=51^{\circ}$ & $99.8 \%$ & $99.7 \%$ & $87.6 \%$ & $99.6 \%$ \\
\hline Refinement method & $\begin{array}{l}\text { Full matrix LS on } \\
\qquad \mathrm{F}^{2}\end{array}$ & Full matrix LS on $\mathrm{F}^{2}$ & $\begin{array}{l}\text { Full matrix LS } \\
\text { on } \mathrm{F}^{2}\end{array}$ & Full matrix LS on $\mathrm{F}^{2}$ \\
\hline Data/restrains/parameters & $5471 / 0 / 340$ & $4058 / 2 / 603$ & $11187 / 67 / 446$ & $5961 / 0 / 385$ \\
\hline Goodness-of-fit on $\mathrm{F}^{2}$ & 0.986 & 0.902 & 1.075 & 1.266 \\
\hline Final $R$ indices $[I>2 \sigma(I)]$ & $\begin{array}{c}R=0.0268 \\
\mathrm{w} R=0.0542\end{array}$ & $\begin{array}{c}R=0.0386 ; \\
\mathrm{w} R=0.1078\end{array}$ & $\begin{array}{c}R=0.1078 \\
\mathrm{w} R=0.3308\end{array}$ & $\begin{array}{c}R=0.0744 ; \\
\mathrm{w} R=0.1295\end{array}$ \\
\hline$R$ indices (all data) & $\begin{array}{c}R=0.0462 \\
\mathrm{w} R=0.0607\end{array}$ & $\begin{array}{c}R=0.0623 \\
\mathrm{w} R=0.1256\end{array}$ & $\begin{array}{c}R=0.2241 \\
\mathrm{w} R=0.3748\end{array}$ & $\begin{array}{c}R=0.0999 \\
\mathrm{w} R=0.1363\end{array}$ \\
\hline Largest diff. peak and hole & $-0.459 / 0.369$ & $-0.462 / 0.522$ & $-2.151 / 3.861$ & $-3.073 / 1.215$ \\
\hline
\end{tabular}

Table 9. Er-N and Er-O distances in $\AA$ for the complexes with 2,4-hexanedione

\begin{tabular}{ccccc} 
Bond & {$\left[\operatorname{Er}(\mathrm{h})_{3}(\mathrm{bipy})\right]$} & {$\left[\operatorname{Er}(\mathrm{h})_{2}(\mathrm{bipy})\left(\mathrm{NO}_{3}\right)\right]$} & {$\left[\operatorname{Er}(\mathrm{h})_{3}(\mathrm{bath})\right]$} & {$\left[\operatorname{Er}(\mathrm{h})_{3}\left(5 \mathrm{NO}_{2} \mathrm{phen}\right)\right]$} \\
\hline Er1-N1 & $2.539(2)$ & $2.533(11)$ & $2.582(7)$ & $2.531(8)$ \\
Er1-N2 & $2.577(2)$ & $2.503(11)$ & $2.546(9)$ & $2.628(8)$ \\
Er1-01 & $2.2994(17)$ & $2.424(9)$ & $2.272(8)$ & $2.288(7)$ \\
Er1-02 & $2.3042(16)$ & $2.460(9)$ & $2.289(8)$ & $2.281(7)$ \\
Er1-03 & $2.2841(19)$ & $2.266(10)$ & $2.3349(17)$ & $2.283(7)$ \\
Er1-04 & $2.3023(19)$ & $2.243(12)$ & $2.262(4)$ & $2.313(7)$ \\
Er1-05 & $2.3144(17)$ & $2.227(9)$ & $2.303(8)$ & $2.301(7)$ \\
Er1-06 & $2.2839(17)$ & $2.248(9)$ & $2.316(7)$ & $2.298(7)$ \\
Er1-N avg. & 2.558 & 2.518 & 2.564 & 2.5795 \\
Er1-0 avg. & 2.298 & 2.311 & 2.296 & 2.294 \\
\hline
\end{tabular}


13. [Er(hd) $)_{3}$ (bipy)] crystallizes in a triclinic centrosymmetric cell with four complexes per unit cell, out of which two of them are symmetry independent (Figure 44, Table 10). Within each complex, the lanthanide $\operatorname{Er}(\mathrm{III})$ ion is eight-coordinated with $\mathrm{N}$ and $\mathrm{O}$ neighbours forming a square antiprismatic structure. For the Er1 complex the set N1A, N2A, O1B, O2B and the set 01A, 02A, 01BC, 02C form two approximate squares, respectively (Figure 44). In the center of the antiprism lies the trivalent erbium with a distance of 1.3654(1) A to the face containing the $\mathrm{N}$ atoms and 1.1936(1) $\AA$ to the opposite face containing exclusively oxygen atoms. Similarly, for the Er2 complex, the set N1B, N2B, O1D, O2D and the set O1E, O2E, O1F, O2F form two approximates squares, respectively (Figure 44). In the center of the antiprism lies the trivalent erbium with a distance of $1.3867(1) \AA$ to the face containing the $\mathrm{N}$ atoms and 1.1845(1) to the opposite face containing exclusively oxygen atoms.

The Er1-N distances are 2.537(4) $\AA$ and 2.541(4) $\AA$ and the Er1-0 distances are in the range of 2.265(3)-2.300(3) Å. The Er2-N distances are 2.545(5) $\AA$ and 2.557(4) $\AA$ and the Er2-O distances are in the range 2.281(3)-2.319(3) $\AA$. The bite angle N-Er1-N is 62.51(15) ${ }^{\circ}$ and the bite angle N-Er2-N is 62.42(15) ${ }^{\circ}$ (Table 11).

The aromatic rings of the 2,2'-bipyridine do not share the same plane. For the Er1 complex, the least-squares plane of the ring that contain the N1A makes a dihedral angle of $7.8(2)^{\circ}$ with the least-squares plane of the ring containing N2A. In the Er2 complex, those two leastsquares planes, defined by the rings that contain N1B and N2B respectively, make a dihedral angle of $12.4(2)^{\circ}$.
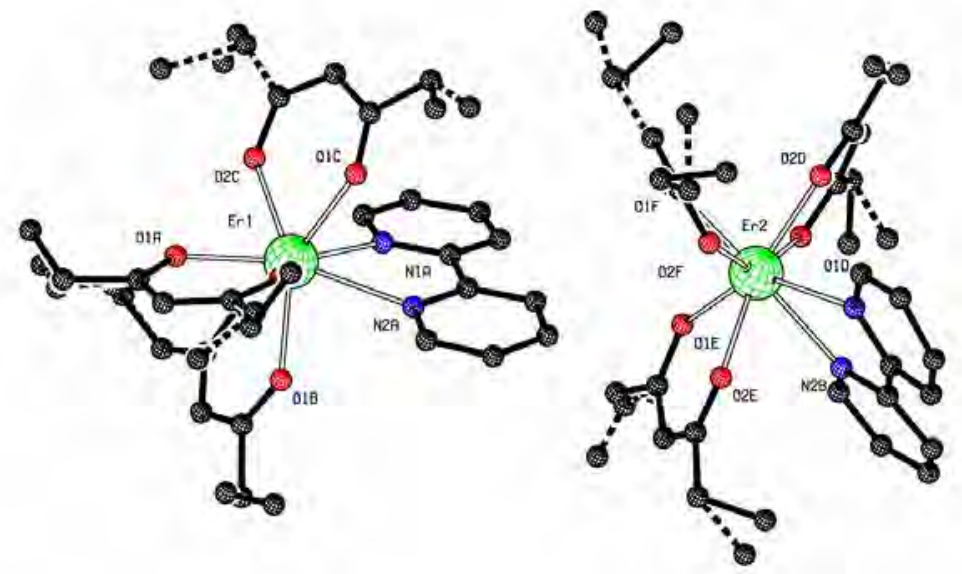

Figure 44. Structural diagram of two independent $\left[\operatorname{Er}(\mathrm{hd})_{3}(\right.$ bipy)] complexes, showing the antisquare prismatic conformation.

There are signs of disorder in the structure: some terminal methyl groups of 3,5heptanedionate exhibit a large displacement factor. For both complexes, two positions could be found for each terminal group with occupation of roughly 50\% (Figure 45). 


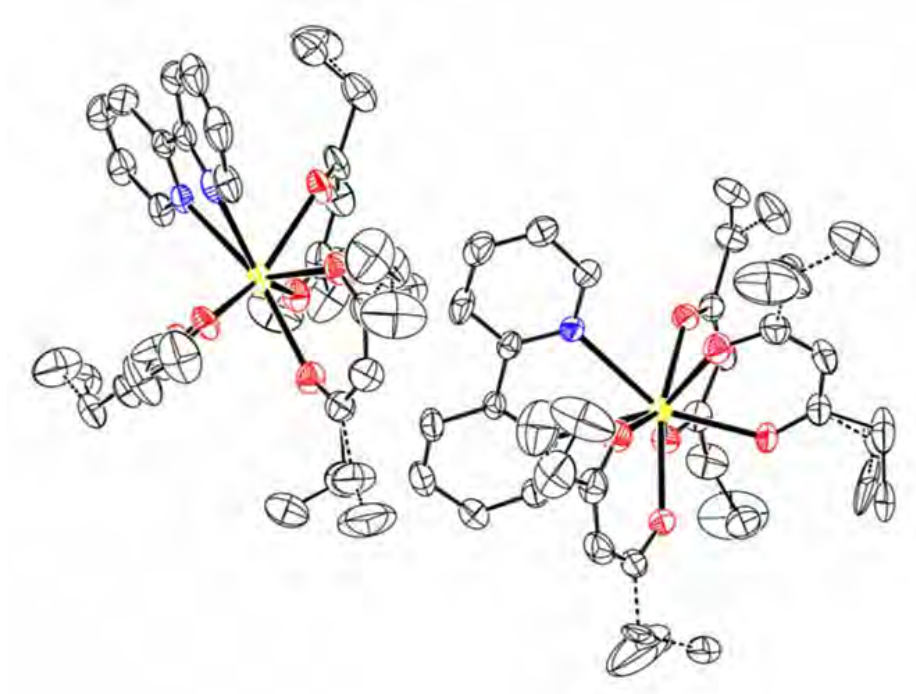

Figure 45. ORTEP plot of two independent $\left[\operatorname{Er}(\text { hd })_{3}(\right.$ bipy)] complexes. For clarity, displacement ellipsoids are drawn at the $20 \%$ level and all hydrogen atoms were omitted.

Table 10. Crystal data and structure refinement parameters for [ $\left.\operatorname{Er}(\mathrm{hd})_{3}(\mathrm{bipy})\right]$

\begin{tabular}{|c|c|}
\hline Complex & {$\left[\operatorname{Er}(\text { hd })_{3}(\right.$ bipy $\left.)\right]$} \\
\hline Empirical formula & $\mathrm{C}_{31} \mathrm{H}_{41} \mathrm{ErN}_{2} \mathrm{O}_{6}$ \\
\hline Formula weight & 704.92 \\
\hline Temperature (K) & $293(2)$ \\
\hline Wavelength $(\AA)$ & 0.71073 \\
\hline Crystal system & Triclinic \\
\hline Space group & $P-1$ \\
\hline$a(\AA)$ & $10.2331(5)$ \\
\hline$b(\AA)$ & $15.8663(8)$ \\
\hline$c(\AA)$ & 21.5723 \\
\hline$\alpha\left(^{\circ}\right)$ & $82.185(3)$ \\
\hline$\beta\left(^{\circ}\right)$ & $79.555(3)$ \\
\hline$\gamma\left({ }^{\circ}\right)$ & $73.638(3)$ \\
\hline Volume $\left(\AA^{3}\right)$ & $3291.6(3)$ \\
\hline$Z$ & 4 \\
\hline Calculated density $\left(\mathrm{g} / \mathrm{cm}^{3}\right)$ & 1.422 \\
\hline Absorption coefficient $\left(\mathrm{mm}^{-1}\right)$ & $2.590 \mathrm{~mm}^{-1}$ \\
\hline$F(000)$ & 1428 \\
\hline Crystal size $\left(\mathrm{mm}^{3}\right)$ & $0.40 \times 0.10 \times 0.05$ \\
\hline$\theta$ range for data collection $\left({ }^{\circ}\right)$ & $1.34-25.95$ \\
\hline Index ranges & $-12<h<12 ;-19<k<19 ;-26<l<26$ \\
\hline Reflections collected/unique ( $\left.R_{\mathrm{int}}\right)$ & $46851 / 12677(0.0475)$ \\
\hline Completeness to $\theta=25^{\circ}$ & $98.4 \%$ \\
\hline Refinement method & Full matrix LS on $\mathrm{F}^{2}$ \\
\hline Data/restrains/parameters & $12677 / 8 / 912$ \\
\hline Goodness-of-fit on $F^{2}$ & 0.912 \\
\hline Final $R$ indices $[I>2 \sigma(I)]$ & $R_{1}=0.0338 ; \mathrm{w} R_{2}=0.0747$ \\
\hline$R$ indices (all data) & $R_{1}=0.0704 ; \mathrm{w} R_{2}=0.0870$ \\
\hline Largest difference in peak and hole $\left(\mathrm{e} \AA^{-}{ }^{-3}\right)$ & $-0.467 / 0.399$ \\
\hline
\end{tabular}


Table 11. Selected bond lengths $(\AA ̊)$ and angles $\left(^{\circ}\right)$ at room temperature for $\left[\operatorname{Er}(\mathrm{hd})_{3}(\right.$ bipy $\left.)\right]$

\begin{tabular}{|c|c|c|c|}
\hline Bond & Er1 & Bond & Er2 \\
\hline Er1-01A & $2.262(2)$ & Er2-01D & $2.290(2)$ \\
\hline Er1-02A & $2.300(2)$ & Er2-02D & $2.281(2)$ \\
\hline Er1-01B & $2.291(2)$ & Er2-01E & $2.282(2)$ \\
\hline Er1-02B & $2.276(2)$ & Er2-02E & $2.319(2)$ \\
\hline Er1-01C & $2.283(2)$ & Er2-01F & $2.302(2)$ \\
\hline Er1-02C & $2.301(2)$ & Er2-02F & $2.299(2)$ \\
\hline Er1-N1A & $2.541(2)$ & Er2-N1B & $2.548(3)$ \\
\hline Er1-N2A & $2.539(2)$ & Er2-N2B & $2.554(2)$ \\
\hline Er1-0 avg. & 2.286 & Er2-0 avg. & 2.296 \\
\hline Er1-N avg. & 2.540 & Er2-N avg. & 2.551 \\
\hline N1A $\cdots$ Er1 $\cdots$ N2A & $62.52(8)$ & N1B $\cdots$ Er2 $\cdots$ N2B & $62.42(9)$ \\
\hline $\mathrm{N} 1 \mathrm{~A} \cdots \operatorname{Er} 1 \cdots \mathrm{O} 2 \mathrm{~B}$ & $73.84(8)$ & N1B $\cdots E r 2 \cdots 02 D$ & $71.60(9)$ \\
\hline N1A $\cdots$ Er1 $\cdots$ O2C & $78.09(8)$ & N1B $\cdots E r 2 \cdots 02 E$ & $74.36(9)$ \\
\hline N2A $\cdots \operatorname{Er} 1 \cdots 01 B$ & $73.81(8)$ & N2B $\cdots \operatorname{Er} 2 \cdots 01 D$ & $73.52(8)$ \\
\hline N2A $\cdots$ Er1 $\cdots$ O1C & $71.11(8)$ & N2B $\cdots$ Er2 $\cdots 01 E$ & $83.71(8)$ \\
\hline $01 \mathrm{~A} \cdots \mathrm{Er} 1 \cdots 02 \mathrm{~A}$ & $73.30(8)$ & 01D $\cdots E r 2 \cdots 02 D$ & 74.14(8) \\
\hline 01B $\cdots \operatorname{Er} 1 \cdots 02 B$ & $78.42(9)$ & 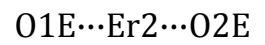 & 73.88(9) \\
\hline 01C $\cdots \operatorname{Er} 1 \cdots 02 C$ & $72.94(9)$ & $01 \mathrm{~F} \cdots \mathrm{Er} 2 \cdots 02 \mathrm{~F}$ & $74.01(9)$ \\
\hline $01 \mathrm{~A} \cdots \operatorname{Er} 1 \cdots 02 \mathrm{~B}$ & $78.42(9)$ & 01D $\cdots$ Er2 $\cdots 01 F$ & $82.41(8)$ \\
\hline $01 \mathrm{~A} \cdots \operatorname{Er} 1 \cdots 02 \mathrm{C}$ & $75.53(8)$ & $01 \mathrm{E} \cdots \mathrm{Er} 2 \cdots 01 \mathrm{~F}$ & $76.03(9)$ \\
\hline 01B $\cdots \operatorname{Er} 1 \cdots 02 A$ & $76.03(9)$ & O2E $\cdots \mathrm{Er} 2 \cdots 02 \mathrm{~F}$ & 74.61(8) \\
\hline 01C $\cdots$ Er1 $\cdots 02 \mathrm{~A}$ & $75.05(9)$ & O2D $\cdots$ Er2 $\cdots 02 F$ & $79.77(8)$ \\
\hline
\end{tabular}

14. The mononuclear complexes in $\left[\operatorname{Er}(\mathbf{d m h})_{3}(\right.$ bipy)] crystallize in a triclinic unit cell with the P-1 space group (Figure 46, Table 12) and two symmetry independent complexes. In each molecule the metal ion is octa-coordinated by six oxygen atoms of the three bidentate diketonate ligands and two nitrogen atoms of a bidentate $\mathrm{N}, \mathrm{N}^{\prime}$ aromatic ligand. These atoms lie at the apexes of a slightly distorted square antiprism. The 0 -face (formed exclusively by oxygen atoms) and the $\mathrm{N}, \mathrm{O}$-face (formed by two nitrogen atoms and two oxygen atoms) are almost parallel, with a dihedral angle between mean planes of $1.03(6)^{\circ}$ and $1.36(7)^{\circ}$, for the two complexes. The trivalent ion sits approximately in the middle of the prisms distancing $1.39 / 1.17$ and $1.37 / 1.18 \AA$ to the antiprismatic square faces. No intermolecular interactions other than Van der Waals can be detected for this compound. The structure shows a packing index of 60.8 (percentage of filled space) and some small voids of less than $53 \AA^{3}$.
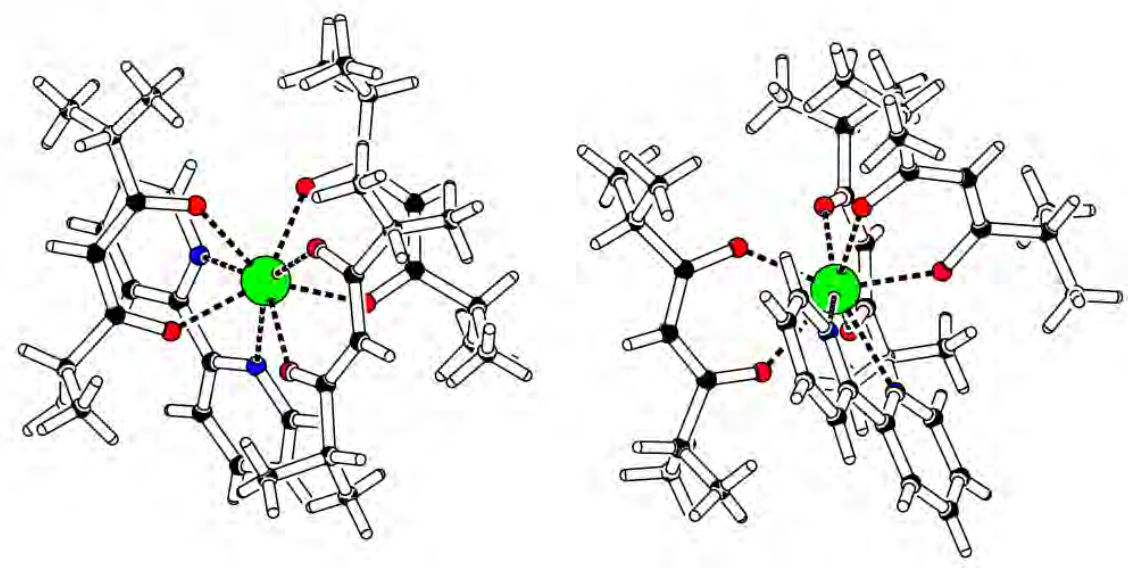

Figure 46. Structural diagram of $\left[\operatorname{Er}(\mathrm{dmh})_{3}(\right.$ bipy $\left.)\right]$ showing the two symmetry independent complexes in the unit cell 
15. [Er(dmh $)_{3}$ (bath)] crystallizes in the monoclinic space group $P 2_{1} / c$ with four complexes in the unit cell (Figure 47, Table 12). In each complex, the three anionic $\beta$-diketonates and one bulkier bathophenanthroline surround the lanthanide three-positive ion. The atoms close to the lanthanide, six $\mathrm{O}$ atoms and two $\mathrm{N}$ atoms, occupy the apexes of an anti-prism. The erbium ion occupies the centre of that anti-prism with a distance of $1.37 \AA$ to the square face containing the $\mathrm{N}$ atoms and $1.18 \AA$ to the opposite face. The terminal phenyl rings in the bathophenanthroline moiety make an angle of near $50^{\circ}$ with the fused rings mean plane. The structure shows a packing index of 59.2 (percentage of filled space) and some small voids of less than $35 \AA^{3}$.

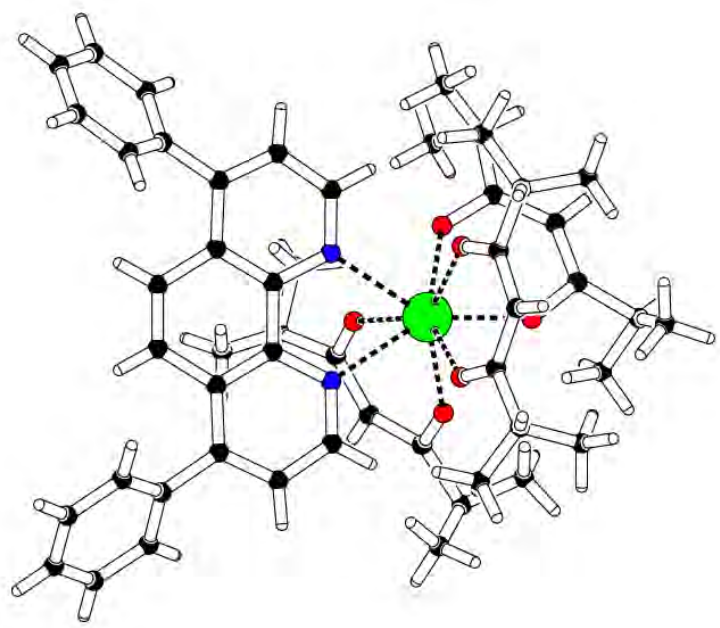

Figure 47. Structural diagram of $\left[\operatorname{Er}(\mathrm{dmh})_{3}(\right.$ bath $\left.)\right]$

16. [Er(dmh $)_{3}\left(\mathbf{5 N O}_{2}\right.$ phen)] crystallizes in the triclinic space group P-1 with three independent complexes of formula $\mathrm{C}_{39} \mathrm{H}_{52} \mathrm{ErN}_{3} \mathrm{O}_{8}$ in the asymmetric unit (Figure 48, Table 12). In each complex, the $\mathrm{Er}^{3+}$ ion is eight times coordinated with six oxygen atoms from the $\beta$-diketonates and two nitrogen atoms from the 5-nitro-1,10-phenanthroline moiety forming a distorted square-antiprismatic prism. The erbium ion occupies the centre of the prism with a distance of 1.36/1.39/1.40 $\AA$ to the square face containing the $N$ atoms and 1.19/1.17/1.16 $\AA$ to the opposite face. There are no classic hydrogen bonds between the complexes due to the lack of donors; only some weak $\mathrm{C}-\mathrm{H} . . . \pi$ intermolecular contacts can be discerned. The complexes pack leaving no solvent accessible voids.

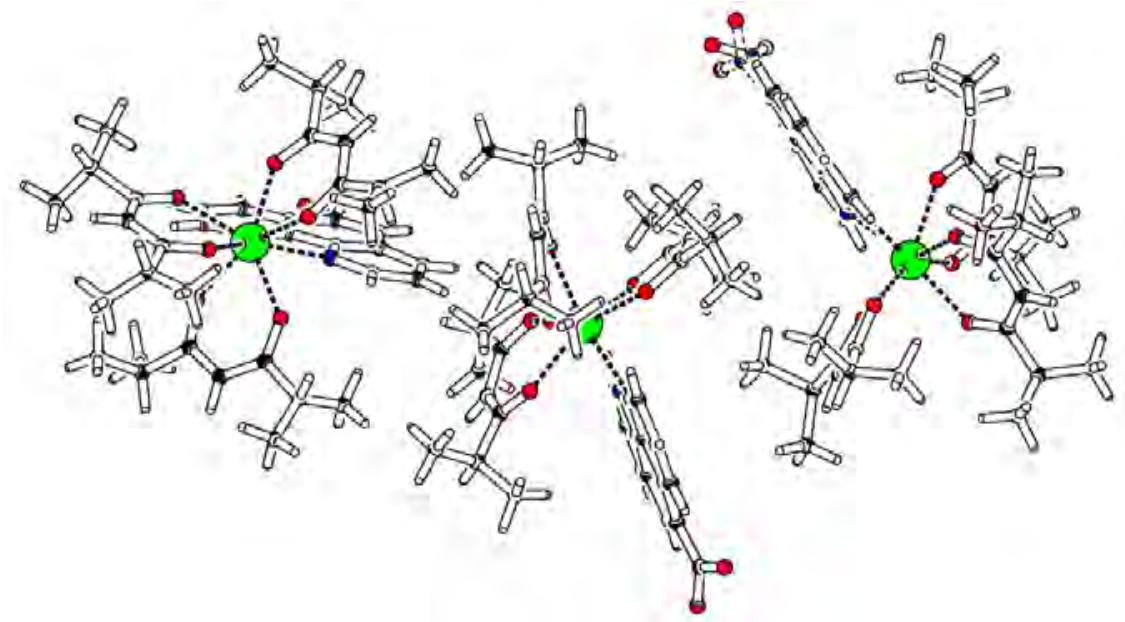

Figure 48. Structural diagram of $\left[\operatorname{Er}(\mathrm{dmh})_{3}\left(5 \mathrm{NO}_{2}\right.\right.$ phen $\left.)\right]$ showing the three symmetry independent complexes in the unit cell 
Table 12. Crystal data and structure refinement for the complexes with 2,6-dimethyl-3,5heptanedione

\begin{tabular}{|c|c|c|c|}
\hline Complex & {$\left[\operatorname{Er}(\mathrm{dmh})_{3}(\right.$ bipy $\left.)\right]$} & {$\left[\operatorname{Er}(\mathrm{dmh})_{3}(\right.$ bath $\left.)\right]$} & {$\left[\operatorname{Er}(\mathrm{dmh})_{3}\left(5 \mathrm{NO}_{2}\right.\right.$ phen $\left.)\right]$} \\
\hline Empirical formula & $\mathrm{C}_{37} \mathrm{H}_{53} \mathrm{ErN}_{2} \mathrm{O}_{6}$ & $\mathrm{C}_{51} \mathrm{H}_{61} \mathrm{ErN}_{2} \mathrm{O}_{6}$ & $\mathrm{C}_{39} \mathrm{H}_{52} \mathrm{ErN}_{3} \mathrm{O}_{8}$ \\
\hline Formula weight & 789.07 & 965.28 & 858.10 \\
\hline Temperature (K) & $293(3)$ & $293(3)$ & 293(3) \\
\hline Wavelength $(\AA)$ & 0.71073 & 0.71073 & 0.71073 \\
\hline Crystal system & Triclinic & Monoclinic & Triclinic \\
\hline Space group & P-1 & $\mathrm{P} 21 / \mathrm{c}$ & $\mathrm{P}-1$ \\
\hline$a(\AA)$ & $13.2245(3)$ & $14.3822(4)$ & $13.7978(9)$ \\
\hline$b(\AA)$ & $16.7752(4)$ & $15.6172(5)$ & $17.4765(12)$ \\
\hline$c(\AA)$ & $18.0529(4)$ & $23.5197(6)$ & $26.1660(17)$ \\
\hline$\alpha(\underline{0})$ & $92.3120(10)$ & 90.00 & $83.044(5)$ \\
\hline$\beta(\underline{o})$ & $94.6010(10)$ & $105.3801(14)$ & $79.390(5)$ \\
\hline$\gamma(\underline{o})$ & $92.7510(10)$ & 90.00 & $88.249(5)$ \\
\hline Volume $\left(\AA^{3}\right)$ & 3983.48(16) & $5093.6(3)$ & $6155.9(7)$ \\
\hline $\mathrm{Z}$ & 4 & 4 & 6 \\
\hline Calculated density $\left(\mathrm{g} / \mathrm{cm}^{3}\right)$ & 1.316 & 1.259 & 1.389 \\
\hline Absorption coefficient $\left(\mathrm{mm}^{-1}\right)$ & 2.148 & 1.693 & 2.096 \\
\hline$F(000)$ & 1620.0 & 1988 & 2634.0 \\
\hline Crystal size $\left(\mathrm{mm}^{3}\right)$ & $0.24 \times 0.10 \times 0.08$ & $0.20 \times 0.06 \times 0.02$ & $0.20 \times 0.10 \times 0.04$ \\
\hline$\theta$ range for data collection $(\underline{o})$ & $2.23-20.50$ & $2.22-29.51$ & $1.5-26.0$ \\
\hline Reflections collected & 54617 & 151284 & 120371 \\
\hline Independent reflections $[I>2 \sigma(I)]$ & 8924 & 10217 & 7866 \\
\hline Completeness to $2 \theta=510$ & $99.6 \%$ & $99.7 \%$ & $97 \%$ \\
\hline Refinement method & Full matrix LS on $\mathrm{F}^{2}$ & Full matrix LS on $\mathrm{F}^{2}$ & Full matrix LS on $\mathrm{F}^{2}$ \\
\hline Data/restrains/parameters & $15197 / 28 / 853$ & $14160 / 208 / 537$ & $23408 / 7 / 1411 / 8$ \\
\hline Goodness-of-fit on $F^{2}$ & 0.987 & 1.067 & 0.939 \\
\hline Final $R$ indices $[\mathrm{I}>2 \sigma(\mathrm{I})]$ & 0.0409 & 0.0484 & 0.0812 \\
\hline$w R$ indices (all data) & 0.1052 & 0.1659 & 0.2190 \\
\hline Largest diff. peak and hole & $0.750 /-0.415$ & $1.736 /-0.786$ & $1.795 /-0.911$ \\
\hline
\end{tabular}

Table 13. Er-N and Er-0 distances in Å for the complexes with 2,6-dimethyl-3,5-heptanedione

\begin{tabular}{cccc} 
Bond & {$\left[\operatorname{Er}(\mathrm{dmh})_{3}(\right.$ bipy $\left.)\right]$} & {$\left[\operatorname{Er}(\mathrm{dmh})_{3}(\right.$ bath $\left.)\right]$} & {$\left[\operatorname{Er}(\mathrm{dmh})_{3}\left(5 \mathrm{NO}_{2}\right.\right.$ phen $\left.)\right]$} \\
\hline Average Er-O & $2.290(5)$ & $2.279(30)$ & $2.274(7)$ \\
Average Er-N & $2.554(3)$ & $2.560(17)$ & $2.547(3)$ \\
Average N-Er-N & $62.10(7)$ & $62.6(3)$ & $63.34(8)$ \\
\hline
\end{tabular}

17. The asymmetric unit of [Er(thd) $)_{3}$ (bath)] contains only one $\operatorname{Er}(\mathrm{III})$ complex. The Er(III) ion lies in a general position in the middle of a square-antiprismatic environment made up of four $\mathrm{O}$ atoms from three bidentate $\beta$-diketonate ligands and two $\mathrm{N}$ atoms from one $\mathrm{N}, \mathrm{N}$ donor (bathophenathroline) (Figure 49, Table 14). The Er-O and Er-N bond distances are in the ranges 2.2178(14)-2.3014(14) $\AA$ and 2.5501(17)-2.603(2) $\AA$, respectively, in good agreement with their average values [159].

The lanthanide ion is displaced by $1.4067(1) \AA$ from the N2/N2/05/06 face, and by 1.1462(1) A from the opposite face.

In the bathophenanthroline molecule there is a rotation of the two terminal phnenyl groups with respect to the central phenanthroline units, dihedral angles 72.95(7)을 and 61.15(8) . There is also aslight torsion in the phenanthroline unit: the angle between the mean planes of the two pyridine rings is $4.72(12)^{\circ}$. 
All the 2,2,6,6-tetramethyl-3,5-heptanedionate ligands show signs of disorder. In the three of them one terminal $\mathrm{C}(\mathrm{CH})_{3}$ groups was modelled in two staggered positions roughly with $60 / 40$ occupancies.

The bulky complexes pack in the centrosymmetric P2 1 /c space group leaving voids of $23 \AA^{3}$ and $172 \AA^{3}$.

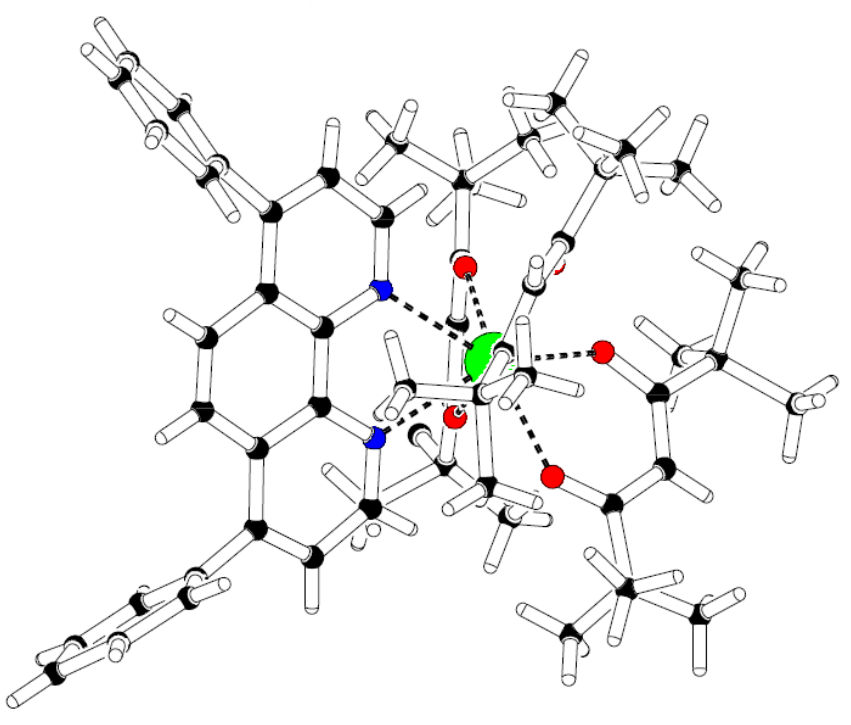

Figure 49. Structural diagram of $\left[\operatorname{Er}(\text { thd })_{3}(\right.$ bath $\left.)\right]$

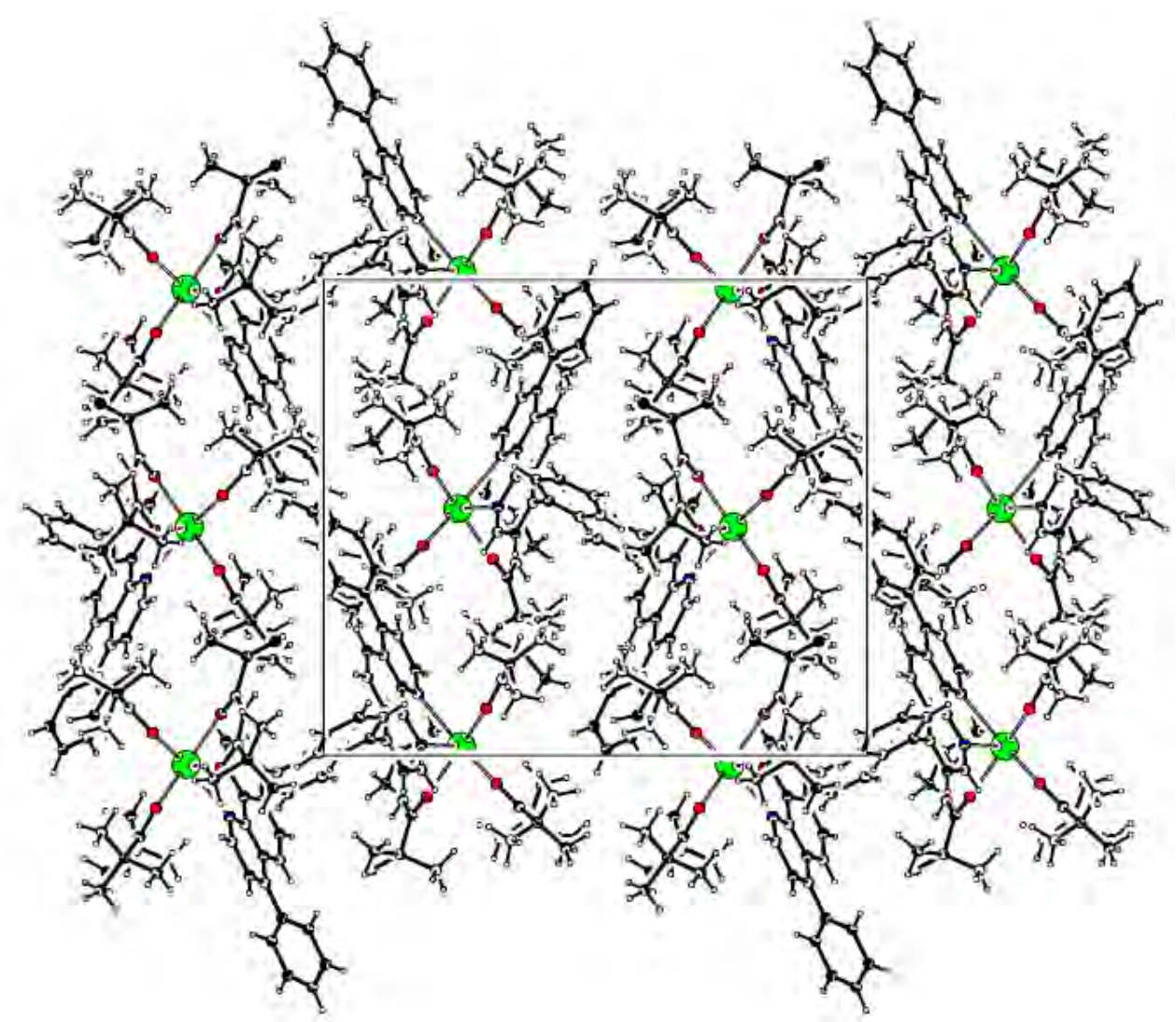

Figure 50. Packing diagram of $\left[\operatorname{Er}(\text { thd })_{3}(\right.$ bath $\left.)\right]$ 
Table 14. Crystal data and structure refinement for [Er(thd $)_{3}($ bath)]

\begin{tabular}{|c|c|}
\hline Complex & {$\left[\operatorname{Er}(\text { thd })_{3}\right.$ (bath) $]$} \\
\hline Empirical formula & $\mathrm{C}_{57} \mathrm{H}_{73} \mathrm{ErN}_{2} \mathrm{O}_{6}$ \\
\hline Formula weight & 1049.43 \\
\hline Temperature $(\mathrm{K})$ & $293(2)$ \\
\hline Wavelength $(\AA)$ & 0.71073 \\
\hline Crystal system & Monoclinic \\
\hline Space group & $P 2_{1} / c$ \\
\hline$a(\AA)$ & $16.8783(6)$ \\
\hline$b(\AA)$ & $20.1277(6)$ \\
\hline$c(\AA)$ & $22.9904(7)$ \\
\hline$\alpha(\underline{\mathrm{o}})$ & 90.00 \\
\hline$\beta(\underline{\mathrm{o}})$ & $130.024(2)$ \\
\hline$\gamma(\underline{\mathrm{o}})$ & 90.00 \\
\hline Volume $\left(\AA^{3}\right)$ & $5981.0(3)$ \\
\hline$Z$ & 4 \\
\hline Calculated density $\left(\mathrm{g} \mathrm{cm}^{-3}\right)$ & 1.165 \\
\hline Absorption coefficient $\left(\mathrm{mm}^{-1}\right)$ & 1.447 \\
\hline$F(000)$ & 2180 \\
\hline Crystal size $\left(\mathrm{mm}^{3}\right)$ & $0.25 \times 0.10 \times 0.12$ \\
\hline$\theta$ range for data collection $(\stackrel{\circ}{ })$ & $1.54-25.86$ \\
\hline Index ranges & $-20<h<20 ;-24<k<24 ;-28<l<28$ \\
\hline Reflections collected & 85886 \\
\hline Independent reflections & 5689 \\
\hline Completeness to $2 \theta=51^{\circ} \mathrm{o}$ & $99.2 \%$ \\
\hline Refinement method & Full matrix LS on $F^{2}$ \\
\hline Data/restrains/parameters & $11479 / 42 / 604$ \\
\hline Goodness-of-fit on $F^{2}$ & 0.973 \\
\hline Final $R$ indices $[I>2 \sigma(I)]$ & $R=0.0714 ; \mathrm{w} R=0.1897$ \\
\hline$R$ indices (all data) & $R=0.1418 ; \mathrm{w} R=0.2148$ \\
\hline Largest diff. peak and hole & $-1.451 / 2.224$ \\
\hline
\end{tabular}

Table 15. Selected distances and angles $(\AA ̊$, og $)$ for $\left[\operatorname{Er}(\text { thd })_{3}(\right.$ bath $\left.)\right]$

\begin{tabular}{cccc} 
Bond & Distance & Bonds & Angle \\
\hline Er1-01 & $2.260(2)$ & 01-Er1-05 & $149.08(7)$ \\
Er1-02 & $2.3014(14)$ & 01-Er1-04 & $77.56(6)$ \\
Er1-03 & $2.2630(19)$ & 05-Er1-03 & $80.60(6)$ \\
Er1-04 & $2.2178(14)$ & 04-Er1-02 & $122.43(6)$ \\
Er1-05 & $2.2970(15)$ & O3-Er1-02 & $76.03(6)$ \\
Er1-06 & $2.264(2)$ & 01-Er1-N1 & $78.91(6)$ \\
Er1-N1 & $2.5501(17)$ & O6-Er1-N1 & $71.10(6)$ \\
Er1-N2 & $2.603(2)$ & O6-Er1-N2 & $110.28(7)$ \\
Er1-O avg. & 2.267 & 05-Er1-N2 & $73.00(6)$ \\
Er1-N avg. & 2.576 & & \\
\hline
\end{tabular}


19. [Er(od) $)_{3}($ bath)] crystallizes in the triclinic space group P-1, with cell parameters $a=12.2423(3) \AA, \quad b=12.9649(4) \AA, \quad c=15.1860(4) \AA, \quad \alpha=97.250(2)^{\circ}, \quad \beta=91.861(2)^{\circ}$, $\gamma=105.927(2)^{\circ}$ and $V=2293.57(11) \AA^{3}$ (Figure 51, Table 16).

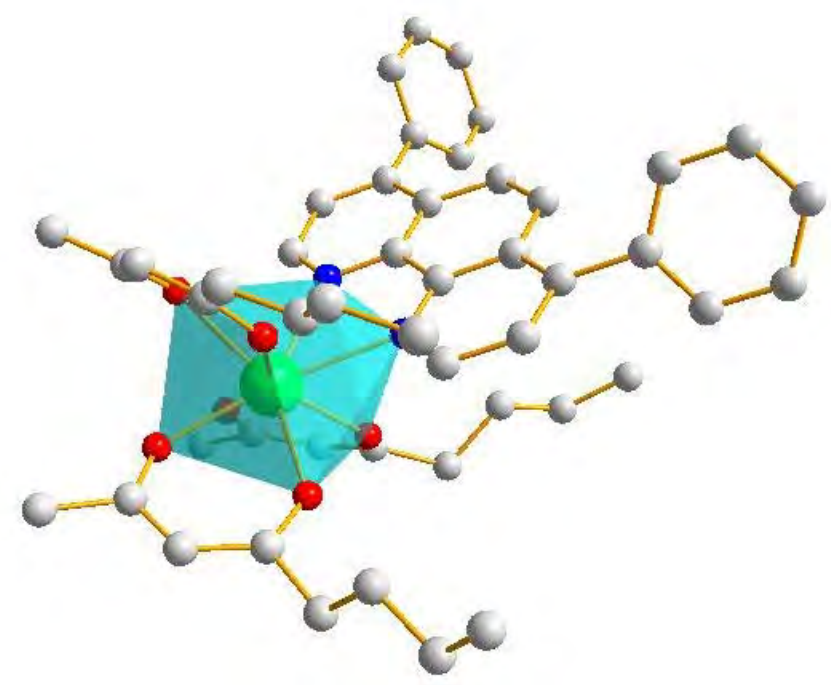

Figure 51. Structural diagram of $\left[\operatorname{Er}(\mathrm{od})_{3}(\right.$ bath $\left.)\right]$

20. [Er(od $)_{3}\left(\mathbf{N N O}_{2}\right.$ phen)] crystallizes in the monoclinic $\mathrm{P} 2_{1} / \mathrm{c}$ space group, with four complexes in the unit cell and cell parameters $a=20.8893(5) \AA, b=9.5161(2) \AA ⿻$, $c=19.3390(5)$ $\AA, \alpha=90.00^{\circ}, \beta=106.230(2)^{\circ}, \gamma=90.00^{\circ}$ a and $V=3691.09(15) \AA^{3}$ (Figure 52, Table 16).

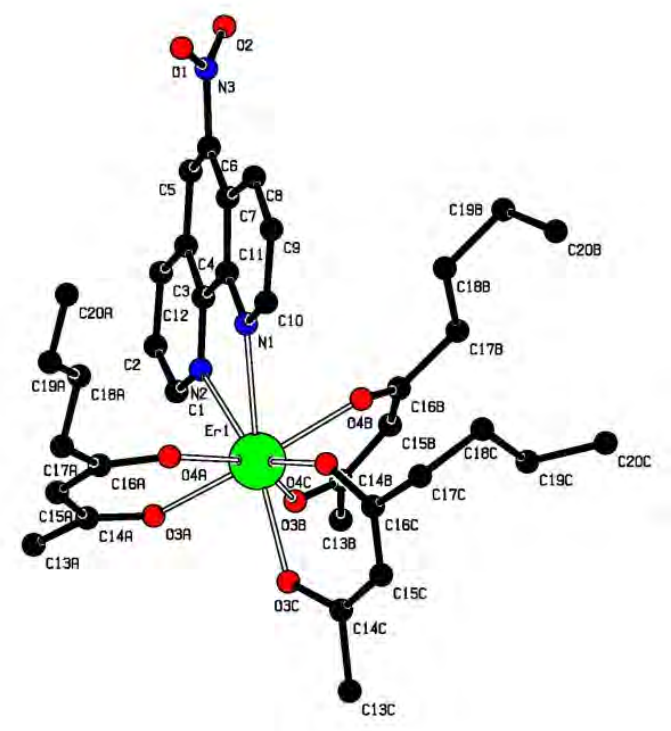

Figure 52. Structural diagram of $\left[\operatorname{Er}(\mathrm{od})_{3}(5 \mathrm{NO} 2 \mathrm{phen})\right]$ 
Table 16. Crystal data and structure refinement for the complexes with 2,4-octanedione

\begin{tabular}{|c|c|c|}
\hline Complex & {$\left[\operatorname{Er}(\mathrm{od})_{3}(\mathrm{bath})\right]$} & {$\left[\operatorname{Er}(\mathrm{od})_{3}\left(5 \mathrm{NO}_{2}\right.\right.$ phen $\left.)\right]$} \\
\hline Empirical formula & $\mathrm{C}_{48} \mathrm{H}_{55} \mathrm{ErN}_{2} \mathrm{O}_{6}$ & $\mathrm{C}_{36} \mathrm{H}_{46} \mathrm{ErN}_{3} \mathrm{O}_{8}$ \\
\hline Formula weight & 923.22 & 816.02 \\
\hline Temperature (K) & $293(3)$ & $293(3)$ \\
\hline Wavelength $(\AA)$ & 0.71073 & 0.71073 \\
\hline Crystal system & Triclinic & Monoclinic \\
\hline Space group & $P-1$ & $P 2_{1} / c$ \\
\hline$a(\AA)$ & $12.2423(3)$ & $20.8893(5)$ \\
\hline$b(\AA)$ & $12.9649(4)$ & $9.5161(2)$ \\
\hline$c(\AA)$ & $15.1860(4)$ & $19.3390(5)$ \\
\hline$\alpha(\underline{0})$ & $97.250(2)$ & 90.00 \\
\hline$\beta(\underline{0})$ & $91.861(2)$ & $106.230(2)$ \\
\hline$\gamma(\underline{\mathrm{o}})$ & $105.927(2)$ & 90.00 \\
\hline Volume $\left(\AA^{3}\right)$ & $2293.57(11)$ & $3691.09(15)$ \\
\hline$Z$ & 1 & 4 \\
\hline Calculated density $\left(\mathrm{g} \mathrm{cm}^{-3}\right)$ & 1.328 & 1.468 \\
\hline Absorption coefficient $\left(\mathrm{mm}^{-1}\right)$ & 1.876 & 2.326 \\
\hline$F(000)$ & 934 & 1660 \\
\hline Crystal size $\left(\mathrm{mm}^{3}\right)$ & $0.33 \times 0.04 \times 0.09$ & - \\
\hline$\theta$ range for data collection & $1.98-25.80^{\circ}$ & $2.03-25.75^{\circ}$ \\
\hline Index ranges & $-14<h<14 ;-15<k<15 ;-18<l<18$ & $-25<h<25 ;-11<k<11 ;-23<l<23$ \\
\hline Reflections collected & 49625 & 69344 \\
\hline Independent reflections & 3567 & 3747 \\
\hline Completeness to $2 \theta=51^{\mathrm{o}}$ & $98.7 \%$ & $99.7 \%$ \\
\hline Refinement method & Full matrix LS on $\mathrm{F}^{2}$ & Full matrix LS on $\mathrm{F}^{2}$ \\
\hline Data/restrains/parameters & $8703 / 1 / 522$ & $7055 / 21 / 439$ \\
\hline Goodness-of-fit on $\mathrm{F}^{2}$ & 0.981 & 1.029 \\
\hline Final $R$ indices $[I>2 \sigma(I)]$ & $R=0.0624 ; \mathrm{w} R=0.2182$ & $R=0.0496 ; \mathrm{w} R=0.1291$ \\
\hline$R$ indices (all data) & $R=0.1288 ; \mathrm{w} R=0.2263$ & $R=0.1300 ; \mathrm{w} R=0.2048$ \\
\hline Largest diff. peak and hole & $-1.500 / 1.099$ & $-1.534 / 0.877$ \\
\hline
\end{tabular}

Table 17. Er-N and Er-O distances in $\AA$ for the complexes with 2,4-octanedione

\begin{tabular}{ccc} 
Bond & {$\left[\operatorname{Er}(\text { od })_{3}(\right.$ bath $\left.)\right]$} & {$\left[\operatorname{Er}(\text { od })_{3}\left(5 \mathrm{NO}_{2}\right.\right.$ phen $\left.)\right]$} \\
\hline Er-N1 & $2.5235(16)$ & $2.575(5)$ \\
Er-N2 & $2.5220(16)$ & $2.627(5)$ \\
Er-01 & $2.3218(12)$ & $2.305(6)$ \\
Er-O2 & $2.2914(13)$ & $2.319(4)$ \\
Er-O3 & $2.3057(13)$ & $2.324(5)$ \\
Er-04 & $2.2599(12)$ & $2.262(5)$ \\
Er-05 & $2.2961(13)$ & $2.301(5)$ \\
Er-06 & $2.2785(14)$ & $2.307(5)$ \\
Er-N avg. & 2.5228 & 2.601 \\
Er-0 avg. & 2.2922 & 2.303 \\
\hline
\end{tabular}

22. The crystal structure of Tris(4,4,4-trifluoro-1-(2-furyl)-1,3-butanedionate)mono(2,2'bipyridine)erbium(III) consists of neutral [Er(tfa) $)_{3}$ (bipy)] complexes compactly packed, since the structure shows no solvent accessible voids. Due to the lack of conventional H-bond donors only weak intermolecular interactions join the complexes together. The lanthanide ion, being eight coordinated, is surrounded by six oxygen atoms belonging to three bidentate betadiketonates anions and two nitrogen atoms belonging to the Schiff base ligand. The coordination polyhedron is a square antiprismatic structure in which the set N1, N2, 04,05 and the set 01, 02, 07, 08 form two approximate squares, respectively, with a dihedral angle of just $2.40(17)^{\mathrm{o}}$ between their mean planes (Figure 53). The lanthanide ion lies 
approximately in the center of the anti-prism with a distance of $1.3768(3) \AA$ to the face containing the $\mathrm{N}$ atoms and 1.1627(3) Å to the opposite face.

The Er-O bond distances span the range 2.288(4)-2.334(4) $\AA$, and the Er-N 2.512(5) 2.526(5) Å (Table 19).

In the anionic ligands the furyl rings share approximately the same plane as the central diketonate moiety. Also, in the bipyridine ligand the two aromatic rings are only slightly rotated with a 5o dihedral angle between their mean planes. In the crystal structure the complexes pack in dimers with the bipyridine ring systems exhibiting a short centroid...centroidi distance 3.749(4) $\AA$ ( $i:-x,-y,-z)$, as it is shown in Figure 54.

Apart from $\pi \ldots \pi$ interactions, a few weak C-H...O,F intermolecular interactions can be spotted, shown in Table 20.

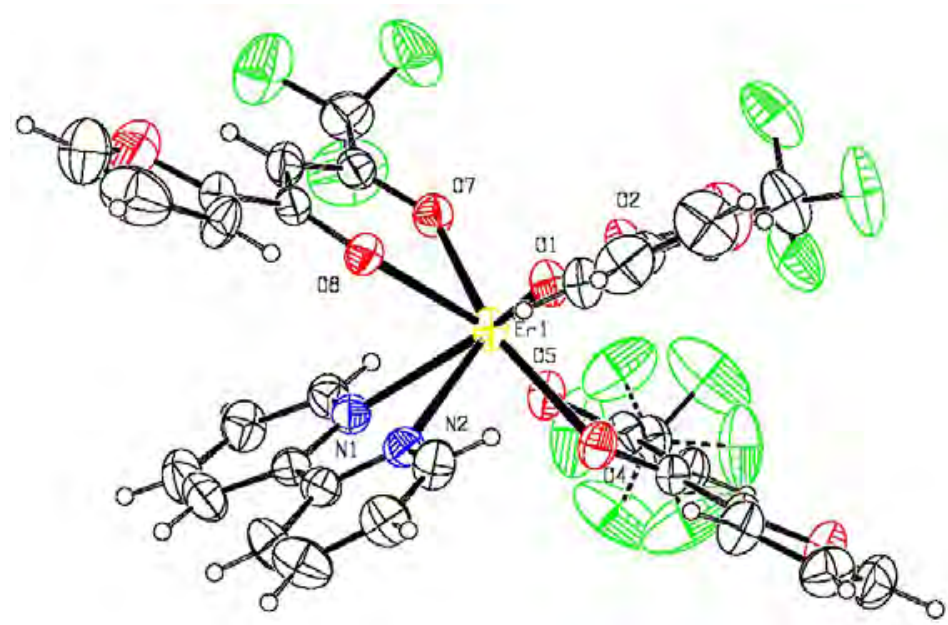

Figure 53. A thermal ellipsoid plot of $\left[\operatorname{Er}(\mathrm{tfa})_{3}(\mathrm{bipy})\right]$, showing the anti-square prismatic conformation around the $\mathrm{Er}^{3+}$ ion.

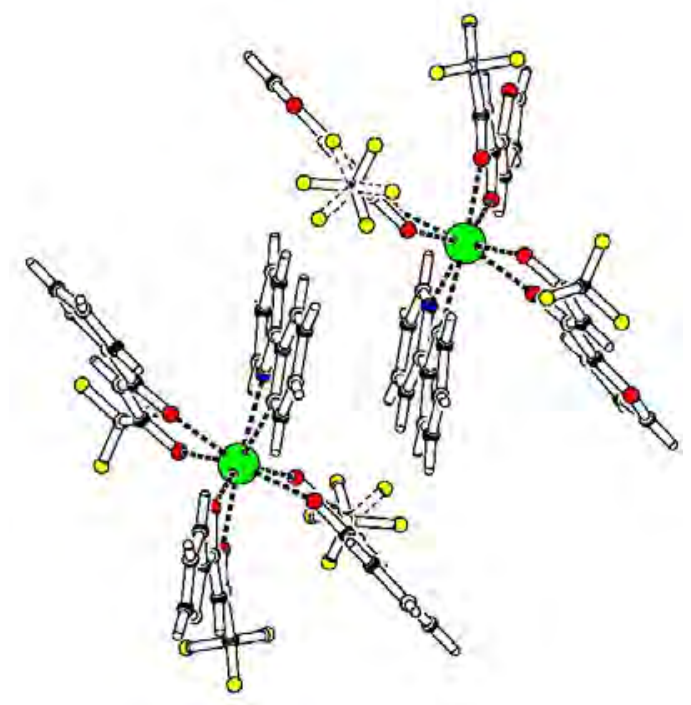

Figure 54. Two neighboring complexes joined via $\pi \ldots \pi$ intermolecular interactions 
Table 18. Crystal data and structure refinement for [Er(tfa $)_{3}($ bipy)]

\begin{tabular}{|c|c|}
\hline Complex & {$\left[\operatorname{Er}(\mathrm{tfa})_{3}(\mathrm{bipy})\right]$} \\
\hline Empirical formula & $\mathrm{C}_{34} \mathrm{H}_{20} \mathrm{ErF}_{9} \mathrm{~N}_{2} \mathrm{O}_{9}$ \\
\hline Formula weight & 938.78 \\
\hline Temperature (K) & $293(2)$ \\
\hline Wavelength $(\AA)$ & 0.71073 \\
\hline Crystal system & Monoclinic \\
\hline Space group & $P 2_{1} / c$ \\
\hline$a(\AA)$ & $10.124(3)$ \\
\hline$b(\AA)$ & $16.991(6)$ \\
\hline$c(\AA)$ & $21.418(7)$ \\
\hline$\alpha(\underline{\mathrm{o}})$ & 90 \\
\hline$\beta(\underline{\mathrm{o}})$ & $104.820(7)$ \\
\hline$\gamma(\underline{\mathrm{o}})$ & 90 \\
\hline Volume $\left(\AA^{3}\right)$ & $3562(2)$ \\
\hline$Z$ & 4 \\
\hline Calculated density $\left(\mathrm{g} \mathrm{cm}^{-3}\right)$ & 1.751 \\
\hline Absorption coefficient $\left(\mathrm{mm}^{-1}\right)$ & 2.459 \\
\hline$F(000)$ & 1836.0 \\
\hline Crystal size $\left(\mathrm{mm}^{3}\right)$ & $0.22 \times 0.15 \times 0.10$ \\
\hline$\theta$ range for data collection $(\stackrel{\circ}{ })$ & $1.97-28.36$ \\
\hline Index ranges & $-13<h<13 ;-22<k<22 ;-28<l<28$ \\
\hline Reflections collected & 58504 \\
\hline Independent reflections & 8884 \\
\hline Completeness to $2 \theta=51^{\circ} \mathrm{o}$ & $99.6 \%$ \\
\hline Refinement method & Full matrix LS on $\mathrm{F}^{2}$ \\
\hline Data/restrains/parameters & $4901 / 0 / 526$ \\
\hline Goodness-of-fit on $\mathrm{F}^{2}$ & 0.958 \\
\hline Final $R$ indices $[I>2 \sigma(I)]$ & $R=0.0486 ; \mathrm{w} R=0.0970$ \\
\hline$R$ indices (all data) & $R=0.1111 ; \mathrm{w} R=0.1195$ \\
\hline Largest diff. peak and hole & $-0.667 / 1.188$ \\
\hline
\end{tabular}

Table 19. Selected distances and angles $(\AA ̊,-0)$ for $\left[\operatorname{Er}(\operatorname{tfa})_{3}(\right.$ bipy $\left.)\right]$

\begin{tabular}{cccc} 
Bond & Distance & Bonds & Angle \\
\hline Er1-01 & $2.295(4)$ & 01-Er1-05 & $138.22(13)$ \\
Er1-02 & $2.288(4)$ & 01-Er1-07 & $119.56(14)$ \\
Er1-04 & $2.303(4)$ & O2-Er1-04 & $82.98(14)$ \\
Er1-05 & $2.334(4)$ & 02-Er1-05 & $76.08(14)$ \\
Er1-07 & $2.309(4)$ & 04-Er1-07 & $151.78(14)$ \\
Er1-08 & $2.310(4)$ & 01-Er1-N1 & $141.37(15)$ \\
Er1-N1 & $2.512(5)$ & 08-Er1-N1 & $79.01(14)$ \\
Er1-N2 & $2.526(5)$ & 08-Er1-N2 & $70.59(14)$ \\
Er1-0 avg. & 2.3065 & 07-Er1-N2 & $131.15(14)$ \\
Er1-N avg. & 2.519 & & \\
\hline
\end{tabular}

Table 20. H-bond geometry $(\AA, \stackrel{\circ}{\circ})$ for $\left[\operatorname{Er}(\operatorname{tfa})_{3}(\right.$ bipy $\left.)\right]$

\begin{tabular}{cccccc} 
D & H & A & H...A & D...A & D - H...A \\
\hline C8 & H8 & F1i & 2.4600 & $3.194(12)$ & 136.00 \\
C16 & H16 & 08ii & 2.5200 & $3.261(9)$ & 137.00 \\
\hline
\end{tabular}

$i: \mathrm{x}-1, \mathrm{y}, \mathrm{z} ;$ ii: $-\mathrm{x},-1 / 2+\mathrm{y}, 1 / 2-\mathrm{z}$ 
23. A summary of $X$-ray crystallographic data for $\left[\operatorname{Er}(\operatorname{tfn} \mathbf{b})_{3}(\mathbf{b i p y})\right]$ is reported in Table 21. $\left[\operatorname{Er}(\operatorname{tfnb})_{3}(\right.$ bipy)] crystallizes in the centrosymmetric $C 2 / c$ space group. The unit cell contains eight complex molecules. Each Er ion eight-coordinated with $\mathrm{N}$ and $\mathrm{O}$ neighbors forming a square anti-prismatic structure in which the set N1, N2, 03, 04 and the set 01, 02, 05, 06 form two approximate squares, respectively (Figure 55). In the center of the anti-prism lies the trivalent erbium with a distance of 1.3358(4) $\AA$ to the face containing the $\mathrm{N}$ atoms and $1.1946(4) \AA$ to the opposite face containing exclusively oxygens.

The Er-N distances are identical within uncertainty, 2.496(7) Å to N1 and 2.497(8) Å to N2. The Er-O distances are in the range 2.270(6)-2.311(7) $\AA$ (Table 22).

The aromatic rings of the fluorinated ligands do not share the same plane as the $\beta$-diketonate moieties. The two planes of the ligands make dihedral angles of $14^{\circ}$ except in one of them. The ligand containing the disordered $\mathrm{CF}_{3}$ group (that was refined over two positions) shows a larger $\left[21.6(4)^{\circ}\right]$ dihedral angle between the aromatic naphthalene moiety and the planar diketonate central part of ligand. This larger twisting promotes a short distance between the centroid of the ring C33-C38 and the centroid of the same ring in a symmetry related complex molecule (centroidi, where $i: 1 / 2-x, 1 / 2-y, 1-z$ ), as shown in Figure 56 [centroid...centroid ${ }^{i}$ distance $=3.671(6) \AA$, slippage $=1.267 \AA$ ] . Apart from $\pi \ldots \pi$ interactions and due to the lack of donors there are no conventional intermolecular $\mathrm{H}$-bonds linking the molecules together. There is a total potential solvent volume of $918.4 \AA^{3}$ in each unit cell comprising $9.3 \%$ of the total volume. The solvent accessible volume is divided over several pores of 109 and $87 \AA^{3}$.

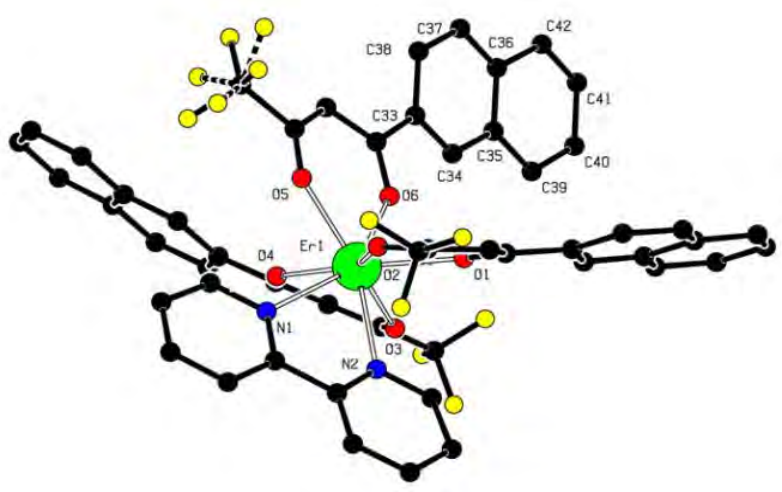

Figure 55. Structural diagram of the $\left[\operatorname{Er}(\operatorname{tfnb})_{3}(\right.$ bipy $\left.)\right]$ complex, showing the anti-square prismatic conformation of the first coordination shell the Er(III) ion.

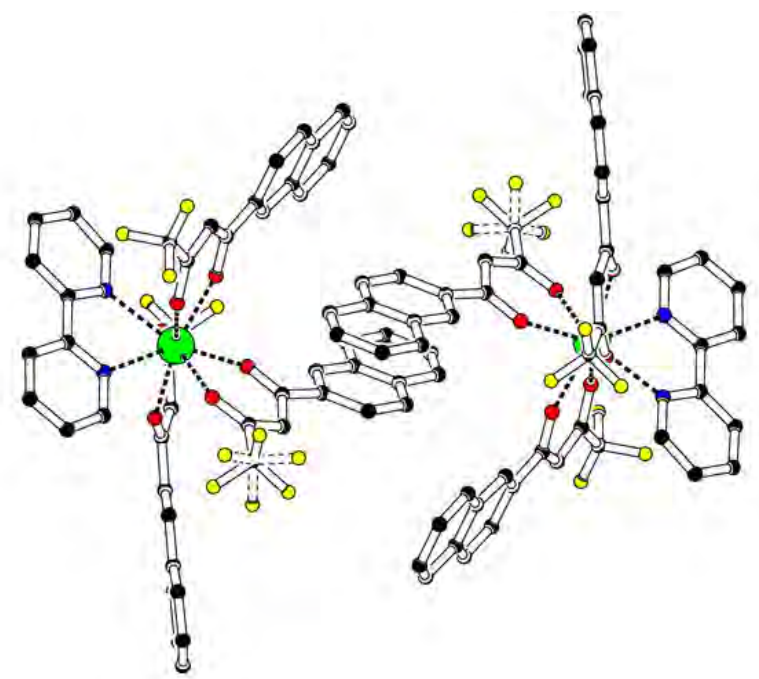

Figure 56. Two neighboring complexes joined via $\pi . . . \pi$ intermolecular interactions. 
Table 21. Crystal data and structure refinement of complex [Er(tfnb) $\left.)_{3}(\mathrm{bipy})\right]$

\begin{tabular}{|c|c|}
\hline Complex & {$\left[\operatorname{Er}(\mathrm{tfnb})_{3}(\mathrm{bipy})\right]$} \\
\hline Empirical formula & $\mathrm{C}_{52} \mathrm{H}_{32} \mathrm{ErF}_{9} \mathrm{~N}_{2} \mathrm{O}_{6}$ \\
\hline Formula weight & 1119.06 \\
\hline Temperature (K) & $293(2)$ \\
\hline Wavelength $(\AA)$ & 0.71073 \\
\hline Crystal system & Monoclinic \\
\hline Space group & $C 2 / c$ \\
\hline$a(\AA)$ & $22.301(2)$ \\
\hline$b(\AA)$ & $22.919(3)$ \\
\hline$c(\AA)$ & $20.220(2)$ \\
\hline$\beta(\underline{\mathrm{o}})$ & $107.657(2)$ \\
\hline Volume $\left(\AA^{3}\right)$ & $9847.6(18)$ \\
\hline$Z$ & 8 \\
\hline Calculated density $\left(\mathrm{g} \mathrm{cm}^{-3}\right)$ & 1.510 \\
\hline Absorption coefficient $\left(\mathrm{mm}^{-1}\right)$ & 1.788 \\
\hline$F(000)$ & 4440 \\
\hline Crystal size $\left(\mathrm{mm}^{3}\right)$ & $0.28 \times 0.14 \times 0.12$ \\
\hline$\theta$ range for data collection $(\stackrel{\circ}{ })$ & $1.49-25.70$ \\
\hline Index ranges & $-19<h<27 ;-24<k<27 ;-24<l<17$ \\
\hline Reflections collected & 25752 \\
\hline Independent reflections & 9328 \\
\hline Completeness to $2 \theta=51^{\circ}$ & $99.6 \%$ \\
\hline Refinement method & Full matrix LS on $\mathrm{F}^{2}$ \\
\hline Data/restrains/parameters & $3885 / 0 / 629$ \\
\hline Goodness-of-fit on $\mathrm{F}^{2}$ & 0.917 \\
\hline Final $R$ indices $[I>2 \sigma(I)]$ & $R=0.0612 ; \mathrm{w} R=0.1000$ \\
\hline$R$ indices (all data) & $R=0.1841 ; \mathrm{w} R=0.1353$ \\
\hline Largest diff. peak and hole & $-0.611 / 0.641$ \\
\hline
\end{tabular}

Table 22. Selected distances $(\AA ̊)$ and angles $\left(\stackrel{\circ}{)}\right.$ ) for [ $\operatorname{Er}(\operatorname{tfnb})_{3}($ bipy $\left.)\right]$

\begin{tabular}{cccc} 
Bond & Distance & Bonds & Angle \\
\hline Er1-01 & $2.270(6)$ & 01-Er1-05 & $115.2(2)$ \\
Er1-05 & $2.275(7)$ & 01-Er1-04 & $143.8(2)$ \\
Er1-06 & $2.292(6)$ & 05-Er1-03 & $142.8(2)$ \\
Er1-04 & $2.293(6)$ & $04-E r 1-02$ & $143.3(2)$ \\
Er1-03 & $2.306(6)$ & $03-E r 1-02$ & $138.6(2)$ \\
Er1-02 & $2.311(7)$ & $01-E r 1-N 1$ & $137.4(2)$ \\
Er1-N1 & $2.496(7)$ & 06-Er1-N1 & $147.2(3)$ \\
Er1-N2 & $2.497(8)$ & 06-Er1-N2 & $146.2(3)$ \\
Er1-0 avg. & 2.2912 & 05-Er1-N2 & $139.4(3)$ \\
Er1-N avg. & 2.4965 & & \\
\hline
\end{tabular}

24, 25 and 26. [Er(tfac) $)_{3}(\mathbf{N}, \mathbf{N}-$ donor)] complexes. In the three structures (Figure 57, Figure 58 and Figure 59), three negative $\beta$-diketonate ligands and one $\mathrm{N}, \mathrm{N}$-donor moiety coordinate the $\mathrm{Er}^{3+}$ ion. The environment around the lanthanide is a slightly distorted square-antiprismatic configuration, with one top square face containing the two $\mathrm{N}$ atoms and two $\mathrm{O}$ atoms and the bottom square face containing the remaining four $\mathrm{O}$ atoms. The angle between the mean planes of such faces ranges between $2^{\circ}$ and $4^{\circ}$ in the three compounds.

All compounds show some signs of structural disorder: [ $\left.\operatorname{Er}(\operatorname{tfac})_{3}(\mathrm{bipy})\right]$ has a random interchange of the bipyridine moiety with one (1,1,1-trifluoro-2,4-pentanedione), simulating an inversion symmetry around the erbium ion, and its $\mathrm{CF}_{3}$ groups are also disordered; in $\left[\operatorname{Er}(\mathrm{tfac})_{3}(\mathrm{bath})\right]$ the terminal $\mathrm{CF}_{3}$ groups are disordered over the two ends of the molecule, and disordered in two conformations in each end, resulting in four possible conformations 
for each group in each diketonate ion; and $\left[\operatorname{Er}(\operatorname{tfac})_{3}\left(5 \mathrm{NO}_{2}\right.\right.$ phen $\left.)\right]$ evidences mild disorder with large terminal ellipsoids.

5-nitro-1,10-phenanthroline and bathophenanthroline show similar Er-N and Er-O distances (Table 24). Due to disorder in 2,2'-bipyridine, the Er-N and Er-O distances are more similar to each other.

None of the three compounds show intermolecular interactions other than Van der Waals.

$\left[\operatorname{Er}(\operatorname{tfac})_{3}\right.$ (bipy)] is the complex which packs more efficiently out of the three, leaving no solvent accessible voids. $\left[\operatorname{Er}(\operatorname{tfac})_{3}\left(5 \mathrm{NO}_{2}\right.\right.$ phen $\left.)\right]$ shows voids up to $34 \AA^{3}$. [ $\operatorname{Er}(\operatorname{tfac})_{3}$ (bath)] packs less efficiently: the molecular disposition allows for several voids up to $102 \AA^{3}$, where solvent molecules can reside. However, these solvent molecules would be distant from the lanthanide coordination sphere.

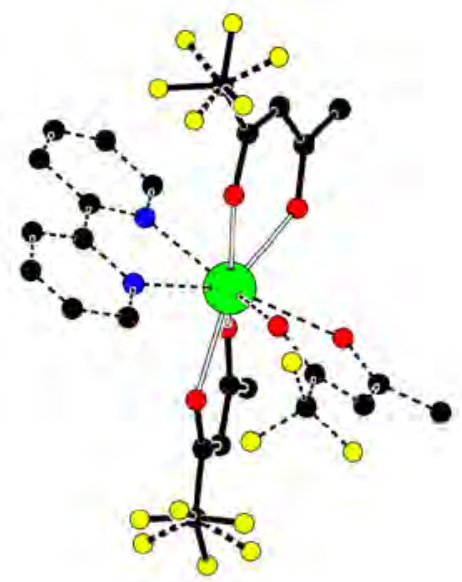

Figure 57. Structural diagram of $\left[\operatorname{Er}(\operatorname{tfac})_{3}(\right.$ bipy) $]$. $\mathrm{H}$ atoms were omitted for clarity. Only one of the alternative conformations is shown.

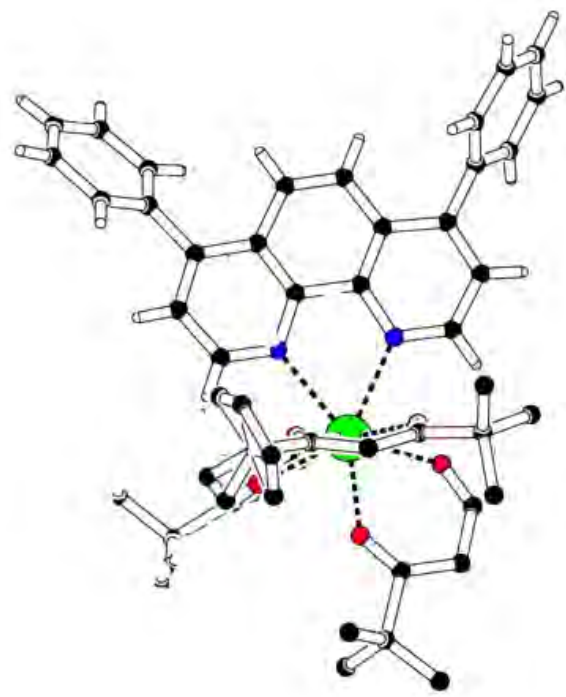

Figure 58. Structural diagram of $\left[\operatorname{Er}(\mathrm{tfac})_{3}(\right.$ bath) $]$. H atoms were omitted for clarity. 


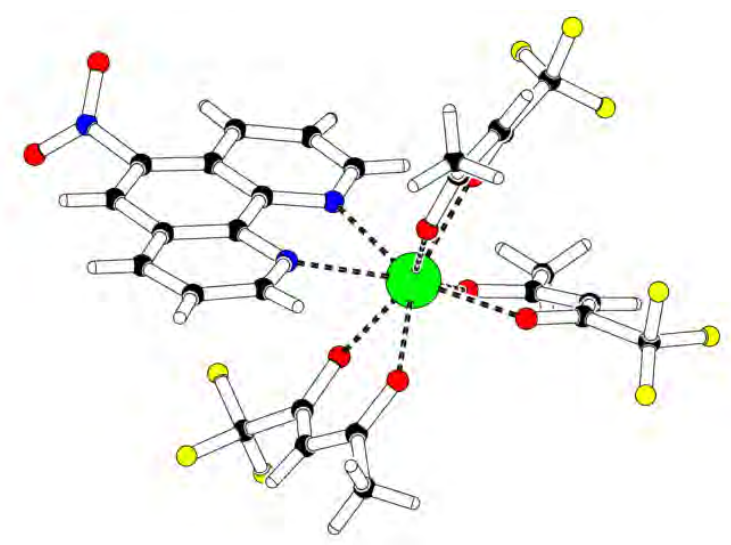

Figure 59. Structural diagram of $\left[\operatorname{Er}(\mathrm{tfac})_{3}\left(5 \mathrm{NO}_{2}\right.\right.$ phen $\left.)\right]$. $\mathrm{H}$ atoms were omitted for clarity.

Table 23. Crystal data and structure refinement for the complexes with 1,1,1-trifluoro-2,4pentanedione

\begin{tabular}{|c|c|c|c|}
\hline Complex & {$\left[\mathrm{Er}(\mathrm{tfac})_{3}(\mathrm{bipy})\right]$} & {$\left[\mathrm{Er}(\mathrm{tfac})_{3}(\mathrm{bath})\right]$} & {$\left[\operatorname{Er}(\mathrm{tfac})_{3}\left(5 \mathrm{NO}_{2}\right.\right.$ phen $\left.)\right]$} \\
\hline Empirical formula & $\mathrm{C}_{25} \mathrm{H}_{20} \mathrm{ErF}_{9} \mathrm{~N}_{2} \mathrm{O}_{6}$ & $\mathrm{C}_{39} \mathrm{H}_{28} \mathrm{ErF}_{9} \mathrm{~N}_{2} \mathrm{O}_{6}$ & $\mathrm{C}_{27} \mathrm{H}_{19} \mathrm{ErF}_{9} \mathrm{~N}_{3} \mathrm{O}_{8}$ \\
\hline Formula weight & 782.69 & 958.89 & 851.71 \\
\hline Temperature $(\mathrm{K})$ & $293(2)$ & $293(2)$ & $293(2)$ \\
\hline Wavelength $(\AA)$ & 0.71073 & 0.71073 & 0.71073 \\
\hline Crystal system & Monoclinic & Monoclinic & Monoclinic \\
\hline Space group & $\mathrm{P} 2{ }_{1} / \mathrm{c}$ & $\mathrm{P} 2{ }_{1} / \mathrm{c}$ & $\mathrm{P} 2{ }_{1} / \mathrm{c}$ \\
\hline$a(\AA)$ & $22.705(5)$ & $21.5991(8)$ & $9.7956(9)$ \\
\hline$b(\AA)$ & $9.2115(18)$ & $14.2783(5)$ & $14.8137(15)$ \\
\hline$c(\AA)$ & $15.646(3)$ & $16.4522(6)$ & $24.0161(18)$ \\
\hline$\alpha(\underline{\mathrm{o}})$ & 90.00 & 90.00 & 90.00 \\
\hline$\beta(\underline{0})$ & $118.042(3)$ & $99.848(2)$ & $114.072(3)$ \\
\hline$\gamma(\underline{\mathrm{o}})$ & 90.00 & 90.00 & 90.00 \\
\hline Volume $\left(\AA^{3}\right)$ & $2888.2(10)$ & 4999.1(3) & $3181.9(5)$ \\
\hline$Z$ & 4 & 4 & 4 \\
\hline Density (calculated, $\mathrm{g} \mathrm{cm}^{-3}$ ) & 1.800 & 1.274 & 1.778 \\
\hline Absorption coefficient $\left(\mathrm{mm}^{-1}\right)$ & 3.005 & 1.749 & 2.741 \\
\hline$F(000)$ & 1524 & 1892 & 1660 \\
\hline Crystal size $\left(\mathrm{mm}^{3}\right)$ & $0.2 \times 0.12 \times 0.04$ & $0.12 \times 0.06 \times 0.04$ & $0.4 \times 0.10 \times 0.10$ \\
\hline$\theta$ range for data collection $(\stackrel{\circ}{)}$ & $2.0-25.7$ & $1.7-25.8$ & $1.7-26.2$ \\
\hline Index ranges & $\begin{array}{c}-27<h<25 ;-11<k<10 ; \\
-8<l<19\end{array}$ & $\begin{array}{c}-12<h<12 ; 18-<k<18 \\
-29<l<29\end{array}$ & $\begin{array}{c}-26<h<26 ;-17<k<17 ; \\
-20<l<20\end{array}$ \\
\hline Reflections collected & 6054 & 71286 & 64512 \\
\hline Independent reflections & 1826 & 3831 & 3246 \\
\hline Completeness to $2 \theta=51^{\circ} \mathrm{o}$ & $99 \%$ & $99 \%$ & $98 \%$ \\
\hline Refinement method & Full matrix LS on $\mathrm{F}^{2}$ & Full matrix LS on $\mathrm{F}^{2}$ & Full matrix LS on $\mathrm{F}^{2}$ \\
\hline Data/restrains/parameters & $2714 / 27 / 220$ & $9551 / 57 / 568$ & $6250 / 0 / 436$ \\
\hline Goodness-of-fit on $\mathrm{F}^{2}$ & 1.049 & 1.003 & 1.037 \\
\hline Final $R$ indices $[I>2 \sigma(I)]$ & 0.0725 & 0.0886 & 0.0670 \\
\hline $\mathrm{w} R$ indices (all data) & 0.2395 & 0.2670 & 0.1960 \\
\hline Largest diff. peak and hole & $1.426 /-2.643$ & $1.360 /-0.696$ & $0.687 /-1.837$ \\
\hline
\end{tabular}

Table 24. Selected distances $(\AA ̊)$ for the complexes with 1,1,1-trifluoro-2,4-pentanedione

\begin{tabular}{cccc} 
Bond & {$\left[\mathrm{Er}(\mathrm{tfac})_{3}(\mathrm{bipy})\right]$} & {$\left[\mathrm{Er}(\mathrm{tfac})_{3}(\mathrm{bath})\right]$} & {$\left[\mathrm{Er}(\mathrm{tfac})_{3}\left(5 \mathrm{NO}_{2} \mathrm{phen}\right]\right.$} \\
\hline Average Er-N & $2.365(12)$ & $2.513(5)$ & $2.550(2)$ \\
Average Er-O & $2.387(71)$ & $2.297(50)$ & $2.301(15)$ \\
\hline
\end{tabular}


27. [Er(tpm) $)_{3}$ (bipy)] crystallizes in a triclinic centrosymmetric cell with two complexes per unit cell (Figure 60, Table 25). Within each complex, the lanthanide ion is coordinated in a distorted square anti-prismatic fashion by six $\mathrm{O}$ atoms and two $\mathrm{N}$ atoms from the fluorinated $\beta$-diketonates and the ancillary ligand 2,2'-bipyridine, respectively. The Er- $\mathrm{N}$ distances are 2.515(4) and 2.542(4) $\AA$ and the Er-O distances are in the range 2.282(4)-2.327(4) $\AA$. The NEr-N bite angle is $63^{\circ}$. The lanthanide ion is 1.41 and $1.16 \AA$ out of the planes of the top (containing $\mathrm{N}$ atoms) and of the bottom square faces. The angle between the least-squares plane of such faces is just 3‥ Molecules pack in dimers joined by $\pi-\pi$ interactions with a distance between centroids of 3.811(4) $\AA$ and a slippage of $1.577 \AA$. This packing causes solvent accessible voids in the structure with volume of $44 \AA^{3}$.

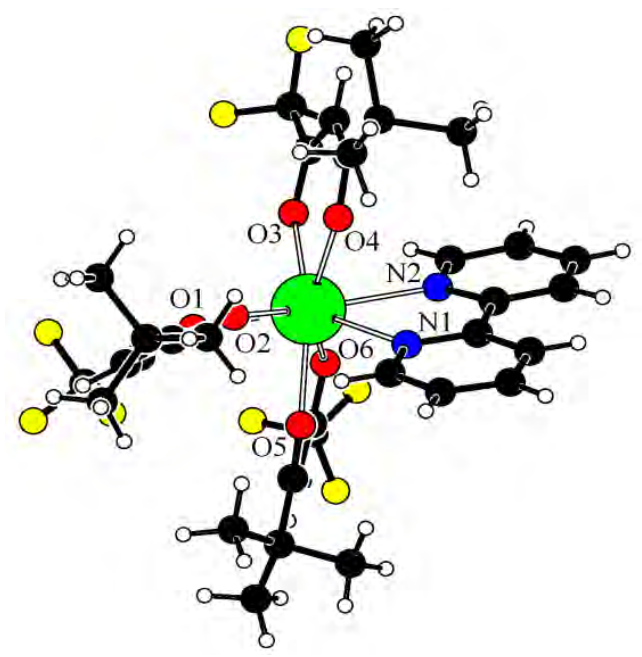

Figure 60. Structural diagram of $\left[\operatorname{Er}(\operatorname{tpm})_{3}(\right.$ bipy $\left.)\right]$

28. The mononuclear complexes in $\left[\operatorname{Er}(\mathbf{t p m})_{3}(\right.$ bath)] crystallize in a monoclinic unit cell with the $P 2_{1} / a$ space group (Figure 61, Table 25). For each $\operatorname{Er}(\mathrm{III})$, the coordination environment includes six $\mathrm{O}$ atoms from the fluorinated sensitizing ligands and two $\mathrm{N}$ atoms from the bathophenanthroline neutral antenna, in a distorted square-antiprismatic geometry (Table 26). The bite angle N-Er-N is 64을 and the N-Er distances are 2.512(5) and 2.524(5) $\AA$. The trivalent ion sits approximately in the middle of the prism distancing 1.38/1.20 $\AA$ to the antiprismatic square faces. Such faces are nearly parallel making an angle of less than $3 \%$. The substituent phenyl rings do not share the central aromatic plane of phenanthroline, as seen by the torsion angles $\mathrm{C} 2-\mathrm{C} 3-\mathrm{C} 19-\mathrm{C} 24$ [64.7- $]$ and $\mathrm{C} 7-\mathrm{C} 10-\mathrm{C} 13-\mathrm{C} 18$ [47.8 ${ }^{\circ}$. This rotation allows some $\mathrm{C}-\mathrm{H} \ldots$...centroid intermolecular contacts such as $\mathrm{C} 18-\mathrm{H} 18 \ldots \mathrm{Cg}^{\mathrm{i}}(\mathrm{Cg} 5$ is the centroid of the ring C19-C24 and $i:-1 / 2+x, 1 / 2-y, z)$. Solvent accessible voids with $71 \AA^{3}$ of volume exist in the crystal structure. 


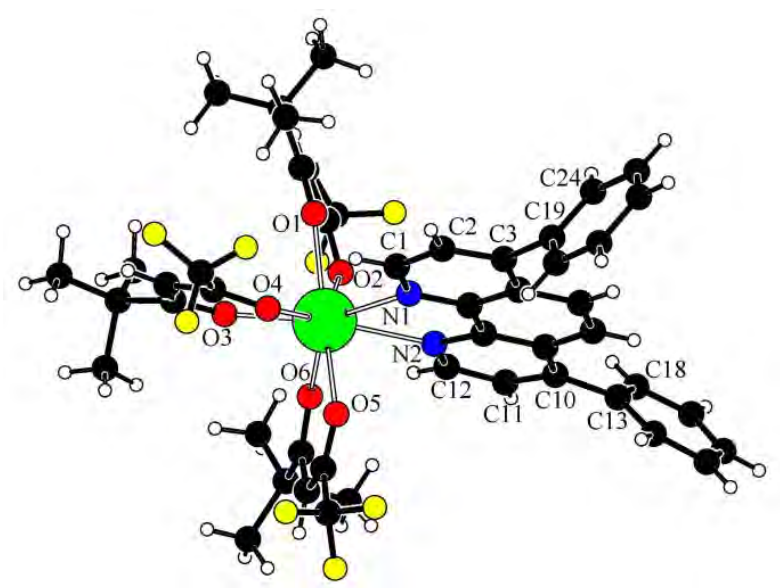

Figure 61. Structural diagram of $\left[\operatorname{Er}(\mathrm{tpm})_{3}(\right.$ bath $\left.)\right]$

30. [Er(tpm $)_{3}\left(\mathbf{5 N O}_{2}\right.$ phen)] crystallizes in the monoclinic space group $P 2_{1} / n$ with four complexes in the unit cell (Figure 62, Table 25). In each complex, the $\mathrm{Er}^{3+}$ ion is eight times coordinated with six oxygen atoms from the fluorinated $\beta$-diketonates and two nitrogen atoms from the 5-nitro-1,10-phenanthroline moiety forming a distorted squareantiprismatic prism. There are signs of disorder in the structure: the terminal fluoro and methyl groups exhibit large displacement factors and two positions could be found for the 5nitro-1,10-phenanthroline with occupations of roughly 50\%. The erbium ion occupies the centre of the prism with a distance of $1.38 \AA$ to the square face containing the $\mathrm{N}$ atoms and $1.18 \AA$ to the opposite face. The bite angle is $62 / 67^{\circ}$ for the N1A-Er-N2A and for the N1B-ErN2B, respectively. The Er-X distances range from 2.266(4) to 2.322(4) A for the 0 atoms and 2.415(13)-2.452(16) for the $\mathrm{N}$ atoms (Table 26). There are no classic hydrogen bonds between the complexes due to the lack of donors; only some weak $\mathrm{C}-\mathrm{H}$...X intermolecular contacts can be discerned. The complexes pack very efficiently in the crystal leaving no solvent accessible voids.

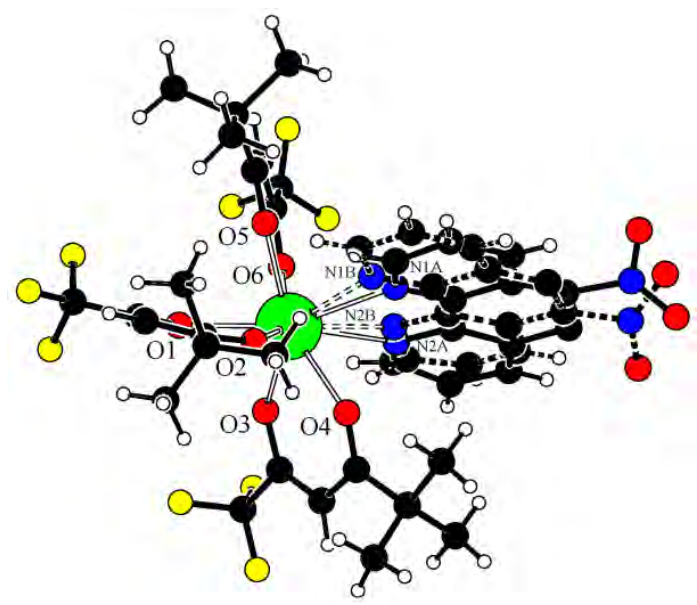

Figure 62. Structural diagram of $\left[\operatorname{Er}(\mathrm{tpm})_{3}\left(5 \mathrm{NO}_{2}\right.\right.$ phen $\left.)\right]$ 
Table 25. Crystal data and structure refinement for the complexes with 1,1,1-trifluoro-5,5-dimethyl2,4-hexanedione

\begin{tabular}{|c|c|c|c|}
\hline Complex & {$\left[\mathrm{Er}(\mathrm{tpm})_{3}(\mathrm{bipy})\right]$} & {$\left[\mathrm{Er}(\mathrm{tpm})_{3}(\mathrm{bath})\right]$} & {$\left[\operatorname{Er}(\operatorname{tpm})_{3}\left(5 \mathrm{NO}_{2}\right.\right.$ phen $\left.)\right]$} \\
\hline Empirical formula & $\mathrm{C}_{34} \mathrm{H}_{38} \mathrm{ErF}_{9} \mathrm{~N}_{2} \mathrm{O}_{6}$ & $\mathrm{C}_{48} \mathrm{H}_{46} \mathrm{ErF}_{9} \mathrm{~N}_{2} \mathrm{O}_{6}$ & $\mathrm{C}_{36} \mathrm{H}_{37} \mathrm{ErF}_{9} \mathrm{~N}_{3} \mathrm{O}_{8}$ \\
\hline Formula weight & 908.92 & 1085.13 & 976.94 \\
\hline Temperature $(\mathrm{K})$ & $293(3)$ & $293(3)$ & $293(3)$ \\
\hline Wavelength $(\AA)$ & 0.71073 & 0.71073 & 0.71073 \\
\hline Crystal system & Triclinic & Monoclinic & Monoclinic \\
\hline Space group & $P-1$ & $P 2_{1} / a$ & $P 2_{1} / n$ \\
\hline$a(\AA)$ & $9.6862(5)$ & $16.4630(5)$ & $14.2828(4)$ \\
\hline$b(\AA)$ & $12.0313(6)$ & $14.2801(4)$ & $16.9860(5)$ \\
\hline$c(\AA)$ & $18.1634(10)$ & $21.6052(7)$ & $18.2384(5)$ \\
\hline$\alpha(\underline{0})$ & $100.086(3)$ & 90 & 90 \\
\hline$\beta(\underline{0})$ & $103.810(3)$ & $99.901(2)$ & $109.5580(11)$ \\
\hline$\gamma(\underline{\mathrm{o}})$ & $101.682(3)$ & 90 & 90 \\
\hline Volume $\left(\AA^{3}\right)$ & $1956.59(18)$ & $5003.6(3)$ & $4169.5(2)$ \\
\hline$Z$ & 2 & 4 & 4 \\
\hline Calculated density $\left(\mathrm{g} \mathrm{cm}^{-3}\right)$ & 1.543 & 1.440 & 1.556 \\
\hline Erbium density $\left(\mathrm{cm}^{-3}\right)$ & $0.978 \times 10^{21}$ & $1.250 \times 10^{21}$ & $1.042 \times 10^{21}$ \\
\hline Absorption coefficient $\left(\mathrm{mm}^{-1}\right)$ & 2.230 & 1.757 & 2.103 \\
\hline$F(000)$ & 906 & 2180 & 1944 \\
\hline Crystal size $\left(\mathrm{mm}^{3}\right)$ & $0.25 \times 0.12 \times 0.10$ & $0.15 \times 0.10 \times 0.08$ & $0.15 \times 0.07 \times 0.07$ \\
\hline$\theta$ range for data collection & $1.78-25.94^{\mathrm{o}}$ & $1.72-25.83^{\circ}$ & $1.58-25.78^{\circ}$ \\
\hline Index ranges & $\begin{array}{c}-11<h<11 ;-14<k<14 ; \\
-22<l<22\end{array}$ & $\begin{array}{c}-20<h<20 ;-17<k<17 ; \\
-26<l<26\end{array}$ & $\begin{array}{c}-17<h<17 ;-20<k<20 ; \\
-22<l<22\end{array}$ \\
\hline Reflections collected & 19836 & 40965 & 40051 \\
\hline Independent reflections & 7513 & 9488 & 7975 \\
\hline Completeness to $2 \theta=51^{\circ}$ & $99.6 \%$ & $98.2 \%$ & $99.9 \%$ \\
\hline Refinement method & Full matrix LS on $F^{2}$ & Full matrix LS on $F^{2}$ & Full matrix LS in $F^{2}$ \\
\hline Data/restrains/parameters & $5907 / 0 / 477$ & $4816 / 0 / 604$ & $5008 / 6 / 503$ \\
\hline Goodness-of-fit on $\mathrm{F}^{2}$ & 1.024 & 0.943 & 1.012 \\
\hline Final $R$ indices $[I>2 \sigma(I)]$ & $R=0.0442 ; \mathrm{w} R=0.1061$ & $R=0.0519 ; \mathrm{w} R=0.0969$ & $R=0.0436 ; \mathrm{w} R=0.0959$ \\
\hline$R$ indices (all data) & $R=0.0640 ; \mathrm{w} R=0.1203$ & $R=0.1331 ; \mathrm{w} R=0.1248$ & $R=0.0865 ; \mathrm{w} R=0.1122$ \\
\hline Largest diff. peak and hole & $-1.649 / 1.526$ & $-0.552 / 0.650$ & $-0.409 / 0.661$ \\
\hline
\end{tabular}

Table 26. Er-N and Er-O distances in $\AA$ for the complexes with 1,1,1-trifluoro-5,5-dimethyl-2,4hexanedione

\begin{tabular}{cccc} 
Bond & {$\left[\mathrm{Er}(\mathrm{tpm})_{3}(\mathrm{bipy})\right]$} & {$\left[\mathrm{Er}(\mathrm{tpm})_{3}(\mathrm{bath})\right]$} & {$\left[\mathrm{Er}(\mathrm{tpm})_{3}\left(5 \mathrm{NO}_{2}\right.\right.$ phen $\left.)\right]$} \\
\hline Er-N1 & $2.515(4)$ & $2.512(5)$ & $2.415(13)$ \\
Er-N2 & $2.542(4)$ & $2.524(5)$ & $2.452(16)$ \\
Er-01 & $2.298(4)$ & $2.338(5)$ & $2.287(4)$ \\
Er-02 & $2.304(4)$ & $2.287(5)$ & $2.271(4)$ \\
Er-03 & $2.282(4)$ & $2.242(5)$ & $2.267(4)$ \\
Er-04 & $2.314(3)$ & $2.325(5)$ & $2.322(4)$ \\
Er-05 & $2.309(4)$ & $2.283(5)$ & $2.313(4)$ \\
Er-O6 & $2.327(4)$ & $2.301(5)$ & $2.266(4)$ \\
Er-N avg. & 2.5285 & 2.518 & 2.4335 \\
Er-O avg. & 2.3057 & 2.296 & 2.2877 \\
\hline
\end{tabular}


31. [Er(fod) $)_{3}$ (bipy)] crystallizes in the monoclinic $P 2_{1} /$ c group, with cell parameters $a=13.8124(3) \AA, \quad b=20.3813(5) \quad \AA, \quad c=18.1497(5) \quad \AA, \quad \alpha=90^{\circ}, \quad \beta=107.342(2)^{\circ}, \quad \gamma=90^{\circ}$, $\mathrm{V}=4877.1(2) \AA^{3}, \mathrm{Z}=4$ (Figure 63, Table 27).

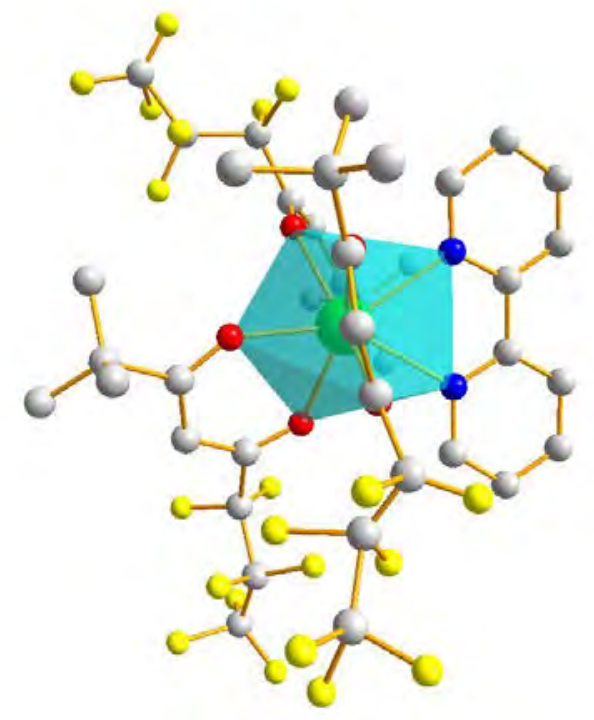

Figure 63. Structural diagram for [Er(fod) $)_{3}($ bipy)]

32. [Er(fod) $)_{3}$ (bath)] structure has only been resolved down to $R=0.1399$ and further refinement is still required prior to its submission to CCDC, as a consequence of the inherent disorder induced by fluorine atoms (Figure 64). Some preliminary data is summarized in Table 27 and Table 28.

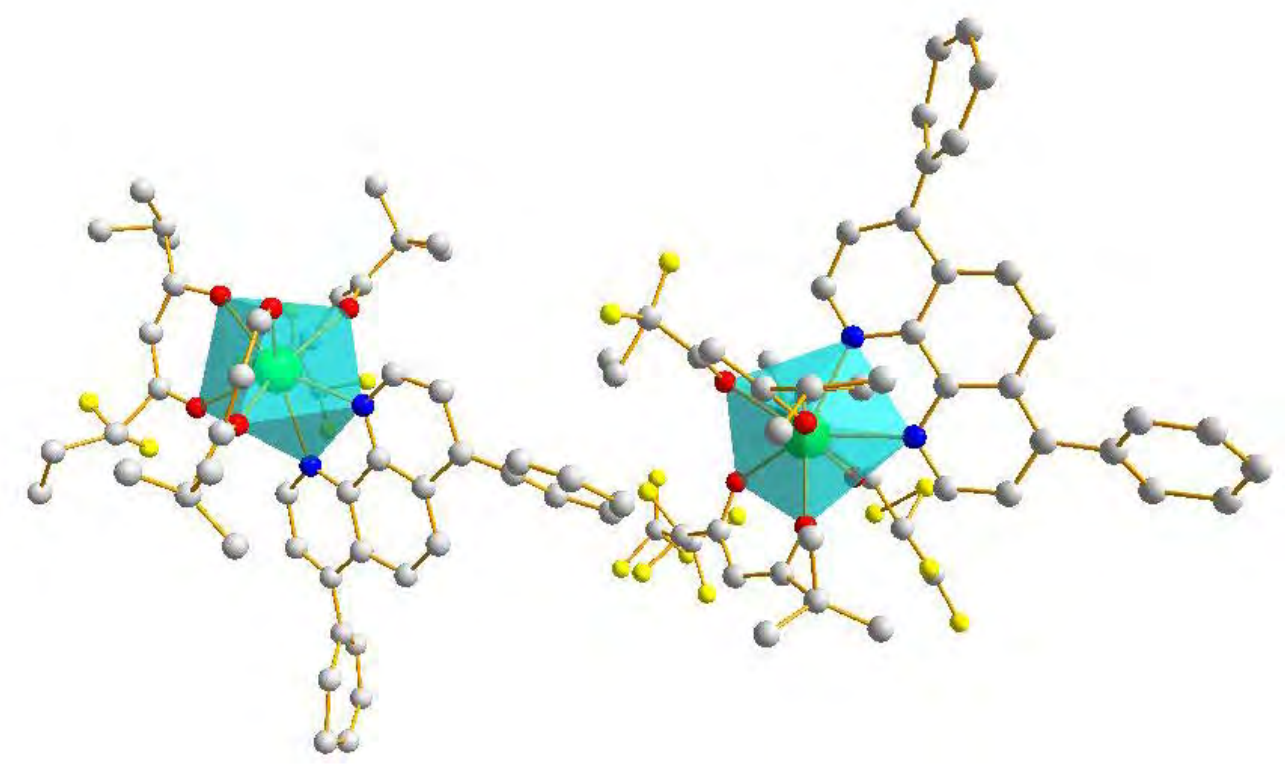

Figure 64. Preliminary structural diagram for $\left[\operatorname{Er}(\mathrm{fod})_{3}(\right.$ bath $\left.)\right]$ (not fully refined) 
Table 27. Crystal data and structure refinement for the complexes with 6,6,7,7,8,8,8-heptafluoro-2,2dimethyl-3,5-octanedione

\begin{tabular}{|c|c|c|}
\hline Complex & {$\left[\mathrm{Er}\left(\mathrm{fod}_{3}\right.\right.$ (bipy) $]$} & {$\left[\operatorname{Er}(\text { fod })_{3}\right.$ (bath)] } \\
\hline Empirical formula & $\mathrm{C}_{40} \mathrm{H}_{38} \mathrm{ErF}_{21} \mathrm{~N}_{2} \mathrm{O}_{6}$ & $\mathrm{C}_{54} \mathrm{H}_{46} \mathrm{ErF}_{21} \mathrm{~N}_{2} \mathrm{O}_{6}$ \\
\hline Formula weight & 1208.96 & 1385.18 \\
\hline Temperature (K) & $293(3)$ & $293(3)$ \\
\hline Wavelength $(\AA)$ & 0.71073 & 0.71073 \\
\hline Crystal system & Monoclinic & Orthorhombic \\
\hline Space group & $P 2_{1} / c$ & $P$ \\
\hline$a(\AA)$ & $13.8124(3)$ & $25.5335(6)$ \\
\hline$b(\AA)$ & $20.3813(5)$ & $28.8665(7)$ \\
\hline$c(\AA)$ & $18.1497(5)$ & $32.5392(8)$ \\
\hline$\alpha(\underline{0})$ & 90.00 & 90.00 \\
\hline$\beta(\underline{o})$ & $107.342(2)$ & 90.00 \\
\hline$\gamma(\underline{\mathrm{o}})$ & 90.00 & 90.00 \\
\hline Volume $\left(\AA^{3}\right)$ & $4877.1(2)$ & 23983.4(10) \\
\hline$Z$ & 4 & - \\
\hline Calculated density $\left(\mathrm{g} \mathrm{cm}^{-3}\right)$ & 2.191 & 0.350 \\
\hline Absorption coefficient $\left(\mathrm{mm}^{-1}\right)$ & 1.811 & 0.364 \\
\hline$F(000)$ & 3152 & 2460 \\
\hline Crystal size $\left(\mathrm{mm}^{3}\right)$ & $0.44 \times 0.44 \times 0.09$ & $0.55 \times 0.16 \times 0.11$ \\
\hline$\theta$ range for data collection & $1.84-25.77^{\circ} \underline{\mathrm{o}}$ & $1.41-25.78^{\circ}$ \\
\hline Index ranges & $-16<h<16 ;-24<k<24 ;-22<l<22$ & $-31<h<31 ;-35<k<35 ;-39<l<39$ \\
\hline Reflections collected & 97420 & 448030 \\
\hline Independent reflections & 6848 & 8503 \\
\hline Completeness to $2 \theta=51^{\mathrm{o}}$ & 0.996 & 0.995 \\
\hline Refinement method & Full matrix LS on $\mathrm{F}^{2}$ & Full matrix LS on $\mathrm{F}^{2}$ \\
\hline Data/restrains/parameters & $9310 / 28 / 672$ & $22917 / 81 / 906$ \\
\hline Goodness-of-fit on $\mathrm{F}^{2}$ & 4.388 & 2.100 \\
\hline Final $R$ indices $[I>2 \sigma(I)]$ & $R=0.0576 ; \mathrm{w} R=0.1259$ & $R=0.1399 ; \mathrm{w} R=0.3909$ \\
\hline$R$ indices (all data) & $R=0.0815 ; \mathrm{w} R=0.1293$ & $R=0.277 ; \mathrm{w} R=0.4318$ \\
\hline Largest diff. peak and hole & $-0.821 / 1.704$ & $-1.882 / 2.359$ \\
\hline
\end{tabular}

Table 28. Er-N and Er-O distances in Å for the complexes with 6,6,7,7,8,8,8-heptafluoro-2,2-dimethyl3,5-octanedione

\begin{tabular}{ccc} 
Bond & $[\text { Er(fod) })_{3}($ bipy $\left.)\right]$ & $[\text { Er(fod })_{3}($ bath $\left.)\right]$ \\
\hline Er-N1 & $2.5235(16)$ & $2.514(11)$ \\
Er-N2 & $2.5220(16)$ & $2.490(10)$ \\
Er-01 & $2.3218(12)$ & $2.235(9)$ \\
Er-02 & $2.2914(13)$ & $2.284(8)$ \\
Er-03 & $2.3057(13)$ & $2.328(10)$ \\
Er-04 & $2.2599(12)$ & $2.477(12)$ \\
Er-05 & $2.2961(13)$ & $2.259(9)$ \\
Er-O6 & $2.2785(14)$ & $2.236(8)$ \\
Er-N avg. & 2.5228 & 2.502 \\
Er-O avg. & 2.2922 & 2.303 \\
\hline
\end{tabular}

34 and 42. [Na $\left.(\mathrm{OH})_{4}\left(\mathrm{H}_{2} \mathbf{O}\right)_{14} \mathrm{Ln}\left(\mathrm{NO}_{3}\right)_{4}\right]$, where $\mathrm{Ln}=\mathrm{Er}(\mathrm{III}), \mathrm{Yb}(\mathrm{III})$. In tetradecaaquatetrahydroxo-pentasodium tetranitrato erbium(III) and ytterbium(III) (Figure 65, Table 29), the lanthanide ions occupy general positions, their coordination number is 8 and their coordination polyhedron is a distorted square antiprism. 8 oxygen atoms (from 4 nitrate ions) are in the immediate environment of the lanthanide ions. The $\mathrm{Ln}(\mathrm{III})$-O distances are in the range 2.32-2.38 $\AA$ for Er(III) and 2.30-2.36 $\AA$ for Yb(III), see Table 30. There are not common vertices in consecutive lanthanide polyhedra. 
The sodium ions are coordinated by the water molecules, located at the vertices of octahedrons. Some of the water molecules coordinate to more than one ion. Na1 and $\mathrm{Na} 2$ ions are assembled in chains along $b$ axis through the sharing of two coordinating oxygen atoms (Figure 66), in the equatorial plane. A similar chain, with $\mathrm{Na} 3$ and $\mathrm{Na} 4$ ions, is assembled along the a axis.

In the coordination sphere of the remaining sodium ion (Na5) there are axial oxygen atoms from the two above described chains and also an oxygen atom from a nitrate group. Therefore all alkaline metal and lanthanide ions are bridged in a 3D network.

A very intricate 3D H-bond network further links all ions together (Table 32, Figure 67).

There are no solvent accessible voids in this structure.

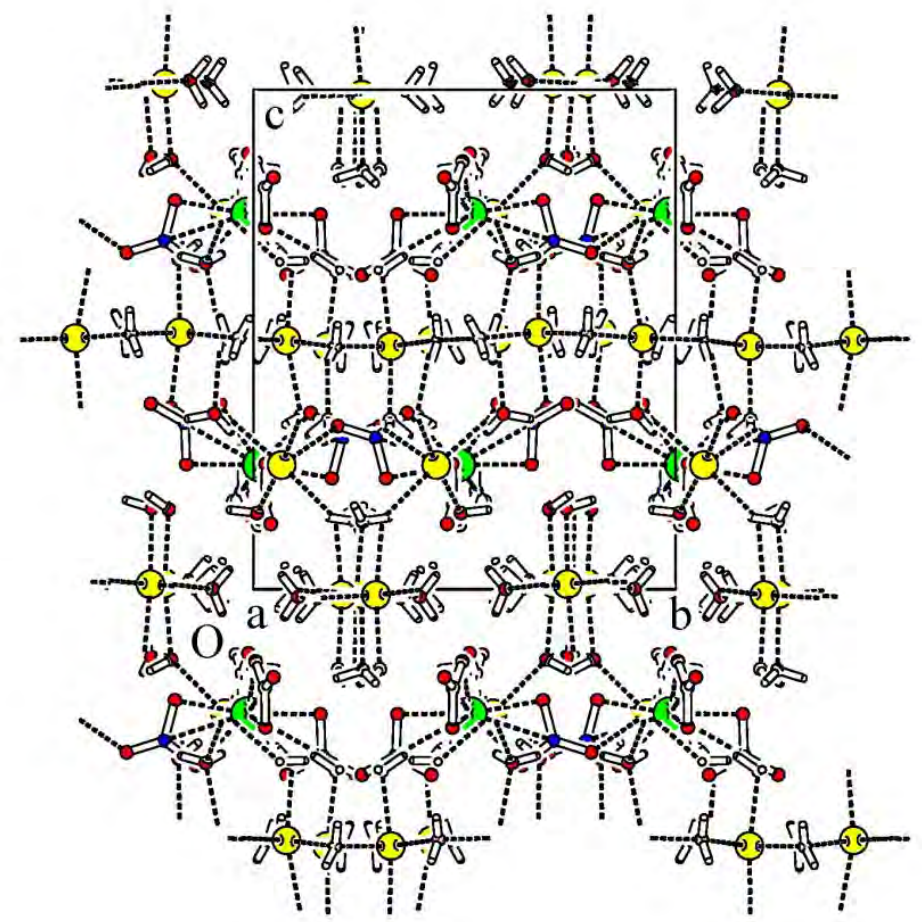

Figure 65. Packing diagram of $\left[\mathrm{Na}_{5}(\mathrm{OH})_{4}\left(\mathrm{H}_{2} \mathrm{O}\right)_{14} \mathrm{Ln}\left(\mathrm{NO}_{3}\right)_{4}\right](\mathrm{Ln}=\mathrm{Er}, \mathrm{Yb})$ in the unit cell

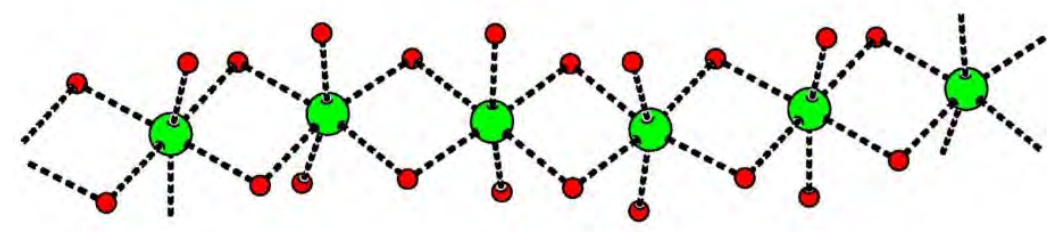

Figure 66. One of the sodium chains that run along the $b$ axis. 


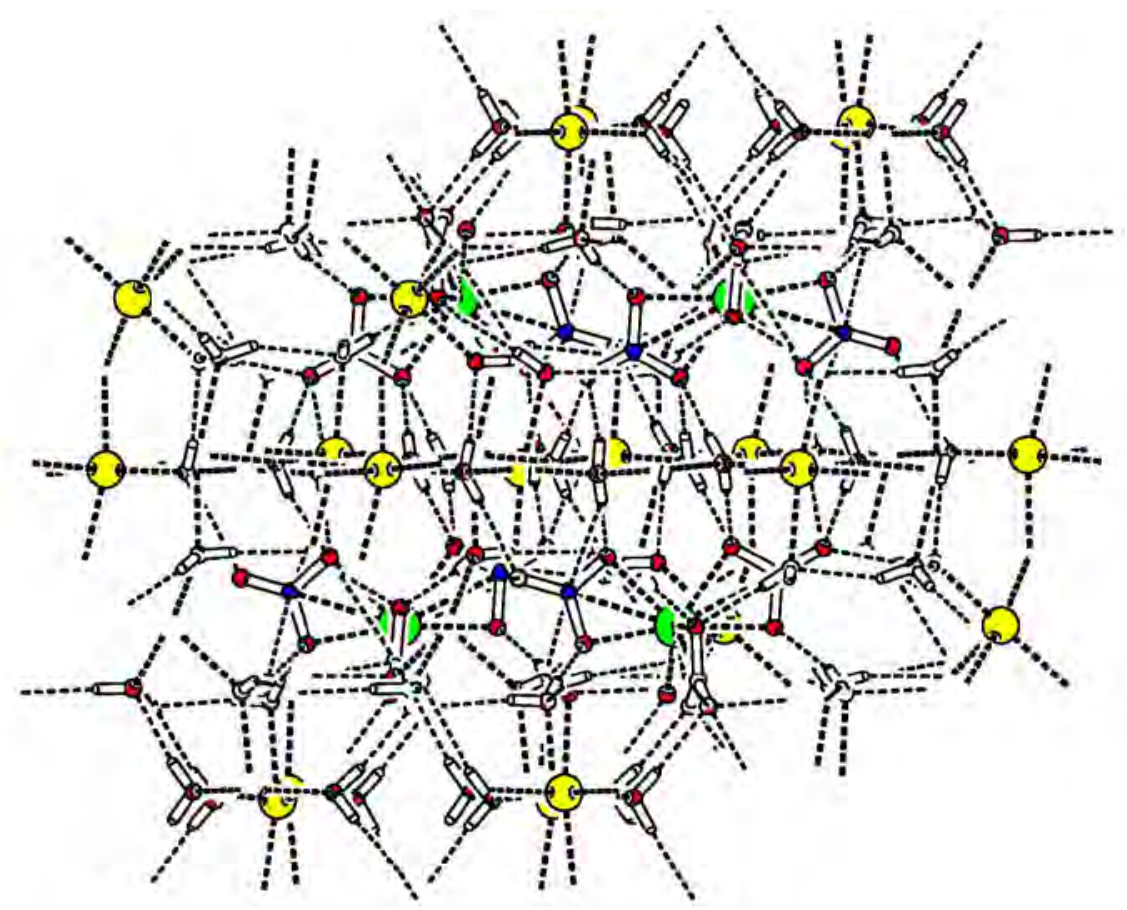

Figure 67. H-bond network depicted as dashed lines

Table 29. Crystal data and structure refinement of the two alkali-lanthanide heterometallic coordination polymers

\begin{tabular}{|c|c|c|}
\hline & Er(III) complex & Yb(III) complex \\
\hline Empirical formula & $\mathrm{H}_{32} \mathrm{ErN}_{4} \mathrm{Na}_{5} \mathrm{O}_{30}$ & $\mathrm{H}_{32} \mathrm{~N}_{4} \mathrm{Na}_{5} \mathrm{O}_{30} \mathrm{Yb}$ \\
\hline Formula weight & 850.51 & 856.29 \\
\hline Temperature (K) & $293(2)$ & $293(2)$ \\
\hline Wavelength $(\AA)$ & 0.71073 & 0.71073 \\
\hline Crystal system & Monoclinic & Monoclinic \\
\hline Space group & $P 2_{1} / a$ & $P 2_{1} / a$ \\
\hline$a(\AA)$ & $13.3515(17)$ & $13.3083(4)$ \\
\hline$b(\AA)$ & $13.4173(18)$ & $13.3707(4)$ \\
\hline$c(\AA)$ & $15.8777(19)$ & $15.8756(5)$ \\
\hline$\alpha(\underline{\mathrm{o}})$ & 90.00 & 90.00 \\
\hline$\beta(\underline{o})$ & $93.585(9)$ & $93.5842(17)$ \\
\hline$\gamma(\underline{\mathrm{o}})$ & 90.00 & 90.00 \\
\hline Volume $\left(\AA^{3}\right)$ & $2838.8(6)$ & $2819.40(15)$ \\
\hline$Z$ & 4 & 4 \\
\hline Calculated density $\left(\mathrm{g} / \mathrm{cm}^{3}\right)$ & 1.990 & 2.017 \\
\hline Absorption coefficient $\left(\mathrm{mm}^{-1}\right)$ & 3.150 & 3.512 \\
\hline$F(000)$ & 1692 & 1700 \\
\hline Crystal size $\left(\mathrm{mm}^{3}\right)$ & $0.30 \times 0.13 \times 0.11$ & $0.24 \times 0.21 \times 0.18$ \\
\hline$\theta$ range for data collection $(\stackrel{\circ}{)}$ & $1.99-31.18$ & $1.99-29.92$ \\
\hline Index ranges & $-19<h<19,-19<k<19,-22<l<23$ & $-18<h<18,-18<k<18,-22<l<22$ \\
\hline Reflections collected & 40994 & 63344 \\
\hline Independent reflections $[I>2 \sigma(I)]$ & 7439 & 5665 \\
\hline Completeness to $2 \theta=51^{\circ}$ & $98.4 \%$ & $99.7 \%$ \\
\hline Refinement method & Full matrix LS on $F^{2}$ & Full matrix LS on $F^{2}$ \\
\hline Data/restrains/parameters & $9047 / 36 / 473$ & $8145 / 36 / 473$ \\
\hline Goodness-of-fit on $F^{2}$ & 1.046 & 1.000 \\
\hline Final $R$ indices $[I>2 \sigma(I)]$ & $R=0.0272 ; \mathrm{w} R=0.0611$ & $R=0.0343 ; \mathrm{w} R=0.0609$ \\
\hline$R$ indices (all data) & $R=0.0384 ; \mathrm{w} R=0.0653$ & $R=0.0609 ; \mathrm{w} R=0.0743$ \\
\hline Largest diff. peak and hole & $-1.026 / 0.720$ & $-1.245 / 1.367$ \\
\hline CCDC number & 942379 & 942380 \\
\hline
\end{tabular}


Table 30. Selected distances $(\AA)$ and angles $(\stackrel{\circ}{)}$ ) for the $\operatorname{Ln}(\mathrm{III})(\mathrm{Ln}=\mathrm{Er}, \mathrm{Yb})$ coordination environment in the two alkali-lanthanide heterometallic coordination polymers

\begin{tabular}{cccc} 
Bond & Er(III) complex & Bond & Yb(III) complex \\
\hline Er1-01 & $2.3487(19)$ & Yb1-01 & $2.319(3)$ \\
Er1-03 & $2.3336(19)$ & Yb1-03 & $2.311(3)$ \\
Er1-04 & $2.3609(18)$ & Yb1-04 & $2.334(3)$ \\
Er1-05 & $2.3286(18)$ & Yb1-05 & $2.311(3)$ \\
Er1-07 & $2.3808(19)$ & Yb1-07 & $2.364(3)$ \\
Er1-08 & $2.3332(18)$ & Yb1-08 & $2.313(3)$ \\
Er1-010 & $2.3328(19)$ & Yb1-010 & $2.301(3)$ \\
Er1-011 & $2.364(2)$ & Yb1-011 & $2.340(3)$ \\
\hline
\end{tabular}

Table 31. Selected distances $(\AA ̊)$ and angles $(\stackrel{\circ}{)})$ for the sodium coordination spheres in the two alkalilanthanide heterometallic coordination polymers

Er(III) complex

\begin{tabular}{|c|c|c|c|c|c|c|c|c|c|}
\hline Bond & Na1 & Bond & $\mathrm{Na} 2$ & Bond & $\mathrm{Na} 3$ & Bond & $\mathrm{Na} 4$ & Bond & $\mathrm{Na} 5$ \\
\hline $\mathrm{Na1-013}$ & $2.329(2)$ & $\mathrm{Na2-013}$ & $2.356(2)$ & $\mathrm{Na3}-015$ & $2.437(2)$ & $\mathrm{Na} 4-015$ & $2.426(2)$ & $\mathrm{Na} 5-\mathrm{O} 2$ & $2.504(2)$ \\
\hline Na1-C & $2.455(2)$ & $\mathrm{Na} 2$ & $2.425(2)$ & 16 & $2.406(2)$ & 16 & $2.322(2)$ & & $577(2)$ \\
\hline $\mathrm{Na1-019}$ & $2.318(2)$ & $\mathrm{Na} 2$ & $2.506(2)$ & $\mathrm{Na} 3$ & $2.385(2)$ & $\mathrm{Na}$ & $2.460(2)$ & & $2.348(2)$ \\
\hline $\mathrm{Na1-022}$ & $2.353(2)$ & $\mathrm{Na} 2-026$ & $2.408(2)$ & $\mathrm{Na3}-018$ & $2.531(2)$ & $\mathrm{Na} 4$ & $2.302(2)$ & 23 & $2.510(2)$ \\
\hline $\mathrm{Na1-028}$ & $2.406(2)$ & $\mathrm{Na} 2-027$ & $2.435(3)$ & $\mathrm{Na} 3-020$ & $2.418(2)$ & $\mathrm{Na} 4-$ & $2.388(2)$ & $\mathrm{Na5}-\mathrm{O} 27$ & $2.412(2)$ \\
\hline $\mathrm{Na1-030}$ & $2.545(3)$ & $\mathrm{Na2}-030$ & $2.347(3)$ & $\mathrm{Na3}-023$ & $2.425(2)$ & $\mathrm{Na} 4-025$ & $2.417(2)$ & Na5-029 & $2.441(2)$ \\
\hline
\end{tabular}

$\mathrm{Yb}$ (III) complex

\begin{tabular}{|c|c|c|c|c|c|c|c|c|c|}
\hline Bond & $\mathrm{Na} 1$ & Bond & $\mathrm{Na} 2$ & Bond & $\mathrm{Na3}$ & Bond & $\mathrm{Na} 4$ & Bond & $\mathrm{Na} 5$ \\
\hline $\mathrm{Na1-013}$ & $2.323(3)$ & $\mathrm{Na2-013}$ & $2.355(3)$ & Na3-015 & $2.434(3)$ & $\mathrm{Na} 4-015$ & $2.416(3)$ & $\mathrm{Na5}-\mathrm{O} 2$ & $2.495(4)$ \\
\hline 14 & $2.450(3)$ & 014 & $2.419(3)$ & 16 & 2.403 & & 2.319 & & $2.578(4)$ \\
\hline Na1- & $2.308(3)$ & $\mathrm{Na} 2$ & $2.498(3)$ & $\mathrm{Na}$ & 2.379 & $\mathrm{Na}^{2}$ & $2.451(3)$ & 021 & $2.351(3)$ \\
\hline $\mathrm{Na} 1-022$ & $2.350(4)$ & $\mathrm{Na2}-026$ & $2.406(4)$ & $\mathrm{Na3}-018$ & $2.528(3)$ & $\mathrm{Na} 4$ & $2.300(3)$ & -023 & $2.503(4)$ \\
\hline $\mathrm{Na} 1-028$ & $2.403(4)$ & $\mathrm{Na} 2-027$ & $2.436(4)$ & $\mathrm{Na3}-020$ & $2.416(4)$ & $\mathrm{Na} 4$ & $2.383(4)$ & $\mathrm{Na} 5-027$ & $2.410(3)$ \\
\hline $\mathrm{Na1-030}$ & $2.538(3)$ & $\mathrm{Na2}-030$ & $2.341(4)$ & $\mathrm{Na3}-\mathrm{O} 23$ & $2.419(4)$ & $\mathrm{Na} 4-025$ & $2.417(4)$ & $\mathrm{Na5}-029$ & $2.435(3)$ \\
\hline
\end{tabular}

Table 32. $\mathrm{H}$ bond parameters in $\AA$ and $\stackrel{0}{ }$ for the two alkali-lanthanide heterometallic coordination polymers

\begin{tabular}{ccccccc} 
& \multicolumn{3}{c}{ Er(III) complex } & \multicolumn{3}{c}{ Yb(III) complex } \\
\cline { 2 - 6 } & H...O & O... & Angle & H... & O... & Angle \\
\hline O13-H13B..O3 & $1.906(14)$ & $2.751(3)$ & $172(2)$ & $1.91(2)$ & $2.756(4)$ & $174(3)$ \\
O14-H14A..O6 & $2.083(14)$ & $2.930(3)$ & $173(2)$ & $2.09(2)$ & $2.939(4)$ & $178(6)$ \\
O15-H15A..09 & $2.055(19)$ & $2.902(3)$ & $174.9(17)$ & $2.06(3)$ & $2.908(4)$ & $172(2)$ \\
O16-H16A..09 & $1.946(12)$ & $2.782(3)$ & $168(4)$ & $1.953(19)$ & $2.783(4)$ & $165(4)$ \\
O17-H17A..021 & $2.156(17)$ & $2.979(3)$ & $163.1(17)$ & $2.14(2)$ & $2.971(4)$ & $164(2)$ \\
O18-H18B..08 & $2.010(14)$ & $2.860(3)$ & $178(3)$ & $2.008(13)$ & $2.855(4)$ & $173(5)$ \\
O19-H19A..05 & $2.033(13)$ & $2.878(3)$ & $172.3(13)$ & $2.034(17)$ & $2.874(4)$ & $170(3)$ \\
O20-H20B..025 & $2.221(10)$ & $3.017(3)$ & $156(2)$ & $2.218(15)$ & $3.002(4)$ & $154(3)$ \\
O21-H21A..020 & $2.017(11)$ & $2.849(3)$ & $166(4)$ & $2.026(15)$ & $2.845(4)$ & $162(5)$ \\
O21-H21B..09 & $1.900(12)$ & $2.736(3)$ & $167(5)$ & $1.935(15)$ & $2.727(4)$ & $154(4)$ \\
O22-H22B..07 & $1.933(19)$ & $2.773(3)$ & $169.6(18)$ & $1.92(3)$ & $2.769(4)$ & $176(4)$ \\
O23-H23B..024 & $2.009(11)$ & $2.857(3)$ & $176(5)$ & $2.002(12)$ & $2.847(4)$ & $173(6)$ \\
O24-H24B..09 & $2.058(13)$ & $2.872(3)$ & $161(3)$ & $2.08(2)$ & $2.868(4)$ & $155(4)$ \\
O25-H25A..04 & $2.055(16)$ & $2.900(3)$ & $173(2)$ & $2.06(2)$ & $2.898(4)$ & $173(3)$ \\
O26-H26A..07 & $2.024(19)$ & $2.854(3)$ & $165.2(16)$ & $2.01(3)$ & $2.847(4)$ & $170(3)$ \\
O26-H26B..028 & $2.090(11)$ & $2.899(3)$ & $159(3)$ & $2.148(16)$ & $2.893(3)$ & $146(3)$ \\
O27-H27A..010 & $1.89(2)$ & $2.704(3)$ & $161.1(17)$ & $1.89(3)$ & $2.704(4)$ & $162(3)$ \\
O27-H27B..022 & $2.124(13)$ & $2.944(3)$ & $162(3)$ & $2.106(18)$ & $2.929(4)$ & $163(4)$ \\
O28-H28B..010 & $1.93(2)$ & $2.772(3)$ & $175(2)$ & $1.93(3)$ & $2.774(4)$ & $171(2)$ \\
O30-H30A..029 & $2.044(10)$ & $2.883(3)$ & $169(3)$ & $2.066(16)$ & $2.883(4)$ & $161(2)$ \\
\hline
\end{tabular}


39. The mononuclear complexes in [Yb(tfac $)_{3}\left(\mathbf{5 N O}_{2}\right.$ phen $\left.)\right]$ crystallize in a monoclinic unit cell with the $\mathrm{P} 2{ }_{1} / c$ space group (Figure 68 , Table 33 ). For each $\mathrm{Yb}(\mathrm{III})$, the coordination environment includes six $\mathrm{O}$ atoms from the fluorinated sensitizing ligands and two $\mathrm{N}$ atoms from the 5-nitro-1,10-phenanthroline neutral antenna, in a distorted square-antiprismatic geometry. The bite angle $\mathrm{N}-\mathrm{Yb}-\mathrm{N}$ is $64^{\circ}$ and the $\mathrm{N}-\mathrm{Yb}$ distances are 2.527(9) and 2.539(10) $\AA$ (Table 34). The trivalent ion sits approximately in the middle of the prism distancing $1.35 / 1.16 \AA$ to the antiprismatic square faces. Such faces are nearly parallel making an angle of less than $2^{\circ}$. The molecules pack joined by weak C-H...O bonds with a packing index of 65.3 (percentage filled space). Only $140 \AA^{3}$ (per unit cell) are available for solvent molecules divided in four voids of $35 \AA^{3}$ each (for comparison, a water molecule needs an empty space of $\left.40 \AA^{3}\right)$.

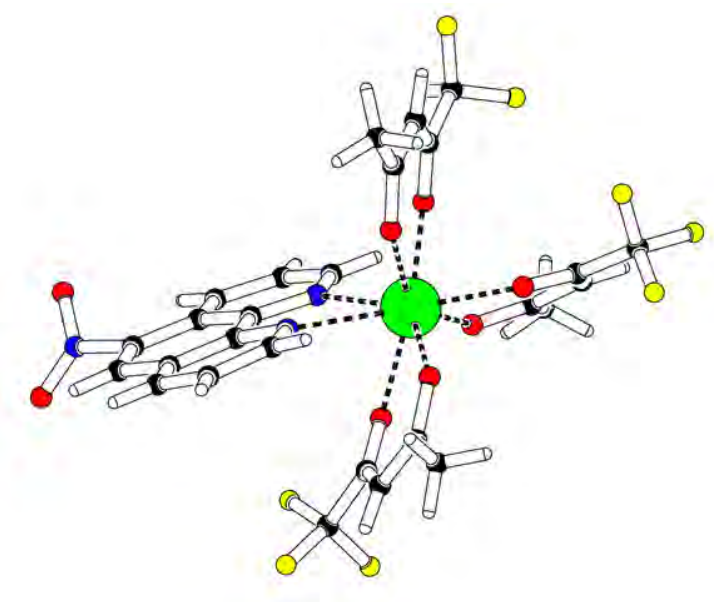

Figure 68. Structural diagram of $\left[\mathrm{Yb}(\mathrm{tfac})_{3}\left(5 \mathrm{NO}_{2} \mathrm{phen}\right]\right.$

40. [Yb(tpm $)_{3}\left(\mathbf{5 N O}_{2}\right.$ phen)] crystallizes in the monoclinic space group $\mathrm{P} 2_{1} / c$ with eight complexes in the unit cell (Figure 69, Figure 70, Table 33) and two independent complexes in the asymmetric unit. In each complex, the $\mathrm{Yb}^{3+}$ ion is eight times coordinated with six oxygen atoms from the fluorinated $\beta$-diketonates and two nitrogen atoms from the 5-nitro-1,10phenanthroline moiety forming a distorted square-antiprismatic prism. There are signs of disorder in the structure: the terminal fluoro and methyl groups exhibit large displacement factors and two positions could be found for the 5-nitro-1,10-phenanthroline with occupations of roughly $50 \%$ in one of the complexes. The erbium ion occupies the center of the prism with a distance of $1.36 / 1.31 \AA$ to the square face containing the $\mathrm{N}$ atoms and $1.18 / 1.18 \AA$ to the opposite face. The Yb-X distances range from 2.248(5) to 2.299(6) $\AA$ for the $\mathrm{O}$ atoms and 2.230(17)-2.804(15) for the $\mathrm{N}$ atoms (Table 34). There are no classic hydrogen bonds between the complexes due to the lack of donors; only some weak C-H...O intermolecular contacts can be discerned. The complexes pack leaving several solvent accessible voids, two of which have a volume of $340 \AA^{3}$. 


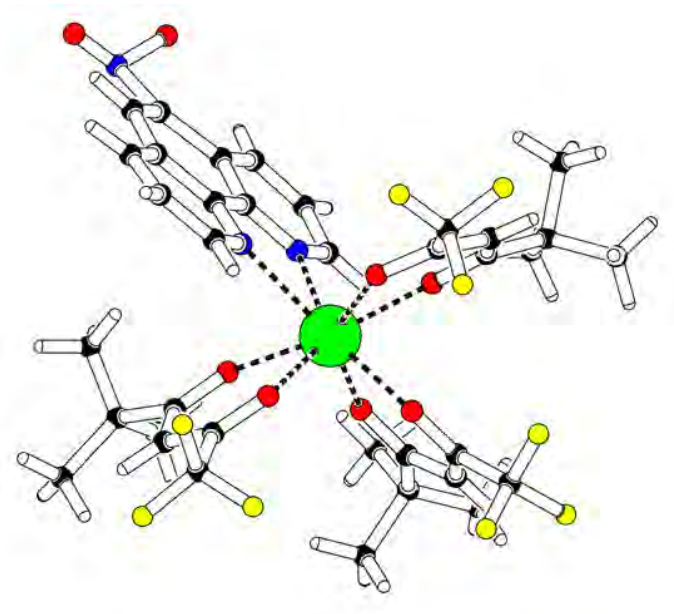

Figure 69. Structural diagram of $\left[\mathrm{Yb}(\mathrm{tpm})_{3}\left(5 \mathrm{NO}_{2}\right.\right.$ phen $\left.)\right]$

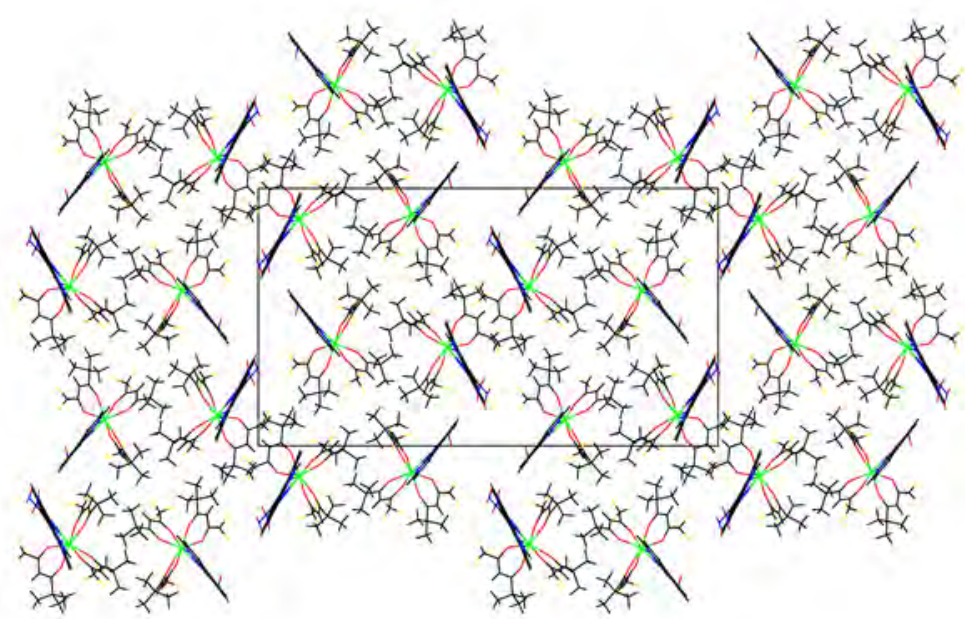

Figure 70. Packing diagram of $\left[\mathrm{Yb}(\mathrm{tpm})_{3}\left(5 \mathrm{NO}_{2}\right.\right.$ phen $\left.)\right]$

41. [Yb(fhd $)_{3}\left(\mathbf{5 N O}_{2}\right.$ phen] crystallizes in a monoclinic centrosymmetric cell with $4176 \AA^{3}$ of volume (Figure 71, Table 33). There is a high degree of disorder in the structure and a data collection was performed at low temperature $(150 \mathrm{~K})$. Although the quality of the data slightly improved, the disorder remained, showing that the disorder is a structural not a dynamical effect. Within each complex, the lanthanide ion is coordinated in a distorted square anti-prismatic fashion by six $\mathrm{O}$ atoms and two $\mathrm{N}$ atoms from the fluorinated $\beta$ diketonates and the ancillary ligand 5-nitro-1,10-phenanthroline, respectively. The $\mathrm{Yb}-\mathrm{N}$ distances are in the range 2.413(10)-2.549(11) $\AA$ and the $\mathrm{Yb}-0$ distances are in the range 2.278(3)-2.316(3) $\AA$ (Table 34). The $\mathrm{N}-\mathrm{Yb}-\mathrm{N}$ bite angle is 64/66- The lanthanide ion is 1.34 and $1.19 \AA$ A out of the planes of the top (containing $\mathrm{N}$ atoms) and of the bottom square faces. The angle between the least-squares plane of such faces is just $1 \stackrel{0}{ }$. These complexes are joined by weak intermolecular interactions of the type $\mathrm{C}-\mathrm{H}$...F, and the packing leaves no solvent accessible voids in the structure. 


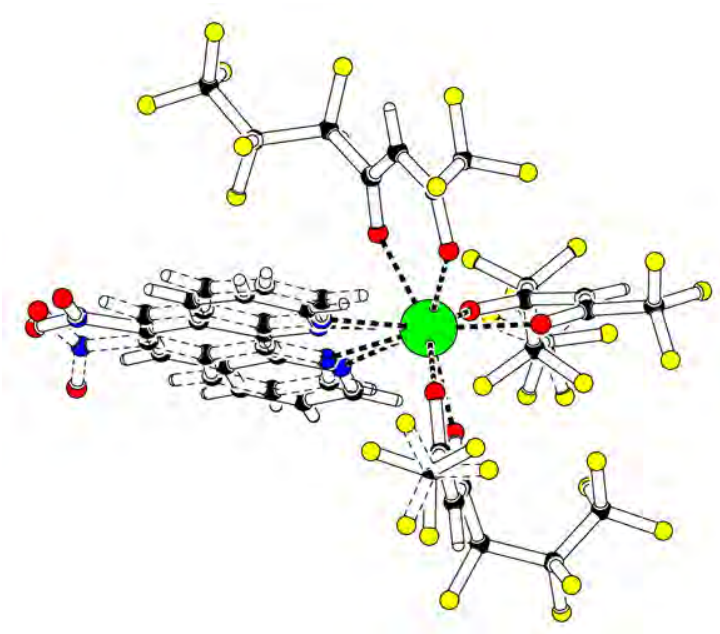

Figure 71. Structural diagram of $\left[\mathrm{Yb}(\mathrm{tpm})_{3}\left(5 \mathrm{NO}_{2}\right.\right.$ phen $]$.

Table 33. Crystal data and structure refinement for the $\left[\mathrm{Yb}(\beta \text {-diketonate })_{3}\left(5 \mathrm{NO}_{2}\right.\right.$ phen $\left.)\right]$ complexes

\begin{tabular}{|c|c|c|c|}
\hline Complex & {$\left[\mathrm{Yb}(\mathrm{tfac})_{3}\left(5 \mathrm{NO}_{2}\right.\right.$ phen $\left.)\right]$} & {$\left[\mathrm{Yb}(\mathrm{tpm})_{3}\left(5 \mathrm{NO}_{2}\right.\right.$ phen $\left.)\right]$} & {$\left[\mathrm{Yb}(\mathrm{fhd})_{3}\left(5 \mathrm{NO}_{2}\right.\right.$ phen $\left.)\right]$} \\
\hline Empirical formula & $\mathrm{C}_{27} \mathrm{H}_{19} \mathrm{~F}_{9} \mathrm{~N}_{3} \mathrm{O}_{8} \mathrm{Yb}$ & $\mathrm{C}_{36} \mathrm{H}_{37} \mathrm{~F}_{9} \mathrm{~N}_{3} \mathrm{O}_{8} \mathrm{Yb}$ & $\mathrm{C}_{33} \mathrm{H}_{10} \mathrm{~F}_{30} \mathrm{~N}_{3} \mathrm{O}_{8} \mathrm{Yb}$ \\
\hline Formula weight & 857.49 & 983.73 & 1319.48 \\
\hline Temperature (K) & $293(2)$ & $293(2)$ & $150(1)$ \\
\hline Wavelength $(\AA)$ & 0.71073 & 0.71073 & 0.71073 \\
\hline Crystal system & Monoclinic & Monoclinic & Monoclinic \\
\hline Space group & $\mathrm{P} 2{ }_{1} / c$ & $\mathrm{P} 2{ }_{1} / c$ & $\mathrm{P} 2{ }_{1} / n$ \\
\hline$a(\AA)$ & $9.7806(16)$ & $9.9036(3)$ & $10.0391(3)$ \\
\hline$b(\AA)$ & $14.750(2)$ & $22.3251(7)$ & $25.3149(7)$ \\
\hline$c(\AA)$ & $23.952(3)$ & $39.8599(11)$ & $18.4810(5)$ \\
\hline$\alpha(\underline{0})$ & 90.00 & 90.00 & 90.00 \\
\hline$\beta(\underline{0})$ & $114.101(5)$ & $90.782(2)$ & $117.213(2)$ \\
\hline$\gamma(\underline{0})$ & 90.00 & 90.00 & 90.00 \\
\hline Volume $\left(\AA^{3}\right)$ & $3154.2(8)$ & $8812.2(5)$ & $4176.9(2)$ \\
\hline$Z$ & 4 & 8 & 4 \\
\hline $\begin{array}{l}\text { Calculated density } \\
\qquad\left(\mathrm{g} \mathrm{cm}^{-3}\right)\end{array}$ & 1.806 & 1.483 & 2.098 \\
\hline Absorption coefficient $\left(\mathrm{mm}^{-1}\right)$ & 3.070 & 2.208 & 2.428 \\
\hline$F(000)$ & 1668 & 3912 & 2532 \\
\hline Crystal size $\left(\mathrm{mm}^{3}\right)$ & $0.28 \times 0.07 \times 0.05$ & $0.26 \times 0.08 \times 0.07$ & $0.27 \times 0.18 \times 0.16$ \\
\hline$\theta$ range for data collection & $3.12-26.28^{\circ}$ & $2.28-25.88^{\circ}$ & $1.61-30.23^{\circ}$ \\
\hline Index ranges & $\begin{array}{c}-11<h<12 ;-17<k<17 ; \\
-29<l<29\end{array}$ & $\begin{array}{c}-12<h<12 ;-27<k<27 ; \\
-48<l<48\end{array}$ & $\begin{array}{c}-13<h<14 ;-35<k<34 ; \\
-26<l<26\end{array}$ \\
\hline Reflections collected & 56609 & 148952 & 95198 \\
\hline Independent reflections & 6096 & 16971 & 12348 \\
\hline Completeness to $2 \theta=51^{\mathrm{o}}$ & $99.8 \%$ & $99.8 \%$ & $99.9 \%$ \\
\hline Refinement method & Full matrix LS on $\mathrm{F}^{2}$ & Full matrix LS on $\mathrm{F}^{2}$ & Full matrix LS on $\mathrm{F}^{2}$ \\
\hline Data/restrains/parameters & $3130 / 0 / 436$ & 7034/32/1199 & 9195/0/886 \\
\hline Goodness-of-fit on $\mathrm{F}^{2}$ & 0.962 & 0.957 & 1.045 \\
\hline Final $R$ indices $[I>2 \sigma(I)]$ & $R=0.0655 ; \mathrm{w} R=0.1618$ & $R=0.0764 ; \mathrm{w} R=0.1754$ & $R=0.0454 ; \mathrm{w} R=0.0883$ \\
\hline$R$ indices (all data) & $R=0.1434 ; \mathrm{w} R=0.2096$ & $R=0.2008 ; \mathrm{w} R=0.2150$ & $R=0.0712 ; \mathrm{w} R=0.0994$ \\
\hline Largest diff. peak and hole & $-2.675 / 0.797$ & $-0.747 / 0.654$ & $-1.484 / 1.276$ \\
\hline
\end{tabular}


Table 34. $\mathrm{Yb}-\mathrm{N}$ and $\mathrm{Yb}-\mathrm{O}$ distances in $\AA$ for the SC-XRD structures of the $[\mathrm{Yb}(\beta-$ diketonate $)_{3}\left(5 \mathrm{NO}_{2}\right.$ phen $\left.)\right]$ complexes

\begin{tabular}{|c|c|c|c|c|c|}
\hline Bond & {$\left[\mathrm{Yb}(\mathrm{tfac})_{3}\left(5 \mathrm{NO}_{2}\right.\right.$ phen $\left.)\right]$} & Bond & {$\left[\mathrm{Yb}(\mathrm{tpm})_{3}\left(5 \mathrm{NO}_{2}\right.\right.$ phen $\left.)\right]$} & Bond & {$\left[\mathrm{Yb}(\mathrm{fhd})_{3}\left(5 \mathrm{NO}_{2}\right.\right.$ phen $\left.)\right]$} \\
\hline Yb1-N1 & $2.539(10)$ & Yb1-N1 & $2.522(7)$ & $\mathrm{Yb} 1-\mathrm{N} 1_{\mathrm{A} / \mathrm{B}}$ & $2.471(9) / 2.475(13)$ \\
\hline Yb1-N2 & $2.527(9)$ & Yb1-N2 & $2.488(6)$ & $\mathrm{Yb} 1-\mathrm{N} 2 \mathrm{~A} / \mathrm{B}$ & $2.413(10) / 2.549(11)$ \\
\hline Yb1-01 & $2.316(8)$ & Yb1-01 & $2.261(5)$ & Yb1-01 & $2.283(3)$ \\
\hline $\mathrm{Yb} 1-02$ & $2.259(9)$ & $\mathrm{Yb} 1-02$ & $2.268(6)$ & Yb1-02 & $2.297(3)$ \\
\hline Yb1-03 & $2.289(7)$ & Yb1-03 & $2.271(5)$ & Yb1-03 & $2.284(3)$ \\
\hline Yb1-04 & $2.252(8)$ & Yb1-04 & $2.284(5)$ & Yb1-04 & $2.316(3)$ \\
\hline Yb1-05 & $2.285(9)$ & Yb1-05 & $2.293(5)$ & Yb1-05 & $2.287(3)$ \\
\hline Yb1-06 & $2.276(9)$ & Yb1-06 & $2.299(6)$ & Yb1-06 & $2.278(3)$ \\
\hline Yb1-N avg. & 2.533 & Yb1-N avg. & 2.505 & $\mathrm{Yb} 1-\mathrm{N}_{\mathrm{A} / \mathrm{B}}$ avg. & $2.442 / 2.512$ \\
\hline Yb1-O avg. & 2.279 & Yb1-O avg. & 2.279 & Yb1-0 avg. & 2.291 \\
\hline
\end{tabular}




\section{Semiempirical models}

18. [Er(od) $)_{3}\left(\right.$ bipy)] crystallizes in the monoclinic $P 2_{1} / c$ space group, with cell parameters $a=20.928 \AA, b=9.615 \AA, c=19.032 \AA, \alpha=90.00^{\circ}, \beta=105.76^{\circ}, \gamma=90.00^{\circ}$ and $\mathrm{V}=3685.20 \AA^{3}$ (Figure 72, Table 35).

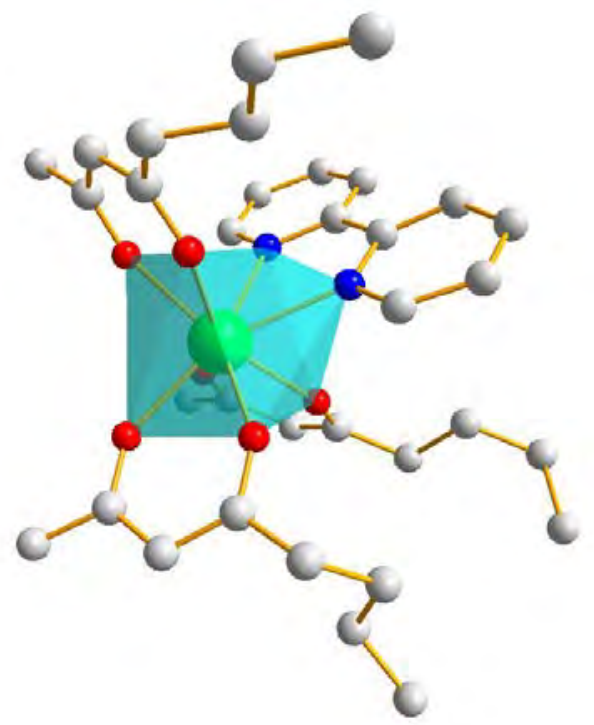

Figure 72. PM7/Sparkle ground state geometry for [Er(od) $)_{3}($ bipy)]

Table 35. Cell parameters for $\left[\operatorname{Er}(\mathrm{od})_{3}(\right.$ bipy)] (calculated using PM7/Sparkle)

\begin{tabular}{|c|c|}
\hline Complex & $\left.\operatorname{Er}(\mathrm{od})_{3}(\mathrm{bipy})\right]$ \\
\hline Empirical formula & $\mathrm{C}_{34} \mathrm{H}_{47} \mathrm{ErN}_{2} \mathrm{O}_{6}$ \\
\hline Formula weight & 747.00 \\
\hline Crystal system & Monoclinic \\
\hline Space group & $P 2_{1} / c$ \\
\hline$a(\AA)$ & 20.928 \\
\hline$b(\AA)$ & 9.615 \\
\hline$c(\AA)$ & 19.032 \\
\hline$\alpha(\underline{o})$ & 90 \\
\hline$\beta(\stackrel{o}{)})$ & 105.76 \\
\hline$\gamma(\underline{o})$ & 90 \\
\hline Volume $\left(\AA^{3}\right)$ & 3685.20 \\
\hline$Z$ & 4 \\
\hline Calculated density $\left(\mathrm{g} \mathrm{cm}^{-3}\right)$ & 1.346 \\
\hline
\end{tabular}

Table 36. Er-N and Er-O distances in Å for the PM7/Sparkle model of [Er(od) $)_{3}($ bipy)]

\begin{tabular}{cc} 
Bond & $[\text { Er(od })_{3}($ bipy $\left.)\right]$ \\
\hline Er-N1 & 2.4716 \\
Er-N2 & 2.4749 \\
Er-01 & 2.2794 \\
Er-O2 & 2.2921 \\
Er-03 & 2.2935 \\
Er-04 & 2.2885 \\
Er-05 & 2.2887 \\
Er-06 & 2.2957 \\
Er-N avg. & 2.473 \\
Er-O avg. & 2.290 \\
\hline
\end{tabular}


21. [Er(nd) $)_{3}$ (bipy)] crystallizes in the triclinic P-1 space group, with cell parameters $a=25.55 \AA, b=25.55 \AA, c=21.467 \AA, \alpha=90.56^{\circ}, \beta=103.69^{\circ}, \gamma=82.28^{\circ}$ and $V=5073.49^{3}{ }^{3}$ (Figure 74, Table 37).
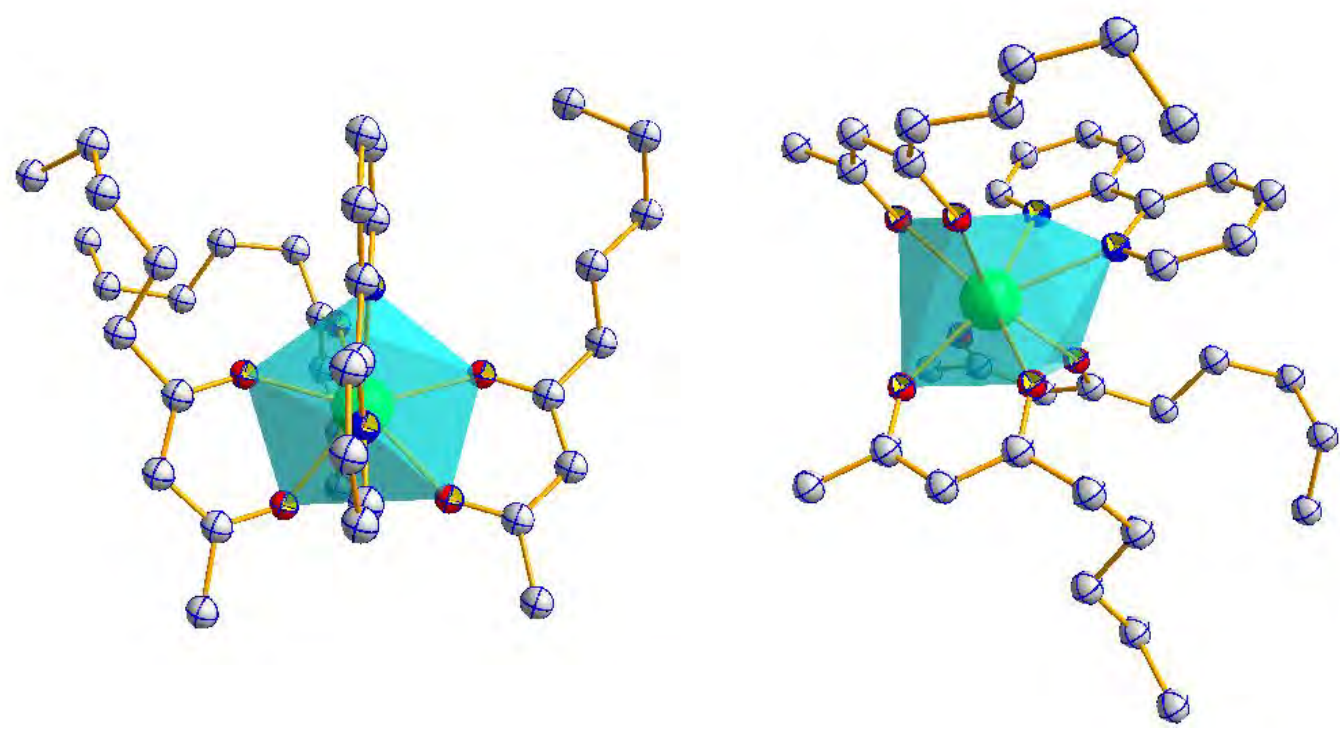

Figure 73. Two views of the Sparkle/PM7 ground state geometry. H atoms have been omitted for clarity.

Table 37. Crystal data and structure refinement of complex [Er(nd) $)_{3}($ bipy)]

\begin{tabular}{|c|c|}
\hline Complex & $\left.\operatorname{Er}(\mathrm{nd})_{3}(\mathrm{bipy})\right]$ \\
\hline Empirical formula & $\mathrm{C}_{37} \mathrm{H}_{53} \mathrm{ErN}_{2} \mathrm{O}_{6}$ \\
\hline Formula weight & 789.08 \\
\hline Temperature (K) & 293(2) \\
\hline Wavelength $(\AA)$ & 0.71073 \\
\hline Crystal system & Triclinic P \\
\hline Space group & P-1 \\
\hline$a(\AA)$ & 25.550 \\
\hline$b(\AA)$ & 25.550 \\
\hline$c(\AA)$ & 21.467 \\
\hline$\alpha(\underline{o})$ & 90.56 \\
\hline$\beta(\stackrel{\circ}{)})$ & 103.69 \\
\hline$\gamma(\stackrel{\circ}{)})$ & 82.28 \\
\hline Volume $\left(\AA^{3}\right)$ & 5073.49 \\
\hline
\end{tabular}

Table 38. Selected distances $(\AA ̊)$ and angles $(\stackrel{\circ}{)})$

\begin{tabular}{cc} 
Bond & $[\text { Er(nd })_{3}($ bipy $\left.)\right]$ \\
\hline Er-N1 & 2.4797 \\
Er-N2 & 2.4737 \\
Er-O1 & 2.2784 \\
Er-O2 & 2.2845 \\
Er-O3 & 2.2877 \\
Er-04 & 2.2904 \\
Er-O5 & 2.2916 \\
Er-O6 & 2.2925 \\
Er-N avg. & 2.4767 \\
Er-O avg. & 2.2875 \\
\hline
\end{tabular}


33. [Er(fod) $)_{3}$ (5NO2phen)] crystallizes in the monoclinic $\mathrm{P} 2_{1} / \mathrm{c}$ space group, with cell parameters $a=14.284 \AA, b=20.868 \AA, c=19.666 \AA, \alpha=90.00^{\circ}, \beta=107.76^{\circ}, \gamma=90.00^{\circ}=$ and $\mathrm{V}=5582.29 \AA^{3}$ (Figure 74, Table 39).

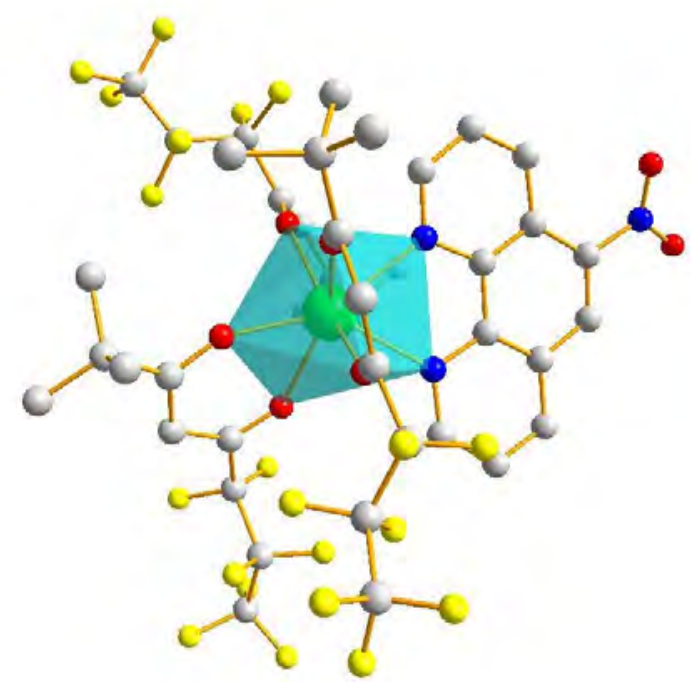

Figure 74. PM7/Sparkle ground state geometry for [ $\operatorname{Er}(\text { fod })_{3}\left(5 \mathrm{NO}_{2}\right.$ phen)]

Table 39. Cell parameters for $\left[\operatorname{Er}(\text { fod })_{3}\left(5 \mathrm{NO}_{2}\right.\right.$ phen)] (calculated using PM7/Sparkle)

\begin{tabular}{|c|c|}
\hline Complex & $\operatorname{Er}(\text { fod })_{3}\left(5 \mathrm{NO}_{2}\right.$ phen $\left.)\right]$ \\
\hline Empirical formula & $\mathrm{C}_{42} \mathrm{H}_{37} \mathrm{ErF}_{21} \mathrm{~N}_{3} \mathrm{O}_{8}$ \\
\hline Formula weight & 1277.98 \\
\hline Crystal system & Monoclinic \\
\hline Space group & $P 2_{1} / c$ \\
\hline$a(\AA ̊)$ & 14.284 \\
\hline$b(\AA)$ & 20.868 \\
\hline$c(\AA)$ & 19.666 \\
\hline$\alpha(\stackrel{\circ}{)})$ & 90.00 \\
\hline$\beta(\underline{o})$ & 107.76 \\
\hline$\gamma(\stackrel{\circ}{\circ})$ & 90.00 \\
\hline Volume $\left(\AA^{3}\right)$ & 5582.29 \\
\hline$Z$ & 4 \\
\hline Calculated density $\left(\mathrm{g} \mathrm{cm}^{-3}\right)$ & 1.521 \\
\hline
\end{tabular}

Table 40. Er-N and Er-O distances in Å for the PM7/Sparkle model of [ $\operatorname{Er}(\text { fod })_{3}\left(5 \mathrm{NO}_{2}\right.$ phen $\left.)\right]$

\begin{tabular}{cc} 
Bond & {$\left[\mathrm{Er}(\mathrm{fod})_{3}\left(5 \mathrm{NO}_{2}\right.\right.$ phen $\left.)\right]$} \\
\hline Er-N1 & 2.4715 \\
Er-N2 & 2.4668 \\
Er-O1 & 2.2955 \\
Er-O2 & 2.2996 \\
Er-O3 & 2.2957 \\
Er-04 & 2.3060 \\
Er-O5 & 2.2747 \\
Er-O6 & 2.3081 \\
Er-N avg. & 2.467 \\
Er-O avg. & 2.297 \\
\hline
\end{tabular}


Structural diagrams for complexes $35,36,37$ and $38, \quad\left[\mathrm{Yb}(\mathrm{h})_{3}\left(\mathrm{NNO}_{2}\right.\right.$ phen $\left.)\right]$, $\left[\mathrm{Yb}(\mathrm{dmh})_{3}\left(\mathbf{5 N O}_{2}\right.\right.$ phen $\left.)\right],\left[\mathrm{Yb}(\mathrm{tfa})_{3}\left(\mathbf{5 N O}_{2}\right.\right.$ phen $\left.)\right]$ and [Yb(tfnb $)_{3}\left(\mathbf{5 N O}_{2}\right.$ phen $\left.)\right]$, respectively, are shown in Figure 75, Figure 76 and Figure 77. Crystallographic data for the four $\mathrm{Yb}(\mathrm{III})$ complexes is summarized in Table 41.
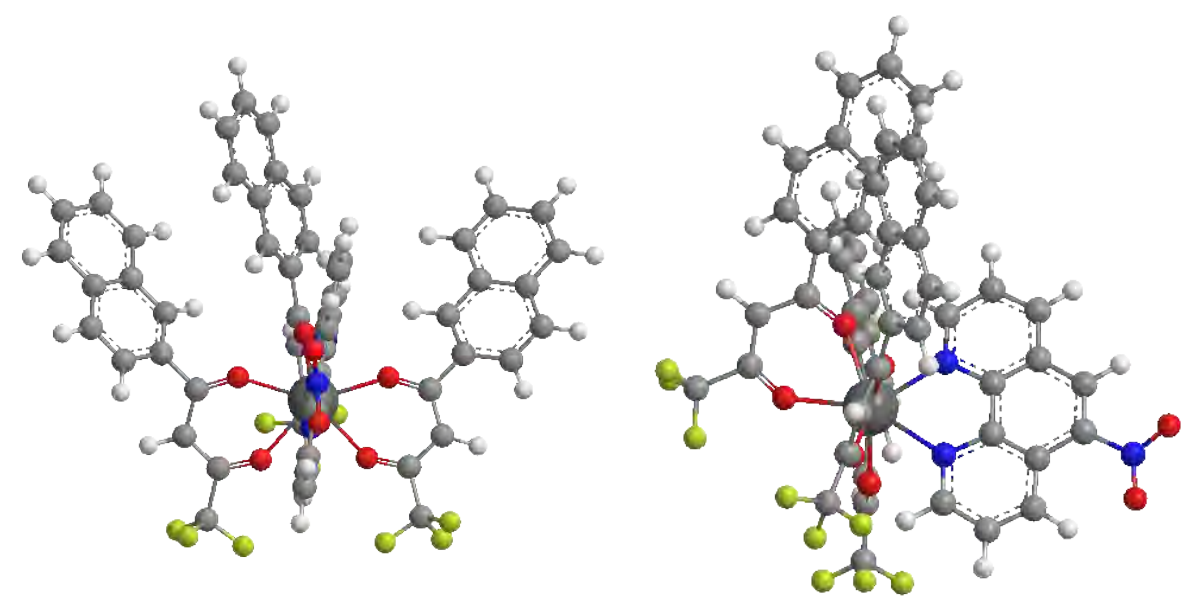

Figure 75. Two views of the PM6/Sparkle ground state geometry of $\left[\mathrm{Yb}(\mathrm{tfnb})_{3}\left(5 \mathrm{NO}_{2}\right.\right.$ phen $\left.)\right]$
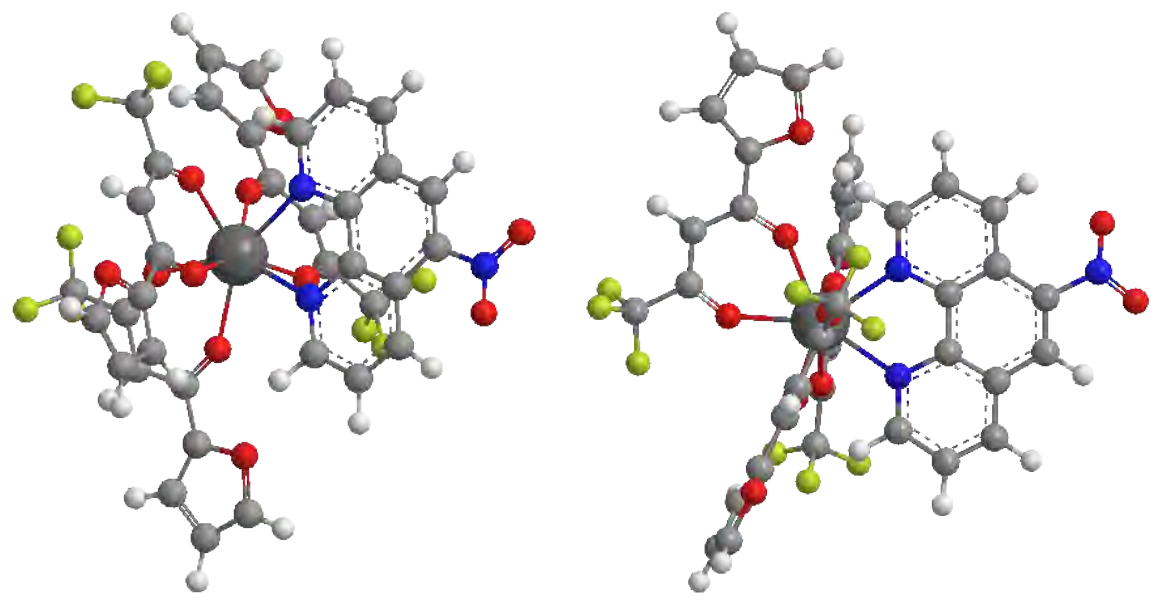

Figure 76. Two views of the PM6/Sparkle ground state geometry of $\left[\mathrm{Yb}(\mathrm{tfa})_{3}\left(5 \mathrm{NO}_{2}\right.\right.$ phen $\left.)\right]$
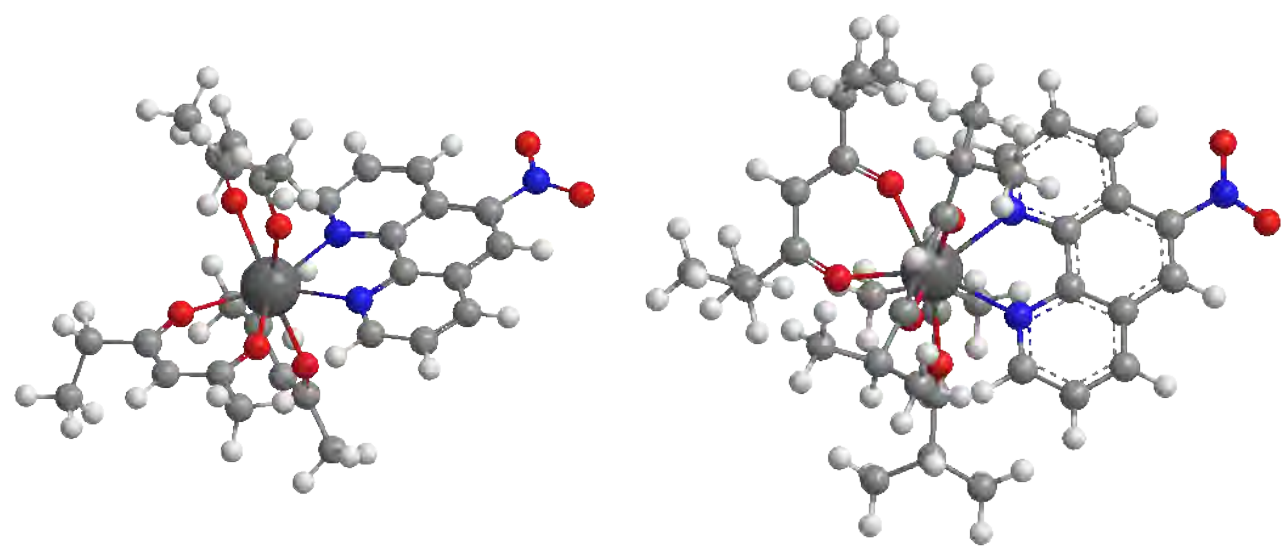

Figure 77. PM6/Sparkle ground state geometries of $\left[\mathrm{Yb}(\mathrm{h})_{3}\left(5 \mathrm{NO}_{2} \mathrm{phen}\right)\right]($ left $)$ and $\left[\mathrm{Yb}(\mathrm{dmh})_{3}\left(5 \mathrm{NO}_{2}\right.\right.$ phen $\left.)\right]($ right $)$ 
Table 41. $\mathrm{Yb}-\mathrm{N}$ and $\mathrm{Yb}-\mathrm{O}$ distances in $\AA$ for the PM6/Sparkle models of the $[\mathrm{Yb}(\beta-$ diketonate $)_{3}\left(5 \mathrm{NO}_{2}\right.$ phen $\left.)\right]$ complexes

\begin{tabular}{ccccc} 
Bond & {$\left[\mathrm{Yb}(\mathrm{tfnb})_{3}\left(5 \mathrm{NO}_{2}\right.\right.$ phen $\left.)\right]$} & {$\left[\mathrm{Yb}(\mathrm{tfa})_{3}\left(5 \mathrm{NO}_{2}\right.\right.$ phen $\left.)\right]$} & {$\left[\mathrm{Yb}(\mathrm{h})_{3}\left(5 \mathrm{NO}_{2}\right.\right.$ phen $\left.)\right]$} & {$\left[\mathrm{Yb}(\mathrm{dmh})_{3}\left(5 \mathrm{NO}_{2}\right.\right.$ phen $\left.)\right]$} \\
\hline $\mathrm{Yb}-\mathrm{N} 1$ & 2.389 & 2.389 & 2.397 & 2.399 \\
$\mathrm{Yb}-\mathrm{N} 2$ & 2.387 & 2.384 & 2.396 & 2.393 \\
$\mathrm{Yb}-01$ & 2.326 & 2.328 & 2.322 & 2.319 \\
$\mathrm{Yb}-02$ & 2.323 & 2.322 & 2.319 & 2.317 \\
$\mathrm{Yb}-03$ & 2.319 & 2.324 & 2.312 & 2.324 \\
$\mathrm{Yb}-04$ & 2.326 & 2.325 & 2.325 & 2.318 \\
$\mathrm{Yb}-05$ & 2.322 & 2.322 & 2.318 & 2.322 \\
$\mathrm{Yb}-06$ & 2.328 & 2.331 & 2.322 & 2.314 \\
$\mathrm{Yb}-\mathrm{N}$ avg. & 2.388 & 2.387 & 2.397 & 2.396 \\
$\mathrm{Yb}-\mathrm{O}$ avg. & 2.324 & 2.325 & 2.320 & 2.319 \\
\hline
\end{tabular}

\section{Assessment of the accuracy of semiempirical quantum chemistry methods}

So as to evaluate the accuracy of the ground state geometries predicted by semiempirical methods, the actual experimental crystallographic data of [Er(acac) 3 (bath)] has been compared to the ground state geometries obtained using the Sparkle/AM1, Sparkle/PM3, Sparkle/PM6 and Sparkle/PM7 models.

The comparison between the results obtained with the four models studied reveal that a substantial improvement in accuracy is obtained with the recently put forward $[124,133]$ Sparkle/PM7 model. The unsigned mean error (UME) in the calculations for a single unit cell is, with the Sparkle/PM7 model, of $\mathrm{UME}_{a, b, c}=0.89 \%$ for the $a, b$ and $c$ parameters and $\mathrm{UME}_{\alpha, \beta, \gamma}=0.39 \%$ for the $\alpha, \beta$ and $\gamma$ parameters. For the Sparkle/AM1, UME $E_{a, b, c}=7.61 \%$ and $\mathrm{UME}_{\alpha, \beta, \gamma}=3.12 \%$, for Sparkle/PM3 we obtained $\mathrm{UME}_{a, b, c}=0.97 \%$ and $\mathrm{UME}_{\alpha, \beta, \gamma}=3.32 \%$ and for Sparkle/PM6 $\mathrm{UME}_{a, b, c}=2.82 \%$ and $\mathrm{UME}_{\alpha, \beta, \gamma}=1.39 \%$. Regarding the percent error for the volume, it was $25.5 \%$ for Sparkle/AM1, 3.60\% for Sparkle/PM3, $6.82 \%$ for Sparkle/PM6 and $2.01 \%$ for Sparkle/PM7.

The size of the unit cell, having a large number of atoms, guarantees enough accuracy of the calculations according to the criteria given in [160]. Nevertheless, we repeated the calculations with $2 \times 1 \times 1,1 \times 2 \times 1,1 \times 1 \times 2,2 \times 2 \times 1$ and $2 \times 2 \times 2$ unit cells. In all cases, the results were consistent with those for the $1 \times 1 \times 1$ case (Table 42 , Table 43 , Table 44 , Table 45 , Table 46, Table 47) with very similar values of $\mathrm{UME}_{a, b, c}$ and $\mathrm{UME}_{\alpha, \beta, \gamma}$ for each model at all computation sizes. For the two largest computations with $2 \times 2 \times 1$ and $2 \times 2 \times 2$ unit cells, the results from the $1 \times 1 \times 1$ computations for each model were used as pre-optimized initial conditions, which permitted a significant speedup of the calculations. In these two largest cases, either with or without pre-optimization, the PM7 calculations showed worse behavior and failed to converge within the allowed time.

Apart from these accuracy considerations, it should also be noted that there are significant differences in the computation times, which range from 2 hours 11 minutes for Sparkle/AM1 to only 16 minutes for Sparkle/PM7 (for a single unit cell). These differences in the running times increase with the number of unit cells (e.g. for $2 \times 1 \times 1$, Sparkle/AM1 requires 8 hours 33 minutes, Sparkle/PM3 needs 12 hours 14 minutes and Sparkle/PM6 takes 6 hours 14 minutes, while Sparkle/PM7 requires only 1 hour 38 minutes). Consequently, Sparkle/PM7 not only provides higher accuracy than the other semi-empirical methods, but also proves to be much faster. 
Table 42. Comparison of crystallographic and calculated cell parameters for $1 \times 1 \times 1$ unit cell

\begin{tabular}{|c|c|c|c|c|c|c|c|c|c|}
\hline Cell parameters & Crystallographic & Sparkle/AM1 & Error $(\%)$ & Sparkle/PM3 & Error $(\%)$ & Sparkle/PM6 & Error $(\%)$ & Sparkle/PM7 & Error (\%) \\
\hline$a(\AA)$ & 11.8685 & 13.351 & 12.49 & 11.913 & 0.37 & 11.486 & 3.22 & 11.779 & 0.75 \\
\hline$b(\AA)$ & 12.1053 & 12.797 & 5.71 & 12.255 & 1.24 & 11.837 & 2.22 & 12.075 & 0.25 \\
\hline$c(\AA)$ & 14.2355 & 14.893 & 4.62 & 14.422 & 1.31 & 13.805 & 3.02 & 13.999 & 1.66 \\
\hline$\alpha(\underline{\mathrm{o}})$ & 96.6630 & 89.58 & 7.33 & 100.58 & 4.05 & 95.30 & 1.41 & 95.90 & 0.79 \\
\hline$\beta(\underline{o})$ & 105.0080 & 106.33 & 1.26 & 106.48 & 1.40 & 105.92 & 0.87 & 104.97 & 0.04 \\
\hline$\gamma(\underline{\mathrm{o}})$ & 110.1770 & 111.04 & 0.78 & 105.22 & 4.50 & 108.10 & 1.89 & 109.80 & 0.34 \\
\hline Volume $\left(\AA^{3}\right)$ & 1806.0500 & 2266.61 & 25.50 & 1871.01 & 3.60 & 1682.85 & 6.82 & 1769.73 & 2.01 \\
\hline $\mathrm{UME}_{a, b, c}$ & - & 7.61 & - & 0.97 & - & 2.82 & - & 0.89 & - \\
\hline $\mathrm{UME}_{\alpha, \beta, \gamma}$ & - & 3.12 & - & 3.32 & - & 1.39 & - & 0.39 & - \\
\hline Computation time (s) & - & 7869 & - & 5619 & - & 4721 & - & 1013 & - \\
\hline
\end{tabular}

Table 43. Comparison of crystallographic and calculated cell parameters for $2 \times 1 \times 1$ unit cell

\begin{tabular}{|c|c|c|c|c|c|c|c|c|c|}
\hline Cell parameters & Crystallographic & Sparkle/AM1 & Error (\%) & Sparkle/PM3 & Error (\%) & Sparkle/PM6 & Error (\%) & Sparkle/PM7 & Error $(\%)$ \\
\hline$a(\AA)$ & 11.8685 & 12.903 & 8.72 & 11.871 & 0.02 & 11.730 & 1.17 & 11.837 & 0.27 \\
\hline$b(\AA)$ & 12.1053 & 12.894 & 6.52 & 12.270 & 1.36 & 11.697 & 3.37 & 12.120 & 0.12 \\
\hline$c(\AA)$ & 14.2355 & 14.929 & 4.87 & 14.307 & 0.50 & 13.802 & 3.05 & 13.951 & 2.00 \\
\hline$\alpha(\underline{\mathrm{o}})$ & 96.6630 & 88.64 & 8.30 & 98.68 & 2.09 & 95.20 & 1.51 & 96.29 & 0.39 \\
\hline$\beta(\underline{o})$ & 105.0080 & 105.88 & 0.83 & 106.27 & 1.20 & 107.61 & 2.48 & 105.46 & 0.43 \\
\hline$\gamma(\underline{\mathrm{o}})$ & 110.1770 & 109.49 & 0.62 & 106.12 & 3.68 & 108.01 & 1.97 & 109.08 & 1.00 \\
\hline Volume $\left(\AA^{3}\right)$ & 1806.0500 & 2245.72 & 24.34 & 1861.79 & 3.09 & 1680.81 & 6.93 & 1780.07 & 1.44 \\
\hline $\mathrm{UME}_{a, b, c}$ & - & 6.70 & - & 0.63 & - & 2.53 & - & 0.80 & - \\
\hline $\mathrm{UME}_{\alpha, \beta, \gamma}$ & - & 3.25 & - & 2.32 & - & 1.99 & - & 0.60 & - \\
\hline Computation time (s) & - & 30836 & - & 44085 & - & 22466 & - & 5898 & - \\
\hline
\end{tabular}


Table 44. Comparison of crystallographic and calculated cell parameters for $1 \times 2 \times 1$ unit cell

\begin{tabular}{|c|c|c|c|c|c|c|c|c|c|}
\hline Cell parameters & Crystallographic & Sparkle/AM1 & Error (\%) & Sparkle/PM3 & Error (\%) & Sparkle/PM6 & Error (\%) & Sparkle/PM7 & Error (\%) \\
\hline$a(\AA)$ & 11.8685 & 14.000 & 17.96 & 11.852 & 0.14 & 11.403 & 3.92 & 11.889 & 0.17 \\
\hline$b(\AA)$ & 12.1053 & 12.297 & 1.58 & 12.195 & 0.74 & 11.942 & 1.35 & 12.165 & 0.49 \\
\hline$c(\AA)$ & 14.2355 & 14.963 & 5.11 & 14.600 & 2.56 & 13.815 & 2.95 & 13.893 & 2.41 \\
\hline$\alpha(\underline{\mathrm{o}})$ & 96.6630 & 87.06 & 9.93 & 100.02 & 3.47 & 95.54 & 1.16 & 96.37 & 0.30 \\
\hline$\beta(\stackrel{\mathrm{o}}{)}$ & 105.0080 & 109.52 & 4.30 & 106.07 & 1.01 & 105.22 & 0.20 & 105.44 & 0.41 \\
\hline$\gamma(\underline{\mathrm{o}})$ & 110.1770 & 111.05 & 0.79 & 105.84 & 3.94 & 108.27 & 1.73 & 109.13 & 0.95 \\
\hline Volume $\left(\AA^{3}\right)$ & 1806.0500 & 2259.03 & 25.08 & 1878.47 & 4.01 & 1690.67 & 6.39 & 1785.97 & 1.11 \\
\hline $\mathrm{UME}_{a, b, c}$ & - & 8.22 & - & 1.15 & - & 2.74 & - & 1.02 & - \\
\hline $\mathrm{UME}_{\alpha, \beta, \gamma}$ & - & 5.01 & - & 2.81 & - & 1.03 & - & 0.55 & - \\
\hline Computation time (s) & - & 31563 & - & 33537 & - & 16507 & - & 6055 & - \\
\hline
\end{tabular}

Table 45. Comparison of crystallographic and calculated cell parameters for $1 \times 1 \times 2$ unit cell

\begin{tabular}{|c|c|c|c|c|c|c|c|c|c|}
\hline Cell parameters & Crystallographic & Sparkle/AM1 & Error $(\%)$ & Sparkle/PM3 & Error (\%) & Sparkle/PM6 & Error (\%) & Sparkle/PM7 & Error $(\%)$ \\
\hline$a(\AA)$ & 11.8685 & 14.000 & 17.96 & 11.804 & 0.54 & 11.422 & 3.76 & 12.002 & 1.12 \\
\hline$b(\AA)$ & 12.1053 & 12.337 & 1.91 & 12.204 & 0.82 & 11.812 & 2.42 & 12.107 & 0.01 \\
\hline$c(\AA)$ & 14.2355 & 14.192 & 0.31 & 14.521 & 2.01 & 14.041 & 1.37 & 14.150 & 0.60 \\
\hline$\alpha(\stackrel{\mathrm{o}}{)})$ & 96.6630 & 96.18 & 0.50 & 99.49 & 2.92 & 95.83 & 0.86 & 97.05 & 0.40 \\
\hline$\beta(\underline{\mathrm{o}})$ & 105.0080 & 99.36 & 5.38 & 105.64 & 0.60 & 105.50 & 0.47 & 106.18 & 1.12 \\
\hline$\gamma(\underline{\mathrm{o}})$ & 110.1770 & 110.12 & 0.05 & 106.08 & 3.72 & 108.28 & 1.72 & 109.00 & 1.07 \\
\hline Volume $\left(\AA^{3}\right)$ & 1806.0500 & 2235.28 & 23.77 & 1869.53 & 3.51 & 1697.34 & 6.02 & 1814.80 & 0.48 \\
\hline $\mathrm{UME}_{a, b, c}$ & - & 6.73 & - & 1.12 & - & 2.52 & - & 0.58 & - \\
\hline $\mathrm{UME}_{\alpha, \beta, \gamma}$ & - & 1.98 & - & 2.42 & - & 1.02 & - & 0.86 & - \\
\hline Computation time (s) & - & 18719 & - & 43366 & - & 23214 & - & 7314 & - \\
\hline
\end{tabular}


Table 46. Comparison of crystallographic and calculated cell parameters for $2 \times 2 \times 1$ unit cell

\begin{tabular}{|c|c|c|c|c|c|c|c|}
\hline Cell parameters & Crystallographic & Sparkle/AM1 & Error $(\%)$ & Sparkle/PM3 & Error (\%) & Sparkle/PM6 & Error $(\%)$ \\
\hline$a(\AA)$ & 11.8685 & 13.347 & 12.46 & 11.905 & 0.31 & 11.504 & 3.07 \\
\hline$b(\AA)$ & 12.1053 & 12.842 & 6.09 & 12.335 & 1.90 & 11.846 & 2.14 \\
\hline$c(\AA)$ & 14.2355 & 14.867 & 4.44 & 14.34 & 0.73 & 13.782 & 3.19 \\
\hline$\alpha(\underline{\mathrm{o}})$ & 96.6630 & 89.75 & 7.15 & 100.71 & 4.19 & 95.17 & 1.54 \\
\hline$\beta(\underline{\mathrm{o}})$ & 105.0080 & 106.38 & 1.31 & 106.28 & 1.21 & 106.07 & 1.01 \\
\hline$\gamma(\underline{\mathrm{o}})$ & 110.1770 & 111 & 0.75 & 104.9 & 4.79 & 107.95 & 2.02 \\
\hline Volume $\left(\AA^{3}\right)$ & 1806.0500 & 2269.05 & 25.64 & 1876.16 & 3.88 & 1685.04 & 6.70 \\
\hline $\mathrm{UME}_{a, b, c}$ & - & 7.66 & - & 0.98 & - & 2.80 & - \\
\hline $\mathrm{UME}_{\alpha, \beta, \gamma}$ & - & 3.07 & - & 3.40 & - & 1.53 & - \\
\hline
\end{tabular}

Table 47. Comparison of crystallographic and calculated cell parameters for $2 \times 2 \times 2$ unit cell

\begin{tabular}{|c|c|c|c|c|c|c|c|}
\hline Cell parameters & Crystallographic & Sparkle/AM1 & Error $(\%)$ & Sparkle/PM3 & Error $(\%)$ & Sparkle/PM6 & Error $(\%)$ \\
\hline$a(\AA)$ & 11.8685 & 13.337 & 12.37 & 11.896 & 0.23 & 11.497 & 3.13 \\
\hline$b(\AA)$ & 12.1053 & 12.897 & 6.54 & 12.354 & 2.05 & 11.849 & 2.12 \\
\hline$c(\AA)$ & 14.2355 & 14.876 & 4.50 & 14.331 & 0.67 & 13.79 & 3.13 \\
\hline$\alpha(\stackrel{\mathrm{o}}{)})$ & 96.6630 & 89.86 & 7.04 & 100.78 & 4.26 & 95.2 & 1.51 \\
\hline$\beta(\underline{\mathrm{o}})$ & 105.0080 & 106.44 & 1.36 & 106.27 & 1.20 & 105.97 & 0.92 \\
\hline$\gamma(\underline{\mathrm{o}})$ & 110.1770 & 110.1 & 0.07 & 104.76 & 4.92 & 107.92 & 2.05 \\
\hline Volume $\left(\AA^{3}\right)$ & 1806.0500 & 2275.51 & 25.99 & 1877.83 & 3.97 & 1686.67 & 6.61 \\
\hline $\mathrm{UME}_{a, b, c}$ & - & 7.80 & - & 0.99 & - & 2.79 & - \\
\hline $\mathrm{UME}_{\alpha, \beta, \gamma}$ & - & 2.82 & - & 3.46 & - & 1.49 & - \\
\hline
\end{tabular}




\section{Remarks on structural parameters}

Although in all the structures the coordination polyhedron is formed by three terminal $\beta$ diketonate oxygens and two terminal pyridyl nitrogens, the rearrangement of the monomer species in the crystal lattice is very different within the studied complexes series. These differences are due to variations in the nature and properties of the ligands (rigidity of the $\mathrm{N}, \mathrm{N}$-donor molecule -higher for bathophenanthroline than for 2,2'-bipyridine-, electronwithdrawing behaviour of the $\beta$-diketonate ligand -higher in the fluorinated ligands due to the inductive effect of the $\mathrm{CF}_{3}$ groups-, etc.) and they are the cause of the versatility of physical properties (and applications) shown by the claimed series of complexes.

Thus, fluorination of the $\beta$-diketonate ligand is a favourable factor: transfer of electron density to the $\mathrm{CF}_{3}$ groups results in a relatively more positively charged $\mathrm{Er}^{3+}$ ion and in stronger and shorter Er-N bonds (the Er-N average is $2.56 \AA$ for non-fluorinated complexes vs. $2.50 \AA$ for fluorinated ones).

Regarding the effect of the N,N-donor choice, while there are no significant differences in the average Er-N distances amongst the non-fluorinated complexes, for fluorinated ones the Er$\mathrm{N}$ bonds are remarkably shorter for 5-nitro-1,10-phenanthroline adducts $(2.44 \AA \bar{\alpha} v$ s. $2.52 \AA$ for 2,2'-bipyridine and bathophenanthroline ones).

Although a significant correlation between Er- $\mathrm{N}$ and Er-O bonds lenght is not observed, their addition leads to a constant: $4.83 \AA \bar{A}$, a larger value than that obtained by semiempirical methods (in these, the sum of Er-N and Er-O distances is $4.72 \AA$ ). For $\mathrm{Yb}(\mathrm{III})$ complexes, the

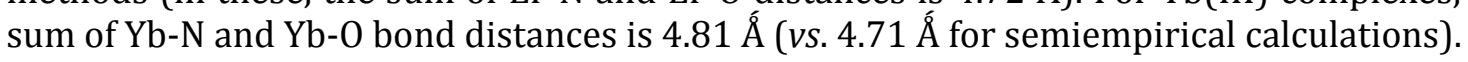

On the other hand, an excellent relationship $\left(r=-0.86 ; r^{2}=0.73\right)$ was found when the average bond distance Er-N is plotted against the bond angle N-Er-N:

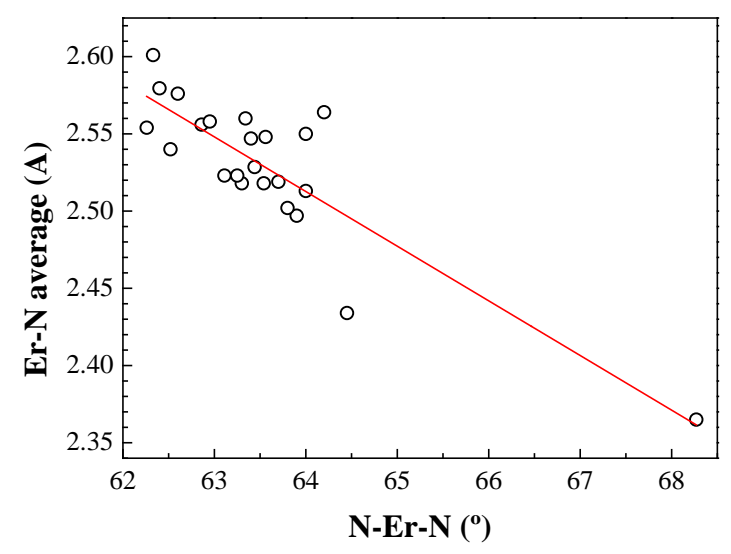

Figure 78. Er-N bond lengths vs. N-Er-N angles

With regard to $\mathrm{Yb}(\mathrm{III})$ complexes, the slight differences in average $\mathrm{Yb}-\mathrm{O}$ and $\mathrm{Yb}-\mathrm{N}$ bond distances in comparison to Er(III) ones (0.01-0.02 $\AA$ shorter for Yb(III)) can be explained by its smaller ionic radius. 
Table 48. Average Ln- $\mathrm{N}$ and Ln-O distances and N-Ln-N and O-Ln-O bond angles

\begin{tabular}{|c|c|c|c|c|}
\hline Complex & Ln-N average $(\AA ̊)$ & $\mathrm{N}-\operatorname{Ln}-\mathrm{N}(\underline{\mathrm{o}})$ & Ln-O average $(\AA ̊)$ & Ln-N + Ln-O (尺́) \\
\hline$[\text { Er(acac) })_{3}($ bath $\left.)\right]$ & 2.548 & 63.56 & 2.302 & 4.85 \\
\hline$\left[\operatorname{Er}(\mathrm{dbm})_{3}(\mathrm{bipy})\right]$ & 2.556 & 62.86 & 2.293 & 4.85 \\
\hline$\left[\operatorname{Er}(\mathrm{dnm})_{3}(\mathrm{bipy})\right]$ & 2.527 & 62.3 & 2.279 & 4.81 \\
\hline$\left[\operatorname{Er}(\mathrm{dnm})_{3}\left(5 \mathrm{NO}_{2}\right.\right.$ phen $\left.)\right]$ & 2.562 & 63.28 & 2.291 & 4.85 \\
\hline$\left[\operatorname{Er}(\mathrm{h})_{3}(\right.$ bipy $\left.)\right]$ & 2.558 & 62.95 & 2.298 & 4.86 \\
\hline$\left[\mathrm{Er}(\mathrm{h})_{2}(\mathrm{bipy})\left(\mathrm{NO}_{3}\right)\right]$ & 2.518 & 63.3 & 2.313 & 4.83 \\
\hline$\left[\operatorname{Er}(\mathrm{h})_{3}(\mathrm{bath})\right]$ & 2.564 & 64.2 & 2.296 & 4.86 \\
\hline$\left[\operatorname{Er}(\mathrm{h})_{3}\left(5 \mathrm{NO}_{2}\right.\right.$ phen $\left.)\right]$ & 2.580 & 62.4 & 2.294 & 4.87 \\
\hline$\left[\operatorname{Er}(\mathrm{hd})_{3}(\mathrm{bipy})\right]$ & 2.540 & 62.52 & 2.286 & 4.83 \\
\hline$\left[\mathrm{Er}(\mathrm{dmh})_{3}(\mathrm{bipy})\right]$ & 2.554 & 62.26 & 2.290 & 4.84 \\
\hline$\left[\mathrm{Er}(\mathrm{dmh})_{3}(\mathrm{bath})\right]$ & 2.560 & 63.34 & 2.279 & 4.84 \\
\hline$\left[\mathrm{Er}(\mathrm{dmh})_{3}\left(5 \mathrm{NO}_{2}\right.\right.$ phen $\left.)\right]$ & 2.547 & 63.4 & 2.274 & 4.82 \\
\hline$\left[\operatorname{Er}(\text { thd })_{3}(\right.$ bath $\left.)\right]$ & 2.576 & 62.6 & 2.267 & 4.84 \\
\hline$\left[\operatorname{Er}(\mathrm{od})_{3}(\mathrm{bath})\right]$ & 2.523 & 63.11 & 2.292 & 4.82 \\
\hline$\left[\operatorname{Er}(\mathrm{od})_{3}\left(5 \mathrm{NO}_{2}\right.\right.$ phen $\left.)\right]$ & 2.601 & 62.33 & 2.303 & 4.90 \\
\hline$\left[\operatorname{Er}(\mathrm{tfa})_{3}(\mathrm{bipy})\right]$ & 2.519 & 63.7 & 2.307 & 4.83 \\
\hline$\left[\mathrm{Er}(\mathrm{tfnb})_{3}(\mathrm{bipy})\right]$ & 2.497 & 63.9 & 2.291 & 4.79 \\
\hline$\left[\mathrm{Er}(\mathrm{tfac})_{3}(\mathrm{bipy})\right]$ & 2.365 & 68.27 & 2.387 & 4.75 \\
\hline$\left[\mathrm{Er}(\mathrm{tfac})_{3}(\mathrm{bath})\right]$ & 2.550 & 64.0 & 2.301 & 4.85 \\
\hline$\left[\operatorname{Er}(\mathrm{tfac})_{3}\left(5 \mathrm{NO}_{2}\right.\right.$ phen $\left.)\right]$ & 2.513 & 64.0 & 2.297 & 4.81 \\
\hline$\left[\operatorname{Er}(\operatorname{tpm})_{3}(\right.$ bipy)$]$ & 2.529 & 63.44 & 2.306 & 4.83 \\
\hline$\left[\operatorname{Er}(\operatorname{tpm})_{3}(\right.$ bath $\left.)\right]$ & 2.518 & 63.54 & 2.296 & 4.81 \\
\hline$\left[\operatorname{Er}(\operatorname{tpm})_{3}\left(5 \mathrm{NO}_{2}\right.\right.$ phen $\left.)\right]$ & 2.434 & 64.45 & 2.288 & 4.72 \\
\hline$\left[\operatorname{Er}(\text { fod })_{3}(\right.$ bipy $\left.)\right]$ & 2.523 & 63.25 & 2.292 & 4.81 \\
\hline$\left[\operatorname{Er}(\text { fod })_{3}\right.$ (bath) $]$ & 2.502 & 63.8 & 2.303 & 4.81 \\
\hline$\left[\mathrm{Yb}(\mathrm{tfac})_{3}\left(5 \mathrm{NO}_{2}\right.\right.$ phen $\left.)\right]$ & 2.533 & 63.8 & 2.279 & 4.81 \\
\hline$\left[\mathrm{Yb}(\mathrm{tpm})_{3}\left(5 \mathrm{NO}_{2}\right.\right.$ phen $\left.)\right]$ & 2.505 & 64.5 & 2.279 & 4.78 \\
\hline$\left[\mathrm{Yb}(\text { fhd })_{3}\left(5 \mathrm{NO}_{2}\right.\right.$ phen $\left.)\right]$ & 2.512 & 64.0 & 2.291 & 4.80 \\
\hline
\end{tabular}

Finally, by way of guidance, some Ln-N and Ln-O bond distances for other complexes reported in the literature are summarized in Table 49. All values are in the same ranges as those indicated above.

Table 49. Average Ln- $\mathrm{N}$ and Ln-O distances for analogous complexes found in the literature

\begin{tabular}{|c|c|c|c|}
\hline & Ln-N $(\AA ́)$ & Ln-O $(\AA ̊)$ & Reference \\
\hline$[\text { Er(acac) })_{3}($ phen $\left.)\right]$ & 2.546 & 2.304 & Neelgund, GM; et al. [161] \\
\hline$\left[\operatorname{Er}(\mathrm{pfnp})_{3}(\mathrm{phen})\right]$ & 2.520 & 2.299 & \multirow{2}{*}{ Feng, J; et al. [162] } \\
\hline$\left[\mathrm{Yb}(\mathrm{pfnp})_{3}(\mathrm{phen})\right]$ & 2.498 & 2.297 & \\
\hline$\left[\operatorname{Er}(\mathrm{fth})_{3}(\mathrm{phen})\right]$ & 2.520 & 2.296 & \multirow{2}{*}{ Sun, LN; et al. [163] } \\
\hline$\left[\mathrm{Yb}(\mathrm{fth})_{3}(\mathrm{phen})\right]$ & 2.501 & 2.276 & \\
\hline$\left[\operatorname{Er}(\mathrm{fpd})_{3}(\right.$ phen $\left.)\right]$ & 2.475 & 2.296 & Van Staveren, DR; et al. [164] \\
\hline$\left[\operatorname{Er}_{2}(\mathrm{pba})_{3}(\text { phen })_{2}\right]$ & 2.510 and 2.595 & 2.316 & Song, L; et al. [165] \\
\hline$[\text { Er(acac })_{3}($ dmobipy $\left.)\right]$ & 2.548 & 2.320 & Bellusci, A; et al. [166] \\
\hline
\end{tabular}

pfnp $=4,4,5,5,5$-pentafluoro-1-(2-naphthyl)-1,3-butanedione $\mathrm{fth}=4,4,5,5,6,6,6$-heptafluoro-1-(2-thienyl)hexane-1,3-dione fpd $=1,1,1,5,5,5$-hexafluoropentane-2,4-dionate pba $=4$-pentylbenzoate dmobipy = 4,4'-dimethoxy-2,2'-bipyridine 


\subsubsection{DSC study on the thermal stability}

DSC curves of the different complexes under study are shown in Figure 79. The main thermal effects are melting and boiling. Nevertheless, for the $\left[\operatorname{Er}(\mathrm{dmh})_{3}(\mathrm{~N}, \mathrm{~N}-\right.$ donor $\left.)\right]$, $\left[\operatorname{Er}(\mathrm{dbm})_{3}(\mathrm{bipy})\right]$ and $\left[\operatorname{Er}(\mathrm{tfac})_{3}(\mathrm{~N}, \mathrm{~N}\right.$-donor $\left.)\right]$ complexes, a partial decomposition takes place due to the lability of $\mathrm{Hdmh}, \mathrm{Hdbm}$ and $\mathrm{Htfac} \beta$-diketones. The final decomposition occurs in two steps: the loss of the $\beta$-diketonate ligand occurs first, followed by the loss of the $\mathrm{N}, \mathrm{N}$ donor ligand.

All thermal effects shown in the DSC curves of the ternary complexes are mainly due to the nature of the $\beta$-diketonate ligand. When the complexes are grouped by the shared common $\mathrm{N}, \mathrm{N}$-donor ligand (Table 50 ), it is possible to observe significant differences in temperature for the same thermal effects as a function of the $\beta$-diketonate ligands, depending on whether they are fluorinated or not, and on the different aliphatic chain lengths. Thus, it is evinced that fluorination diminishes the melting points: whereas complexes with non-fluorinated $\beta$ diketone ligands, such as $\left[\operatorname{Er}(\text { acac })_{3}(\right.$ bath $\left.)\right]$, melt at $250^{\circ} \mathrm{C}$ (the analogous $\left[\operatorname{Er}(\text { acac })_{3}(\right.$ phen)] complex melts at $229^{\circ} \mathrm{C}$ [167]), the ternary perfluorinated erbium $\beta$-diketonate complexes melt at temperature below $200^{\circ} \mathrm{C}$. In addition, we can also observe that ternary perfluoroalkyl- $\beta$-diketonate complexes with long alkyl chains have lower melting points than analogous complexes with short alkyl chains, in agreement with [13]: [Er(fod $)_{3}\left(5 \mathrm{NO}_{2}\right.$ phen $\left.)\right]$ octanedione complex melts at $94^{\circ} \mathrm{C}$, whereas $\left[\operatorname{Er}(\operatorname{tpm})_{3}\left(5 \mathrm{NO}_{2}\right.\right.$ phen $\left.)\right]$ hexanedione complex melts at $134^{\circ} \mathrm{C}$.

When a general comparison for ternary fluorinated $\beta$-diketonates is carried out on the basis of the bidentate nitrogen donor ligands, it should be noticed that the melting point temperatures decrease as follows: 2,2'-bipyridine > bathophenanthroline > 5-nitro-1,10phenanthroline, as it occurs for $\left[\operatorname{Er}(\operatorname{tpm})_{3}(\mathrm{~N}, \mathrm{~N}-\right.$ donor $\left.)\right]$ and $\left[\operatorname{Er}(\mathrm{dmh})_{3}(\mathrm{~N}, \mathrm{~N}-\right.$ donor $\left.)\right]$ families.

As important as the melting point is the width of the interval between the meeting point and the start of the ebullition. The large temperature interval for $\left[\operatorname{Er}(\operatorname{tpm})_{3}\left(5 \mathrm{NO}_{2}\right.\right.$ phen)] and $\left[\mathrm{Er}(\mathrm{fod})_{3}\left(5 \mathrm{NO}_{2}\right.\right.$ phen)] complexes (over $200^{\circ} \mathrm{C}$ ) facilitates engineering processing of these complexes by chemical vapor deposition (CVD).

In a comparison among the end decomposition points for [ $\operatorname{Er}(\text { acac) })_{3}\left(\right.$ bath)] (above $400^{\circ} \mathrm{C}$ ), [Er(acac) $)_{3}$ phen] (383을 [167] and [Er(acac) ${ }_{3}$ (bipy)] (235으) [168], it is interesting to observe that the temperature dependence is much stronger for Er(acac $)_{3}$ adducts with phen or bath than for those with bipy. This can be explained by the greater rigidity of bath or phen in comparison to bipy: bipy can rotate fragments around the C-C bond and, therefore, it can fill the voids in the $\operatorname{Er}(\mathrm{acac})_{3}$ structure in a more flexible manner than phen or bath.

The final product of the thermal transformations above $400^{\circ} \mathrm{C}$ is $\mathrm{Er}_{2} \mathrm{O}_{3}$ in all cases.

Table 50. Main DSC termal effects for selected new complexes

\begin{tabular}{|c|c|c|c|c|c|c|}
\hline & $\begin{array}{l}\text { Pre- } \\
\text { melting } \\
\text { (endo) }\end{array}$ & $\begin{array}{l}\text { Melting } \\
\text { point } \\
\text { (endo) }\end{array}$ & $\begin{array}{c}\text { Partial } \\
\text { decomposition } \\
\text { (exo) }\end{array}$ & $\begin{array}{l}\text { Pre- } \\
\text { boiling } \\
\text { (endo) }\end{array}$ & $\begin{array}{l}\text { Boiling } \\
\text { (endo) }\end{array}$ & $\begin{array}{l}\text { Decomposition } \\
\text { (exo) }\end{array}$ \\
\hline$\left[\mathrm{Er}(\mathrm{acac})_{3}(\mathrm{bath})\right]$ & & $250^{\circ} \mathrm{C}$ & & & $280^{\circ} \mathrm{C}$ & $>400^{\circ} \mathrm{C}$ \\
\hline$\left[\operatorname{Er}(\mathrm{dbm})_{3}(\mathrm{bipy})\right]$ & & $110^{\circ} \mathrm{C}$ & $157^{\circ} \mathrm{C}$ & & & $283^{\circ} \mathrm{C}$ \\
\hline$(\mathrm{tba})\left[\operatorname{Er}(\mathrm{dbm})_{4}\right]$ & $77^{\circ} \mathrm{C}$ & $154^{\circ} \mathrm{C}$ & $157^{\circ} \mathrm{C}$ & & & $356^{\circ} \mathrm{C}$ \\
\hline$\left[\operatorname{Er}(\mathrm{h})_{3}(\mathrm{bipy})\right]$ & $134^{\circ} \mathrm{C}$ & $165^{\circ} \mathrm{C}$ & $180^{\circ} \mathrm{C}$ & & & \\
\hline$\left[\operatorname{Er}(\mathrm{h})_{3}(\right.$ bath $\left.)\right]$ & & $165^{\circ} \mathrm{C}$ & $200,248^{\circ} \mathrm{C}$ & & & \\
\hline$\left[\operatorname{Er}(\mathrm{h})_{3}\left(5 \mathrm{NO}_{2}\right.\right.$ phen $\left.)\right]$ & & $165^{\circ} \mathrm{C}$ & $219^{\circ} \mathrm{C}$ & & & \\
\hline$\left[\operatorname{Er}(\mathrm{hd})_{3}(\right.$ bipy $\left.)\right]$ & $138^{\circ} \mathrm{C}$ & $150^{\circ} \mathrm{C}$ & & & & \\
\hline$\left[\operatorname{Er}(\mathrm{dmh})_{3}(\right.$ bipy $\left.)\right]$ & $\left(150^{\circ} \mathrm{C}\right)$ & $200^{\circ} \mathrm{C}$ & $212^{\circ} \mathrm{C}$ & & & \\
\hline$\left[\operatorname{Er}(\mathrm{dmh})_{3}(\right.$ bath $\left.)\right]$ & & $157^{\circ} \mathrm{C}$ & $204^{\circ} \mathrm{C}$ & & & \\
\hline$\left[\mathrm{Er}(\mathrm{dmh})_{3}\left(5 \mathrm{NO}_{2}\right.\right.$ phen $\left.)\right]$ & & $147^{\circ} \mathrm{C}$ & $229^{\circ} \mathrm{C}$ & & & \\
\hline
\end{tabular}


[Er(thd) $)_{3}$ (bath)]

[Er(tfa) 3 (bipy)]

[Er(tfnb) $)_{3}$ (bipy)]

[Er(tfac) $)_{3}$ (bipy)]

[Er(tfac) ${ }_{3}$ (bath)]

[ $\mathrm{Er}(\mathrm{tfac})_{3}\left(5 \mathrm{NO}_{2}\right.$ phen $\left.)\right]$

[Er(tpm) $)_{3}$ (bipy)]

[Er(tpm) $)_{3}$ (bath)]

$\left[\operatorname{Er}(\operatorname{tpm})_{3}\left(5 \mathrm{NO}_{2}\right.\right.$ phen $\left.)\right]$

[Er(fhd $)_{3}\left(5 \mathrm{NO}_{2}\right.$ phen $\left.)\right]$

$\left[\operatorname{Er}(\text { fod })_{3}\left(5 \mathrm{NO}_{2}\right.\right.$ phen $\left.)\right]$ $220^{\circ} \mathrm{C}$

$200^{\circ} \mathrm{C}$

$140^{\circ} \mathrm{C}$

$187^{\circ} \mathrm{C}$

$120^{\circ} \mathrm{C}$

$171^{\circ} \mathrm{C}$

$140^{\circ} \mathrm{C} \quad 208^{\circ} \mathrm{C}$

$165^{\circ} \mathrm{C}$

$134^{\circ} \mathrm{C}$

$106^{\circ} \mathrm{C}$

$94^{\circ} \mathrm{C}$ $>350^{\circ} \mathrm{C}$

$305,321,342^{\circ} \mathrm{C}$

$310^{\circ} \mathrm{C}$

$275^{\circ} \mathrm{C} \quad 335^{\circ} \mathrm{C}$

$333^{\circ} \mathrm{C}$

$334^{\circ} \mathrm{C}$

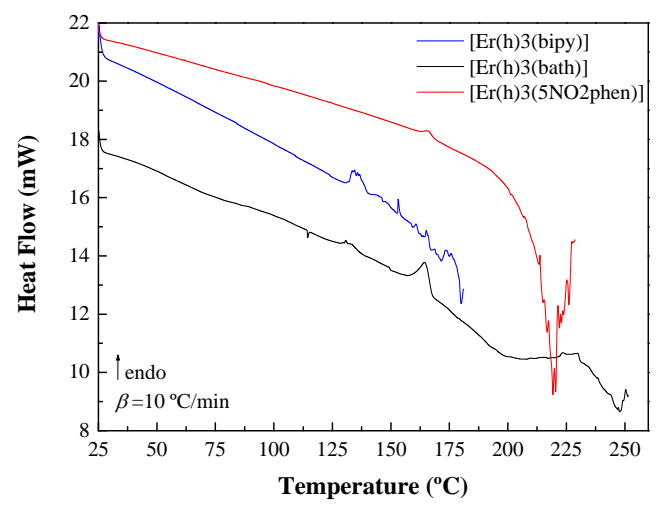

[Er(h) $3(\mathrm{~N}, \mathrm{~N}-$ donor $)]$

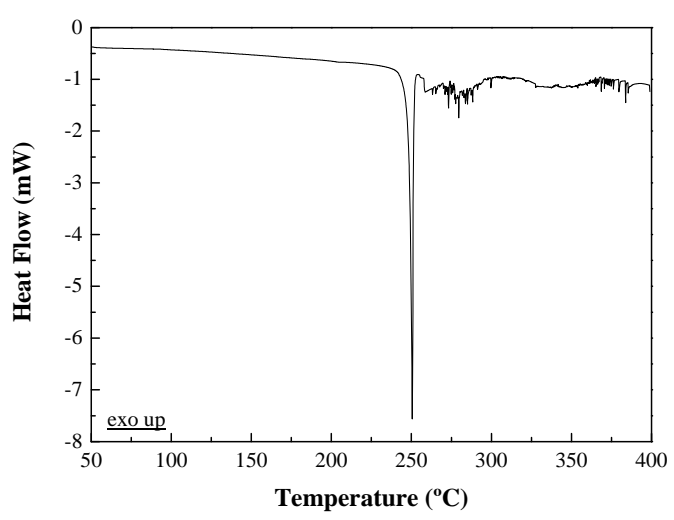

[Er(acac) 3 (bath)]

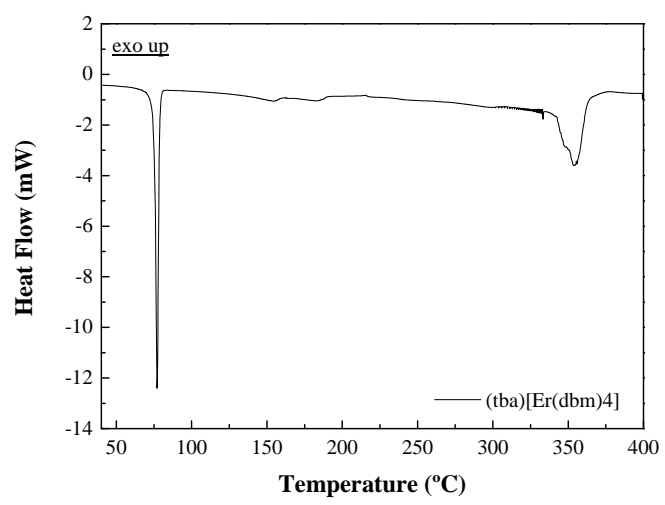

(tba) $\left[\operatorname{Er}(\mathrm{dbm})_{4}\right]$

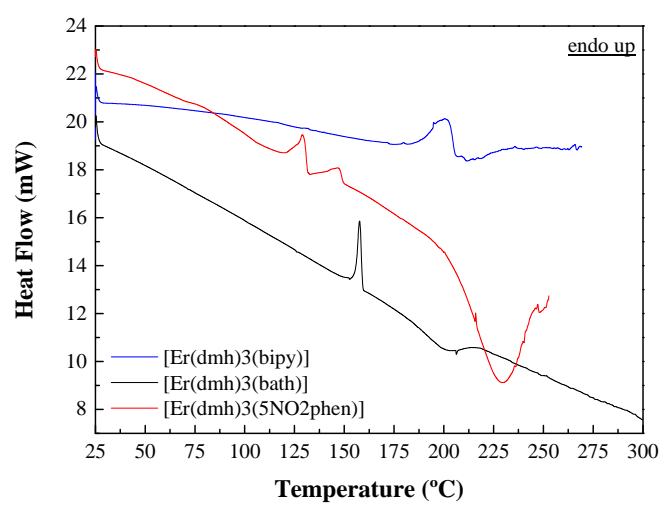

[Er(dmh) ${ }_{3}(\mathrm{~N}, \mathrm{~N}-$ donor $\left.)\right]$

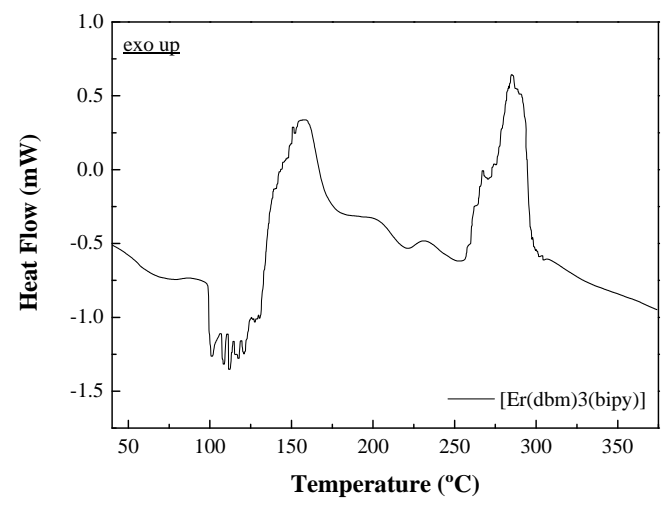

[Er(dbm) $)_{3}($ bipy)]

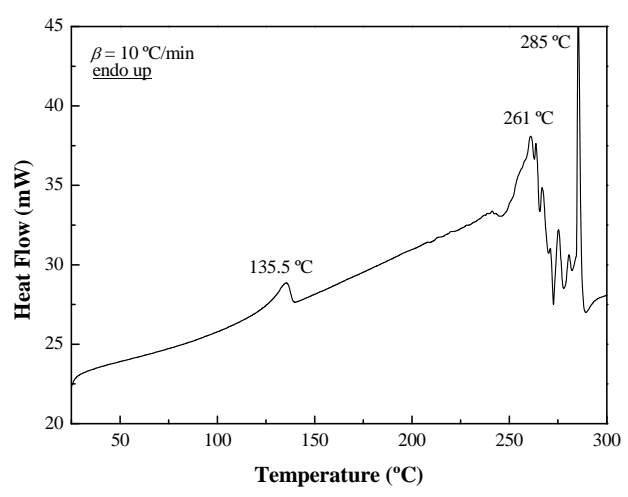

[Er(hd)3(bipy)] 


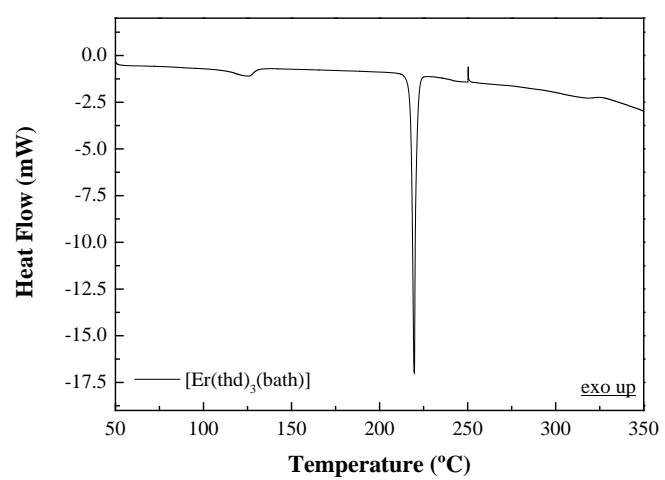

[Er(thd) $)_{3}$ (bath)]

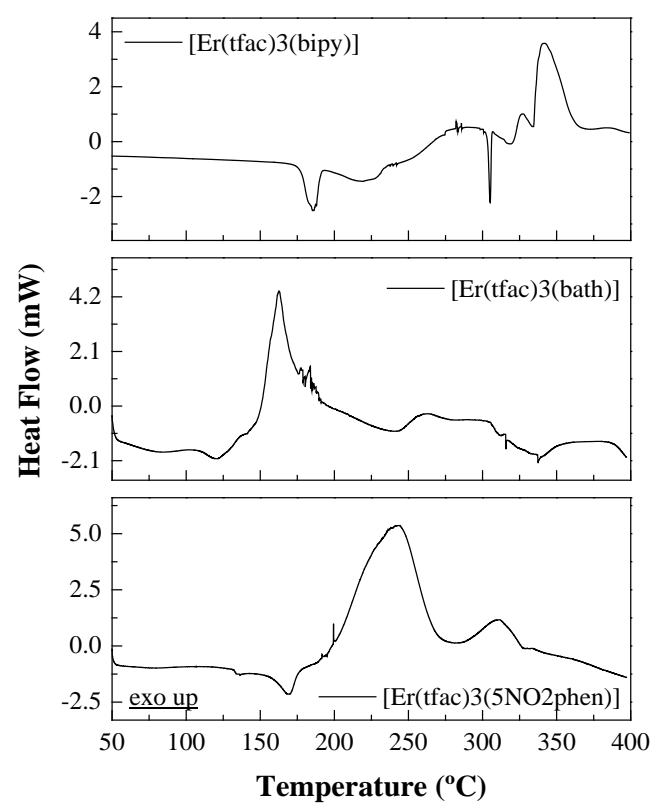

$\left[\operatorname{Er}(\mathrm{tfac})_{3}(\mathrm{~N}, \mathrm{~N}-\right.$ donor $\left.)\right]$

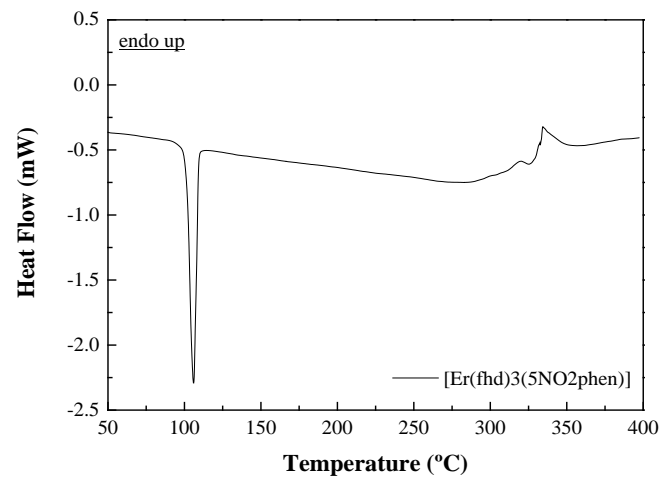

[Er(fhd $)_{3}\left(5 \mathrm{NO}_{2}\right.$ phen $\left.)\right]$

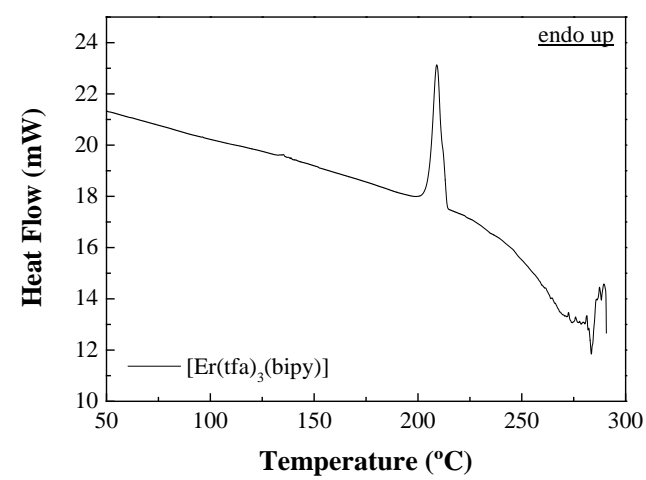

[Er(tfa) $)_{3}$ (bipy)]

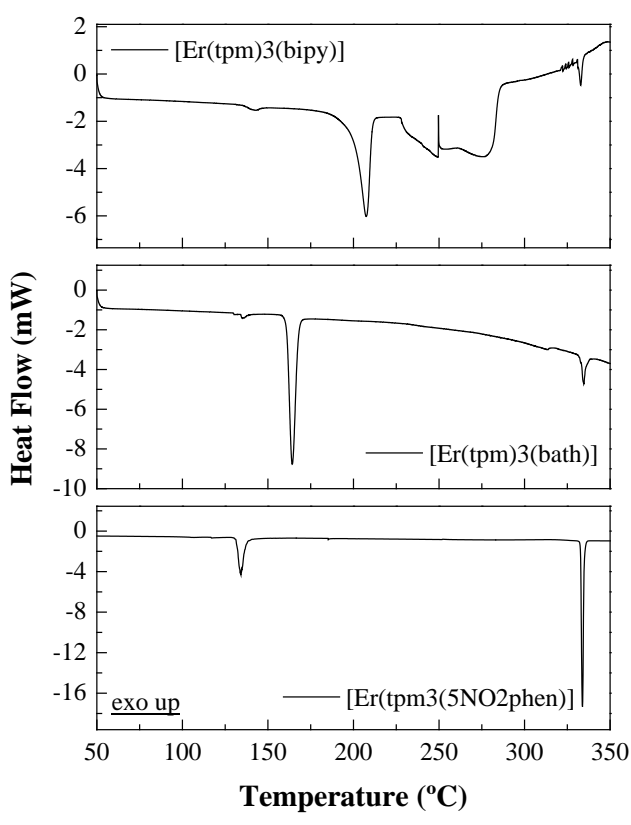

$\left[\operatorname{Er}(\mathrm{tpm})_{3}(\mathrm{~N}, \mathrm{~N}-\right.$ donor $\left.)\right]$

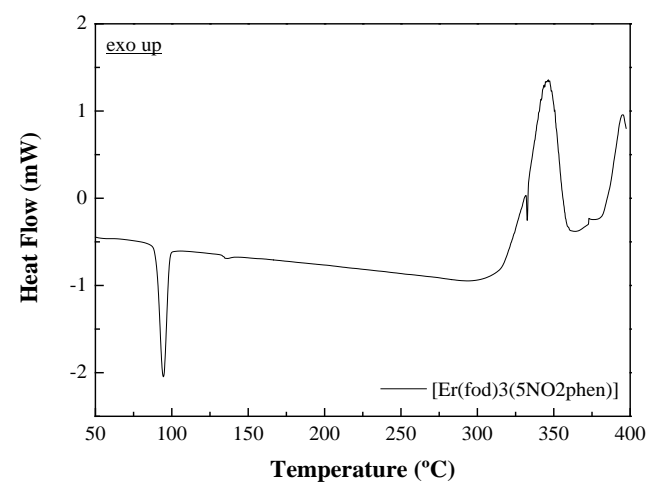

$\left[\operatorname{Er}(\text { fod })_{3}\left(5 \mathrm{NO}_{2}\right.\right.$ phen $\left.)\right]$

Figure 79. DSC curves 


\section{Applications of the results of DSC study}

Provided that the decomposition of the non-fluorinated erbium complexes does not occur below $250^{\circ} \mathrm{C}$ (actually, depending on their chemical composition, it may occur between $280^{\circ} \mathrm{C}$ and $330^{\circ} \mathrm{C}$ ), they can be heated up to that temperature without fearing for their stability.

This finding is useful in OLEDs manufacturing for adjusting the working temperature to the temperature of the components of other layers of the device, as it is the case, for example, for the PEDOT:PSS mixture, in order to achieve optimum performances. Thus, recent annealing studies on the PEDOT:PSS films [169], with temperatures ranging from $120^{\circ} \mathrm{C}$ to $400^{\circ} \mathrm{C}$, showed an optimum device performance, in particular regarding EQE and $V_{\text {oc }}$, at $250^{\circ} \mathrm{C}$. This optimum performance was found to be associated with loss of water from the PSS shell of the PEDOT:PSS grains. For annealing temperatures above $260^{\circ} \mathrm{C}$, device performance was dramatically reduced. This was associated with chemical decomposition leading to loss of sulfonic acid, although this did not significantly affect the in-plane conductivity.

The results on the decomposition temperature of the octacoordinated erbium complexes are also useful when it comes to EDFAs manufacturing because, interestingly, they coincide with the maximum decomposition temperature of mesoporous silica (Figure 80). The products of decomposition of the erbium complexes above $280^{\circ} \mathrm{C}$ are, in fact, erbium oxides or oxyfluorides and the annealing of mesoporous silica doped with erbium at these high temperatures leads to a solid solution of such erbium oxides or oxyfluorides in silica.

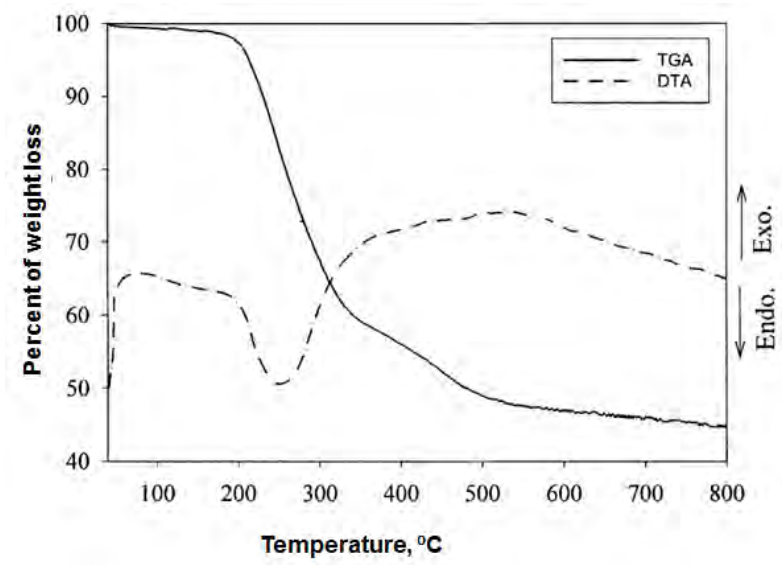

Figure 80. TGA and DTA (heating rate $10^{\circ} \mathrm{C} / \mathrm{min}$ ) of mesoporous silica fiber

\section{Assessment of the presumable lanthanidomesogen behavior of some of the complexes}

Lantanidemesogen complexes have been shown to offer an alternative to the luminescent liquid crystals that are obtained by doping luminescent non-mesomorphic lanthanide(III) complexes in a liquid-crystalline host matrix (further information for the interested reader is available in Section 4.6 in [113]).

Since some $\operatorname{Ln}(\mathrm{III})$ tris( $\beta$-diketonate) complexes have been reported to exhibit smectic mesomorphism [170], an assessment of this behavior has been conducted for some of the novel complexes, which exhibited similar consecutive endothermic peaks in their DSC curves (such as $\left[\operatorname{Er}(\mathrm{hd})_{3}(\mathrm{bipy})\right],\left[\operatorname{Er}(\mathrm{tfac})_{3}(\mathrm{bipy})\right]$ or $\left[\operatorname{Er}(\mathrm{dmh})_{3}\left(5 \mathrm{NO}_{2}\right.\right.$ phen)]). Upon heating the crystalline solid phase, it melts into the liquid crystal phase (first peak, melting point), and this mesophase then fully looses its ordering at a higher temperature. This is called the clearing point, at which the turbid liquid becomes clear and all order disappears.

Figure 81 (left) shows two DSC traces: one for a complex of a lanthanide from the beginning of the lanthanide series (Pr(III), bottom trace) and the other for a heavier lanthanide ion (so 
having a smaller ionic radius: Er(III), upper trace). The effect of the lanthanide contraction leads to closer endothermic peaks. This feature is also shown in the DSC curve of [Er(hd) ${ }_{3}$ (bipy)] (Figure 81 (right)): the first endothermic peak at $115^{\circ} \mathrm{C}$ corresponds to the melting point, and the second endothermic peak at $131^{\circ} \mathrm{C}$ is presumably associated to the clearing point.
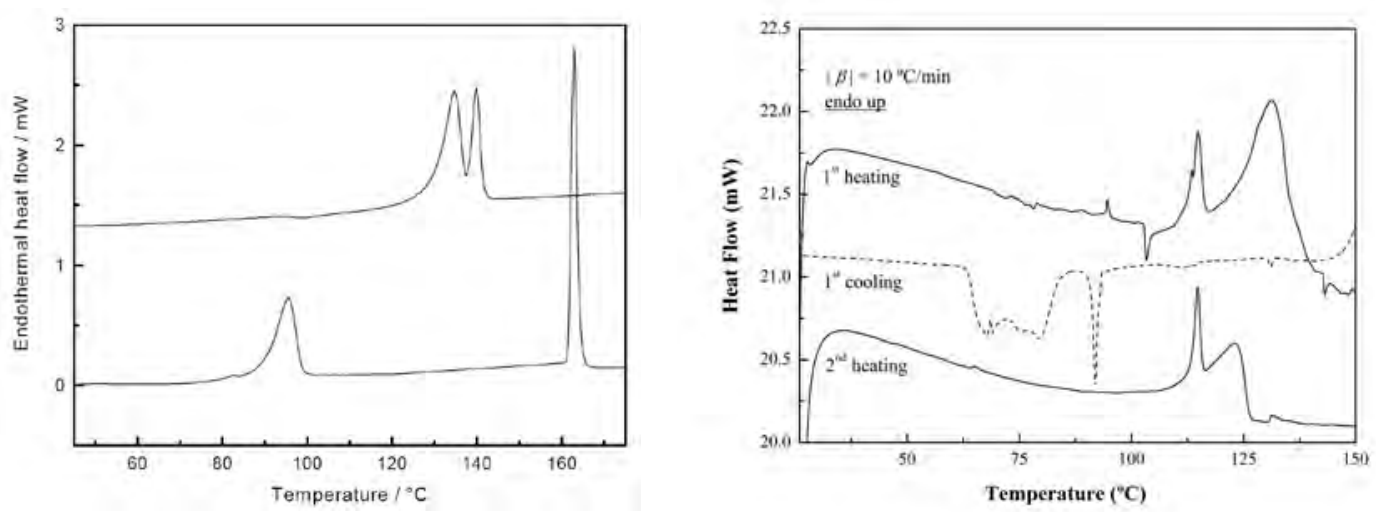

Figure 81. DSC curves of two lanthanidemesogen complexes according to R. van Deun's PhD Thesis

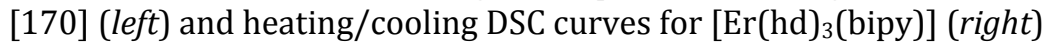

Nevertheless, a similar DSC behavior is not conclusive and complementary assays by hotstage microscopy are needed. Although lanthanidemesogen complexes are not liquidcrystalline and melt directly to give isotropic liquids, a monotropic mesophase can be observed upon cooling (i.e., on further cooling, the lanthanide(III) complexes do not crystallize but form a glass). This feature was found by Knyazev et al. [171] for $\left[\operatorname{Er}\left(\mathrm{C}_{12} \mathrm{C}_{16} \mathrm{dbm}\right)_{3}(\right.$ phen $\left.)\right]$ and $\left[\mathrm{Yb}\left(\mathrm{C}_{12} \mathrm{C}_{16} \mathrm{dbm}\right)_{3}(\right.$ phen $\left.)\right]$ complexes, for which the mesophase was identified as a smectic A phase by hot-stage polarizing optical microscopy. Upon cooling from the isotropic melt, typical bâtonnets coalesced to give rise to a focal conic-like texture in which large pseudo-isotropic domains occur due to homeotropic alignment of the molecules in these domains.

Figure 82 shows aforementioned thermomicroscopy study for [ $\operatorname{Er}(\mathrm{hd})_{3}($ bipy)]: a first quick melting step takes place around $113-115^{\circ} \mathrm{C}$, followed by a second incomplete melting at 127 $149^{\circ} \mathrm{C}$. No smetic phase was clearly observed. The close proximity of these two thermal effects minimizes the possible mesogenic behavior vs. those reported in the literature.

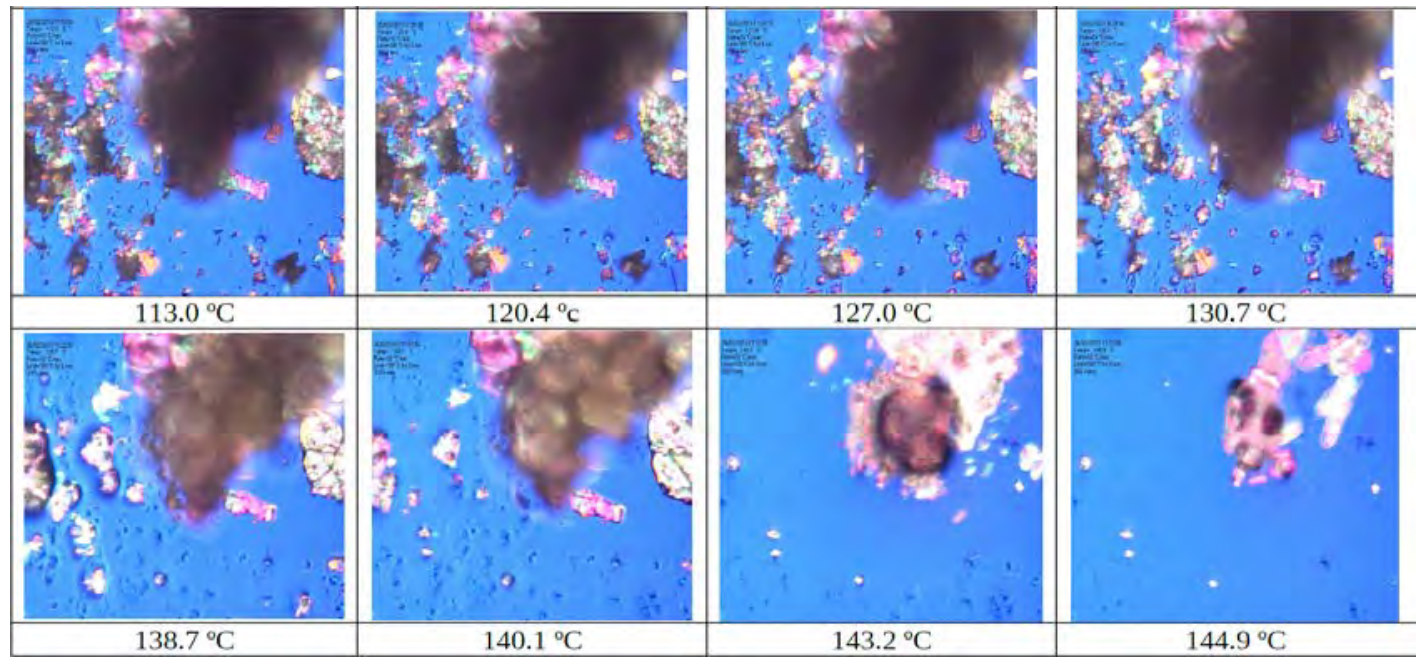

Figure 82. Thermomicroscopy study of [Er(h) $\left.{ }_{3}(\mathrm{bipy})\right]$ 


\section{On the special thermal behavior of dibenzoylmethanate complexes in methanol solution and their applications in chemical vapor deposition and sonochemistry}

Whereas most rare-earth complexes have a very low vapor pressure and it is difficult to transform them from the solid or liquid state into the vapor phase, the dibenzoylmethane complexes in solution can be sublimated without decomposition at temperatures under $100^{\circ} \mathrm{C}$ at atmospheric pressure. Combination of two properties, volatility and stability, makes these complexes a good choice for chemical deposition vapor processes in the microelectronic industry [172] and as new precursors for the sonochemical synthesis of nanosized materials [173].

\subsubsection{Infrared and Raman spectroscopies}

\section{IR spectra for erbium ternary complexes}

\section{Region below $900 \mathrm{~cm}^{-1}$ (low frequency region)}

IR spectra of the complexes in the $900-575 \mathrm{~cm}^{-1}$ region exhibit the absorption bands characteristic of N,N-donor molecules: 575, 629, 702, 742, 764 and $792 \mathrm{~cm}^{-1}$ for complexes containing bathophenanthroline ancillary ligand; 635, 737, 752 and $835 \mathrm{~cm}^{-1}$ for complexes with $5 \mathrm{NO}_{2}$ phen as the $\mathrm{N}, \mathrm{N}$-donor ligand; and 644,739 and $766 \mathrm{~cm}^{-1}$ for complexes containing bipyridyl.

Among these bands, the $\mathrm{CH}$ twisting bending vibrations at around 840 and $750 \mathrm{~cm}^{-1}$ (this later attributed to a ring breathing mode) are appreciably red shifted (vs. those of free ligands) due to the perturbation induced by their coordination to the metal ion.

Some bands under $640 \mathrm{~cm}^{-1}$ also offer evidence of the fact that coordination bonds have been formed between erbium and $\mathrm{N}, \mathrm{N}$-donor and erbium and $\beta$-diketonate, respectively, such as the one between 635 and $560 \mathrm{~cm}^{-1}$, assigned to a $v(E r-N)$ vibration (Table 52), and that which appears around $432 \mathrm{~cm}^{-1}$, which corresponds to $v($ Er-O) vibration.

\section{0-3500 $\mathrm{cm}^{-1}$ region (fingerprint and functional group regions)}

IR spectra of the complexes in this region also show the expected absorption bands for $\mathrm{N}, \mathrm{N}$ donor molecules and $\beta$-diketonate ligands. The absorption bands were identified in accordance with the literature [174].

The bands assigned to $\mathrm{C}=\mathrm{N}$ stretching mode and $\mathrm{C}=\mathrm{C}$ stretching mode (slightly coupled with the $\mathrm{CH}$ in-plane bending mode) of nitrogen donors molecules are observed in the 1542-1405 $\mathrm{cm}^{-1}$ region (in the ranges 1542-1494 and 1485-1405 $\mathrm{cm}^{-1}$, respectively). Those assigned to ring 'breathing' modes appear at 1039-1006 $\mathrm{cm}^{-1}$. All bands are red shifted in comparison with those of free ligands, suggesting the involvement of heteroaromatic nitrogens in the complex formation with the $\operatorname{Er}(\mathrm{III})$ ion $[174,175]$. Moreover, a shift can be also observed in the bands at around $1090 \mathrm{~cm}^{-1}: 1115 \mathrm{~cm}^{-1}$ for fluorinated complexes and below $1093 \mathrm{~cm}^{-1}$ for non-fluorinated ones.

Another good evidence for the complexation is obtained from the observation of the $\mathrm{C}=\mathrm{O}$ stretching band. The carbonyl stretching frequency of the keto form of the $\beta$-diketonate ligand should appear at $1728 \mathrm{~cm}^{-1}$, while that of the enol form should appear at $1630 \mathrm{~cm}^{-1}$. These bands are shifted to lower wavenumbers in the complexes $\left(\sim 1625\right.$ and $\left.\sim 1600 \mathrm{~cm}^{-1}\right)$ indicating the involvement of carbonyl oxygen in the complex formation with the $\operatorname{Er}($ III) ion. Complexes containing fluorinated $\beta$-diketonate ligands can be distinguished from nonfluorinated ones by a shift in the main absorption band: between 1630 and $1617 \mathrm{~cm}^{-1}$ for those which are fluorinated and between 1621 and $1599 \mathrm{~cm}^{-1}$ for those which are non- 
fluorinated $[176,118]$. In addition, the large band shown in free diketonate ligands becomes narrower in the complexes [177].

An important feature common to all fluorinated complexes is the occurrence of strong absorption bands from 1139 to $1105 \mathrm{~cm}^{-1}$, assigned to $\mathrm{CF}_{3}$ as (C-F) stretching modes [178].

Absorption bands at $2972-2851 \mathrm{~cm}^{-1}$ and around $3050 \mathrm{~cm}^{-1}$ are attributed to aliphatic C- $\mathrm{H}$ and aromatic C-H vibrations, respectively. Finally, the absorption in the region 3500-3410 $\mathrm{cm}^{-1}$, attributed to $\mathrm{OH}$ stretching vibrations, appears for non-fluorinated complexes (band at $3446 \mathrm{~cm}^{-1}$ ) but does not appear for most fluorinated complexes.

From the comparison of the spectra of complexes with the same $\beta$-diketonate ligand and different N,N-donors (for example, comparing $\left[\operatorname{Er}(\mathrm{h})_{3}(\mathrm{bath})\right]$ to $\left[\operatorname{Er}(\mathrm{h})_{3}(\mathrm{bipy})\right]$; $\left[\operatorname{Er}(\mathrm{dmh})_{3}(\right.$ bath $\left.)\right]$ to $\left[\operatorname{Er}(\mathrm{dmh})_{3}\right.$ (bipy)]; $\left[\operatorname{Er}(\text { fod })_{3}\left(5 \mathrm{NO}_{2}\right.\right.$ phen $\left.)\right]$ to $\left[\operatorname{Er}(\text { fod })_{3}(\right.$ bath $\left.)\right] ;$ or $\left[\operatorname{Er}(\operatorname{tpm})_{3}\left(5 \mathrm{NO}_{2}\right.\right.$ phen $\left.)\right]$ to $\left[\operatorname{Er}(\operatorname{tpm})_{3}(\right.$ bath $\left.\left.)\right]\right)$ a shift in the absorptions to higher wavenumbers, for most of the bands, can be seen when bath is replaced either by bipy or $5 \mathrm{NO}_{2}$ phen.

Finally, it should be noticed that there is a good correlation between the $v($ Er- $N)$ wavenumbers and the Er- $\mathrm{N}$ bond distances determined by single crystal X-ray diffraction $(r=0.824)$, as shown in Table 52 .

Table 51. Main IR bands for erbium ternary complexes $\left(\mathrm{cm}^{-1}\right)$

\begin{tabular}{|c|c|c|c|c|c|c|c|c|c|c|c|c|}
\hline \multirow{4}{*}[\operatorname{Er}(\mathrm{acac})_{3}(\mathrm{bath})]{} & 3441 & 2960 & 1620 & 1519 & 1464 & 1389 & 1261 & 1191 & 1018 & 919 & 764 & 657 \\
\hline & & & 1599 & & 1414 & 1358 & 1233 & 1091 & & 855 & 743 & 629 \\
\hline & & & & & & & & & & 834 & 703 & 575 \\
\hline & & & & & & & & & & & & 530 \\
\hline \multirow{3}{*}[\mathrm{Er}(\mathrm{dbm})_{3}(\mathrm{bipy})]{} & 3057 & 2958 & 1594 & 1516 & 1456 & 1394 & 1219 & 1173 & 1022 & 942 & 755 & 610 \\
\hline & 3028 & & 1553 & & 1419 & 1312 & & 1061 & 1012 & & 723 & 530 \\
\hline & & & & & & & & & & & 687 & 419 \\
\hline \multirow{4}{*}{$(\mathrm{tba})\left[\operatorname{Er}(\mathrm{dbm})_{4}\right]$} & & & 1591 & 1510 & 1453 & 1305 & 1226 & 1192 & 1022 & 998 & 749 & 606 \\
\hline & & & & 1483 & 1426 & & & 1102 & & 925 & 703 & \\
\hline & & & & & & & & 1057 & & 833 & 677 & \\
\hline & & & & & & & & & & 811 & & \\
\hline \multirow{4}{*}[\operatorname{Er}(\mathrm{h})_{3}(\text{bipy})]{} & 3411 & 2965 & 1597 & 1523 & 1465 & 1384 & 1259 & 1179 & 1015 & 884 & 779 & 644 \\
\hline & & 2932 & & 1497 & 1438 & 1359 & & 1157 & & & 762 & 627 \\
\hline & & & & & & 1300 & & & & & 740 & 536 \\
\hline & & & & & & & & & & & & 411 \\
\hline \multirow{4}{*}[\operatorname{Er}(\mathrm{h})_{3}(\text{bath})]{} & 3446 & 2963 & 1619 & 1519 & 1463 & 1355 & 1255 & 1171 & 1020 & 881 & 763 & 629 \\
\hline & & 2929 & 1595 & & 1419 & 1321 & 1233 & 1091 & & 854 & 742 & 575 \\
\hline & & & 1560 & & & & & 1067 & & 834 & 703 & 548 \\
\hline & & & & & & & & & & 807 & & \\
\hline \multirow{3}{*}[\operatorname{Er}(\mathrm{h})_{3}(5\mathrm{NO}_{2}\text{phen})]{} & 3446 & 2964 & 1596 & 1521 & 1464 & 1357 & 1257 & 1174 & 1019 & 835 & 752 & 635 \\
\hline & & 2930 & & & & & & & & & 737 & \\
\hline & & & & & & & & & & & 668 & \\
\hline $\multirow{3}{*}{\text { Er(hd) }}_{3}($ bipy)] & & 2968 & 1617 & 1516 & 1457 & 1384 & 1245 & 1173 & 1012 & 855 & 770 & 642 \\
\hline & & 2933 & 1598 & & & 1343 & & 1068 & & 807 & 757 & 623 \\
\hline & & 2872 & & & & 1307 & & & & & 739 & \\
\hline \multirow{3}{*}[\operatorname{Er}(\mathrm{dmh})_{3}(\mathrm{bipy})]{} & 3425 & 2963 & 1603 & 1532 & 1485 & 1384 & 1240 & 1176 & 1007 & 920 & 788 & 645 \\
\hline & & 2927 & 1587 & 1500 & 1439 & 1357 & & 1156 & & 816 & 769 & 627 \\
\hline & & 2867 & 1576 & & & 1314 & & & & & 739 & 431 \\
\hline \multirow{4}{*}[\operatorname{Er}(\mathrm{dmh})_{3}(\text{bath})]{} & 3424 & 2962 & 1621 & 1527 & 1464 & 1356 & 1236 & 1160 & & 921 & 793 & 629 \\
\hline & & 2926 & 1595 & 1501 & 1429 & 1313 & & 1091 & & 854 & 765 & 575 \\
\hline & & 2866 & & & & & & & & 835 & 742 & 432 \\
\hline & & & & & & & & & & & 703 & \\
\hline \multirow{3}{*}[\mathrm{Er}(\mathrm{dmh})_{3}(5\mathrm{NO}_{2}\text{phen})]{} & & 2965 & 1618 & 1592 & 1502 & 1348 & & 1162 & 1091 & 920 & 784 & 636 \\
\hline & & 2927 & & 1532 & 1457 & 1313 & & & & 835 & 755 & \\
\hline & & 2867 & & 1517 & & & & & & 814 & 735 & \\
\hline \multirow{2}{*}{$\operatorname{Er}(\text { thd })_{3}$ (bath)] } & 3440 & 2950 & 1611 & 1521 & 1454 & 1387 & 1226 & 1179 & & 868 & 764 & 629 \\
\hline & & 2863 & 1588 & 1505 & 1429 & 1357 & & 1142 & & 855 & 742 & 575 \\
\hline
\end{tabular}




\begin{tabular}{|c|c|c|c|c|c|c|c|c|c|c|c|c|}
\hline & & & 1576 & & & & & & & 834 & 701 & 477 \\
\hline $\multirow{3}{*}{\text { Er(od) }}_{3}($ bipy)] & 3432 & 2955 & 1617 & 1520 & 1460 & 1384 & 1262 & 1174 & 1013 & 925 & 762 & 645 \\
\hline & & 2928 & 1594 & & 1439 & 1356 & & & & & 739 & 535 \\
\hline & & 2870 & & & 1419 & 1316 & & & & & & \\
\hline \multirow{4}{*}[\operatorname{Er}(\mathrm{od})_{3}(\text{bath})]{} & & 2953 & 1617 & 1517 & 1457 & 1358 & 1263 & 1172 & 1085 & 910 & 761 & 628 \\
\hline & & 2926 & 1592 & & 1419 & & 1233 & & 1019 & 882 & 744 & 575 \\
\hline & & 2868 & 1560 & & & & & & & 852 & 701 & 538 \\
\hline & & & & & & & & & & 834 & & \\
\hline \multirow{3}{*}[\operatorname{Er}(\mathrm{od})_{3}(5\mathrm{NO}_{2}\text{phen})]{} & 3446 & 2956 & 1616 & 1520 & 1464 & 1384 & 1263 & 1174 & 1017 & 921 & 752 & 668 \\
\hline & & 2928 & 1593 & & 1424 & 1354 & & 1087 & & 835 & 737 & 635 \\
\hline & & 2870 & 1576 & & & & & & & & & 541 \\
\hline \multirow{3}{*}{ [Er(nd)3(bipy)] } & 3418 & 2955 & 1617 & 1518 & 1462 & 1384 & 1251 & 1172 & 1013 & 929 & 762 & 645 \\
\hline & & 2926 & 1593 & & 1439 & 1313 & & 1058 & & & 739 & 537 \\
\hline & & 2857 & 1576 & & 1419 & & & & & & & \\
\hline \multirow{4}{*}{ [Er(tfa)3(bipy)] } & 3446 & & 1620 & 1521 & 1459 & 1308 & 1228 & 1187 & 1015 & 948 & 777 & 673 \\
\hline & & & 1540 & & & & & 1140 & & 854 & 756 & 645 \\
\hline & & & & & & & & & & 801 & 738 & 615 \\
\hline & & & & & & & & & & & & 585 \\
\hline \multirow{2}{*}[\mathrm{Er}(\mathrm{tfnb})_{3}(\mathrm{bipy})]{} & & & 1613 & 1531 & & 1384 & & 1139 & 1016 & & 762 & 571 \\
\hline & & & & 1510 & & & & & & & & \\
\hline \multirow{3}{*}{ [Er(tfac) $\left.)_{3}(\mathrm{bipy})\right]$} & & & 1630 & 1529 & 1478 & 1384 & 1214 & 1189 & 1137 & 852 & 764 & 646 \\
\hline & & & 1605 & 1495 & 1441 & 1361 & & & 1015 & & 740 & 561 \\
\hline & & & 1579 & & & 1299 & & & & & 726 & \\
\hline \multirow{3}{*}[\operatorname{Er}(\mathrm{tfac})_{3}(\mathrm{bath})]{} & 3446 & 2965 & 1621 & 1532 & 1456 & 1384 & & & 1162 & 835 & 788 & 637 \\
\hline & & & 1594 & 1517 & & 1356 & & & 922 & 811 & 755 & 432 \\
\hline & & & & 1502 & & 1313 & & & & & 735 & \\
\hline \multirow{4}{*}[\mathrm{Er}(\mathrm{tfac})_{3}(5\mathrm{NO}_{2}\text{phen})]{} & & & 1630 & 1530 & 1485 & 1364 & 1225 & 1188 & 1142 & 854 & 780 & 610 \\
\hline & & & & & & 1300 & & & & 836 & 749 & 561 \\
\hline & & & & & & & & & & & 737 & \\
\hline & & & & & & & & & & & 726 & \\
\hline \multirow{3}{*}{$\operatorname{Er}(\operatorname{tpm})_{3}($ bipy$\left.)\right]$} & & 2669 & 1629 & 1542 & 1489 & 1395 & 1252 & 1186 & 1017 & 849 & 764 & 645 \\
\hline & & & 1604 & 1517 & 1474 & 1365 & & 1138 & & 796 & 740 & 627 \\
\hline & & & & & 1441 & 1302 & & & & & 692 & 574 \\
\hline \multirow{4}{*}{$\operatorname{Er}(\mathrm{tpm})_{3}($ bath)] } & & 2967 & 1622 & 1515 & 1475 & 1393 & 1252 & 1188 & & 850 & 766 & 690 \\
\hline & & & 1594 & 1494 & 1431 & 1364 & & 1158 & & 836 & 746 & 630 \\
\hline & & & 1558 & & & 1305 & & 1139 & & 795 & 702 & 596 \\
\hline & & & & & & & & & & & & 574 \\
\hline \multirow{3}{*}[\operatorname{Er}(\operatorname{tpm})_{3}(5\mathrm{NO}_{2}\text{phen})]{} & & 2971 & 1626 & 1542 & 1473 & 1394 & 1253 & 1188 & & 849 & 798 & 574 \\
\hline & & & & & 1428 & 1357 & & 1138 & & 836 & 749 & 522 \\
\hline & & & & & & 1304 & & 1115 & & & 737 & 465 \\
\hline \multirow{4}{*}[\mathrm{Er}(\mathrm{fhd})_{3}(5\mathrm{NO}_{2}\text{phen})]{} & & & & 1542 & 1498 & 1384 & 1232 & 1153 & & 965 & $\begin{array}{l}783 \\
\end{array}$ & 664 \\
\hline & & & & 1524 & 1472 & 1354 & & 1124 & & 931 & 749 & 625 \\
\hline & & & & & 1429 & 1320 & & 1103 & & 900 & 735 & 579 \\
\hline & & & & & & & & 1072 & & 837 & & 531 \\
\hline \multirow{4}{*}{ [Er(fod) $)_{3}($ bipy)] } & & 2974 & 1626 & 1535 & 1479 & 1393 & 1283 & 1156 & 1017 & 966 & 760 & 646 \\
\hline & & & 1580 & 1509 & 1441 & 1384 & 1223 & 1119 & & 940 & 739 & \\
\hline & & & & & & 1346 & & 1106 & & 830 & 687 & \\
\hline & & & & & & & & 1064 & & 790 & & \\
\hline & & 2969 & 1624 & 1509 & 1476 & 1394 & 1285 & 1157 & 1021 & 968 & 765 & 630 \\
\hline & & & 1582 & & 1431 & 1384 & 1228 & 1122 & & 941 & 755 & 576 \\
\hline$\left[\operatorname{Er}(\text { fod })_{3}(\right.$ bath $\left.)\right]$ & & & 1560 & & & 1346 & & 1106 & & 857 & 742 & 480 \\
\hline & & & & & & & & 1071 & & 835 & 702 & \\
\hline & & & & & & & & & & 791 & & \\
\hline & 3446 & 2971 & 1626 & 1537 & 1475 & 1394 & 1284 & 1151 & 1021 & 968 & 735 & 640 \\
\hline & & & 1588 & 1509 & 1429 & 1347 & 1230 & 1121 & & 941 & 695 & 473 \\
\hline$\left[\mathrm{Er}(\mathrm{fod})_{3}\left(5 \mathrm{NO}_{2}\right.\right.$ phen $\left.)\right]$ & & & & & & & & 1106 & & 836 & & \\
\hline & & & & & & & & 1069 & & 816 & & \\
\hline & & & & & & & & & & 793 & & \\
\hline
\end{tabular}


Table 52. Main peaks in the vibrational IR regions and Er-N bond lengths for the $\operatorname{Er}(I I I)$ complexes $\left(\mathrm{cm}^{-1}\right)$

\begin{tabular}{|c|c|c|}
\hline Complex & $v(\mathrm{Er}-\mathrm{N})$ & Er-N bond length \\
\hline$\left[\operatorname{Er}(\text { acac })_{3}(\right.$ bath $\left.)\right]$ & 629 & 2.548 \\
\hline$\left[\mathrm{Er}(\mathrm{dbm})_{3}(\mathrm{bipy})\right]$ & 610 & 2.556 \\
\hline$\left[\operatorname{Er}(\mathrm{h})_{3}(\mathrm{bipy})\right]$ & 644 & 2.558 \\
\hline$\left[\operatorname{Er}(\mathrm{h})_{3}(\mathrm{bath})\right]$ & 629 & 2.564 \\
\hline$\left[\operatorname{Er}(\mathrm{h})_{3}\left(5 \mathrm{NO}_{2}\right.\right.$ phen $\left.)\right]$ & 635 & 2.58 \\
\hline$\left[\operatorname{Er}(\mathrm{hd})_{3}(\mathrm{bipy})\right]$ & 623 & 2.54 \\
\hline$\left[\operatorname{Er}(\mathrm{dmh})_{3}(\mathrm{bipy})\right]$ & 642 & 2.554 \\
\hline$\left[\operatorname{Er}(\mathrm{dmh})_{3}(\right.$ bath $\left.)\right]$ & 629 & 2.56 \\
\hline$\left[\mathrm{Er}(\mathrm{dmh})_{3}\left(5 \mathrm{NO}_{2} \mathrm{phen}\right)\right]$ & 636 & 2.547 \\
\hline$\left[\operatorname{Er}(\text { thd })_{3}(\right.$ bath $\left.)\right]$ & 629 & 2.576 \\
\hline$\left[\operatorname{Er}(\mathrm{od})_{3}(\right.$ bath $\left.)\right]$ & 628 & 2.523 \\
\hline$\left[\mathrm{Er}(\mathrm{od})_{3}\left(5 \mathrm{NO}_{2}\right.\right.$ phen $\left.)\right]$ & 668 & 2.601 \\
\hline$\left[\operatorname{Er}(\mathrm{tfa})_{3}(\right.$ bipy $\left.)\right]$ & 615 & 2.519 \\
\hline$\left[\operatorname{Er}(\mathrm{tfnb})_{3}(\mathrm{bipy})\right]$ & 571 & 2.497 \\
\hline$\left[\operatorname{Er}(\operatorname{tfac})_{3}(\mathrm{bipy})\right]$ & 561 & 2.365 \\
\hline$\left[\operatorname{Er}(\operatorname{tfac})_{3}(\right.$ bath $\left.)\right]$ & 637 & 2.55 \\
\hline$\left[\mathrm{Er}(\mathrm{tfac})_{3}\left(5 \mathrm{NO}_{2}\right.\right.$ phen $\left.)\right]$ & 610 & 2.513 \\
\hline$\left[\operatorname{Er}(\operatorname{tpm})_{3}(\right.$ bipy $\left.)\right]$ & 627 & 2.529 \\
\hline$\left[\operatorname{Er}(\operatorname{tpm})_{3}(\right.$ bath $\left.)\right]$ & 596 & 2.518 \\
\hline$\left[\mathrm{Er}(\mathrm{tpm})_{3}\left(5 \mathrm{NO}_{2}\right.\right.$ phen $\left.)\right]$ & 522 & 2.434 \\
\hline$\left[\operatorname{Er}(\text { fod })_{3}(\right.$ bipy $\left.)\right]$ & 646 & 2.523 \\
\hline$\left[\operatorname{Er}(\text { fod })_{3}\right.$ (bath) $]$ & 576 & 2.502 \\
\hline
\end{tabular}

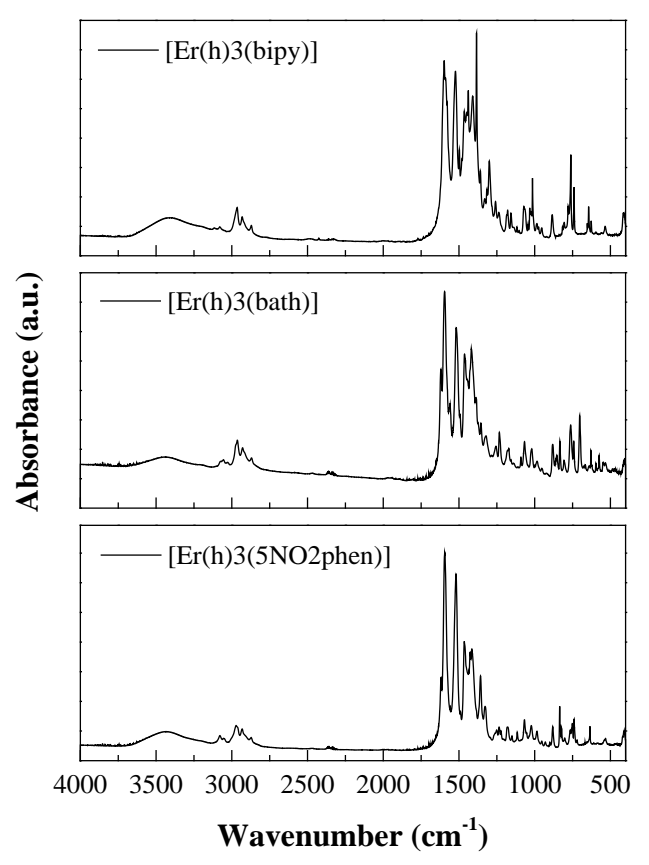

[Er(h) $)_{3}(\mathrm{~N}, \mathrm{~N}-$ donor $\left.)\right]$

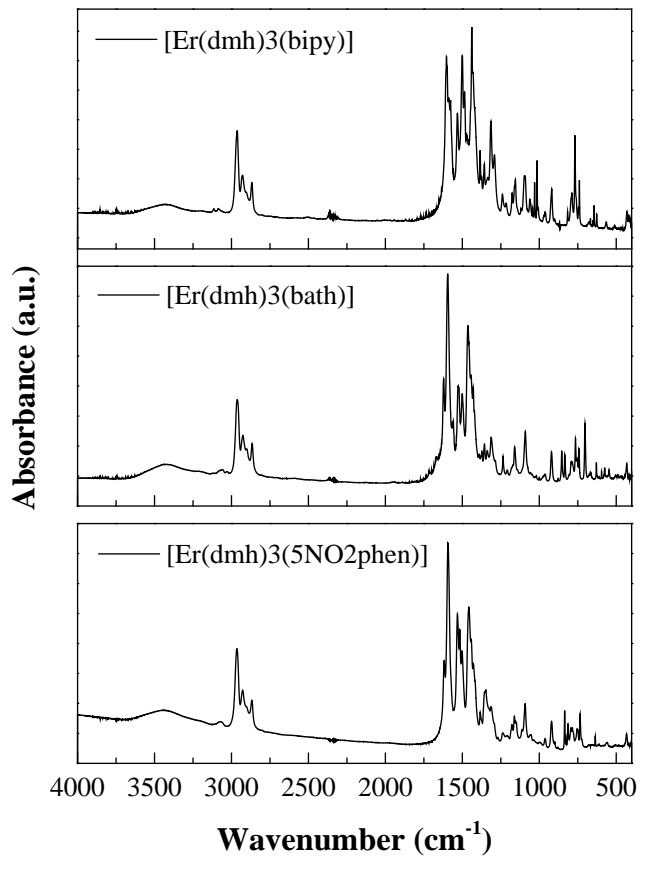

[Er(dmh) $)_{3}(\mathrm{~N}, \mathrm{~N}-$ donor $\left.)\right]$ 

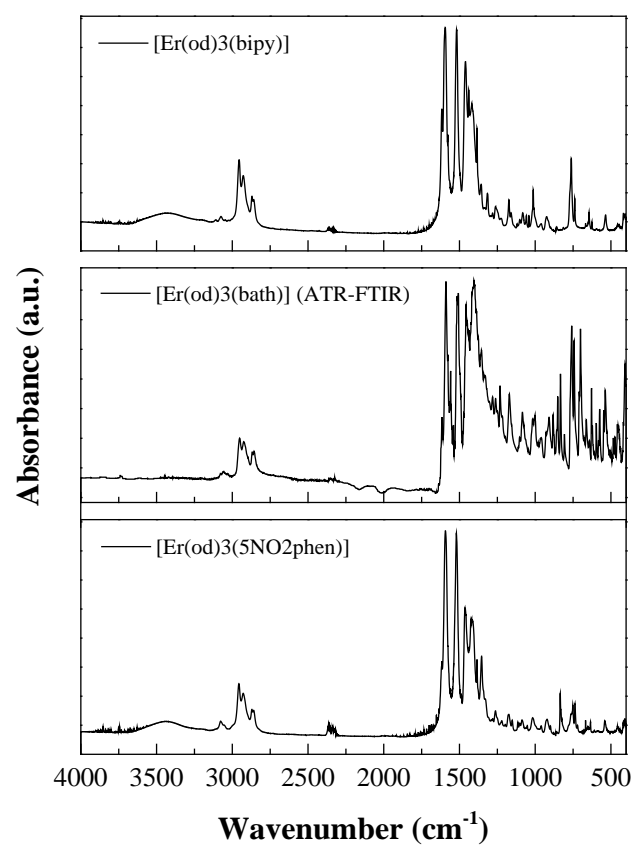

[Er(od) $)_{3}(\mathrm{~N}, \mathrm{~N}-$ donor $\left.)\right]$

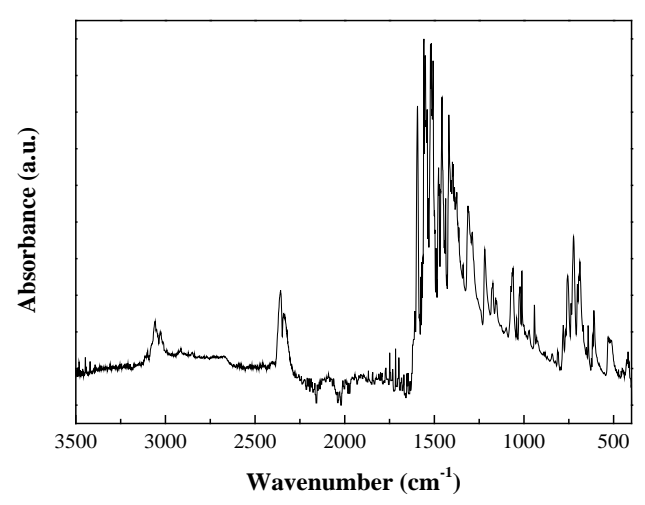

[Er(dbm) ${ }_{3}($ bipy)]

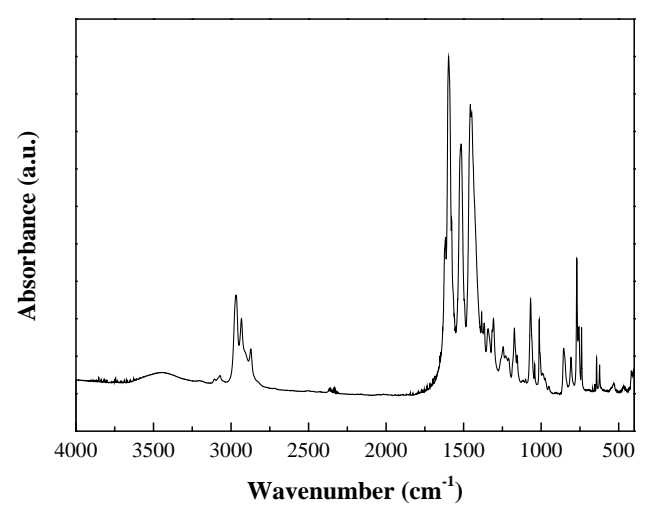

[Er(hd) ${ }_{3}$ (bipy)]

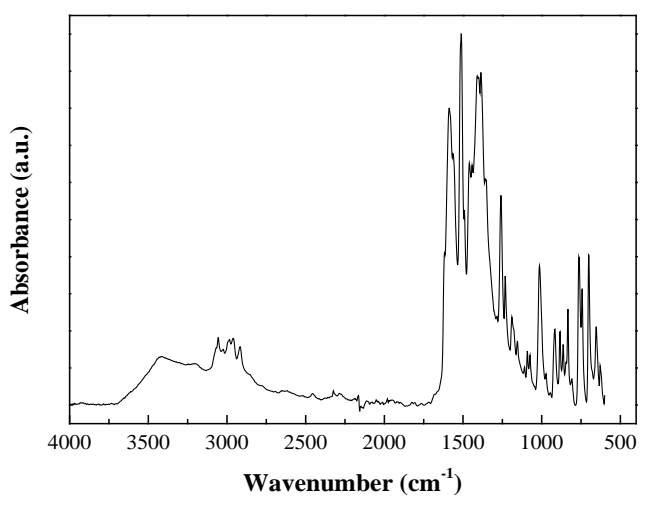

[Er(acac)3(bath)]

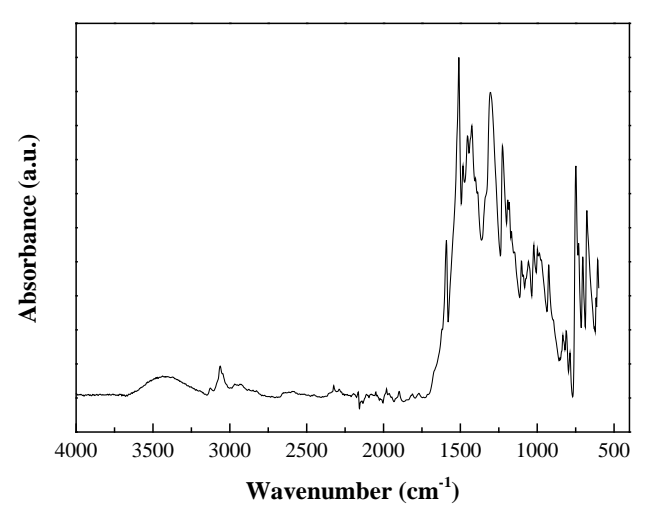

(tba) $\left[\operatorname{Er}(\mathrm{dbm})_{4}\right]$

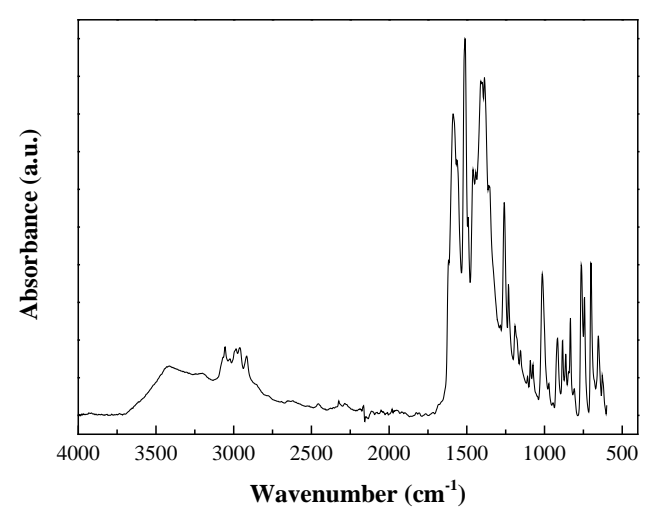

[Er(thd) ${ }_{3}$ (bath)] 


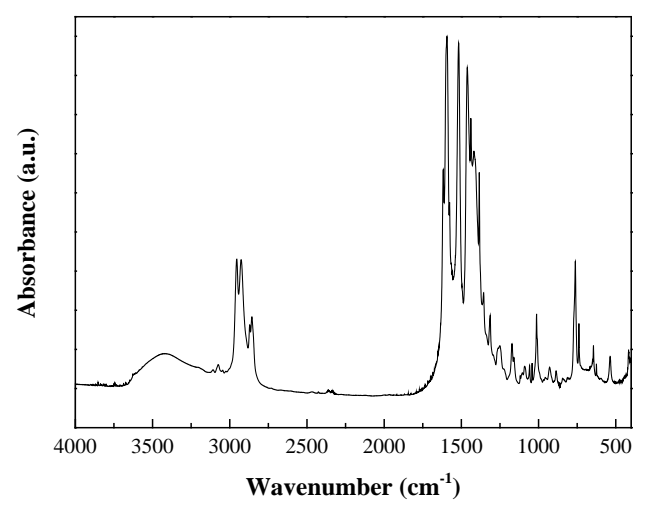

[Er(nd) $)_{3}$ (bipy)]

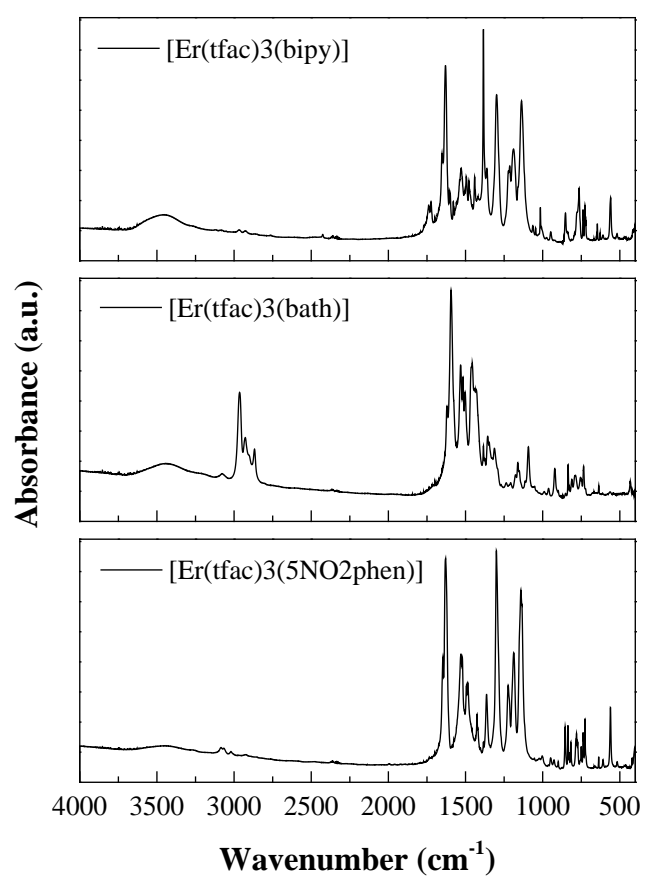

[Er(tfac) $3(\mathrm{~N}, \mathrm{~N}-$ donor $)]$

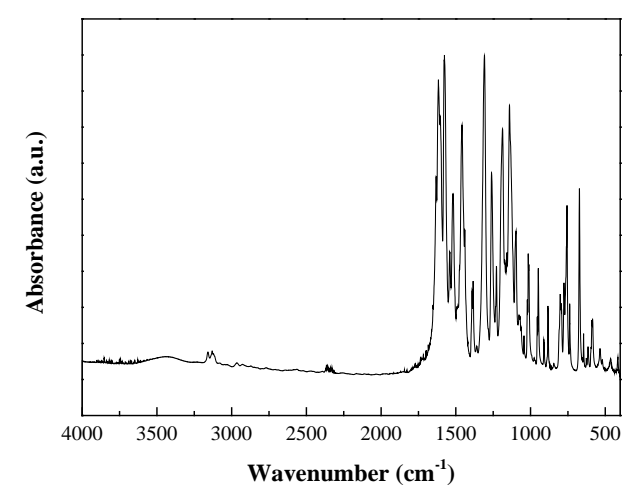

[Er(tfa)3(bipy)]

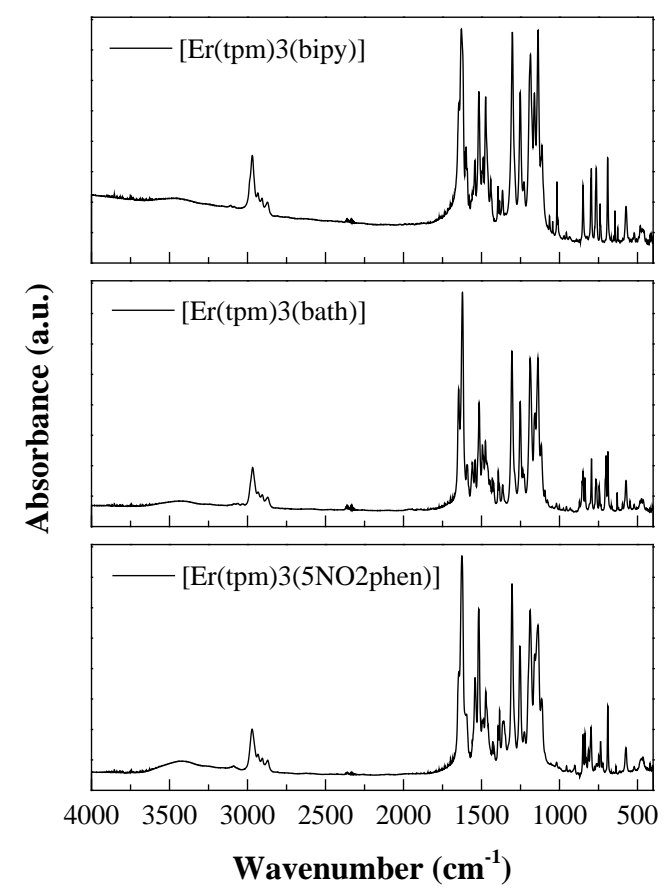

$\left[\operatorname{Er}(\operatorname{tpm})_{3}(\mathrm{~N}, \mathrm{~N}-\right.$ donor $\left.)\right]$

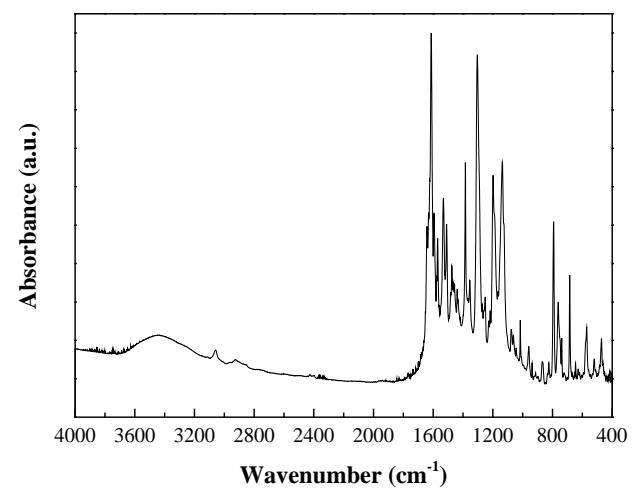

[Er(tfnb) $)_{3}$ (bipy)] 


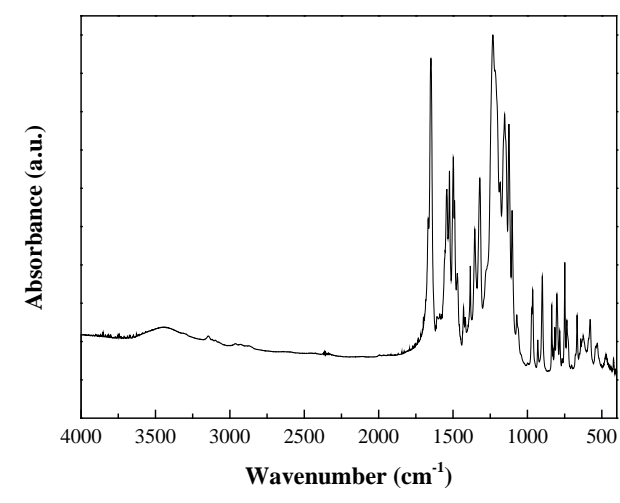

[Er(fhd $)_{3}\left(5 \mathrm{NO}_{2}\right.$ phen $\left.)\right]$

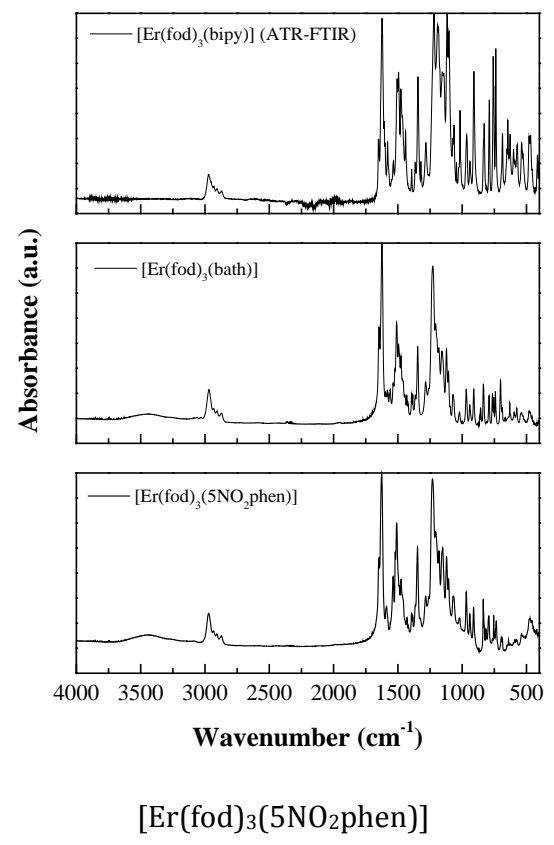

[Er(fod) $)_{3}\left(5 \mathrm{NO}_{2}\right.$ phen $\left.)\right]$

Figure 83. FTIR spectra of $\operatorname{Er}($ III) complexes

\section{IR spectra for selected Yb(III) complexes with 5-nitro-1,10-phenanthroline}

ATR-FTIR spectrum of the complexes offers evidence for the coordination bonds formation between $\mathrm{Yb}^{3+}$ and $5 \mathrm{NO}_{2}$ phen, and $\mathrm{Yb}^{3+}$ and $\beta$-diketonates, respectively.

The bands assigned to $5 \mathrm{NO}_{2}$ phen $\mathrm{CN}$ and $\mathrm{CC}$ ring stretching modes can be observed in the $1540-1500 \mathrm{~cm}^{-1}$ range and in the $1030-1000 \mathrm{~cm}^{-1}$ range, respectively [179]. They are shifted in comparison with those of the free ancillary ligand, suggesting that $5 \mathrm{NO}_{2}$ phen is in fact coordinated to ytterbium(III) $[179,174]$.

Another good evidence for the complexation is obtained by observation of the $\mathrm{C}=0$ stretching band: it is a large band in the free $\beta$-diketonate ligand (enolic and diketone forms are possible) at $1600 \mathrm{~cm}^{-1}$ and becomes narrower in the complexes (just diketone form is expected) at $1639-1578 \mathrm{~cm}^{-1}$ [175].

An important feature for the fluorinated complexes is the occurrence of strong carbonfluorine bands in the 1000 to $1300 \mathrm{~cm}^{-1}$ range, mainly the one which appears between 1125 and $1140 \mathrm{~cm}^{-1}$, assigned to $\mathrm{CF}_{3}$ as (C-F) stretching modes [177].

Analysis of the region below $1100 \mathrm{~cm}^{-1}$ allows us to see the absorption bands characteristic of coordinated 5-nitro-1,10-phenanthroline at 733-760 and 638-645 $\mathrm{cm}^{-1}$. The band that appears between 583 and $540 \mathrm{~cm}^{-1}$, which should assigned to a $v(\mathrm{Yb}-\mathrm{N})$ vibration, also offers evidence of the fact that the coordination bonds have been formed between $\mathrm{Yb}^{3+}$ and $5 \mathrm{NO}_{2}$ phen [178].

The bands between 2990 and $2350 \mathrm{~cm}^{-1}$ can be assigned to aliphatic $\mathrm{C}-\mathrm{H}$ and aromatic $\mathrm{C}-\mathrm{H}$, respectively.

Finally, the absence of absorption in the $3500-3000 \mathrm{~cm}^{-1}$ region, attributed to $\mathrm{OH}$ stretching vibrations, is the expected for a low amount of $\mathrm{OH}$ groups, as planned in the preparative conditions. 
Table 53. Main ATR-FTIR peaks for $\left[\mathrm{Yb}(\beta \text {-diketonate })_{3}\left(5 \mathrm{NO}_{2}\right.\right.$ phen $\left.)\right]$ complexes

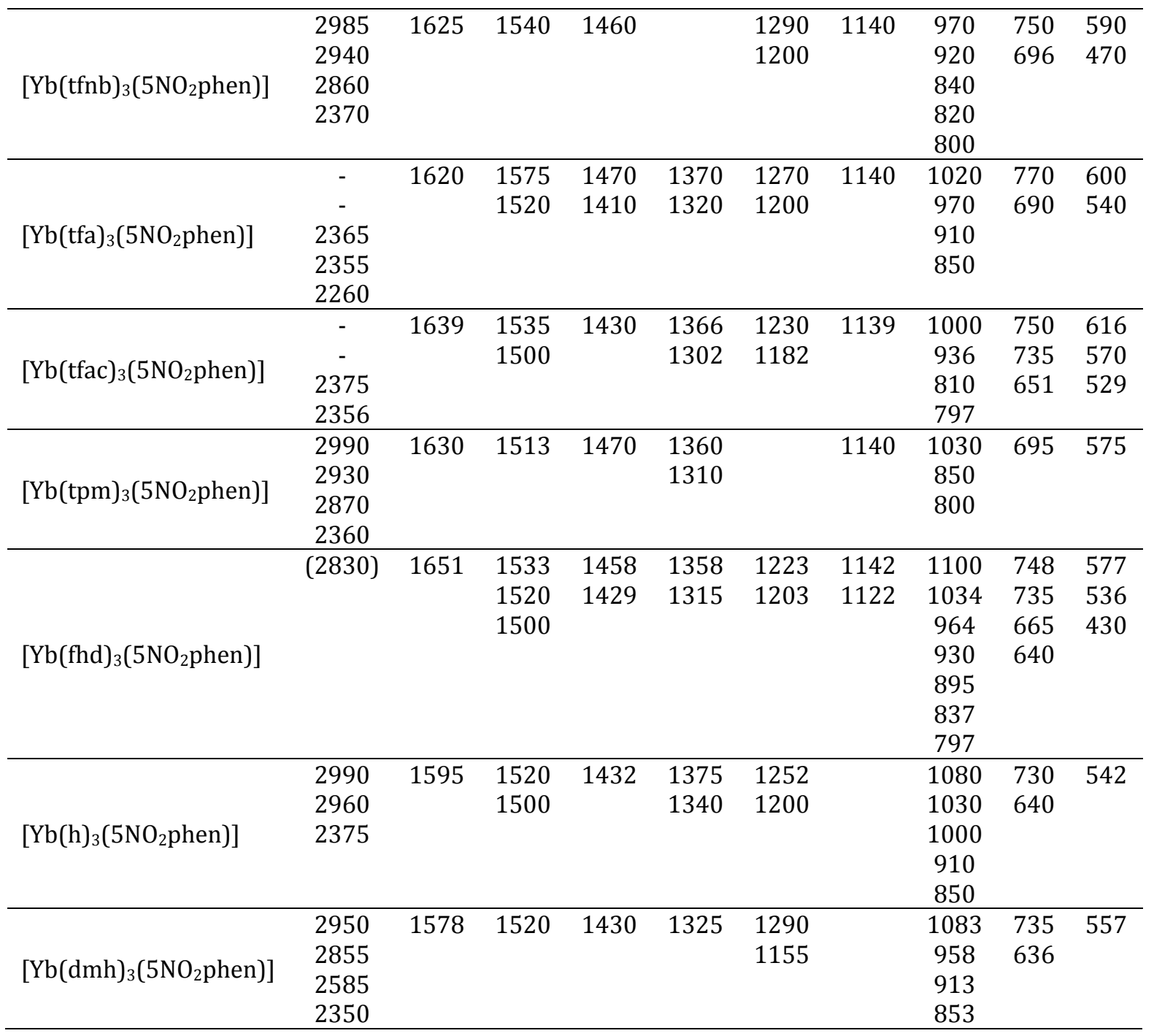

\section{Simulated vs. experimental infrared spectra}

In general terms, the simulated infrared spectra -although less resolved- exhibit a morphology similar to that of those obtained experimentally by ATR-FTIR (Figure 84) in all the fingerprint region (1800-400 $\mathrm{cm}^{-1}$ ), supporting the good accuracy of the semi-empirical quantum chemical methods used to predict the ground state geometries of the two complexes.

Theoretical calculations of the vibrational frequencies tend to deviate from experimental ones in a systematic way and, even after scale factor corrections have been applied [136], frequency shifts in the $1000-400 \mathrm{~cm}^{-1}$ region can still be detected. Nonetheless, it should be noted that some of these differences may in fact be related to the use of the ATR technique in the experimental spectra, which introduces distortion of the relative intensities of bands and absolute shifts in frequency [180], not to the simulations. 

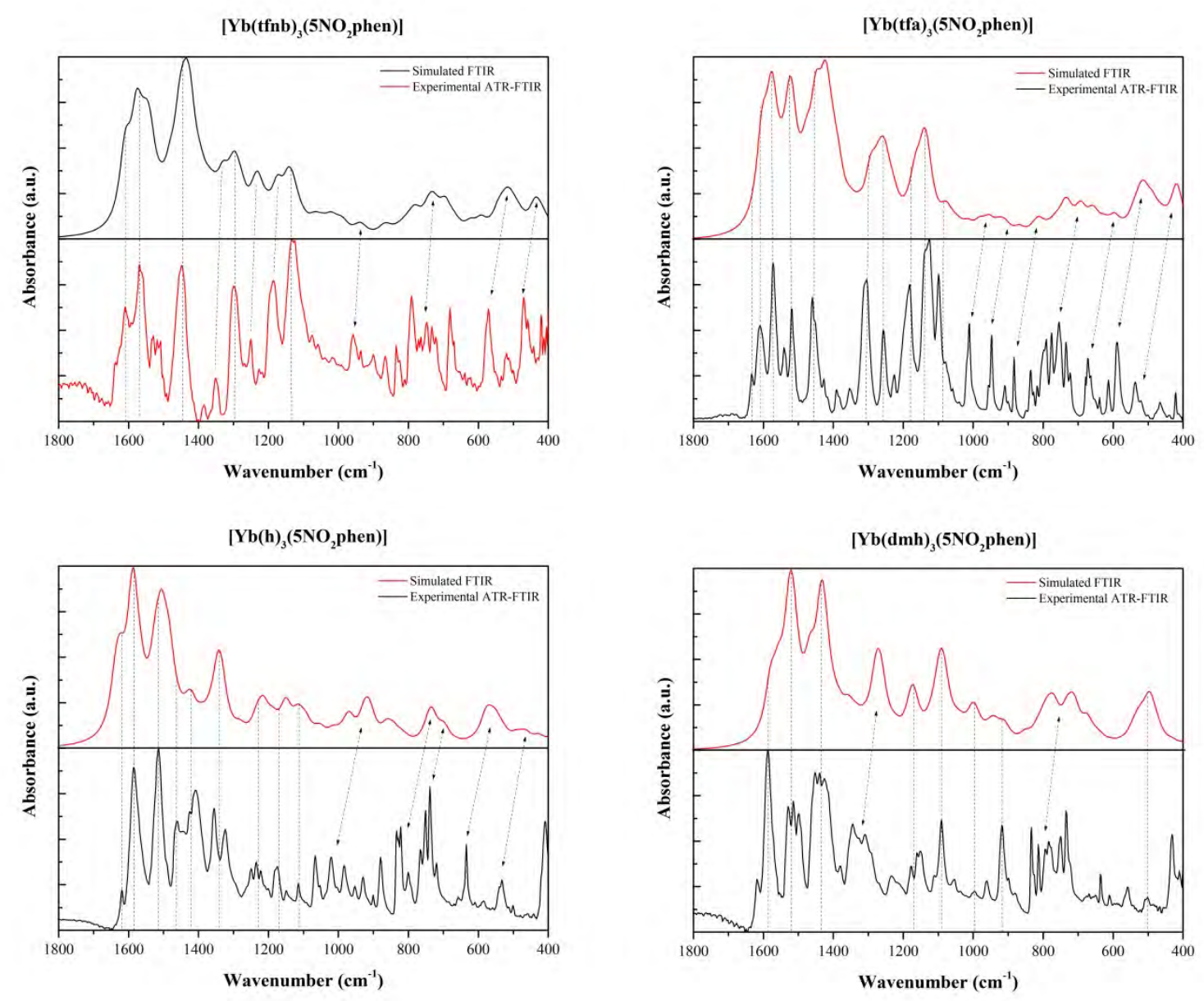

Figure 84. Comparison of simulated FTIR spectra with experimental ATR-FTIR spectra for $[\mathrm{Yb}(\beta$ diketonate $)_{3}\left(5 \mathrm{NO}_{2}\right.$ phen $\left.)\right]$ complexes

\section{IR spectra for other $\mathrm{Yb}(\mathrm{III})$ complexes}

The wealth of functional groups in Vildagliptin (mainly, hydroxy-1-adamantyl and pyrrolidine) and their high representation in the complex determined that most of the bands of the spectrum can be assigned to this ligand. However, there is also evidence of coordination of the 2,2'-bipyridine ancillary ligand and, in the case of $\left[\mathrm{Yb}(\mathrm{vilda})_{2}\right.$ (acac)(bipy)] complex, of the formation of $\mathrm{Yb}^{3+}$-acetylacetonate coordination bonds (Table 54).

Thus, the bands observed between $1465-1450 \mathrm{~cm}^{-1}$ and $1060-1045 \mathrm{~cm}^{-1}$ are assigned, respectively, to ring stretching modes $\mathrm{CN}$ and CC from 2,2'-bipyridine [174]. They are shifted in comparison with those of the free ligand, suggesting that 2,2'-bipyridine is in fact coordinated to Ytterbium(III) [174,175]. Analysis of the region below $1100 \mathrm{~cm}^{-1}$ also allows us to see the absorption bands characteristic of coordinated 2,2'-bipyridine at $762 \mathrm{~cm}^{-1}(\mathrm{C}-\mathrm{H}$ out of plane bending mode) and at $657 \mathrm{~cm}^{-1}$ (ring deformation mode). The bands that appear between 584 and $535 \mathrm{~cm}^{-1}$, which should assigned to a $v(\mathrm{Yb}-\mathrm{N})$ vibration, offer additional evidence of the fact that the coordination bonds have been formed between $\mathrm{Yb}^{3+}$ and bipy [179].

Other bands of interest in the fingerprint region are: $1653 \mathrm{~cm}^{-1}$ (either a Vildagliptin $\mathrm{C}=0$ (enol form) stretching mode or a $\mathrm{C}=\mathrm{N}$ stretching mode); $1620 \mathrm{~cm}^{-1}$ (acetylacetonate $\mathrm{C}=0$ (enol form) stretching mode); $1407-1404 \mathrm{~cm}^{-1}\left(\mathrm{CH}_{3}\right.$ asymmetric deformation mode); 1353$1348 \mathrm{~cm}^{-1}$ (C=0 stretching mode); $1258-1256 \mathrm{~cm}^{-1}$ (C-O stretching mode); $1148 \mathrm{~cm}^{-1}$ ( $\mathrm{CH}$ in 
plane bending mode; $1012-970 \mathrm{~cm}^{-1}\left(\mathrm{C}-\mathrm{H}\right.$ rocking mode); and $915-913 \mathrm{~cm}^{-1}\left(\mathrm{C}^{-} \mathrm{CH}_{3}\right.$ stretching mode).

In the 3500-2100 $\mathrm{cm}^{-1}$ region, bands between 2975 and $2800 \mathrm{~cm}^{-1}$ were assigned to $\mathrm{C}-\mathrm{H}$ of adamantyl and that at $2238 \mathrm{~cm}^{-1}$, to nitrile CN stretching. Finally, the small absorption in the 3500-3000 $\mathrm{cm}^{-1}$ region, attributed to $\mathrm{OH}$ stretching vibrations, is the expected for a low amount of $\mathrm{OH}$ groups, as planned in the preparative conditions.

Table 54. Main ATR-FTIR peaks in the spectra for Yb(III) complexes with Vildagliptin

\begin{tabular}{|c|c|c|c|c|c|c|c|c|c|c|}
\hline$\left[\mathrm{Yb}(\text { vilda })_{3}\right.$ (bipy)] & 1653 & $\begin{array}{l}1450 \\
1407\end{array}$ & $\begin{array}{l}1353 \\
1308\end{array}$ & $\begin{array}{l}1256 \\
1217\end{array}$ & $\begin{array}{l}1187 \\
1148 \\
1114\end{array}$ & 1054 & 913 & 762 & 603 & $\begin{array}{l}584 \\
549 \\
517\end{array}$ \\
\hline$\left[\mathrm{Yb}(\text { vilda })_{2}(\mathrm{acac})(\mathrm{bipy})\right]$ & 1624 & $\begin{array}{l}1465 \\
1404\end{array}$ & $\begin{array}{l}1348 \\
1314\end{array}$ & 1258 & $\begin{array}{l}1192 \\
1173 \\
1159\end{array}$ & $\begin{array}{c}1060 \\
1045 \\
1012 \\
970\end{array}$ & 915 & $\begin{array}{l}762 \\
755 \\
735\end{array}$ & $\begin{array}{l}654 \\
643 \\
623\end{array}$ & $\begin{array}{l}535 \\
526\end{array}$ \\
\hline
\end{tabular}

\section{Comparison of simulated FTIR spectra vs. experimental ATR-FTIR spectra}

The simulated infrared spectra exhibit a morphology similar to those obtained experimentally by ATR-FTIR (Figure 85), supporting the good accuracy of the semi-empirical quantum chemical methods used to predict the ground state geometries of the two complexes. As mentioned above, some of the differences between the two may be in fact related to the ATR technique, which introduces distortion of the relative intensities of bands and absolute shifts in frequency [180].
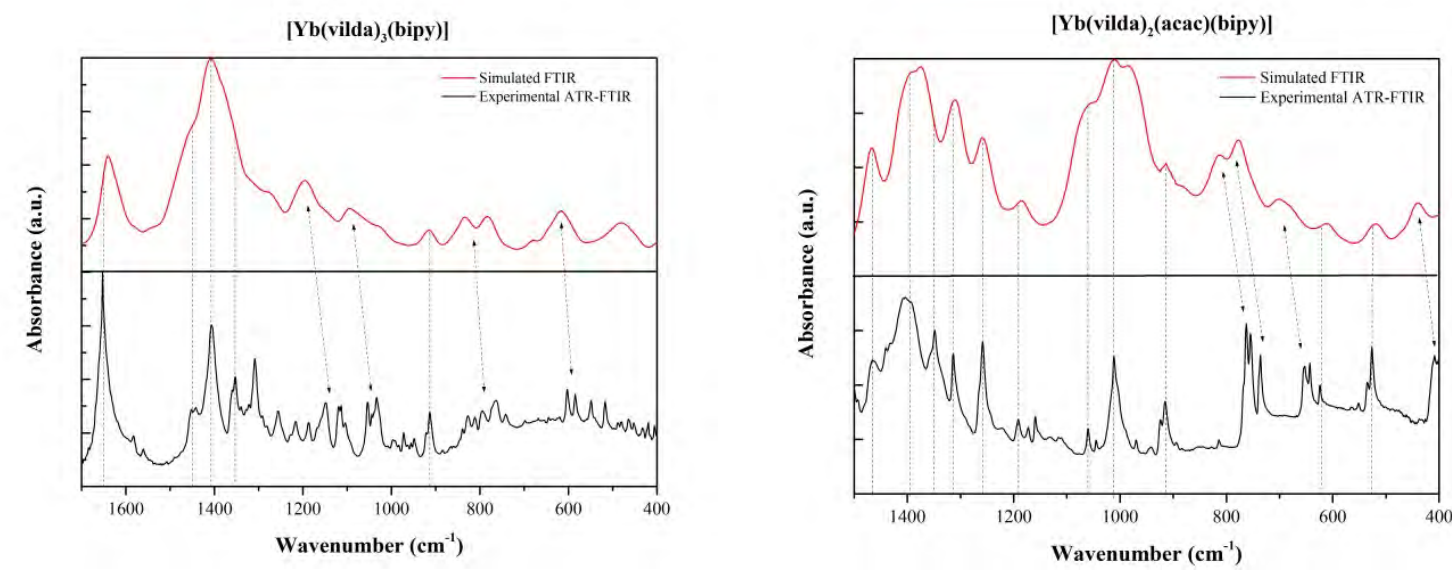

Figure 85. Comparison of simulated FTIR (red) vs. experimental ATR-FTIR (black) for the Yb(III) complexes with Vildagliptin 


\section{Raman spectroscopy}

Figure 86 shows the Raman spectra of some of the novel erbium(III) complexes. Four spectral regions are reported: (I) anti-Stokes and Stokes low frequency $\left(<200 \mathrm{~cm}^{-1}\right)$ region; (II) the region between 250 and $1500 \mathrm{~cm}^{-1}$; (III) the 1500-1600 $\mathrm{cm}^{-1}$ region and (IV) the region between 3500 and $3800 \mathrm{~cm}^{-1}$.

The Raman spectra in regions I and II have peaks at 114-86, 266-250, 422-410, 755-716, 1017-986, 1067-1050, 1350-1267 and 1457-1388 $\mathrm{cm}^{-1}$. Bipy adducts present characteristic emission peaks at 352 and $250 \mathrm{~cm}^{-1}$; bath ones show peaks at 410 and $254 \mathrm{~cm}^{-1}$; and those with $5 \mathrm{NO}_{2}$ phen exhibit peaks at 420,266 and $90 \mathrm{~cm}^{-1}$.

In fluorinated complexes, $v \mathrm{C}-\mathrm{F}$ modes appear at 1051-1064 $\mathrm{cm}^{-1}$. Whereas complexes with $5 \mathrm{NO}_{2}$ phen ligand show peaks at 716, 986-1000 and $1051 \mathrm{~cm}^{-1}$, those with bipy and bath show peaks at higher wavenumbers. For the peak at around $1300 \mathrm{~cm}^{-1}[v(\mathrm{C}=\mathrm{C})+\delta(\mathrm{CH})]$, the behavior is reversed: it appears at higher wavenumbers for $5 \mathrm{NO}_{2}$ phen complexes than for bipy and bath ones. The peaks at around $1448 \mathrm{~cm}^{-1}$ are assigned to $v(\mathrm{C}=\mathrm{C}, \mathrm{C}=\mathrm{N})$ vibrations.

In region III all the studied complexes (including (tba)[Er(dbm) $\left.)_{4}\right]$ ) showed strong multiple peaks at $1600-1526 \mathrm{~cm}^{-1}$ (with maxima for bipy adducts). It is known that the introduction of additional neutral ligands such as phen, bath or bipy into the inner sphere weakens the coordination to erbium of the main $\beta$-diketonate ligand, strengthening the $\mathrm{C}=0$ bond (diketone form). This weakening of the erbium-oxygen (diketonate) bond to facilitate the coordination erbium-nitrogen ( $\mathrm{N}, \mathrm{N}$-donor molecule) resulted in very stable octa-coordinated complexes.

In region IV three peaks appear at 3074-3090, 2964 and 2917-2931 cm-1.

Table 55. Main peaks in the I-III Raman spectral regions of the new erbium complexes $\left(\mathrm{cm}^{-1}\right)$

\begin{tabular}{|c|c|c|c|c|c|c|c|c|}
\hline$\left[\operatorname{Er}(\mathrm{acac})_{3}(\mathrm{bath})\right]$ & 1602 & $\begin{array}{l}1446 \\
1396\end{array}$ & $\begin{array}{l}1290 \\
1270\end{array}$ & & 1000 & 670 & 408 & $\begin{array}{l}258 \\
210 \\
104\end{array}$ \\
\hline & 1600 & 1450 & 1328 & 1045 & 1017 & 795 & 425 & 195 \\
\hline [Er(dbm)3(bipy)] & $\begin{array}{l}1580 \\
1510\end{array}$ & 1400 & 1300 & & 1005 & 615 & & $\begin{array}{c}114 \\
98\end{array}$ \\
\hline$(\mathrm{tba})\left[\operatorname{Er}(\mathrm{dbm})_{4}\right]$ & 15981500 & & $\begin{array}{l}1300 \\
1200\end{array}$ & & $\begin{array}{c}1000 \\
930\end{array}$ & $\begin{array}{l}790 \\
695\end{array}$ & & $\begin{array}{c}225 \\
99\end{array}$ \\
\hline$\left[\operatorname{Er}(\mathrm{h})_{3}\right.$ (bipy)] & $\begin{array}{l}1598 \\
1575 \\
1500 \\
\end{array}$ & $\begin{array}{l}1440 \\
1360\end{array}$ & $\begin{array}{l}1315 \\
1275\end{array}$ & $\begin{array}{l}1075 \\
1045\end{array}$ & 1010 & 763 & 405 & 170 \\
\hline$\left[\operatorname{Er}(\mathrm{h})_{3}(\right.$ bath $\left.)\right]$ & $\begin{array}{l}1610 \\
1600\end{array}$ & $\begin{array}{l}1444 \\
1398\end{array}$ & $\begin{array}{l}1300 \\
1285\end{array}$ & 1070 & 1001 & & 410 & 230 \\
\hline$\left[\operatorname{Er}(\mathrm{h})_{3}\left(5 \mathrm{NO}_{2}\right.\right.$ phen $\left.)\right]$ & $\begin{array}{l}1607 \\
1584\end{array}$ & $\begin{array}{l}1453 \\
1418 \\
1360 \\
\end{array}$ & 1293 & 1054 & 996 & $\begin{array}{l}753 \\
720\end{array}$ & 421 & 186 \\
\hline [Er(hd)3(bipy)] & $\begin{array}{c}16021587 \\
1500\end{array}$ & 1450 & 1320 & 1080 & 1020 & $\begin{array}{l}780 \\
630 \\
\end{array}$ & 400 & 140 \\
\hline$\left[\operatorname{Er}(\mathrm{dmh})_{3}(\mathrm{bipy})\right]$ & $\begin{array}{c}16001570 \\
1499\end{array}$ & 1460 & $\begin{array}{l}1315 \\
1265 \\
\end{array}$ & $\begin{array}{l}1103 \\
1064 \\
\end{array}$ & $\begin{array}{c}1017 \\
963 \\
\end{array}$ & $\begin{array}{l}628 \\
769 \\
\end{array}$ & 437 & $\begin{array}{l}191 \\
265 \\
\end{array}$ \\
\hline$\left[\operatorname{Er}(\mathrm{dmh})_{3}(\right.$ bath $\left.)\right]$ & 16121602 & $\begin{array}{l}1450 \\
1398\end{array}$ & $\begin{array}{l}1300 \\
1280\end{array}$ & 1100 & $\begin{array}{c}1002 \\
920\end{array}$ & 670 & 415 & $\begin{array}{l}270 \\
220 \\
105 \\
\end{array}$ \\
\hline$\left[\operatorname{Er}(\mathrm{dmh})_{3}\left(5 \mathrm{NO}_{2}\right.\right.$ phen $\left.)\right]$ & 1604 & $\begin{array}{l}1451 \\
1416 \\
1350 \\
\end{array}$ & $\begin{array}{l}1291 \\
1212\end{array}$ & $\begin{array}{l}1096 \\
1052\end{array}$ & $\begin{array}{l}992 \\
961\end{array}$ & $\begin{array}{l}880 \\
722\end{array}$ & & \\
\hline$\left[\operatorname{Er}(\text { thd })_{3}(\right.$ bath $\left.)\right]$ & $\begin{array}{l}1600 \\
1584 \\
1490\end{array}$ & $\begin{array}{l}1434 \\
1376 \\
1345\end{array}$ & $\begin{array}{l}1314 \\
1283 \\
1260\end{array}$ & $\begin{array}{l}1158 \\
1058\end{array}$ & $\begin{array}{c}1025 \\
998\end{array}$ & $\begin{array}{l}874 \\
768 \\
659\end{array}$ & $\begin{array}{l}408 \\
344\end{array}$ & $\begin{array}{l}304 \\
247 \\
221 \\
132 \\
\end{array}$ \\
\hline
\end{tabular}




\begin{tabular}{|c|c|c|c|c|c|c|c|c|}
\hline$\left[\operatorname{Er}(\mathrm{od})_{3}(\right.$ bipy $\left.)\right]$ & $\begin{array}{c}16041575 \\
1503\end{array}$ & 1453 & 1320 & 1062 & $\begin{array}{c}1018 \\
955\end{array}$ & $\begin{array}{l}775 \\
630\end{array}$ & 408 & $\begin{array}{l}260 \\
130\end{array}$ \\
\hline$\left[\operatorname{Er}(\mathrm{nd})_{3}(\right.$ bipy) $]$ & $\begin{array}{c}16051580 \\
1505 \\
\end{array}$ & $\begin{array}{l}1448 \\
1360 \\
\end{array}$ & $\begin{array}{l}1300 \\
1273 \\
\end{array}$ & 1058 & 1004 & 790 & 408 & 255 \\
\hline $\left.\operatorname{Er}(\mathrm{tfa})_{3}(\mathrm{bipy})\right]$ & $\begin{array}{c}16001583 \\
1500\end{array}$ & $\begin{array}{l}1450 \\
1398\end{array}$ & 1325 & $\begin{array}{l}1109 \\
1080\end{array}$ & $\begin{array}{c}1025 \\
953\end{array}$ & $\begin{array}{l}890 \\
780\end{array}$ & 360 & $\begin{array}{l}288 \\
104\end{array}$ \\
\hline $\left.\operatorname{Er}(\mathrm{tfa})_{3}(\mathrm{bath})\right]$ & 16031494 & 1450 & 1300 & $\begin{array}{l}1105 \\
1098 \\
\end{array}$ & $\begin{array}{c}1002 \\
950 \\
\end{array}$ & 780 & & $\begin{array}{l}270 \\
220\end{array}$ \\
\hline $\operatorname{Er}(\operatorname{tfnb})_{3}($ bipy $\left.)\right]$ & 16301600 & $\begin{array}{l}1475 \\
1380 \\
\end{array}$ & $\begin{array}{l}1350 \\
1302 \\
\end{array}$ & 1060 & $\begin{array}{c}1028 \\
975 \\
\end{array}$ & 765 & 512 & \\
\hline [Er(tfac) 3 (bipy)] & $\begin{array}{c}16001575 \\
1500\end{array}$ & 1395 & $\begin{array}{l}1330 \\
1300\end{array}$ & 1070 & $\begin{array}{c}1020 \\
950\end{array}$ & 765 & $\begin{array}{l}405 \\
350\end{array}$ & $\begin{array}{c}295 \\
201 \\
99 \\
\end{array}$ \\
\hline$\left[\operatorname{Er}(\mathrm{tfac})_{3}(\mathrm{bath})\right]$ & $\begin{array}{l}1605 \\
1540\end{array}$ & $\begin{array}{l}1449 \\
1423\end{array}$ & $\begin{array}{l}1357 \\
1300\end{array}$ & $\begin{array}{l}1100 \\
1055\end{array}$ & & $\begin{array}{l}890 \\
750\end{array}$ & 433 & $\begin{array}{l}280 \\
212 \\
135 \\
\end{array}$ \\
\hline$\left[\mathrm{Er}(\mathrm{tfac})_{3}\left(5 \mathrm{NO}_{2}\right.\right.$ phen $\left.)\right]$ & $\begin{array}{l}1630 \\
1530\end{array}$ & 1485 & $\begin{array}{l}1364 \\
1300\end{array}$ & $\begin{array}{l}1188 \\
1142\end{array}$ & & $\begin{array}{l}854 \\
836\end{array}$ & & \\
\hline$\left[\operatorname{Er}(\operatorname{tpm})_{3}(\right.$ bipy $\left.)\right]$ & $\begin{array}{l}16001583 \\
15221500\end{array}$ & & 1302 & $\begin{array}{l}1065 \\
1055\end{array}$ & $\begin{array}{c}1014 \\
960\end{array}$ & $\begin{array}{l}775 \\
700\end{array}$ & 465 & $\begin{array}{l}263 \\
200 \\
170 \\
\end{array}$ \\
\hline$\left[\operatorname{Er}(\operatorname{tpm})_{3}(\right.$ bath $\left.)\right]$ & 1605 & $\begin{array}{l}1455 \\
1395\end{array}$ & $\begin{array}{l}1301 \\
1280\end{array}$ & & $\begin{array}{c}1001 \\
970\end{array}$ & $\begin{array}{l}855 \\
700 \\
678 \\
\end{array}$ & $\begin{array}{l}428 \\
380\end{array}$ & $\begin{array}{l}325 \\
260 \\
230 \\
\end{array}$ \\
\hline$\left[\operatorname{Er}(\operatorname{tpm})_{3}\left(5 \mathrm{NO}_{2}\right.\right.$ phen $\left.)\right]$ & 16081520 & $\begin{array}{l}1456 \\
1415 \\
\end{array}$ & $\begin{array}{l}1355 \\
1346 \\
\end{array}$ & 1053 & & 711 & 416 & $\begin{array}{c}105 \\
89\end{array}$ \\
\hline$\left[\operatorname{Er}(\mathrm{fhd})_{3}\left(5 \mathrm{NO}_{2}\right.\right.$ phen $\left.)\right]$ & 16031526 & $\begin{array}{l}1448 \\
1426 \\
1402\end{array}$ & 1344 & 1051 & 986 & 719 & 416 & 86 \\
\hline$\left[\operatorname{Er}(\text { fod })_{3}(\right.$ bath $\left.)\right]$ & & 1438 & $\begin{array}{l}1388 \\
1286\end{array}$ & 1102 & 999 & $\begin{array}{l}748 \\
659\end{array}$ & 401 & 246 \\
\hline$\left[\operatorname{Er}(\text { fod })_{3}\left(5 \mathrm{NO}_{2}\right.\right.$ phen $\left.)\right]$ & 1604 & $\begin{array}{l}1470 \\
1420\end{array}$ & $\begin{array}{l}1350 \\
1300\end{array}$ & 1049 & & $\begin{array}{l}820 \\
750 \\
725\end{array}$ & 421 & $\begin{array}{c}265 \\
240 \\
198 \\
100 \\
94\end{array}$ \\
\hline
\end{tabular}




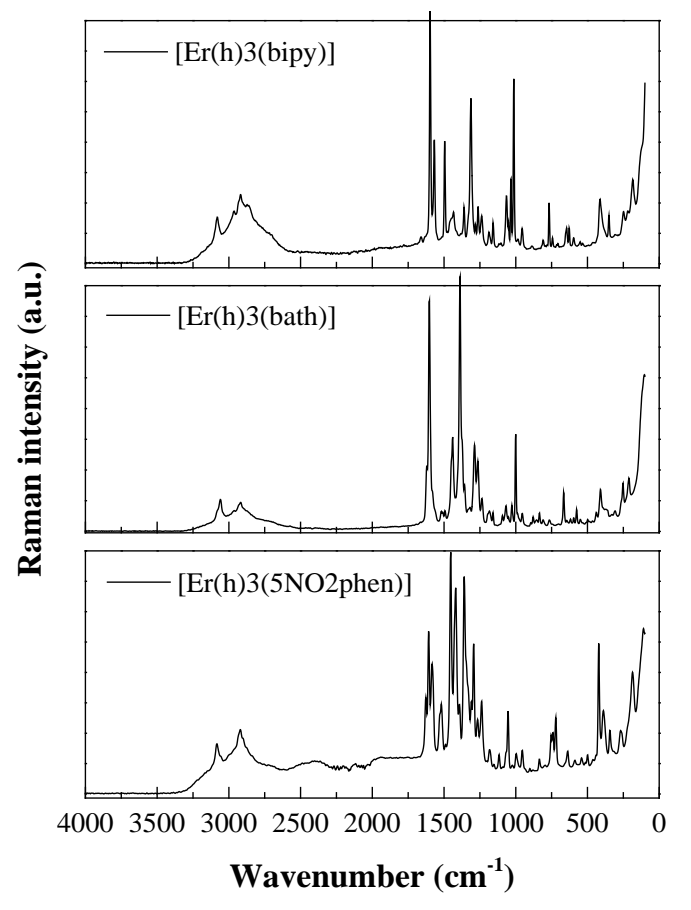

[Er(h) ${ }_{3}(\mathrm{~N}, \mathrm{~N}-$ donor $\left.)\right]$

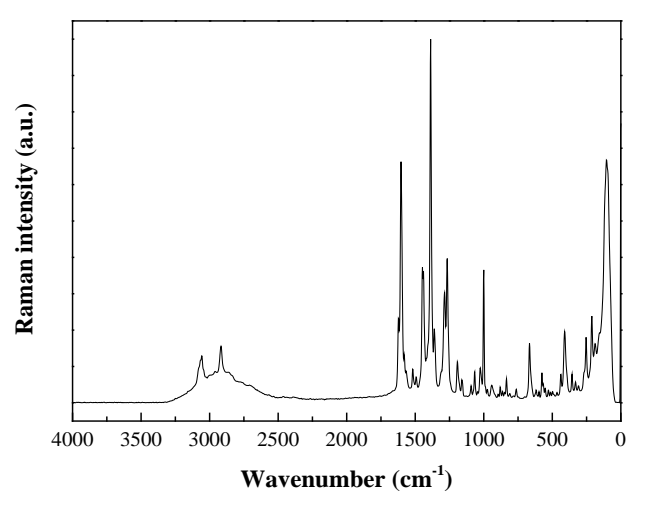

[Er(acac) 3 (bath)]

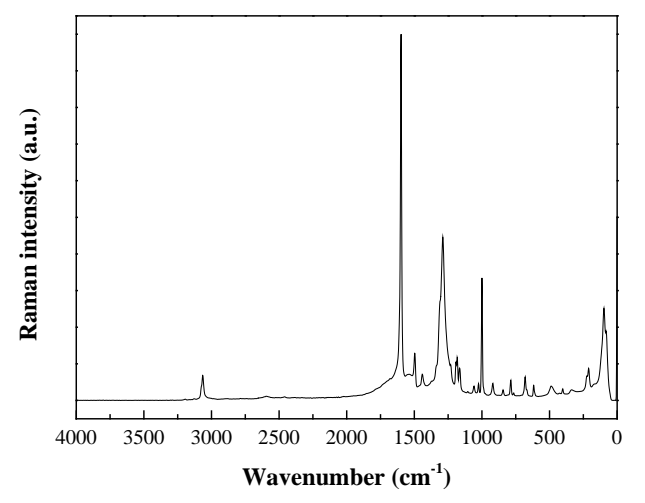

(tba) $\left[\operatorname{Er}(\mathrm{dbm})_{4}\right]$

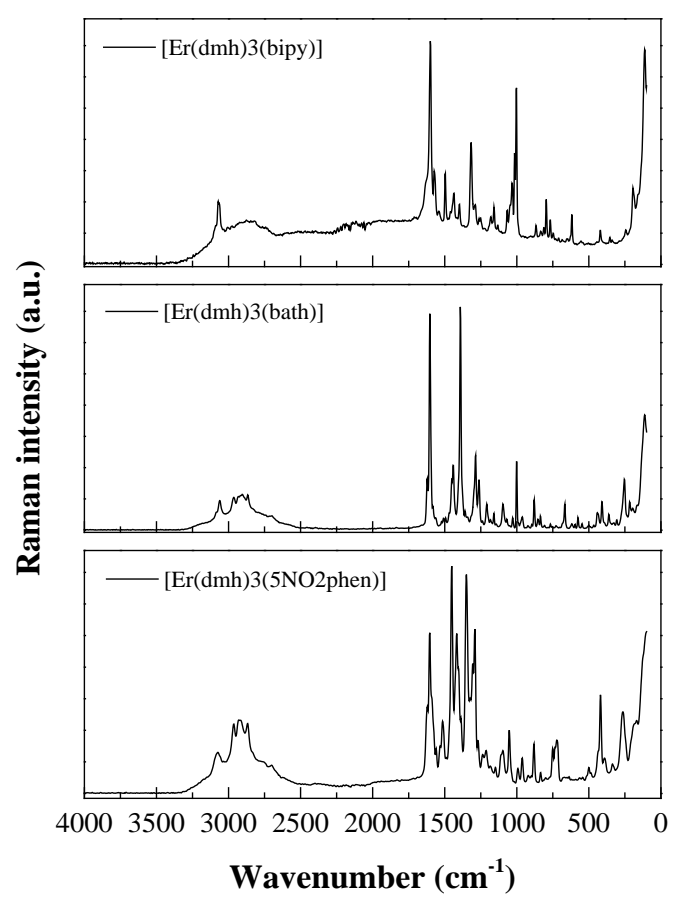

$\left[\operatorname{Er}(\mathrm{dmh})_{3}(\mathrm{~N}, \mathrm{~N}-\mathrm{donor})\right]$

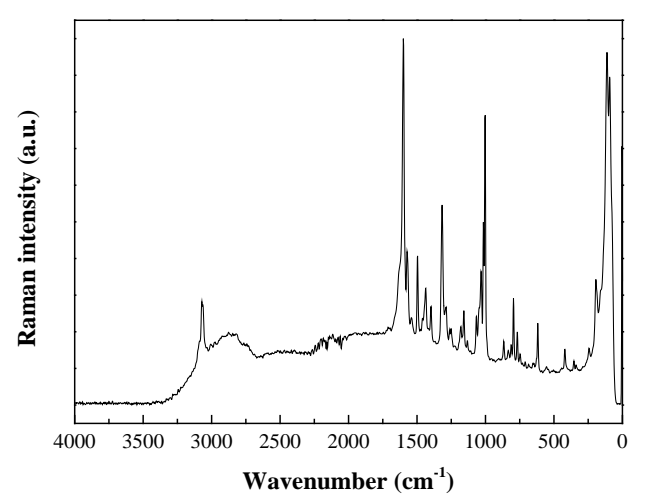

[Er(dbm) $)_{3}($ bipy)]

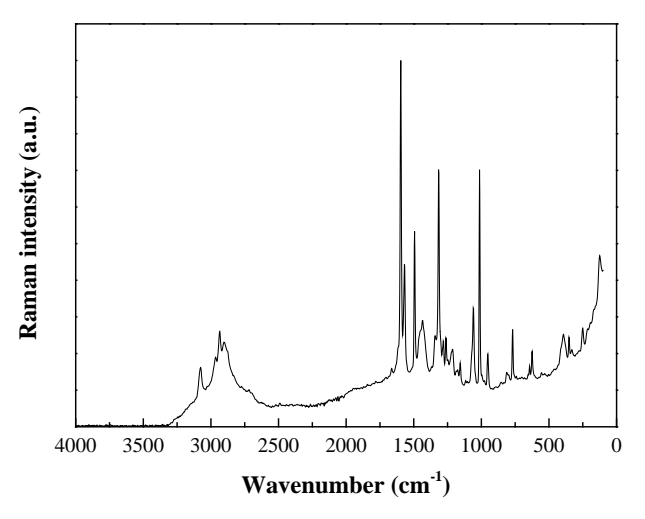

[Er(hd) $)_{3}$ (bipy)] 


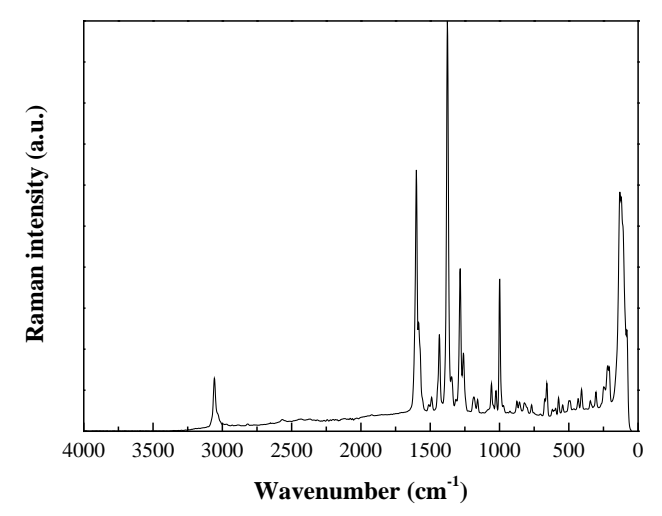

[Er(thd) ${ }_{3}$ (bath)]

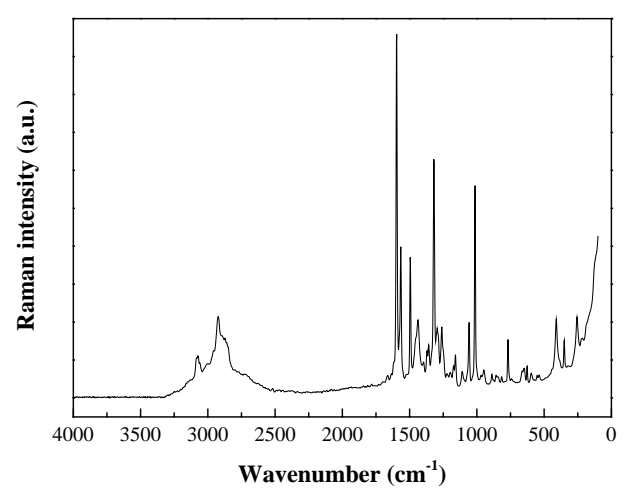

[Er(nd) $)_{3}$ (bipy)]

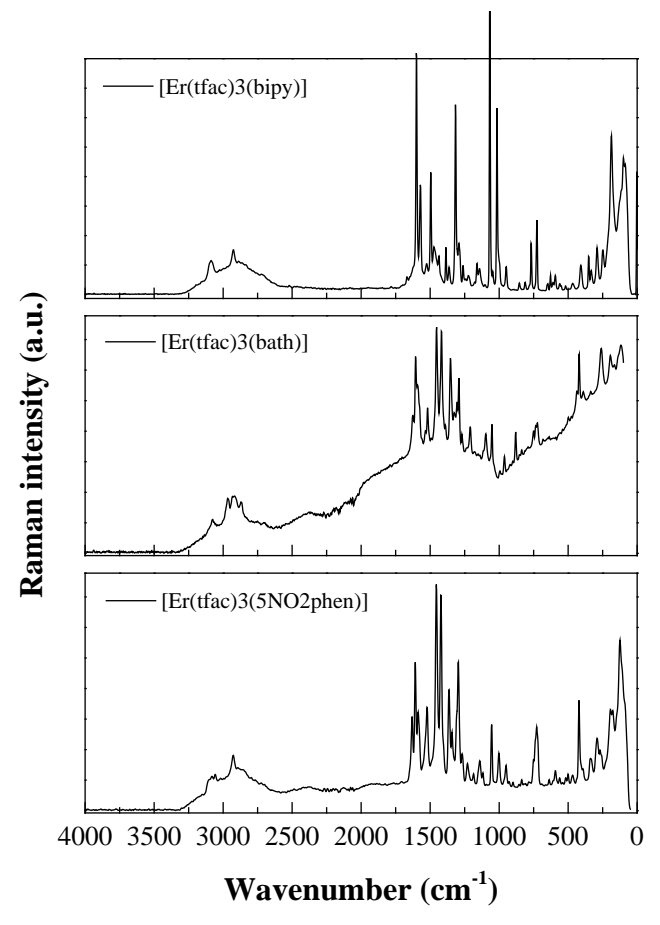

[Er(tfac) $)_{3}(\mathrm{~N}, \mathrm{~N}$-donor $\left.)\right]$

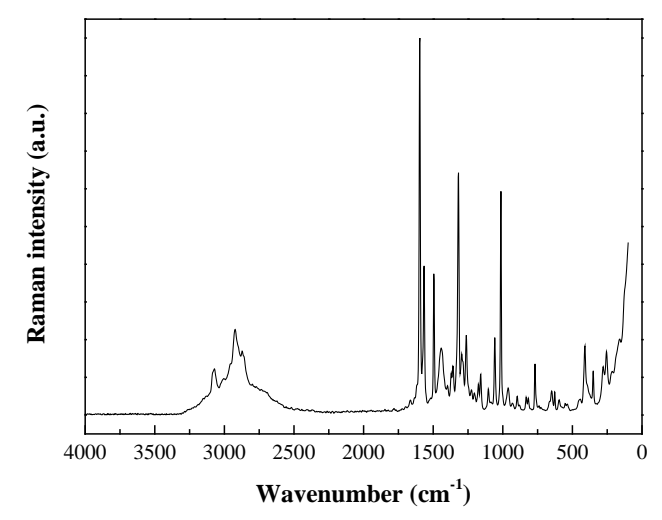

[Er(od) $)_{3}$ (bipy)]

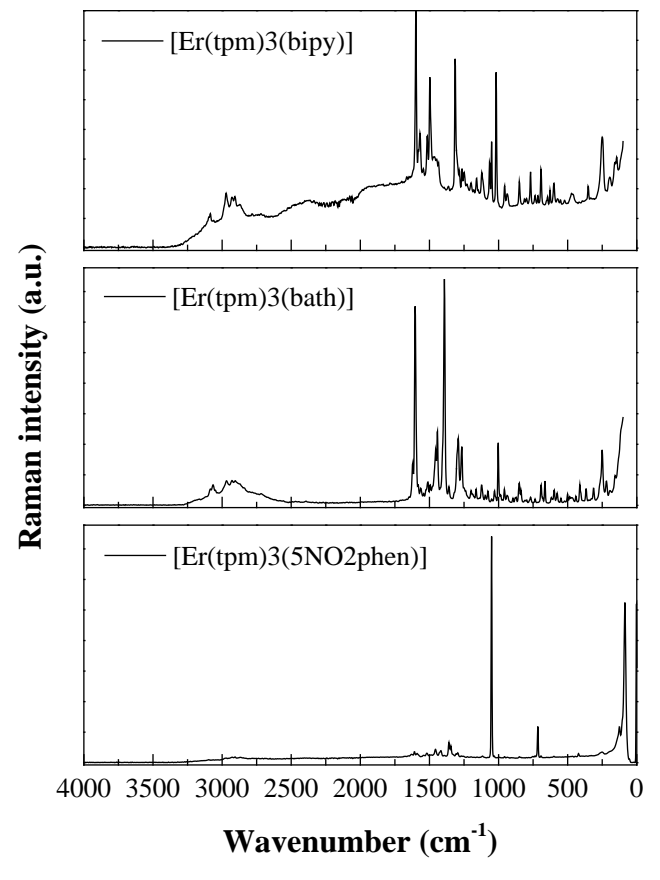

[Er(tpm) $)_{3}(\mathrm{~N}, \mathrm{~N}-$ donor $\left.)\right]$ 


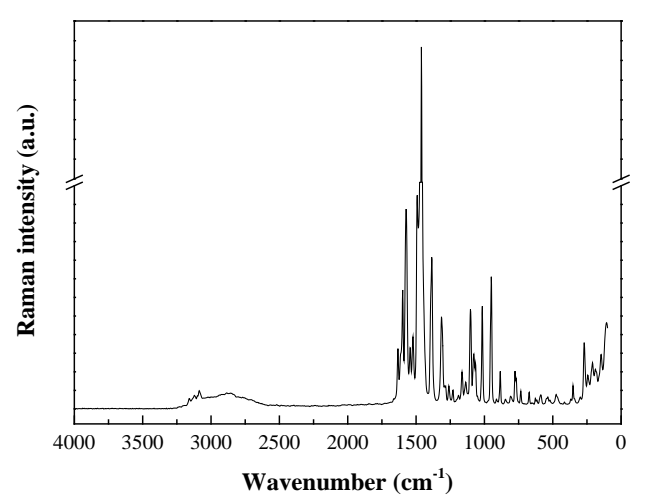

[Er(tfa) $)_{3}$ (bipy)]

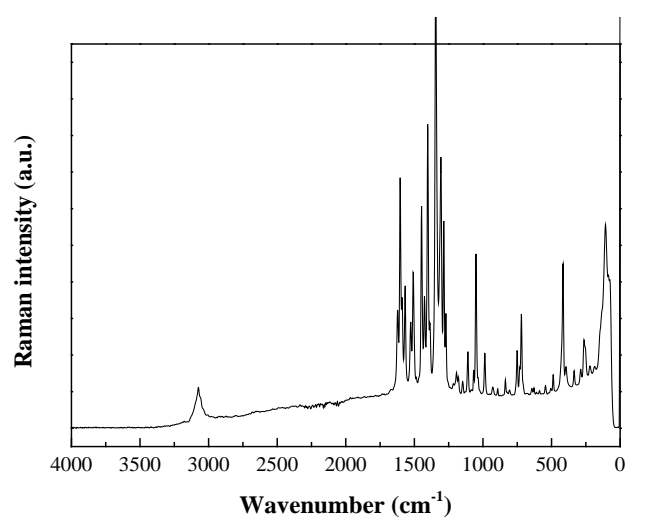

[Er(fhd $)_{3}\left(5 \mathrm{NO}_{2}\right.$ phen $\left.)\right]$

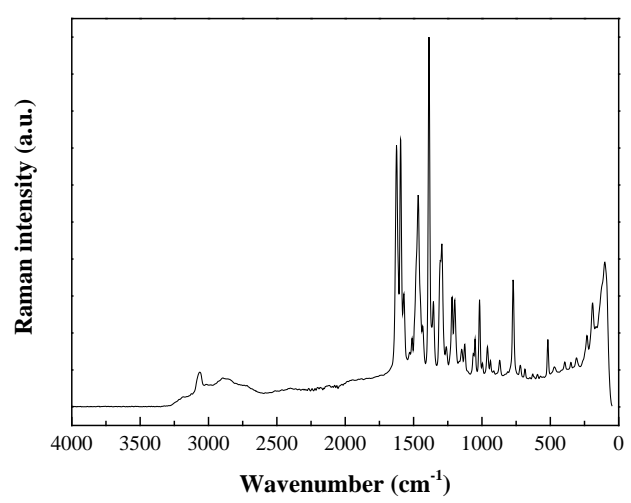

$\left[\operatorname{Er}(\mathrm{tfnb})_{3}(\right.$ bipy) $]$

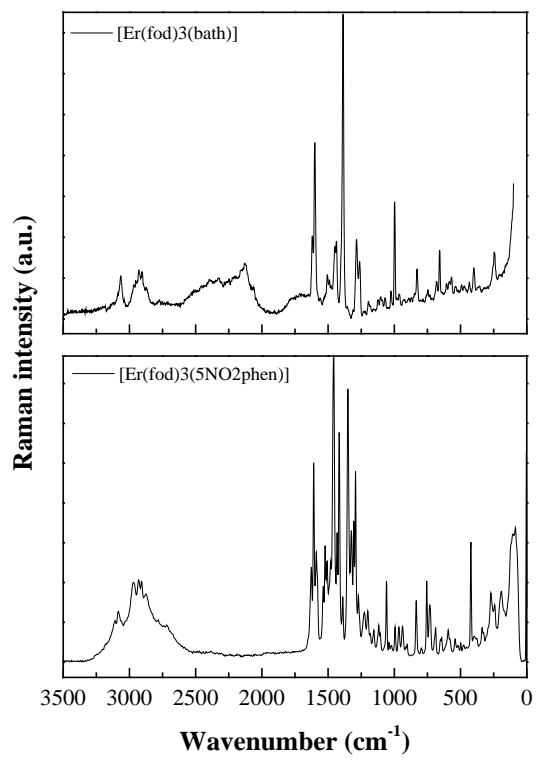

[Er(fod) $)_{3}$ (N,N-donor)]

Figure 86. Raman spectra for the $\left[\operatorname{Er}(\beta \text {-diketonate })_{3}(\mathrm{~N}, \mathrm{~N}-\right.$ donor $\left.)\right]$ complexes 


\subsubsection{NMR and EPR spectroscopic studies}

\section{NMR spectroscopy}

\section{${ }^{1}$ H-NMR spectra}

The spectra of the $\left[\operatorname{Er}(\beta \text {-diketonate })_{3}(\mathrm{~N}, \mathrm{~N}\right.$-donor $\left.)\right]$ complexes display one signal due to methine protons of $\beta$-diketonate and the rest due to heterocyclic diimines. The signal at 6 $\operatorname{ppm}(\delta)$ can be tentatively assigned to the methine group of the $\beta$-diketonates. The signals due to coordinated $\mathrm{N}, \mathrm{N}$-donor are observed at 13.5-13.3 ppm $(\delta)$ and 10.3-9.7 ppm $(\delta)$ for coordinated bath in $\left[\operatorname{Er}(\operatorname{tpm})_{3}(\right.$ bath $\left.)\right]$ and $\left[\operatorname{Er}(\text { fod })_{3}(\text { bath })\right]^{\prime}$; and at $12.6 \mathrm{ppm}(\delta)$ and 11.3 ppm $(\delta)$ for coordinated bipy in $\left[\operatorname{Er}(\mathrm{tfa})_{3}(\right.$ bipy)]. Another signals due to coordinated bipy are observed at 8.8-8.5 ppm $(\delta)$ and 8.0-7.7 ppm $(\delta)$ in $\left[\operatorname{Er}(\mathrm{tfa})_{3}(\mathrm{bipy})\right]$; and at 8.2-8.1 ppm $(\delta)$ and $7.7 \mathrm{ppm}(\delta)$ in $\left[\operatorname{Er}(\mathrm{tfnb})_{3}(\right.$ bipy $\left.)\right]$.

Signals at 8.5 and $7.9 \mathrm{ppm}(\delta)$ in $\left[\operatorname{Er}(\mathrm{tfa})_{3}(\mathrm{bipy})\right]$ are assigned to furyl-H protons; and the signal at $5.3 \mathrm{ppm}(\delta)$ in $\left[\operatorname{Er}(\mathrm{tfnb})_{3}(\mathrm{bipy})\right]$ is assigned to naphthyl-H protons.

The weak absorption in the region from $\delta=1.9$ to $\delta=2.7 \mathrm{ppm}$ is indicative of the protons adjacent to a carbonyl group in the $\beta$-diketonate. The chemical shift at around $1.2 \mathrm{ppm}$ is typical of $\mathrm{RCH}_{3}$.

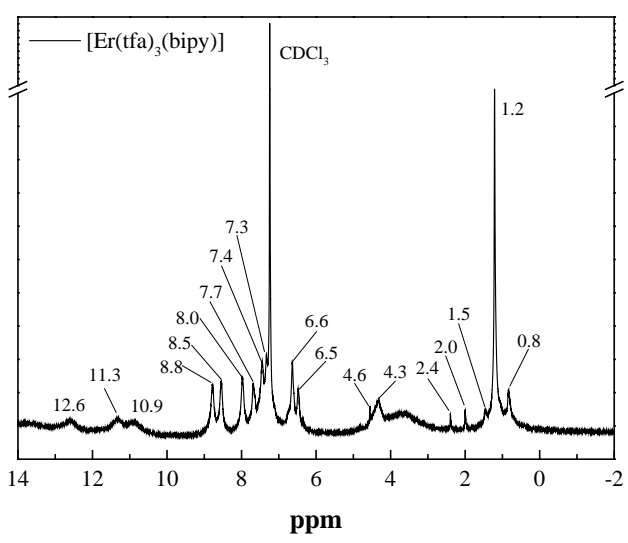

$\left[\operatorname{Er}(\mathrm{tfa})_{3}(\mathrm{bipy})\right]$

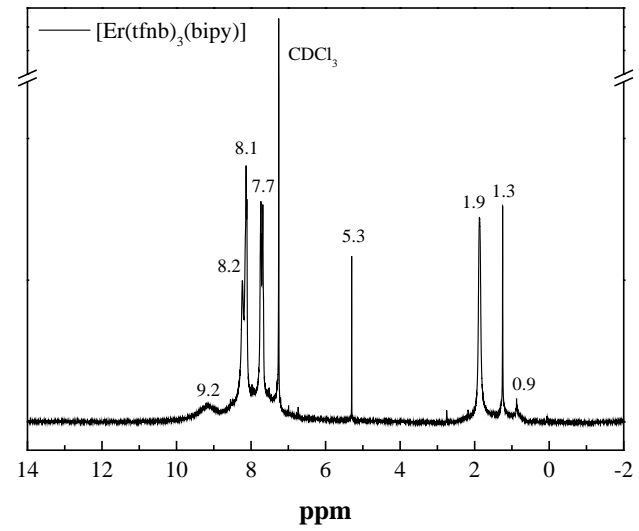

$\left[\operatorname{Er}(\mathrm{tfnb})_{3}(\mathrm{bipy})\right]$

\footnotetext{
${ }^{1}$ This is an interesting feature, since such downfield chemical shifts make these two complexes useful as NMR shift reagents. In fact, tris( $\beta$-diketonate) $\operatorname{Ln}(\mathrm{III})$ complexes are the most popular shift reagents for the study of achiral substrates. Complexes with $\mathrm{Eu}, \mathrm{Er}, \mathrm{Yb}$, and $\mathrm{Tm}$ are downfield shift reagents with a relative ordering $\mathrm{Tm}>$ $\mathrm{Er}>\mathrm{Yb}>\mathrm{Eu}$. One might conclude that $\mathrm{Tm}$ would be the best option to use, but this lanthanide ion is seldom applied in shift reagents because of too severe line broadening -which blurs the fine structure in the proton NMR spectrum- and because it causes such large shifts that can make finding and assigning all the resonances difficult. The shifts induced by the less powerful lanthanide ions are still large enough to produce first-order proton NMR spectra, while line broadening is less a problem. Even though Eu(III) complexes have usually been the preferred choice, $\operatorname{Er}(\operatorname{tpm})_{3}\left(5 \mathrm{NO}_{2}\right.$ phen $\left.)\right]$ and $\left[\operatorname{Er}(\text { fod })_{3}(\right.$ bath $\left.)\right]$ can also be used as downfield shift reagents.
} 


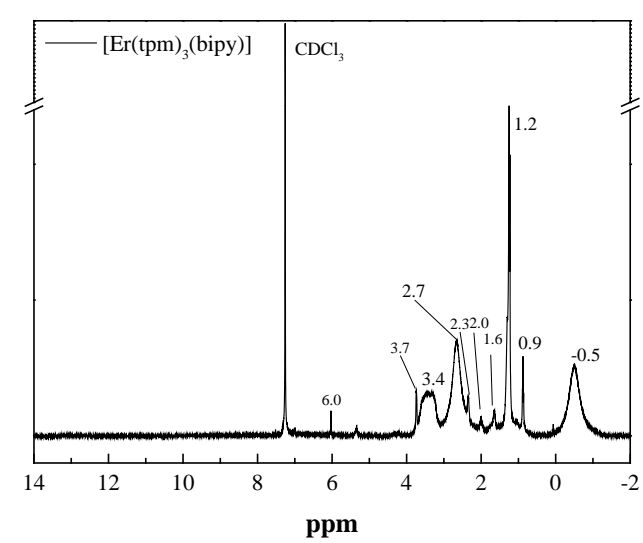

[Er(tpm) $)_{3}$ (bipy)]

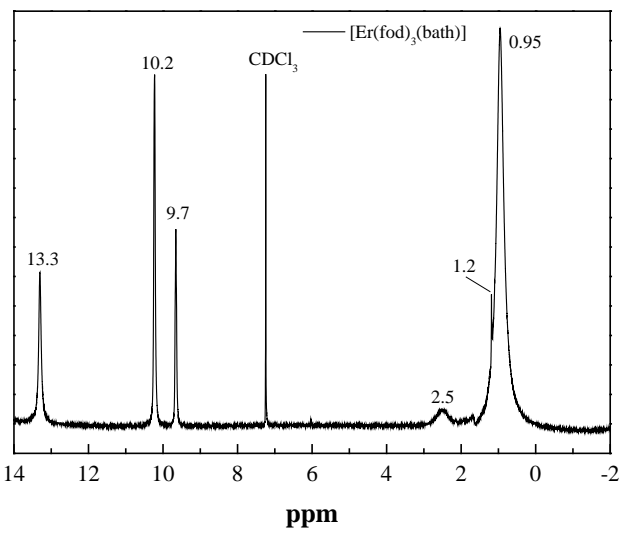

$\left[\operatorname{Er}(\text { fod })_{3}(\right.$ bath $\left.)\right]$

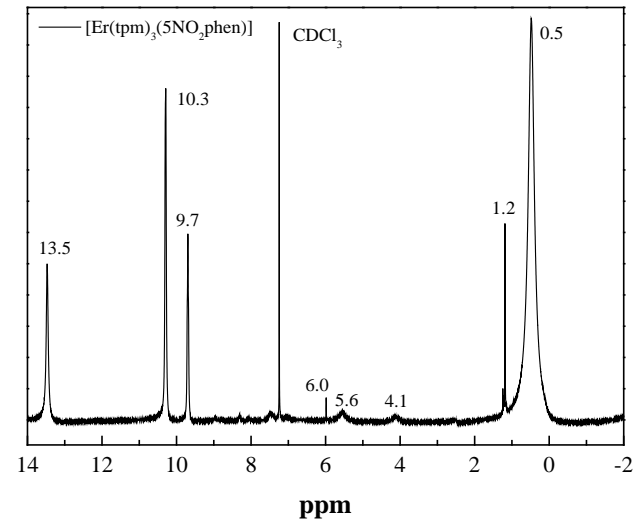

[Er(tpm) $)_{3}($ bath)]

Figure 87. ${ }^{1} \mathrm{H}-\mathrm{NMR}$ spectra

\section{${ }^{13} \mathrm{C}$-NMR spectra}

${ }^{13} \mathrm{C}-\mathrm{NMR}$ spectra of $\left[\operatorname{Er}(\operatorname{tpm})_{3}\left(5 \mathrm{NO}_{2}\right.\right.$ phen $\left.)\right]$, [Er(fhd $)_{3}\left(5 \mathrm{NO}_{2}\right.$ phen $\left.)\right]$ and [Er(fod $)_{3}\left(5 \mathrm{NO}_{2}\right.$ phen $\left.)\right]$ show signals at $88.6 \mathrm{ppm}, 91.6 \mathrm{ppm}$ and $86.2 \mathrm{ppm}$, respectively. In addition, all these complexes show a common signal (triplet) at $80.3 \mathrm{ppm}$.

${ }^{13} \mathrm{C}-\mathrm{NMR}$ spectra of $\left[\operatorname{Er}(\mathrm{tpm})_{3}(\mathrm{bath})\right]$ and $\left[\operatorname{Er}(\text { fod })_{3}(\right.$ bath $\left.)\right]$ show signals at $25.7 \mathrm{ppm}$ and 26.1 ppm, in good agreement with analogous signals at $35.5 \mathrm{ppm}$ (and $23.7 \mathrm{ppm}$ ) found for $\left[\operatorname{Er}(\text { fod })_{3}\left(5 \mathrm{NO}_{2}\right.\right.$ phen $\left.)\right]$.

Above signals should be assigned to different $\beta$-diketonate ligands in accordance with the assignations made for acetylacetonate ligand found in the literature.

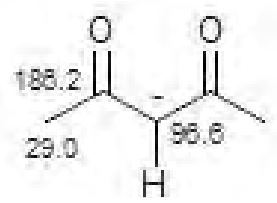

The ${ }^{13} \mathrm{C}$-NMR spectrum of [Er(tfa) $)_{3}$ (bipy)] shows a multiplet signal at around $44.9 \mathrm{ppm}$, in good agreement with that which also appears at $40.22 \mathrm{ppm}$ for $\left[\operatorname{Er}(\operatorname{tpm})_{3}\left(5 \mathrm{NO}_{2}\right.\right.$ phen $\left.)\right]$.

Signals in the 144-130 ppm region should be referred to $\mathrm{CH}$ in phenanthroline for bathophenanthroline and 5-nitro-1,10-phenanthroline complexes. 


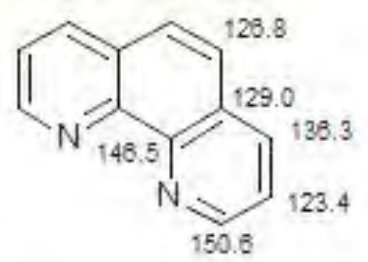

Signals at 77.2 can be assigned to $\mathrm{C}_{6} \mathrm{H}_{4} \mathrm{Cl}_{2}$, which was used as a reference.

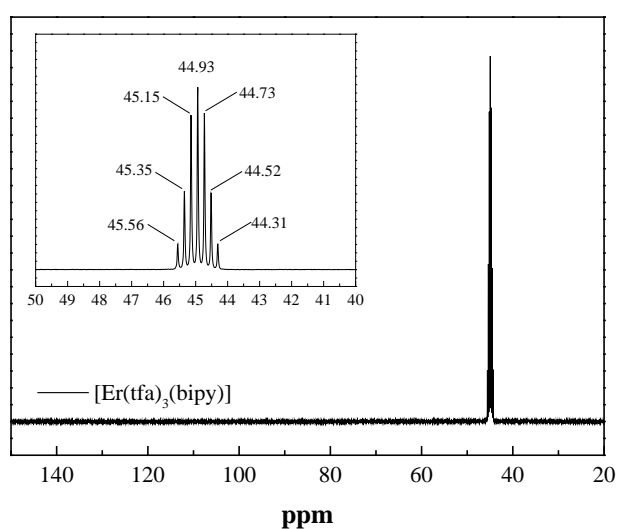

[Er(tfa) 3 (bipy)]

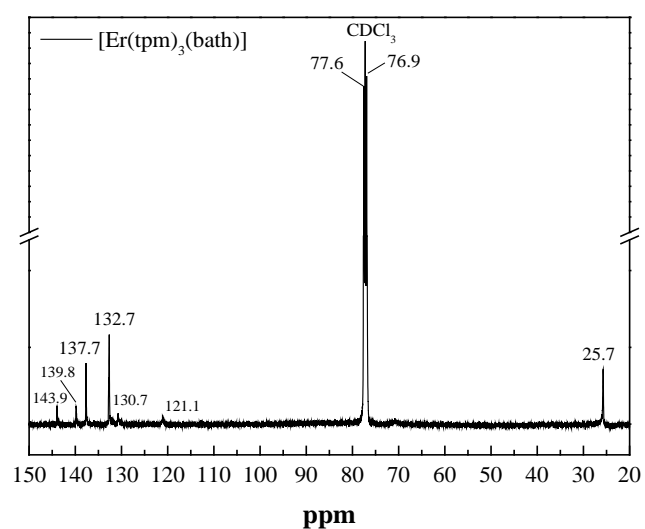

[Er(tpm) $)_{3}($ bath)]

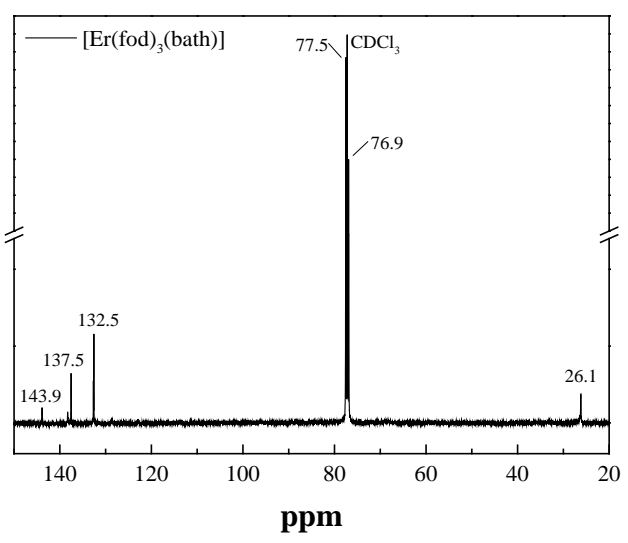

[Er(fod) $)_{3}($ bath)]

Figure 88. ${ }^{13} \mathrm{C}$-NMR spectra for $\left[\operatorname{Er}(\operatorname{tfa})_{3}(\right.$ bipy $\left.)\right],\left[\operatorname{Er}(\operatorname{tpm})_{3}(\right.$ bath $\left.)\right]$ and $\left[\operatorname{Er}(\text { fod })_{3}(\right.$ bath $\left.)\right]$ 

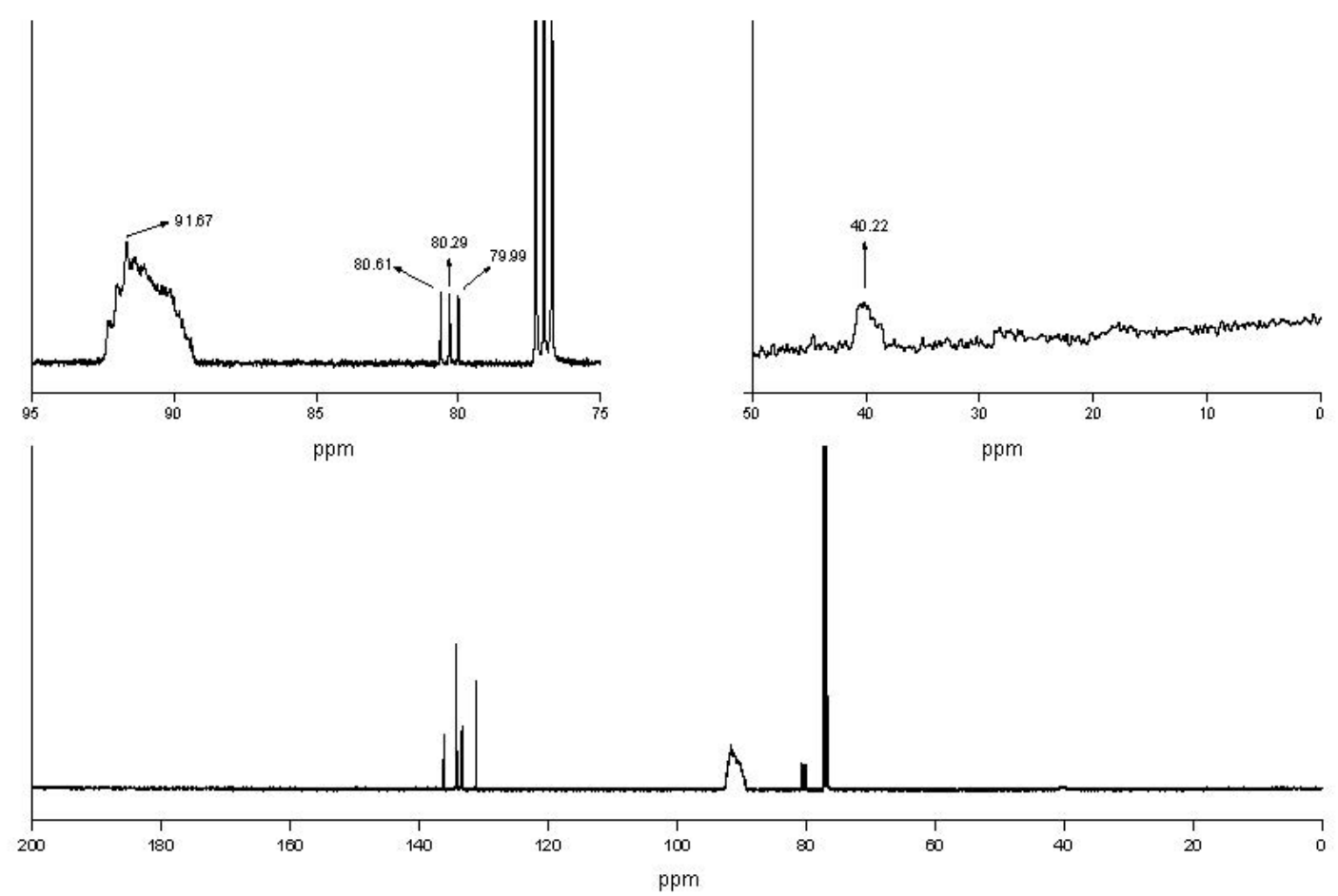

Figure 89. ${ }^{13} \mathrm{C}-\mathrm{NMR}\left[\operatorname{Er}(\mathrm{tpm})_{3}\left(5 \mathrm{NO}_{2}\right.\right.$ phen $\left.)\right]$
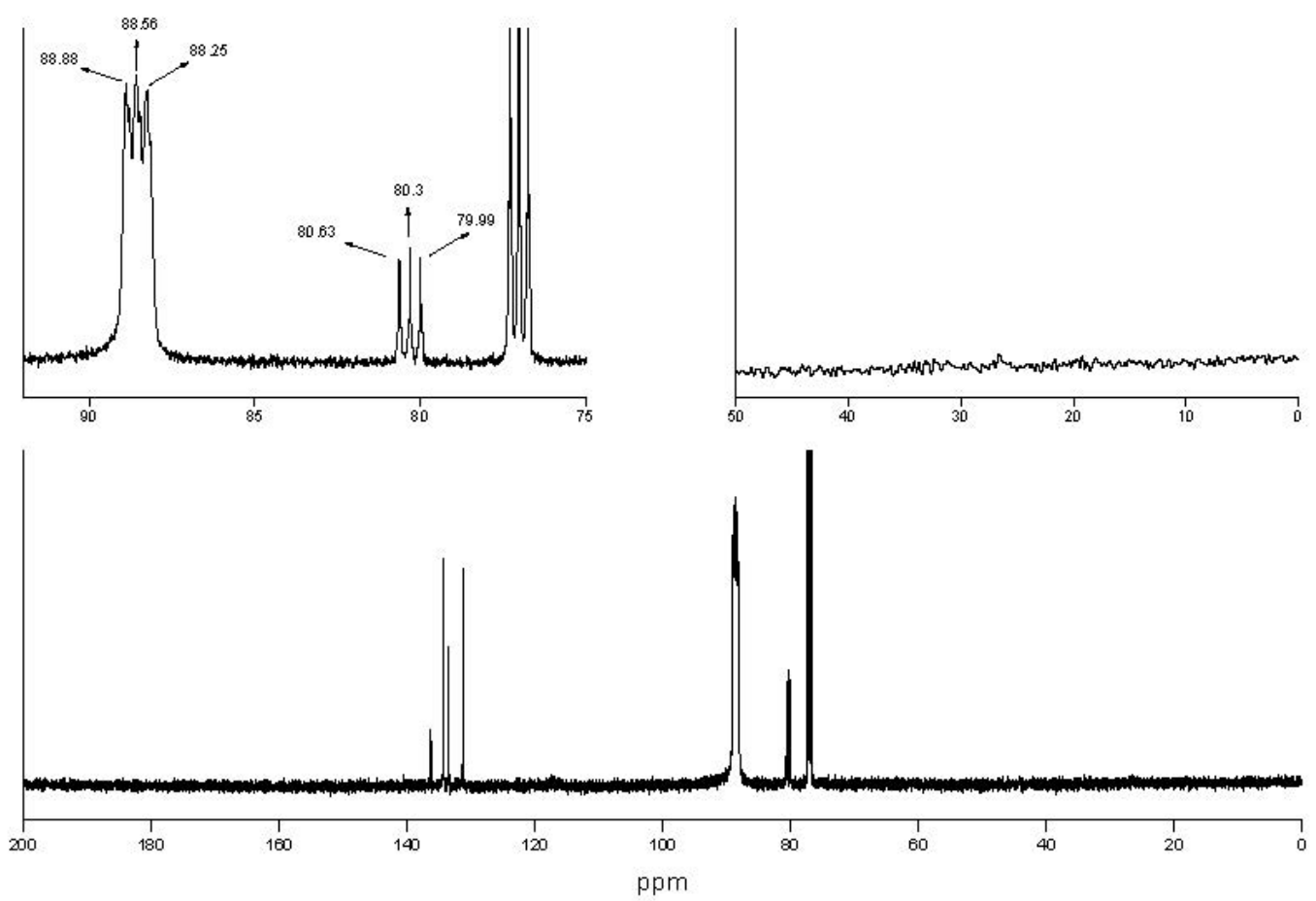

Figure 90. ${ }^{13} \mathrm{C}-\mathrm{NMR}\left[\mathrm{Er}(\mathrm{fhd})_{3}\left(5 \mathrm{NO}_{2}\right.\right.$ phen $\left.)\right]$ 

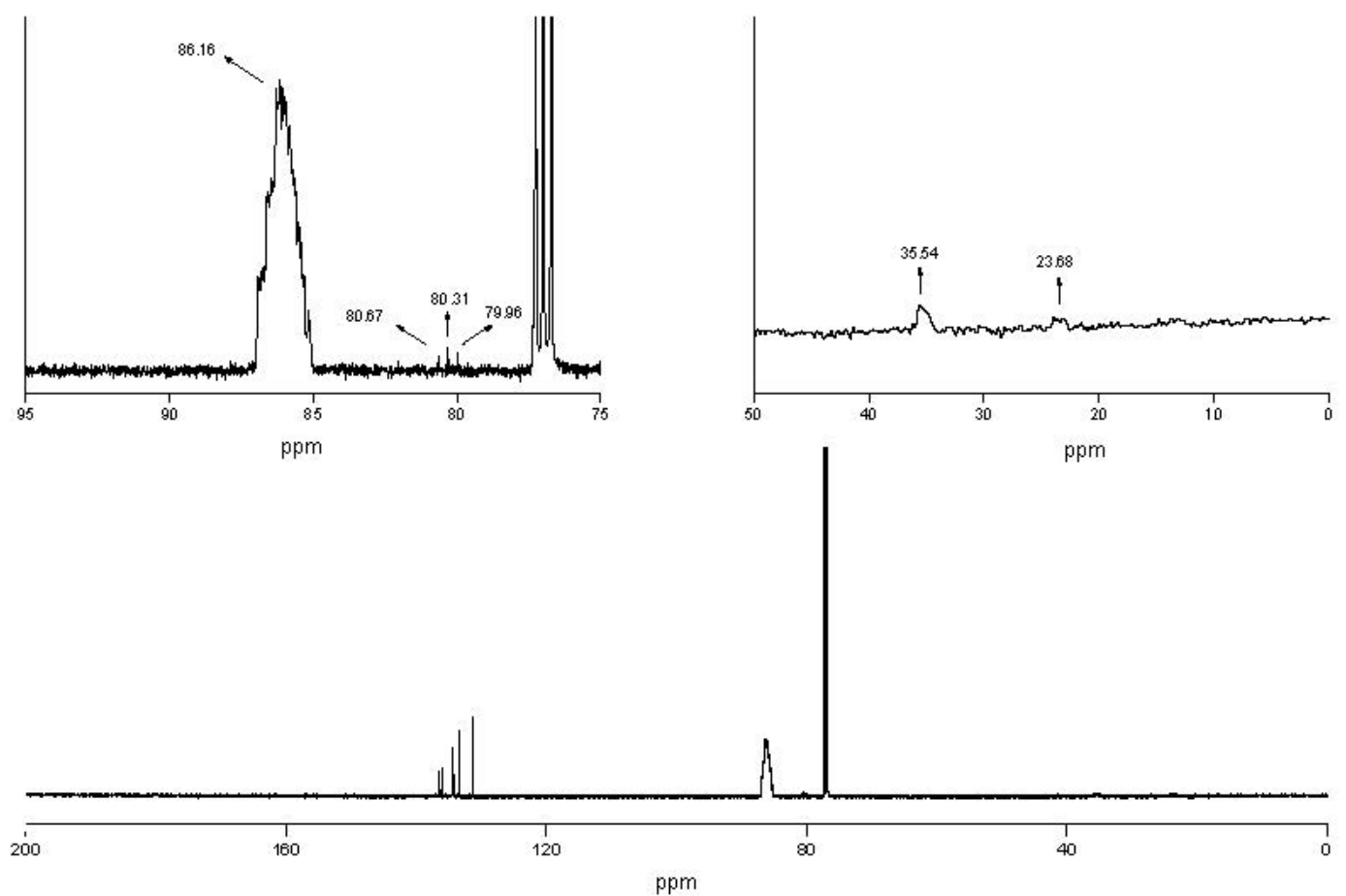

Figure $91 .{ }^{13} \mathrm{C}-\mathrm{NMR}\left[\mathrm{Er}(\mathrm{fod})_{3}\left(5 \mathrm{NO}_{2}\right.\right.$ phen $\left.)\right]$

\section{${ }^{19}$ F-NMR spectra}

The four spectra of the $\left[\operatorname{Er}(\text { fluorinated } \beta \text {-diketonate })_{3}(\mathrm{~N}, \mathrm{~N}-\right.$ donor $\left.)\right]$ complexes reported below display one strong signal between -78 and $-97 \mathrm{ppm}$ due to $-\mathrm{CF}_{3}$ groups and, in the case of complexes with longer alkyl chains (fhd and fod ligands), an additional doublet of medium intensity around $-123 \mathrm{ppm}$ assignable to $-\mathrm{CF}_{2}$ groups. In the particular case of $\left[\mathrm{Er}(\mathrm{fhd})_{3}\left(5 \mathrm{NO}_{2}\right.\right.$ phen $\left.)\right]$, in which there are two $-\mathrm{CF}_{3}$ groups, an additional signal appears at -71 ppm.

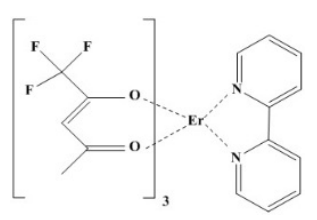

$\left[\operatorname{Er}(\operatorname{tfac})_{3}(\right.$ bipy $\left.)\right]$

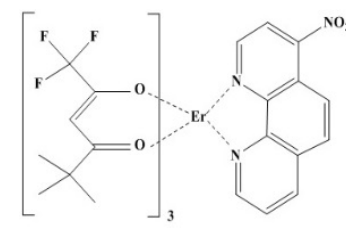

$\left[\operatorname{Er}(\operatorname{tpm})_{3}\left(5 \mathrm{NO}_{2}\right.\right.$ phen $\left.)\right]$

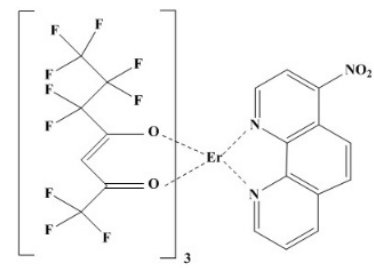

$\left[\operatorname{Er}(\text { fhd })_{3}\left(5 \mathrm{NO}_{2}\right.\right.$ phen $\left.)\right]$

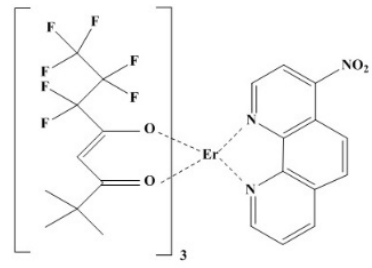

$\left[\operatorname{Er}(\text { fod })_{3}\left(5 \mathrm{NO}_{2}\right.\right.$ phen $\left.)\right]$ 


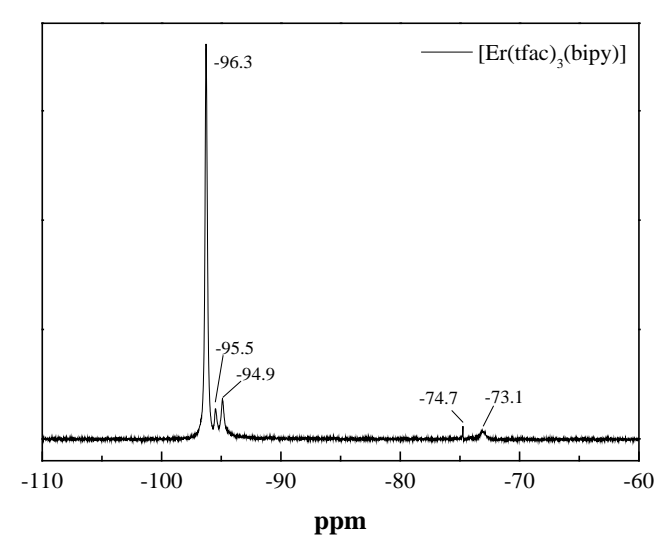

$\left[\operatorname{Er}(\operatorname{tfac})_{3}(\right.$ bipy $\left.)\right]$

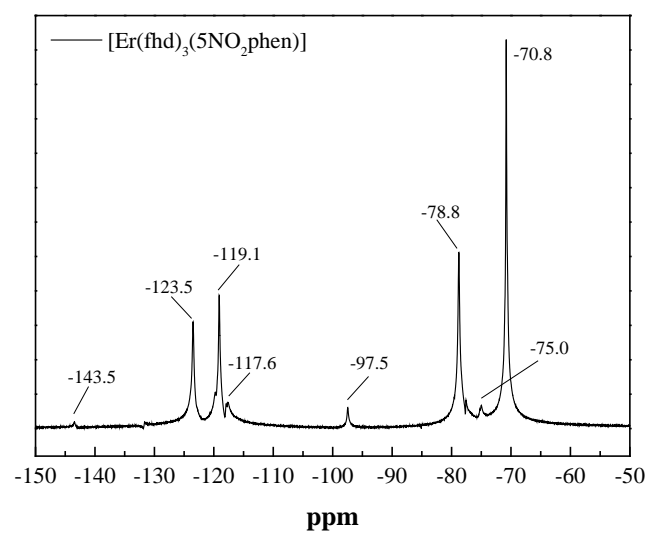

[Er(fhd $)_{3}\left(5 \mathrm{NO}_{2}\right.$ phen $\left.)\right]$

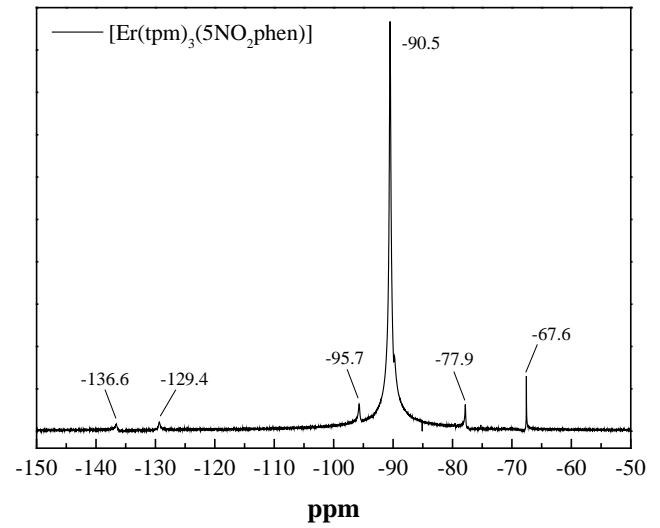

$\left[\operatorname{Er}(\operatorname{tpm})_{3}\left(5 \mathrm{NO}_{2}\right.\right.$ phen $\left.)\right]$

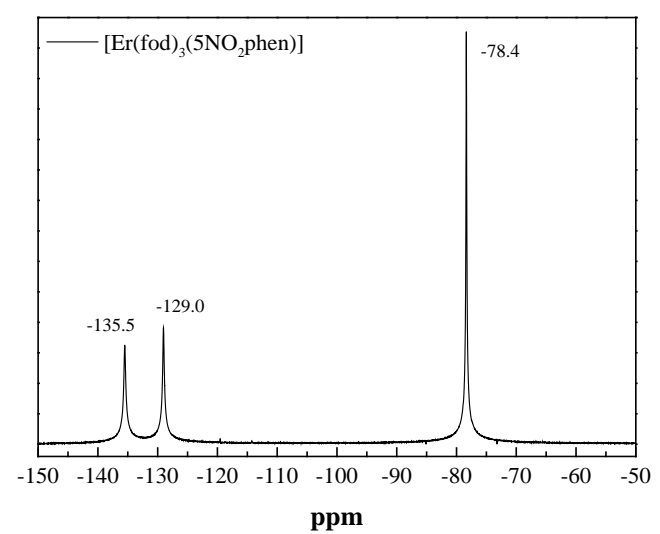

$\left[\mathrm{Er}(\mathrm{fod})_{3}\left(5 \mathrm{NO}_{2}\right.\right.$ phen $\left.)\right]$

Figure 92. ${ }^{19} \mathrm{~F}-\mathrm{NMR}$ spectra

\section{EPR spectroscopy}

Microcrystalline powder of the Er(III) complexes were used to measure spectra at X band frequency at USC facilities. The registered spectra are poor (Figure 93) and require both instrumental optimization and computational simulation to corroborate them. Our aim was to obtain an estimation on the zero-field-splitting parameters, $D^{*}$, from the spectra.

The ZFS parameters of Er-bipy, Er-bath and Er-5 $\mathrm{NO}_{2}$ phen are expected to be smaller than those of $\operatorname{Er}-\beta$-diketonate, as it has been referred for other $\operatorname{Ln}(\mathrm{III})$ in the literature [181]. This would imply that the donating nitrogens from the $\mathrm{N}, \mathrm{N}$-donors have weaker ligand-fields than those of the donating oxygens from the $\beta$-diketonates, according to Ryo et al. [181].

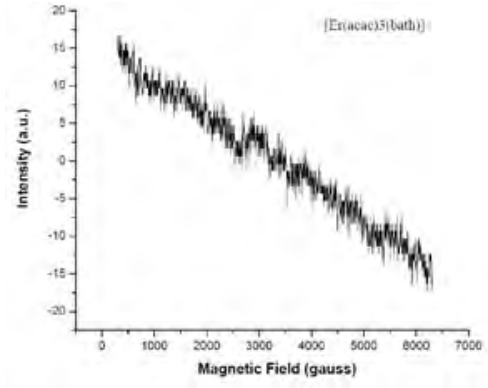

$\left[\operatorname{Er}(\mathrm{acac})_{3}(\mathrm{bath})\right]$

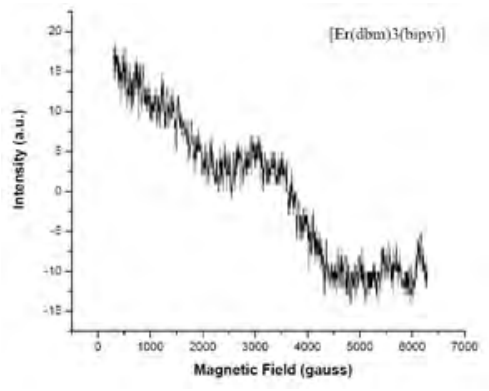

[Er(dbm) $)_{3}$ (bipy)]

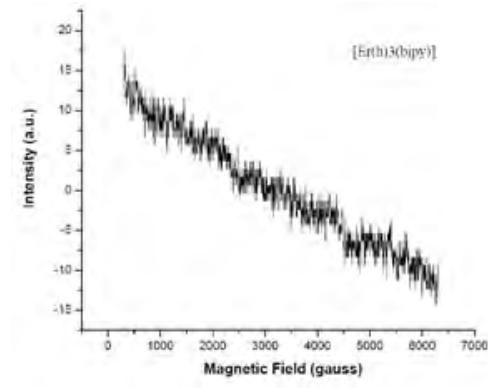

[Er(h) $)_{3}($ bipy)] 


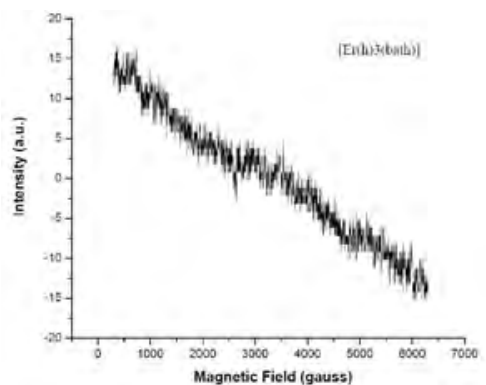

[Er(h) $)_{3}($ bath)]

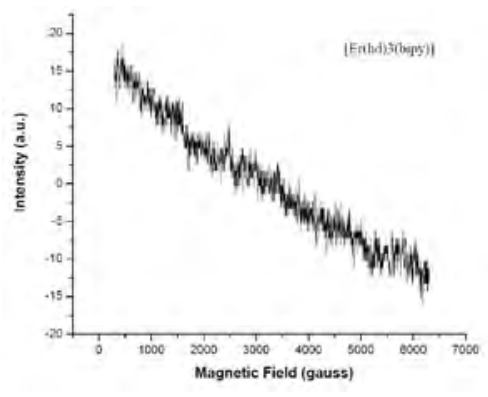

[Er(hd) $)_{3}($ bipy)]

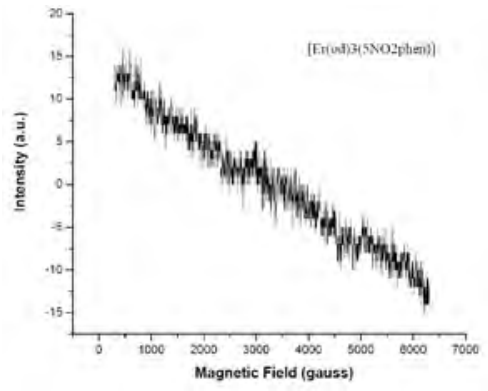

$\left[\operatorname{Er}(\mathrm{od})_{3}\left(5 \mathrm{NO}_{2}\right.\right.$ phen $\left.)\right]$

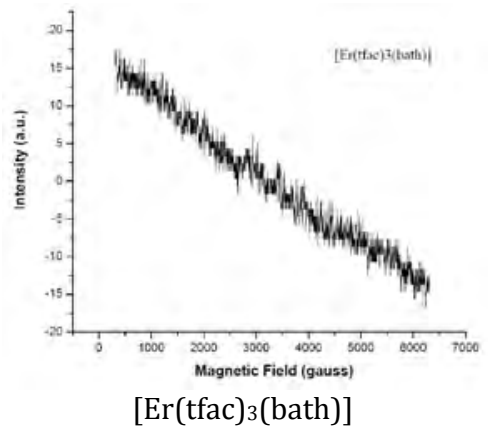

$\left[\operatorname{Er}(\mathrm{tfac})_{3}(\mathrm{bath})\right]$
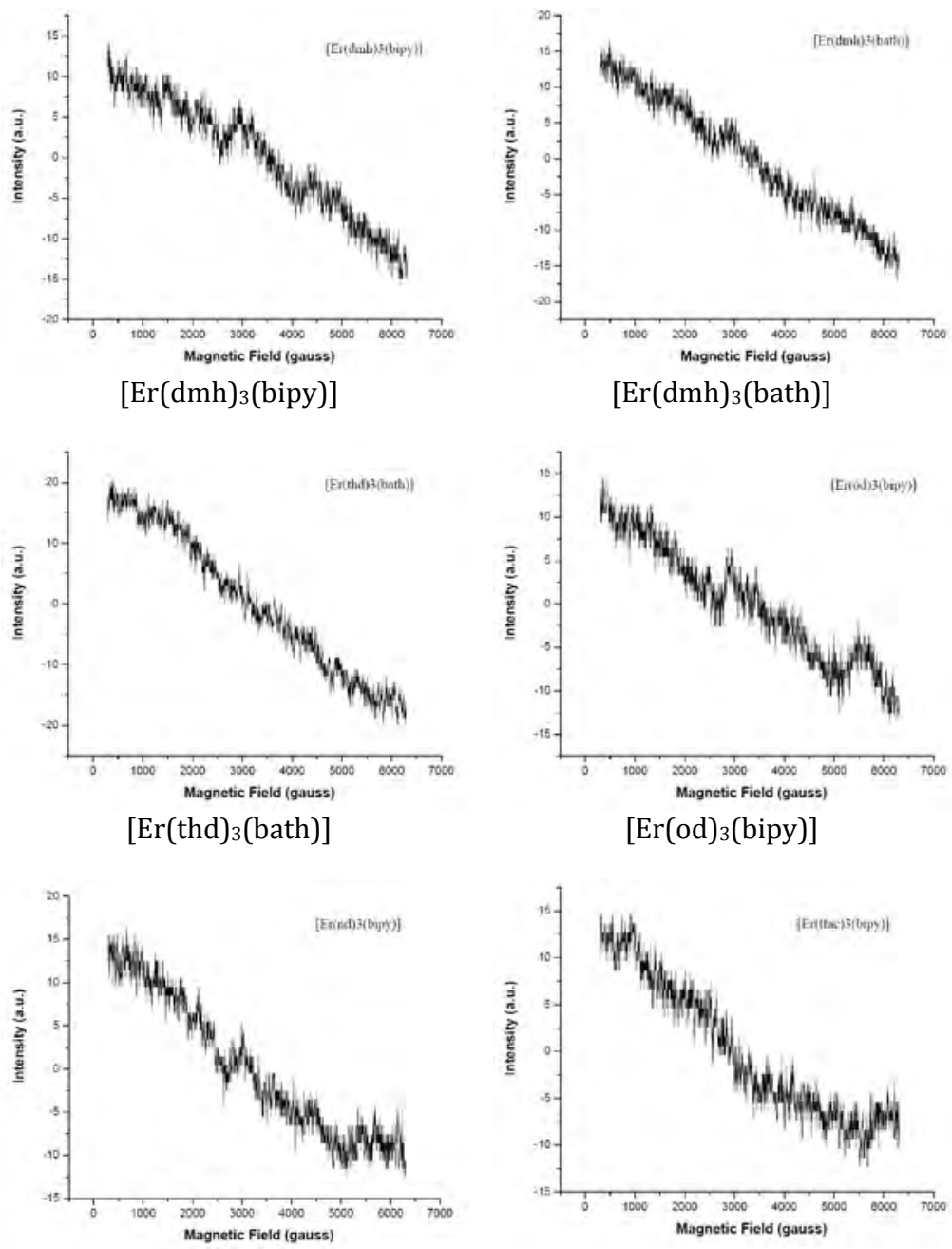

$\left[\operatorname{Er}(\text { nd) })_{3}(\right.$ bipy) $]$

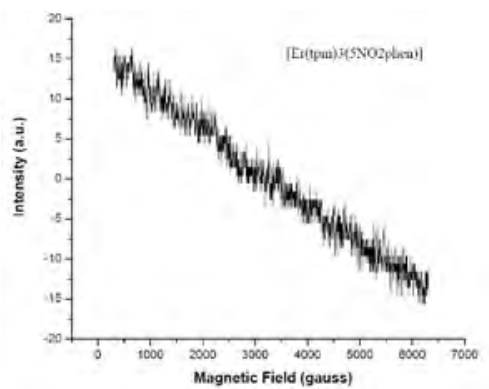

$\left[\operatorname{Er}(\operatorname{tpm})_{3}\left(5 \mathrm{NO}_{2}\right.\right.$ phen $\left.)\right]$

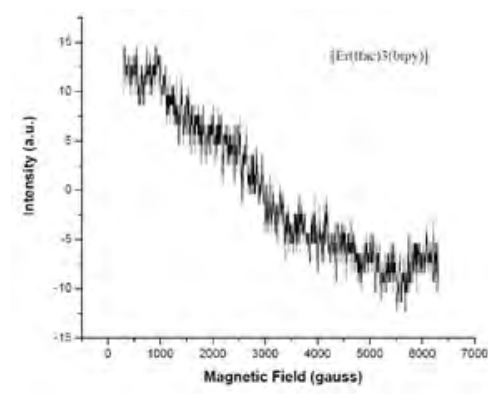

[Er(tfac) $)_{3}($ bipy)]

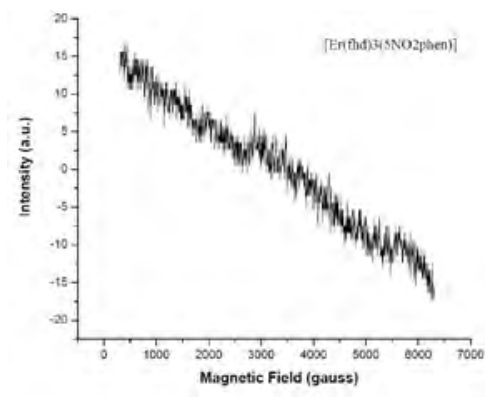

[Er(fhd $)_{3}\left(5 \mathrm{NO}_{2}\right.$ phen $\left.)\right]$

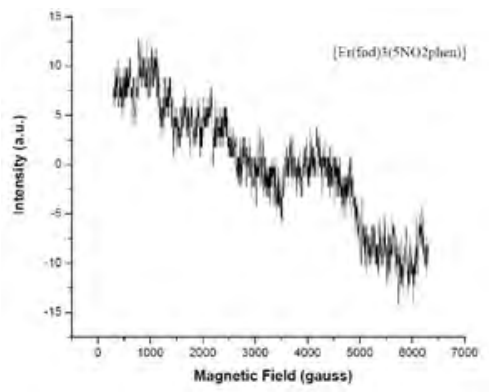

$\left[\operatorname{Er}(\text { fod })_{3}\left(5 \mathrm{NO}_{2}\right.\right.$ phen $\left.)\right]$

Figure 93. EPR spectra of different for ternary erbium(III) $\beta$-diketonate complexes 


\subsubsection{Optical absorption, excitation and photoluminescence studies}

\section{UV-Vis absorption spectra for Er(III) complexes}

Figure 94 shows the optical absorption spectra for several fluorinated and non-fluorinated complexes, in $10^{-5} / 10^{-6} \mathrm{M}$ and in $10^{-3} \mathrm{M}$ methanol diluted solutions.

The absorption spectrum is always dominated by the spin allowed $\pi-\pi^{*}$ transitions of the $\beta$ diketone and the $\mathrm{N}, \mathrm{N}$-donor in the ultraviolet-visible region. Their absorption bands are red shifted in the complexes and show stabilization of the ligand orbitals after complex formation. Nonetheless, the spectral shapes of the complex and free ligands in solution tend to be pretty similar, suggesting that the coordination of the $\mathrm{Er}^{3+}$ ion does not have a significant influence on the $\pi-\pi^{*}$ state energy.

Increasing the solution concentration up to $10^{-3} \mathrm{M}$ allows us to observe that the complex also exhibits the typical parity-forbidden narrow absorption bands, which originate from ground state ${ }^{4} \mathrm{I}_{15 / 2}$ to the various excited states of $\mathrm{Er}^{3+}$ ion, overlapped with the organic ligands absorption. The transitions observed are (a) ${ }^{4} \mathrm{I}_{15 / 2} \rightarrow{ }^{2} \mathrm{G}_{11 / 2}(376 \mathrm{~nm})$, (b) ${ }^{4} \mathrm{I}_{15 / 2} \rightarrow\left({ }^{2} \mathrm{G},{ }^{4} \mathrm{~F},{ }^{2} \mathrm{H}\right)_{9 / 2}$ (405 nm), (c) ${ }^{4} \mathrm{I}_{15 / 2} \rightarrow\left({ }^{4} \mathrm{~F}_{5 / 2},{ }^{4} \mathrm{~F}_{3 / 2}\right)(450 \mathrm{~nm}),(d){ }^{4} \mathrm{I}_{15 / 2} \rightarrow{ }^{4} \mathrm{~F}_{7 / 2}(486.5 \mathrm{~nm}),(e){ }^{4} \mathrm{I}_{15 / 2} \rightarrow{ }^{2} \mathrm{H}_{11 / 2}$ (hypersensitive transition, $522 \mathrm{~nm}$ ), (f) ${ }^{4} \mathrm{I}_{15 / 2} \rightarrow{ }^{4} \mathrm{~S}_{3 / 2}(540 \mathrm{~nm})$ and (g) ${ }^{4} \mathrm{I}_{15 / 2} \rightarrow{ }^{4} \mathrm{~F}_{9 / 2}(650.5 \mathrm{~nm})$, but it should be noted that not all of them can be distinguished in most cases.
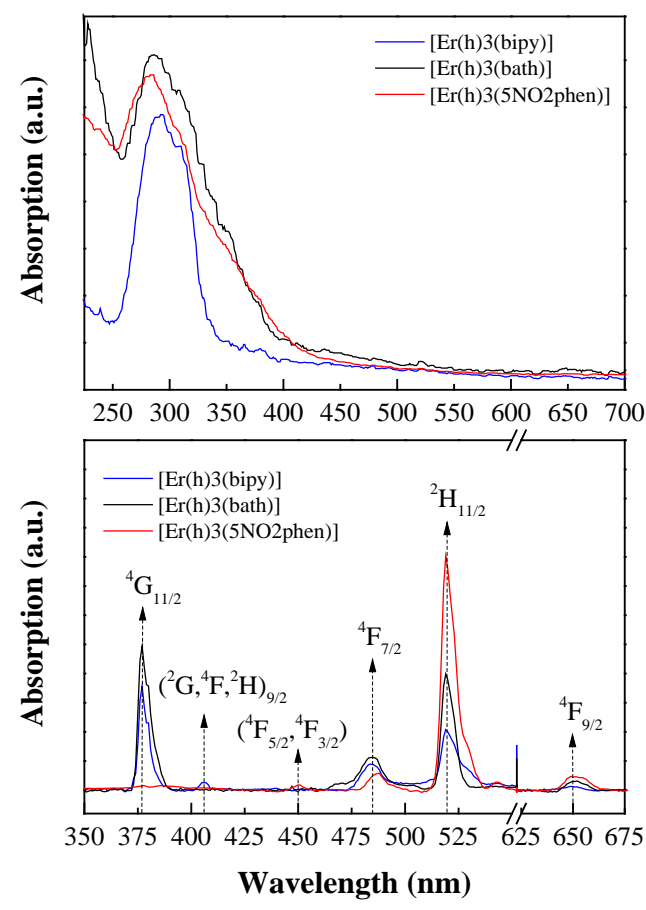

[Er(h)3(N,N-donor)]

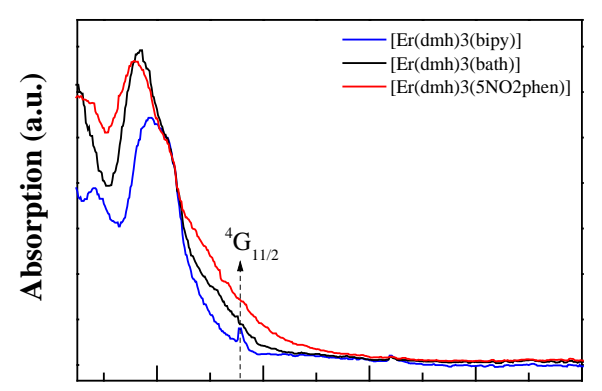

250300350400450500550600650700

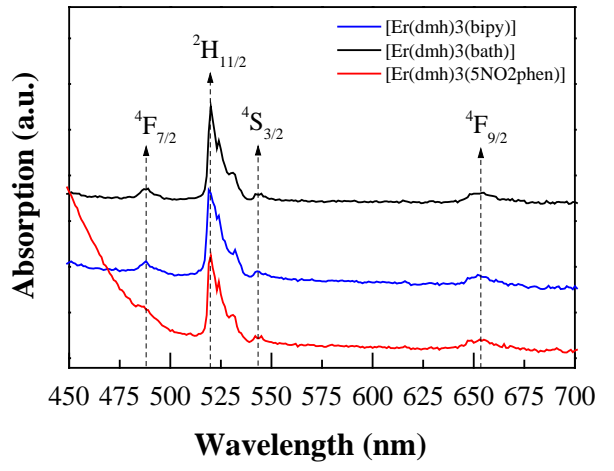

[Er(dmh) $)_{3}(\mathrm{~N}, \mathrm{~N}-$ donor $\left.)\right]$ 


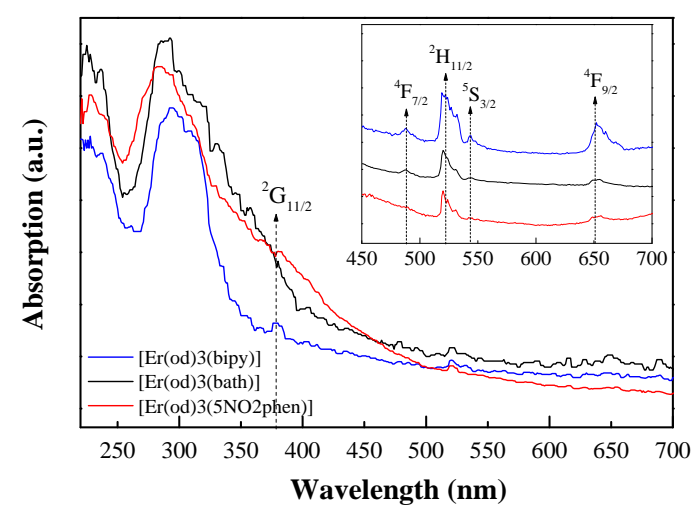

[Er(od) $3(\mathrm{~N}, \mathrm{~N}-$ donor $)]$

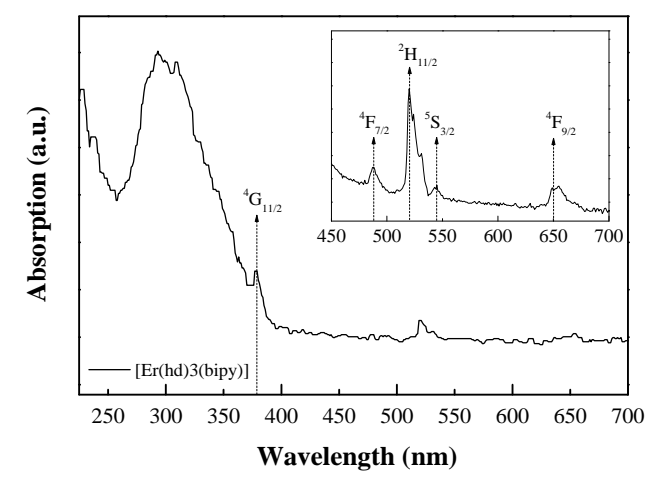

[Er(hd) ${ }_{3}$ (bipy)]

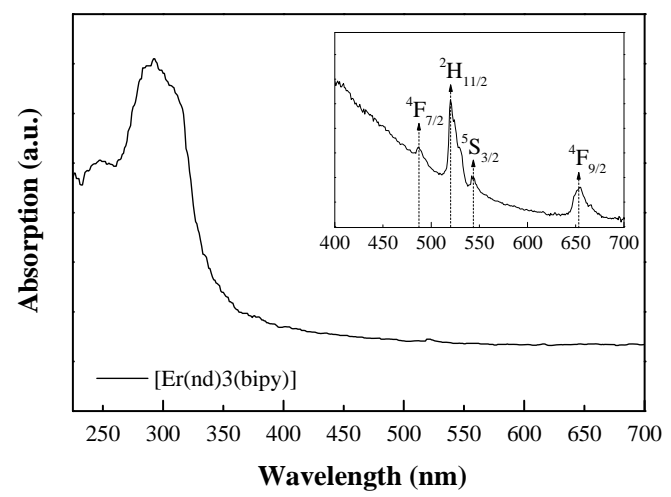

[Er(nd)3(bipy)]

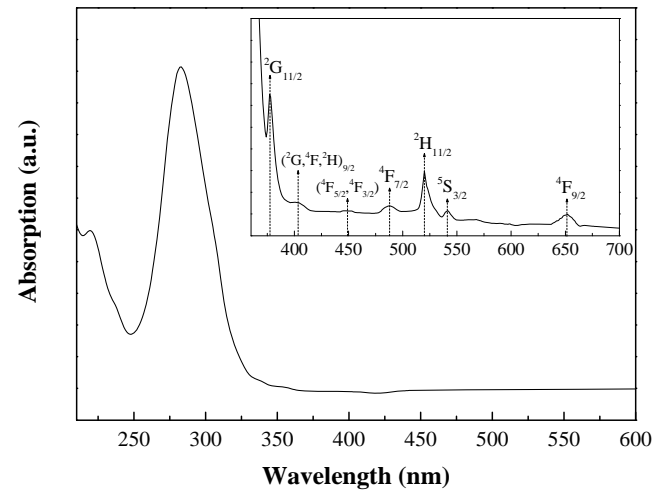

[Er(acac) 3 (bath)]

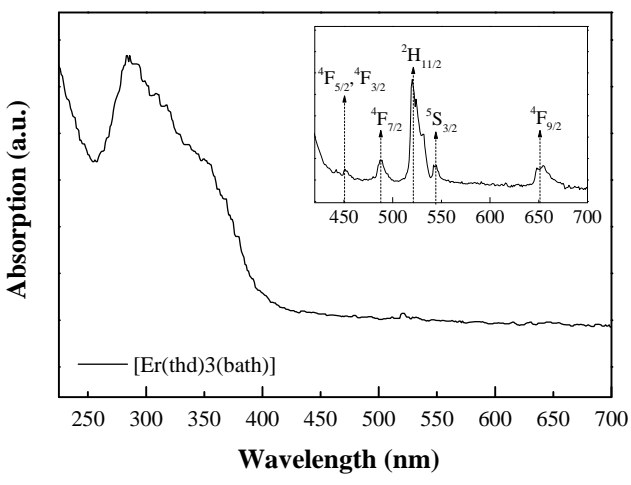

[Er(thd) ${ }_{3}$ (bath)] 

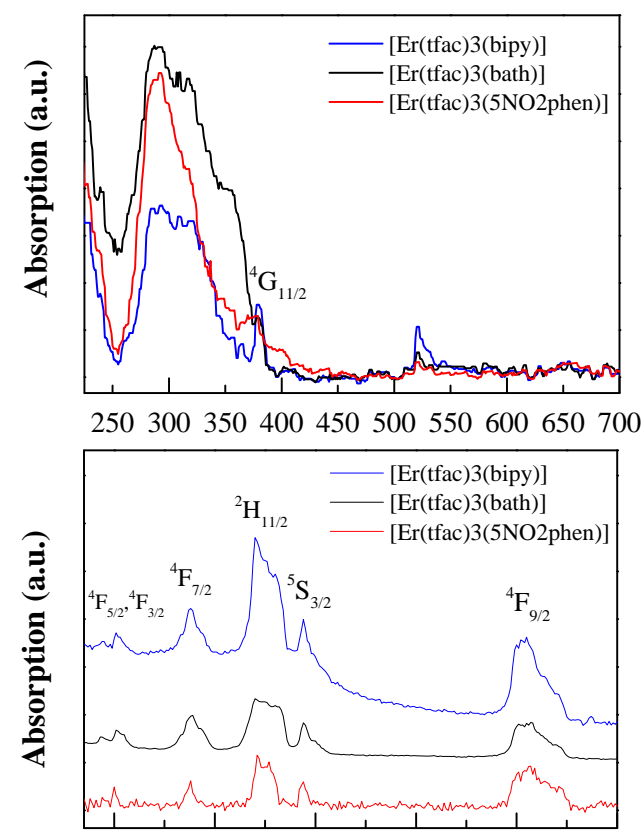

450475500525550575600625650675700

Wavelength (nm)

$\left[\operatorname{Er}(\mathrm{tfac})_{3}(\mathrm{~N}, \mathrm{~N}\right.$-donor $\left.)\right]$

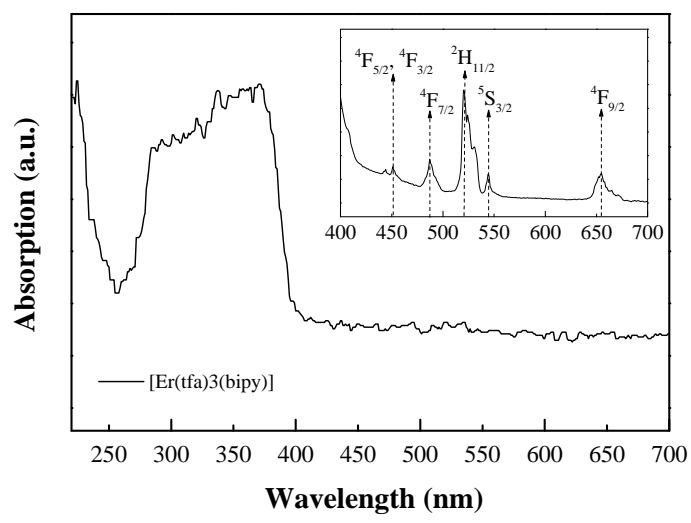

[Er(tfa) $)_{3}($ bipy)]
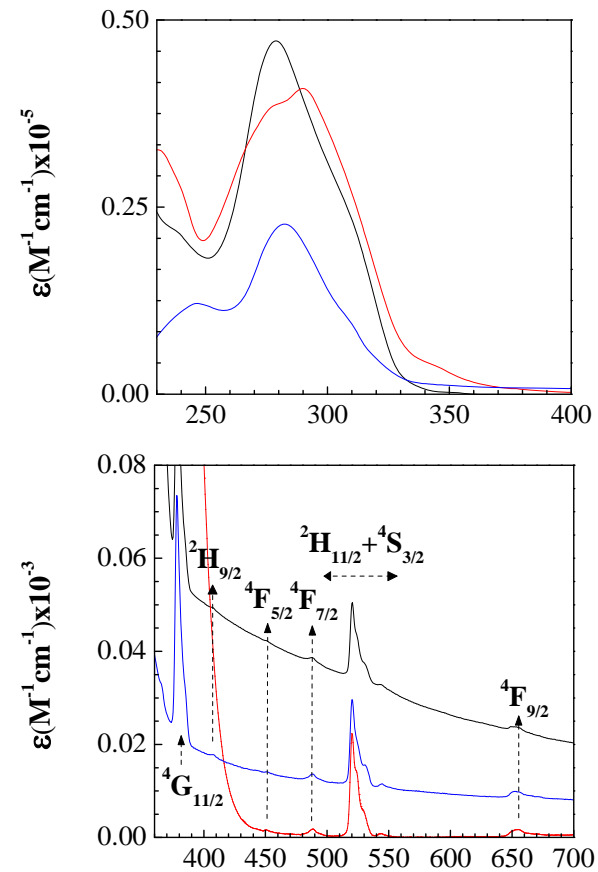

Wavelength (nm)

$\left[\operatorname{Er}(\operatorname{tpm})_{3}(\mathrm{~N}, \mathrm{~N}-\right.$ donor $\left.)\right]$

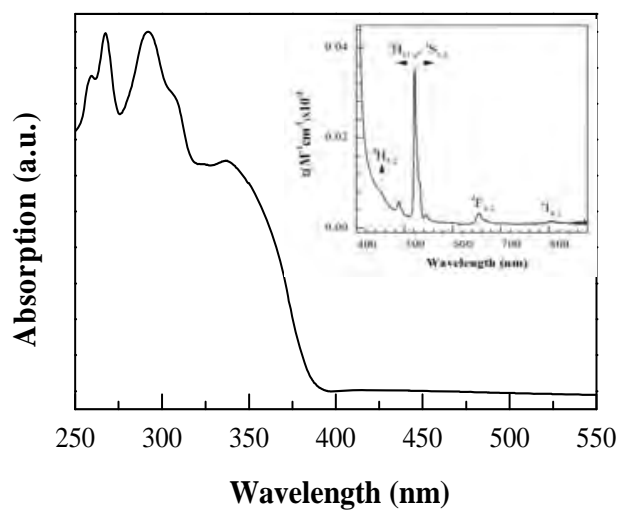

[Er(tfnb) $)_{3}$ (bipy)]

Figure 94. UV-Vis absorption spectra

\section{Remarks on the solvent effect on the $4 f-4 f$ absorption properties}

The band shape of the ${ }^{4} \mathrm{I}_{15 / 2} \rightarrow{ }^{2} \mathrm{H}_{11 / 2},{ }^{4} \mathrm{~S}_{3 / 2}$ hypersensitive transition does not show a significant modification upon changing the solvent (shown for $\left[\operatorname{Er}(\text { thd })_{3}(\right.$ bath)] in Figure 95). This indicates that the complexes are largely inert towards the solvents and this may be due to close packing of the ligands around Er(III), in agreement with the structural data elucidated by single crystal X-ray diffraction. The rigidly planar structure of the $\mathrm{N}, \mathrm{N}$-donors might be helpful in achieving this. 


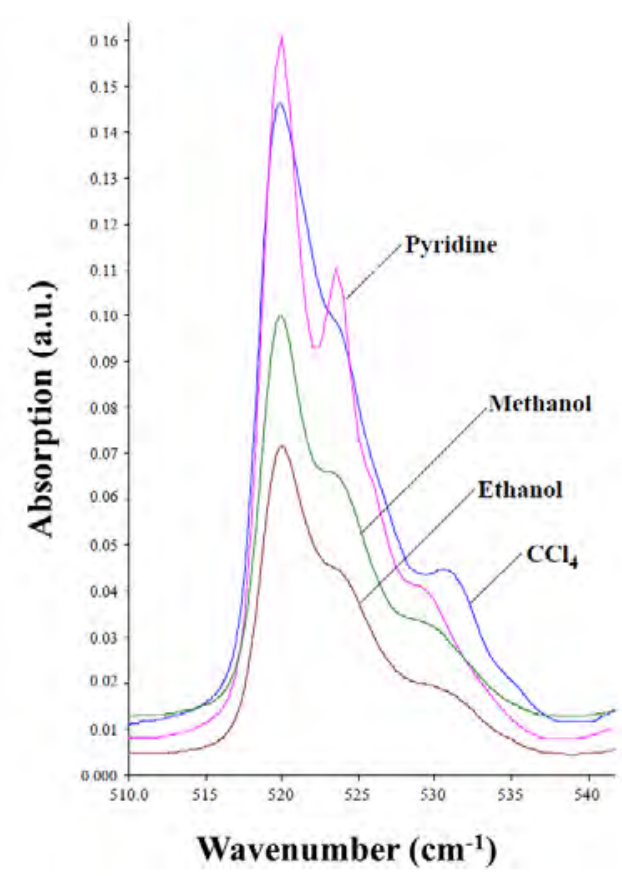

Figure 95. Example of the solvent effect on the band shapes of hypersensitive transition of $\left[\operatorname{Er}(\text { fod })_{3}\right.$ (bath)]

Nonetheless, it should be noted that the basicity of the heterocyclic ligands (bipy, bath and $5 \mathrm{NO}_{2}$ phen) may actually lead to a dissimilar solvent effect. Bathophenanthroline is more basic (pKa=4.67) than 2,2'-bipyridine (pKa=4.38), while 5-nitro-1,10-phenanthroline has the lowest basicity (pKa=3.33) of the three. Being most basic, bath would be expected to contribute more electron density through Er-N bond making the $\mathrm{Er}^{3+}$ ion more electron rich. As a result, the $\mathrm{Er}^{3+}$ shows less attraction for electron donor solvents like methanol, ethanol and pyridine. On the contrary, $5 \mathrm{NO}_{2}$ phen is a weaker base and, after coordination, the $\mathrm{Er}^{3+}$ ion would seek more electron richness and electron donor solvents may enter into the coordination sphere of the metal ion. Prudence suggests the use of non-coordinating solvents (such as methanol or chloroform) so as to retain the bulk composition of the complexes in solution.

\section{UV-Vis spectra for selected $\mathrm{Yb}(\mathrm{III})$ complexes with 5-nitro-1,10-phenanthroline}

As in the Er(III) complexes above, the absorption is dominated by spin allowed $\pi-\pi^{*}$ transitions of the various $\beta$-diketones and 5-nitro-1,10-phenanthroline in the ultravioletvisible region (Figure 96). Absorption bands of the organic ligands are red-shifted in the complex and show stabilization of the ligand orbitals after complex formation. At least two absorption bands, one centered about 270-280 $\mathrm{nm}$, and the second around 320-360 $\mathrm{nm}$, are observed with different relative intensities in the compounds (Table 56).

Table 56. UV-Vis absorption features of the complexes

\begin{tabular}{cccc} 
Complex & Peak wavelength $(\mathrm{nm})$ & Reported for analogous complexes & Reference \\
\hline$\left[\mathrm{Yb}(\mathrm{tfnb})_{3}\left(5 \mathrm{NO}_{2}\right.\right.$ phen $\left.)\right]$ & 270,335 & 267,330 & {$[182]$} \\
{$\left[\mathrm{Yb}(\mathrm{tfa})_{3}\left(5 \mathrm{NO}_{2}\right.\right.$ phen $\left.)\right]$} & 350 & 340 & {$[183]$} \\
{$\left[\mathrm{Yb}(\mathrm{tfac})_{3}\left(5 \mathrm{NO}_{2}\right.\right.$ phen $\left.)\right]$} & 275,342 & 346 & {$[184]$} \\
{$\left[\mathrm{Yb}(\mathrm{tpm})_{3}\left(5 \mathrm{NO}_{2}\right.\right.$ phen $\left.)\right]$} & 283 & $280-283$ & {$[185]$} \\
{$\left[\mathrm{Yb}(\mathrm{fhd})_{3}\left(5 \mathrm{NO}_{2}\right.\right.$ phen $\left.)\right]$} & 281,314 & - & - \\
{$\left[\mathrm{Yb}(\mathrm{h})_{3}\left(5 \mathrm{NO}_{2}\right.\right.$ phen $\left.)\right]$} & 282 & 280 & {$[186]$} \\
{$\left[\mathrm{Yb}(\mathrm{dmh})_{3}\left(5 \mathrm{NO}_{2}\right.\right.$ phen $\left.)\right]$} & 279 & 277 & {$[187]$} \\
\hline
\end{tabular}




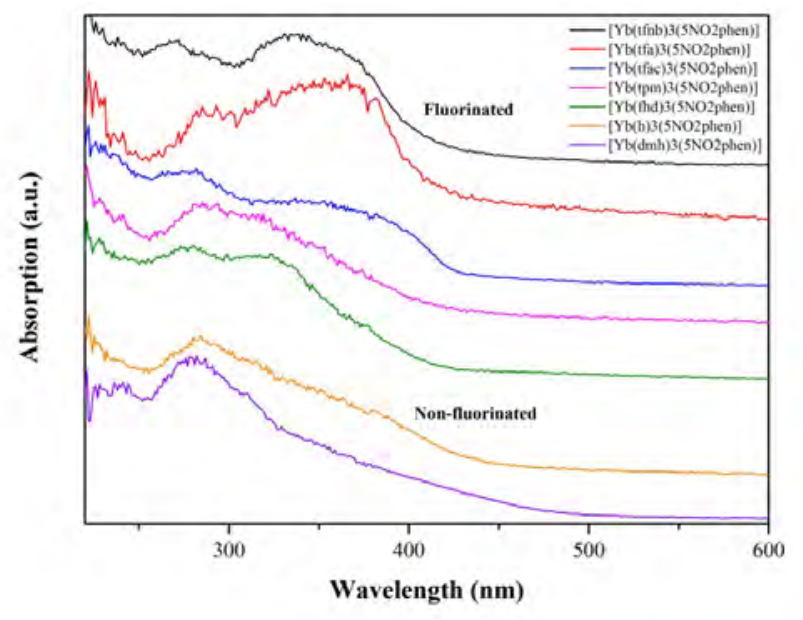

Figure 96. UV-Vis absorption spectra

\section{NIR absorption spectra for Er(III) complexes}

Figure 97 shows the absorption spectra in $\mathrm{KBr}$ pellets for the $900-2000 \mathrm{~nm}$ region for various non-fluorinated and fluorinated complexes.

Several band sets corresponding either to f-f Er transitions or to $\mathrm{C}-\mathrm{H}$ vibrations can be identified: ${ }^{4} \mathrm{I}_{15 / 2} \rightarrow{ }^{4} \mathrm{I}_{11 / 2,13 / 2}$ bands appear at around $980 \mathrm{~nm}$ and $1500-1600 \mathrm{~nm}$, respectively, with some band structure associated to the Stark's components for the ground ${ }^{4} \mathrm{I}_{15 / 2}$ multiplet; while the bands which appear near $1675 \mathrm{~nm}$ and $1140 \mathrm{~nm}$ can be assigned to the second and third overtones of aromatic $\mathrm{C}$-H stretching vibration, respectively.

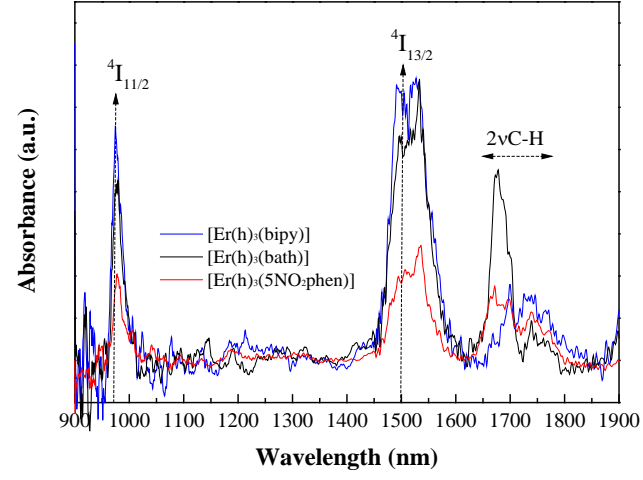

[Er(h) $3(\mathrm{~N}, \mathrm{~N}$-donor $)]$

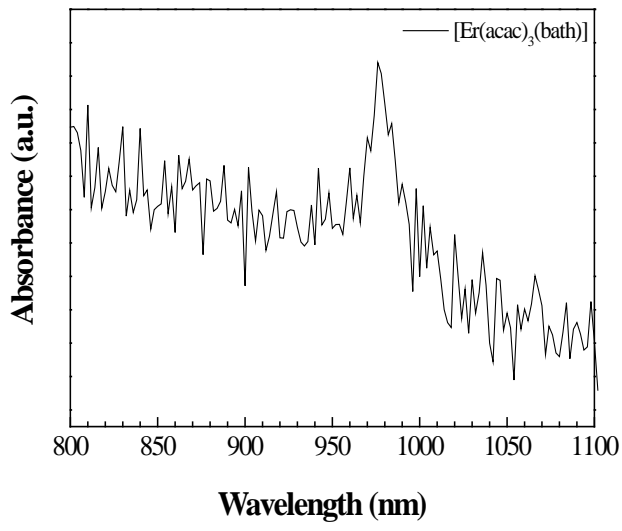

[Er(acac) $\left.)_{3}(\mathrm{bath})\right]$

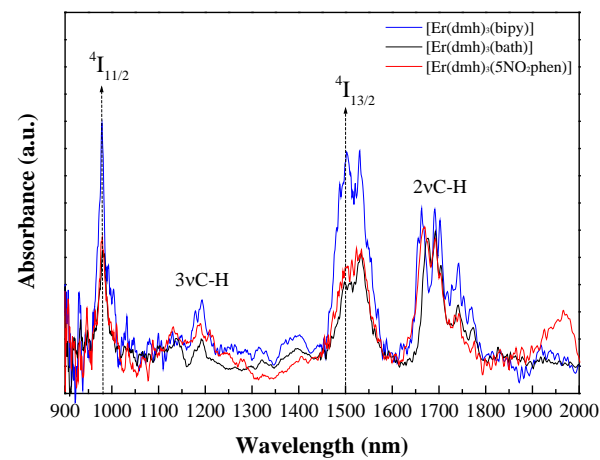

$\left[\operatorname{Er}(\mathrm{dmh})_{3}(\mathrm{~N}, \mathrm{~N}-\right.$ donor $\left.)\right]$

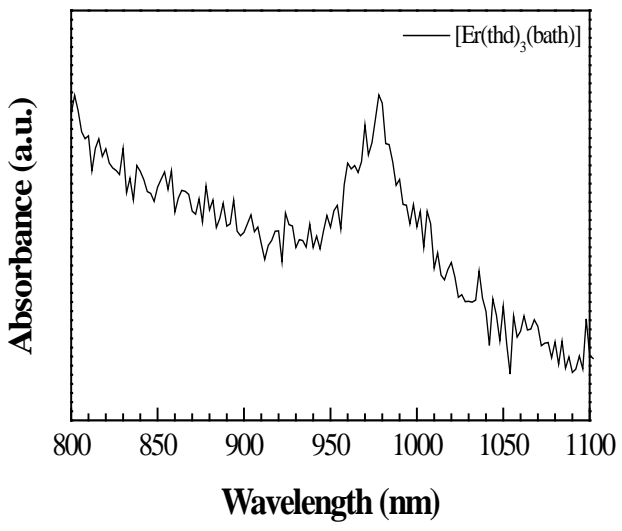

[Er(thd) 3 (bath)] 


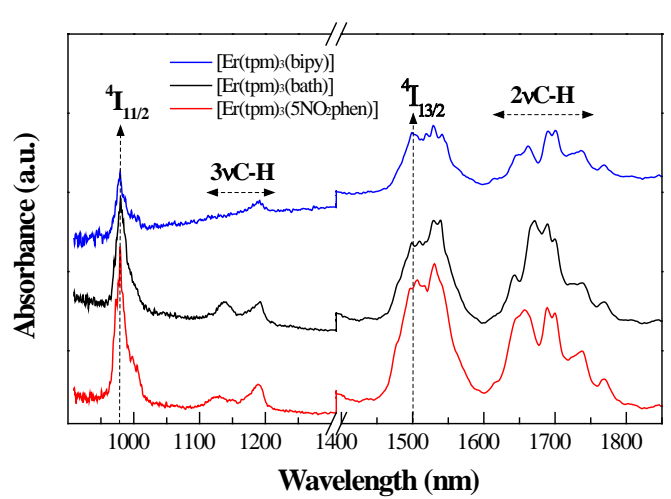

$[\operatorname{Er}(\operatorname{tpm}) 3(\mathrm{~N}, \mathrm{~N}-$ donor $)]$

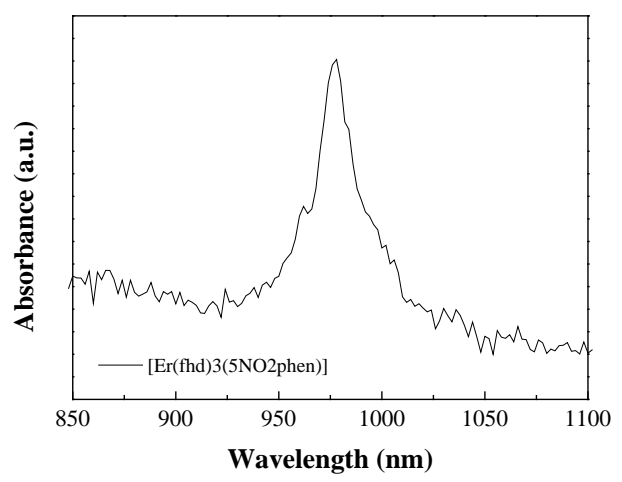

[Er(fhd $)_{3}\left(5 \mathrm{NO}_{2}\right.$ phen $\left.)\right]$

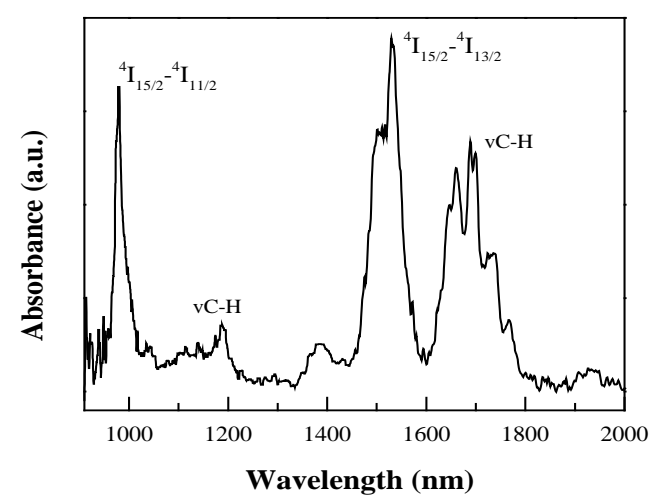

[Er(tfnb) $)_{3}($ bipy) $]$

Figure 97. NIR absorption spectra in $\mathrm{KBr}$ pellets

\section{Excitation spectra}

Excitation spectra obtained by fluorescence spectroscopy, whereby the emission light is held at a constant wavelength (1532 $\mathrm{nm}$ for Er(III) complexes and $980 \mathrm{~nm}$ for $\mathrm{Yb}(\mathrm{III})$ complexes) and the excitation light is scanned through many different wavelengths (via a monochromator), are complementary of those obtained by absorption spectroscopy, that is, one can think of an excitation spectrum as "fluorescence detected absorption".

The processes involved are the same: the electronic transition from the ground state to the first and second excited states. Because of this, the two spectra have generally the same shape and are almost superimposable. Nevertheless, absorption and excitation spectra do not correspond if there are more species in the ground state, or if the sole present species has different forms in the the ground state (aggregates, complexes, tautomeric forms, etc). In such cases the comparison of the two spectra can provide very useful information.

Since for the complexes herein refered we have the first situation, only a spectrum is provided for illustrative purposes $\left(\left[\operatorname{Er}(\mathrm{acac})_{3}(\right.\right.$ bath $\left.)\right]$, Figure 98). It consists of a broad band $(325-450 \mathrm{~nm})$ with maximum at $378 \mathrm{~nm}$, which corresponds to the excitation of the organic chromophores (Hacac and bathophenanthroline), and weaker intraconfigurational $f-f$ transitions from the ${ }^{4} \mathrm{I}_{15 / 2}$ ground state to the ${ }^{4} \mathrm{~F}_{7 / 2}(486 \mathrm{~nm})$ and ${ }^{2} \mathrm{H}_{11 / 2}(522 \mathrm{~nm})$ levels of the Er(III) ion. The excitation spectrum reveals that the energy transfer from the ligand to Er(III) centre is efficient since the $S_{0} \rightarrow S_{1}$ excitation band is dominant in comparison to the $f-f$ transitions. Therefore, both ligands, Hacac and bath, are efficient in transferring energy to erbium which leads to strong sensitization of Er(III) emission, discussed below.

In the case of $\mathrm{Yb}(\mathrm{III})$ complexes, the excitation spectra are similar, except for the $f-f$ transitions (Figure 99). 


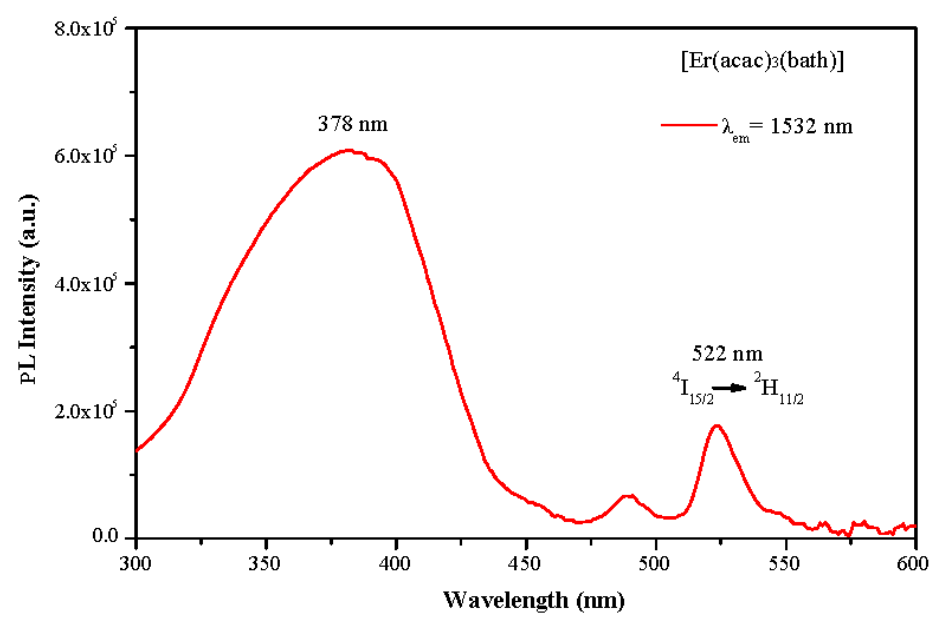

Figure 98. Excitation spectrum for $\left[\operatorname{Er}(\mathrm{acac})_{3}(\right.$ bath $\left.)\right]$ under detection at $1532 \mathrm{~nm}$

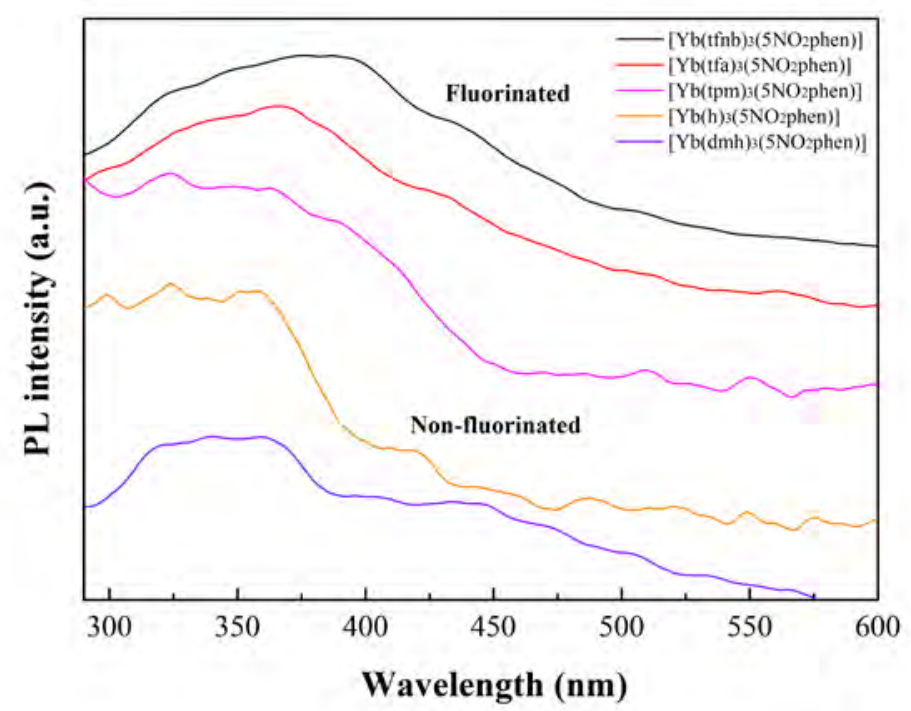

Figure 99. Excitation spectra of some of the $\mathrm{Yb}(\mathrm{III})$ complexes under detection at $980 \mathrm{~nm}$

\section{PL emission in the visible region}

The emission in the visible region (410-700 $\mathrm{nm}$ ) has generally been studied under direct excitation of the organic ligands at $\lambda=405 \mathrm{~nm}$ (Figure 100). The organic part of the complexes exhibits a broad emission with maxima at around $420 \mathrm{~nm}$. Weak $\mathrm{Er}^{3+}$-associated visible emission, arising from ${ }^{4} \mathrm{~S}_{3 / 2} \rightarrow{ }^{4} \mathrm{I}_{15 / 2}$ transition, can also be observed.

Since the emission spectrum has usually been measured in powder, it is expected that it may be slightly red-shifted in comparison to that obtained in solution (such as those for the $\left[\operatorname{Er}(\operatorname{tpm})_{3}(\mathrm{~N}, \mathrm{~N}-\right.$ donor $\left.)\right]$ complexes, obtained upon excitation at $\left.\lambda=270 \mathrm{~nm}\right)$, as a result of the aggregation state.

Regarding the emission intensities, even though the intensities of the spectra in powder form cannot be strictly compared, assuming the same incident energy density, we can qualitatively observe the same behaviour as in solution (e.g. for $\left[\operatorname{Er}(\operatorname{tpm})_{3}(\mathrm{~N}, \mathrm{~N}-\right.$ donor $\left.)\right]$ complexes, in 
which visible PL emission was measured both in solution and in powder). Furthermore, these visible emission intensities are in good agreement with the visible emission decay data (vide infra).

The quenching of the visible emission of the complexes is related to the efficiency of the energy transfer capability from the ligand to $\mathrm{Er}^{3+}$ ion, the aforementioned antenna effect. At the same time, it is to be expected that the characteristic NIR emission of the Er: ${ }^{4} I_{13 / 2} \rightarrow{ }^{4} I_{15 / 2}$ transition is favored.

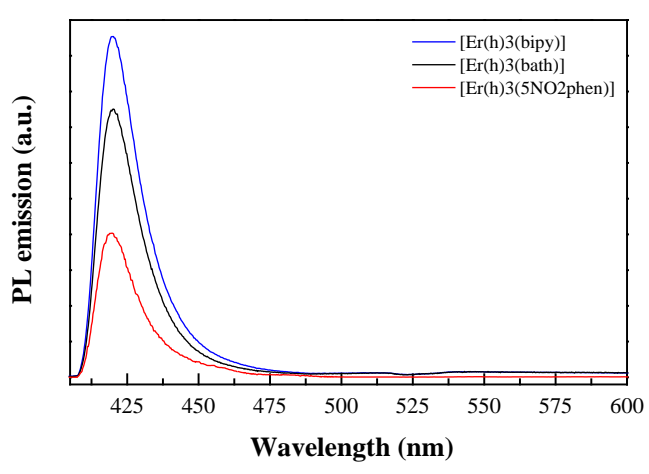

$\left[\operatorname{Er}(\mathrm{h})_{3}(\mathrm{~N}, \mathrm{~N}-\right.$ donor $\left.)\right]$

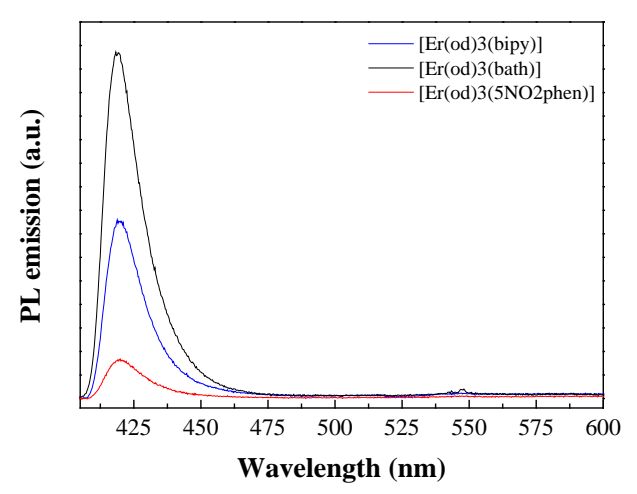

$\left[\operatorname{Er}(\mathrm{od}){ }_{3}(\mathrm{~N}, \mathrm{~N}-\right.$ donor $\left.)\right]$

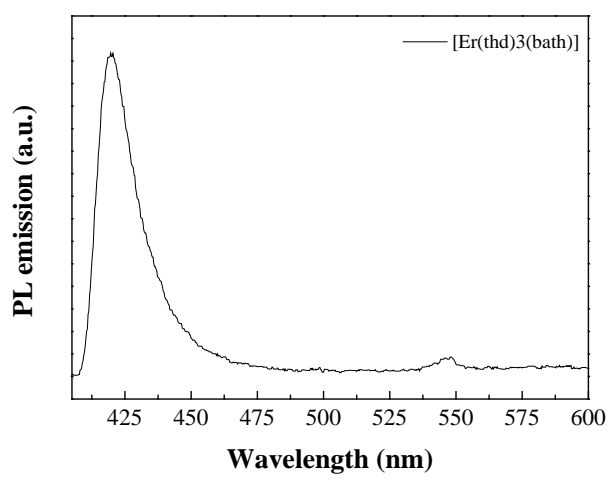

$\left[\operatorname{Er}(\text { thd })_{3}(\right.$ bath $\left.)\right]$

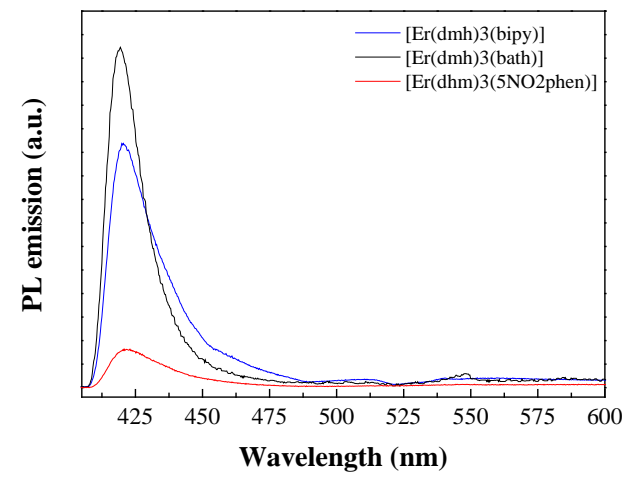

$\left[\operatorname{Er}(\mathrm{dmh})_{3}(\mathrm{~N}, \mathrm{~N}-\right.$ donor $\left.)\right]$

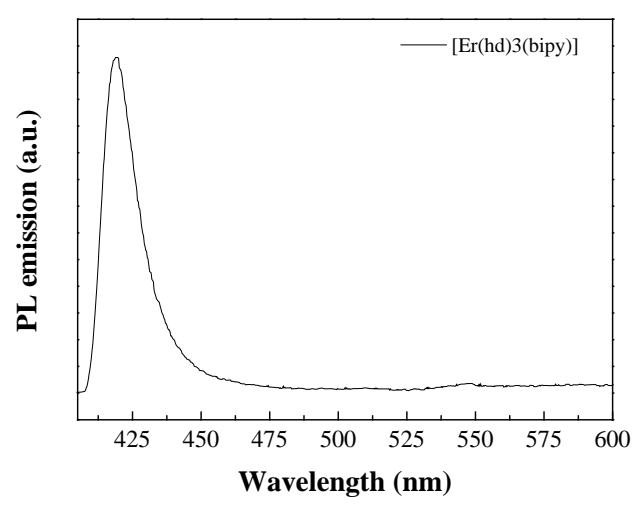

[Er(hd)3(bipy)]

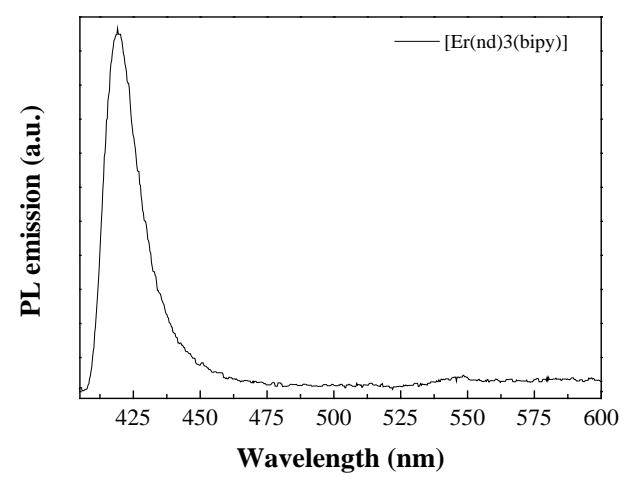

[Er(nd) ${ }_{3}($ bipy)] 


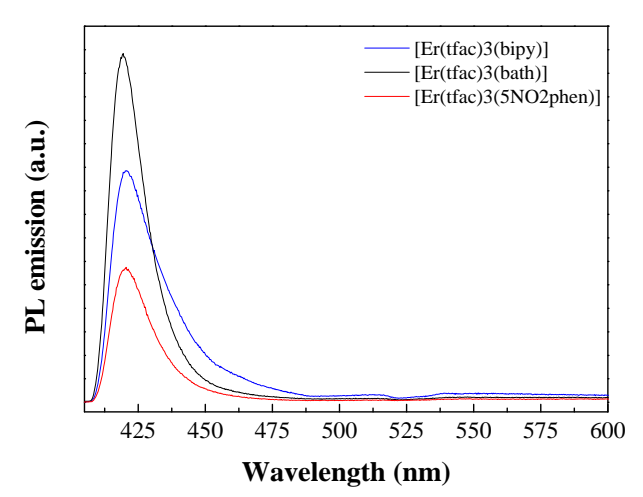

$\left[\operatorname{Er}(\operatorname{tfac})_{3}(\mathrm{~N}, \mathrm{~N}-\right.$ donor $\left.)\right]$

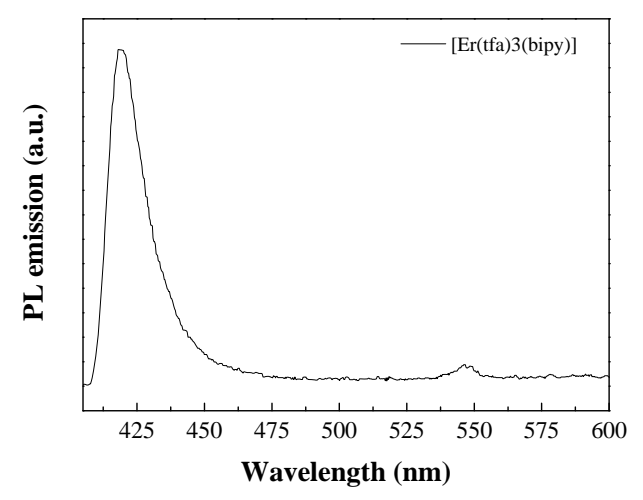

[Er(tfa) $)_{3}($ bipy) $]$

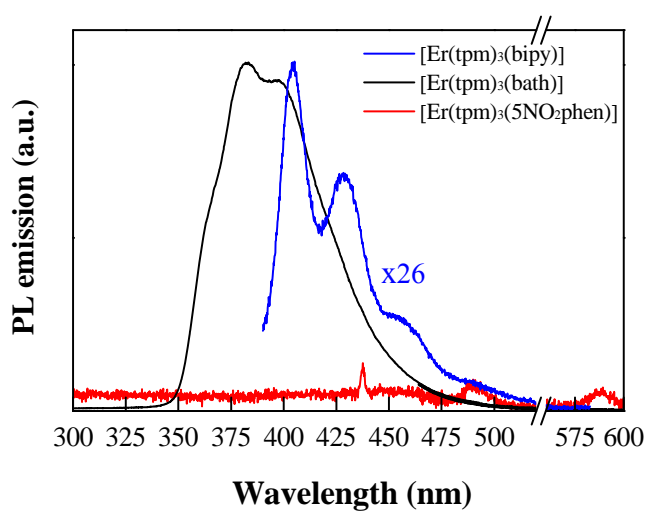

$\left[\operatorname{Er}(\operatorname{tpm})_{3}(\mathrm{~N}, \mathrm{~N}-\right.$ donor $\left.)\right]$

Figure 100. PL emission spectra upon ligand excitation (at $\lambda=405 \mathrm{~nm}$ or at $\lambda=270 \mathrm{~nm}$ )

\section{NIR PL emission for Er(III) complexes}

Figure 101 shows a comparison between the NIR emission (Er ${ }^{3+}:{ }^{4} I_{13 / 2} \rightarrow{ }^{4} I_{15 / 2}$ transition) at room temperature under excitation of the organic ligand at $\lambda=337 \mathrm{~nm}$ and under direct excitation of $\mathrm{Er}^{3+}:{ }^{2} \mathrm{H}_{11 / 2}$ state $(532 \mathrm{~nm})$ for some selected complexes (namely $\left[\operatorname{Er}(\mathrm{h})_{3}(\mathrm{~N}, \mathrm{~N}-\right.$ donor $)], \quad\left[\operatorname{Er}(\mathrm{dmh})_{3}(\mathrm{~N}, \mathrm{~N}-\right.$ donor $\left.)\right], \quad\left[\operatorname{Er}(\mathrm{tfac})_{3}(\mathrm{~N}, \mathrm{~N}-\mathrm{donor})\right], \quad\left[\operatorname{Er}(\mathrm{tfa})_{3}(\mathrm{bipy})\right] \quad$ and $\left[\operatorname{Er}(\operatorname{tfnb})_{3}(\right.$ bipy)] $)$. We may observe that -as expected- both excitation methods yield the same results, provided that the structures of these bands are very similar. The emission band shows a maximum at around $1532 \mathrm{~nm}$ and some structure related with the Stark energy level and electron population distributions of ${ }^{4} \mathrm{I}_{13 / 2}$ and ${ }^{4} \mathrm{I}_{15 / 2}$ multiplets.

There are some exceptions, always for complexes with 5-nitro-1,10-phenanthrolinem, in which the ligand mediated emission is accompanied by strong reabsorption associated to ${ }^{4} \mathrm{I}_{13 / 2}$ level $(1500.5 \mathrm{~nm})$, but we have been able to confirm that this is actually related to the measurement procedure, since a change in the orientation of the sample has an impact on the false minimum intensity, making it more (less) pronounced when the sample is perpendicular (parallel) to the excitation. Nonetheless, this effect does not appear in the

$\mathrm{m}$ In the three families of complexes mentioned above, a qualitative comparison of the emission intensities upon Er(III) direct excitation (Figure 103) indicated that the most intense emission arose from the $5 \mathrm{NO}_{2}$ phen complexes, so the reabsorption phenomenon probably becomes apparent as a result of their stronger emission (i.e. it may also be present in bipy and bath complexes, but is hidden by their weaker emission). 
electroluminescence spectra, so it is not a concern for the application of the materials to OLED devices.

Apart from the band shapes, it is also important to compare the relative emission intensities upon excitation at the two chosen wavelengths (for the same complex) and for different complexes with the same $\beta$-diketone and different $\mathrm{N}, \mathrm{N}$-donor moieties (at the same excitation wavelength).

Regarding the former, since the luminescence intensity is not only proportional to the luminescence quantum yield but also to the amount of light absorbed, it is necessary to evidence the importance of the ligand sensitization effect ( $v s$. a more limited amount of radiation absorbed by direct excitation in the $4 \mathrm{f}$ levels). Thus, for $\left[\operatorname{Er}(\mathrm{acac})_{3}(\mathrm{bath})\right]$ and $\left[\operatorname{Er}(\mathrm{fhd})_{3}\left(5 \mathrm{NO}_{2}\right.\right.$ phen)] complexes, the emission spectra have been recorded (and corrected for the different source intensities) at $350 \mathrm{~nm}$ and at $532 \mathrm{~nm}$. It can be observed that the shape of the spectrum is again the same in both cases, but upon excitation at $532 \mathrm{~nm}$ the PL intensity is half or a third than that gained upon excitation at $350 \mathrm{~nm}$ (Figure 102).

With regard to the impact of the ancillary ligand choice on the NIR PL intensity, Figure 103 presents several qualitative comparisons, for $\left[\operatorname{Er}(\mathrm{h})_{3}(\mathrm{~N}, \mathrm{~N}-\right.$ donor $\left.)\right],\left[\operatorname{Er}(\mathrm{dmh})_{3}(\mathrm{~N}, \mathrm{~N}-\right.$ donor $\left.)\right]$, $\left[\operatorname{Er}(\mathrm{od})_{3}(\mathrm{~N}, \mathrm{~N}-\right.$ donor $\left.)\right]$ and $\left[\operatorname{Er}(\mathrm{tfac})_{3}(\mathrm{~N}, \mathrm{~N}-\right.$ donor $\left.)\right]$ complexes upon $\operatorname{Er}(\mathrm{III})$ direct excitation at $522 \mathrm{~nm}$. [Er(tpm) $3(\mathrm{~N}, \mathrm{~N}$-donor)] complexes case is particularly illustrative, since the comparison has been conducted not only upon $\mathrm{Er}^{3+}: 4 \mathrm{I}_{11 / 2}$ level excitation $(980 \mathrm{~nm})$, but also upon excitation of the organic ligand at $\lambda_{\text {exc }}=337 \mathrm{~nm}\left(\mathrm{~N}_{2}\right.$ laser $) .\left[\operatorname{Er}(\mathrm{tpm})_{3}\left(5 \mathrm{NO}_{2}\right.\right.$ phen $\left.)\right]$ complex emission is much higher, in agreement with the structural findings: absence of solvent-accessible voids and shorter Er-N distances. This confirms again the behaviour observed for visible emission: an inverse relationship between the intensity of $\mathrm{Er}^{3+}$ : ${ }^{4} \mathrm{I}_{13 / 2} \rightarrow{ }^{4} \mathrm{I}_{15 / 2}$ emission and visible emission of the organic ligand. Similar behaviour is observed for the other families.

Finally, in order to assess the goodness of the chosen approach for the design of the complexes, a quantitative comparison was conducted for 6 samples: two of the novel $\beta$ diketonate complexes (a non-fluorinated complex and a fluorinated one) vs. a highly hydrated and hydroxilated tetranitrate pentasodium-tetrahydroxide tetradecahydrate Er(III) complex (in solid state, $\mathrm{CCl}_{3}$ and $\mathrm{D}_{2} \mathrm{O}$ ) and an eneahydrated fluorocomplex trifluoromethanesulfonate $\operatorname{Er}(\mathrm{III})$ complex. Figure 105 shows that the most intense emission is achieved for the decafluorinated $\left[\mathrm{Er}(\mathrm{fhd})_{3}\left(5 \mathrm{NO}_{2}\right.\right.$ phen $\left.)\right]$, followed by that of $\left[\operatorname{Er}(\mathrm{acac})_{3}(\mathrm{bath})\right]$, whereas for the other samples no emission is appreciable. Thus, the effects of the replacement of hydrogen with fluorine on the triplet state energy of the ligands are evinced: fluorine, as expected according to the discussion presented in Section 1.1, minimizes quenching by $\mathrm{O}-\mathrm{H}$ and $\mathrm{N}-\mathrm{H}$ oscillators and significanty improves the luminescent properties of the complex. 

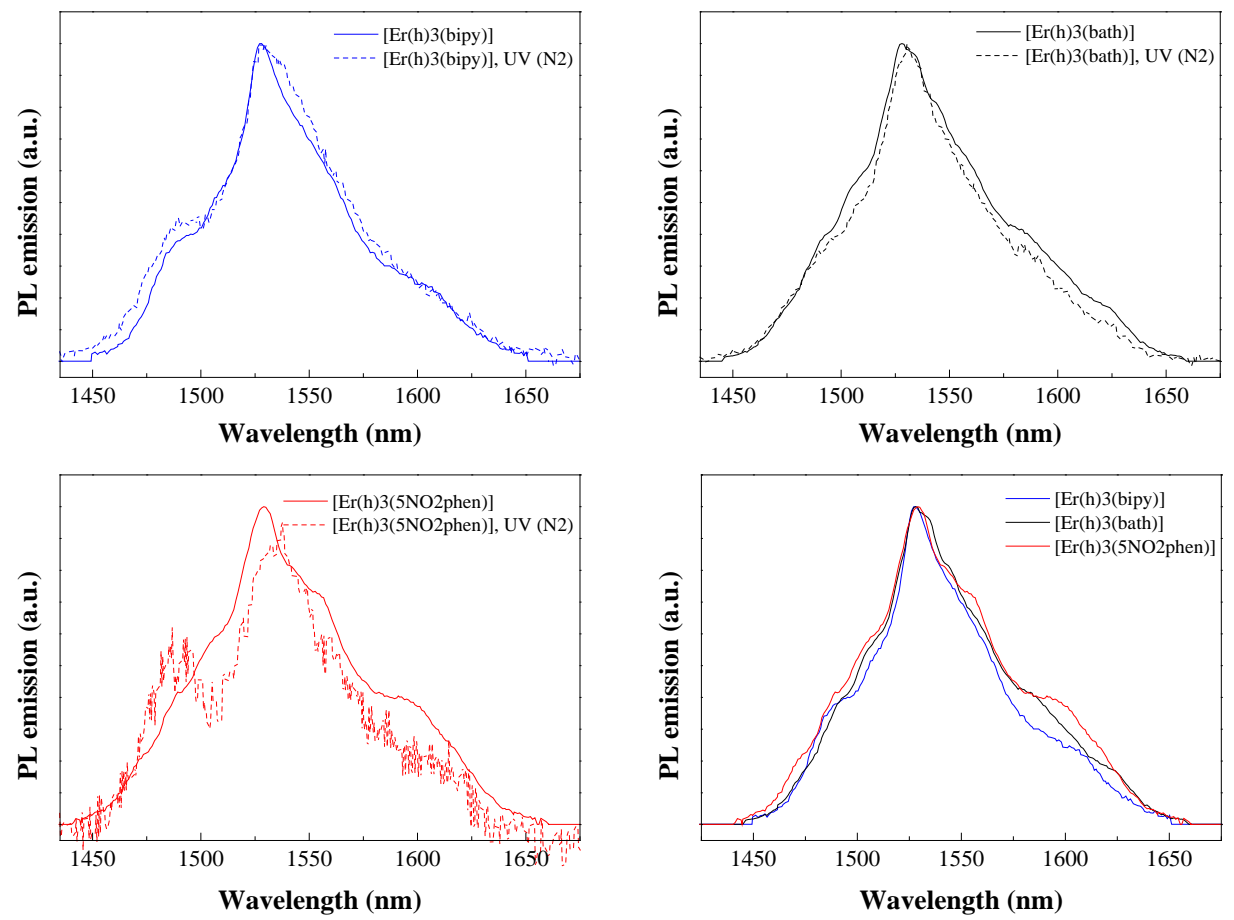

[Er(h) $)_{3}(\mathrm{~N}, \mathrm{~N}-$ donor $\left.)\right]$
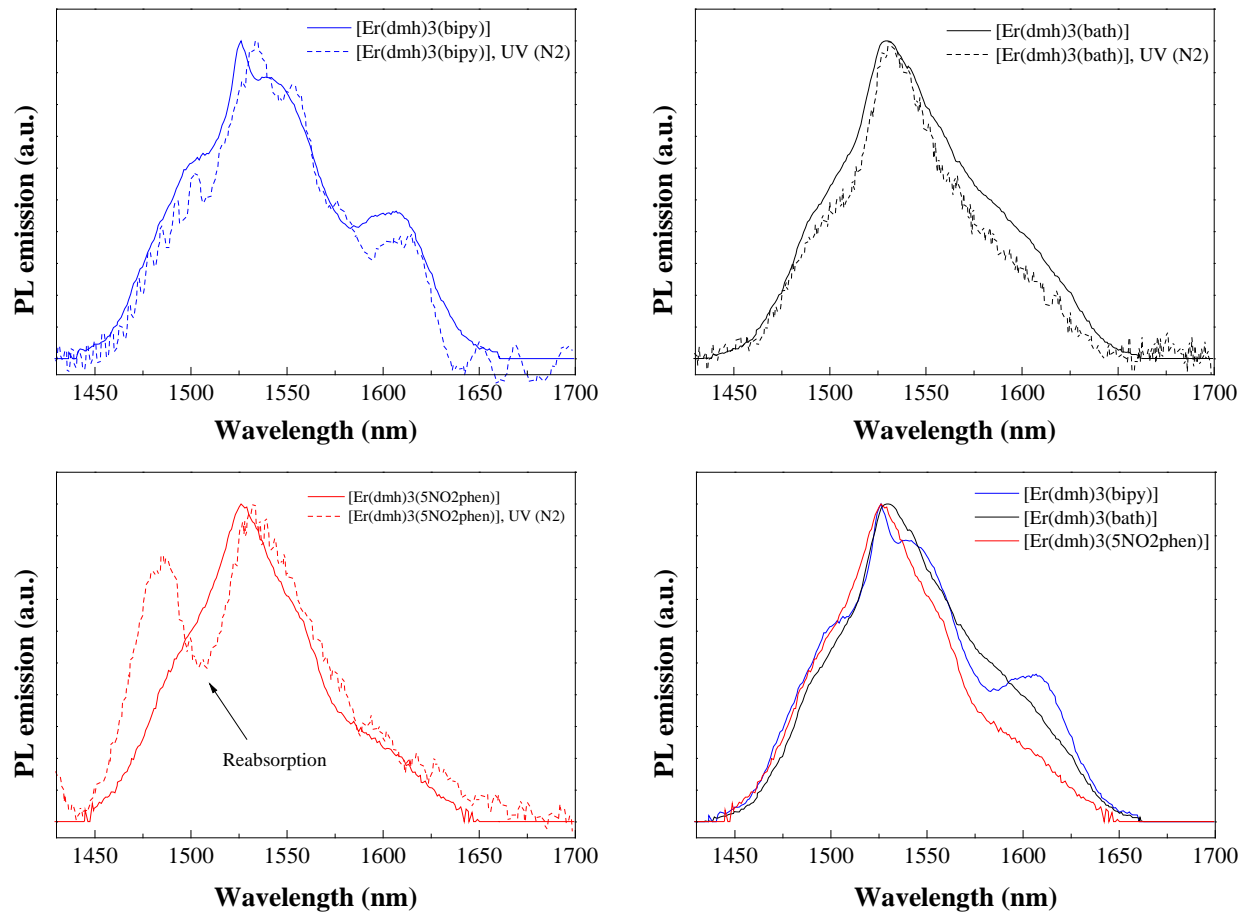

[Er(dmh) $)_{3}(\mathrm{~N}, \mathrm{~N}-$ donor $\left.)\right]$ 

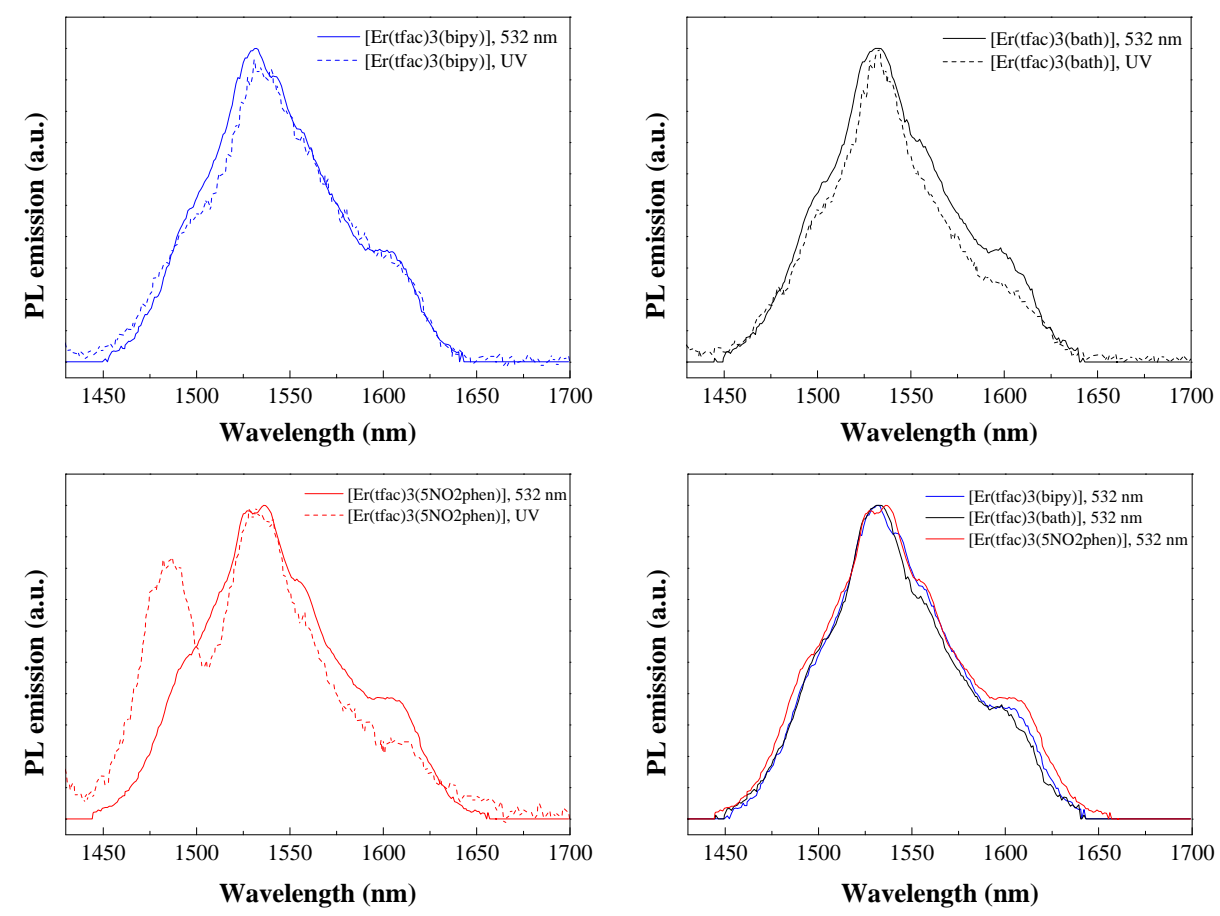

[Er(tfac) $3(\mathrm{~N}, \mathrm{~N}-$ donor $)]$

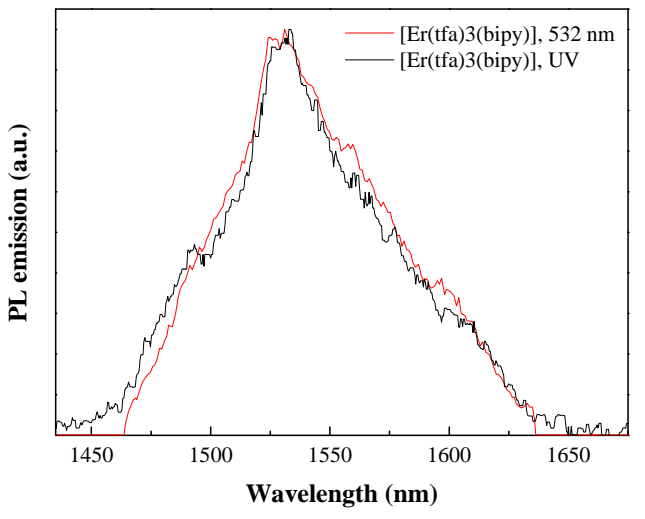

[Er(tfa) $)_{3}($ bipy) $]$

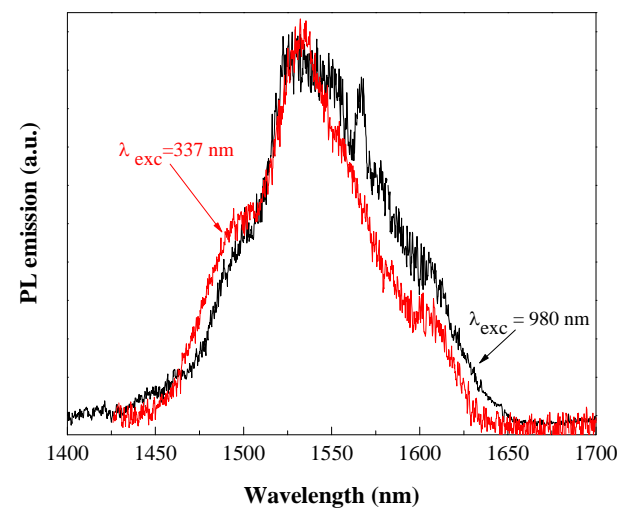

[Er(tfnb) $)_{3}($ bipy)]

Figure 101. Comparison of the infrared ${ }^{4} \mathrm{I}_{13 / 2} \rightarrow{ }^{4} \mathrm{I}_{15 / 2}$ emission spectra upon ligand excitation in the UV $(\lambda=337 \mathrm{~nm})$ and upon $\operatorname{Er}(\mathrm{III})$ direct excitation (at $\lambda=522 \mathrm{~nm})$
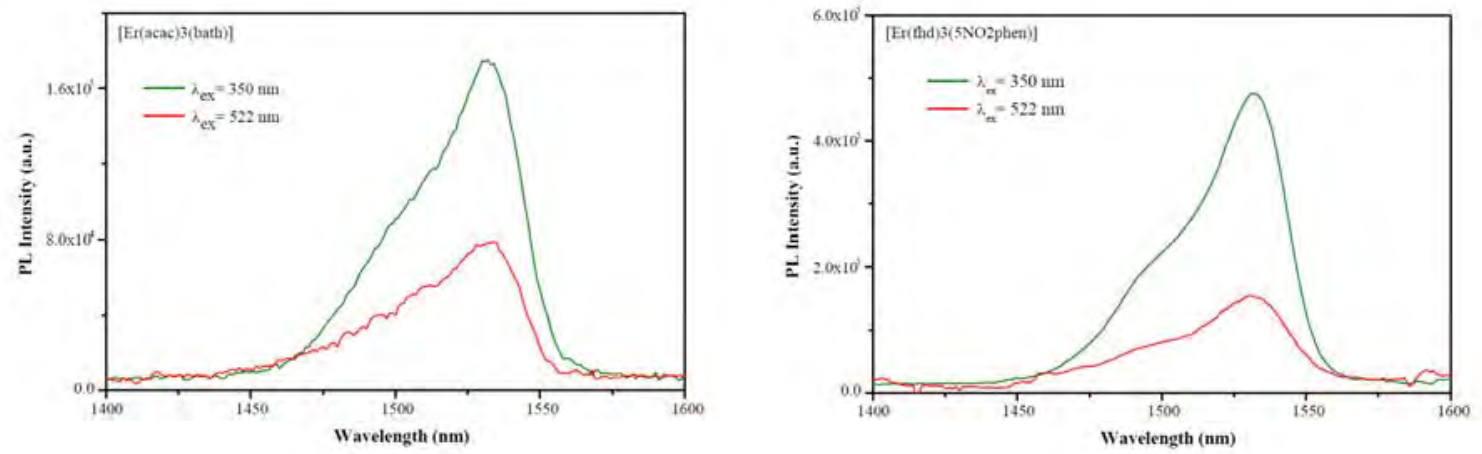

Figure 102. Comparison between the NIR emission intensities with excitation at $350 \mathrm{~nm}$ and at 522 $\mathrm{nm}$ for $\left[\mathrm{Er}(\mathrm{acac})_{3}(\mathrm{bath})\right]$ (left) and $\left[\mathrm{Er}(\mathrm{fhd})_{3}\left(5 \mathrm{NO}_{2}\right.\right.$ phen)] (right) 


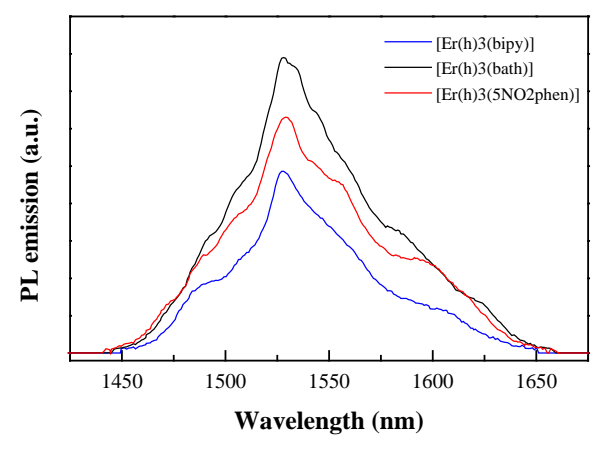

$\left[\operatorname{Er}(\mathrm{h})_{3}(\mathrm{~N}, \mathrm{~N}-\right.$ donor $\left.)\right]$

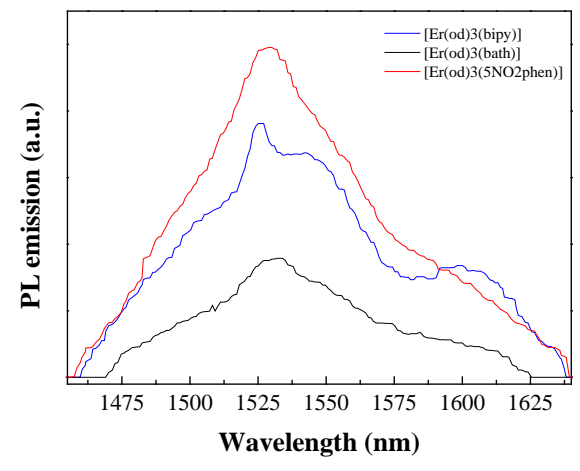

$\left[\operatorname{Er}(\mathrm{od})_{3}(\mathrm{~N}, \mathrm{~N}-\right.$ donor $\left.)\right]$

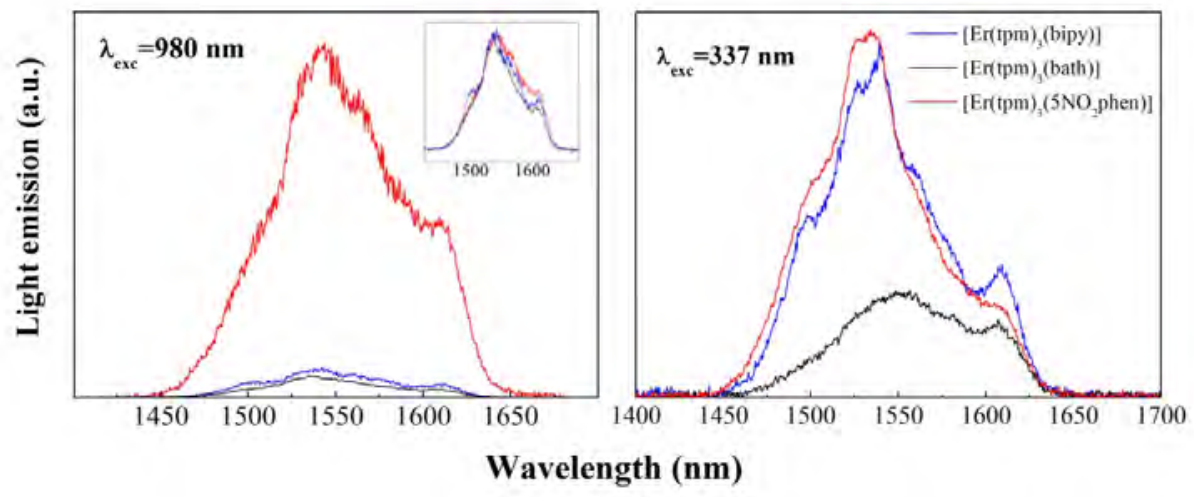

$\left[\operatorname{Er}(\operatorname{tpm})_{3}(\mathrm{~N}, \mathrm{~N}-\right.$ donor $\left.)\right]$

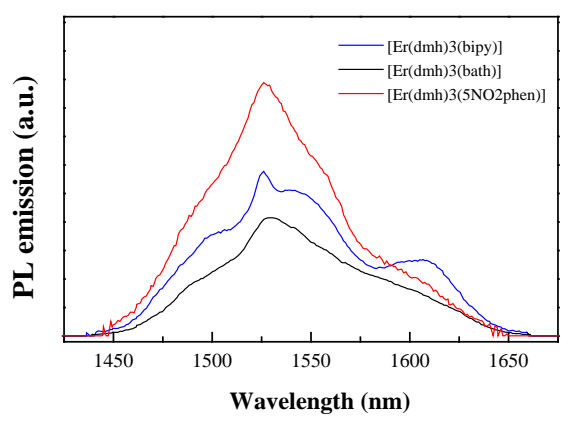

$\left[\operatorname{Er}(\mathrm{dmh})_{3}(\mathrm{~N}, \mathrm{~N}-\mathrm{donor})\right]$

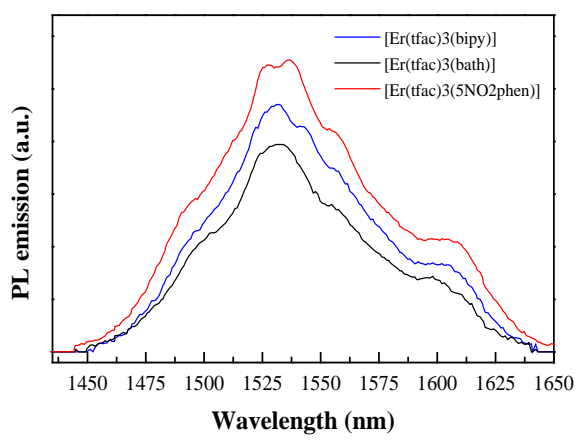

$\left[\operatorname{Er}(\mathrm{tfac})_{3}(\mathrm{~N}, \mathrm{~N}-\right.$ donor $\left.)\right]$

Figure 103. NIR emission intensity comparisons for $\operatorname{Er}(\mathrm{III})$ complexes with different $\mathrm{N}, \mathrm{N}$-donors

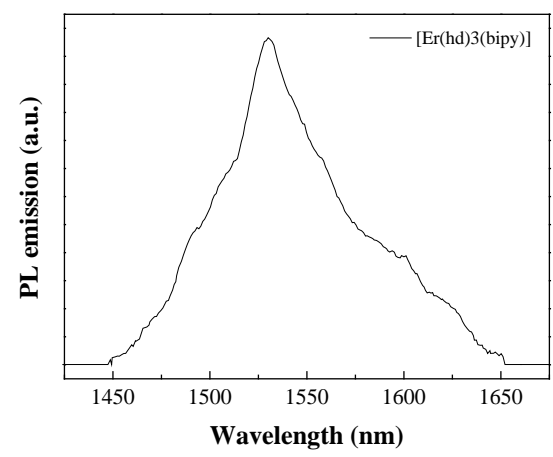

[Er(hd) $)_{3}($ bipy)]

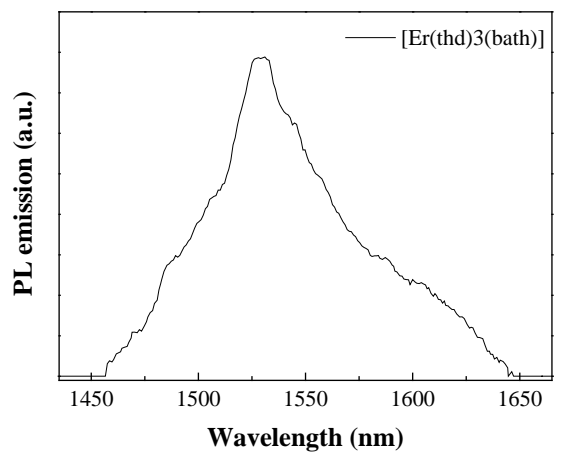

[Er(thd) $3($ bath $)]$ 


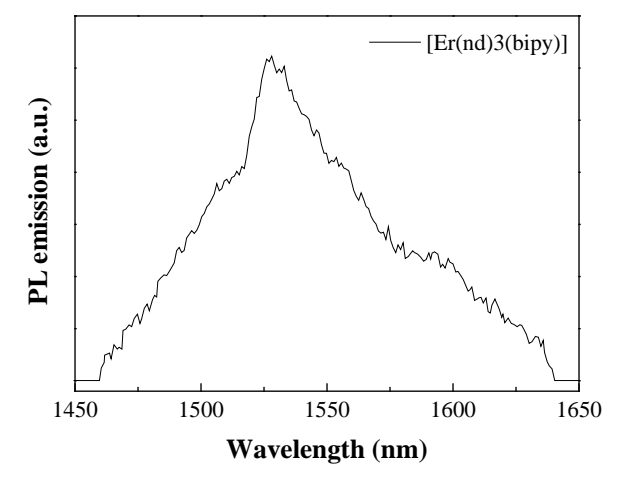

[Er(nd) $)_{3}($ bipy)]

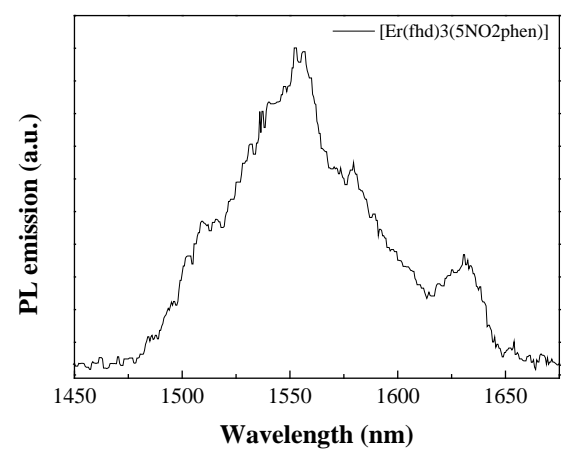

[Er(fhd $)_{3}\left(5 \mathrm{NO}_{2}\right.$ phen $\left.)\right]$

Figure 104. NIR spectra for other Er(III) complexes

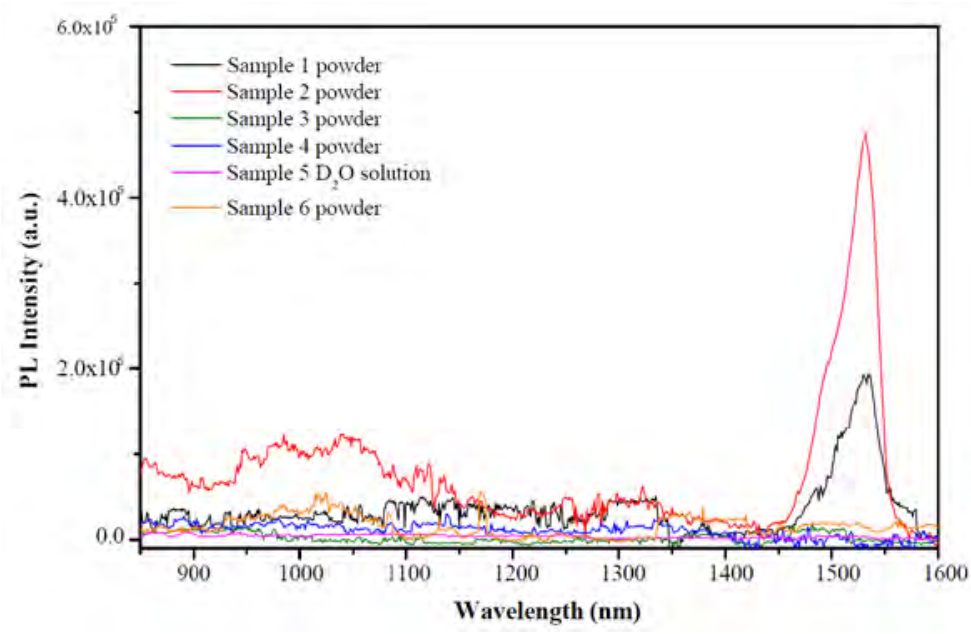

Figure 105. Comparison of PL emission intensities in NIR region $(850-1600 \mathrm{~nm})$ for several $\operatorname{Er}(\mathrm{III})$ complexes

1. $\quad\left[\operatorname{Er}(\mathrm{acac})_{3}(\mathrm{bath})\right]$

2. $\quad\left[\operatorname{Er}(\mathrm{fhd})_{3}(5 \mathrm{NO} 2 \mathrm{phen})\right]$

3-5. Alkali-lanthanide heterometallic coordination polymers

6. $\left[\mathrm{Er}\left(\mathrm{CF}_{3} \mathrm{SO}_{3}\right)_{3} \cdot\left(\mathrm{H}_{2} \mathrm{O}\right)_{9}\right.$

\section{NIR PL emission for selected Yb(III) complexes with 5-nitro-1,10-phenanthroline}

In previous section, the effect of the different $\mathrm{N}, \mathrm{N}$-donor moieties with the same $\beta$-diketone was studied for several $\operatorname{Er}(\mathrm{III})$ complexes. Nonetheless, the reverse study (i.e., the effect of changing the $\beta$-diketone while keeping the same $\mathrm{N}, \mathrm{N}$-donor) was not conducted. Thus, the effect of the $\beta$-diketone chain length on the emission intensity has been assessed for the $\mathrm{Yb}(\mathrm{III})$-complexes with chemical formula $\left[\mathrm{Yb}(\beta \text {-diketonate })_{3}\left(5 \mathrm{NO}_{2}\right.\right.$ phen $\left.)\right]$, where the $\beta$ diketone is either 4,4,4-trifluoro-1-(2-naphthyl)-1,3-butanedione (Htfnb), 4,4,4-trifluoro-1(2-furyl)-1,3-butanedione ( $\mathrm{Htfa}$ ), 1,1,1-trifluoro-2,4-pentanedione (Htfac), 1,1,1-trifluoro5,5-dimethyl-2,4-hexanedione (Htpm), 1,1,1,5,5,6,6,7,7,7-decafluoro-2,4-heptanedione (Hfhd), 2,4-hexanedione (Hh) or 2,6-dimethyl-3,5-heptanedione (Hdmh), and $5 \mathrm{NO}_{2}$ phen is 5nitro-1,10-phenanthroline.

Upon direct excitation of the ligands at $405 \mathrm{~nm}$, all the studied $\mathrm{Yb}^{3+}$ complexes show a nearinfrared peak at $977 \mathrm{~nm}\left(10235 \mathrm{~cm}^{-1}\right)$ with a shoulder at $1014 \mathrm{~nm}\left(9860 \mathrm{~cm}^{-1}\right)$ both assigned 
to the ${ }^{2} \mathrm{~F}_{5 / 2} \rightarrow{ }^{2} \mathrm{~F}_{7 / 2}$ transition. Figure 106 shows a comparison of the emission intensities for the fluorinated complexes. Even though the intensities of the spectra in powder form cannot be strictly compared, given the good reproducibility of the results and assuming the same incident energy density, we may qualitatively observe that, for the same $\mathrm{N}, \mathrm{N}$-donor moiety, the emission intensities are directly related to the $\beta$-diketone chain length: shorter chain lengths lead to a more efficient emission in the NIR region, that is, $\mathrm{Htfnb}>\mathrm{Htfa}>\mathrm{Htfac}>$ Htpm > Hfhd.

In the particular case of Htfnb and Htfa, which have the same chain length, the differences can be attributed to the favorable presence of two aromatic groups in the former, vs. only one in the latter.

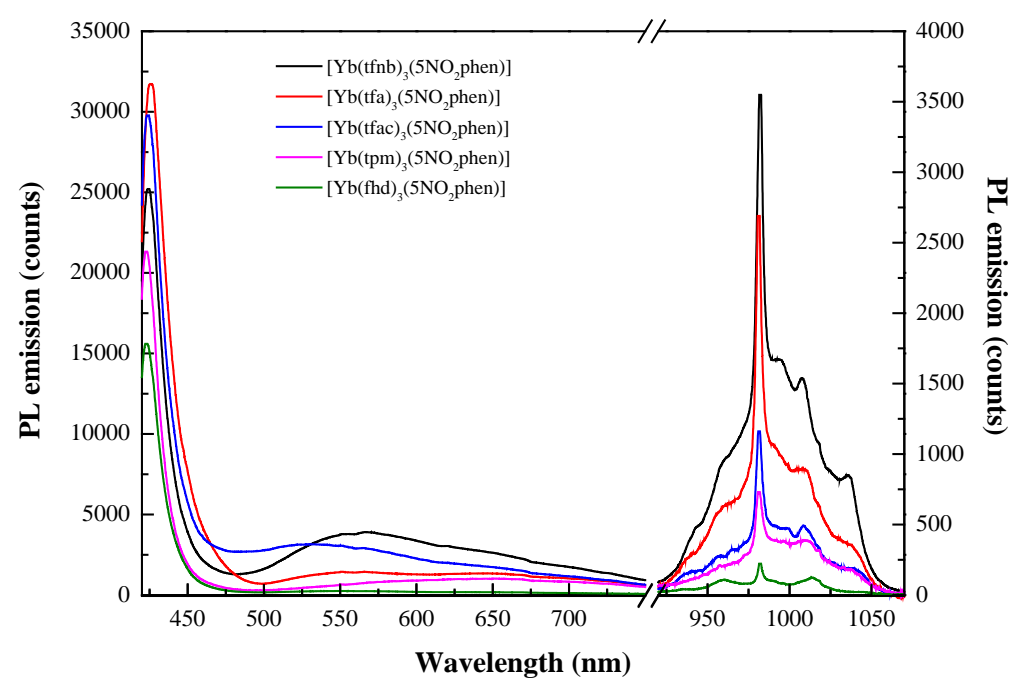

Figure 106. Vis-NIR photoluminescence spectra for [Yb(fluorinated $\beta$-diketonate $)_{3}\left(5 \mathrm{NO}_{2}\right.$ phen $\left.)\right]$ complexes under pumping at $405 \mathrm{~nm}$.

Regarding the effect of fluorination, contrary to what has been reported for $\mathrm{Er}^{3+}$ complexes, in these $\mathrm{Yb}^{3+}$ complexes we have observed that fluorination of the $\beta$-diketone offers no advantages: it should be taken into consideration that no $\mathrm{C}-\mathrm{H}$ overtone is close to the ${ }^{2} \mathrm{~F}_{7 / 2}$ (neither $v=3$ nor $v=4$ ), and the same applies to $\mathrm{C}-\mathrm{D}, \mathrm{C}-\mathrm{O}$ and $\mathrm{C}-\mathrm{C}$ overtones (the closest overtone would be $v=3$ for $\mathrm{O}-\mathrm{H}$, which are not present in the coordination sphere).

Figure 107 shows that for hexanedione (left) and heptanedione (right) there are slight differences between the complexes with fluorinated and non-fluorinated $\beta$-diketones, more evident in the heptanedione case. Lifetime measurements, discussed later on, provide additional information which advises against fluorination. However, if more complex systems co-doped with isostructural $\mathrm{Er}^{3+}$ and $\mathrm{Yb}^{3+}$ compounds are envisaged, such as YEDFAs, fluorinated $\beta$-diketones should be chosen (since fluorination is essential in order to provide efficient luminescence from $\mathrm{Er}^{3+}$ ). 

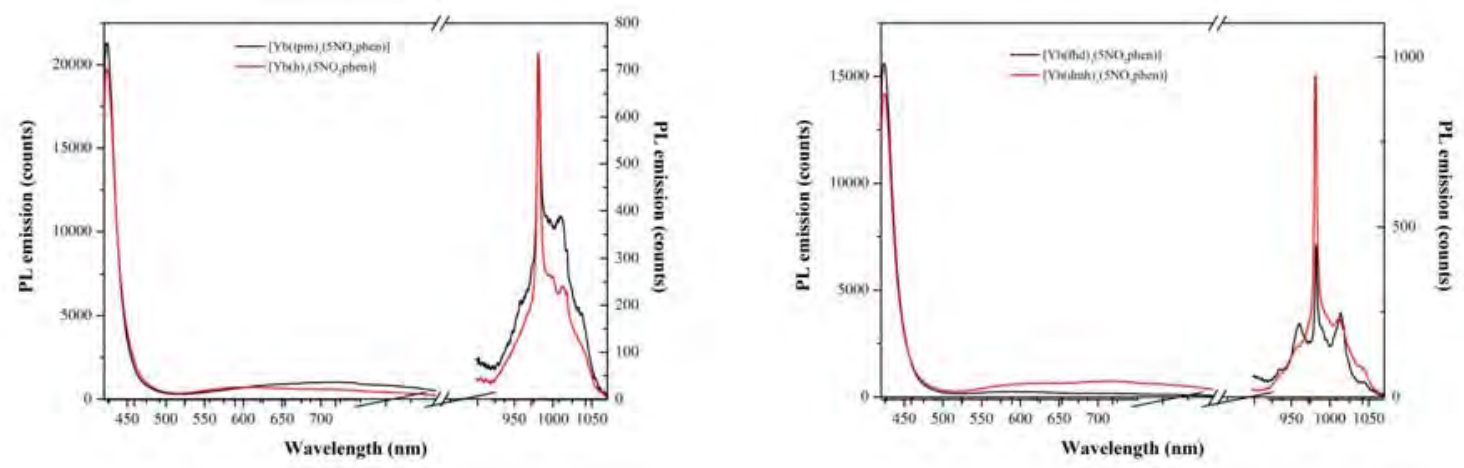

Figure 107. Comparison of fluorinated vs. non-fluorinated $\mathrm{Yb}(\mathrm{III}) \beta$-diketonate complexes photoluminescence emission for the same chain-lengths: (left) Htpm vs. Hh; (right) Hfhd vs. Hdmh.

\section{Visible PL emission lifetimes}

The PL decay of the organic ligands has been measured after excitation with a highrepetition rate pulsed picosecond laser at $\lambda=405 \mathrm{~nm}$ (Figure 108). Under this direct excitation, ground state absorption $S_{0} \rightarrow S_{1}$ in the ligand moiety occurs, followed by fast vibrational relaxation to the lowest excited singlet level, from which it can relax radiatively emitting a photon at around $430 \mathrm{~nm}$ or it may undergo intersystem crossing to the triplet level due to spin reorientation (from which subsequent resonance energy transfer to $\mathrm{Ln}^{3+}$ may take place). The studied $S_{1} \rightarrow S_{0}$ radiative decay times -determined using FAST ${ }^{\circledR}$ software for the deconvolution of the instrument response function (IRF) and the exponential component analysis- are summarized in Table 57.

The decay times for Er(III) complexes with bipy and $5 \mathrm{NO}_{2}$ phen tend to be significantly shorter than for complexes with bath ancillary ligand. In fact, for some $5 \mathrm{NO}_{2}$ phen complexes the decay is almost comparable to the IRF, thus preventing an accurate deconvolution (labelled as N/A). These ligand lifetimes are in good agreement with the intensities of the PL spectra discussed above: longer ligand lifetimes lead to more intense emission in the visible region. Conversely, the complexes which show the most efficient antenna effect have the shortest ligand decay time values (usually complexes with $5 \mathrm{NO}_{2}$ phen ancillary ligand), provided that excited states are very efficiently transferred to the $\operatorname{Ln}^{3+}$ (by ISC and subsequent RET).

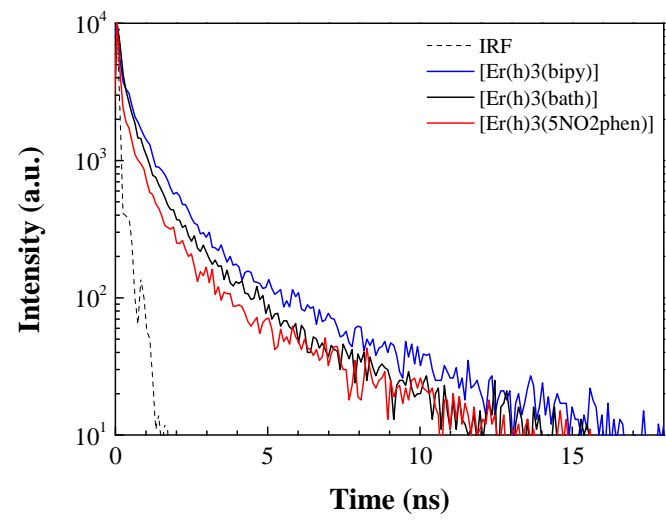

$\left[\operatorname{Er}(\mathrm{h})_{3}(\mathrm{~N}, \mathrm{~N}-\right.$ donor $\left.)\right]$

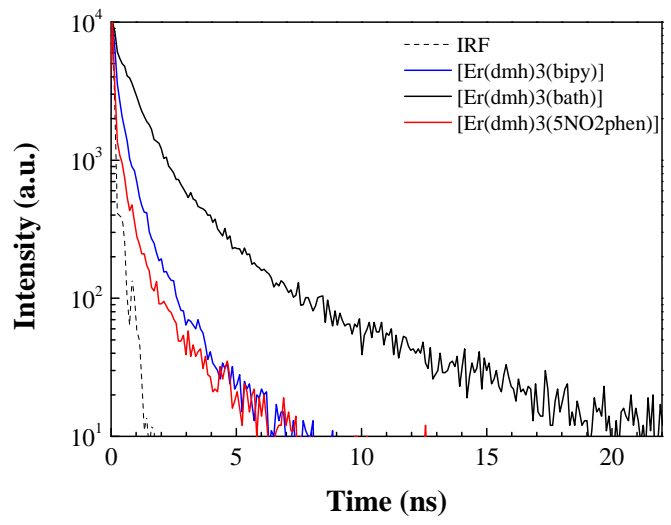

[Er(dmh) $3(\mathrm{~N}, \mathrm{~N}$-donor $)]$ 


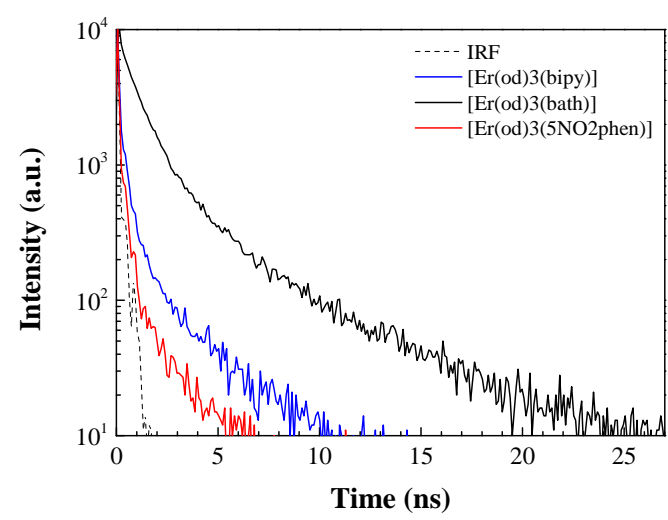

[Er(od) $)_{3}(\mathrm{~N}, \mathrm{~N}-$ donor $\left.)\right]$

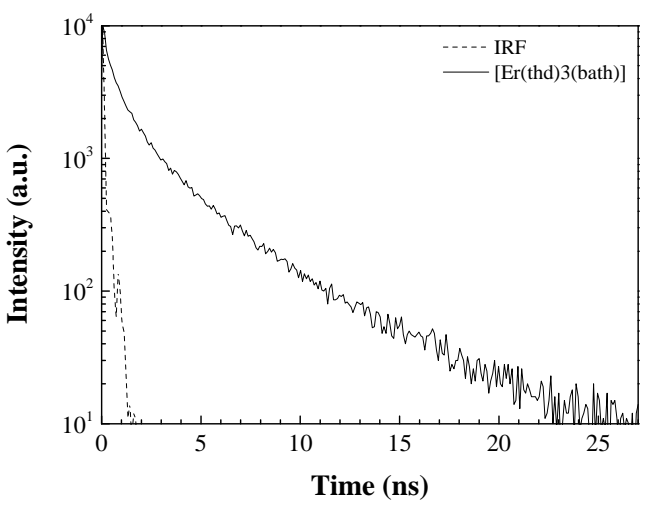

[Er(thd) $)_{3}$ (bath)]

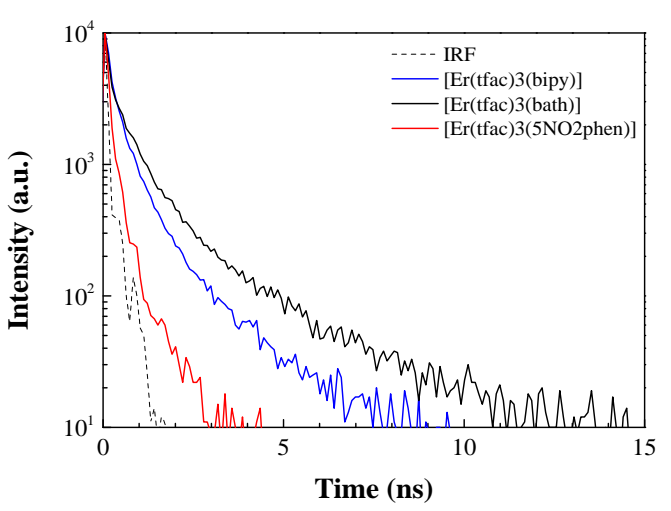

[Er(tfac) $3(\mathrm{~N}, \mathrm{~N}-$ donor $)]$

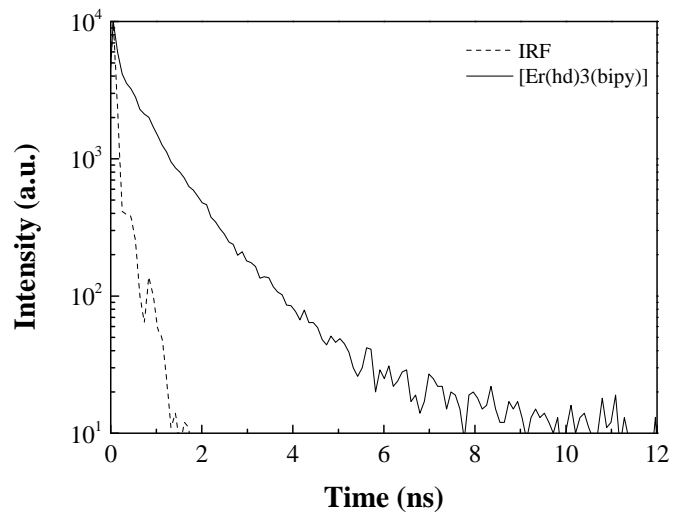

[Er(hd) ${ }_{3}$ (bipy)]

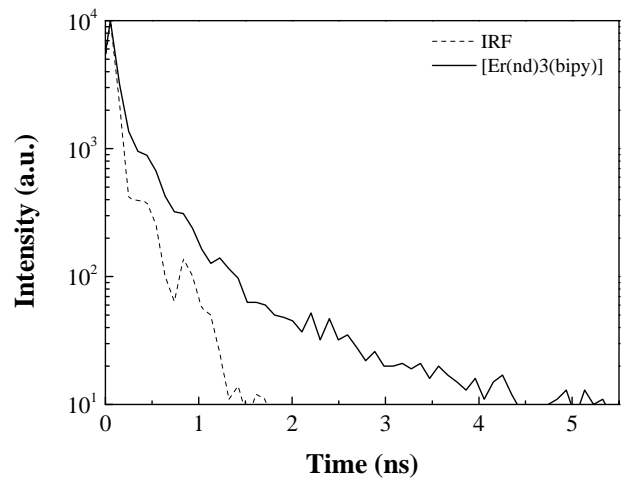

[Er(nd)3(bipy)]

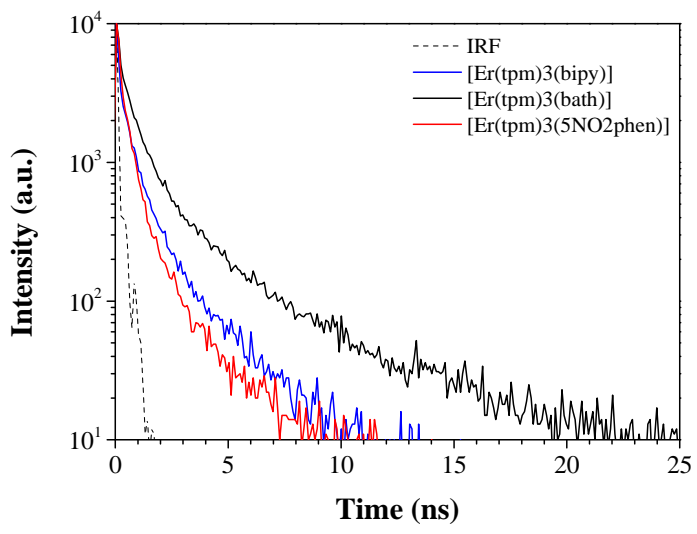

[Er(tpm) $3(\mathrm{~N}, \mathrm{~N}-$ donor $)]$ 


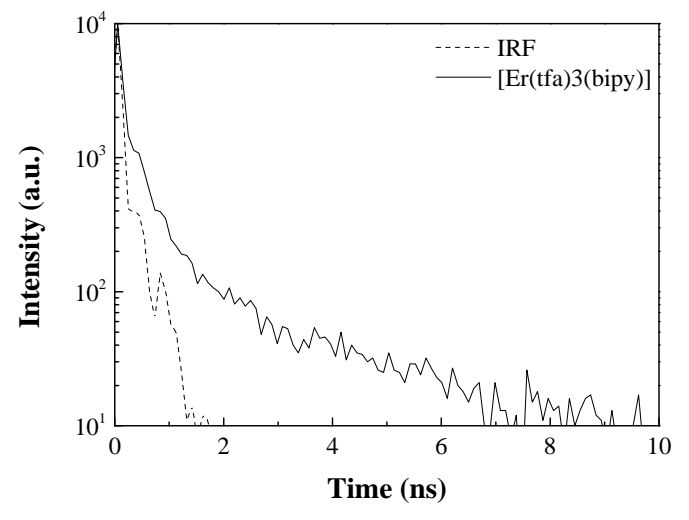

[Er(tfa) 3 (bipy)]

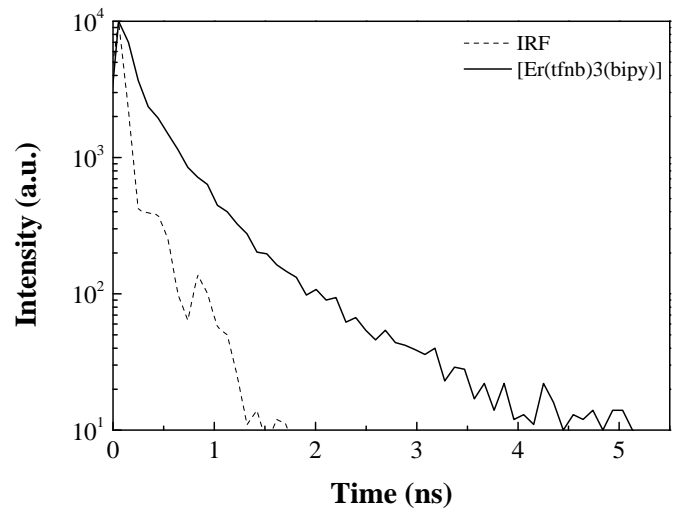

[Er(tfnb) 3 (bipy)]

Figure 108. Ligand-associated visible PL emission lifetimes for Er(III) complexes

Table 57. Visible PL emission lifetime values for Er(III) complexes

\begin{tabular}{|c|c|c|c|c|c|}
\hline Complexes & $\mathrm{f}_{1}$ & $t_{1}$ & $\mathrm{f}_{2}$ & $t_{2}$ & $\tau_{\text {effective }}(\mathrm{ns})$ \\
\hline$\left[\operatorname{Er}(\mathrm{h})_{3}(\right.$ bipy) $]$ & 69.931 & 0.618 & 30.069 & 3.038 & 1.35 \\
\hline$\left[\operatorname{Er}(\mathrm{h})_{3}(\right.$ bath $\left.)\right]$ & 58.011 & 0.325 & 41.989 & 1.971 & 1.02 \\
\hline$\left[\operatorname{Er}(\mathrm{h})_{3}\left(5 \mathrm{NO}_{2}\right.\right.$ phen $\left.)\right]$ & 65.696 & 0.428 & 34.304 & 2.327 & 1.08 \\
\hline$\left[\operatorname{Er}(\mathrm{dmh})_{3}(\mathrm{bipy})\right]$ & 68.415 & 0.135 & 31.585 & 0.853 & 0.36 \\
\hline$\left[\operatorname{Er}(\mathrm{dmh})_{3}(\mathrm{bath})\right]$ & 71.008 & 0.714 & 28.992 & 2.952 & 1.36 \\
\hline$\left[\mathrm{Er}(\mathrm{dmh})_{3}\left(5 \mathrm{NO}_{2}\right.\right.$ phen $\left.)\right]$ & 96.213 & 0.04 & 3.787 & 1.246 & 0.09 \\
\hline$\left[\operatorname{Er}(\text { od })_{3}(\right.$ bipy $\left.)\right]$ & 73.526 & 0.139 & 26.474 & 1.585 & 0.52 \\
\hline$\left[\operatorname{Er}(\mathrm{od})_{3}(\mathrm{bath})\right]$ & 81.952 & 0.817 & 18.048 & 3.561 & 1.31 \\
\hline$\left[\mathrm{Er}(\mathrm{od})_{3}\left(5 \mathrm{NO}_{2}\right.\right.$ phen $\left.)\right]$ & 97.012 & 0.046 & 2.988 & 1.379 & N/A \\
\hline$\left[\operatorname{Er}(\mathrm{hd})_{3}(\mathrm{bipy})\right]$ & 59.549 & 0.513 & 40.451 & 1.179 & 0.78 \\
\hline$\left[\operatorname{Er}(\mathrm{nd})_{3}(\right.$ bipy $\left.)\right]$ & 82.726 & 0.238 & 17.274 & 1.118 & 0.39 \\
\hline$\left[\operatorname{Er}(\text { thd })_{3}(\right.$ bath $\left.)\right]$ & 50.107 & 0.549 & 49.893 & 3.391 & 1.97 \\
\hline$\left[\operatorname{Er}(\operatorname{tfac})_{3}(\mathrm{bipy})\right]$ & 67.349 & 0.257 & 32.651 & 1.194 & 0.56 \\
\hline$\left[\operatorname{Er}(\operatorname{tfac})_{3}(\mathrm{bath})\right]$ & 66.33 & 0.465 & 33.67 & 2.106 & 1.02 \\
\hline$\left[\mathrm{Er}(\mathrm{tfac})_{3}\left(5 \mathrm{NO}_{2} \mathrm{phen}\right)\right]$ & $2.60 \times 10^{-6}$ & 0.061 & 100 & - & $\mathrm{N} / \mathrm{A}$ \\
\hline$\left[\operatorname{Er}(\operatorname{tpm})_{3}(\right.$ bipy $\left.)\right]$ & 54.779 & 0.22 & 45.221 & 1.395 & 0.75 \\
\hline$\left[\operatorname{Er}(\operatorname{tpm})_{3}(\right.$ bath $\left.)\right]$ & 53.884 & 0.41 & 46.116 & 2.796 & 1.51 \\
\hline$\left[\mathrm{Er}(\mathrm{tpm})_{3}\left(5 \mathrm{NO}_{2}\right.\right.$ phen $\left.)\right]$ & 70.684 & 0.159 & 29.317 & 1.063 & 0.42 \\
\hline$\left[\operatorname{Er}(\mathrm{tfa})_{3}(\mathrm{bipy})\right]$ & 73.23 & 0.276 & 26.77 & 2.031 & 0.75 \\
\hline$\left[\operatorname{Er}(\operatorname{tfnb})_{3}(\mathrm{bipy})\right]$ & 69.312 & 0.187 & 30.688 & 0.726 & 0.35 \\
\hline
\end{tabular}

Again, the assessment of the $\beta$-diketone choice (keeping the same $\mathrm{N}, \mathrm{N}$-donor) has been conducted for the $\left[\mathrm{Yb}(\beta \text {-diketonate })_{3}\left(5 \mathrm{NO}_{2}\right.\right.$ phen $\left.)\right]$ complexes (Figure 109). No significant differences can be detected amongst fluorinated $\beta$-diketones.

Conversely, it can be observed that the ligand averaged lifetimes are slightly shorter for fluorinated than for non-fluorinated $\mathrm{Yb}(\mathrm{III}) \beta$-diketonate complexes. Nevertheless, globally they are all in the ns range, ranging from $0.79 \mathrm{~ns}$ for $\left[\mathrm{Yb}(\mathrm{fhd})_{3}\left(5 \mathrm{NO}_{2}\right.\right.$ phen $\left.)\right]$ to $1.81 \mathrm{~ns}$ $\left[\mathrm{Yb}(\mathrm{h})_{3}\left(5 \mathrm{NO}_{2}\right.\right.$ phen $\left.)\right]$. This is indicative of an efficient antenna effect, since the excitation energy photogenerated in the organic ligands is quickly transferred to the $\mathrm{Yb}^{3+}$ core. It should also be noticed that the differences in these values are relative small from one complex to another, which are justified because the ligand relaxation is dominated by the $\mathrm{N}, \mathrm{N}$-donor (which is the same in all complexes, 5-nitro-1,10-phenanthroline), while, as explained above, the choice of the $\beta$-diketone mainly affects the absorption and emission intensity. 


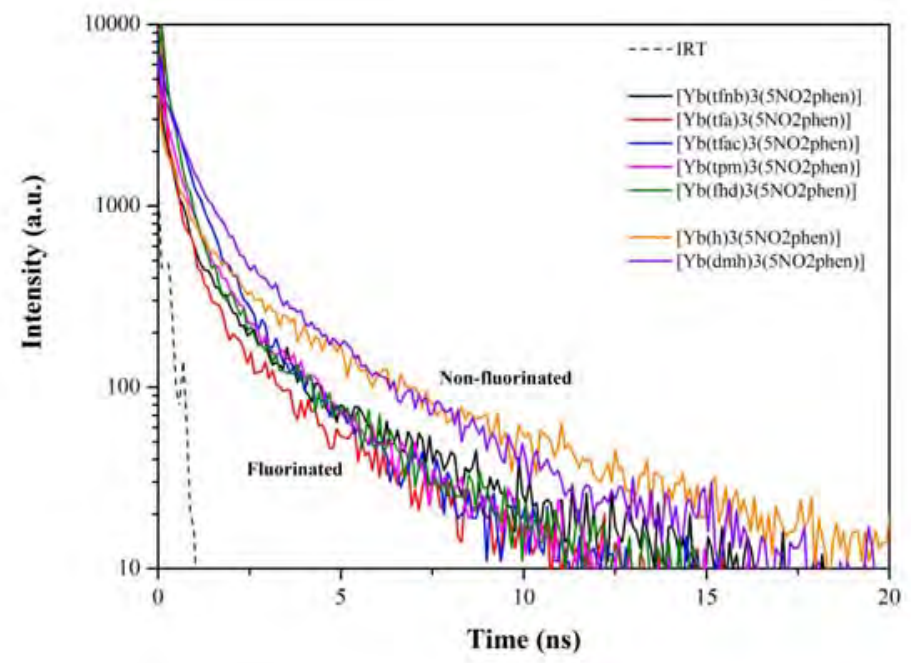

Figure 109. Comparison of ligand VIS emission lifetimes upon excitation at $405 \mathrm{~nm}$ for both fluorinated and non-fluorinated $\mathrm{Yb}(\mathrm{III}) \beta$-diketonate complexes

\section{NIR PL emission lifetimes for Er(III) complexes}

The PL decay of the ${ }^{4} \mathrm{I}_{13 / 2}$ multiplet was measured after ${ }^{4} \mathrm{I}_{11 / 2}$ excitation (Figure 110). In spite of the possible PL reabsorption, the obtained decays show single exponential behaviour, with the time constants summarized in Table 58. These values, typical of lanthanide complexes (and far smaller than the emission decay time of the isolated ion, $\tau \approx 8 \mathrm{~ms}$ ), are due to vibronic coupling with high energy C-H stretching vibrations in the neighbourhood of the $\mathrm{Er}^{3+}$ ion (originated from the ligands), which lead to quenching of the excited state (because of the relatively small energy gap between the excited state ${ }^{4} \mathrm{I}_{13 / 2}$ and the ground state). Nonetheless, it must be noted that all these complexes show a significant reduction of the non-radiative losses caused by $\mathrm{O}-\mathrm{H}$ and $\mathrm{N}-\mathrm{H}$ oscillators, which are the ones which have the most deleterious effects on the emission. Partial fluorination of the $\beta$-diketones (with a subsequent reduction of $\mathrm{C}$ - $\mathrm{H}$ vibrations) leads to an increase in the lifetime ranging from $30 \%$ to $60 \%$.

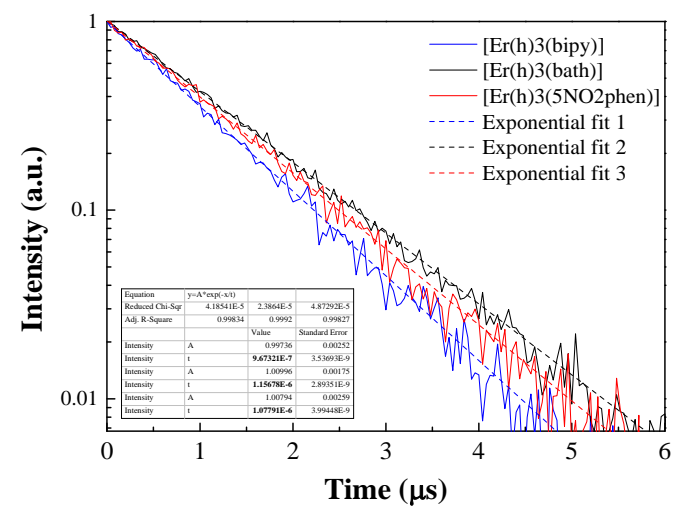

[Er(h)3(N,N-donor)]

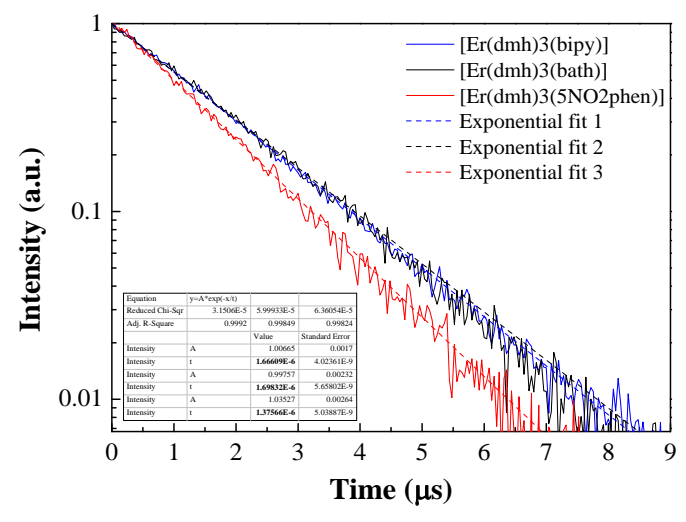

[Er(dmh)3(N,N-donor)] 


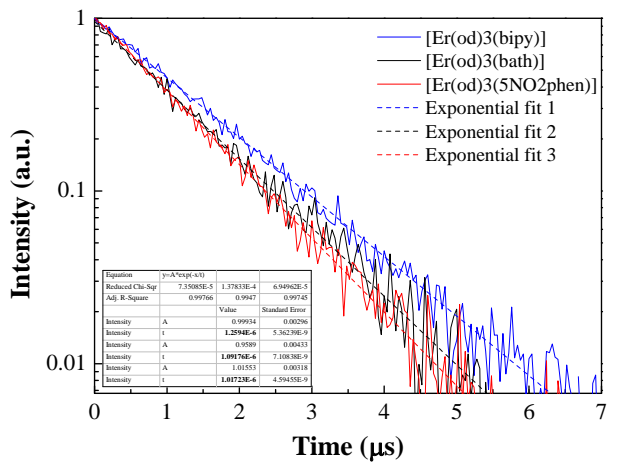

[Er(od)3(N,N-donor)]

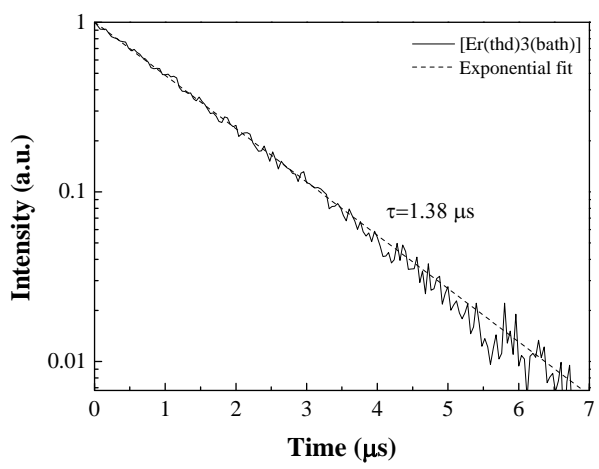

[Er(thd)3(bath)]

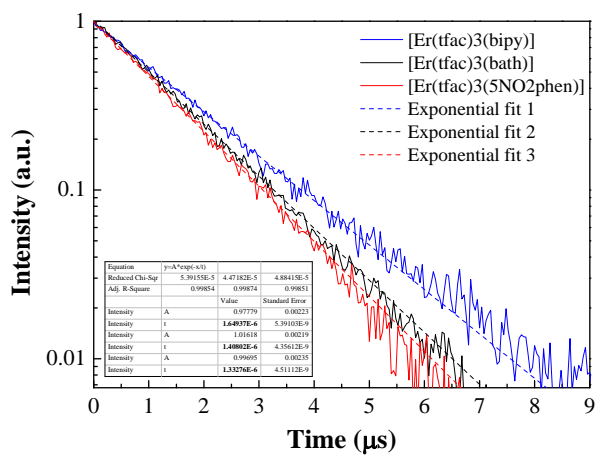

[Er(tfac)3(N,N-donor)]

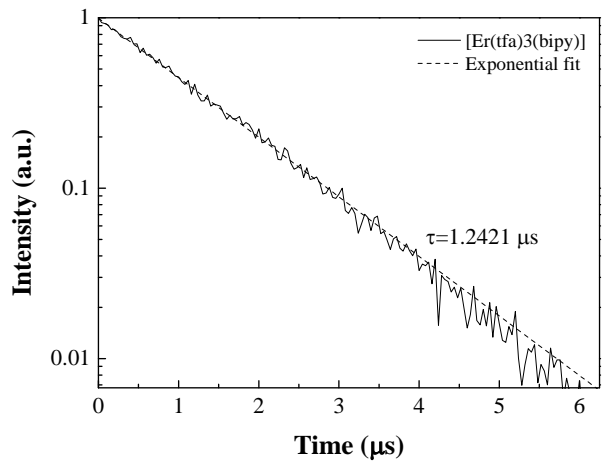

[Er(tfa)3(bipy)]

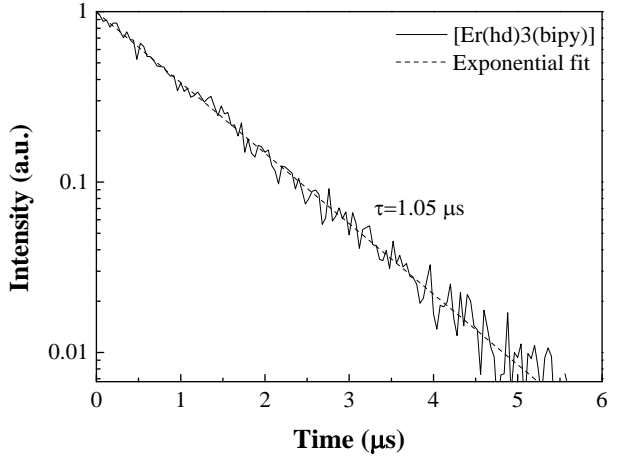

[Er(hd)3(bipy)]

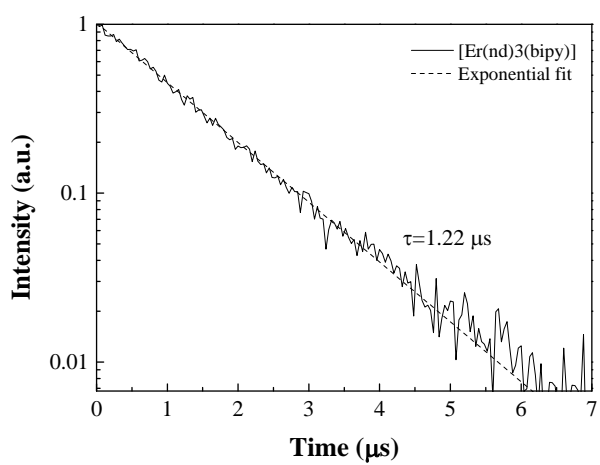

[Er(nd)3(bipy)]

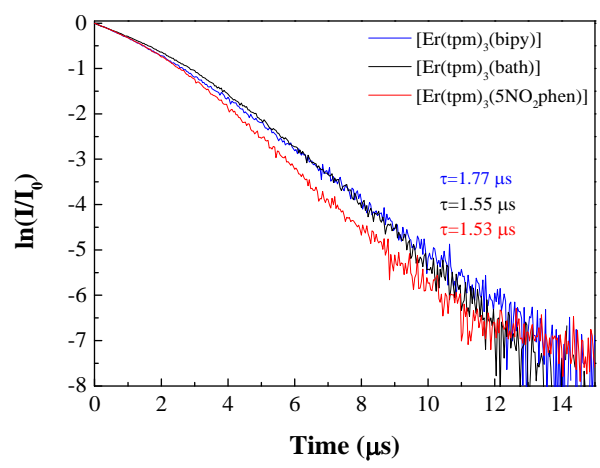

[Er(tpm)3(N,N-donor)]

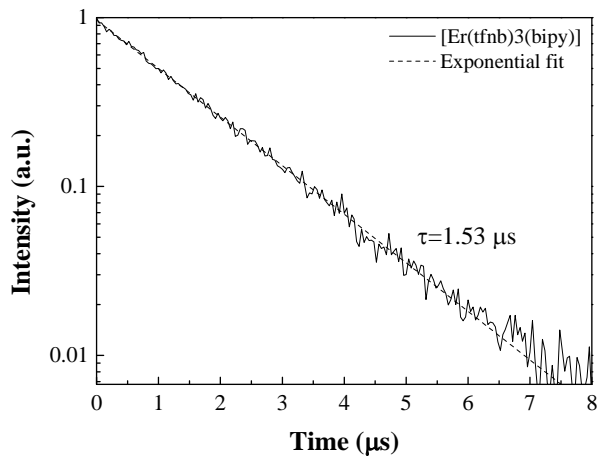

[Er(tfnb)3(bipy)]

Figure 110. Room temperature PL decays of the ${ }^{4} I_{13 / 2}$ multiplet measured under $980 \mathrm{~nm}$ excitation of ${ }^{4} \mathrm{I}_{11 / 2} \mathrm{Er}^{3+}$ level 
Table 58. PL decay values of the ${ }^{4} I_{13 / 2}$ multiplet

\begin{tabular}{|c|c|}
\hline Complexes & NIR emission lifetimes upon $\mathrm{Er}^{3+}$ direct excitation at $980 \mathrm{~nm}(\mu \mathrm{s})$ \\
\hline$\left[\mathrm{Er}(\mathrm{acac})_{3}(\mathrm{bath})\right]$ & 1.02 \\
\hline$\left[\operatorname{Er}(\mathrm{h})_{3}(\mathrm{bipy})\right]$ & 0.97 \\
\hline$\left[\operatorname{Er}(\mathrm{h})_{3}(\mathrm{bath})\right]$ & 1.15 \\
\hline$\left[\mathrm{Er}(\mathrm{h})_{3}\left(5 \mathrm{NO}_{2}\right.\right.$ phen $\left.)\right]$ & 1.08 \\
\hline$\left[\operatorname{Er}(\mathrm{dmh})_{3}(\mathrm{bipy})\right]$ & 1.67 \\
\hline$\left[\mathrm{Er}(\mathrm{dmh})_{3}(\mathrm{bath})\right]$ & 1.70 \\
\hline$\left[\mathrm{Er}(\mathrm{dmh})_{3}\left(5 \mathrm{NO}_{2}\right.\right.$ phen $\left.)\right]$ & 1.38 \\
\hline$\left[\operatorname{Er}(\mathrm{hd})_{3}(\right.$ bipy $\left.)\right]$ & 1.05 \\
\hline$\left[\operatorname{Er}(\text { thd })_{3}(\right.$ bath $\left.)\right]$ & 1.38 \\
\hline$\left[\operatorname{Er}(\mathrm{od})_{3}(\mathrm{bipy})\right]$ & 1.26 \\
\hline$\left[\operatorname{Er}(\mathrm{od})_{3}(\mathrm{bath})\right]$ & 1.09 \\
\hline$\left[\mathrm{Er}(\mathrm{od})_{3}\left(5 \mathrm{NO}_{2}\right.\right.$ phen $\left.)\right]$ & 1.02 \\
\hline$\left[\operatorname{Er}(\text { nd })_{3}(\right.$ bipy $\left.)\right]$ & 1.22 \\
\hline$\left[\mathrm{Er}(\mathrm{tfa})_{3}(\mathrm{bipy})\right]$ & 1.24 \\
\hline$\left[\mathrm{Er}(\mathrm{tfnb})_{3}(\mathrm{bipy})\right]$ & 1.51 \\
\hline$\left[\mathrm{Er}(\mathrm{tfac})_{3}(\mathrm{bipy})\right]$ & 1.65 \\
\hline$\left[\operatorname{Er}(\mathrm{tfac})_{3}(\mathrm{bath})\right]$ & 1.41 \\
\hline$\left[\operatorname{Er}(\mathrm{tfac})_{3}\left(5 \mathrm{NO}_{2}\right.\right.$ phen $\left.)\right]$ & 1.33 \\
\hline$\left[\operatorname{Er}(\operatorname{tpm})_{3}(\right.$ bipy $\left.)\right]$ & 1.77 \\
\hline$\left[\operatorname{Er}(\operatorname{tpm})_{3}(\mathrm{bath})\right]$ & 1.55 \\
\hline$\left[\operatorname{Er}(\operatorname{tpm})_{3}\left(5 \mathrm{NO}_{2}\right.\right.$ phen $\left.)\right]$ & 1.53 \\
\hline$\left[\mathrm{Er}(\mathrm{fhd})_{3}\left(5 \mathrm{NO}_{2}\right.\right.$ phen $\left.)\right]$ & 1.45 \\
\hline$\left[\operatorname{Er}(\text { fod })_{3}(\right.$ bath $\left.)\right]$ & 1.39 \\
\hline$\left[\mathrm{Er}(\text { fod })_{3}\left(5 \mathrm{NO}_{2}\right.\right.$ phen $\left.)\right]$ & 1.40 \\
\hline
\end{tabular}

\section{NIR PL emission lifetimes for selected Yb(III) complexes with $5 \mathrm{NO}_{2}$ phen}

Lifetime measurements upon $\mathrm{Yb}^{3+}$ direct excitation at $940 \mathrm{~nm}$

For the series of $\mathrm{Yb}(\mathrm{III})$ fluorinated complexes, a good correspondence of the $\mathrm{Yb}^{3++}{ }^{2} \mathrm{~F}_{5 / 2} \rightarrow{ }^{2} \mathrm{~F}_{7 / 2}$ lifetime values with the emission intensities in Figure 108 is observed: shorter $\beta$-diketone chain lengths have longer associated $\mathrm{Yb}^{3+}$ lifetimes and stronger emissions (Figure 111). The decay curves have been fitted to single exponential decays and the simulated decay time is given in Table 59 (right).

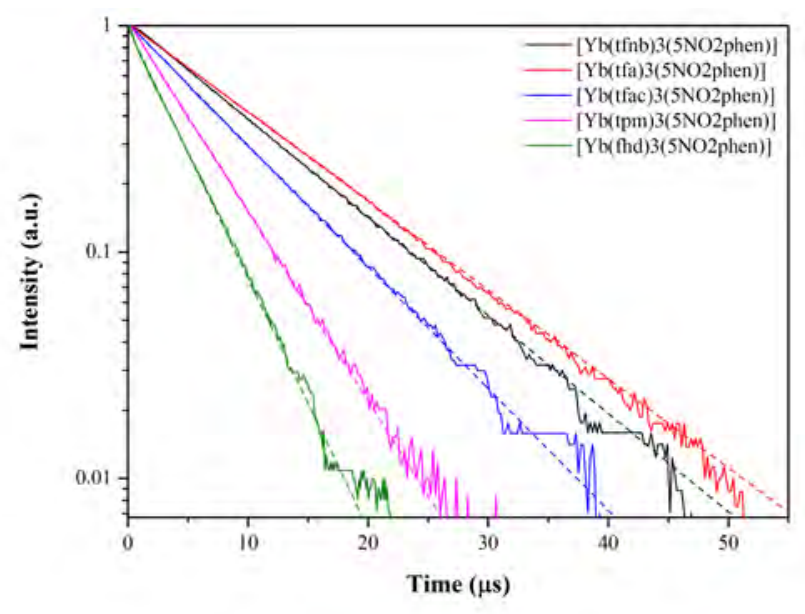

Figure 111. Comparison of luminescence decay curves (solid) for the fluorinated $\beta$-diketonate complexes upon $\mathrm{Yb}^{3+}$ direct excitation. Fittings to monoexponential decays are given (dotted). 
Upon comparison of the luminescence decay curves for the fluorinated and non-fluorinated hexanedione and heptanedione complexes (Figure 112), the effect of fluorination is more obvious. Fluorinated complexes have appreciably shorter decay times: $5.2 \mu \mathrm{s}$ for $\left[\mathrm{Yb}(\mathrm{tpm})_{3}\left(5 \mathrm{NO}_{2}\right.\right.$ phen $\left.)\right] \quad$ vs. $6.5 \mu \mathrm{s}$ for $\left[\mathrm{Yb}(\mathrm{h})_{3}\left(5 \mathrm{NO}_{2}\right.\right.$ phen $\left.)\right]$, and $3.9 \mu \mathrm{s}$ for $\left[\mathrm{Yb}(\mathrm{fhd})_{3}\left(5 \mathrm{NO}_{2}\right.\right.$ phen $\left.)\right] v s .6 .3 \mu \mathrm{s}$ for $\left[\mathrm{Yb}(\mathrm{dmh})_{3}\left(5 \mathrm{NO}_{2}\right.\right.$ phen $\left.)\right]$.

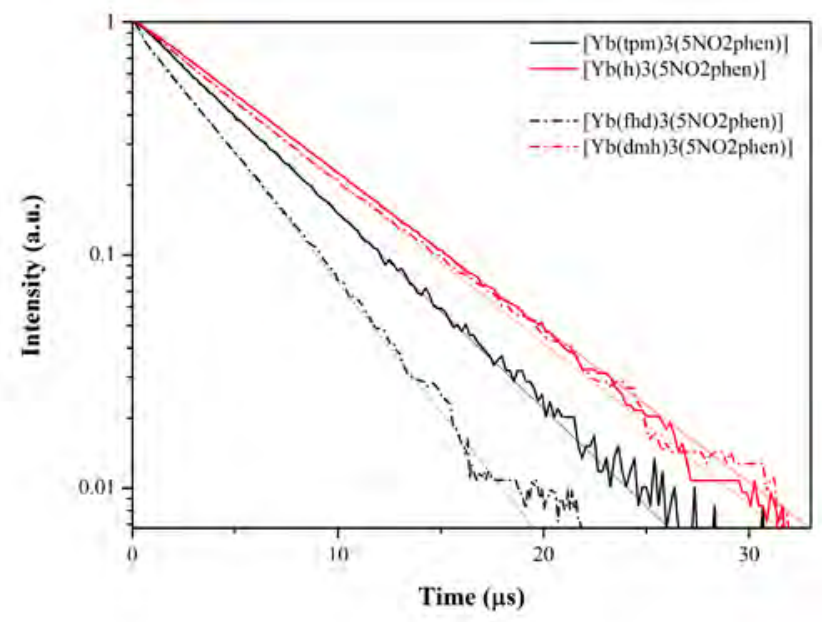

Figure 112. Comparison of $\mathrm{Yb}^{3+}$ luminescence decay curves for complexes with fluorinated (black) vs. non-fluorinated (red) $\beta$-diketonates

\section{Lifetime measurements upon ligand excitation}

We propose the following excitation and energy transfer scheme (Figure 113). Under direct excitation around $405 \mathrm{~nm}$, ground state absorption $S_{0} \rightarrow S_{1}$ in the ligand moiety occurs. Fast vibrational relaxation to the lowest excited singlet level takes place, from which it can relax radiatively emitting a photon at around $430 \mathrm{~nm}$ or it may undergo intersystem crossing to the triplet level due to spin reorientation. According to the Dexter model [9], the energy transfer probability due to dipole-dipole interaction is proportional to the overlap between the emission cross section of the donor and the absorption cross section of the acceptor. However, in the case of the $\mathrm{Yb}^{3+}$ ions, there is a significant energy gap between the triplet state of the ligand and the excited state of the $\mathrm{Yb}^{3+}$ ion: the fluorescent ${ }^{2} \mathrm{~F}_{5 / 2}$ level lies at around $\sim 10000 \mathrm{~cm}^{-1}$ above the $2 \mathrm{~F}_{7 / 2}$ ground state, that is, substantially lower than the energy of the lowest triplet states of the selected $\beta$-diketones (Figure 113). This fact has already been mentioned for other organo-metallic systems and it has been suggested to be a vibronic assisted interaction or due to internal electron transfer mechanism from the ligand to the metal [45]. In our system, the relatively short lifetimes measured for the $\mathrm{Yb}^{3+}$ ions (typical radiative lifetimes are of the order of $1 \mathrm{~ms}$ [45]), indicate that vibronic relaxation of the $\mathrm{Yb}^{3+}$ ions could play a significant role in the $\mathrm{Yb}^{3+}$ relaxation processes. Therefore, we suggest that the vibronically-assisted energy transfer from the ligand triplet state to the excited $\mathrm{Yb}^{3+}$ level occurs and explains the population of the $\mathrm{Yb}^{3+}$ excited state, which may relax radiatively to the ground state by emission of photons at around $980 \mathrm{~nm}$. 


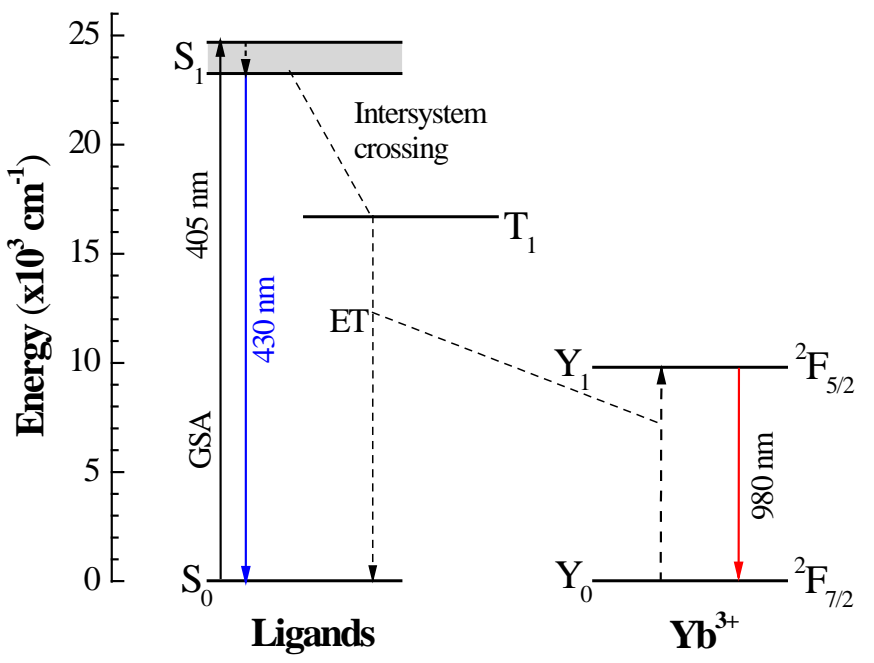

Figure 113. Energy level diagram of $\left[\mathrm{Yb}(\beta \text {-diketonate })_{3}\left(5 \mathrm{NO}_{2}\right.\right.$ phen $\left.)\right]$ complexes. Ligand fluorescence is indicated with a blue arrow. Ligand to metal energy transfer mechanism is indicated by dashed lines. IR radiative transition of $\mathrm{Yb}^{3+}$ ions is shown in red.

This model can be confirmed by a rate equation analysis:

$$
\begin{aligned}
& \frac{d S_{1}}{d t}=\sigma \Phi S_{0}-\left[W_{r a d}\left(S_{1}\right)+W_{n o n-r a d}\left(S_{1}\right)+W_{I S C}\right] S_{1} \\
& \frac{d T_{1}}{d t}=W_{I S C} S_{1}-\left[W_{\text {rad }}\left(T_{1}\right)+W_{\text {non-rad }}\left(T_{1}\right)+W_{L-Y b}\right] T_{1} \\
& \frac{d Y_{1}}{d t}=W_{L-Y b} T_{1}-\left[W_{\text {rad }}\left(Y_{1}\right)+W_{\text {non-rad }}\left(Y_{1}\right)\right] Y_{1}
\end{aligned}
$$

where $\sigma$ is the ligand ground state absorption cross-section, $\Phi$ is the pump flux, $\mathrm{W}_{\mathrm{rad}}\left(\mathrm{S}_{1}\right)$ and $\mathrm{W}_{\text {non-rad }}\left(\mathrm{S}_{1}\right)$ represent the radiative and non-radiative relaxation rate of the $\mathrm{S}_{1}$ excited state, respectively, and, analogously for the $\mathrm{T}_{1}$ and $\mathrm{Y}_{1}$ excited states. $\mathrm{W}_{\mathrm{ISC}}$ means the $\mathrm{S}_{1} \rightarrow \mathrm{T}_{1}$ intersystem crossing rate, and $\mathrm{W}_{\mathrm{L}-\mathrm{Yb}}$ stands for the ligand to metal energy transfer rate.

The total relaxation probability of an energy level is inversely proportional to its average decay time. Therefore, the decay time of $S_{1}, T_{1}$ and $Y_{1}$ excited levels can be expressed as:

$$
\begin{aligned}
& \frac{1}{\tau\left(S_{1}\right)}=W_{\text {rad }}\left(S_{1}\right)+W_{\text {non-rad }}\left(S_{1}\right)+W_{I S C} \\
& \frac{1}{\tau\left(T_{1}\right)}=W_{\text {rad }}\left(T_{1}\right)+W_{\text {non-rad }}\left(T_{1}\right)+W_{L-Y b} \\
& \frac{1}{\tau\left(Y_{1}\right)}=W_{\text {rad }}\left(Y_{1}\right)+W_{\text {non-rad }}\left(Y_{1}\right)
\end{aligned}
$$

After the excitation pump pulse, $\Phi$ equals zero, and Eq. (1) simplifies to

$$
\frac{d S_{1}}{d t}=-\frac{1}{\tau\left(S_{1}\right)} \cdot S_{1}
$$

Consequently, the temporal evolution of $S_{1}$ is given by: 


$$
S_{1}(t)=S_{1}(0) \cdot e^{-\frac{t}{\tau\left(S_{1}\right)}}
$$

Taking this into account Eq. (2) can be expressed as:

$$
\frac{d T_{1}}{d t}=W_{I S C} \cdot S_{1}(0) \cdot e^{-\frac{t}{\tau\left(S_{1}\right)}}-\frac{1}{\tau\left(T_{1}\right)} T_{1}
$$

Eq. (9) has analytical solution given by:

$$
T_{1}(t)=K_{1}\left[e^{-\frac{t}{\tau\left(T_{1}\right)}}-e^{-\frac{t}{\tau\left(S_{1}\right)}}\right]
$$

where $K_{1}=\left[W_{I S c} \cdot S_{1}(0) \cdot \tau\left(S_{1}\right) \cdot \tau\left(T_{1}\right)\right] /\left[\tau\left(T_{1}\right)-\tau\left(S_{1}\right)\right]$. The average lifetime of $S_{1}$ is in the nanosecond range, while that of $\mathrm{T}_{1}$ is several orders of magnitude larger. Subsequently, a few nanoseconds after the excitation pump pulse, $\mathrm{T}_{1}(\mathrm{t})$ can be approximated by:

$$
T_{1}(t)=K_{1} \cdot e^{-\frac{t}{\tau\left(T_{1}\right)}}
$$

Finally, Eq. (3) can be expressed as :

$$
\frac{d Y_{1}}{d t}=W_{L-Y b} K_{1} e^{-\frac{t}{\tau\left(T_{1}\right)}}-\frac{1}{\tau\left(Y_{1}\right)} Y_{1}
$$

which admits the analytical solution,

$$
Y_{1}(t)=K_{2}\left[e^{-\frac{t}{\tau\left(Y_{1}\right)}}-e^{-\frac{t}{\tau\left(T_{1}\right)}}\right]
$$

where $\mathrm{K}_{2}$ is a constant given by $\mathrm{K}_{2}=\left[\mathrm{W}_{\mathrm{L}-\mathrm{Yb}} \cdot \mathrm{W}_{\mathrm{ISC}} \cdot \mathrm{S}_{1}(0) \cdot \tau\left(\mathrm{S}_{1}\right) \cdot \tau^{2}\left(\mathrm{~T}_{1}\right) \cdot \tau\left(\mathrm{Y}_{1}\right)\right] /\left[\left(\tau\left(\mathrm{T}_{1}\right)-\tau\left(\mathrm{S}_{1}\right)\right) \cdot\left(\tau\left(\mathrm{Y}_{1}\right)-\right.\right.$ $\left.\left.\tau\left(\mathrm{T}_{1}\right)\right)\right]$.

Eq. (13) describes the typical temporal evolution of the luminescence due to an energy transfer process, in which there is a rise of the luminescence, which is governed by $\tau\left(\mathrm{T}_{1}\right)$, followed by a decay at a rate given by $\tau\left(\mathrm{Y}_{1}\right)$. In fact, the temporal evolutions of the $\mathrm{Yb}^{3+}$ luminescence under excitation of the ligand using a $10 \mathrm{~ns}$ pulsed OPO laser tuned at $350 \mathrm{~nm}$ (Figure 114) shows this behavior. However, the temporal resolution of our experimental setup is limited to about $1 \mu \mathrm{s}$. Therefore, the fast rise time of the luminescence associated to $\tau\left(\mathrm{T}_{1}\right)$ cannot be accurately determined. Instead of that, we have fitted the decay of the luminescence curves, taking into account that for $t » \tau\left(\mathrm{T}_{1}\right)$, Eq.(13) can be approximated to

$$
Y_{1}(t)=K_{2} e^{-\frac{t}{\tau\left(Y_{1}\right)}}
$$

We have chosen the criterion to fit the decay data for $t>2 \mu$ s to Eq. (14). The best fitting has been obtained for the values given in Table 59 (left). 


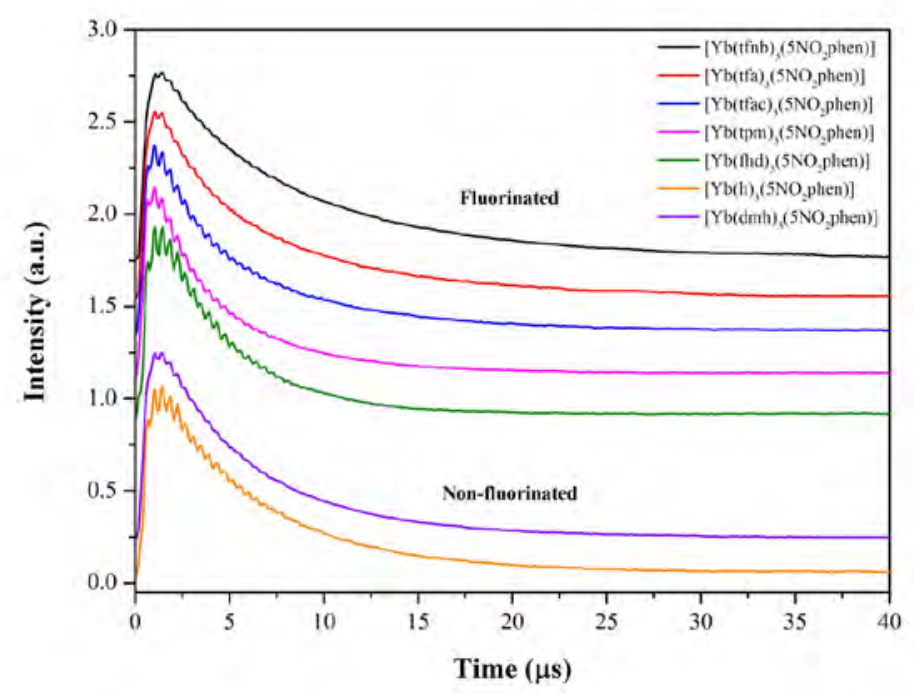

Figure 114. $\mathrm{Yb}^{3+}$ lifetimes upon ligand excitation in the UV region

Table 59 shows a comparison of the $\mathrm{Yb}^{3+}$ lifetime values estimated using the procedure indicated above and those determined by analyzing the intensity decay upon $\mathrm{Yb}^{3+}$ direct excitation at $\lambda=940 \mathrm{~nm}$. The first approach yields lower values, but in fact the correspondence with the emission behavior observed upon ligand excitation (Figure 106) is better and the lifetime value-emission intensity relation is strictly preserved, while in the second approach $\mathrm{Htfa}$ has a slightly higher value than $\mathrm{Htfnb}$. Surprisingly, the $\mathrm{Yb}^{3+}$ excited state lifetime upon ligand excitation is systematically shorter than under direct excitation. A possible explanation to this behavior would be related to structural disorder. Since there is some disorder in the coordination polyhedra, there are slightly different $\mathrm{N}-\mathrm{Yb}$ or $\mathrm{O}-\mathrm{Yb}$ distances. Energy transfer from the ligand to the $\mathrm{Yb}^{3+}$ ion strongly depends on the distance between them. Therefore, ligand to metal energy transfer would preferentially occur in those centers with shorter distances, which could account for the difference in the measured lifetime values [188].

Table 59. Comparison of $\mathrm{Yb}^{3+}:{ }^{2} \mathrm{~F}_{5 / 2} \rightarrow{ }^{2} \mathrm{~F}_{7 / 2}$ lifetime values obtained upon ligand excitation at $\lambda=350 \mathrm{~nm}$ vs. upon $\mathrm{Yb}^{3+}$ direct excitation at $\lambda=940 \mathrm{~nm}$

Complex Lifetime upon ligand excitation ( $\mu \mathrm{s}) \quad$ Lifetime upon $\mathrm{Yb}^{3+}$ direct excitation $(\mu \mathrm{s})$

\begin{tabular}{ccc}
{$\left[\mathrm{Yb}(\mathrm{tfnb})_{3}\left(\mathrm{SNO}_{2}\right.\right.$ phen $\left.)\right]$} & 7.2 & 10 \\
{$\left[\mathrm{Yb}(\mathrm{tfa})_{3}\left(5 \mathrm{NO}_{2}\right.\right.$ phen $\left.)\right]$} & 5.8 & 11 \\
{$\left[\mathrm{Yb}(\mathrm{tfac})_{3}\left(5 \mathrm{NO}_{2}\right.\right.$ phen $\left.)\right]$} & 4.7 & 8.1 \\
{$\left[\mathrm{Yb}(\mathrm{tpm})_{3}\left(5 \mathrm{NO}_{2}\right.\right.$ phen $\left.)\right]$} & 3.6 & 5.2 \\
{$\left[\mathrm{Yb}(\mathrm{fhd})_{3}\left(5 \mathrm{NO}_{2}\right.\right.$ phen $\left.)\right]$} & 3.3 & 3.9 \\
{$\left[\mathrm{Yb}(\mathrm{h})_{3}\left(5 \mathrm{NO}_{2}\right.\right.$ phen $\left.)\right]$} & 5.2 & 6.5 \\
{$\left[\mathrm{Yb}(\mathrm{dmh})_{3}\left(5 \mathrm{NO}_{2}\right.\right.$ phen $\left.)\right]$} & 5.0 & 6.3 \\
\hline
\end{tabular}




\subsubsection{NLO properties of $\mathrm{Yb}(\mathrm{III})$ complexes with Vildagliptin ligand}

For $L=1 \mathrm{~mm}$ samples, the transmittance curves obtained by Z-scan and P-scan techniques for $\left[\mathrm{Yb}(\text { vilda })_{3}\right.$ (bipy)] and $\left[\mathrm{Yb}\left(\right.\right.$ vilda) ${ }_{2}$ (acac)(bipy)] show a defocusing response (red lines in Figure 115 and Figure 116, respectively). The numerical calculations of the Z-scan curves (red solid lines) were obtained in accordance with the Z-scan theory described by Bahae et al. [153], where $n_{2}$ is the value calculated from the experimental data considering an equivalent Kerr medium. The value of $\theta$ was determined by using a numerical fit [189]. The experimental values obtained from P-scan are in agreement with those obtained from Z-scan (Figure 117 and Figure 118). The results of these measurements are summarized in Table 60.

As it has been indicated above, it is possible to obtain either focusing or defocusing response by working with different sample thicknesses. Figure 115 and Figure 116 (black lines) show that the thin film samples $(L \approx 5 \mu \mathrm{m})$ of the two complexes display a focusing behavior, since in this case thermal effects are not accumulated. The settings for P-scans obtained with samples of $L \approx 5 \mu \mathrm{m}$ are also presented in Figure 117 and Figure 118. The obtained values for Z-scan and P-scan in this regime are shown in Table 60 too.

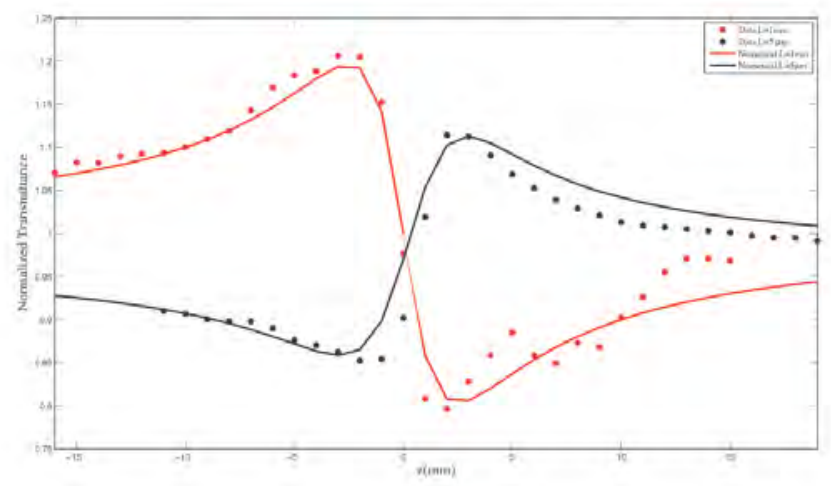

Figure 115. Z-scan closed-aperture measurements for [Yb(vilda) ${ }_{3}($ bipy $\left.)\right]$ samples with thickness $L=1$ $\mathrm{mm}$ (red dotted line) and $L=5 \mu \mathrm{m}$ (black dotted line) and their respective numerical calculations in the defocusing (red solid line) and focusing regime (black solid line).

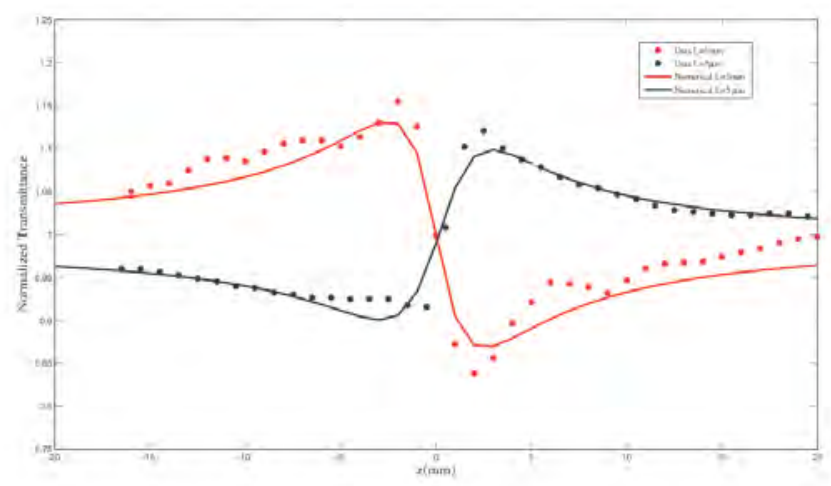

Figure 116. Z-scan closed-aperture measurements for [Yb(vilda) ${ }_{2}$ (acac)(bipy)] samples with thickness $L=1 \mathrm{~mm}$ (red dotted line) and $L=5 \mu \mathrm{m}$ (black dotted line) and their respective numerical calculations in the defocusing (red solid line) and focusing regime (black solid line). 


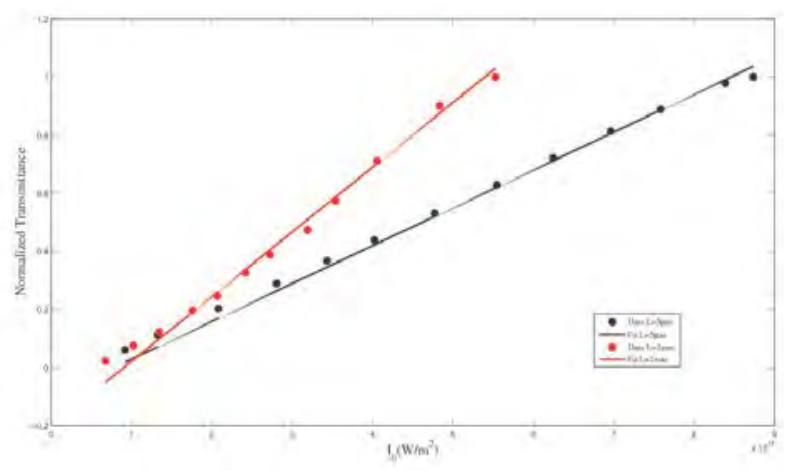

Figure 117. P-scan measurements for $L=1 \mathrm{~mm}$ (red) and $L=5 \mu \mathrm{m}$ (black) [Yb(vilda) $)_{3}($ bipy)] samples.

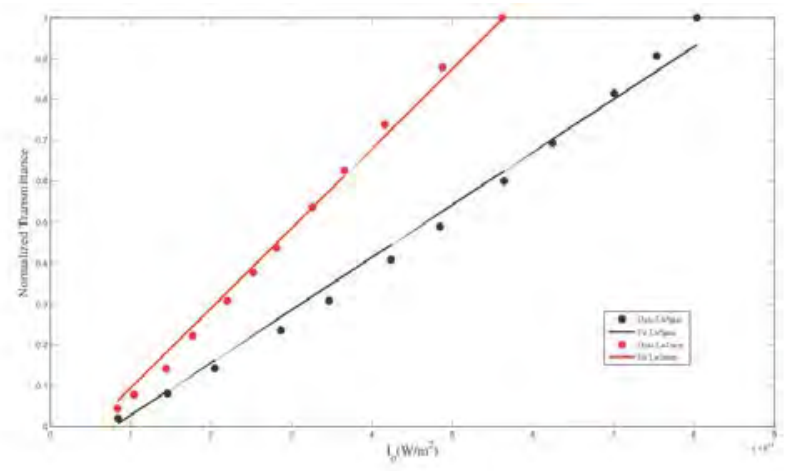

Figure 118. P-scan measurements for $L=1 \mathrm{~mm}$ (red) and $L=5 \mu \mathrm{m}$ (black) [Yb(vilda) ${ }_{2}$ (acac)(bipy)] samples.

Several considerations should be raised in view of Table 60: while in the defocusing regime the nonlinear refractive index of the Vildagliptin complexes is in the same order of magnitude as that of $\mathrm{CS}_{2}$, the $n_{2}$ for both complexes is two orders of magnitude higher $(\times 116$ and $\times 129$ for $\left[\mathrm{Yb}(\text { vilda) })_{3}\right.$ (bipy)] and [Yb(vilda) ${ }_{2}$ (acac)(bipy)], respectively).

In the complex [Yb(vilda) $)_{3}$ (bipy)], the value of the focusing Kerr index has been increased by $38 \%$ vs. that of pure Vildagliptin (already two orders of magnitude higher than that of $\mathrm{CS}_{2}$ ), while the negligible nonlinear absorbance properties are preserved. Nevertheless, against the odds, the complex in which a Vildagliptin unit had been substituted by a $\beta$-diketone (Hacac), [Yb(vilda) 2 (acac)(bipy)], evinces a higher $n_{2}$ value $(11 \%$ higher than that of $[\mathrm{Yb}$ (vilda) 3 (bipy)] and $54 \%$ higher than that of pure Vildagliptin). We consider that this could be attributed, more than to a favorable effect of Hacac, to a structural factor: the higher symmetry in the $\left[\mathrm{Yb}\right.$ (vilda) ${ }_{2}$ (acac)(bipy)] molecule may have a beneficial effect.

These findings are indicative of the promising behavior of Vildagliptin and its derivatives for the development of ultrafast photonics devices. Furthermore, it is noteworthy that $\left[\mathrm{Yb}\left(\mathrm{vilda}_{3}\right.\right.$ (bipy)] shows a negligible nonlinear absorbance, while this feature is not present when one of the Vildagliptin units is substituted by other ligands, such as Hacac (Figure 119).

Table 60. Optical characterization of Vildagliptin complexes in methanol

\begin{tabular}{ccccc} 
& {$\left[\mathrm{Yb}(\text { vilda })_{3}(\right.$ bipy $\left.)\right]\left[\mathrm{Yb}(\text { vilda })_{2}(\mathrm{acac})(\mathrm{bipy})\right]$} & Vildagliptin & $\mathrm{CS}_{2}[154]$ \\
\hline$n_{0}$ & 1.449 & 1.464 & 1.389 & 1.6295 \\
Focusing Kerr index $\left(\mathrm{m}^{2} / \mathrm{W}\right)$ & $3.6 \pm 0.5 \times 10^{-17}$ & $4 \pm 0.5 \times 10^{-17}$ & $2.6 \pm 0.5 \times 10^{-17}$ & $3.1 \pm 1 \times 10^{-19}$ \\
Defocusing Kerr index $\left(\mathrm{m}^{2} / \mathrm{W}\right)$ & $-1.5 \pm 0.5 \times 10^{-18}$ & $-1.5 \pm 0.5 \times 10^{-18}$ & $-1 \pm 0.5 \times 10^{-18}$ & $-1 \pm 0.2 \times 10^{-18}$ \\
$\theta$ & 0.38 & 0.25 & 0.21 & ---- \\
$\beta(\mathrm{m} / \mathrm{W})$ & Not present & $1.2 \pm 0.3 \times 10^{-11}$ & Not present & $1.4 \pm 0.3 \times 10^{-12}$ \\
\hline
\end{tabular}




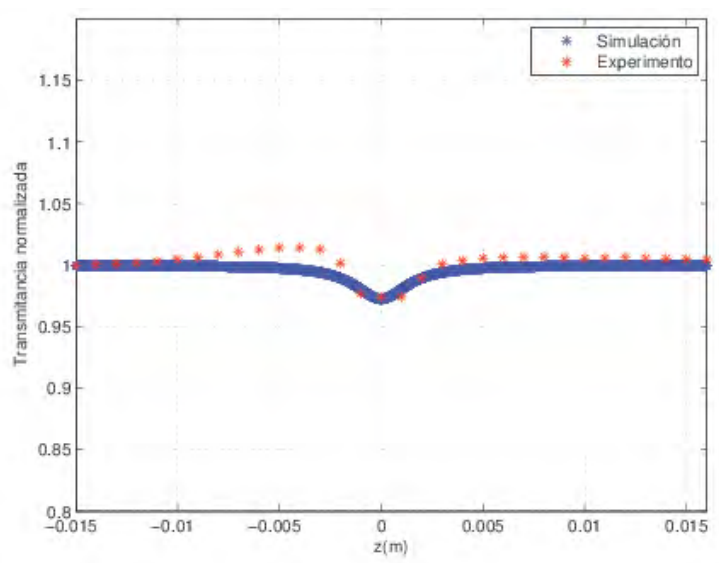

Figure 119. Z-scan open-aperture measurement of the absorbance for [Yb(vilda) ${ }_{2}($ acac)(bipy)] sample $(L=1 \mathrm{~mm}$ ) with $\mathrm{P}=1.734 \mathrm{~W}$ : experimental values (red dots) vs. numerical calculation (blue solid line).

The computed average values over all the tensor components of the second hyperpolarizabilities contributing to the nonlinear refractive index are shown in Table 61. The static values are shown in the first row. The remaining rows of Table 61 show the frequency dependent contributions to the nonlinear refractive index $\gamma_{\mathrm{abcd}}(-\omega ; \omega,-\omega, \omega)$ in atomic units evaluated at different wavelengths within the three telecommunication windows.

Table 61. Second hyperpolarizabilities calculated with MOPAC2012 using the Sparkle/PM6 model (a.u.)

\begin{tabular}{cccc} 
Wavelength & Vildagliptin & {$[$ Yb(vilda) 3 (bipy) $]$} & {$[$ Yb(vilda) 2 (acac)(bipy) $]$} \\
\hline static & $1.4567850 \times 10^{4}$ & $8.7569162 \times 10^{4}$ & $6.3524116 \times 10^{4}$ \\
\hline $800 \mathrm{~nm}$ & $1.9891635 \times 10^{4}$ & $4.1939857 \times 10^{18}$ & $4.6769473 \times 10^{5}$ \\
$810 \mathrm{~nm}$ & $1.9708140 \times 10^{4}$ & $1.3714396 \times 10^{16}$ & $1.8676083 \times 10^{5}$ \\
$850 \mathrm{~nm}$ & $1.9056378 \times 10^{4}$ & $3.3172267 \times 10^{7}$ & $1.2850226 \times 10^{5}$ \\
\hline $1250 \mathrm{~nm}$ & $1.6309816 \times 10^{4}$ & $1.1759568 \times 10^{5}$ & $8.1512673 \times 10^{4}$ \\
$1270 \mathrm{~nm}$ & $1.6248433 \times 10^{4}$ & $1.1626744 \times 10^{5}$ & $8.1312022 \times 10^{4}$ \\
$1300 \mathrm{~nm}$ & $1.6162484 \times 10^{4}$ & $1.1445286 \times 10^{5}$ & $7.9776908 \times 10^{4}$ \\
\hline $1500 \mathrm{~nm}$ & $1.5730899 \times 10^{4}$ & $1.0603732 \times 10^{5}$ & $7.4996519 \times 10^{4}$ \\
$1540 \mathrm{~nm}$ & $1.5668766 \times 10^{4}$ & $1.0486768 \times 10^{5}$ & $7.4254883 \times 10^{4}$ \\
$1560 \mathrm{~nm}$ & $1.5636158 \times 10^{4}$ & $1.0432705 \times 10^{5}$ & $7.3933560 \times 10^{4}$ \\
$1600 \mathrm{~nm}$ & $1.5579700 \times 10^{4}$ & $1.0332402 \times 10^{5}$ & $7.3334652 \times 10^{4}$ \\
\hline
\end{tabular}

Even though these are microscopic molecular results and drawing a relation with the macroscopic susceptibilities should take into consideration the density of molecules and local field effects, a high correlation with the macroscopic nonlinear refractive index is expected [190]. The results for the two new complexes and pure Vildagliptin show a very small dispersion in the nonlinear refractive index in the second and third optical communications windows. For all the wavelengths studied, and also in the static case, the two new complexes display an enhancement of the nonlinear refraction as compared to that of pure Vildagliptin. Moreover, the molecular hyperpolarizability term contributing to the nonlinear refractive index of [Yb(vilda) $)_{3}$ (bipy)] is larger than that of [Yb(vilda) ${ }_{2}($ acac)(bipy)] in all the cases. This apparent inconsistency with the experimental results (which evince a larger $n_{2}$ in [Yb(vilda) $)_{2}$ (acac)(bipy)]) can be explained by the fact that the evaluation of the impact of the microscopic results on the measured value of $n_{2}$ should include corrections such as the molecule size (vide supra), which favors a larger concentration of the pure material in the case of [Yb(vilda) ${ }_{2}$ (acac)(bipy)]. 
The computed nonlinear refractive index of all three species shows a steady spectral growth as we approach the visible part of the spectrum. This happens much more markedly in the case of [Yb(vilda) $)_{3}$ (bipy)], for which the values of the second hyperpolarizability in the first telecomm window are too large to be accepted as realistic. The same type of exponential growth is found for [Yb(vilda) $)_{2}$ (acac)(bipy)] at shorter wavelengths, with a value of $4.767 \times 10^{11}$ a.u. at $750 \mathrm{~nm}$. The calculations for the nonlinear refractive index of Vildagliptin are found to be stable at least up to $650 \mathrm{~nm}$.

Notwithstanding above reported results, it should be noted that this study is at a very early stage of its development: further research is still required so as to explore alternative coordination environments and it would also be convenient to compare the focusing regime values obtained for thin film samples with those obtained using a well-established method (such as using a lower frequency laser source or a chopper) and a thicker sample. Efforts are currently being undertaken, as part of J.A. Nóvoa López's Ph.D. Thesis at Universidade de Vigo (Orense, Spain), so as to continue studying these promising materials and in order to evaluate their potential use in novel types of photonics devices. 


\subsubsection{Magnetic measurements}

A detailed analysis, although still unfinished, of the SIM magnetic behavior of $\left[\operatorname{Er}(\text { thd })_{3}\right.$ (bath)] complex is presented. This is the first $\mathrm{Er}^{3+} \beta$-diketonate complex to display slow magnetic relaxation behavior to date. Although AC susceptibility measurements indicate the presence of significantly fast QTM in zero DC field, the application of a DC field slows down the relaxation and two thermally activated barriers can be discerned. The occurrence of two relaxation mechanisms may be explained by the structural disorder in an otherwise crystallographic unique complex.

Subsequently, an assessment of the N,N-donor choice influence on SIM behavior is reported for $\left[\operatorname{Er}(\mathrm{h})_{3}(\mathrm{~N}, \mathrm{~N}-\right.$ donor $\left.)\right]$ family.

\section{Static magnetic properties of [Er(thd $)_{3}$ (bath)]}

The temperature dependence of the magnetic susceptibility under static field for [Er(thd) $)_{3}$ (bath)] was collected in the range from 4 to $300 \mathrm{~K}$ at $500 \mathrm{G}$ (Figure 120). The $\chi_{\mathrm{M}^{-1}} \mathrm{vs}$. $\mathrm{T}$ plot shows a linear tendency above $15.2 \mathrm{~K}$, confirming the single-lanthanide-ion behaviour. The room temperature $\chi_{M} \mathrm{~T}$ value is $11.42 \mathrm{emu} \cdot \mathrm{mol}^{-1}$, in good agreement with the expected paramagnetic value for $\mathrm{J}=15 / 2,11.48 \mathrm{emu} \cdot \mathrm{mol}^{-1}$ [191]. The decrease of the $\chi_{\mathrm{M}} \mathrm{T}$ values upon cooling can be explained by depopulation of the Stark level split by the ligand field, suggesting the presence of significant magnetic anisotropy.

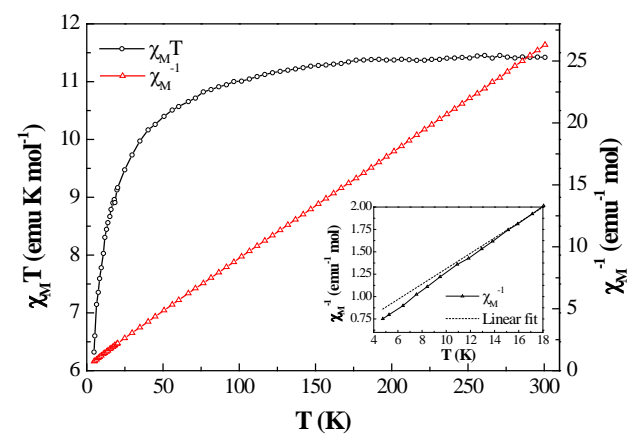

Figure 120. Static magnetic properties of the complex: plots of $\chi_{\mathrm{M}} \mathrm{T}$ (black) and $\chi_{\mathrm{M}^{-1}}$ (red) vs. T in the 4.75 to $300 \mathrm{~K}$ range. The inset shows the diversion of $\chi_{\mathrm{M}^{-1}}$ values from the linear fit at low temperatures.

\section{Dynamic magnetic properties of [Er(thd $)_{3}($ bath)]}

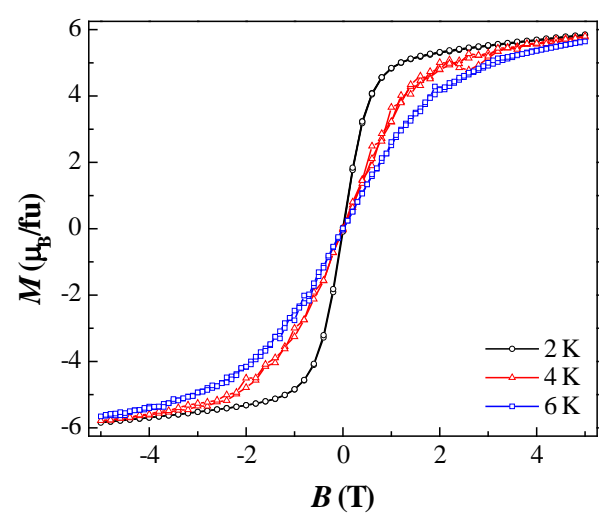

Figure 121. Hysteresis loops at different temperatures for [Er(thd $)_{3}($ bath $\left.)\right]$ complex 
The magnetization dynamics of the complex were probed using AC measurements. In the absence of a static magnetic field, no appreciable out-of-phase component of the AC susceptibility could be observed. By applying a field of $500 \mathrm{G}$, as shown in Figure 122, both in-phase $\left(\chi^{\prime}\right)$ and out-of-phase $\left(\chi^{\prime \prime}\right)$ signals showed strong frequency dependence at $1.7-10 \mathrm{~K}$, with two peaks clearly discerned at some frequencies.

This behavior is possibly due to fast zero-field tunneling of the magnetization between sublevels, which is suppressed with the application of the DC field.
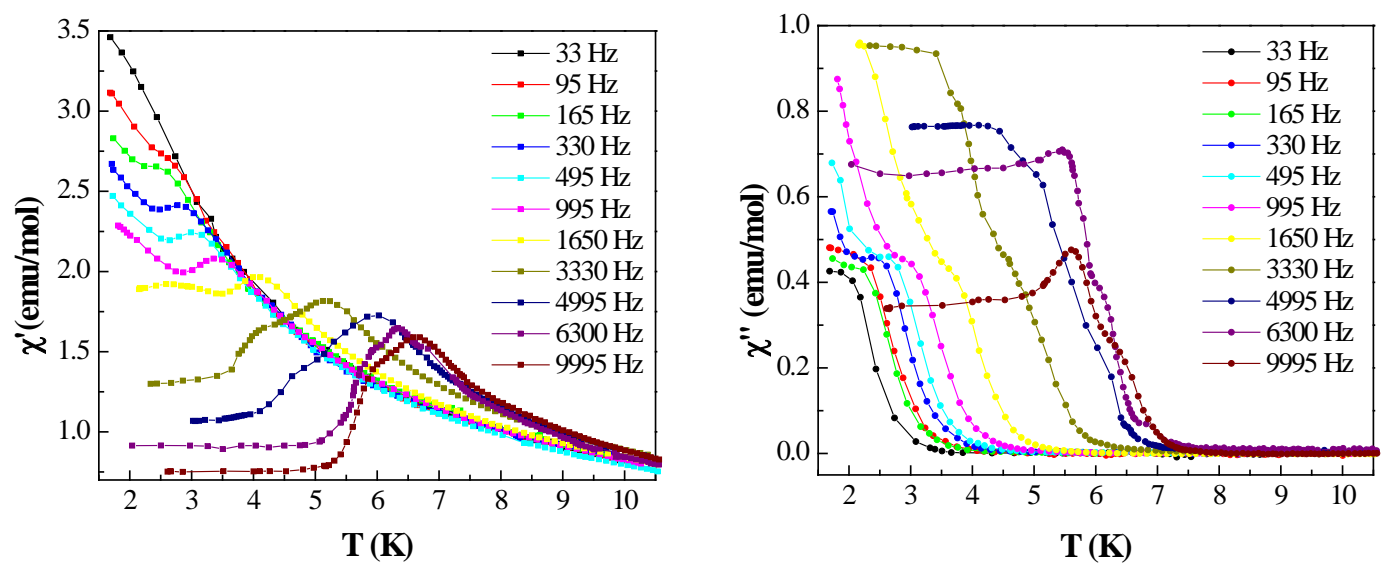

Figure 122. In-phase (left) and out-of-phase (right) components of AC susceptibility at different frequencies in the absence of $\mathrm{dc}$ field in the $1.7 \mathrm{~K}$ to $10 \mathrm{~K}$ temperature range

The observed peaks in the signals correspond to the coincidence of the applied field oscillation with the relaxation rate. Using the values of the temperature where the maximum values occurred ${ }^{\mathrm{n}}$ and considering $\omega \tau=1$, two Arrhenius equations $\left(\tau_{i}(T)=\tau_{0, i}\right.$. $\exp \left(U_{i} / k_{B} T\right)$, for $\mathrm{i}=\mathrm{FR}$ and SR since here we apply it independently to describe the fast or slow relaxation species) were fitted to estimate the magnetization-relaxation parameters. The best fitting parameters afforded a barrier height $\mathrm{U}_{\mathrm{FR}} / \mathrm{k}_{\mathrm{B}}=7.30$ and $\mathrm{U}_{\mathrm{SR}} / \mathrm{k}_{\mathrm{B}}=23.51 \mathrm{~K}$ with $\tau_{0, \mathrm{FR}}=6.58 \times 10^{-6} \mathrm{~s}$ and $\tau_{0, \mathrm{SR}}=4.66 \times 10^{-7} \mathrm{~s}$ (Figure 123), but these values are affected by a large uncertainty due to the difficulty in deconvoluting the peaks in the susceptibility curves.
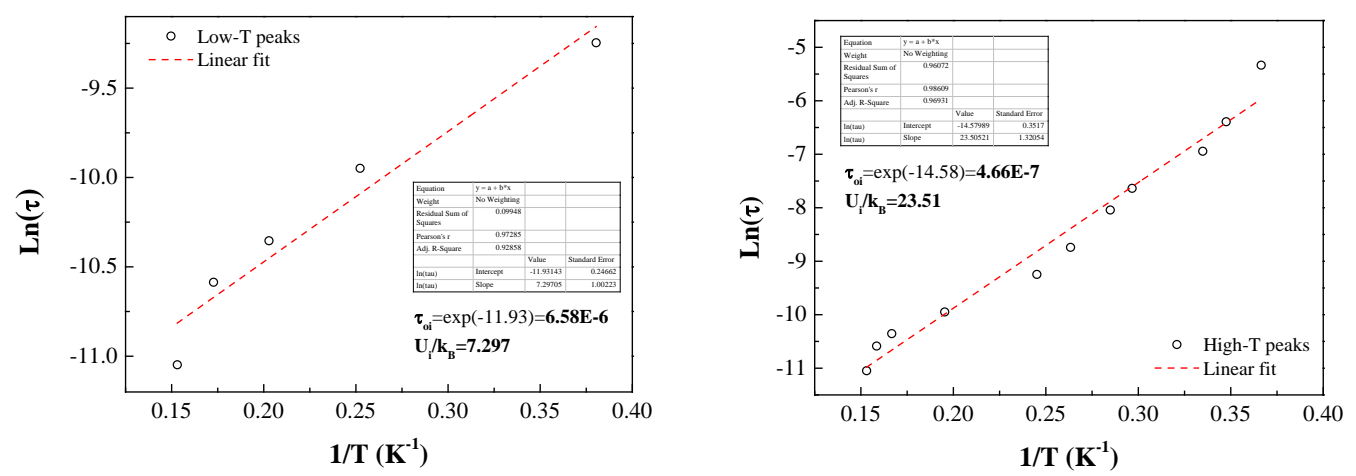

Figure 123. Arrhenius Law fittings for the FR (left) and the SR (right) species of the [Er(thd) $\left.{ }_{3}(\mathrm{bath})\right]$ complex n Gaussian fits superimposed on Curie-Weiss Law curves were used in order to improve the accuracy
of the peak positions estimations. 
Two Curie-Weiss laws were used to fit the in-phase signal above the peaks in order to extract the relative fractions of the fast relaxation and slow relaxation species. The values obtained were 0.66 and 0.33 .

An Argand diagram of the out-of-phase vs. the in-phase susceptibility at low temperatures was constructed. When there is a single relaxation time, the plot has the ideal semi-circular shape; conversely, if there is a distribution of relaxation times, the $\chi(\omega) " / \chi(\omega)^{\prime}$ curve is somewhat distorted semicircle. Therefore, two distorted semicircles are seen when there is a distribution around two different relaxation times, as it is the case here.

To determine the distribution of relaxation processes, we have fitted the complex susceptibility given by a linear combination of two modified Debye models at each temperature, according to [192] (Figure 124):

$$
\chi_{\text {total }}^{*}(\omega)=\chi_{S}+\left(\chi_{T}-\chi_{S}\right)\left[\frac{\beta}{1+\left(i \omega \tau_{F R}\right)^{1-\alpha_{F R}}}+\frac{1-\beta}{1+\left(i \omega \tau_{S R}\right)^{1-\alpha_{S R}}}\right]
$$
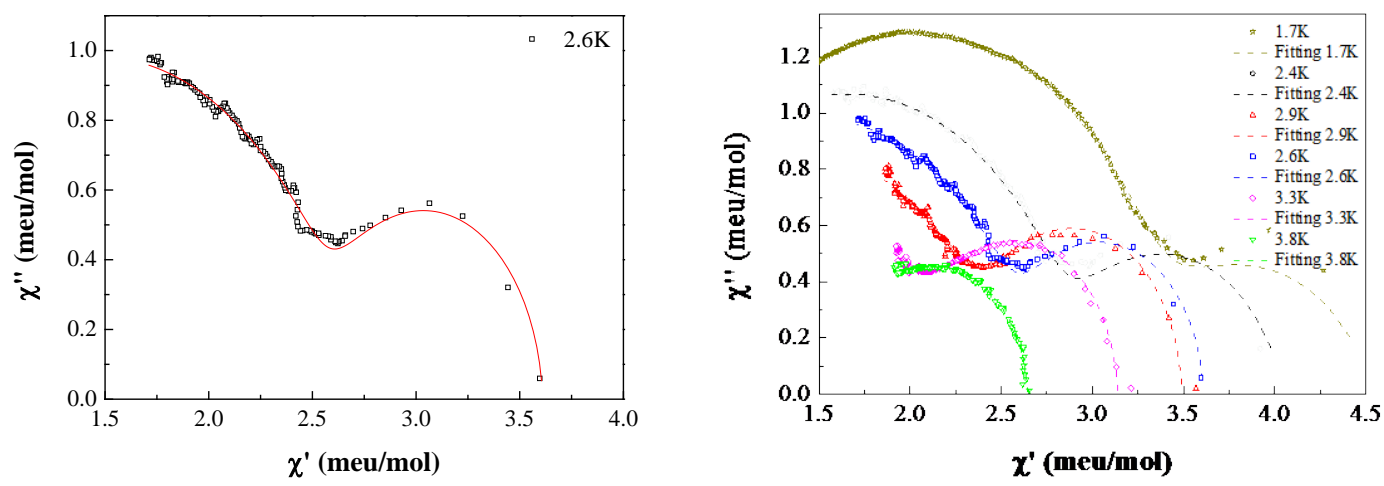

Figure 124. Argand diagram and fitting of the linear combination of two modified Debye models for the $2.6 \mathrm{~K}$ curve (left) and general view of the fittings for all temperatures ranging from $1.7 \mathrm{~K}$ to $3.8 \mathrm{~K}$

(right)

The $\beta$ parameter average value is 0.687 (Table 62). Consequently, the weight ratio of the fast and slow relaxation species is $0.687: 0.313$, very close to the values obtained above from the Curie-Weiss law (0.66:0.33).

Table 62. Modified Debye model fitting parameters from 1.7 to $3.8 \mathrm{~K}$ for [Er(thd $)_{3}($ bath)]

\begin{tabular}{cccccccc}
$\mathrm{T}(\mathrm{K})$ & $\chi_{\mathrm{s}}(\mathrm{emu} / \mathrm{mol})$ & $\chi_{\mathrm{T}}(\mathrm{emu} / \mathrm{mol})$ & $\tau_{1}(\mathrm{~s})$ & $\alpha_{1}$ & $\tau_{2}(\mathrm{~s})$ & $\alpha_{2}$ & $\beta$ \\
\hline 1.7 & 0.630 & 4.543 & $2.743 \times 10^{-5}$ & 0.049 & 0.00195 & 0.259 & 0.685 \\
2.4 & 0.395 & 4.048 & $1.691 \times 10^{-5}$ & 0.103 & 0.00168 & 0.173 & 0.671 \\
2.6 & 0.278 & 3.610 & $1.177 \times 10^{-5}$ & 0.122 & $7.319 \times 10^{-4}$ & 0.025 & 0.702 \\
2.9 & 0.230 & 3.448 & $7.278 \times 10^{-6}$ & 0.196 & $4.531 \times 10^{-4}$ & 0 & 0.707 \\
3.3 & 0.123 & 3.140 & $3.033 \times 10^{-6}$ & 0.269 & $1.950 \times 10^{-4}$ & 0 & 0.700 \\
3.8 & 0.057 & 2.624 & $1.627 \times 10^{-6}$ & 0.323 & $4.241 \times 10^{-5}$ & 0 & 0.652 \\
\hline
\end{tabular}

The two relaxation processes may be explained by the presence of structural disordered complexes in unit cell. The terminal methyl groups, which occupy two main staggered positions (roughly 60/40 occupation), may influence the distribution of orbitals in the lanthanide and therefore the energy barriers to overcome. 


\section{Preliminary assessment of N,N-donor choice influence on SIM behavior}

$\left[\operatorname{Er}(\mathrm{h})_{3}(\mathrm{~N}, \mathrm{~N} \text {-donor)] complexes display a simpler behavior than [Er(thd })_{3}(\right.$ bath $\left.)\right]$, with only one relaxation process. A detailed analysis will be published elsewhere in the next few months, but preliminary results are presented so as to assess the impact of the $\mathrm{N}, \mathrm{N}$-donor choice on the magnetic properties. The most relevant data, namely static magnetic properties (Figure 125), magnetization hysteresis loops (Figure 126), AC susceptibility data at different frequencies (Figure 127), Arrhenius Law fittings (Figure 128) and Argand diagrams (Figure 129) are presented below.

A comparison of the three complexes' behavior indicates that bathophenanthroline ancillary ligand leads to the best magnetic properties (i.e. the highest effective barrier), followed by 5nitro-1,10-phenanthroline and lastly by 2,2 '-bipyridine adducts. The bulkier bath molecule enlarges the crystal field splitting, turning the complex more anisotropic, with a larger difference in the susceptibility of the easy and hard axis and thus a larger energy barrier to overcome. This can be tentatively explained by the fact that the delocalized $\pi$ electrons system of bath moiety can facilitate the generation of higher order uniaxial local symmetry, i.e., its high order single axial field can generate an Ising-type degenerated ground state which can give rise to uniaxial anisotropy and possibly make this molecule behave as a high relaxation energy barrier. Accordingly, this effect is less prominent in $5 \mathrm{NO}_{2}$ phen and even weaker in bipy Lewis bases.

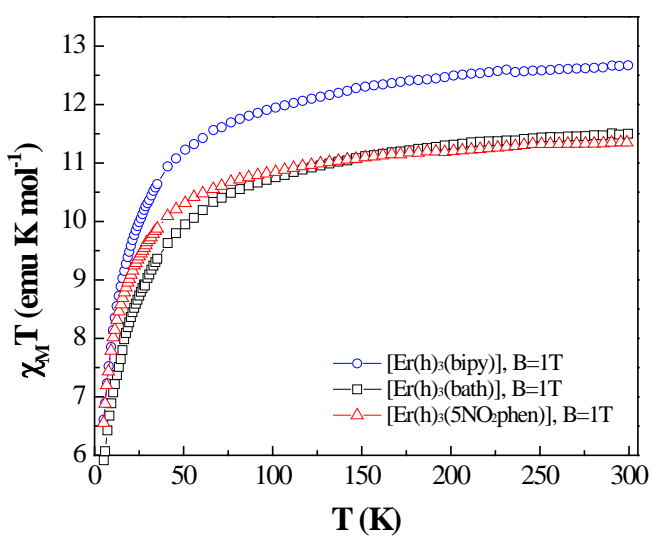

Figure 125. Static magnetic properties of the $\left[\operatorname{Er}(\mathrm{h})_{3}(\mathrm{~N}, \mathrm{~N}-\mathrm{donor})\right]$ complexes: plots of $\chi_{\mathrm{M}} \mathrm{T}$ vs. $\mathrm{T}$ in the $5 \mathrm{~K}$ to $300 \mathrm{~K}$ range.
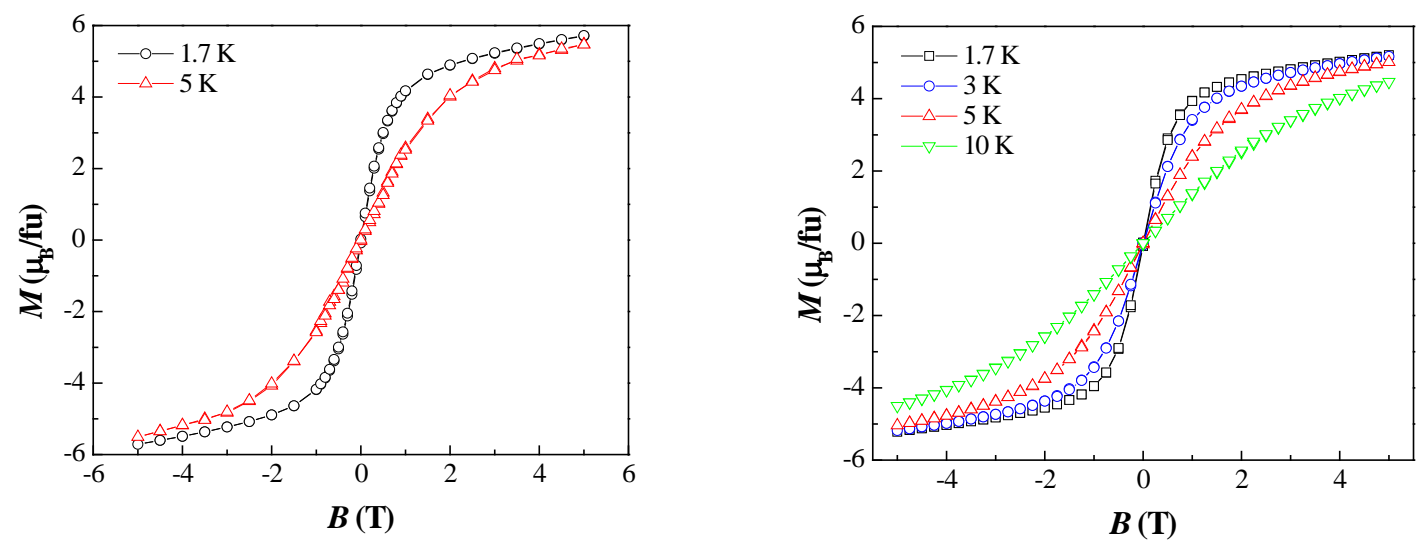


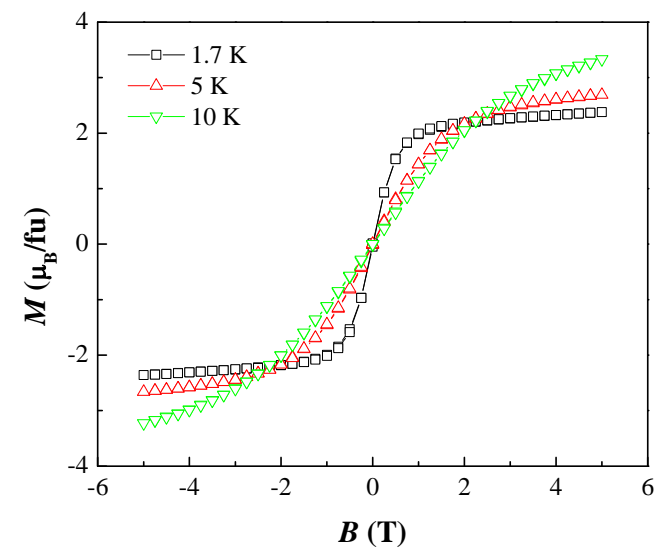

Figure 126. Hysteresis loops at different temperatures for $\left[\operatorname{Er}(\mathrm{h})_{3}(\right.$ bipy) $]$ (top left), $\left[\operatorname{Er}(\mathrm{h})_{3}(\right.$ bath) $]$ (top right) and $\left[\operatorname{Er}(\mathrm{h})_{3}\left(5 \mathrm{NO}_{2}\right.\right.$ phen) $]$ (bottom left).
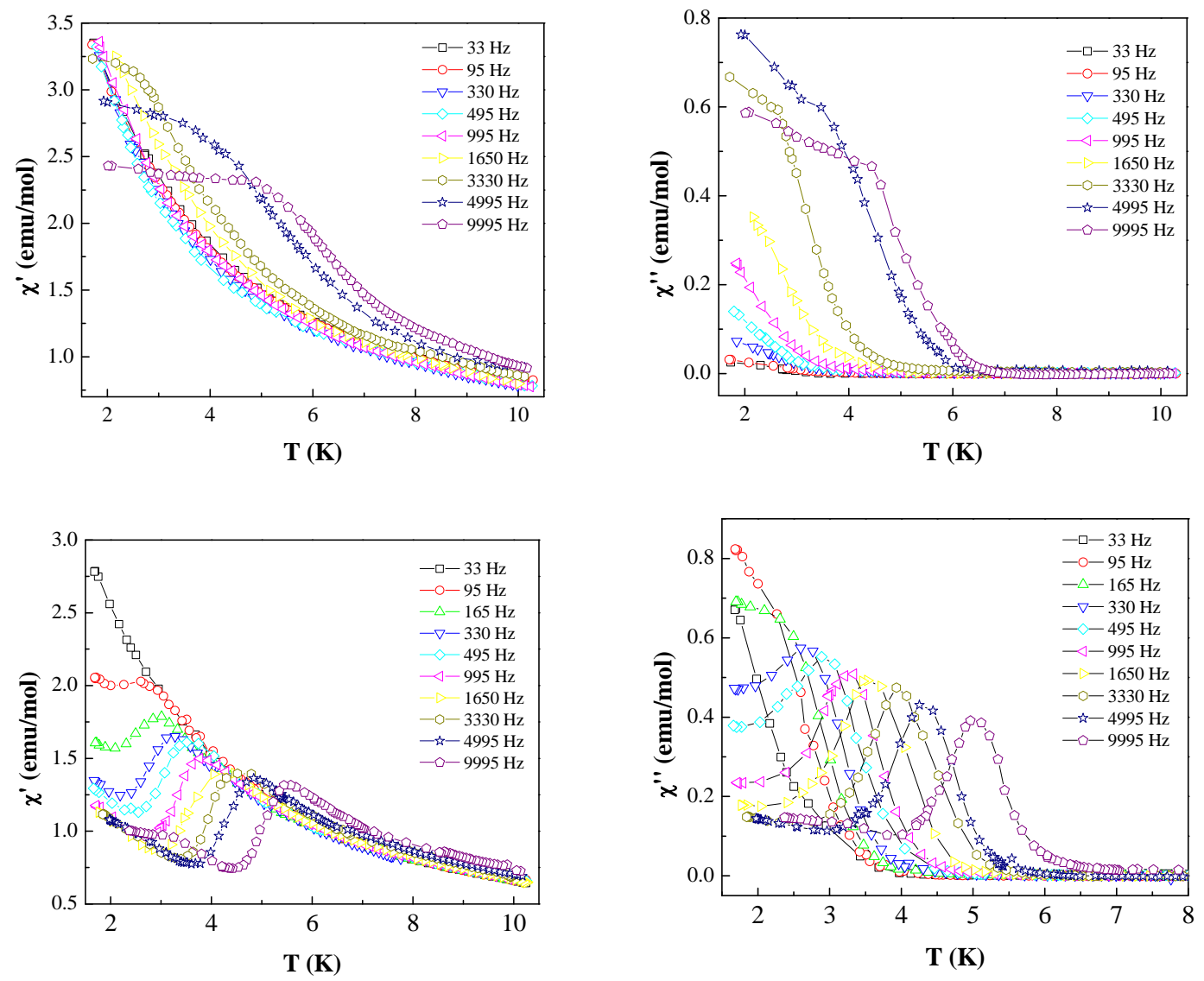

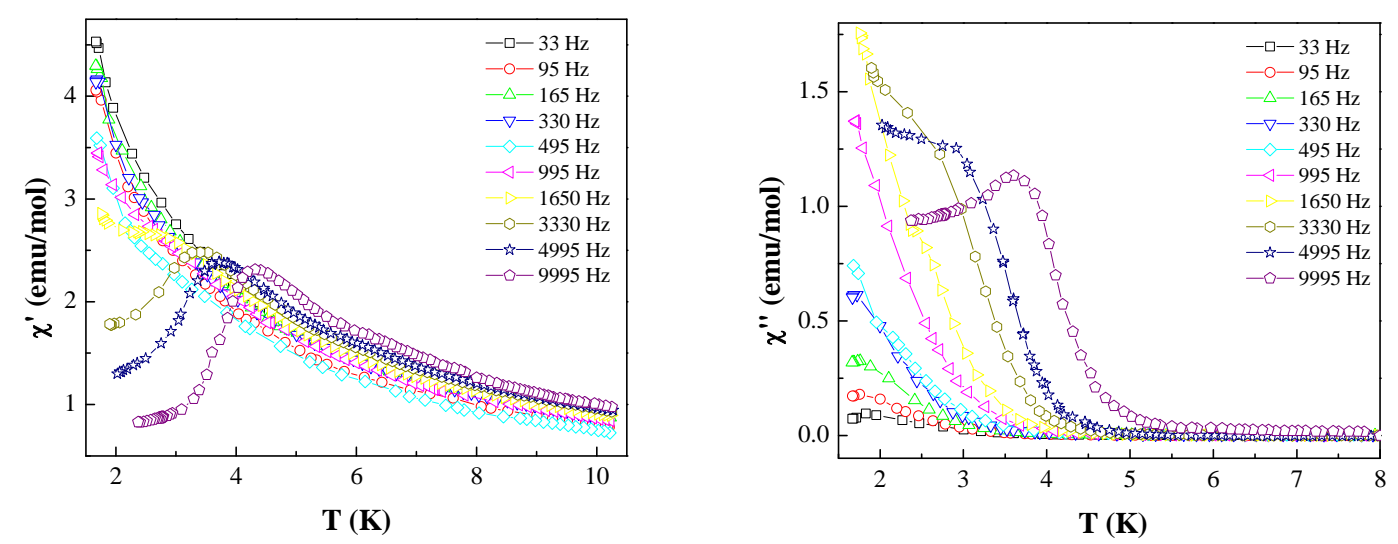

Figure 127. In-phase (left) and out-of-phase (right) components of AC susceptibility at different frequencies in the absence of dc field in the 1.7 to $10 \mathrm{~K}$ temperature range for [ $\left.\operatorname{Er}(\mathrm{h})_{3}(\mathrm{bipy})\right]$ (top), $\left[\operatorname{Er}(\mathrm{h})_{3}(\right.$ bath $\left.)\right]($ center $)$ and $\left[\operatorname{Er}(\mathrm{h})_{3}\left(5 \mathrm{NO}_{2}\right.\right.$ phen $\left.)\right]($ bottom $)$. Hac $=5$ Oe; B=1000 Oe.
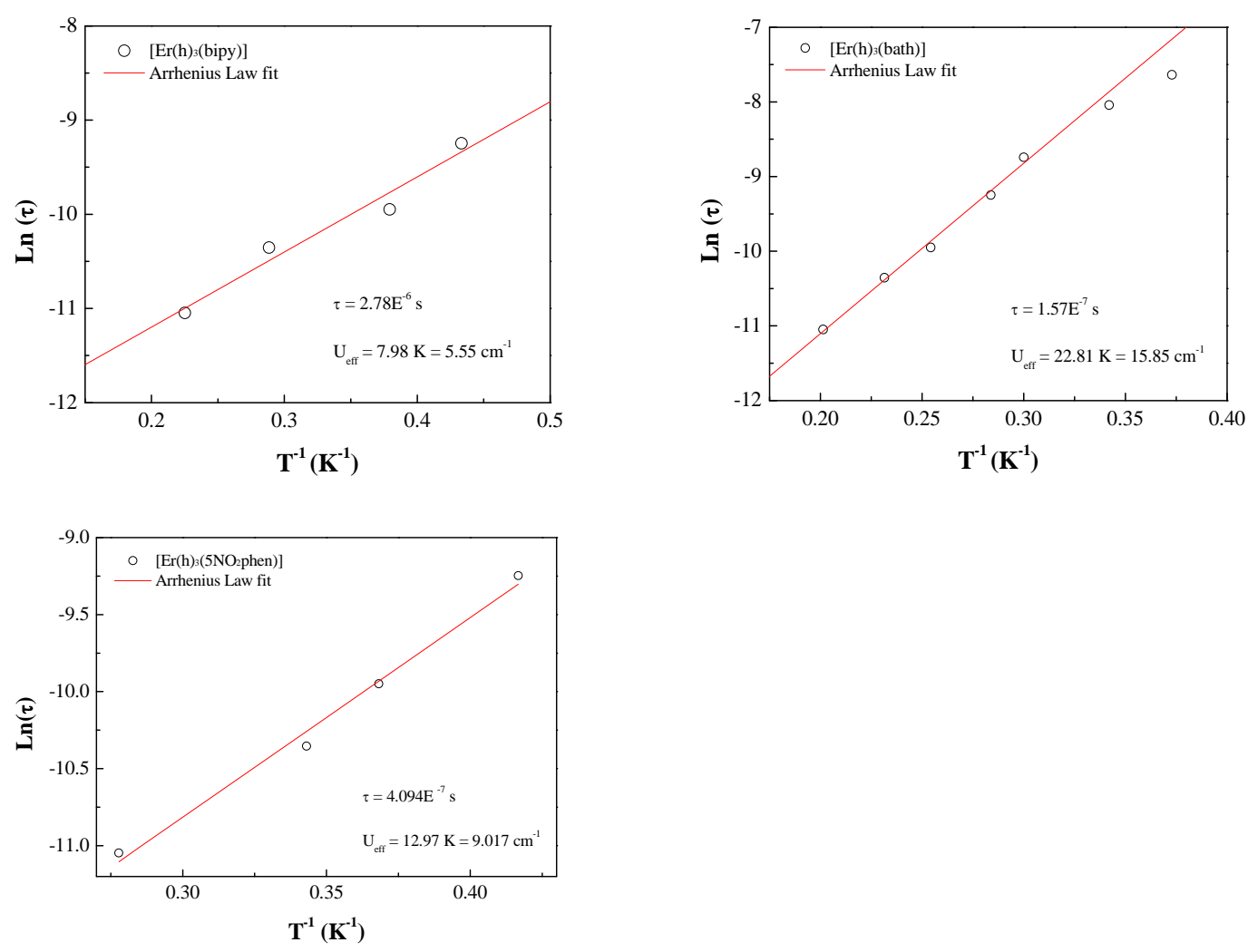

Figure 128. Arrhenius Law fittings for [ $\operatorname{Er}(\mathrm{h})_{3}\left(\right.$ bipy)] (top left), $\left[\operatorname{Er}(\mathrm{h})_{3}(\right.$ bath $\left.)\right]$ (top right) and $\left[\mathrm{Er}(\mathrm{h})_{3}\left(5 \mathrm{NO}_{2}\right.\right.$ phen $\left.)\right]$ (bottom left $)(\mathrm{Hac}=5 \mathrm{Oe} ; \mathrm{B}=1000 \mathrm{Oe})$ 

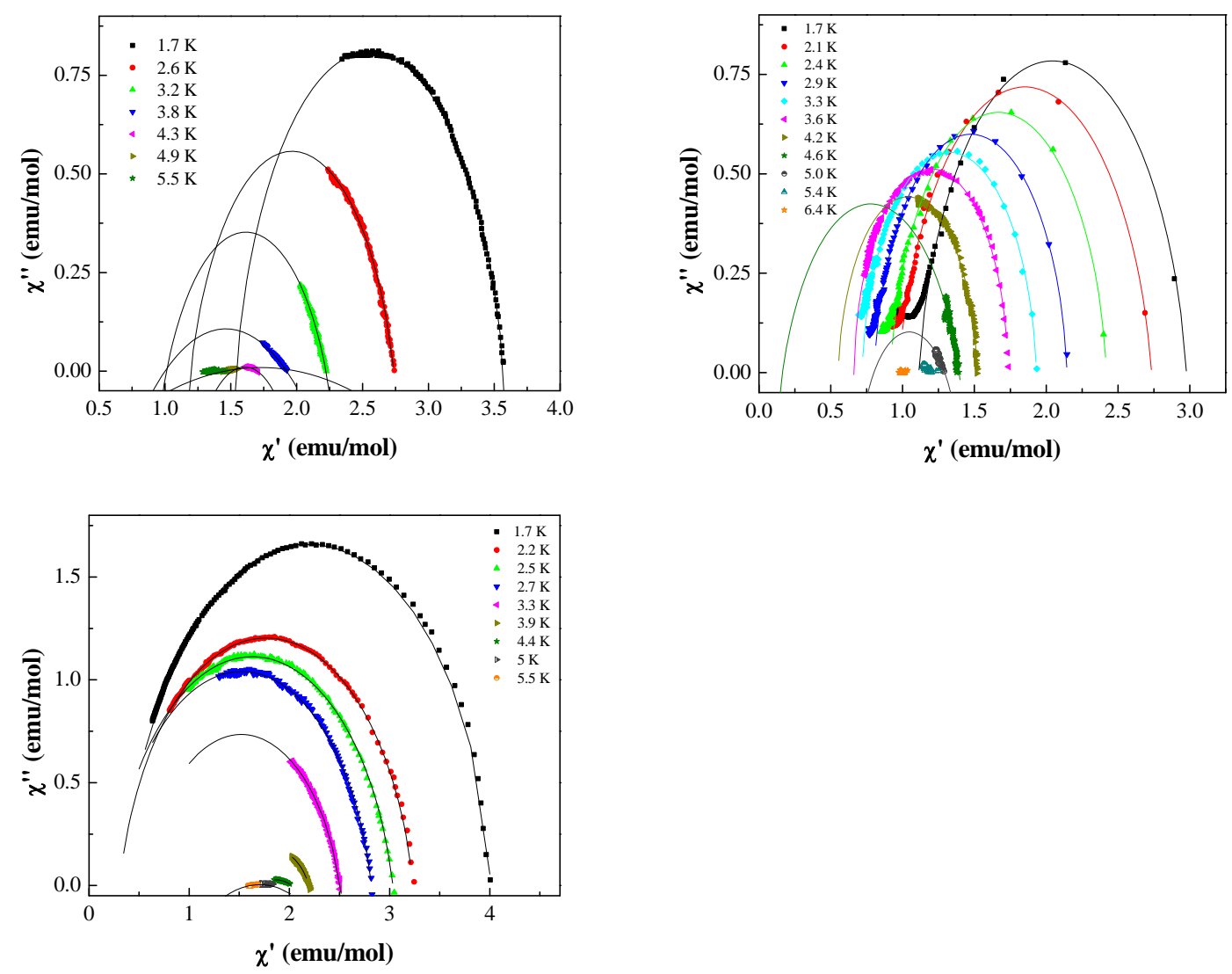

Figure 129. Argand diagrams and Debye fittings for [ $\operatorname{Er}(\mathrm{h})_{3}($ bipy) $]$ (top left), $\left[\operatorname{Er}(\mathrm{h})_{3}(\right.$ bath $\left.)\right]$ (top right) and $\left[\operatorname{Er}(\mathrm{h})_{3}\left(5 \mathrm{NO}_{2}\right.\right.$ phen)] (bottom left). Hac=5 Oe; $\mathrm{B}=1000$ Oe.

Finally, it should be clarified that -given the absence of prior research on the magnetic behavior of $\operatorname{Er}($ III) $\beta$-diketonate complexes- these materials have been chosen so as to be as representative of the entire series as possible and attempting to exclude 'optimized luminescence-oriented' features. Consequently, non-fluorinated $\beta$-diketonates with mediumlength chains (hexanediones and heptanediones) have been selected. In the particular case of the first studied complex, $\left[\operatorname{Er}(\text { thd })_{3}(\right.$ bath $\left.)\right]$, the bathophenanthroline ancillary ligand was chosen on the basis of theoretical assumptions, which have been subsequently proven right for the $\left[\operatorname{Er}(\mathrm{h})_{3}(\mathrm{~N}, \mathrm{~N}-\right.$ donor $\left.)\right]$ complexes. 


\section{IMPLICATIONS OF THE NEW MATERIALS FOR OPTOELECTRONIC APPLICATIONS}

\subsection{Correlation between structural data and photoluminescence properties}

Strictly speaking, the majority of compounds under consideration in this work are phenanthroline-like adducts of the $\beta$-diketonate complexes of $\operatorname{Ln}(\mathrm{III})$ ( $\mathrm{Ln}=\mathrm{Er}, \mathrm{Yb}$ ) and should more appropriately be formulated as $\operatorname{Ln}(\beta \text {-diketonate })_{3} \cdot$ bath, $\operatorname{Ln}(\beta \text {-diketonate })_{3} \cdot$ bipy and $\operatorname{Ln}(\beta \text {-diketonate })_{3} \cdot 5 \mathrm{NO}_{2}$ phen. This is because, in aforementioned compounds, the $\mathrm{Ln}^{3+}$ ion is coordinated by six oxygen atoms of the $\beta$-diketonate and two nitrogen atoms of phenanthroline type $\mathrm{N}, \mathrm{N}$-donor (either bath, bipy or $5 \mathrm{NO}_{2}$ phen).

An analysis of the SC-XRD elucidated structures (30) in relation to their corresponding PL spectra shows that, with a view to protecting the $\mathrm{Ln}^{3+}$ ion from quenchers, the chelating bidentate coordination by $\beta$-diketonate and phenanthroline ligands is more effective than bidentate coordinating by nitrates. Unlike what happens with nitrates, $\beta$-diketonate binding only leaves enough space for the coordination of one bidentate phenanthroline ligand (instead of water molecules) in order to saturate the coordination sphere. Thus, the binding of water molecules, which can deactivate the lanthanide excited state through non-radiative decay (vibronic coupling with modes $\mathrm{OH}$ ), is effectively prevented. It should be kept in mind that erbium(III) is inherently more sensitive to quenching by molecular vibrations in the near infrared that other luminescent ions (e.g. ytterbium(III)), so the prevention of nonradiative deactivation in the erbium(III) complexes is the most important goal that needs to be pursued in this particular case. In this regard, it has been proposed to use fluorinated $\beta$ diketonates rather than non-fluorinated $\beta$-diketonates, replacing the $\mathrm{CH}_{3}$ groups with $\mathrm{CF}_{3}$ groups, so to avoid the effect due to $\mathrm{CH}$ stretching mode, which has a vibrational frequency very close to that of $\mathrm{OH}$.

By correlating the data presented in Section 2.4.1 and Section 2.4.5, partial conclusions can be drawn with a view in ulterior application of the materials. It can be inferred that the improvement in PL emission intensity in the complexes with fluorinated ligands may not be associated only to the lowest vibrational energy of the C-F bonds $v$. the $\mathrm{C}-\mathrm{H}$ bonds, but to a significant decrease in the Lewis base-erbium (III) distance. From the structural data, it can be observed that, while Er-O distances are almost the same in complexes with fluorinated ligands and in those with non-fluorinated ones (2.306 $\AA$ vs. $2.290 \AA)$, Er-N distances are significantly shorter for the fluorinated compounds ( $2.50 \AA$ ss. $2.56 \AA$ ). This shortening of the Er-N distance results in an increased antenna effect, which can be further enhanced by a wise ancillary ligand choice: as indicated in Section 2.4.1, Er-N distances are remarkably shorter for 5-nitro-1,10-phenanthroline adducts $(2.44 \AA$ vs. $2.52 \AA$ for 2,2'-bipyridine and bathophenanthroline ones).

Another interesting observation is that shorter Er- $\mathrm{N}$ distances are associated to shorter $\beta$ diketonate chains (Table 48: e.g., for fluorinated complexes with 2,2'-bipyridine:

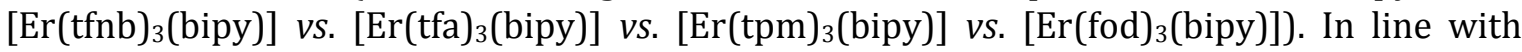
previous paragraph reasoning line, then complexes with shorter $\beta$-diketonante chains should show an increased PL emission intensity, and this should apply not only to Er(III) complexes, but any highly-coordinated Ln(III) complex. The study conducted for Yb(III) complexes with $5 \mathrm{NO}_{2}$ phen (vide supra) also supports this hypothesis. Moreover, it suggests that -for the same $\beta$-diketonate chain length- aromatic substituents (e.g. phenyl, furyl, naphthyl, etc) increase the luminescence. Again, Er-N distances for $\left[\operatorname{Er}(\operatorname{tfnb})_{3}\left(\right.\right.$ bipy)] vs. $\left[\operatorname{Er}(\operatorname{tfa})_{3}(\right.$ bipy)] confirm this premise.

All in all, complexes with short chains and aromatic substituents in the $\beta$-diketonate and with 5-nitro-1,10-phenanthroline as the ancillary ligand should be the most advisable choice when the application places emphasis on the PL emission properties. 


\subsection{Specific role of the organic ligands on the photoluminescence of ternary complexes and their relationship with pumping wavelengths}

As it has already been indicated, the main role of the organic ligands is to saturate the coordination sphere of the $\mathrm{Ln}^{3+}$ ion. Nevertheless, an equally important feature is to efficiently collect the excitation light and transfer this energy to the excited states of $\mathrm{Ln}^{3+}$ ion.

For the Er(III) particular case, upon direct excitation of the $\mathrm{Er}^{3+}$ ion in the ${ }^{4} \mathrm{f}_{11}$ manifold at 520 $\mathrm{nm}$ (population of the ${ }^{2} \mathrm{H}_{11 / 2}$ level), a near-infrared photoluminescence signal centered at around $1520 \mathrm{~nm}$ can be observed at room temperature [37]. This signal corresponds to the transition of the first excited state $\left({ }^{4} \mathrm{I}_{13 / 2}\right)$ to the ground state $\left({ }^{4} \mathrm{I}_{15 / 2}\right)$ of the $\mathrm{Er}^{3+}$ ion. When the excitation wavelength is changed to the UV region, the intensity of the photoluminescence signal increases dramatically by a factor of more than 2 (even 3), as shown in Figure 102. This excitation wavelength $(\sim 300 \mathrm{~nm})$ corresponds to the absorption maximum of the organic ligands. This indicates that a very efficient energy transfer occurs between the organic ligands and the central $\mathrm{Er}^{3+}$ ion.

Same applies to $\mathrm{Yb}^{3+}$, although in this case the energy transfer from the ligand triplet state to the excited $\mathrm{Yb}^{3+}$ level would be vibronically-assisted. Moreover, studies currently under way for $\mathrm{Eu}(\mathrm{III}), \mathrm{Tm}(\mathrm{III})$ and $\mathrm{Tb}(\mathrm{III}) \beta$-diketonate complexes -conducted in collaboration with CEMDRX- further confirm that $\beta$-diketonates are the most versatile choice for the sensitization of $\operatorname{Ln}(\mathrm{III})$ complexes.

Notwithstanding the advantageous effect of changing the excitation wavelength from the erbium band at $520 \mathrm{~nm}$ to the UV in terms of emission intensity (2.5-fold increase), it should also be emphasized that the ability to excite $\mathrm{Yb}(\mathrm{III})$ in the UV-Vis can be particularly useful, provided that $\mathrm{Yb}(\mathrm{III})$ is a special ion within the lanthanide series in that it possesses only two electronic $4 f$ levels $\left({ }^{2} \mathrm{~F}_{5 / 2}\right.$ level lies at around $10000 \mathrm{~cm}^{-1}$ above the ${ }^{2} \mathrm{~F}_{7 / 2}$ ground state) and its excitation is not trivial [45].

In short, sensitization of the $\mathrm{Ln}^{3+}$ ions in the UV region through antenna effect from these organic ligands not only overcomes the relatively low absorptivities of lanthanides and improves their luminescence output, but opens up the door to optical pumping via low-cost pump sources, such as LEDs and low-cost lasers. The PL spectra of the complexes when doped in hybrid matrices should then be pumped at wavelengths in the UV region (see Section 4.1.2). 


\subsection{Applicability of hybrid materials based on lanthanide complexes to optical networks}

A review on the application of these hybrid materials (referred by some authors as OIHs, organic-inorganic hybrid materials) in the next generation of optical networks has been published in 2010 [193], which covers both passive (planar and channel waveguides, couplers and multimode interference splitters) and active (lasers and optical amplifiers) optical architectures for long haul/metro and access/indoor networks.

Amongst other examples, we may highlight that a polymer-based waveguide optical amplifier doped with $\mathrm{Er}^{3+}$ ions (trisnitrato-tris-[4-[4-(3-triethoxysilyl-propoxy)-phenylazooxide]-phenyl-diphenylphosphin]erbium(III) incorporated into a PMMA matrix) was already fabricated and characterized by Le Quang et al. [194] back in 2006. By accounting for overall propagation loss, a net gain of $1.34 \mathrm{~dB}$ at $1540 \mathrm{~nm}$ was demonstrated for a $1.6 \mathrm{~cm}$ long single mode waveguide amplifier. In 2009, Chen et al., using a structure which consisted of an embedded waveguide (made of PMMA co-doped with erbium and ytterbium complexes) and a PMMA-GMA cladding layer, demonstrated relative optical gains of $6.5 \mathrm{~dB}$ in a $12 \mathrm{~mm}$-long device [195].

It is also specially noteworthy that a near-infrared polymer semiconductor laser, exhibiting whispering-gallery-mode laser emission at $825 \mathrm{~nm}$ upon pulsed photoexcitation at $532 \mathrm{~nm}$, has been demonstrated in 2010 by Kobayashi et al. [196]. The gain layer was fabricated by use of PVK containing an electron-transport material, 2-(4-biphenylyl)-5-(4-tertbutylphenyl)-1,3,4-oxadiazole (30\% by weight, abbreviated as PBD), and a near-infraredemitting molecule, 2-(6-(p-dimethylaminophenyl)-2,4-neopentylene-1,3,5-hexatrienyl)-3ethylbenzothiazolium perchlorate ( $1 \%$ by weight). Nonetheless, it should be indicated that the strong light absorption by the $\beta$-diketonate ligands, which is an advantage for sensitizing the luminescence of lanthanide ions by the antenna effect, may limit the usefulness of lanthanide $\beta$-diketonate complexes as laser materials.

In spite of the inherent interest of aforementioned applications, this Thesis will confine the assays only to the preparation of the actual OIHs (Section 4.1). Further applications of the materials discussed therein -analogous to those referred above- have already been envisaged and constitute one of the future research lines.

With regard to light sources for optical communications, precedents in near-infraredemitting OLEDs are thouroughly discussed in Section 4.2.1. A detailed manufacturing procedure, together with electrical and electroluminescent characterization of the fabricated devices, is reported in Section 4.2.2. 


\subsection{Approaches for co-doping in YEDFAs: separate $\mathrm{Er}^{3+}$ and $\mathrm{Yb}^{3+}$ complexes vs. 'Er ${ }^{3+}-\mathrm{Yb}^{3+}$ co-crystalline' complexes}

Despite the fact that the use of UV-Vis absorbing antennas for NIR luminescence offers several important advantages (optical pumping via low cost pump sources, such as LEDs and low cost lasers, and normal glass for substrate handling instead of quartz), using $\mathrm{Yb}^{3+}$ as a sensitizer is also an attractive alternative, as it has been discussed in Section $1.5 .3\left(\mathrm{Yb}^{3+}\right.$ ion could be pumped at $940 \mathrm{~nm}$ or taking advantage of UV-sensitization, as explained in Section $3.2)$.

In this regard, a continuing challenge has been to develop and construct efficient functional systems to achieve $\mathrm{Yb}^{3+}-\mathrm{Er}^{3+}$ energy transfer. The usual approach, the one proposed in this Thesis, is to utilize separate isostructural $\mathrm{Er}^{3+}$ and $\mathrm{Yb}^{3+}$ complexes. A theoretical alternative would be to use $\mathrm{Er}^{3+}-\mathrm{Yb}^{3+}$ co-crystalline complexes.

In several papers $[165,195,197,198]$ Song et al. have reported near-infrared luminescence properties of novel binuclear erbium complexes (for which the structures were actually obtained, such as that in Figure 130) and several dubious erbium-ytterbium co-crystalline complexes based on benzoate (or 4-pentylbenzoate, PBa) and 1,10-phenanthroline ligands (Figure 131). The 1,10-phenanthroline ligand chelated to an erbium ion acts as a lightharvesting moiety and sensitizer, which effectively transfers excitation energy to the erbium ion excited at $320 \mathrm{~nm}$. Compared to $\mathrm{Er}_{2}$ (benzoate) ${ }_{6}(\text { phen })_{2}$, the NIR luminescence of the erbium ion in the $\operatorname{Er}_{1.4} \mathrm{Yb}_{0.6}$ (benzoate) (bhen $_{2}$ adduct is enhanced, excited at both 320 and $975 \mathrm{~nm}$, indicating an efficient energy transfer from $\mathrm{Yb}^{3+}$ to $\mathrm{Er}^{3+}$ (Figure 132).

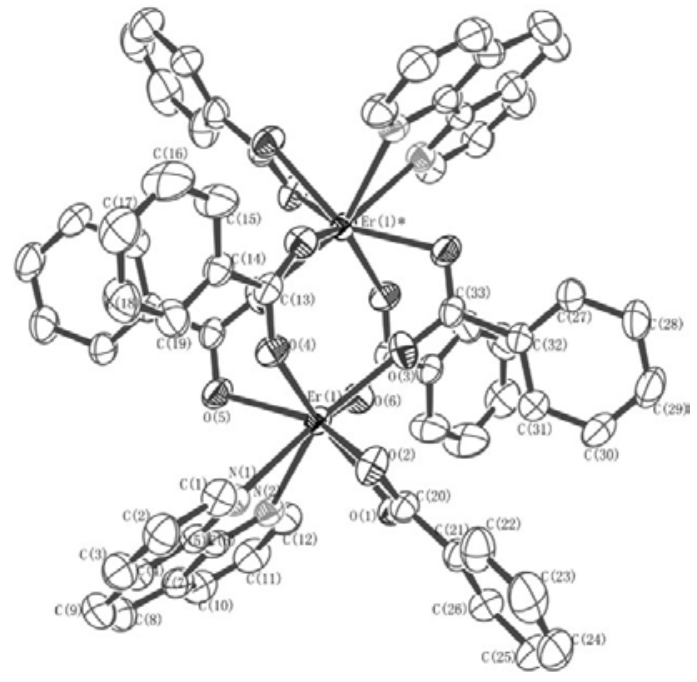

Figure 130. The molecular structure of $\mathrm{C}_{66} \mathrm{H}_{46} \mathrm{~N}_{4} \mathrm{O}_{12} \mathrm{Er}_{2}$ [197]

Similar results had already been presented by Zhong et al. [199] in 2006, reporting another stoichiometrically Er-Yb cocrystalline complex $\operatorname{Er}_{1 / 2} \mathrm{Yb}_{1 / 2}(\mathrm{HFA})_{3}(\mathrm{TPPO})_{2}$ (HFA=hexafluoroacetylacetonate, TPPO=triphenylphosphine oxide), but again no structural data was provided and only PL spectra were presented. The sensitization mechanism (Figure 133 ) is analogous to that of Song et al.'s complexes.

\footnotetext{
- It should be noted that no SC-XRD structures or NMR data were reported (in any of the articles) for the co-crystalline complexes (such as $\operatorname{Er}_{1.4} \mathrm{Yb}_{0.6}$ (benzoate) 6 (phen) $)_{2}$ ), which has cast doubts on the reliability of their studies. The authors only support their claims with elementary analysis and ESI-MS, two techniques which provide bulk information.
} 


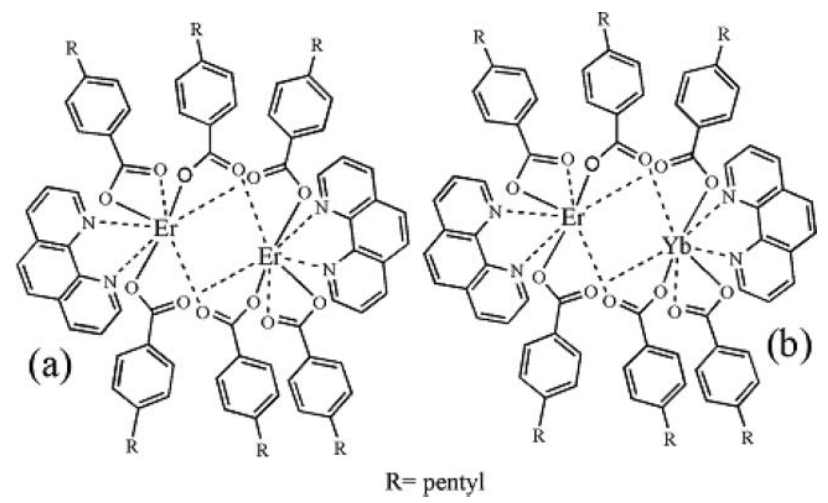

Figure 131. Chemical structures of (a) $\operatorname{Er}_{2}(\mathrm{PBa})_{6}(\text { phen })_{2}$ and (b) $\operatorname{ErYb}(\mathrm{PBa})_{6}(\text { phen })_{2}[165]$

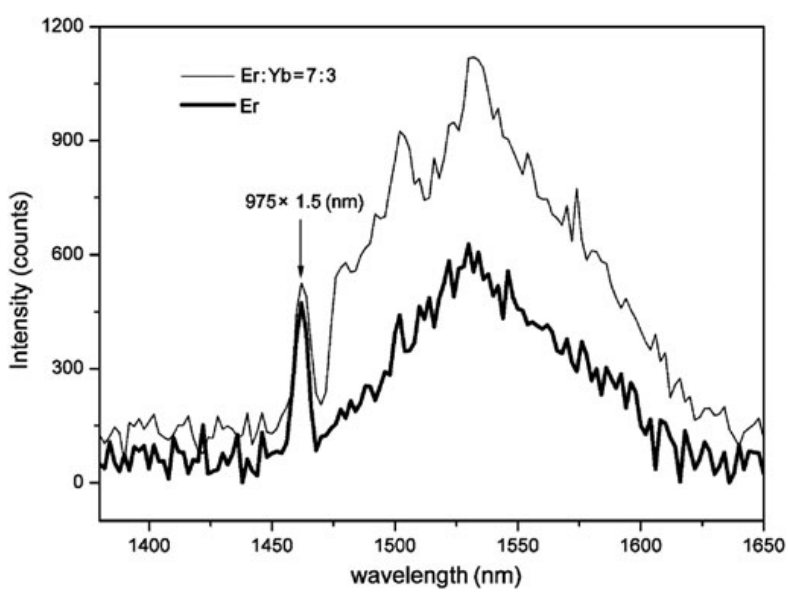

Figure 132. NIR PL spectra of bulky microcrystalline powders of complexes $\operatorname{Er}_{1.4} \mathrm{Yb}_{0.6}(\text { benzoate })_{6}(\mathrm{phen})_{2}$ and $\mathrm{Er}_{2}$ (benzoate) $)_{6}(\text { phen })_{2}$ around $1535 \mathrm{~nm}$, excited at $975 \mathrm{~nm}$ at room temperature [197]

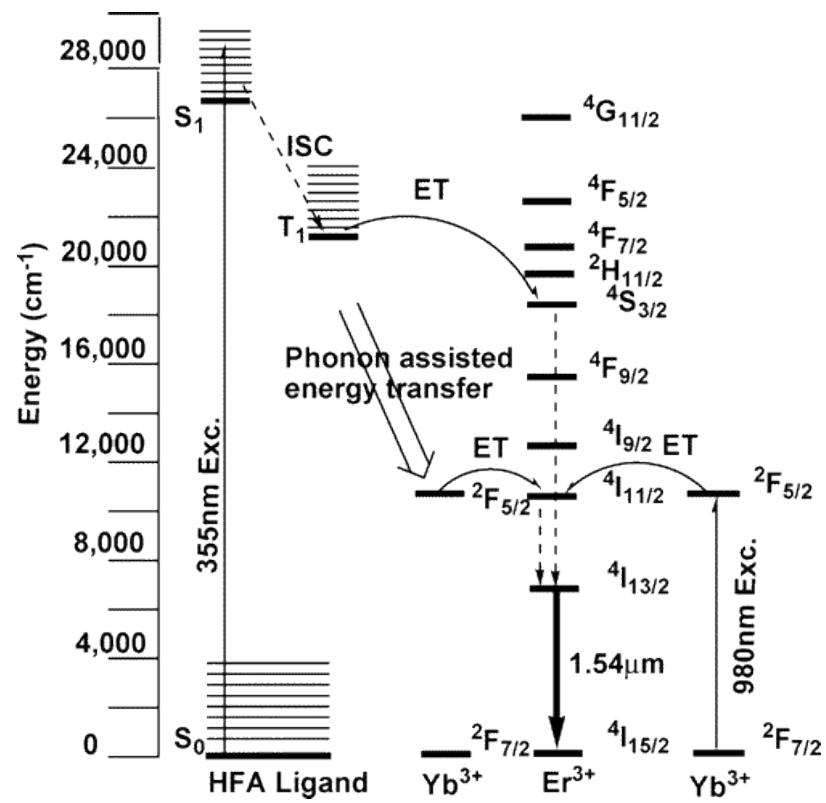

Figure 133. Schematic diagram for sensitization mechanism of the $\mathrm{Er}^{3+}$ ion by $\mathrm{Yb}^{3+}$ ion or HFA ligand [199] 
In spite of aforementioned issues (which actually point out how experimental data based on well documented synthetic strategies and characterization, including structural data, are of crucial importance for the interpretation of the photophysical properties to achieve a reliable structure/property relationship), dinuclear complexes are an active area of research, and promising advances have been made in dimeric homonuclear complexes. Since the first dinuclear methoxo-bridged hexafluoropentanedionate $\mathrm{Er}^{3+}$ complexes with 1,10phenanthroline and 2,2'-bipyridine $\left(\left[\operatorname{Er}(\mathrm{hfpd})_{2}\left(\mu-\mathrm{OCH}_{3}\right)(\text { bipy })\right]_{2}\right.$ and $\left[\operatorname{Er}(\mathrm{hfpd})_{2}(\mu-\right.$ $\left.\mathrm{OCH}_{3}\right)($ phen)] 2 , respectively) were presented by Staveren et al. [200], XRD structures for several other Er and $\mathrm{Yb}$ homodinuclear complexes -apart from the Erbium ones discussed in Song et al.'s aforementioned papers- have also been reported, such as [ $\mathrm{Er}_{2}$ (benzoate) $\left._{6}(\text { bipy) })_{2}\right]$ [201] and $\left[\mathrm{Ln}_{2}(\mathrm{HTH})_{6}\right.$ (bipy)] (where $\mathrm{Ln}=\mathrm{Er}, \mathrm{Yb}$; HTH= 4,4,5,5,6,6,6heptafluroro-1-(2-thienyl)hexane-1,3-dione) [202].

Structural elucidation in solution by means of NMR for two other dinuclear $\mathrm{Er}^{3+}$ complexes has been recently reported by Iftikhar and coworkers: [(acac) $\left.)_{3} \operatorname{Er}(\mu-\mathrm{bpm}) \operatorname{Er}(\mathrm{acac})_{3}\right]$ (where acac $=2,4$-pantanedionate and bpm $=2,2^{\prime}$-bipyrimidine) [203] and $\left[\operatorname{Er}_{2}(\text { fod })_{6}(\mu\right.$-bpm)] (where fod $=6,6,7,7,8,8,8$-heptafluoro-2,2-dimethyl-3,5-octanedionate) [204,205]. In addition, the same group has presented an Er-Ho co-crystalline complex isostructural to the latter: $\left[\operatorname{HoEr}(\text { fod })_{6}(\mu-b p m)\right][206]$.

In this Thesis, even though only Er(III) complexes have been doped in the hybrid matrices (Section 4.1) and no actual co-doping assays have been conducted yet, the involved materials have been developed and optimized. It should be noted that the chosen approach has a major and non-trivial advantage: doping with separate $\mathrm{Er}^{3+}$ and $\mathrm{Yb}^{3+}$ complexes allows control of the Er:Yb proportion, so that it can be tuned to meet the optimum 3:7 pumping ratio, whereas the co-cystalline complexes approach has a fixed 1:1 ratio. 


\section{APPLICATIONS OF THE NOVEL COMPLEXES IN OPTOELECTRONICS AND TELECOMMUNICATIONS}

\subsection{Assessment of the suitability of the new complexes for the fabrication of waveguides and optical amplifiers}

\subsubsection{Studies with mesoporous glass and fluorinated polymers}

Preliminary studies of the suitability of the novel complexes have been conducted during the DAAD-sponsored research stay at MPI, aimed at their application as luminescent materials in either mesoporous silica gel or Teflon hosts.

\section{Assay on the impregnation of mesoporous glass with solutions of erbium complexes}

It has been designed according the descriptions published by Sofer et al. [207] and Tresnakova et al. [208]:

Manufacturing of mesoporous silica. It begins by mixing tetraethylorthosilicate (TEOS) [or tetrametylortosilicate (TMOS)] and hydrochloric acid, followed by stirring for 1 hour. Then methanol is added and the mixture is stirred for 1 additional hour. $\mathrm{N}$ Cetyltrimethylammonium chloride, $\left.\left[\mathrm{CH}_{3} \mathrm{CH}_{2}\right)_{15} \mathrm{~N}\left(\mathrm{CH}_{3}\right)_{3}\right] \mathrm{Cl}$ (CTMACl), is added and further stirring must be carried out for $24 \mathrm{~h}$. After filtering this solution, either a sol-gel spin-coating (if we want to obtain a film) or a yarn (if we want to get a fiber) must be conducted. The drying of both mesoporous fiber and films should be carried out in the sequence: $60^{\circ} \mathrm{C}$, $130^{\circ} \mathrm{C}$ and $200^{\circ} \mathrm{C}$. Finally, mesoporous products should be calcined at $400^{\circ} \mathrm{C}$ for $12 \mathrm{~h}$ in air. The heating rate was controlled to $1^{\circ} \mathrm{C} / \mathrm{min}$ because the mesoporous structure should be protected from collapsing by a rapid change of temperature.

The ATR-FTIR spectra of the mesoporous silica glass prior and after calcination are shown in Figure 134. The main absorption band is related to vibration Si-O-Si in the region of $1000 \mathrm{~cm}^{-1}$.

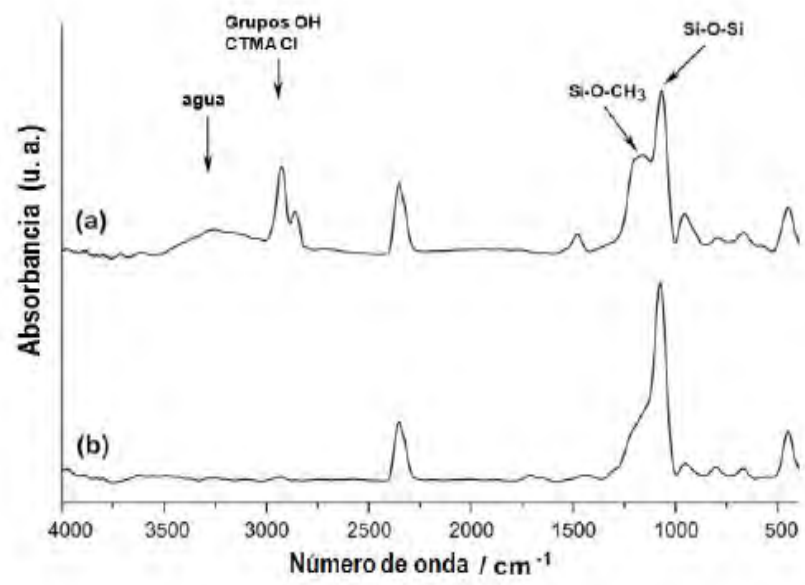

Figure 134. ATR-FTIR spectra of mesoporous silica glass $(a)$ right after synthesis; $(b)$ after calcination at $400^{\circ} \mathrm{C}$ for 12 hours.

Alternatively, mesoporous glass is commercially available as wafers under the trademarks SIMAX (Kavalier Glass) or VYCOR (Corning). 


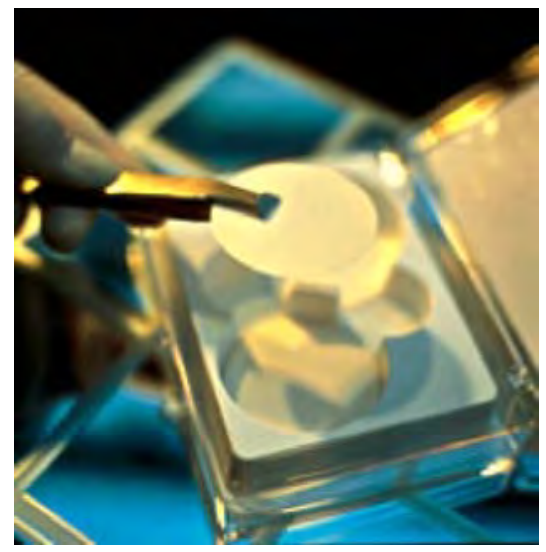

Figure 135. Commercial porous glass wafer

Incorporation of $\mathrm{Er}^{3+}$ and $\mathrm{Yb}^{3+}$ complexes. It is achieved by impregnation from a solution of the $\mathrm{Er}^{3+}$ or $\mathrm{Yb}^{3+}$ complex in methanol or ethanol $\left(1.5 \times 10^{-3}\right.$ and $\left.1.5 \times 10^{-2} \mathrm{M}\right)$, taking $10 \mathrm{~mL}$ of this solution and immersing the mesoporous film or glass wafer in it. Then an ultrasound treatment is applied. We proceed to extract the impregnated film or fiber from the container and remove the excess alcohol by passing a stream of nitrogen.

Subsequently, samples are gradually treated by annealing. At this time, the information from the curves of thermal analysis (Section 2.4.2) is required. The annealing must always start at temperatures below the decomposition temperature for each complex. Given that the decompositions of the complexes are not simple processes and that they take place through the formation of various intermediate products, annealing temperature has to start slightly below that corresponding to the formation of the first intermediate product.

When it comes to reaching the sintering, annealing should continue to over $1000^{\circ} \mathrm{C}$ but at these temperatures, obviously, erbium or ytterbium complexes will have decomposed to their corresponding oxides and oxyfluorides.

A key observation to get a good annealing is that it should be carried out slowly. If the annealing of the impregnated samples is done quickly, decomposition volatiles are trapped in the samples in the form of bubbles that do not disappear by any means. It is therefore necessary to place emphasis on the desirability of a stage by stage annealing.

PL intensity variation with the concentration of erbium complex. In agreement with other authors [209], the mesoporous glass doping should be done with $\mathrm{Er}^{3+}$ ion concentrations between 100 and $500 \mathrm{pmm}$ (between 0.03 and $0.30 \mathrm{wt} \%$ ), corresponding to concentrations of the complexes between $0.0015 \mathrm{~N}$ and $0.015 \mathrm{~N}$. As shown in Figure 136, the PL intensity is optimized for concentrations of erbium complexes close to $0.015 \mathrm{~N}$. 


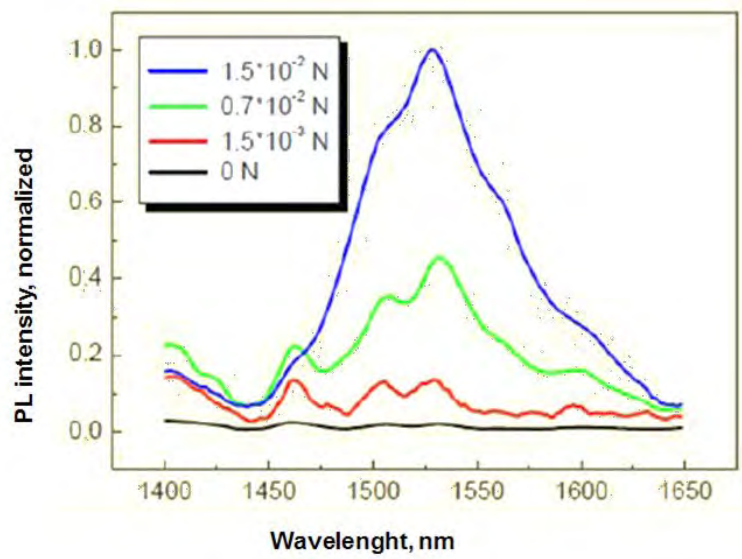

Figure 136. Emission of Er-doped mesoporous glass under 980-nm excitation for different concentrations of the complexes

Regarding the bandwidth gain, FWHM, the value for $0.015 \mathrm{~N}$ was $90 \mathrm{~nm}(1480$ to $1570 \mathrm{~nm})$. These results are consistent with those obtained by Park et al. [210] and Wang et al. [211].

Notwithstanding the above achievements, the response of gain versus wavelength is not sufficiently flat within the band from 1525 to $1560 \mathrm{~nm}$ (over $12 \mathrm{~dB}$.) This effect is detrimental if the EDFA is used in applications such as dense wavelength division multiplexing (DWDM). In this case, an EDFA design on fluoride optical fiber would be required, for which a response with variation of less than $3 \mathrm{~dB}$ in the range of 1525-1560 nm has been advocated. Our first attempts to work in these conditions have been tarnished by problems of surface crystallization when ZBLAN glasses have been used.

\section{Assay on the fabrication of doped fluorinated polymer films}

In this case, Teflon $\mathrm{AF}^{\circledR}$ was the chosen matrix (Figure 137) in which [Er(fhd $)_{3}\left(5 \mathrm{NO}_{2}\right.$ phen)] was dispersed, according to the procedure described by Kuriki and coworkers for [Er(fhdd) 3 ] [249].

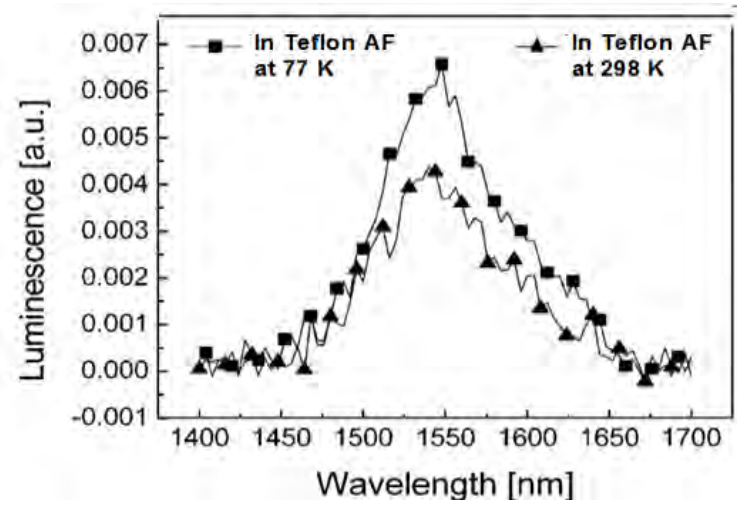

Figure 137. PL of the erbium complex $\left[\operatorname{Er}(f h d)_{3}\left(5 \mathrm{NO}_{2}\right.\right.$ phen $\left.)\right]$ embedded in Teflon $\mathrm{AF}^{\circledR}$ as host matrix

Nonetheless, the fluorinated polymers CYTOP (Cyclic Transparent Optical Polymer, developed by Asahi Glass Company) and poly(hexafluoro isopropyl methacrylate) (PFiPMA), are excellent alternatives. Unfortunately, the high cost of these perfluorinated polymers (above $\$ 1000 / \mathrm{g}$ ) and the toxicity of the initiators required for the polymerization 
reaction of their cheaper precursor monomers prevented further development of this application.<smiles>CC(C)(C)[Te][Te][Te]C(C)(C)C</smiles>

CYTOP<smiles>CC(C)(C)C(OC(=O)C(C)(C)C(C)(C)C)C(F)(F)F</smiles>

P-FiPMA

Figure 138. Alternative fluorinated polymers that can be used as a host matrix for the near-infrared emitting complexes

\subsubsection{Studies with doped ormosils and PMMA}

Two other hybrid matrices have been studied in a preliminary effort for the design of materials suitable for passive (planar and channel waveguides, couplers and multimode interference splitters) and active (lasers and optical amplifiers) optical architectures: organically modified xerogels and polymer matrices. The former have been chosen so as to overcome the problems referred in traditional purely inorganic glasses prepared by the controlled hydrolysis of metal alkoxides by incorporation of organic components in the backbone of the xerogel network. The choice of the tetramethyl orthosilicate (TMOS) - 3glycidoxypropyltrimethoxysilane (GLYMO) over TMOS-DEDMS (diethoxydimethylsilane) is supported not only because the former composition has been reported by Reisfeld et al. to have a high luminescence efficiency [212], but also because it reaches the gel-point faster than the TMOS-DEDMS mixture [213]. With regard to the polymer matrices, poly(methyl methacrylate) (PMMA) has been chosen due to its low optical absorption and low cost.

\section{Organically modified xerogel matrices: preparation of the doped TMOS-GLYMO films}

As it has been stated in Section 1.3, the traditional purely inorganic glasses have some disadvantages to entrap molecular lanthanide complexes, namely low solubility and crystallization of the lanthanide complexes (which leads to loss of transparency), cracking due to the surface tension of the liquid in the pores (according to K. Driesen [214], up to 4 months) and fragility. These disadvantages (illustrated in Figure 139 for a pure TEOS sample) can be overcome by incorporation of organic components in the backbone of the xerogel network [50], as the TMOS-GLYMO mixture chosen here.
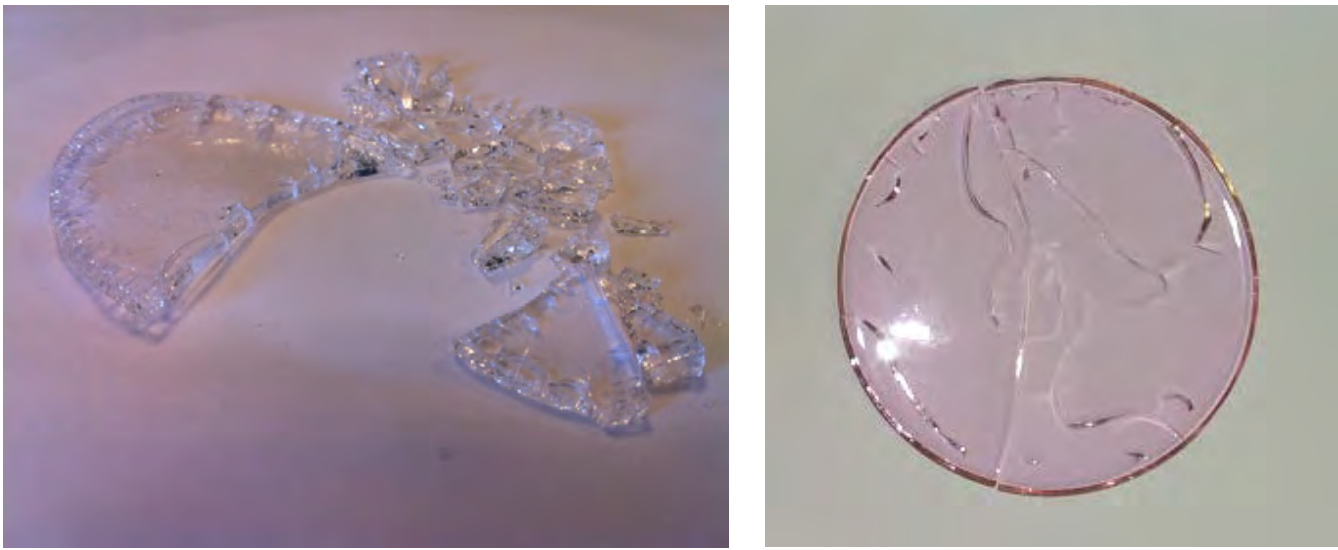

Figure 139. Pure TEOS simple prepared in-house (left) and slow cracking of a Nd(III) doped TMOS (diameter $4 \mathrm{~cm})($ right) reported by Driesen [214] 
The preparation procedure is based on that reported by K. Driesen et al. [213]: firstly, a $0.006 \mathrm{M}$ solution of the selected complex in dimethylformamide (DMF) is prepared. $2 \mathrm{~mL}$ of the resulting solution are mixed with tetramethoxysilane (TMOS, $1 \mathrm{~mL}$ ), 3glycidoxypropyltrimethoxysilane (GLYMO, $1 \mathrm{~mL})$ and water $(0.5 \mathrm{~mL})$ acidified with a drop of $\mathrm{HCl}$. The solution is then stirred for one hour at $50^{\circ} \mathrm{C}$. Thermostable casts are subsequently filled with aliquots of the solution and are covered with Parafilm. After one day, three small holes are pierced in the Parafilm. Monolithic glass samples are obtained after a drying period of 1 week. Before the luminescence measurements are made, the samples are dried for $48 \mathrm{~h}$ at $100^{\circ} \mathrm{C}$.
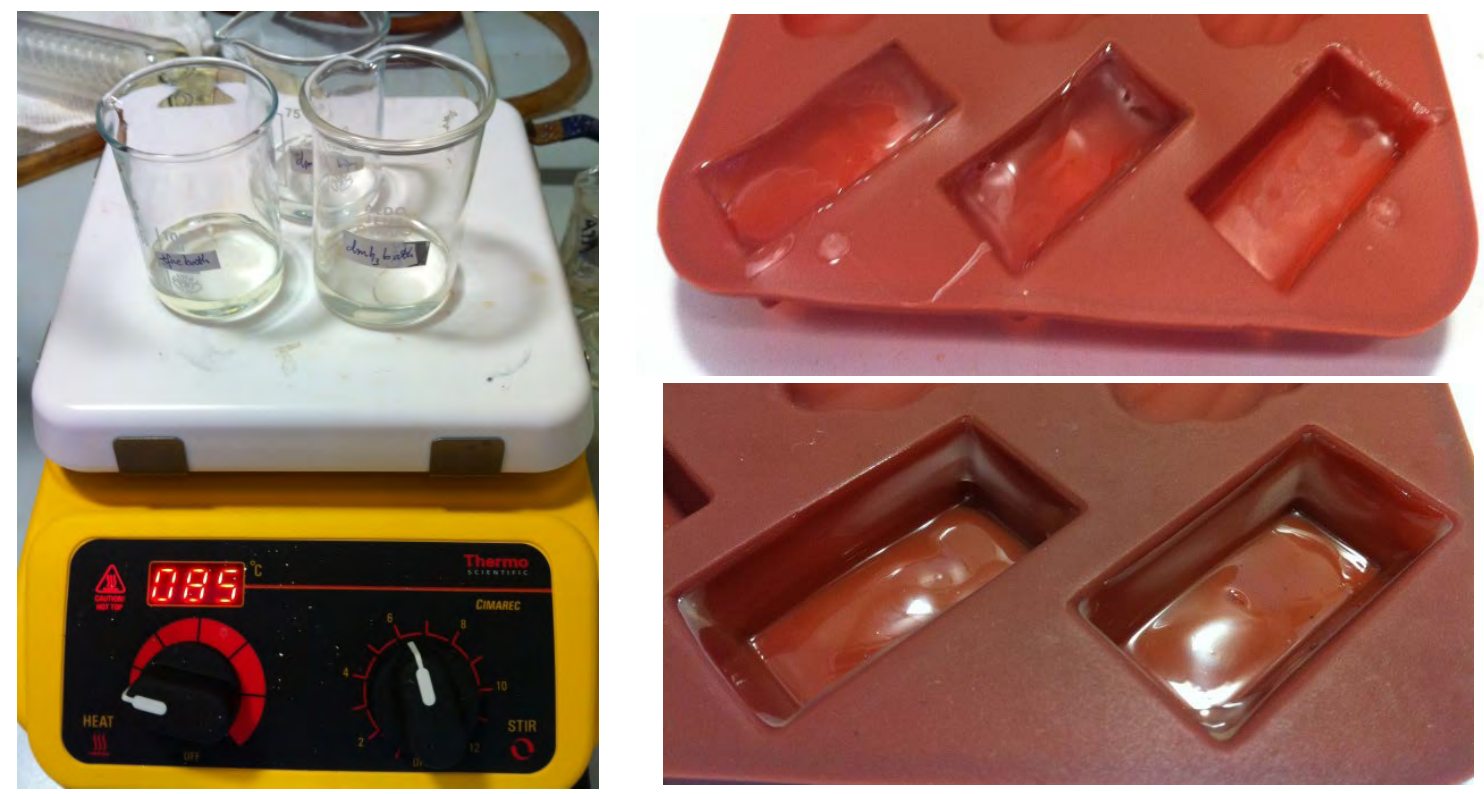

Figure 140. Solutions being stirred (left); casts immediately after being filled with aliquots of the solutions, prior to drying (top right) and same samples during the drying process after 3 days (bottom, right). Parafilm has been removed so as to take the photograph.

\section{Polymer matrices: preparation of the doped PMMA films}

In this case, we have assayed the incorporation of the novel complexes in one of the most popular optically transparent polymers: poly(methyl methacrylate) (PMMA). Doped PMMA polymers are prepared by initially dissolving a known amount $(9 \mathrm{mg})$ of the novel complexes in $2 \mathrm{~mL}$ of chloroform $\left(\mathrm{CHCl}_{3}\right) .90 \mathrm{mg}$ of PMMA are subsequently added under vigorous stirring. This concentration has been reported to be an optimum one by Prajzler et al. [215]. The resulting solutions are concentrated by gentle heating $\left(50^{\circ} \mathrm{C}\right)$ and then transferred to the thermostable casts. The residual solvent is allowed to evaporate overnight at room temperature and then the samples are kept under $10^{-3}$ torr pressure for 6 hours, in agreement with the procedure reported by Bortoluzzi et al. [216]. 

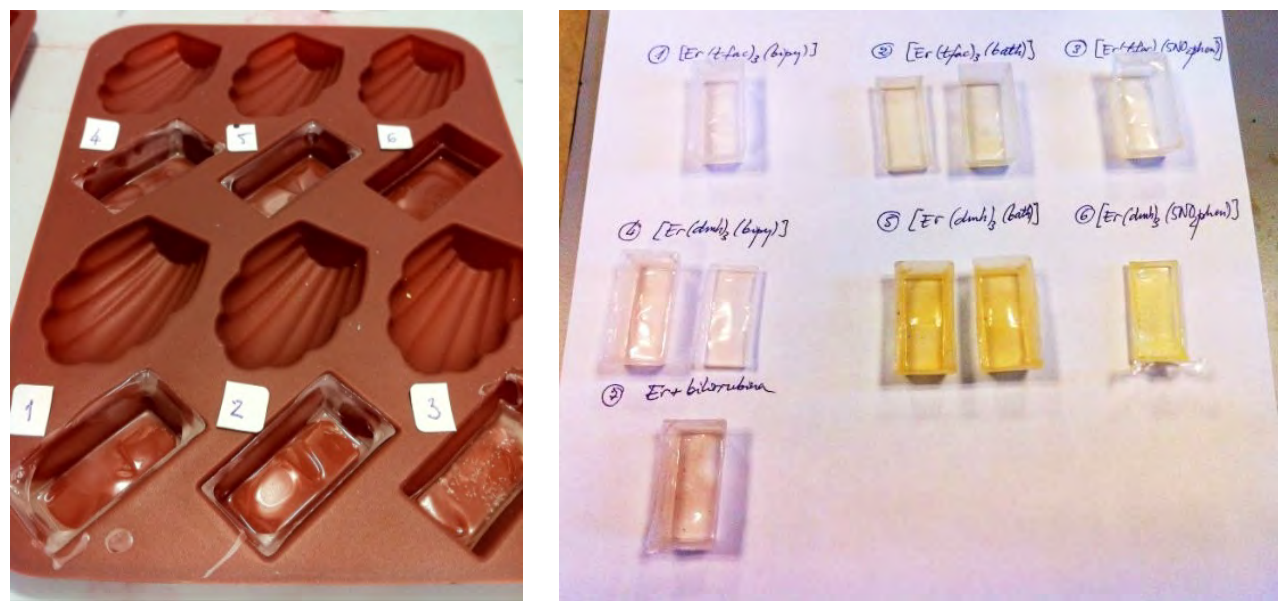

Figure 141. PMMA doped samples after 3 hours, most solvent has already been evaporated (left) and samples after being turned out from the casts (right).
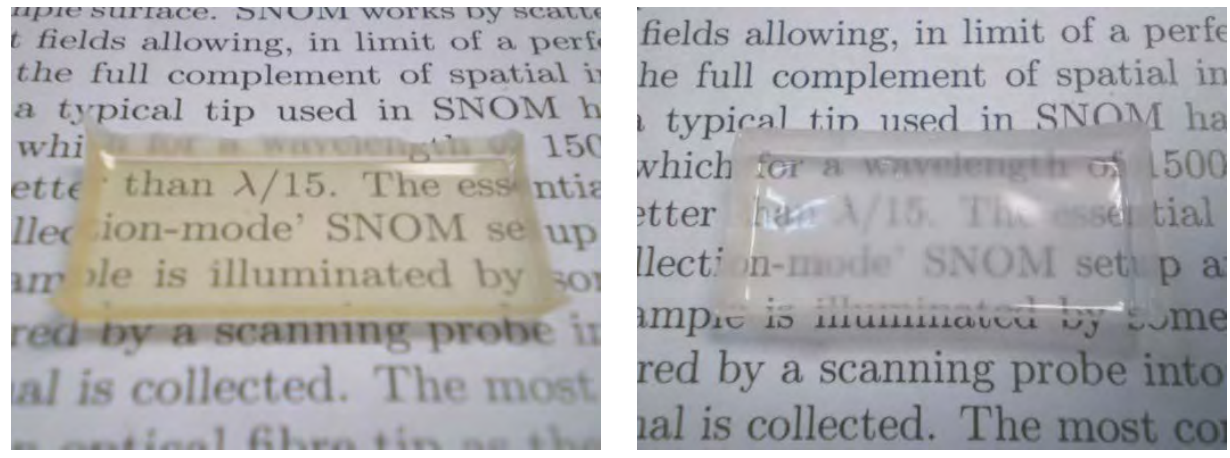

Figure 142. Comparison of ormosil (left) and PMMA (right) samples doped with the novel complexes. The increased turbidity in the PMMA sample is associated to its higher dopant concentration $(\times 20)$.

So as to assess the effect of dispersing the complexes in the matrices, a X-ray powder diffraction study was conducted for one of the complexes, comparing the pattern for the original complex as-is with those of the complex in gel form and the doped ormosil and PMMA films (Figure 143): the two thin films are completely amorphous (even PMMA doped at $10 \% \mathrm{wt}$ ), while the thermally-treated complex in gel form still shows some weak peaks.

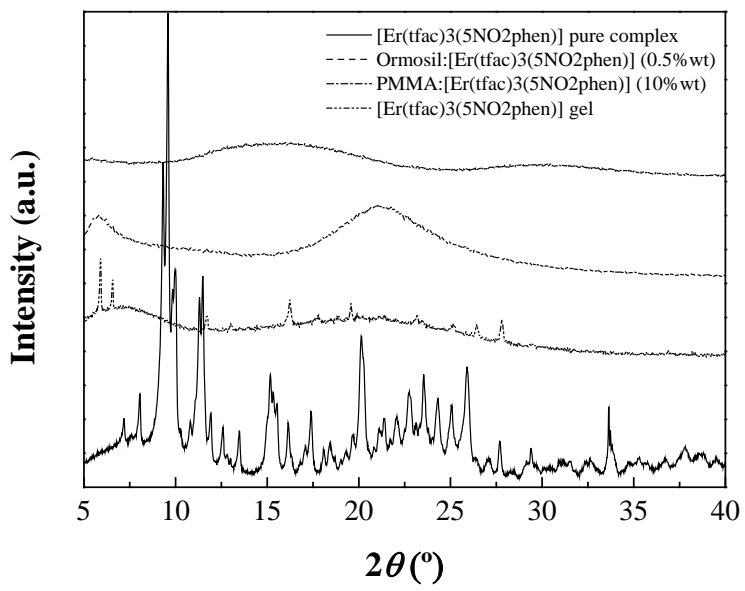

Figure 143. X-ray powder diffraction patter for $\left[\operatorname{Er}(\mathrm{tfac})_{3}\left(5 \mathrm{NO}_{2}\right.\right.$ phen $\left.)\right]$ in crystalline form, after a thermal treatment so as to obtain a gel, doped into ormosil $(0.5 \% \mathrm{wt})$ and doped into PMMA (10\%wt) thin films. 


\section{Photoluminescent properties of the hybrid materials}

The PL spectra in the visible region for PMMA films (Figure 144, right) are very similar to those reported above for the pure complexes (Figure 100), with a much more intense emission from the samples doped with $\left[\operatorname{Er}(\beta \text {-diketonate })_{3}(\right.$ bath $\left.)\right]$ and $[\operatorname{Er}(\beta$ diketonate $)_{3}\left(\right.$ bipy)] than from the samples doped with $\left[\operatorname{Er}(\beta \text {-diketonate })_{3}\left(5 \mathrm{NO}_{2}\right.\right.$ phen $\left.)\right]$. Conversely, for the ormosil-doped films (Figure 144, left), a major change is detected: the emission from $\left[\operatorname{Er}(\beta \text {-diketonate })_{3}\right.$ (bipy)] and $\left[\operatorname{Er}(\beta \text {-diketonate })_{3}\left(5 \mathrm{NO}_{2}\right.\right.$ phen $\left.)\right]$ samples are very weak, while the emission for $\left[\operatorname{Er}(\beta \text {-diketonate })_{3}(\right.$ bath $\left.)\right]$ is much stronger. In addition, there is a significant bathochromic shift, with a maximum at around $525 \mathrm{~nm}$. The dip detected in the $\left[\operatorname{Er}(\mathrm{dmh})_{3}(\mathrm{bipy})\right]$ spectrum at $522 \mathrm{~nm}$ would be associated to $\mathrm{Er}^{3+}$ reabsorption $\left({ }^{2} \mathrm{H}_{11 / 2}\right.$ level).

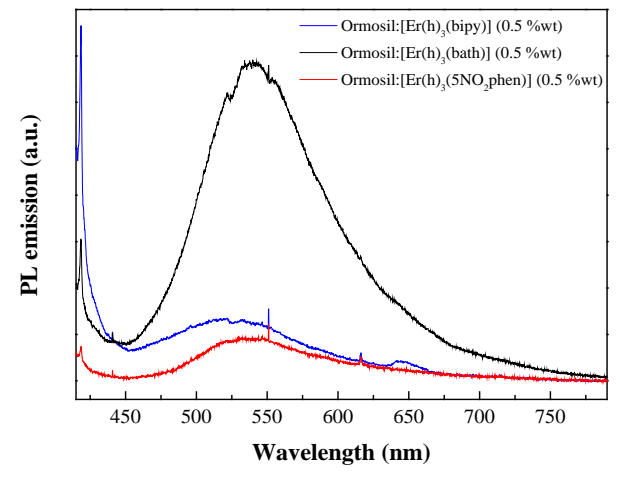

Ormosil:[Er(h) $)_{3}(\mathrm{~N}, \mathrm{~N}-$ donor $\left.)\right](0.5 \% \mathrm{wt})$

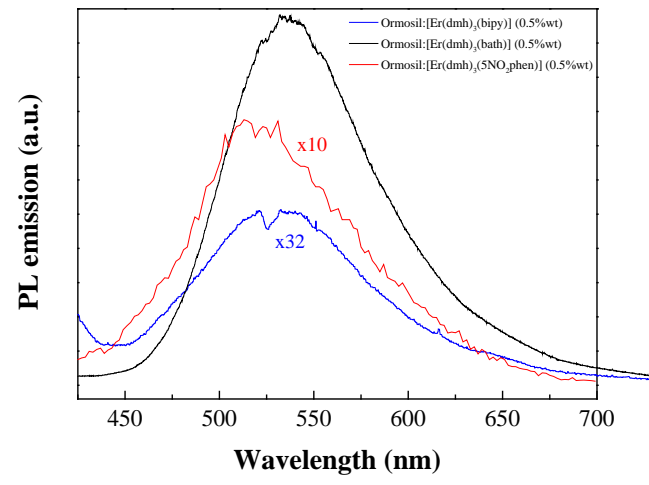

Ormosil:[Er(dmh) $\left.)_{3}(\mathrm{~N}, \mathrm{~N}-\mathrm{donor})\right](0.5 \% \mathrm{wt})$

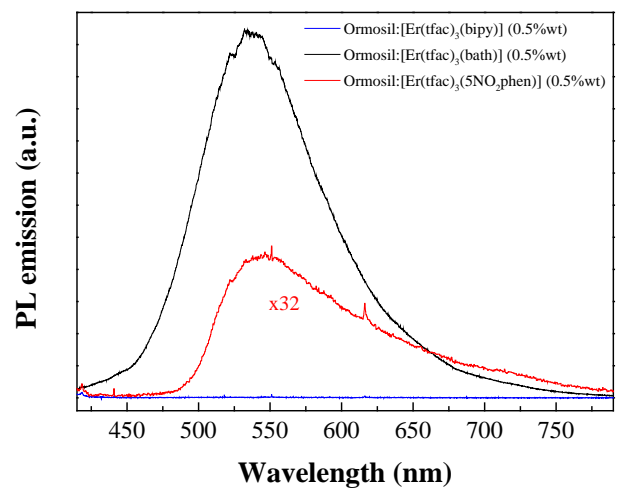

Ormosil:[Er(tfac) 3 (N,N-donor)] (0.5\%wt)

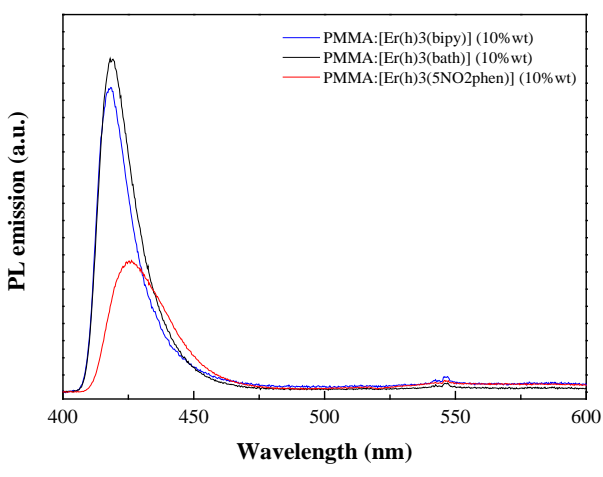

PMMA:[Er(h) 3 (N,N-donor)] $(10 \% \mathrm{wt})$

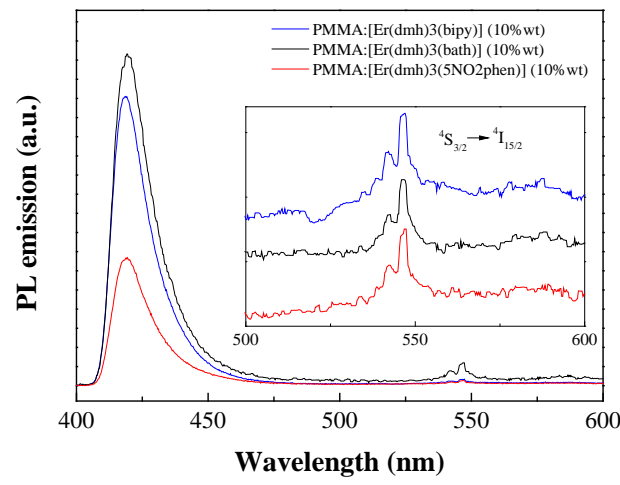

PMMA:[Er(dmh) $)_{3}(\mathrm{~N}, \mathrm{~N}-$ donor $\left.)\right](10 \% \mathrm{wt})$

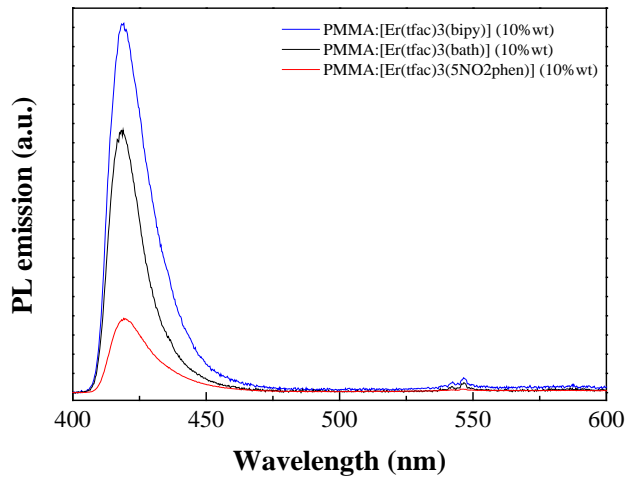

PMMA:[Er(tfac) $)_{3}(\mathrm{~N}, \mathrm{~N}-$ donor $\left.)\right]$ (10\%wt)

Figure 144. PL in the visible range for the ormosil (left) and PMMA (right) samples doped with the novel complexes, doped at $0.5 \% \mathrm{wt}$ and $10 \% \mathrm{wt}$, respectively. 


\section{NIR photoluminescence}

For the ormosil samples doped with [Er(dmh $)_{3}(\mathrm{~N}, \mathrm{~N}-$ donor $\left.)\right]$ and $\left[\operatorname{Er}(\operatorname{tfac})_{3}(\mathrm{~N}, \mathrm{~N}-\right.$ donor $\left.)\right]$, the almost complete quenching of the visible emission for the complexes with bipy and $5 \mathrm{NO}_{2}$ phen leads to a strong emission associated to $\mathrm{Er}^{3+}:{ }^{4} \mathrm{I}_{13 / 2} \rightarrow{ }^{4} \mathrm{I}_{15 / 2}$ transition, while that for the complex with bath is much weaker (i.e., the behavior referred in Section 2.4.5 for the pure complexes in powder is amplified). Conversely, for [ $\operatorname{Er}(\mathrm{h})_{3}(\mathrm{~N}, \mathrm{~N}-$ donor $\left.)\right]$ doped ormosil samples, the NIR emission is almost the same in the three cases, also in agreement with the observations made regarding Figure 103).

In the PMMA samples, the behavior is again very similar to that reported for the pure complexes in powder form (Figure 145).

The observed emission band in the range of 1450 to $1700 \mathrm{~nm}$ centered at $1530 \mathrm{~nm}$ has a FWHM ranging from 60 to $65 \mathrm{~nm}$ for the bipy, bath and $5 \mathrm{NO}_{2}$ phen complexes dispersed in the organically modified xerogel, and of 70-90 nm for the PMMA samples. These FWHM values would enable a wide gain bandwidth for optical applications in comparison with that of other similar $\mathrm{Er}^{3+}$-doped materials [217].

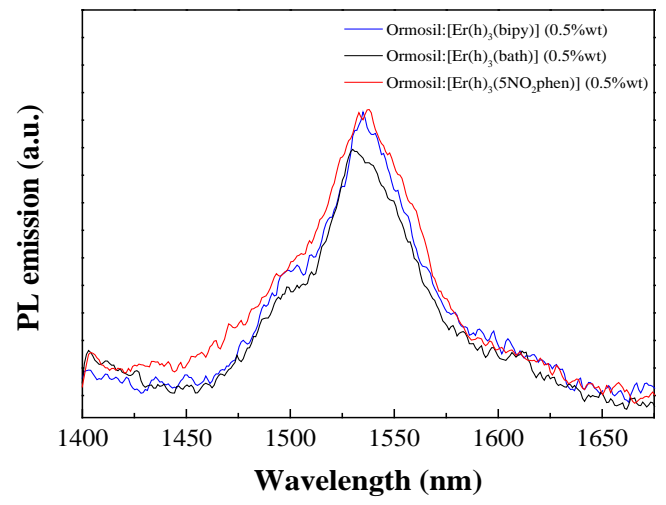

Ormosil:[Er(h) 3 (N,N-donor)] $(0.5 \%$ wt $)$

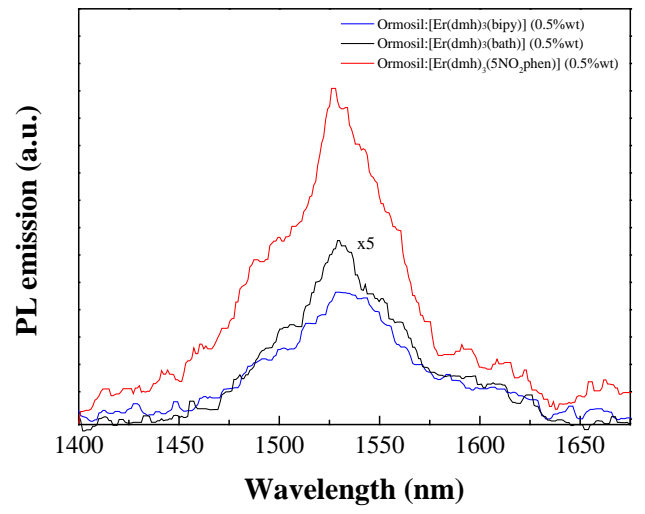

Ormosil:[Er(dmh) $3(\mathrm{~N}, \mathrm{~N}-\mathrm{donor})](0.5 \% \mathrm{wt})$

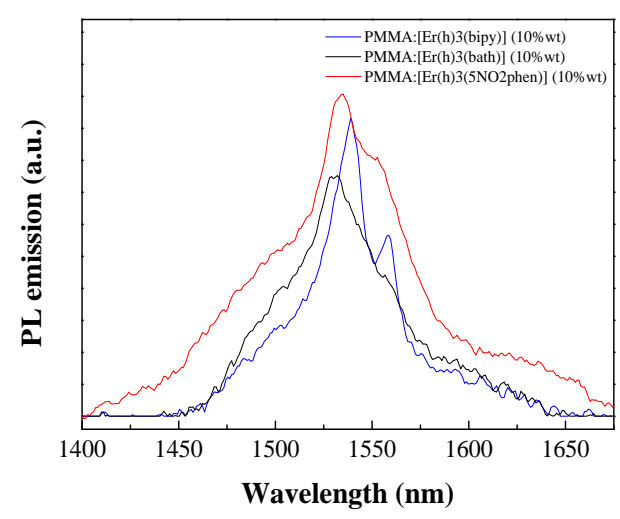

PMMA:[Er(h)3(N,N-donor)] (10\%wt)

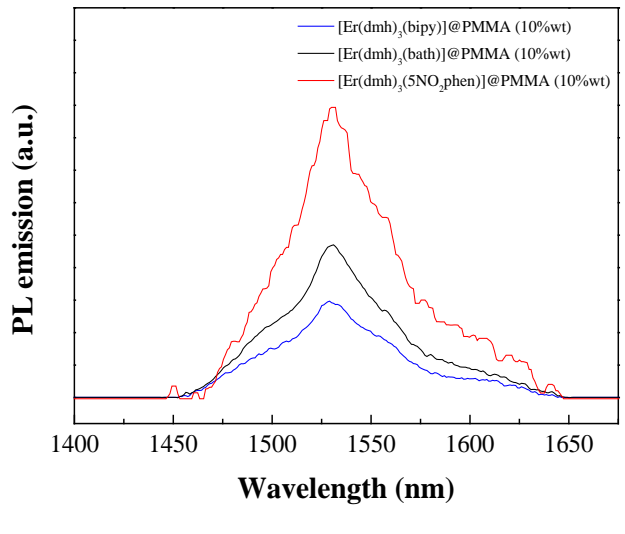

PMMA:[Er(dmh)3(N,N-donor)] $(10 \% w t)$ 


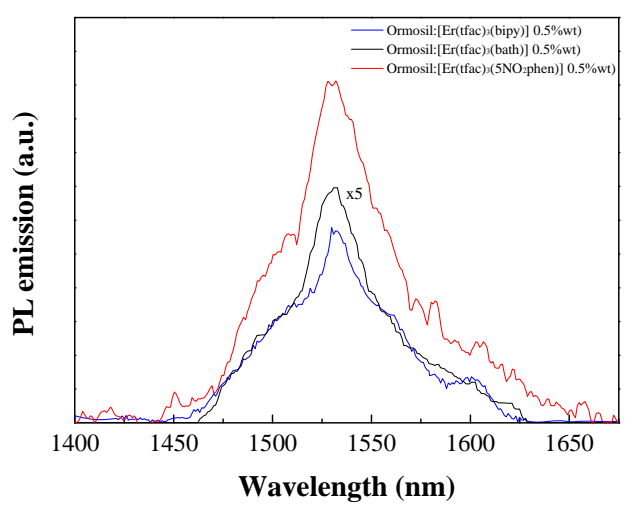

Ormosil:[Er(tfac) $3(\mathrm{~N}, \mathrm{~N}-$ donor $)]$ (0.5\%wt)

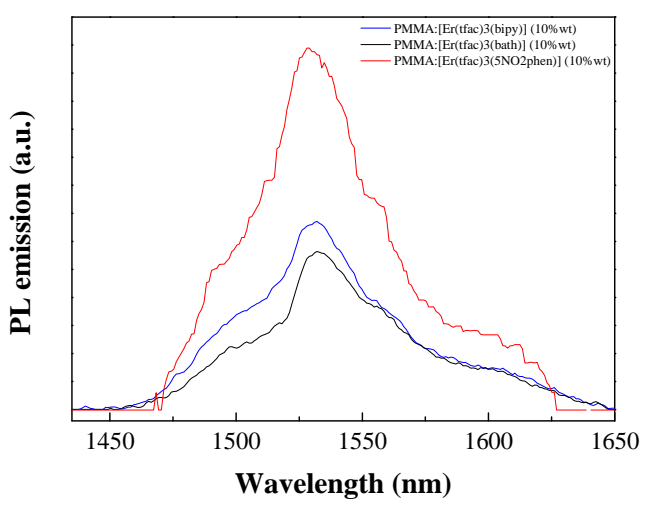

PMMA:[Er(tfac) $3(\mathrm{~N}, \mathrm{~N}$-donor)] (10\%wt)

Figure 145. PL in the NIR range for the ormosil (left) and PMMA (right) samples doped at 0.5\%wt and $10 \% \mathrm{wt}$, respectively

\section{Visible PL lifetime measurements}

The ligand fluorescence lifetime measurements (Figure 146) are in good agreement with the visible PL results: the longer the lifetime, the stronger the intensity of the visible emission, with an associated decrease in the efficiency of the antenna effect and in the NIR PL emission. For ormosil films, the $S_{1} \rightarrow S_{0}$ radiative decay times are summarized in Table 63. Regarding the PMMA matrices, the correspondent $S_{1} \rightarrow S_{0}$ radiative decay times are presented in Table 64. An increase in all the lifetime values, associated to a decrease of donor-donor interactions as a consequence of dispersing the complexes in the hybrid matrices, is expected in all cases. Nonetheless, in the particular case of the $\left[\operatorname{Er}(\beta \text {-diketonate })_{3}(\right.$ bath $\left.)\right]$ doped ormosils, this increase is much more dramatic. The obtained values, ranging from 20 to $25 \mathrm{~ns}$, are in fact close to that of the non-coordinated bathophenanthroline. This suggests that the solvent used for the ormosil preparation, DMF, has a disruptive effect which leads to a partial rupture of the complexes. Similar problems have been reported in the past for other $\mathrm{N}, \mathrm{N}$ donors: e.g., Strek et al. mention that during the synthesis of sol-gel matrices some of the $\mathrm{N}, \mathrm{N}$-donors are not preserved in the first coordination sphere of metal ion [218].

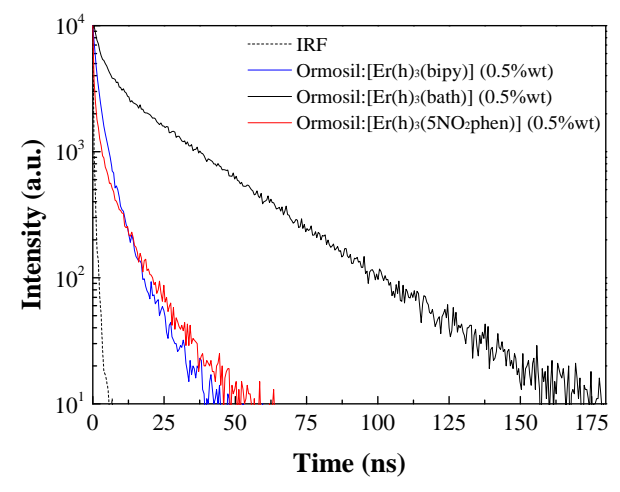

Ormosil:[Er(h) $)_{3}(\mathrm{~N}, \mathrm{~N}-$ donor $\left.)\right](0.5 \% \mathrm{wt})$

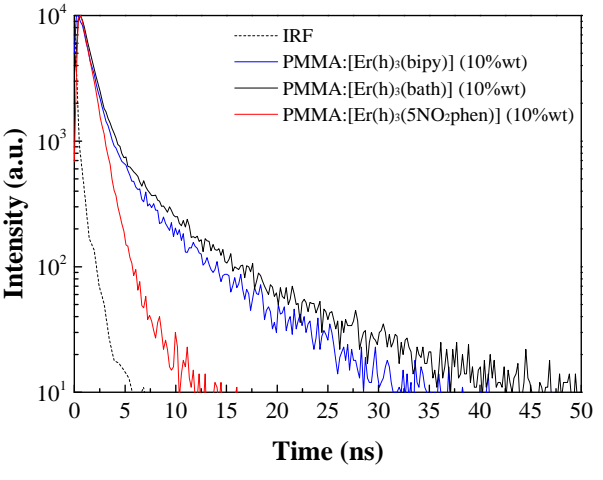

PMMA:[Er(h) 3 (N,N-donor)] (10\%wt) 


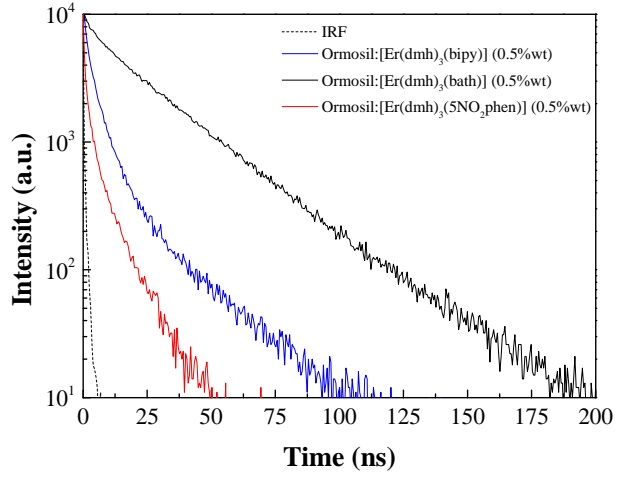

Ormosil:[Er(dmh) $\left.{ }_{3}(\mathrm{~N}, \mathrm{~N}-\mathrm{donor})\right](0.5 \% \mathrm{wt})$

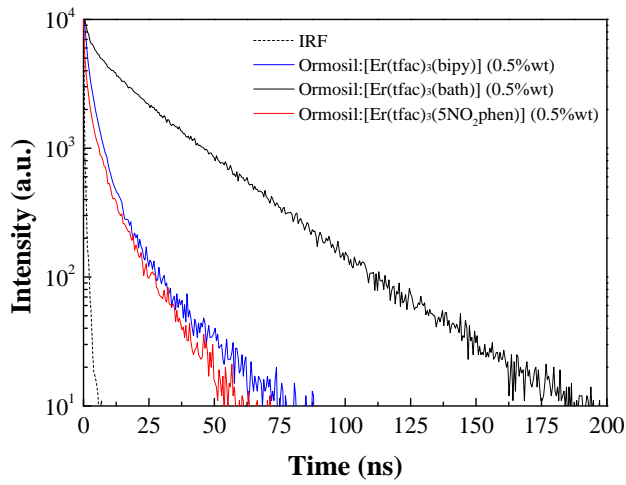

Ormosil:[Er(tfac) $3(\mathrm{~N}, \mathrm{~N}-$ donor $)](0.5 \% \mathrm{wt})$

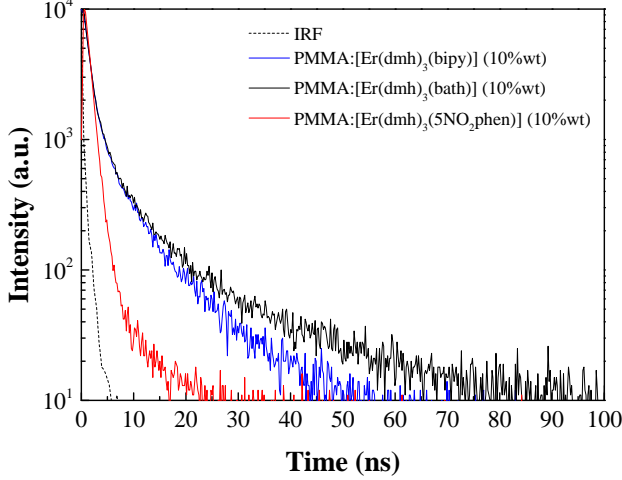

PMMA:[Er(dmh) $3(\mathrm{~N}, \mathrm{~N}-$ donor $)](10 \% \mathrm{wt})$

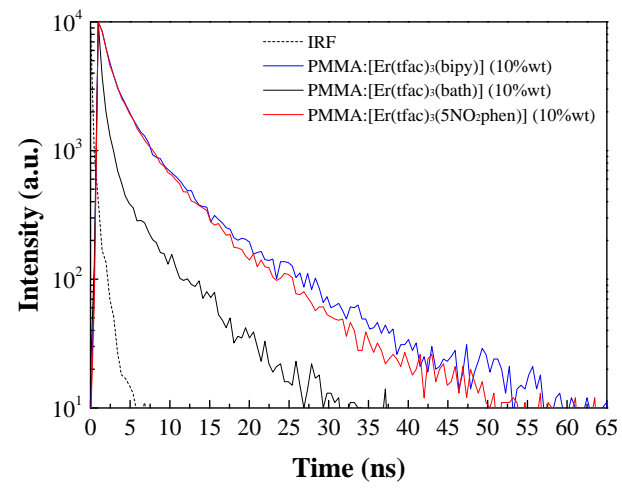

PMMA:[Er(tfac) $)_{3}(\mathrm{~N}, \mathrm{~N}-$ donor $\left.)\right](10 \% \mathrm{wt})$

Figure 146. Ligand emission-associated PL decay times in the visible region for the ormosil (left) and PMMA (right) samples, doped at $0.5 \% \mathrm{wt}$ and $10 \% \mathrm{wt}$, respectively

Table 63. Visible PL emission lifetime values for doped ormosil films

\begin{tabular}{|c|c|c|c|c|c|}
\hline Matrices & $\mathrm{f}_{1}$ & $t_{1}$ & $\mathrm{f}_{2}$ & $t_{2}$ & $\tau_{\text {effective }}(\mathrm{ns})$ \\
\hline Ormosil:[Er(h) ${ }_{3}($ bipy $\left.)\right](0.5 \% \mathrm{wt})$ & 93.011 & 1.266 & 6.989 & 6.116 & 1.60 \\
\hline Ormosil: $\left[\operatorname{Er}(\mathrm{h})_{3}(\right.$ bath $\left.)\right](0.5 \% \mathrm{wt})$ & 25.113 & 3.185 & 74.887 & 26.301 & 20.50 \\
\hline Ormosil:[Er(h) $)_{3}\left(5 \mathrm{NO}_{2}\right.$ phen $\left.)\right](0.5 \% \mathrm{wt})$ & 51.87 & 0.971 & 48.13 & 8.089 & 4.40 \\
\hline Ormosil:[Er(dmh) $)_{3}($ bipy)] $(0.5 \% w t)$ & 70.45 & 3.747 & 29.55 & 17.234 & 7.73 \\
\hline Ormosil: $\left[\operatorname{Er}(\mathrm{dmh})_{3}(\right.$ bath $\left.)\right](0.5 \% \mathrm{wt})$ & 12.31 & 5.638 & 87.69 & 27.147 & 24.50 \\
\hline Ormosil:[Er(dmh $)_{3}\left(5 \mathrm{NO}_{2}\right.$ phen $\left.)\right](0.5 \% \mathrm{wt})$ & 73.103 & 1.613 & 26.897 & 8.755 & 3.53 \\
\hline Ormosil:[Er(tfac) $)_{3}($ bipy)] (0.5\%wt) & 77.105 & 2.425 & 22.895 & 11.778 & 4.57 \\
\hline Ormosil:[Er(tfac $)_{3}($ bath $\left.)\right](0.5 \% w t)$ & 14.8 & 3.772 & 85.2 & 26.85 & 23.43 \\
\hline Ormosil:[Er(tfac $)_{3}\left(5 \mathrm{NO}_{2}\right.$ phen $\left.)\right](0.5 \% \mathrm{wt})$ & 64.005 & 2.127 & 35.995 & 10.221 & 5.04 \\
\hline
\end{tabular}

Table 64. Visible PL emission lifetime values for doped PMMA films

\begin{tabular}{|c|c|c|c|c|c|}
\hline Matrices & $\mathrm{f}_{1}$ & $t_{1}$ & $\mathrm{f}_{2}$ & $t_{2}$ & $\tau_{\text {effective }}(\mathrm{ns})$ \\
\hline PMMA:[Er(h) ${ }_{3}($ bipy)] $(10 \% w t)$ & 77.937 & 1.072 & 22.063 & 5.541 & 2.06 \\
\hline PMMA $\left[\operatorname{Er}(\mathrm{h})_{3}(\right.$ bath $\left.)\right](10 \% \mathrm{wt})$ & 72.987 & 1.102 & 27.013 & 6.594 & 2.59 \\
\hline PMMA: $\left[\operatorname{Er}(\mathrm{h})_{3}\left(5 \mathrm{NO}_{2}\right.\right.$ phen $\left.)\right](10 \% \mathrm{wt})$ & 34.376 & 0.611 & 65.624 & 1.164 & 0.97 \\
\hline PMMA:[Er(dmh) $)_{3}($ bipy $\left.)\right](10 \% w t)$ & 67.812 & 1.182 & 32.188 & 7.222 & 3.13 \\
\hline PMMA:[Er(dmh $)_{3}($ bath $\left.)\right](10 \% w t)$ & 76.097 & 1.341 & 23.903 & 10.185 & 3.45 \\
\hline PMMA: $\left[\operatorname{Er}(\mathrm{dmh})_{3}\left(5 \mathrm{NO}_{2}\right.\right.$ phen $\left.)\right](10 \% \mathrm{wt})$ & 95.785 & 0.965 & 4.215 & 5.358 & 1.15 \\
\hline PMMA:[Er(tfac) $)_{3}($ bipy $\left.)\right](10 \% \mathrm{wt})$ & 67.702 & 1.309 & 32.298 & 6.51 & 2.99 \\
\hline PMMA: $\left[\operatorname{Er}(\mathrm{tfac})_{3}(\mathrm{bath})\right](10 \% \mathrm{wt})$ & 95.856 & 0.931 & 4.144 & 4.671 & 1.09 \\
\hline PMMA:[Er(tfac $)_{3}\left(5 \mathrm{NO}_{2}\right.$ phen $\left.)\right](10 \% \mathrm{wt})$ & 81.154 & 0.654 & 18.846 & 4.658 & 1.41 \\
\hline
\end{tabular}


Table 65. Comparison of visible PL emission lifetime values for the pure complexes in powder and dispersed in ormosil and PMMA matrices

\begin{tabular}{|c|c|c|c|}
\hline Complexes & $\begin{array}{c}\tau_{\text {effective ormosil }} \\
0.5 \% \text { wt }(\mathrm{ns})\end{array}$ & $\begin{array}{c}\tau_{\text {effective }} \text { PMMA } \\
10 \% \mathrm{wt}(\mathrm{ns})\end{array}$ & $\begin{array}{l}\tau_{\text {effective }} \text { pure complex in } \\
\text { powder (ns) }\end{array}$ \\
\hline$\left[\operatorname{Er}(\mathrm{h})_{3}(\right.$ bipy) $]$ & 1.60 & 2.06 & 1.35 \\
\hline$\left[\mathrm{Er}(\mathrm{h})_{3}(\mathrm{bath})\right]$ & 20.50 & 2.59 & 1.02 \\
\hline$\left[\operatorname{Er}(\mathrm{h})_{3}\left(5 \mathrm{NO}_{2}\right.\right.$ phen $\left.)\right]$ & 4.40 & 0.97 & 1.08 \\
\hline $\operatorname{Er}(\mathrm{dmh})_{3}($ bipy $\left.)\right]$ & 7.73 & 3.13 & 0.36 \\
\hline$\left[\operatorname{Er}(\mathrm{dmh})_{3}(\right.$ bath $\left.)\right]$ & 24.50 & 3.45 & 1.36 \\
\hline$\left[\mathrm{Er}(\mathrm{dmh})_{3}\left(5 \mathrm{NO}_{2}\right.\right.$ phen $\left.)\right]$ & 3.53 & 1.15 & $\mathrm{~N} / \mathrm{A}$ \\
\hline$\left[\mathrm{Er}(\mathrm{tfac})_{3}(\mathrm{bipy})\right]$ & 4.57 & 2.99 & 0.56 \\
\hline$\left[\operatorname{Er}(\operatorname{tfac})_{3}(\mathrm{bath})\right]$ & 23.43 & 1.09 & 1.02 \\
\hline$\left[\mathrm{Er}(\mathrm{tfac})_{3}\left(5 \mathrm{NO}_{2}\right.\right.$ phen $\left.)\right]$ & 5.04 & 1.41 & $\mathrm{~N} / \mathrm{A}$ \\
\hline
\end{tabular}

\section{NIR PL lifetime measurements}

The observed lifetimes in PMMA matrices are similar to those reported above for the pure complexes in powder form, indicating that the lanthanide ion is well shielded from its environment and only slightly influenced by changes in the matrix (Figure 147). NIR lifetime measurements were not feasible for the ormosil-doped films, since they are quickly burnt by the incident MOPO laser beam. Nonetheless, it can be expected that the lifetimes will also be similar to those of the pure complexes, in agreement with what has been reported by other authors for analogous highly coordinated lanthanide complexes dispersed in organically modified xerogels [213].

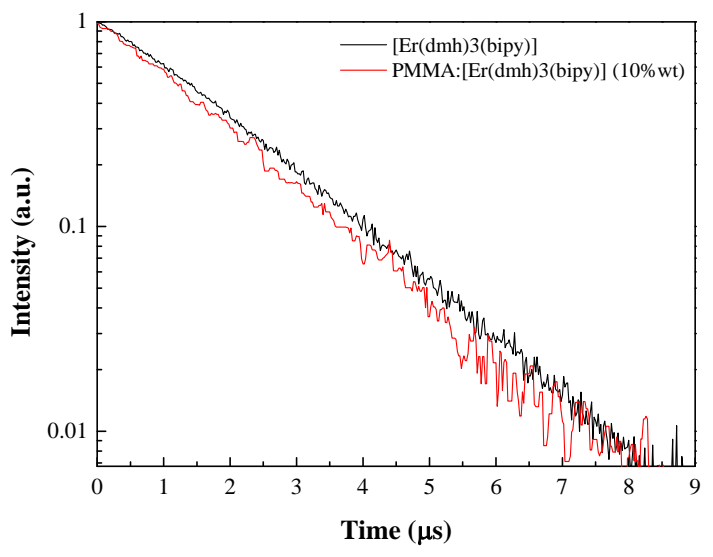

Figure 147. Comparison between the luminescence decay curves of one of the complexes ([Er(dmh) $)_{3}$ (bipy)]) as-is (black) and dispersed in PMMA (red). 


\subsection{Assessment of the suitability of the new complexes for the fabrication of NIR-OLEDs}

\subsubsection{Precedents in the manufacturing of NIR-OLEDs}

To date, only a few emitters show the NIR EL around $1550 \mathrm{~nm}$, and the corresponding OLEDs usually have a non-measurable external quantum efficiency and low power output.

One of the first examples in the fabrication of NIR-OLEDs were OLEDs containing erbium(III) 8-hydroxyquinoline complex (ErQ), still considered a reference erbium complex, which shows a broad emission band at room temperature in the range of $380-750 \mathrm{~nm}$, with a maximum at $600 \mathrm{~nm}$, in addition to emission in the infrared at $1540 \mathrm{~nm}[98,219]$. That first device, with structure ITO/TPD $(50 \mathrm{~nm}) / \mathrm{ErQ}(60 \mathrm{~nm}) / \mathrm{Al}$, emitted under a driving voltage of $25 \mathrm{~V}$ and no efficiency values were reported. A recent review paper [220] has revised the old assumption that these complexes were analogous in structure to $\mathrm{AlQ}_{3}$ and pointed out the specificity of the lanthanide coordination chemistry. In fact, depending on reaction conditions, species with high coordination numbers and differing for nuclearity and stoichiometry were found, and the difficulty of obtaining pure products was stressed. In some instances it has been shown that coordinated water molecules directly bounded to the emitting erbium ion definitely represent the most effective quenchers for the luminescence at $1.5 \mu \mathrm{m}$. Additionally, the combined optical and structural investigation (structures of lanthanide quinolinolates were not available in the literature) of water-free Er-quinolinolate complexes, has allowed Artizzu et al. to conclude that the $\mathrm{C}-\mathrm{H}$ groups sitting in the $\mathrm{Er}^{3+}$ inner coordination sphere represent a very severe limit to the near-infrared emission yield, and that only when hydrogen atoms in the quinolinolate ring are properly substituted (with deuterium or halogen atoms) improvement of luminescence efficiency of this class of complexes can be reached.

The $\beta$-diketonate complexes $\operatorname{Er}(\mathrm{acac})_{3}$ and $\left[\operatorname{Er}(\mathrm{acac})_{3}(\mathrm{phen})\right]$ have also been used instead of ErQ in erbium-doped OLEDs [221,222]. In [221], Si-based organic light emitting diodes (OLED) with structures of $\mathrm{p}-\mathrm{Si} / \mathrm{SiO}_{2} / \mathrm{NPB} / \mathrm{AlQ} / \mathrm{AlQ}: \mathrm{Er}(\mathrm{acac})_{3} / \mathrm{AlQ} / \mathrm{Sm} / \mathrm{Au}$ were manufactured and $1.54 \mu \mathrm{m}$ emission from $\mathrm{Er}^{3+}$ was observed, but no efficiency data is reported. In [222], [Er(acac) $)_{3}($ phen)] was dispersed into a polymer matrix of poly- $\mathrm{N}$ vinylcarbazole (PVK) to fabricate an OLED with an ITO/PVK:Er(acac) 3 (phen)/Al:Li/Ag structure, one of the few Er-based OLEDs manufactured by spin-coating which have been reported to date (the majority of the devices referred in this section were manufactured by evaporation of the active layer). Despite the fact that the device showed infrared EL emission at $1.54 \mu \mathrm{m}$, no external efficiency data were reported.

Besides erbium(III), neodymium(III), [223] ytterbium(III) [224], holmium(III) [224] and praseodymium(III) [225] have also been selected for the construction of near-infraredemitting OLEDs.

According to Buckley [226], K.S. Schanze's group prepared NIR-OLEDs with emission at 977 $\mathrm{nm}$ (arising from ${ }^{2} \mathrm{~F}_{5 / 2} \rightarrow{ }^{2} \mathrm{~F}_{7 / 2}$ transition of the $\mathrm{Yb}^{3+}$ ion) using blue-light-emitting polymers of bis(alkoxy)-substituted poly(p-phenylene) (PPP-OR1) blended with $\mathrm{Yb}$ complexes $\left(\mathrm{Yb}(\mathrm{DBM})_{3}\right.$ phen and $\mathrm{Yb}(\mathrm{DNM})_{3}$ phen, where DBM stands for dibenzoylmethane and DNM is dinaphtoylmethane). The structure of the OLED was ITO/PEDOT:PSS (40 nm)/PPP-OR:Yb complexes $(100 \mathrm{~nm}) / \mathrm{Ca}(5 \mathrm{~nm}) / \mathrm{Al} \quad(200 \mathrm{~nm})$, where PEDOT:PSS was poly(3,4ethylenedioxythiophene) poly(styrenesulfonate). The external efficiencies of the $\mathrm{Yb}(\mathrm{DBM})_{3}$ phen and $\mathrm{Yb}(\mathrm{DNM})_{3}$ phen OLEDs were $7 \times 10^{-5}$ at $6 \mathrm{~V}$ and $0.81 \mathrm{~mA} \cdot \mathrm{mm}^{-2}$ and $4 \times 10^{-4}$ at $7 \mathrm{~V}$ and $0.74 \mathrm{~mA} \cdot \mathrm{mm}^{-2}$, respectively.

When Er(TPP)acac (where TPP is 5,10,15,20-tetraphenylporphine) was blended with MEHPPV in an OLED structure ITO/PEDOT:PSS/MEH-PPV:Er(TPP)acac/Ca/Al, EL peaked at 1560 
$\mathrm{nm}\left({ }^{4} \mathrm{I}_{13 / 2} \rightarrow{ }^{4} \mathrm{I}_{15 / 2}\right)$ [227]. The same complex was also doped into p-conjugated polymers of type poly(arylene-ethynylene) (PAE) by Pizzoferrato et al. [228].

The same group as in [227] subsequently reported a series of NIR-OLED in which the active materials consisted of a series of Ln(TPP) complexes dispersed in polystyrene (PS) as the host matrix, instead of MEH-PPV [229]. The structure of these OLEDs was ITO/PEDOT:PSS/PS:Ln(TPP)L(OEt)/Ca $\quad(5 \mathrm{~nm}) / \mathrm{Al} \quad(200 \mathrm{~nm})$, where L(OEt) was $\left[\left(\mathrm{C}_{5} \mathrm{H}_{5}\right) \mathrm{Co}\left\{\mathrm{P}(\mathrm{O}) \mathrm{Et}_{2}\right\}_{3}\right]^{-}$. The $\mathrm{Yb}^{3+}$ device emitted with a maximum intensity at $977 \mathrm{~nm}$ along with two weaker bands at $923 \mathrm{~nm}$ and $1003 \mathrm{~nm}$. The emission arised from the ${ }^{2} \mathrm{~F}_{5 / 2} \rightarrow{ }^{2} \mathrm{~F}_{7 / 2}$ transition of $\mathrm{Yb}^{3+}$ and the satellite bands arised from crystal field splitting effects of the L(OEt) and TPP ligands. The device which contained Er(TPP)L(OEt) exhibited a strong NIR emission al $1520 \mathrm{~nm}$, which arises from the ${ }^{4} \mathrm{~F}_{13 / 2} \rightarrow{ }^{4} \mathrm{I}_{15 / 2}$ transition of $\mathrm{Er}^{3+}$ ion. PVK was also tested as a host matrix, with similar turn-on voltages and overall near-IR emission characteristics. In addition, poly(paraphenylene) PPP-OR11 was assayed as a suitable host too [230], with modest efficiencies for the Yb-based devices (no efficiency data was provided in any of these articles for the Er-based NIR-OLEDs).

For an up-to-date review of the design, synthesis, structural analysis and photophysical studies dealing with the NIR emission of Ln(III) cations sensitized by a variety of porphyrin chromophores, the reader is advised to check the paper by Bulach et al. [231].

Kawamura et al. [232] fabricated OLED devices having the structure glass/ITO/N,N'diphenyl-N.N'-di(m-tolyl)benzidine/Ln(DBM) ${ }_{3}$ bath(Ln: Nd, Er, Yb)/bathocuproine/Mg:Ag/ Ag back in 2004. The EL efficiencies of the NIR emission were $7 \times 10^{-5}, 3 \times 10^{-4}$, and $1 \times 10^{-5}$ for $\mathrm{Nd}^{3+-}, \mathrm{Yb}^{3+-}$, and $\mathrm{Er}^{3+-}$ doped devices, respectively. Nonetheless, it must be clarified that these efficiencies were not measured directly, but estimated by comparison with the value of the $\mathrm{Eu}(\mathrm{DBM})_{3}$ bath device having the same configuration. Also in 2004, Zang et al. [233] used $\left[\operatorname{Er}(\mathrm{DBM})_{3}(\right.$ bath $\left.)\right]$ complex, together with [Tm(DBM) $)_{3}$ bath], in EL devices so as to obtain a broadened EL emission band ranging from 1.4 to $1.6 \mu \mathrm{m}$, showing the potential application of Tm complexes for optical communication systems. The device structure was Glass/ITO/TPD $(60 \mathrm{~nm}) /[\mathrm{Tm}(\mathrm{DBM}) 3 \mathrm{bath}]:[\operatorname{Er}(\mathrm{DBM}) 3 \mathrm{bath}](60 \mathrm{~nm}$, mass ratio 9:1)/Mg:Ag (10:1, $150 \mathrm{~nm})$. Unfortunately, apart from EL spectra, no additional data regarding the efficiency of the devices is provided.

In 2008, it was shown [234] that the relative intensity of [Er(DBM $)_{3}($ bath)]-based devices can be improved by nearly 4 -fold, by using a phosphorescent sensitizer in the emissive layer in order to harvest both singlet and triplet excitons formed on the host molecules. Devices with structure of ITO/m-MTDATA $(10 \mathrm{~nm}) / \mathrm{NPB}(30 \mathrm{~nm}) / \mathrm{Er}-\mathrm{DB}: \operatorname{Ir}(\mathrm{ppy})_{3}(x$ wt \%, 40 $\mathrm{nm}) /$ Bath $(30 \mathrm{~nm}) / \mathrm{LiF}(1 \mathrm{~nm}) / \mathrm{Al}(120 \mathrm{~nm})$ were constructed, where $m$-MTDATA, NPB, and Bath denote 4,4',4"'-tris[3-methyl-pheny(phenyl)-amino] triphenylamine, 4,4'-bis[ $N$-(1napthyl)- $N$-phenyl-amino]-biphenyl, and 4,7-diphenyl-1,10-phenanthroline (bathophenanthroline), respectively, which work as the hole injection, hole transporting, and the hole blocking/electron transfer layers, respectively, as well $x=0$ and 50, respectively. Nonetheless, no EQE data was presented.

In 2009 Zang et al. [235] have reported on the broadening of the infrared emission bands of OLEDs based on mixing $\mathrm{Er}^{3+}$ and $\mathrm{Nd}^{3+}$ complexes as the active medium. This suggests the possibility of realizing simultaneous multiple IR emissions over a broad spectral band via electrical excitation. To date, there has been little reported work on modulation of NIR emissions from OLEDs. The IR emissions in tris(dibenzoylmethane) mono(bathophenanthroline)erbium(III) (named $\operatorname{Er}(\mathrm{DBM})_{3}$ bath) and tris(dibenzoylmethane) mono(bathophenanthroline)neodimium(III) (or $\mathrm{Nd}(\mathrm{DBM})_{3}$ bath) based OLEDs can be achieved by adjusting the forward bias or varying the configuration of $\operatorname{Er}(\mathrm{DBM})_{3}$ bath/Nd(DBM $)_{3}$ bath layer thickness used in the devices. The emission intensity of the $\mathrm{Nd}^{3+}$ at 1.064 is comparable to that of $\mathrm{Er}^{3+}$ at $1.54 \mu \mathrm{m}$ under the same excitation conditions, matching the spectral windows that are suitable for telecommunication 
applications. The devices were fabricated with a three layer structure: ITO(TPD 60 $\mathrm{nm}) / \mathrm{Nd}(\mathrm{DBM})_{3}$ bath $(x \mathrm{~nm}) / \operatorname{Er}(\mathrm{DBM})_{3}$ bath $(60-x \mathrm{~nm}) / \operatorname{Mg}: \operatorname{Ag}(10: 1,150 \mathrm{~nm})(x=1,5,15,20)$.

In 2010, Wei et al. [99], the same group as in [221], used tris(dibenzoylmethane) mono (phenanthroline)erbium(III) [Er(DBM) $)_{3}$ phen] as the dopant and 4,7-diphenyl-1,10phenanthroline (Bphen) as emissive host and electron transport layer, in a device with structure p-Si substrate/ $\mathrm{SiO}_{2}(1.5 \mathrm{~nm}) / \mathrm{NPB}(60 \mathrm{~nm}) /$ Bphen:Er(DBM $)_{3}$ phen $(20 \mathrm{~nm}) /$ Bphen $(25 \mathrm{~nm}) / \mathrm{CsPh}(15 \mathrm{~nm})$, achieving a maximum NIR power of $0.93 \mu \mathrm{W} / \mathrm{cm}^{2}$. [Er(DBM) $($ phen)] has also been used as a dopant in near-infrared luminescent macroporous materials [236], which could be a promising approach for optical amplifiers.

Using $\operatorname{Er}(\mathrm{PM})_{3}(\mathrm{TP})_{2}\left[\mathrm{PM}=1\right.$-phenyl-3-methyl-4-isobutyryl-5-pyrazolone ${ }^{\mathrm{p}}, \mathrm{TP}=$ triphenyl phosphine oxide], Zhang et al. [237] reported in 2008 a device with structure ITO/NPB (20 $\mathrm{nm}) / \operatorname{Er}(\mathrm{PM})_{3}(\mathrm{TP})_{2}(50 \mathrm{~nm}) / \mathrm{BCP}(20 \mathrm{~nm}) / \mathrm{AlQ}(40 \mathrm{~nm}) / \mathrm{LiF}(1 \mathrm{~nm}) / \mathrm{Al}(120 \mathrm{~nm})$, which shows $1.54 \mu \mathrm{m}$ near-infrared (NIR) emission from $\mathrm{Er}^{3+}$ ion with the maximum NIR irradiance of $0.21 \mu \mathrm{W} / \mathrm{cm}^{2}$. The same group has assayed other complexes, such as $\mathrm{Ln}(\mathrm{DBM}-\mathrm{OH})_{3} \cdot 2 \mathrm{H}_{2} \mathrm{O}$ (where $\mathrm{DBM}-\mathrm{OH}=o$-hydroxydibenzoylmethane, $\mathrm{Ln}=\mathrm{Nd}, \mathrm{Er}, \mathrm{Yb}$, and $\mathrm{Sm}$ ) in sol-gel derived hybrid materials for optical amplifiers, but not in NIR-OLED devices [238].

Recently [239], NIR-OLEDs have been demonstrated by employing erbium fluoride $\left(\mathrm{ErF}_{3}\right)$ doped tris-(8-hydroxyquinoline) aluminum $\left(\mathrm{AlQ}_{3}\right)$ as the emitting layer. The device structure is ITO/N,N'-di-1-naphthyl- $\mathrm{N}, \mathrm{N}^{\prime}$-diphenylbenzidine $\quad(\mathrm{NPB}) / \mathrm{Alq}_{3}: \mathrm{ErF}_{3} / 2,2^{\prime}, 2^{\prime \prime}-(1,3,5-$ phenylene)tris(1-phenyl-1H-benzimidazole) (TPBI)/Alq ${ }_{3} / \mathrm{Al} . \quad$ Room-temperature electroluminescence around $1530 \mathrm{~nm}$ is observed due to the ${ }^{4} \mathrm{I}_{13 / 2}{ }^{4} \mathrm{I}_{15 / 2}$ transition of $\mathrm{Er}^{3+}$. NIR EL intensity from the $\mathrm{ErF}_{3}$-based device is $\sim 4$ times higher than that of $\operatorname{Er}(\mathrm{DBM})_{3}$ phenbased device at the same current (no absolute NIR EL external quantum efficiency measurements are reported).

In 2011, Katkova et al. [240] fabricated devices using $\operatorname{Ln}_{2}(00 N)_{6}(O O N=2-(2$-benzoxyazol-2yl)phenolate) and $\operatorname{Ln}_{2}(\mathrm{SON})_{6}$ ( $\mathrm{SON}=2$-(2-benzothiazol-2-yl)phenolate) complexes, where $\mathrm{Ln}=\operatorname{Pr}(\mathrm{III}), \mathrm{Nd}(\mathrm{III}), \mathrm{Ho}(\mathrm{III}), \operatorname{Er}(\mathrm{III}), \mathrm{Tm}(\mathrm{III})$, and $\mathrm{Yb}(\mathrm{III})$. For $\mathrm{Nd}^{3+-}$ and $\mathrm{Yb}^{3+}$-based devices, the maximum wall plug efficiencies were 0.82 and $1.22 \mathrm{~mW} / \mathrm{W}$. No data for NIR wall plug efficiencies for the $\mathrm{Er}^{3+}$-based OLEDs was provided, since ${ }^{4} \mathrm{I}_{13 / 2} \rightarrow{ }^{4} \mathrm{I}_{15 / 2}(1.5 \mu \mathrm{m})$ transition was reported to be very weak.

Manufacturing approaches for lanthanide-based OLEDs: vacuum deposition vs. solutionprocessed devices [13]

The first types of lanthanide-based OLEDs were prepared by vacuum deposition of the different layers (hole injection layer, emitting layer, electron transport layer, cathode) on the ITO substrate. This technique is applicable only for volatile and thermally stable lanthanide complexes. Unfortunately, most volatile lanthanide $\beta$-diketonate complexes are not the ones with the best luminescence properties. Many $\beta$-diketonate complexes cannot be sublimed without considerable thermal decomposition, or give deposited layers of an inferior qualityq. The films of lanthanide $\beta$-diketonates produced by vacuum deposition have poor chargecarrier properties. Especially the transport of electrons is problematic. Because of the

\footnotetext{
$\mathrm{p}$ The structure of pyrazolone-based ligands is similar to that of $\beta$-diketone ligands, which can enhance luminescent properties of $\mathrm{Ln}^{3+}$ ions well.

q According to Katkova et al. [Russian Chemical Reviews, 2005, 74:12, 1089-1109 and references therein], many low-molecular-weight lanthanide complexes are non-technological because film deposition is often accompanied by undesired crystallisation or aggregation. To solve this problem, attempts were made to synthesise high-molecular-weight lanthanide complexes. However, the simplest procedure is apparently based on the introduction of low-molecular-weight lanthanide compounds into polymeric matrices ('host-guest' systems), with all their drawbacks and advantages.
} 
unbalanced injection and transport of charge carriers, recombination often takes place at sites other than the emitting layer. This not only leads to low electroluminescence efficiency, but also to a reduced lifetime of the OLED.

One approach to improve OLEDs based on lanthanide compounds is to replace the tris $\beta$ diketonate complexes by Lewis base adducts (i.e. by ternary complexes), as we have done. In this way, not only the volatility and the thermal stability can be improved (these adducts show a large temperature interval between the melting point and the onset of thermal decomposition, which facilitates processing of this complex by vacuum vapor deposition), but also the film forming properties and the carrier-transport ability. Although 1,10phenanthroline is often used as Lewis base to form ternary complexes, better results are often obtained when 4,7-diphenyl-1,10-phenanthroline (bathophenanthroline, bath) is used instead $[241,242,243,244,245]$, which supports the choice of this ancillary ligand in this Thesis.

Instead of depositing the emissive layer by vacuum vapor deposition, it is also possible to deposit it from solution. The usual approach is to dope the lanthanide complex into a polymer matrix. In this case the lanthanide complex and the polymer are dissolved in a suitable solvent and the emissive layer is cast directly from solution by spin coating $[246,247,248]$. Further information on research conducted to date regarding the dispersion of lanthanide complexes into polymer (and glass matrices) can be found in $[37,249,250,251,252,253,254,255,98]$.

The doped polymers have several advantages. First of all, the thermal decomposition of the electroluminescent complexes by vacuum sublimation is avoided. Secondly, the processing of the films is simplified. Thirdly, the polymer has better film forming properties than the lowmolecular weight lanthanide complexes. Fourthly, the polymer matrix can have good holeand electron-transporting properties, so that the electroluminescence is improved. Finally, the energy of blue-emitting polymers (such as PVK or PVN) can be transferred to the lanthanide complex. It should be stressed that in order to have a good energy transfer from the polymer to the Ln(III) complex, there must be an overlap between the emission spectrum of the polymer and the absorption spectrum of the ligands.

Most of the complexes presented in this Thesis would be suitable both for vacuum deposition and for doping of a polymer matrix. Nonetheless, despite the fact that the second approach was initially chosen (vida infra), the good mobility properties of the reported complexes enable a third and entirely novel approach: solution-processed devices in which the active layer consists of only the pure complex "as-is". This approach retains the main advantages of the polymer-based OLEDs mentioned above, and is also easier to scale up to large substrates. 


\subsubsection{Fabrication process and device characterization}

Organic electroluminescent devices with the corresponding trivalent erbium complexes as emissive layers in the NIR have been fabricated. The new complexes show very good film forming properties. Solution-processed multiple-layer-type devices with both AZO/PEDOT:PSS/PVK:PBD:Er-complex/Ba/Al and ITO/PEDOT:PSS/Er-complex/Ca/Al structures have been assayed.

\section{NIR-OLEDs with AZO/PEDOT:PSS/PVK:PBD:Er-complex/Ba/Al structure}

For the preparation of phosphorescent polymer OLEDs, the commonly used practice is to blend a low molecular weight phosphorescent dye with a properly chosen polymer matrix. Since a polymer host with a triplet state energy above that of the phosphorescent dye is required to guarantee the confinement of the triplet excited state on the guest, large band gap polymers such as poly(N-vinyl carbazole) (PVK) are commonly utilized for the purpose. It is a well-known fact that the electron-transporting properties of PVK are poor. In order to improve these properties, it has been reported in the literature [256,257] that electrontransporting materials such as 2-(4-biphenylyl)-5-(4-tert-butylphenyl)-1,3,4-oxa-diazole (PBD) can be blended with PVK to insure better efficiencies and low driving voltages.

On the other hand, Yang et al. [258] also reported that by introducing hole-transporting TPD molecules in PVK the driving voltage of polymer OLEDs could be decreased. The first couple of materials are the preferred option, provided that they are better established, but later on $\alpha$-NPD could also be assayed as a hole-transporting molecule (instead of TPD, used by other groups, based on the fact that the hole injection barrier from PEDOT:PSS [poly(3,4ethylenedioxythiophene):poly(styrene sulfonate)] to $\alpha$-NPD is lower than that to TPD: Makinen et al. [259] showed that hole injection barrier from PEDOT:PSS to a-NPD was 0.51 $\mathrm{eV}$ while that to TPD was $0.58 \mathrm{eV}$ ).

\section{Fabrication procedure}

Polymer light emitting diodes with AZO/PEDOT:PSS/PVK:PBD:Er-complex/Ba/Al structure were prepared at the IGM (Institut für Großflächige Mikroelektronik, i.e., Institute for Large Area Microelectronics) facilities in Stuttgart (Figure 148). The inert-gas work stations of the IGM consist of three modules by the company MBRAUN. The first module contains an evaporation tool (Lesker Spectros) with thermal evaporation sources for 4 organic materials and for 2 metals. In the second module solution-based films can be deposited by spincoating. This module also contains a hotplate and a vacuum oven for temper processes. The third module is used for the electric and optic characterization of the processed layers and devices within the inert-gas atmosphere. Furthermore, an ink jet-system (Dimatix) for processes with printed films and structures is also available. 


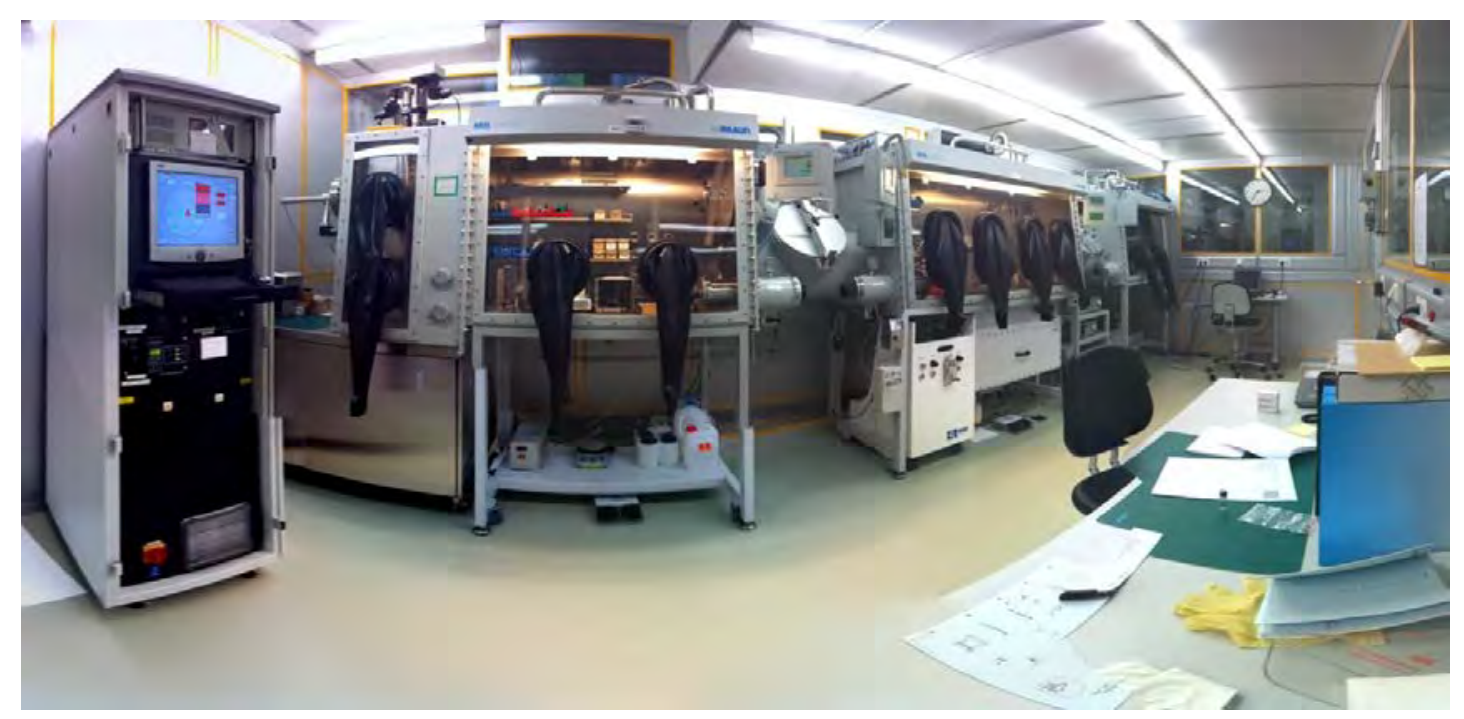

Figure 148. General view of the glove-box system for organic electronics at IGM facilities

The processing steps can be summarized as follows (Figure 149):

Preparation of the substrate. Substrates (Corning Eagle 2000 glass substrates, thickness 0.7 $\mathrm{mm}$ ) were cut to an area of $5 \mathrm{~cm} \times 5 \mathrm{~cm}$ and cleaned using IGM's standard cleaning procedure (ultrasonic cleaning, followed by rinsing with deionized water, trichloroethylene, acetone, and methanol). Sputter etching prior to deposition of the anode was conducted

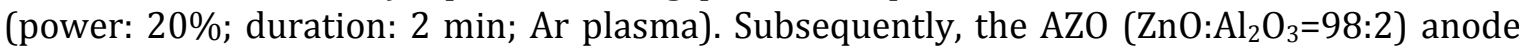
was deposited by sputtering $(150 \mathrm{~nm})$, using a palette driving speed of $0.45 \mathrm{~m} / \mathrm{min}$, followed by a tempering in a convection oven at $250^{\circ} \mathrm{C}$. The anode was patterned using a soft-contact photolithographic process: AZ 650 TFP resist (Clariant) was dispensed through a $1 \mu \mathrm{m}$ PTFE filter and ARPT-5x5 spin-coater program was chosen; the resist was soft-baked using program AZ5x5 and exposed for $4 \mathrm{~s}$, developed in an AZ Developer:deionized water (1:1) solution for aprox. $20 \mathrm{~s}$, rinsed with deionized water for 1 min and blown dry with nitrogen. Then the AZO layer was etched in oxalic acid at $30^{\circ} \mathrm{C}$, and the substrate was rinsed with deionized water for $1 \mathrm{~min}$ and blown dry with nitrogen again. The resist was stripped in acetone and the substrate was blown dry with nitrogen once more. Another lithography step was conducted afterwards so as to define the OLED pixels (edge insulation layer), but this time the resist was not stripped, but hard baked in a convection oven at $140^{\circ} \mathrm{C}$ for $90 \mathrm{~min}$.

OLED processing. After UV- $\mathrm{O}_{3}$ cleaning of the substrates (100W for $5 \mathrm{~min}$ ), Clevios PVP AI 4083 (diluted with deionized water, 1:1) was filtered using a $0.45 \mu \mathrm{m}$ PTFE filter and spin coated using a predefined coater program ( $5000 \mathrm{rpm}$ for 30 seconds), followed by a drying step at $120^{\circ} \mathrm{C}$ for $90 \mathrm{sec}$. on a hot plate +30 minutes at $120^{\circ} \mathrm{C}$ in a vacuum oven. For the deposition of the active layer, the PVK:PBD:Er complex solution (host matrix:guest (95:5), where the host matrix is PVK:PBD (60:40), according to [260]) in toluene was dispensed through a $0.45 \mu \mathrm{m}$ PTFE filter and spin-coated at $2000 \mathrm{rpm}$ onto the subtrates (yielding a film of $\sim 100 \mathrm{~nm}$ thickness), and soft-baked at $100^{\circ} \mathrm{C}$ for 30 minutes on a hot plate. The $\mathrm{Ba} / \mathrm{Al}$ cathode was deposited using the IGM prototype standard shadow mask. Finally, the devices were encapsulated ${ }^{r}$ using Corning Eagle $200038 \mathrm{~mm} \times 38 \mathrm{~mm}$ glass slides as follows: the edges of the glass slide were covered with stripes of tape ( $2 \mathrm{~mm}$ aprox.) and Polytec OG603 was spin-coated on the them, without any filter; the cover slides were stuck face down to the OLED and the glue was cured under UV radiation for $1 \mathrm{~min} 50 \mathrm{sec}$.

${ }^{\mathrm{r}}$ In spite of the fact that the conventional etched glass + dryer with UV epoxy sealing approach has been used, a more reliable encapsulation could have been achieved by using, for example, Barix ${ }^{\mathrm{TM}}$ thin-film barrier (Vitex) or DryFlex ${ }^{\mathrm{TM}}$ (SAES). 


\section{I-V and EL characterization}

Each $5 \mathrm{~cm} \times 5 \mathrm{~cm}$ substrate contains 11 devices, with diameters ranging from $2 \mathrm{~mm}$ to 0.01 $\mathrm{mm}$. The encapsulated OLEDs were mounted on a custom-made sample holder so as to facilitate the connections to the equipment. Keithley source meters and picoampmeters were used for the J-V measurements (examples of some of the obtained J-V curves are shown in Figure 150). The diodes show a good electrical performance, with very low threshold voltages and low noise levels, indicative of the good quality of the deposited layers.

For the EL measurements, an AQ-6315A optical spectrum analyzer was first used, coupling the OLEDs light output to a fiber. This coupling -and the multimode fiber used- led to a signicant attenuation of the signal, forcing us to increase the applied voltage and current in an attempt to increase the light output. Unfortunately, it was not feasible to record any fullrange (1400 $\mathrm{nm}$ to $1700 \mathrm{~nm}$ ) spectra before devices were burnt. An alternative setup, using a $\mathrm{NaCl}$ filter, a chopper at $30 \mathrm{~Hz}$ and a ThorLabs ES111C Pyroelectric Energy Sensor (Broadband Coating, 0.185-25 $\mu \mathrm{m}, 150 \mathrm{~mJ}$ ) connected to a SRS830 lock-in amplifier was also assayed, but also with poor results.
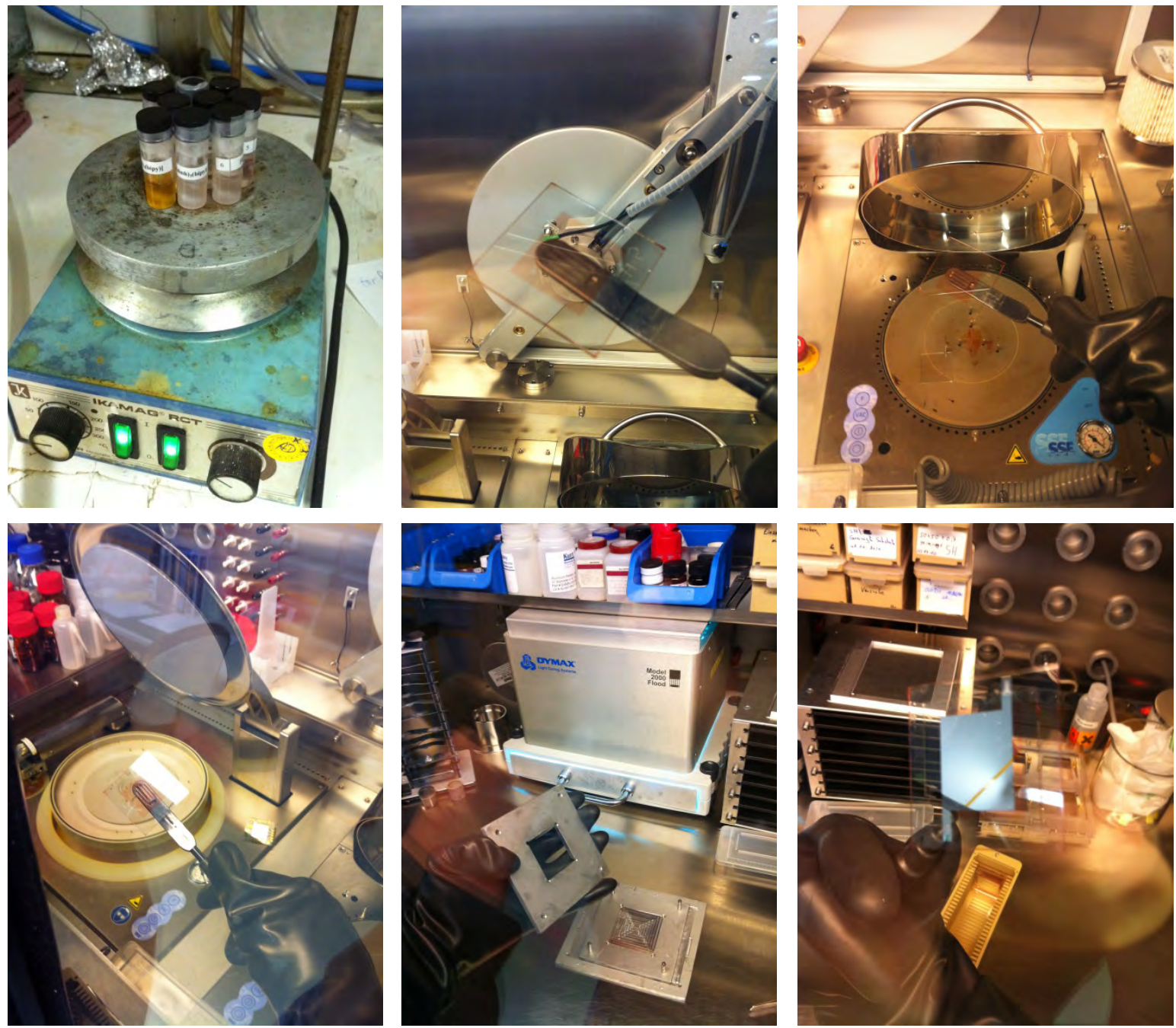

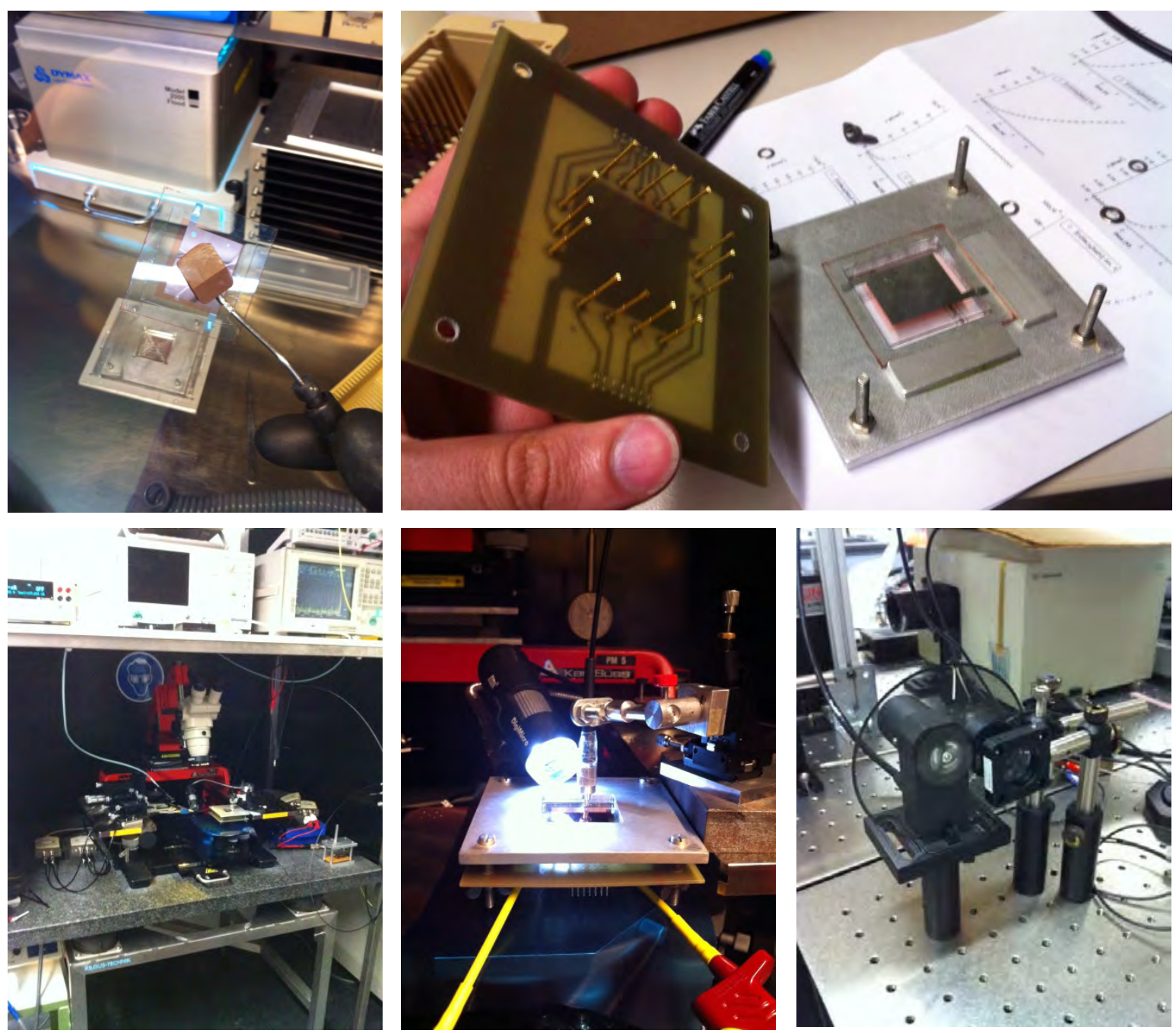

Figure 149. Different steps of the OLED manufacturing and characterization process: preparation of the active layer solutions, substrate after PEDOT:PSS deposition; active layer deposition; hot plate for the soft-baking steps; sample holder for the encapsulation step; two views of the encapsulated device; sample holder used for electrical measurements and EL measurements; setup for EL measurements; detail of the stage for coupling the light to the fiber; alternative setup for EL measurements.

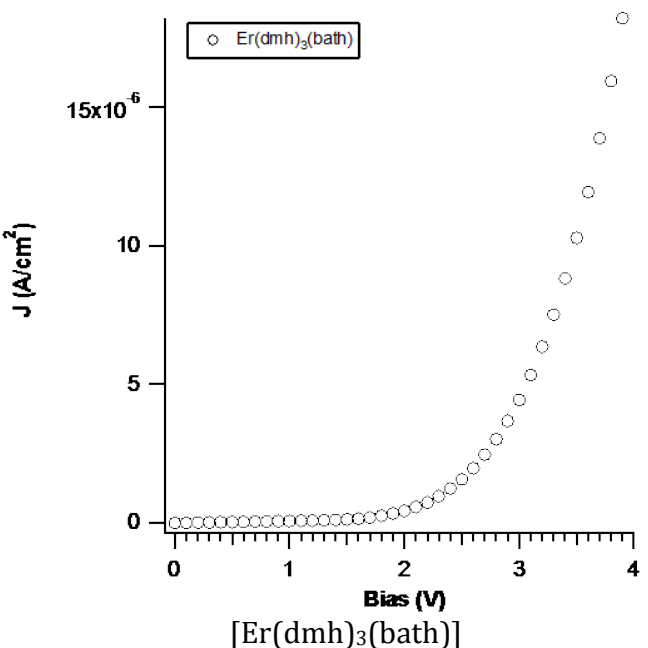

[Er(dmh) $)_{3}$ (bath)]

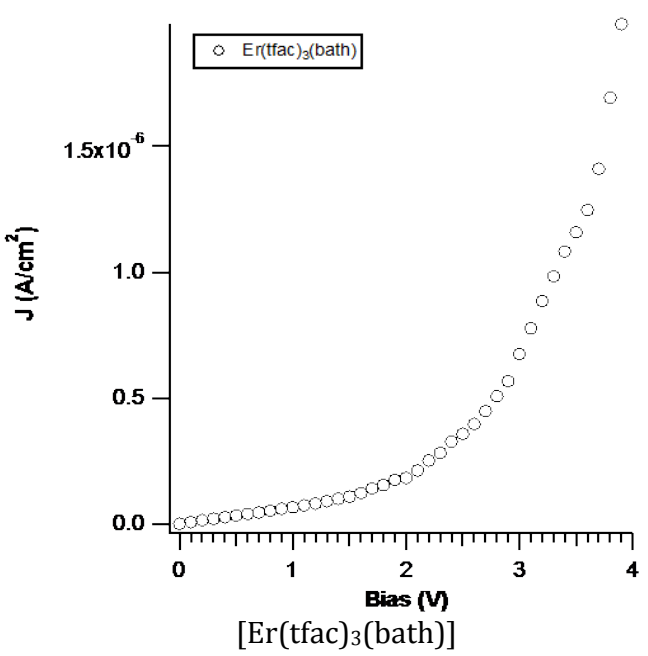

[Er(tfac) ${ }_{3}($ bath) $]$ 


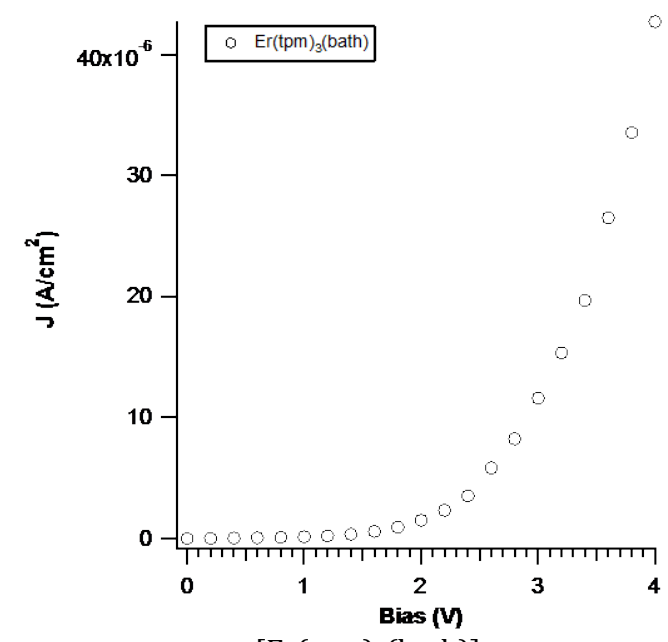

[Er(tpm)3(bath)]

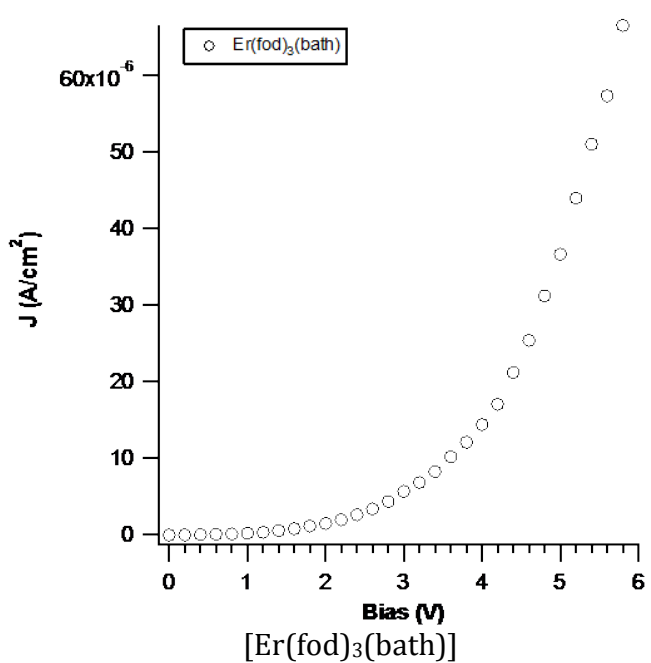

[Er(fod) 3 (bath)]

Figure 150. J-V curves for four devices with $\mathrm{PVK}: \mathrm{PBD}:\left[\operatorname{Er}(\beta \text {-diketonate })_{3}(\right.$ bath $\left.)\right]$ active layer, were the chosen $\operatorname{Er}(\mathrm{III})$ complexes are $\left[\operatorname{Er}(\mathrm{dmh})_{3}(\right.$ bath $),\left[\operatorname{Er}(\mathrm{tfac})_{3}(\right.$ bath $\left.)\right],\left[\operatorname{Er}(\operatorname{tpm})_{3}(\right.$ bath $\left.)\right]$ and $\left[\operatorname{Er}(\text { fod })_{3}(\right.$ bath $\left.)\right]$

\section{NIR-OLEDs with ITO/PEDOT:PSS/Er-complex/Ca/Al structure}

On the basis of the previously reported experience, a different device architecture and manufacturing procedure were designed so as to meet the specificity of these complexes and the non-trivial task of measuring the device EL characteristics. The updated device architecture, shown in Figure 151, basically differs from the one chosen for IGM's prototypes in two key aspects: $(1)$ the $\left[\operatorname{Er}(\beta \text {-diketonate })_{3}(\mathrm{~N}, \mathrm{~N}-\right.$ donor $\left.)\right]$ complex is not dispersed in a host matrix, but used as-is for the active layer; (2) the pixels are now defined by the cathode shadow mask instead of defining them by etching the anode layer and subsequent edge insulation. The former change relies both on good filming properties of the complexes and on the assumption that the materials have an (almost) ambipolar behavior, being able to transport both electrons and holes (vide infra).

The manufacturing process for these NIR-OLEDs has been conducted at the Organic Optoelectronics Group's class 10000 cleanroom at Universidad Rey Juan Carlos (Madrid), and the EL characterization has been carried out at the ISOM facilities at Universidad Politécnica de Madrid.

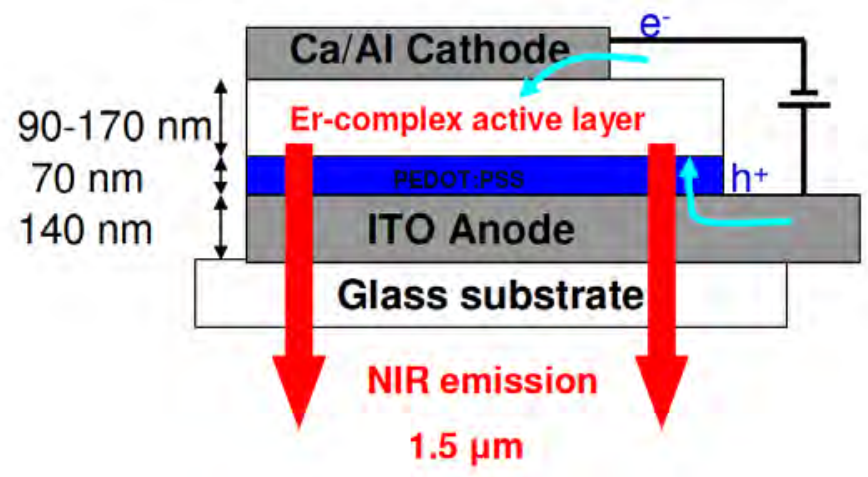

Figure 151. Structure of the NIR-OLEDs manufactured at URJC's cleanroom facilities 
OOG's cleanroom is equipped with a Mbraun glovebox, and Emitech $975 \mathrm{~K}$ turbo pumped thermal evaporator, a Karl Suss spin coater, a Jelight UV-O ${ }_{3}$ cleaner, a Flovigas VA 960120 fume hood, and an Alpha Step D120 stylus profiler (Figure 152).
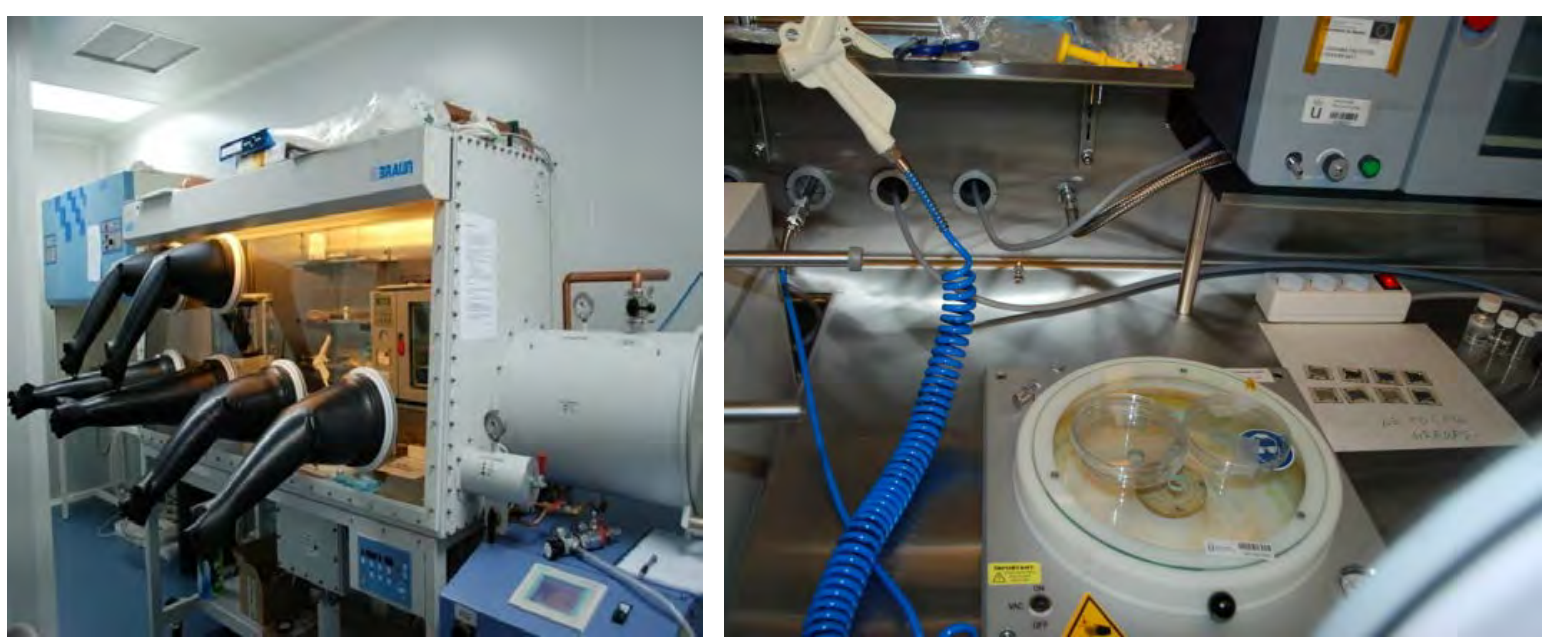

Figure 152. Mbraun glovebox (left) and Karl Suss spin-coater (right) at OOG's $25 \mathrm{~m}^{2}$ class 10000 cleanroom.

\section{Fabrication procedure}

Pre-patterned ITO glass plates with four circular diodes $(1 \mathrm{~mm}$ and $1.5 \mathrm{~mm}$ radii) (Lumtech. Techn.) are extensively cleaned using chemical and UV-ozone methods, right before the deposition of the organic layers. The chemical cleaning is conducted as follows: 5 minutes in trichlorethylene or pyrrolidone, 4 minutes in acetone, 3 minutes sonication in isopropanol (with $\mathrm{N}_{2}$ drying after each step). The $\mathrm{UV}-\mathrm{O}_{3}$ cleaning is then conducted for 20 minutes (so as to passivate the ITO and adjust its work function). This treatment lasts for about 2 hours and it is important to deposit the PEDOT:PSS layer within this time limit.

\section{PEDOT:PSS layer deposition:}

- PEDOT:PSS (Clevios PVP AI 4083) is mixed with isopropyl alcohol (5:1) in order to improve its adhesion properties to ITO [261]. The mixture is stirred for 1 hour and filtered using a $0.2 \mu \mathrm{m}$ hidrofobic filter.

- The solution is kept at $50-60^{\circ} \mathrm{C}$ until it is spin-coated at $2000 \mathrm{rpm}$. Very uniform $70 \mathrm{~nm}$ layers are obtained.

- The layer is cured by heating the sample at $90^{\circ} \mathrm{C}$ for 5 minutes and, subsequently, at $110^{\circ} \mathrm{C}$ for 10 additional minutes.

Emission layer deposition $(\sim 100 \mathrm{~nm})$ :

- The $\left[\operatorname{Er}(\beta \text {-diketonate })_{3}(\mathrm{~N}, \mathrm{~N}\right.$-donor $\left.)\right]$ active layer is deposited by spin-coating at 1500 rpm from a $4 \%$ wt methanol solution. The solution is stirred for 2 hours and then sonicated till it is completely transparent. Subsequently, it is filtered with a $0.2 \mu \mathrm{m}$ PTFE hidrophilic filter (so the $1.5 \mathrm{~mL}$ are slightly diminished). The filtered solution is sonicated for an additional 30 minutes. For $2.5 \mathrm{~cm} \times 2.5 \mathrm{~cm}$ substrates, $0.4 \mathrm{~mL}$ of solution per sample are usually required.

- The layer is slowly cured on a hotplate at $50^{\circ} \mathrm{C}$ for 30 minutes to achieve complete solvent removal. We have found that this slow curing, conducted at a temperature lower than the solvent's boiling point, reduces aggregation and results in an improved film uniformity (leading to highly reflecting surfaces). 
Layer thicknesses are measured using an Alpha Step 200 profilometer (Tenkor Instruments), using control glass slides which are spin-coated in the same conditions as the layers in the device. Therefore, $1.5 \mathrm{~mL}$ of solution is usually enough so as to prepare a control slide and two OLED devices.

The $\mathrm{Ca}$ and $\mathrm{Al}$ cathode is thermally evaporated in an atmosphere of 7-8 $\times 10^{-6}$ Torr on top of the organic layer surface. So as to minimize leakage currents, the four diodes should be isolated from each other (by patterning lines that reach the glass substrate). The structure is finally encapsulated using a glass cover attached by a bead of epoxy adhesive EPO-TEK(302$3 \mathrm{M})$ or EPO-TEK(730).

All the manufacturing process is carried out in an inert atmosphere glove box $\left(<0.1\right.$ p.p.m $\mathrm{O}_{2}$ and $\mathrm{H}_{2} \mathrm{O}$ ).

Thin films on quartz substrates have been spin coated in the same conditions as the active layers of the devices to study the absorption and emission properties. Micrographs for two of these layers at different zoom levels are shown in Figure 153 for illustrative purposes.
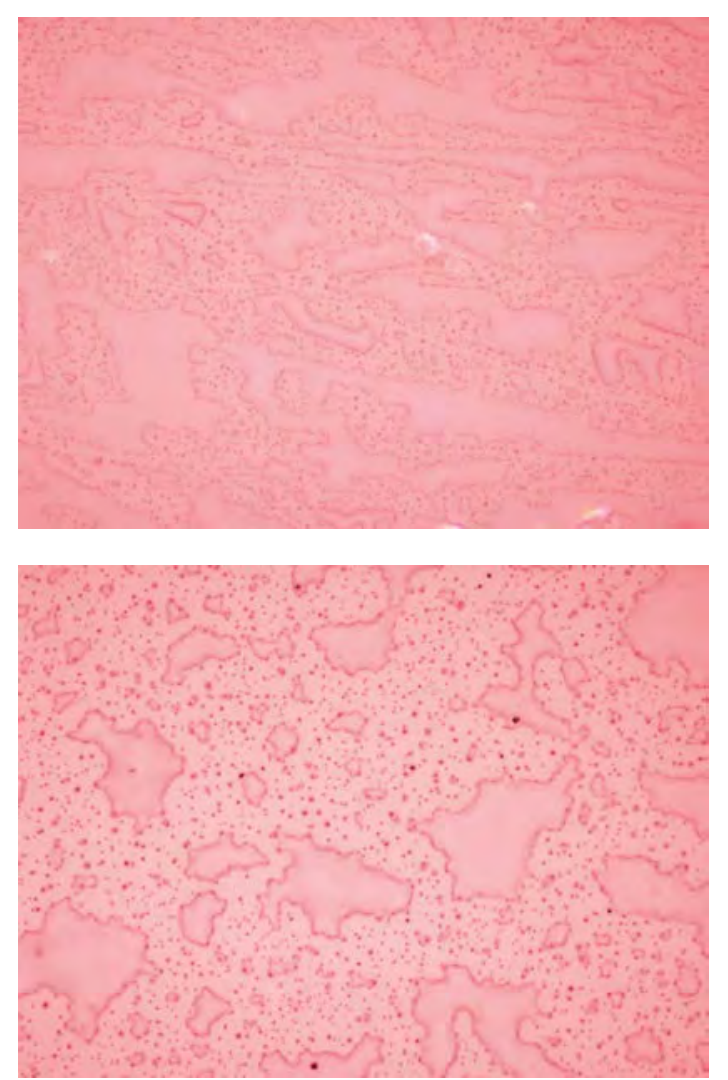
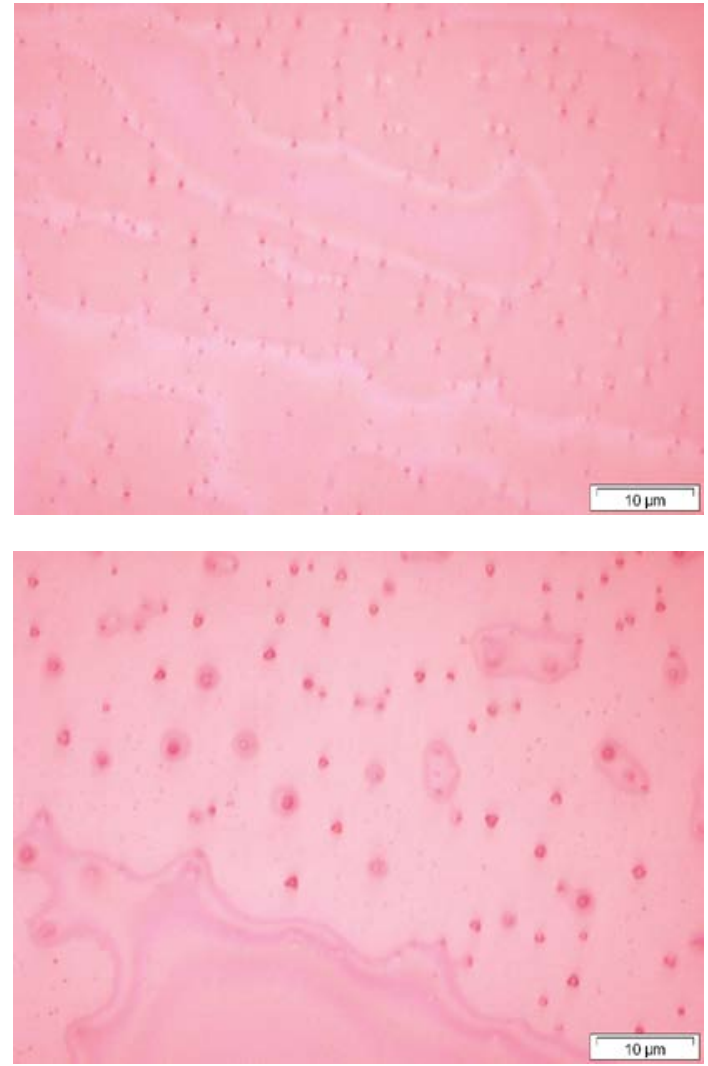

Figure 153. [Er(fod) $)_{3}$ (bath)] $\times 20(365 \mu \mathrm{m} \times 275 \mu \mathrm{m}$, top left $),\left[\operatorname{Er}(\text { fod })_{3}\right.$ (bath)] $\times 100(73 \mu \mathrm{m} \times 55 \mu \mathrm{m}$, top right $),\left[\operatorname{Er}(\text { fod })_{3}\left(5 \mathrm{NO}_{2}\right.\right.$ phen $\left.)\right] \times 20(365 \mu \mathrm{m} \times 275 \mu \mathrm{m}$, bottom left $)$ and $\left[\operatorname{Er}(\text { fod })_{3}\left(5 \mathrm{NO}_{2}\right.\right.$ phen $\left.)\right] \times 100$ $(73 \mu \mathrm{m} \times 55 \mu \mathrm{m}$, bottom right).

\section{Alternative patterning technique}

Instead of using a shadow mask for defining the cathode, the devices can be manufactured avoiding any photolithographic step by using electrical arc erosion () to pattern the successive electrodes of the devices [262], paving the way to the manufacturing of the NIROLEDs in large area format. In this case, the $\mathrm{Ca} / \mathrm{Al}$ cathode is evaporated on the whole sample surface, and the substrate is then re-aligned in the arc erosion apparatus. The 
adequate grooves for the cathode rows conformation are subsequently eroded with the aid of custom-made software.

The setup for micro and nanolithography based on electric arc consists of a XYZ Micropositioner based on PLS-85 PiMICOS linear tables with three-directional repeatability of $1 \mu \mathrm{m}$ and accuracy of $0.1 \mu \mathrm{m}$, for large scale working on a $10 \times 10 \mathrm{~cm}^{2}$ area, a XY nanopositioner based on HERA PiMICOS piezo stage (small scale working, on $100 \times 100 \mu \mathrm{m}$ ) and a $\mathrm{Z}$ nanopositioner based on LISA PiMICOS piezo stage.
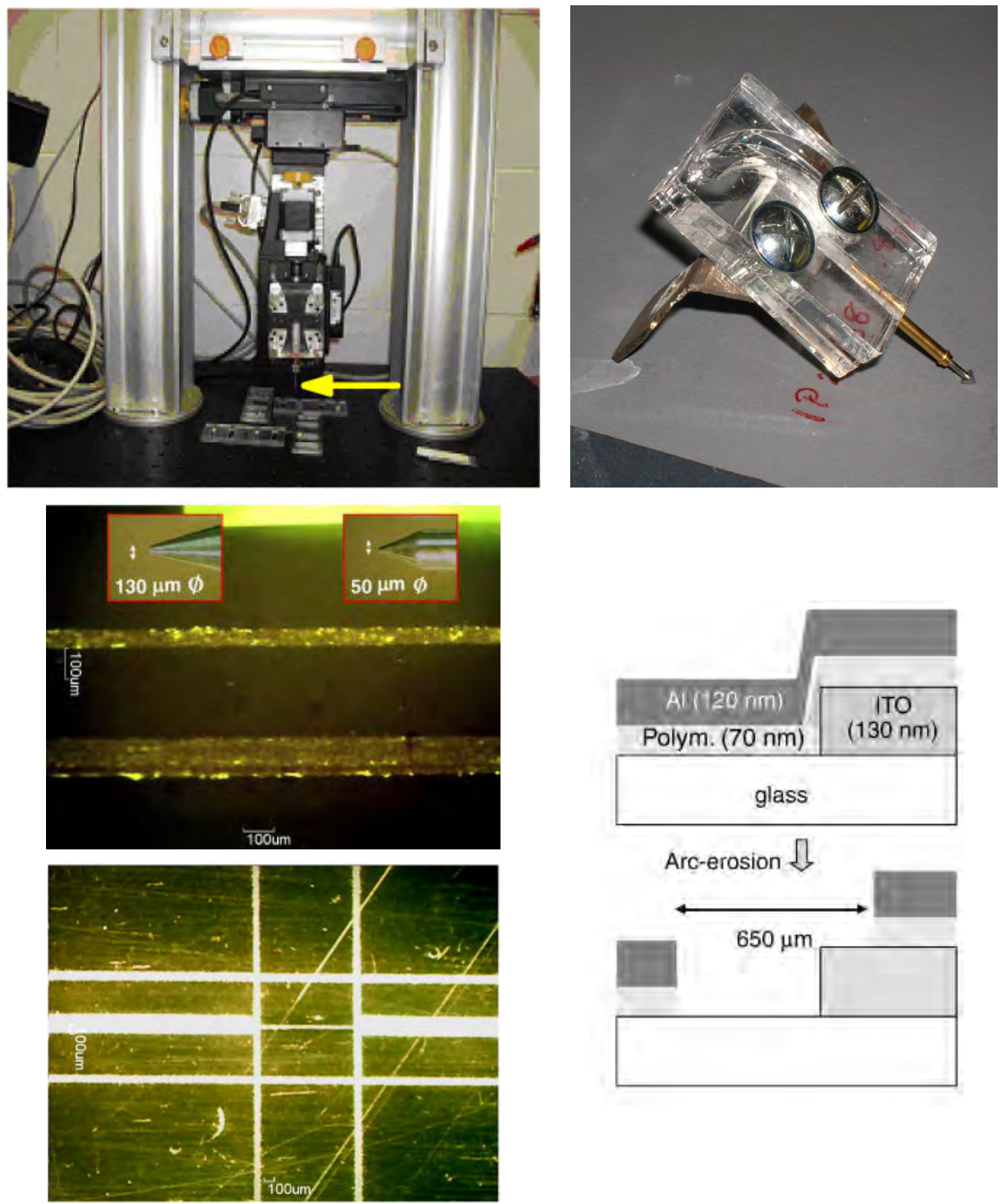


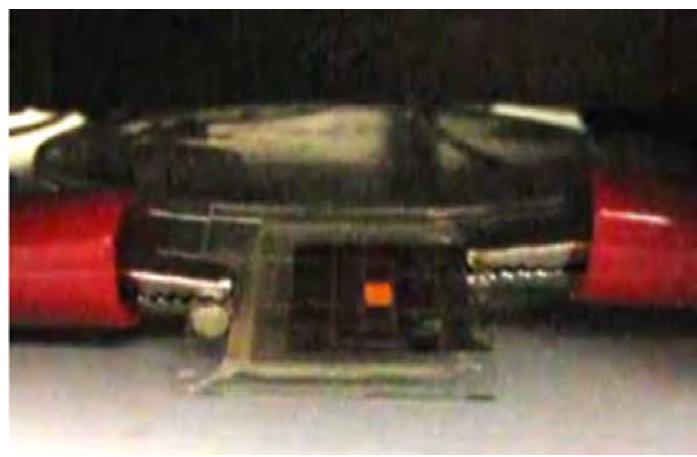

Figure 154. Details of the mask free electrodischarge patterning procedure: tip-assisted ablation equipment, closer view of the spring probe, optical micrograph of two stripes performed on ITO at 12 $\mathrm{V}$, with different tips of $50 \mu \mathrm{m}$ and $130 \mu \mathrm{m}$ (see insets) and grooves performed by arc erosion on a $\mathrm{Au} / \mathrm{Cr}$ film on PET using a tip $40 \mu \mathrm{m}$ diameter (different widths between 40 and $200 \mu \mathrm{m}$ were obtained by changing the continuous operating voltage), sketches of the layer structure of an $\mathrm{Al}(120$ $\mathrm{nm}) /$ Polymer $(70 \mathrm{~nm}$ ) bilayer deposited on top of a step of ITO (130 nm thick) on glass before and after arc erosion, example of a $3 \times 3$ display based on MDMO-PPV commercial red emitter.

\section{Electrical measurements}

After the encapsulation, the OLEDs are characterized in ambient conditions. The device current-voltage characteristics are measured using a semiconductor parameter analyzer Agilent HP4155C and a SMU pulse generator Agilent 41501B. A pulse train is used as the input signal. The duty cycle is set to be $0.2 \%$, thus having a pulse width of $0.5 \mathrm{~ms}$ for a period of $100 \mathrm{~ms}$. Refresh time between two consecutive pulses ensures long time operation without device degradation. Furthermore, the I-V curve stability is achieved by gradually increasing the pulse amplitude up to the point where reproducible measurements are observed. The voltage range is below the onset for electroluminescence (EL).

Following figures show in detail the current-voltage curves of some OLEDs manufactured using aforementioned procedure. The diodes exhibit a good electrical performance, with threshold voltages of a few volts (e.g. $5.5 \mathrm{~V}$ for $\left[\operatorname{Er}(\operatorname{tfnb})_{3}(\right.$ bipy)], Figure 155, and $7 \mathrm{~V}$ for $\left[\operatorname{Er}(\operatorname{tpm})_{3}\left(5 \mathrm{NO}_{2}\right.\right.$ phen)], Figure 156), similar to that reported by Wei et al. [99] for a multilayer vacuum-deposited device and further lower than that reported for the ErQ-based device [263]. Moreover, the very low noise level is indicative of the quality of the layer.

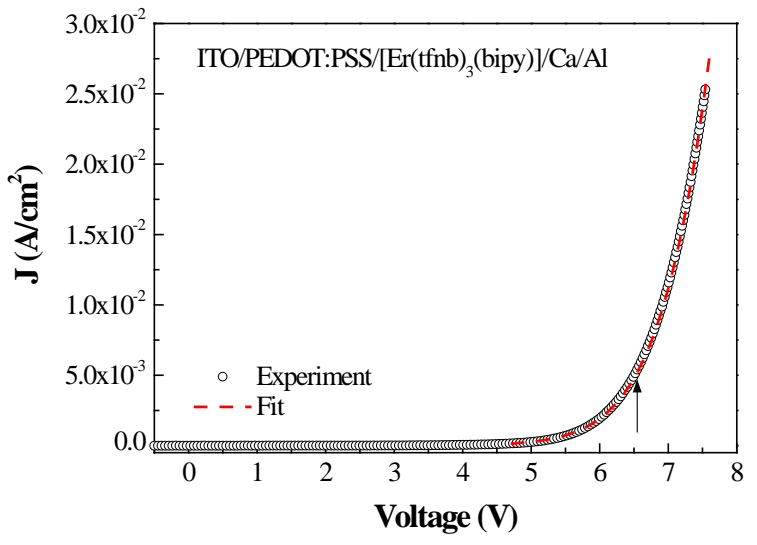

Figure 155. Recorded J-V curve (open symbols) of the $\left[\operatorname{Er}(\mathrm{tfnb})_{3}(\mathrm{bipy})\right]$ based OLED. The arrow marks the infrared EL onset. Solid line represents the fit achieved using the model explained in the text later on. 


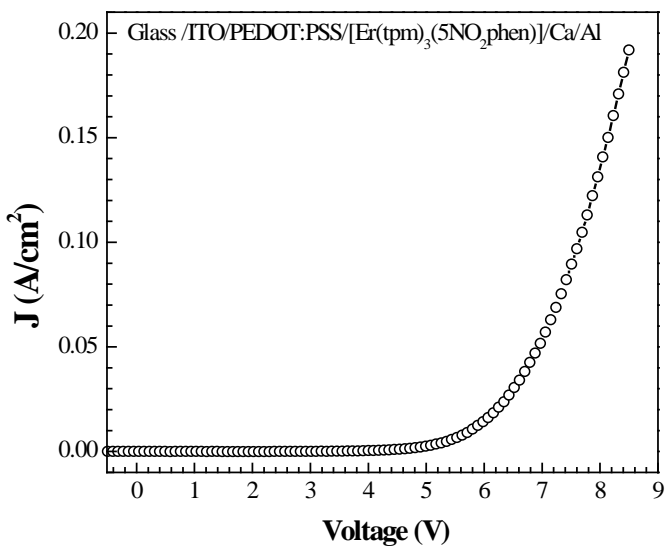

Figure 156. J-V response of the solution processed $\left[\operatorname{Er}(\operatorname{tpm})_{3}\left(5 \mathrm{NO}_{2}\right.\right.$ phen $\left.)\right]$ based OLED
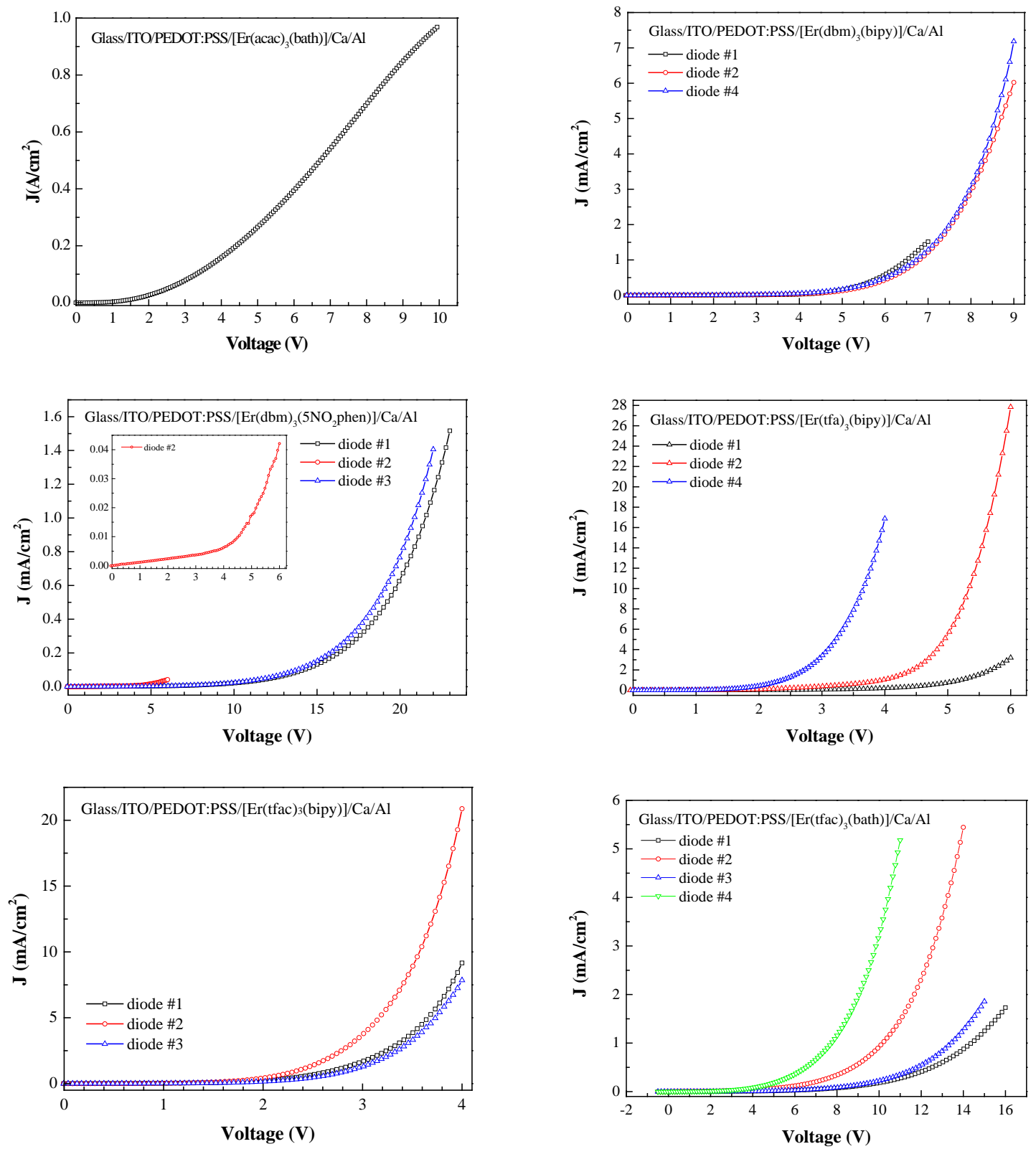

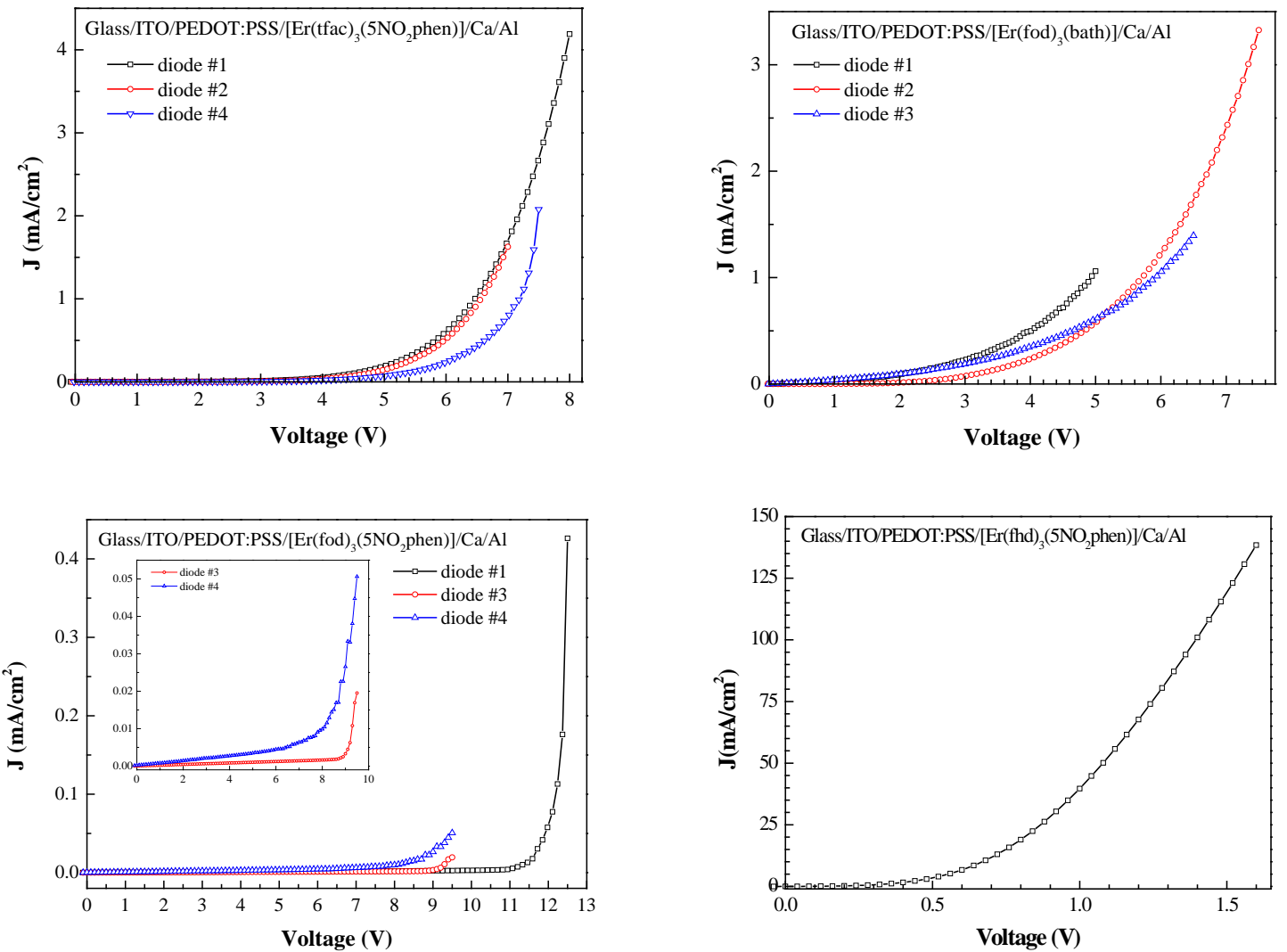

Figure 157. J-V curves of several of the devices with structure ITO/PEDOT:PSS/emission layer/Ca/Al, where the emission layer is either [Er(acac $)_{3}($ bath $\left.)\right],\left[\operatorname{Er}(\mathrm{dbm})_{3}(\right.$ bipy $\left.)\right],\left[\operatorname{Er}(\mathrm{dbm})_{3}\left(5 \mathrm{NO}_{2}\right.\right.$ phen $\left.)\right]$, $\left[\operatorname{Er}(\mathrm{tfa})_{3}(\mathrm{bipy})\right], \quad\left[\operatorname{Er}(\mathrm{tfac})_{3}(\mathrm{bipy})\right], \quad\left[\operatorname{Er}(\mathrm{tfac})_{3}(\mathrm{bath})\right], \quad\left[\operatorname{Er}(\mathrm{tfac})_{3}\left(5 \mathrm{NO}_{2}\right.\right.$ phen $\left.)\right], \quad\left[\operatorname{Er}(\mathrm{fod})_{3}(\mathrm{bath})\right]$, [Er(fod $)_{3}\left(5 \mathrm{NO}_{2}\right.$ phen $\left.)\right]$ or [ $\operatorname{Er}(\mathrm{fhd})_{3}\left(5 \mathrm{NO}_{2}\right.$ phen $\left.)\right]$. Diodes labeled as \#1 or \#3 have $r=1.5 \mathrm{~mm}$ and diodes labeled as \#2 or \#4 have $r=0.75 \mathrm{~mm}$. [ $\operatorname{Er}(\mathrm{acac})_{3}\left(\right.$ bath)] and $\left[\operatorname{Er}(\mathrm{fhd})_{3}\left(5 \mathrm{NO}_{2}\right.\right.$ phen)] devices were manufactured from $1 \%$ wt methanol solutions.

Regarding the influence of the cathode, Figure 158 shows the I-V response measured from OLEDs with identical layer structure, Glass/ITO(130 nm)/PEDOT:PSS (50 nm)/Er-complex $(15 \mathrm{~nm}) /$ cathode $(100 \mathrm{~nm})$, but using different cathode materials. In the $\mathrm{Ca} / \mathrm{Al}$ device, the low threshold voltage may be indeed attributed to the very thin active layer in the absence of barrier for electrons injection, as favored by the low Ca work function. When Ca is removed the effective offset between the Al Fermi level and the Er complex LUMO increases and electron injection is drastically reduced. In any case, the low noise I-V responses are also an indication of the good active layer quality obtained, despite their small thicknesses. 


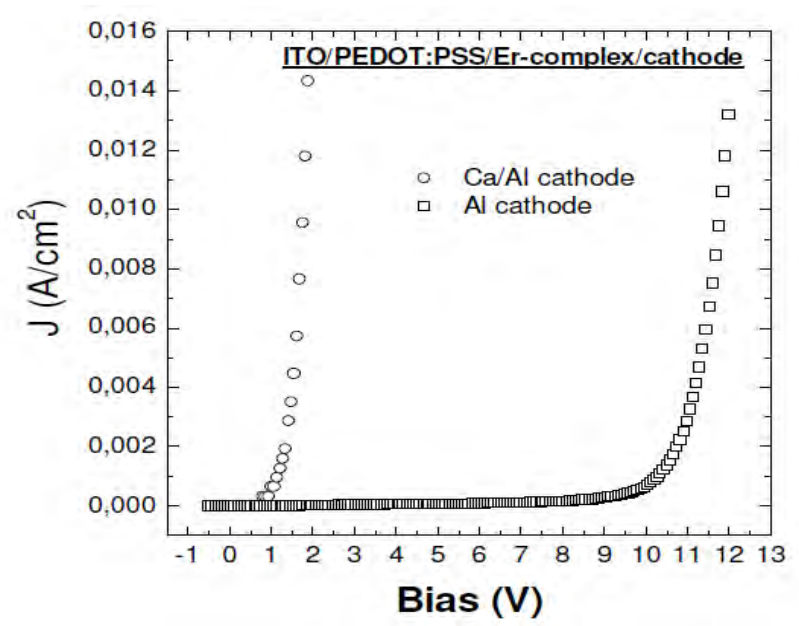

Figure 158. J-V response for two OLED with identical active layer but different cathode materials: $\mathrm{Ca} / \mathrm{Al}$ (open circles) and Al (open squares).

\section{EL characterization}

The infrared electroluminescence (EL) spectra have been analysed using a SPEX 1702/04 $(\mathrm{f}=1 \mathrm{~m})$ monochromator and detected with a $77 \mathrm{~K}$ cooled Ge detector connected to a Stanford Research system SR530 locking amplifier, using 50\% duty cycle electrical excitation waveform from a TTi TG4001 $40 \mathrm{MHz}$ arbitrary waveform generator and a TREK-601C amplifier.

NIR EL of the [Er(tfnb) $)_{3}$ (bipy)] OLED measured at $0.5 \mathrm{~mA}$ driving current $\left(5.3 \mathrm{~mA} / \mathrm{cm}^{2}\right)$ and $6.5 \mathrm{~V}$ is shown in . The emission is assigned to $\operatorname{Er}(\mathrm{III}):{ }^{4} \mathrm{I}_{13 / 2} \rightarrow{ }^{4} \mathrm{I}_{15 / 2}$ transition at $1.5 \mu \mathrm{m}$. This NIR emission can be attributed to the exciton-harvesting processes from the ligands, as no emission from the latter is observed. The EL spectrum matches very well with the photoluminescence spectra, except for a narrowing at the low energy wing of the band. This is most likely related to the increase in the material temperature and consequently to the redistribution of the electronic population in the excited ${ }^{4} \mathrm{I}_{13 / 2}$ multiplet.

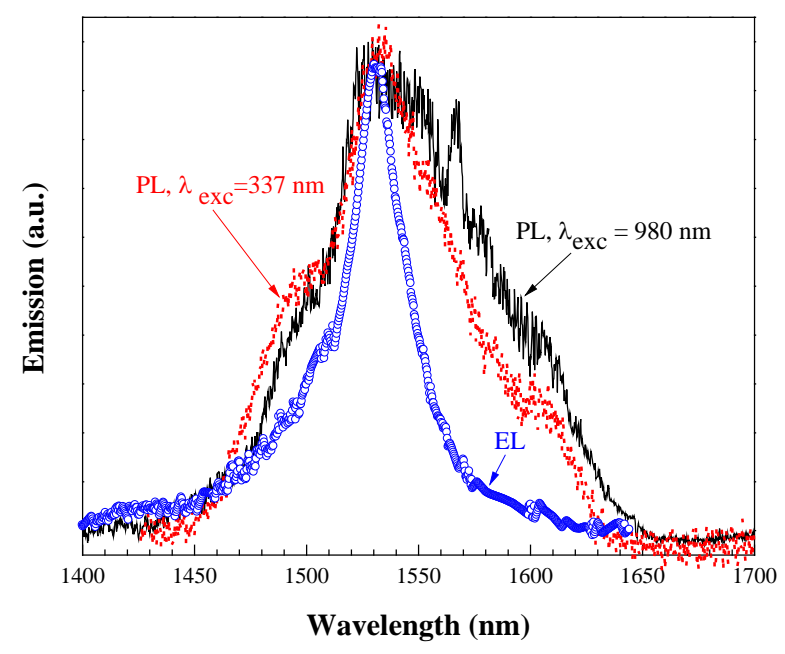

Figure 159. Electroluminescent (open blue squares) $1.5 \mu \mathrm{m}$ emission (Er(III): ${ }^{4} \mathrm{I}_{13 / 2} \rightarrow{ }^{4} \mathrm{I}_{15 / 2}$ transition) from the $\left[\operatorname{Er}(\mathrm{tfnb})_{3}(\right.$ bipy) $]$ solution processed OLED. PL of the complex in powder under ligand excitation $\left(\lambda_{\text {exc }}=337 \mathrm{~nm}\right.$, red dotted line $)$ and under $\operatorname{Er}(\mathrm{III}):{ }^{4} \mathrm{I}_{11 / 2}$ level excitation $\left(\lambda_{\mathrm{exc}}=980 \mathrm{~nm}\right.$, black solid line) are also shown for comparison purposes. 
The same applies to the $\left[\operatorname{Er}(\operatorname{tpm})_{3}\left(5 \mathrm{NO}_{2}\right.\right.$ phen $\left.)\right]$ NIR-OLED, whose EL emission is shown below.

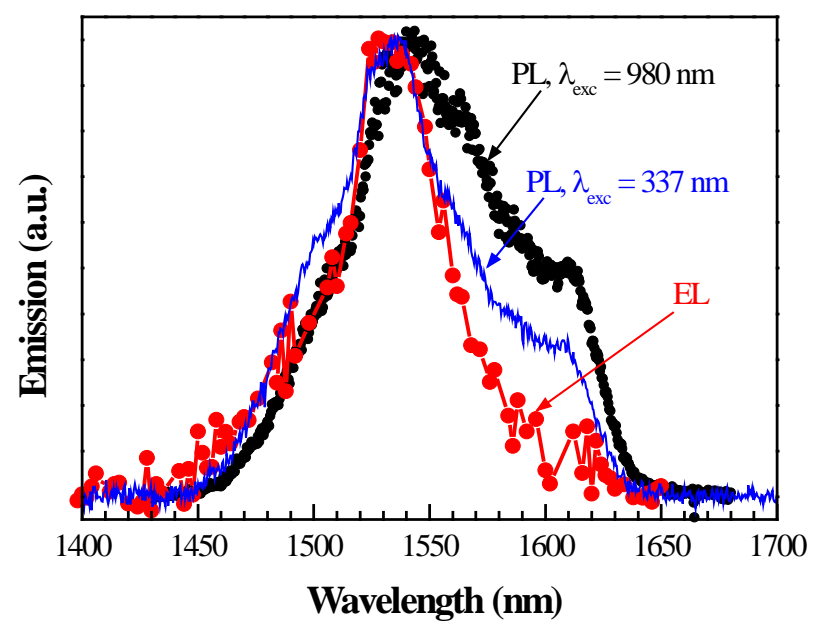

Figure 160. Electroluminescent emission $(r e d)$ for $\left[\operatorname{Er}(\operatorname{tpm})_{3}\left(5 \mathrm{NO}_{2}\right.\right.$ phen $\left.)\right]$. PL emissions excited at $337 \mathrm{~nm}$ (blue) and at $980 \mathrm{~nm}$ (black) are also shown for comparison purposes. All spectra have been normalized.

\section{Evaluation of the carrier mobilities}

As mentioned above, the ability to use of a pure $\left[\operatorname{Er}(\beta \text {-diketonate })_{3}(\mathrm{~N}, \mathrm{~N}\right.$-donor $\left.)\right]$ complex active layer depends on the transport properties of the material. Taking the [Er(tfnb $\left.)_{3}(b i p y)\right]$ device as an example, the current density-voltage $(\mathrm{J}-\mathrm{V})$ response has been studied using a numerical model which includes field dependent carrier mobility under a single carrier approach. This allows us to evaluate the carrier mobility dependence on the applied voltage in this novel complex, and to compare it to that of a commercial conjugated polymer with optical properties close to those of the molecular ligands.

Numerical modelling. A quantitative insight into the transport parameters of materials such as charge carrier mobility can be gained by the interpretation of the current density-voltage $\left(\mathrm{J}_{\mathrm{b}}-V_{\mathrm{b}}\right)$ response with a suitable model. Such a model must include structural characteristics of the device, such as layer thicknesses or active diode area, material parameters such as carrier mobility, $\mu(\mathrm{E})$, and characteristics of interfaces such as potential barriers for carrier injection from the electrodes. The model proposed resolves at the same time space charge effects, together with injection mechanisms and non-vanishing electric fields at the contact interfaces [264,265], so as to avoid making assumptions -as in previous models in the literature- on the conduction regime (injection limited or bulk limited) in order to extract parameters [266]. So as to achieve this goal we have used a simple formulation based on the fundamental equations for the drift current (1), the one-dimensional Poisson equation (2) and the integral expression of the applied external voltage (3) under single carrier approach:

$$
\begin{aligned}
& J_{b}=e \mu(E)\left[p_{n}\right] E(x)=e \mu_{0} \exp (k \sqrt{E(x)})\left[p_{n}\right] E(x) \\
& \frac{d E(x)}{d x}=\frac{e[p]}{\varepsilon \varepsilon_{0}} \\
& V_{b}=\int_{o}^{L} E(x) d x
\end{aligned}
$$

In (1) carrier mobility $\mu(E)$ has been approximated to the empirical field-dependent expression for transport in disordered materials at low carrier concentrations [267,268]: 


$$
\mu(E)=\mu_{0} \exp \left(\sqrt{E / E_{h}}\right)=\mu_{0} \exp (k \sqrt{E})
$$

where $E$ is the internal electric field, $\mu_{0}$ is the mobility at low fields below the critical value $E_{\mathrm{h}}$ from which the effective barrier reduction for trapped carriers becomes significant. $k=\left(E_{\mathrm{h}}\right)^{(-}$ $1 / 2)$ is a phenomenological factor related to the lowering of the effective depth of a trap, as predicted by Frenkel effect [269]. In above equations, $J_{\mathrm{b}}$ is the total current density in the bulk, $e$ is the elementary charge, $\varepsilon_{0}$ is the vacuum permittivity, $\varepsilon$ is the material dielectric constant, $L$ is the active layer thickness, $V_{\mathrm{b}}$ the applied external voltage, $[p]$ the total carrier density and finally $\left[p_{\mathrm{n}}\right]$ is the actual density of carriers contributing to transport. It is remarkable that in disordered materials, transport is mainly accomplished by hoping between localized states. This causes that not all the injected carriers contribute to transport but those with energy in the vicinity of a certain level, so-called transport level. Injected carriers below that level remain as virtually trapped. Thus, the fraction $\theta$ of conductive carriers verifies: $\left[p_{\mathrm{n}}\right]=\theta \times[p]$, where usually $\theta<1[270]$.

In this simple model, the energy relaxation associated to intra-molecular charge transfer, such as the transitions from the singlet LUMO level to a triplet level or Er levels, is to be considered a trapping process, whose effect is included into the $\theta$ factor. Since the determination of this parameter is difficult, from here onwards $\theta$ will be merged into the mobility term, and we shall use an effective mobility $\mu^{*}=\mu_{0} \times \theta$.

By integration of (2) using (1), one should obtain $E\left(\mathrm{x}, L, J_{\mathrm{b}}\right)$. In this case, it is more straightforward to derive the inverse function $\mathrm{x}\left(E_{0}, E_{\mathrm{L}}, J_{\mathrm{b}}\right)$, where the integration limits $E_{0}$ and $\mathrm{E}_{\mathrm{L}}$ are the value of the internal electric fields at both contacts: $E_{0}=E(\mathrm{x}=0), E_{\mathrm{L}}=E(\mathrm{x}=L)$. Further details of these calculations are given elsewhere [264,265]. A second integration of the electric field using (3), provides the electrical response of the diode $V_{\mathrm{b}}\left(E_{0}, E_{\mathrm{L}}, J_{\mathrm{b}}\right)$. The determination of the unknown value $E_{0}$ is carried out numerically via the continuity equation for the current density across the device:

$$
J_{i n j}\left(E_{0}\right)=J_{b}
$$

where $J_{\text {inj }}$ is the injection current according to a certain injection formulation of the injection process. Notice that carrier injection is generally dependent on the electric field at the interface $\left(E_{0}\right)$. In this work we have assumed the injection mechanism proposed by Arkhipov [271], in which carrier transitions from the metal Fermi level to the conduction level in the organic are not modelled by conventional tunnel effect between Bloch wavefunctions, but by incoherent carrier hops between localized states.

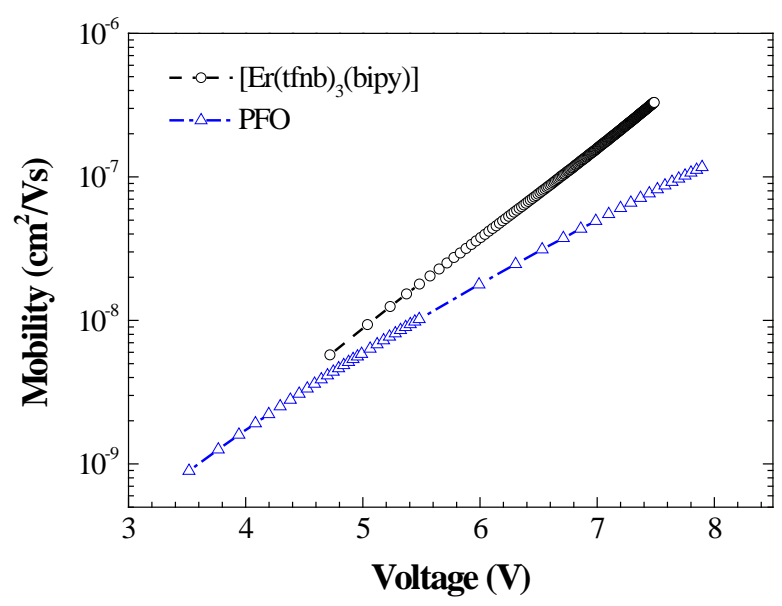

Figure 161. Carrier mobility-applied voltage relationship derived from the model for $\left[\operatorname{Er}(\mathrm{tfnb})_{3}(\mathrm{bipy})\right]$ based (dashed line) and PFO-based OLEDs (dot-dashed). 
The proposed model has been used to fit the experimental J-V curves using a quasi-Newton algorithm, considering the mobility factors, $\mu^{*}$ and $k$, together with an injection barrier $\Phi$ (in $\mathrm{eV}$ ), as the three numerical parameters. In Figure 155, the experimental J-V response (open symbols) of the OLED is shown up to currents slightly above the onset for the infrared EL. At such high currents the effect of the series resistance due to tracks and contacts must also be taken into account. The fit obtained from the conduction model (dashed line in Figure 155) corresponds to $\mu^{*}=8.8 \times 10^{-9} \mathrm{~cm}^{2} / \mathrm{V} \cdot \mathrm{s}$ and $k=2.52 \times 10^{-3}[\mathrm{~V} / \mathrm{m}]^{-1 / 2}$. The fit reproduces very well the experimental data, even beyond the EL onset (marked with an arrow). The additional conductivity term associated with exciton recombination beyond this limit is a physical mechanism not included in the model. In spite of that, the good fitting obtained beyond the EL onset suggests the conduction term associated with exciton recombination is a small fraction of the total current, as advanced above in view of the possible carrier unbalance.

Since our model allows us to determine the electric field $E(\mathrm{x})$ across the layer thickness, the average mobility function can be plotted versus applied voltage, and this is shown in Figure 161. In this figure the material mobility is compared to that of a commercial conjugated polymer, poly(9,9-dioctylfluorene) (PFO) obtained by an analogous procedure. PFO shows an absorption onset similar to that of the material under study, and is a well-known holeconductor. For that purpose an OLED with multilayer structure similar to the previous one (85 $\mathrm{nm}$ active layer thickness) has been manufactured, except for the absence of a Ca layer in the cathode electrode, aimed to promote hole-based conduction (as expected from the moderately high $\mathrm{Al}$ work-function at $-4.3 \mathrm{eV}$ ). In view of the literature results, our method tends to undervalue mobility with respect to experimental techniques like time of flight [272], probably due to its simplicity and the rough mobility expression used in (4), but allows a comparison between different materials. In our case, hole mobility in $\left[\operatorname{Er}(\mathrm{tfnb})_{3}\right.$ (bipy)] results comparable or even slightly higher than that of PFO. Since this molecule exhibits a clear ambipolar character due to the acceptor trifluoroalkyl ligands, electron mobility should also expected to be high. In this sense, a more balanced carrier injection, achievable by adding semiconductor layers for a better energetic level alignment, should result in a promising efficiency.

\section{Expectatives on the application of OLEDs for lighting in greenhouses}

Natural light is essential for plant growth [273,274], but in fact only a small amount of it is used for growth, which is called growth light. The majority of the sunlight is heat. When a supplemental, artificial light is used to enhance growth, its source is expected to produce growth light efficiently.

Since typical inorganic LEDs are small area sources that emit an incoherent narrowspectrum (with the color dependent upon the composition and condition of the semiconducting material used), in order to convert such point light sources into solid-state lighting (SSL) lamps able to produce a uniform and homogeneous light output, it is necessary to use optics which increase the cost, the weight and require more space for the lighting systems. In contrast, OLEDs represent a diffuse source of light and so they are naturally suited to large area general lighting required for growing plants.

Therefore, the aforementioned effiency requirement can be optimally accomplished with OLEDs that exploit the monomer and excimer emissions from single phosphor dopant emitters. These OLEDs must be effective in the simultaneous creation of blue and red emission bands essential for plant growth (Figure 162 and Figure 163). Thus, OLEDs can allow researchers and agriculturists to eliminate those wavelengths within normal white light that are inactive in photosynthesis, thus reducing the amount of energy required to power the plant growth lamps. They can be used in any application - hydroponics or soil, at home or in greenhouses. 

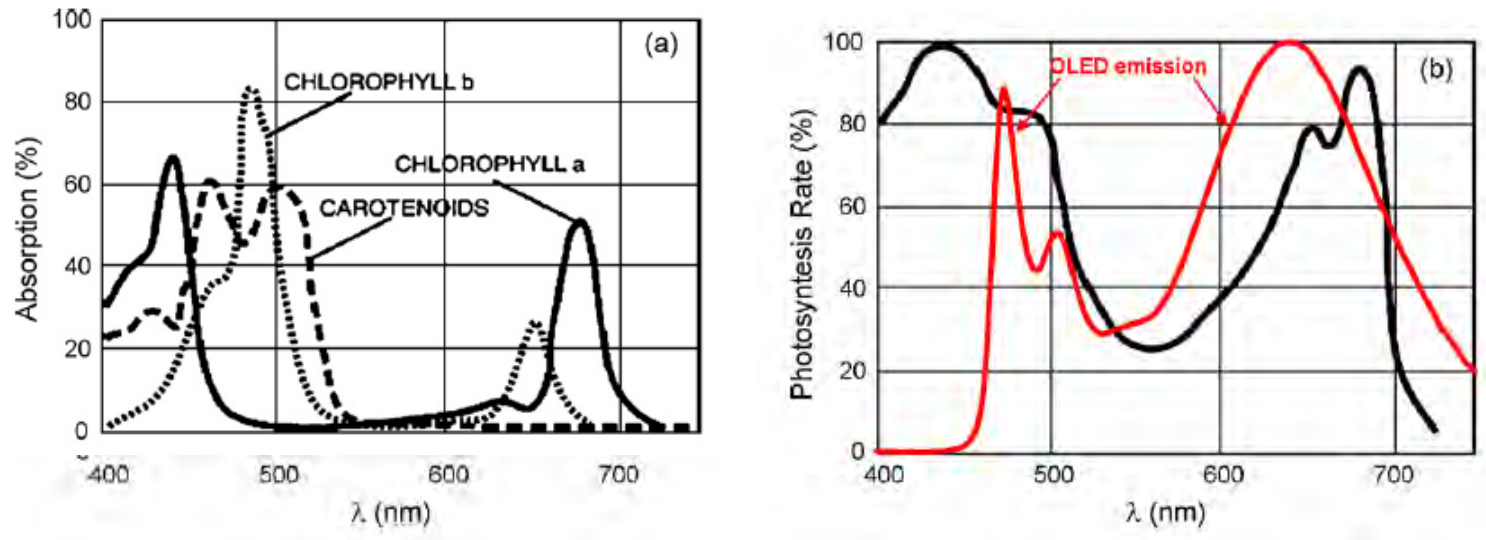

Figure 162. (a) Absorption spectra of chloroplast chlorophyll-a and -b, and carotenoids and action spectrum of photosynthesis; $(b)$ Spectral requirements of OLEDs for lighting in greenhouses. The rate of photosynthesis is related to the number of photons between 400 and $700 \mathrm{~nm}$ of the total incident light flux.
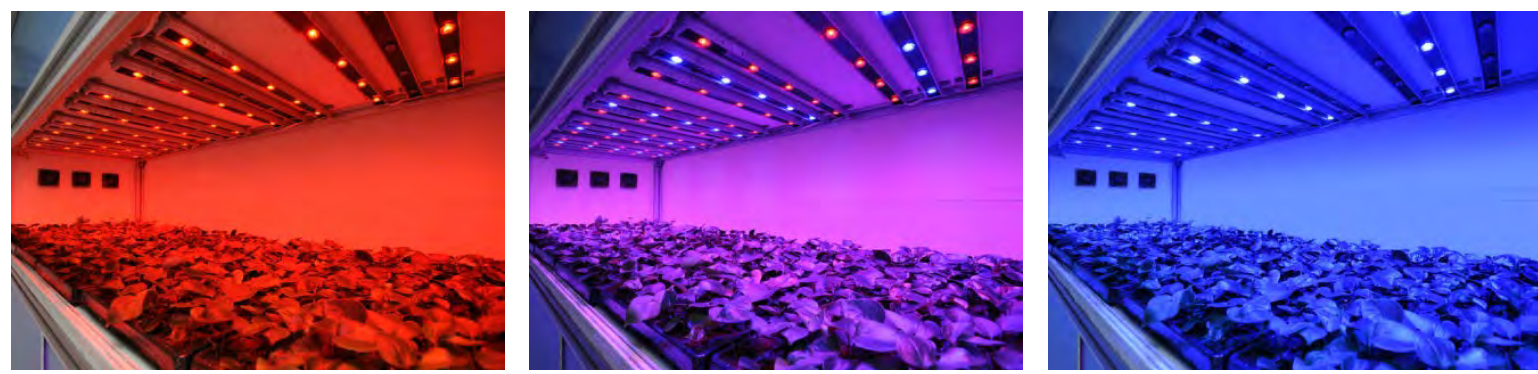

Figure 163. Plant lighting mimetic to than that photosynthesis with blue and red emission bands (Courtesy of Philips)

By varying the concentration of novel phosphorescent dopants selected from a series of newly synthesized platinum complexes [PtL $\left.{ }^{22-25} \mathrm{Cl}\right]$, Fattori et al. [275] manufactured bluebiased OLEDs and red-biased OLEDs (Figure 164) at a high luminance of $500 \mathrm{~cd} / \mathrm{m}^{2}$ and with external electroluminescence quantum efficiency of 15-18\% photon/electron (power efficiency 8-12 lm/W). 


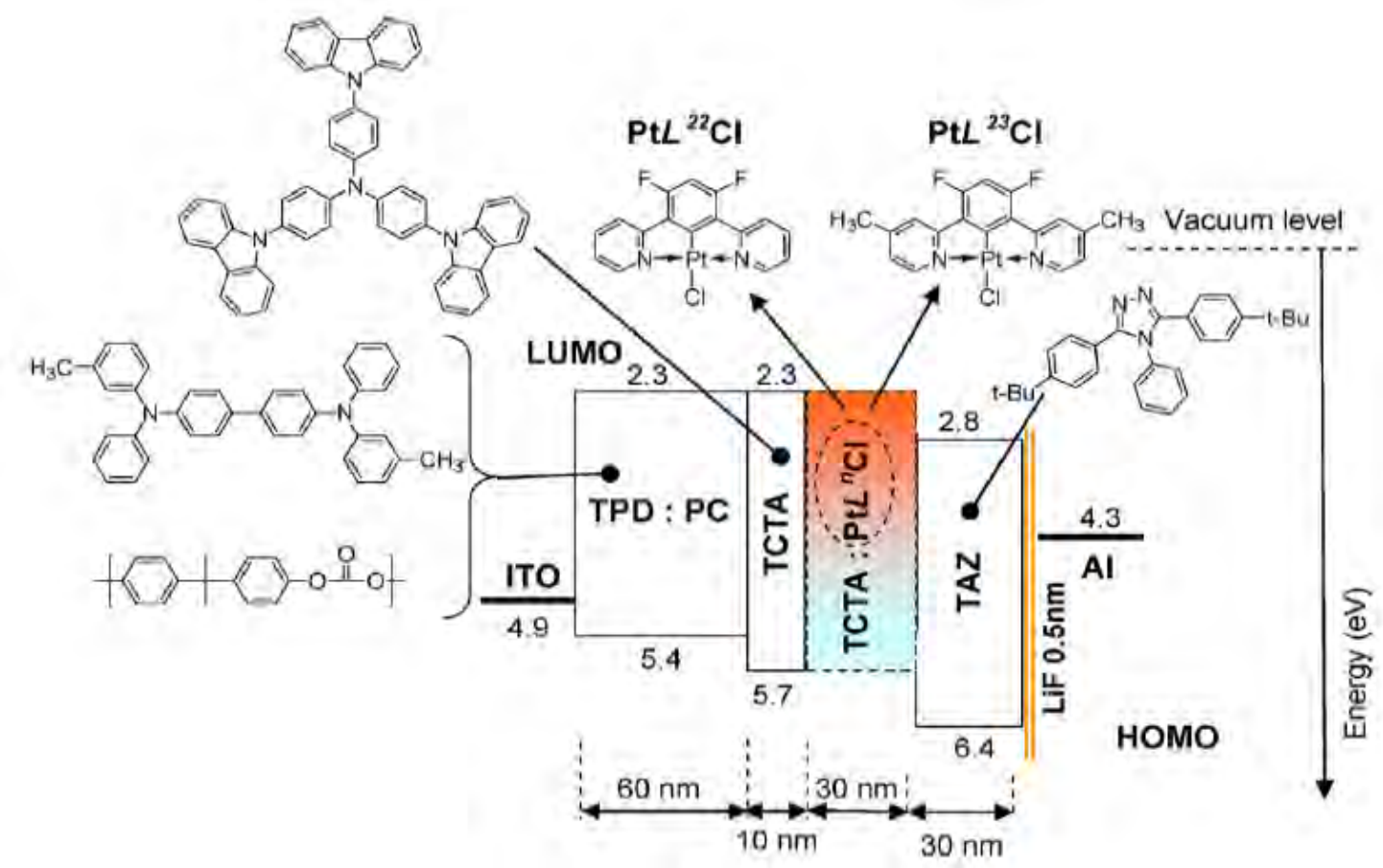

Figure 164. Structure of the OLEDs (and molecular structures of the materials used) manufactured by Fattori et al. [275] for lighting in greenhouses.

A feasible alternative to this structure, based on the novel Eu(III) dimers developed by Joao P. Martins et al. [276] (Figure 165, collaboration on visible OLEDs development with CEMDRX at Universade de Coimbra, Portugal) and on the Er(III) complexes developed in this Thesis, is shown in Figure 166. This solution would have a major advantage in comparison to the device by Fattori and coworkers: the Eu(III) dimers show improved emission in the red region, while the Er(III) complexes emit in the near infrared regions. This contribution is particularly important, since plants that bloom need infrared light wavelengths to produce flowers: plants grown indoors may grow well under fluorescent lights, but will not bloom unless appropriate levels of infrared radiation are introduced $[277,278]$. To date, this has been done either by using special horticultural lights (expensive) or simply by adding incandescent light bulbs (very inefficient), according to the Texas Agricultural Extension Service at Texas A\&M University. Thus, the proposed solution would facilitate the difficult task of achieving a correct infrared balance (extremely important for indoor gardening), while avoiding the problems associated to combining conventional incandescent and fluorescent bulbs.

\footnotetext{
s In addition, the chosen wavelength is not in the far red end of the spectrum, in which it has been shown that too much infrared light may actually damage plants, causing them to experience early growth spurts that reduce their health, or encouraging them to flower too soon.
} 

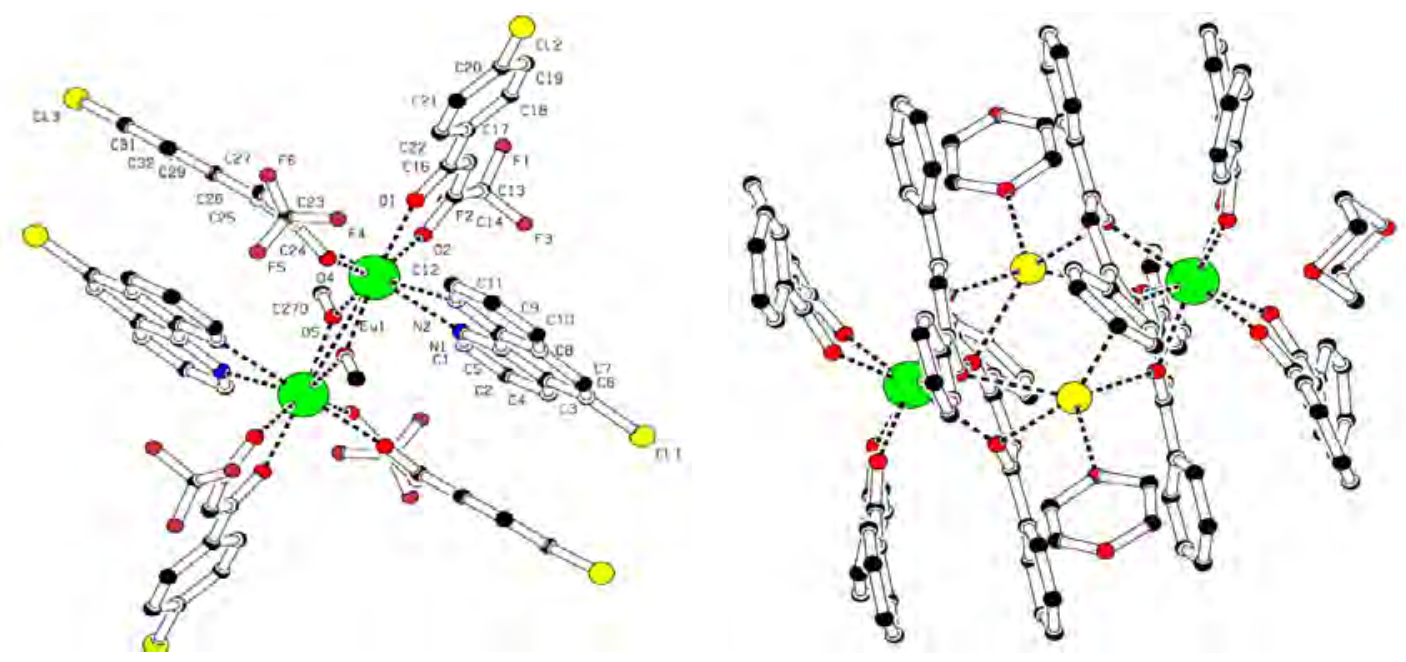

Figure 165. Examples of the novel Eu(III) dimers: tetra(1-(4-Chlorophenyl)-4,4,4-trifluoro-1,3butanedionate) di(5-chloro-1,10-phenanthroline) di-methanol di-Europium(III) (left) and 1,4-dioxane solvated octa(dibenzoylmethanate) di(1,4-dioxane) di-potassium di-Europium(III) (right) [276]

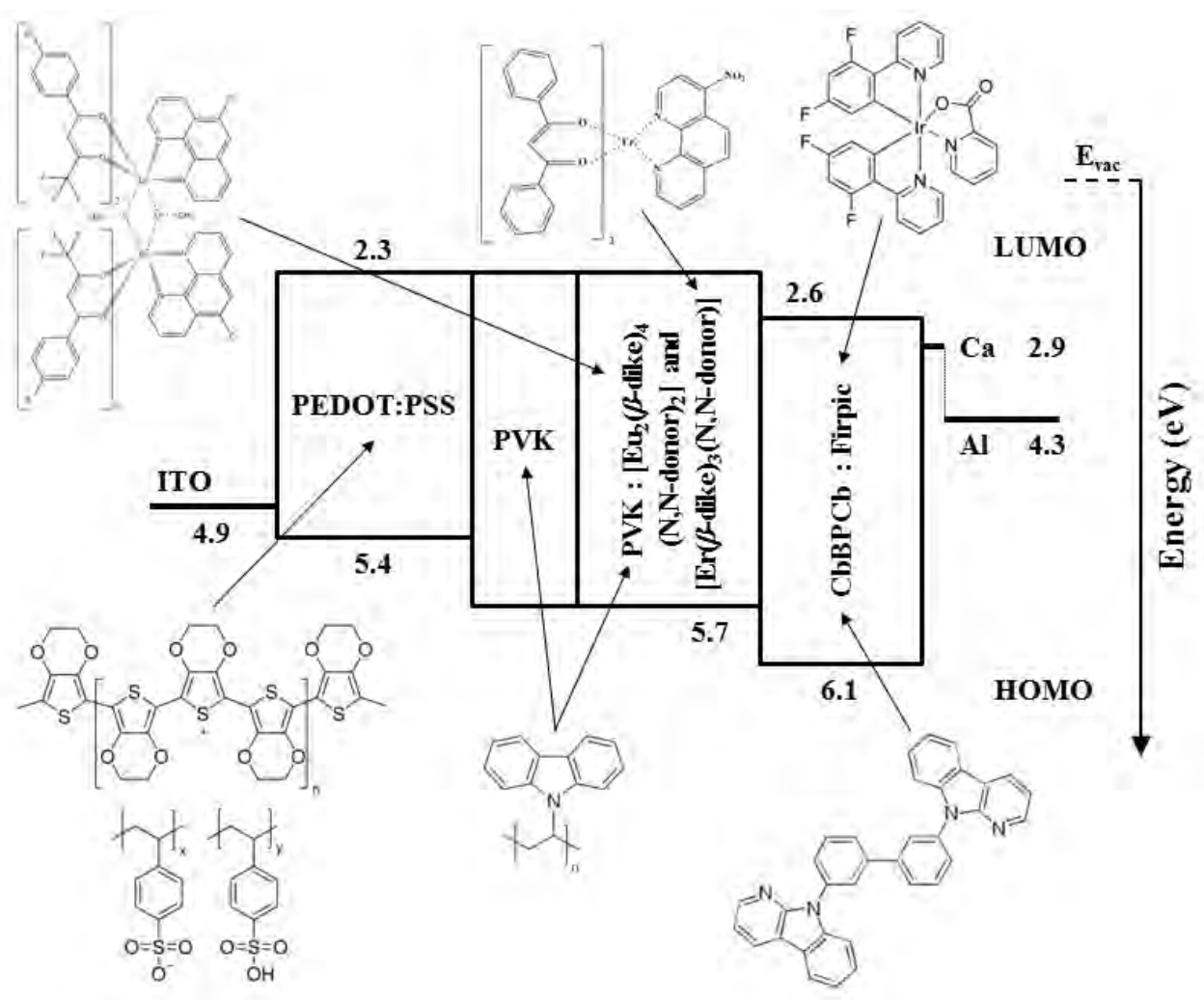

Figure 166. Alternative OLED structure, aimed at optimizing the photosynthesis process.

Because a high luminance is required for this application so as to achieve the desired photosynthetic photon flux (PPF), and since achieving both high-luminance and long-lifetime has always been a major challenge, the proposed devices could profit from Multi-Photon Emission (MPE) stacked device structure, originally developed by Lumiotec for general purpose OLED lighting, which has proven to attain both high luminance and long lifetime. In this method, multiple layers of emission units are laminated with charge generation layers interposed inbetween so that the current efficiency (luminance) is multiplied almost by the number of emission units (the structure and principle are shown in Figure 167). These 
charge generation layers constitute a stable carrier transport structure that produces a blue device (which is the one that has the lowest durability) with a lifetime more than five times longer than that of existing blue devices by maintain carrier balance of electrons and holes over a long period of time [279]. If, in addition, the OXD-7 host used by Fattori et al. is replaced with the novel host material recently developed at Dankook University (DKU) [280], as suggested in Figure 166, external quantum efficiencies for the blue layer of up to $30.1 \%$ efficiency can be achieved.

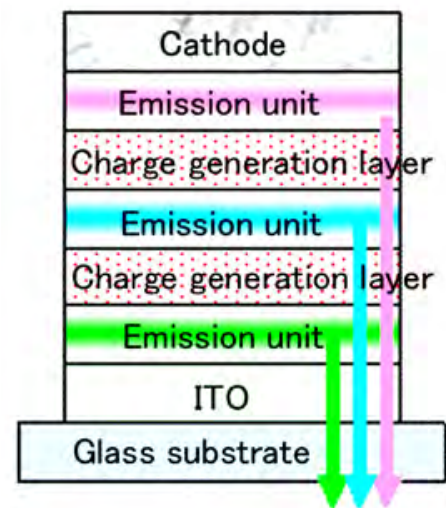

Multi-unit (MPE) type

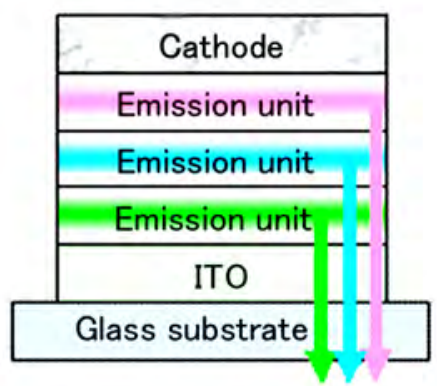

Multi-layer emission type

Figure 167. MPE $v s$. multilayer emission. In MPE, in the case of $n$ laminated units, the luminance is increased nearly $n$-fold; at a fixed luminance, the current is reduced to $1 / n$. The multi-layer emission type is capable of mixing colors with a simple structure but has problems delivering high luminance. 


\section{CONCLUSIONS}

From the results obtained throughout the present study on novel $\operatorname{Er}(\mathrm{III})$ and $\mathrm{Yb}(\mathrm{III})$-based materials for Optoelectronic and Telecommunication applications, the following conclusions have been reached:

1. The chosen synthetic strategy for the novel materials, based on $\beta$-diketonate ligands, has proven to be an excellent option from the sensitization point of view, leading to very efficient energy transfer to the central Ln(III) ion. Moreover, the other parameters governed by the chemical environment choice have also fulfilled the design requirements: the resulting highly coordinated complexes show good thermal stability, good processability and thin-film forming properties, and reasonably well-balanced carrier transport properties.

2. Regarding the particular $\beta$-diketonate choice, contrary to what had been claimed by other authors, longer $\beta$-diketonate chains are not advisable: on the contrary, the shorter the chain is, the higher the emission intensity is achieved. The inclusion of aromatic rings as substituents has also been shown to lead to a stronger emission, as a consequence of their stronger light absorption. Fluorination of the $\beta$-diketonate does not have a very significant impact on the emission intensities, but the associated decay times are systematically shorter. Therefore, it is only recommended for $\operatorname{Er}($ III) complexes, so as to avoid nonradiative losses.

3. With regard to the Lewis base choice, 2,2'-bipyridine can be considered as the preferred ancillary ligand for the assessment of a particular $\beta$-diketonate when novel synthesis is involved, provided that good yields and very crystalline materials are usually obtained, combined with reasonably-good luminescence properties. The bulkier bathophenanthroline $\mathrm{N}, \mathrm{N}$-donor moiety should be used when the emphasis is placed on the SMM/SIM behavior of the complexes, since the induced axial crystal fields promotes the uniaxial anisotropy of $\operatorname{Ln}(\mathrm{III})$ ions. 5-nitro-1,10-phenanthroline is to be chosen when the focus is placed on the luminescent properties and doping of hybrid matrices or NIROLED devices are envisaged.

4. Theoretical studies conducted by semi-empirical quantum methods, and in particular the recently available Sparkle/PM7, are an extremely useful tool for the design of novel Ln(III) complexes, since they are able to predict their ground state geometries and -in combination with INDO/S method and CIS- their electronic properties. These methods are not only substantially faster than $a b$ initio ones, but have also been shown to be very accurate, with unsigned mean errors below $1 \%$.

5. A thorough characterization of the 44 novel $\operatorname{Er}(\mathrm{III})$ and $\mathrm{Yb}(\mathrm{III})$ complexes by X-ray diffraction, DSC, FTIR, Raman, NMR and EPR spectroscopies, optical absorption, excitation and photoluminescence studies has been reported. This corpus constitutes an extremely valuable dataset for the design of highly coordinated Ln(III) complexes with improved luminescence efficiency in the future: experimental data based on well documented synthetic strategies and characterization, including structural data, is of crucial importance for the interpretation of the photophysical properties to achieve the demanded understanding of the structure/property relationship.

6. The novel materials have been assayed as dopants in hybrid films, in a preliminary effort for the design and elaboration of ormosil- or polymer-based materials suitable for passive (planar and channel waveguides, couplers and multimode interference splitters) and active (lasers and optical amplifiers) optical architectures. These photofunctional lanthanide hybrid materials open up new possibilities to produce a new generation of low-cost optical amplifiers in the centimeter-length scale, featuring superior optical and 
mechanical figures of merit, and suitable for the fabrication of photonic integrated circuits.

7. The materials have also been successfully used as an active layer for solution-processed OLEDs in the $1.54 \mu \mathrm{m}$ NIR band. An optimized fabrication method has been developed for the manufacturing these devices, which differs from those reported in the literature in the fact that the emissive layer consists only of the Er(III) complex, instead of a host:guest system based on a polymeric matrix. In addition, the arc-erosion patterning technique developed at URJC avoids any photolithographic or mask step, paving the way to the manufacturing of large area NIR-OLEDs by cost-effective methods.

8. The initial studies on the non-linear properties of the $\mathrm{Yb}(\mathrm{III})$ complexes based on Vildagliptin ligand show the enhancement of the $n_{2}$ in the complexes when compared to that of Vildagliptin, which is already significantly better than that of $\mathrm{CS}_{2}$. This result has been confirmed by extensive numerical quantum chemical computations and the extension of this result to the three telecomm windows.

9. Preliminary studies of the magnetic properties of some of the materials indicate that the complexes show a SIM behavior with high effective barriers, owing to the large magnetic moments and strong anisotropy of lanthanides, which arises from their large unquenched magnetic moments. AC susceptibility measurements reveal the existence of either one or two thermally activated magnetic relaxation processes, depending on the complex. The considerable potential of these materials to be developed for surface deposition and device applications opens up a popular avenue to nanoscale electronic devices, sensors and high-density data storage media at the molecular level. 


\section{REFERENCES}

[1] Weissman, SI. J. Chem. Phys., 1942, 10, 214.

[2] Lehn, JM. Angew. Chem. Int. Ed. Engl., 1990, 29, 1304.

[3] Sabbatini, N; Guardigli, M; Lehn, JM. Coord. Chem. Rev., 1993, 123, 201.

[4] Sa, GF; Malta, OL; Mello Donega, C; Simas, AM; Longo, RL; Santa-Cruz, PA; Silva EF, Coord. Chem. Rev., 2000, 196, 165.

[5] Kido, J; Okamoto, Y. Chem. Rev., 2002, 102, 2357.

[6] Crosby, GA; Whan, RE; Alire, RM. J. Chem. Phys., 1961, 34, 743.

[7] Bhaumik, ML; El-Sayed, MA. J. Phys. Chem.,1965, 69, 275.

[8] Van der Meer, BW; Coker, G; Chen, SYS. Resonance Energy Transfer Theory and Data, Wiley-VCH, New York, 1994.

[9] Dexter, DL. J. Chem. Phys., 1953, 21, 836.

[10] Forster, T. Discuss. Faraday Soc., 1959, 27, 7; Forster, T. Annalen der Physik 2, 1948 55, english translation (1993).

[11] Lowe, MP; Parker, D. Inorg. Chim. Acta, 2001, 317, 163; Vogtle, F; Gorka, M; Vicinelli, V; Ceroni, P; Maestri, M; Balzani, V. Chem. Phys. Chem., 2001, 2, 769.

[12] Wang, H; Qian, G; Wang, M; Zhang, J; Luo, Y. J. Phys. Chem. B, 2004, 108, 8084, and references therein; Tanner, PA; Duan, CK. Coord. Chem. Rev., 2010, 254, 3026, and references therein; Eliseeva, SV; Bunzli, JCG. Chem. Soc. Rev., 2010, 39, 189.

[13] Binnemans, K. Rare-Earth Beta-Diketonates. In Handbook on the Physics and Chemistry of Rare Earths; Gschneidner, KA; Bünzli, JCG; Pecharsky, VK (Eds.); Elsevier: Amsterdam, 2005; Volume 35, Chapter 225, p. 107.

[14] Kleinerman, M. Bull. Am. Phys. Soc. 1964, 9, 265.

[15] Archer, RD; Chen, HY; Thompson, LC. Inorg. Chem., 1998, 37, 2089; Gutierrez, F; Tedeschi, C; Maron, L; Daudey, JP; Poteau, R; Azema, J; Tisnes, P; Picard, C. Dalton Trans., 2004, 91334.

[16] Latva, M; Takalo, H; Mukkala, VM; Matachescu, C; Rodriguez-Ubis, JC; Kankare, J. J. Lumin., 1997, $75,149$.

[17] Lower, K; El-Sayed, MA. Chem. Rev., 1966, 66, 199.

[18] Bunzli, JCG; Chauvin, AS; Kim, HK; Deiters, E; Eliseeva, SV. Coord. Chem. Rev., 2010, 254, 2623.

[19] Görller-Walrand, C; Binnemans, K. Spectral intensities of $f$-f transitions. In Handbook on the Physics and Chemistry of Rare Earths; Gschneidner, KA; Eyring, L (Eds.); Elsevier: Amsterdam, 1998; Volume 25, Chapter 167, p. 101.

[20] Judd, BR. Phys. Rev., 1962, 127, 750.

[21] Ofelt, GS. J. Chem. Phys., 1962, 37, 511.

[22] Axe, JD. J. Chem. Phys., 1963, 39, 1154.

[23] Dieke, GH. Spectra and Energy Levels of Rare Earth Ions in Crystals; Interscience Publishers: New York, 1968.

[24] Wybourne, BG. Spectroscopic Properties of the Rare Earths; Wiley: New York, 1965.

[25] Judd, BR. Operator Techniques in Atomic Spectroscopy; Princeton University Press: Princeton, NJ, 1988.

[26] Hüfner, S. Optical Spectra of Transparent Rare Earths Compounds; Academic Press: New York, 1978.

[27] Smentek, L.; Wybourne, B. G. Optical Spectroscopy of Lanthanides: Magnetic and Hyperfine Interactions; CRC Press: Boca Raton, FL, 2007.

[28] Newman, DG; Ng, B. Crystal Field Handbook; Cambridge University Press: Cambridge, U.K., 2000.

[29] Armelao, L; Quici, S; Barigelletti, F; Accorsi, G; Bottaro, G; Cavazzini, M; Tondello, E. Coordination Chemistry Reviews, 2010, 254, 487.

[30] Liang, C; Li, W; Hong, Z; Liu, X; Peng, J; Liu, L; Liu, Z; Xie, M; Yu, J; Zhao, D. Synth. Met., 1997, 91, 151.

[31] Uekawa, M; Miyamoto, Y; Ikeda, H; Kaifu, K; Nakaya, T. Bull. Chem. Soc. Jpn., 1998, 71, 2253. 
[32] Heil, H; Steiger, J; Schmechel, R; Seggern, H. J. Appl. Phys., 2001, 90, 5357.

[33] Monguzzi, A; Tubino, R; Meinardi, F; et al. Chem. Mater. 2009, 21, 128.

[34] Pietrantoni, S; Pizzoferrato, R; Francini, R; et al. Phys. Stat. Sol. (c), 2007, 4(3), 1048.

[35] Pizzoferrato, R; Francini, R; Pietrantoni, S; et al. J. Phys. Chem. A, 2010, 114, 4163.

[36] Van Deun, R; Fias, P; Nockemann, P; et al. Inorg. Chem., 2004, 43, 8461.

[37] Van Deun, R; Fias, P; Driesen, K; Binnemans, K; Görller-Walrand, C. Phys. Chem. Chem. Phys., 2003, 5, 2754.

[38] Bassett, AP; Van Deun, R; Nockemann, P; et al. Inorg. Chem., 2005, 44, 2754.

[39] (a) Hasegawa, Y.; Murakoshi, K.; Wada, Y.; Yanagida, S.; Kim, J.-H.; Nakashima, N.; Yamanaka, T. Chem. Phys. Lett. 1996, 248, 8; (b) Hasegawa, Y.; Murakoshi, K.; Wada, Y.; Kim, J.-H.; Nakashima, N.; Yamanaka, T.; Yanagida, s. Chem. Phys. Lett. 1996, 260, 173; (c) Hasegawa, Y.; Kimura, Y.; Murakoshi, K.; Wada, Y.; Kim, J.-H.; Nakashima, N.; Yamanaka, T.; Yanagida, S. J. Phys. Chem. 1996, 100, 10201; (d) Iwamuro, M.; Hasegawa, Y.; Wada, Y.; Murakoshi, K.; Kitamura, T.; Nakashima, N.; Yamanaka, T.; Yanagida, S. Chem. Lett. 1997, 1067; (e) Hasegawa, Y.; Iwamuro, M.; Murakoshi, K.; Wada, Y.; Arakawa, R.; Yamanaka, T.; Nakashima, N.; Yanagida, S. Bull. Chem. Soc. Jpn. 1998, 71, 2573; (f) Iwamuro, M.; Wada, Y.; Kitamura, T.; Nakashima, N.; Yanagida, S. Phys. Chem. Chem. Phys. 2000, 2, 2291; (g) Yanagida, S.; Hasegawa, Y.; Wada, Y. J. Lumin. 2000, 87-89, 995; (h) Yanagida, S.; Hasegawa, Y.; Murakoshi, K.; Wada, Y.; Nakashima, N.; Yamanada, T. Coord. Chem. Rev. 1998, 171, 461

[40] Glover, P; Bassett, AP; Nockemann, P; Kariuki, B.M; Deun, RV; Pikramenou, Z. Chem. Eur. J., 2007, 13(22), 6308.

[41] Chen, B; Yang, Y; Zapata, F; Qian, G; Luo, Y; Zhang, J; Lobkovsky, EB. Inorganic Chemistry, 2006, 45(22), 8882.

[42] Moynihan, S; Van Deun, R; Binnemans, K; Redmond, G. Optical Materials, 2007, 29(12), 1821.

[43] Lehn, JM. Design of Organic Complexing Agents: Strategies Towards Properties, Springer, Berlin, Heidelberg, 1973.

[44] Brunet, E; Juanes, O; Rodriguez-Ubis, JC. Curr. Chem. Biol., 2007, 1, 11.

[45] Comby, S; Bünzli, JCG. Lanthanide Near-Infrared Luminescence in Molecular Probes and Devices. In Handbook on the Physics and Chemistry of Rare Earths; Gschneidner, KA; Bünzli, JCG; Pecharsky, VK (Eds.); Elsevier: Amsterdam, 2007; Volume 37, Chapter 235, p. 217.

[46] Yang, XD; Ci, YY; Chang, WB. Anal. Chem., 1994, 66, 2590; Yang, YS; Gong, ML; Li, YY; Lei, HY; Wu, SL. J. Alloys Compds., 1994, 207/208, 112.

[47] Zheng, YX; Liang, JL; Lin, Q; Yu, YN; Meng, QG; Zhou, YH; Wang, SB; Wang, HA; Zhang HJ. J. Mater. Chem., 2001, 11, 2615.

[48] Escribano, P; Julian-Lopez, B; Planelles-Arago, J; Cordoncillo, E; Viana, B; Sanchez, C. J. Mater. Chem., 2008, 18, 23.

[49] Carlos, LD; Ferreira, RAS; Zea Bermudez, V; Ribeiro, SJL. Adv. Mater., 2009, 21, 509.

[50] Binnemans, K. Chem. Rev., 2009, 109, 4283 and references therein.

[51] Tissue, BM. Chem. Mater. 1998, 10, 2837.

[52] Vetrone, F; Capobianco, JA. Int. J. Nanotechnol., 2008, 5, 1306.

[53] Dosev, D; Nichkova, M; Kennedy, IM. J. Nanosci. Nanotechnol., 2008, 8, 1052.

[54] Liu, GK; Chen, XY. Spectroscopic Properties of Lanthanides in Nano-materials. In Handbook on the Physics and Chemistry of Rare Earths; Gschneidner, KA; Bünzli, JCG; Pescharsky, VK (Eds.); Elsevier: Amsterdam, 2007; Volume 37, Chapter 233, p. 99.

[55] Shen, J; Sun, LD; Yan, CH. Dalton Trans., 2008, 42, 5687.

[56] Wang, F; Liu, XG. Chem. Soc. Rev., 2009, 38, 976.

[57] Blochwitz, J. Dissertation zur Erlangung des Doktorgrades der Naturwissenschaften. Technische Universität Dresden, 2001.

[58] Tang, CW; VanSlyke, SA. Appl. Phys. Lett., 1987, 51, 913.

[59] Guan, HS; Cheng, CH; Li, WC; Geng, DF; Fan ZQ; Chang, YC; Zhao, W; Guo, ZQ; Du, GT. Chem. Res. Chinese Universities, 2009, 25(6), 786.

[60] Hamberg, I; Granqvist, GC. J. Appl. Phys., 1986, 60, R123. 
[61] Digonnet, M (Ed.), Rare Earth Doped Fiber Lasers and Amplifiers, 2nd Ed., Marcel Dekker: New York, 2001.

[62] Paschotta, R. The Encyclopedia of Laser Physics and Technology. RP Photonics Consulting GmbH, 2010.

[63] Silversmith, AJ; et al., Appl. Phys. Lett., 1987, 51, 1977.

[64] Pollnau, M; Jackson, SD. IEEE J. Sel. Top. Quantum Electron., 2001, 7(1), 30.

[65] Pedersen, B; et al., IEEE Photon. Technol. Lett., 1991, 3, 1085.

[66] Giles, CR; Desurvire, E. J. Lightwave Technol., 1991, 9(2), 271.

[67] Desurvire, E. Erbium-Doped Fiber Amplifiers: Principles and Applications, John Wiley \& Sons: New York, 1994.

[68] Valley, GC. Opt. Fiber Technol., 2001, 7, 21.

[69] Zhu, X; Jain, R. Opt. Lett., 2008, 33(14), 1578.

[70] Jiang, C; et al., IEEE J. Quantum Electron., 2005, 41(5), 704.

[71] Konkanen, S; et al., Proc. SPIE, 1997, 32, 2996.

[72] Bünzli, JCG; Comby, S; Chauvin, AS; Vandevyver, CDB. Journal of Rare Earths, 2007, 25, 257.

[73] Catchpole, KR; Polman, A. Optic Express, 2008, 16(26), 21793.

[74] Green, MA. Third Generation Photovoltaics. Springer: Berlin, 2003.

[75] Swanson, R. Approaching the 29\% limit efficiency of silicon solar cells. In: 31th PVSC, Orlando, Florida, 2005.

[76] Trupke, T; Green, MA; Würfel, P, J. Appl. Phys., 2002, 92(3), 1668.

[77] Trupke, T; Green, MA; Würfel, P, J. Appl. Phys., 2002, 92(7), 4117.

[78] Shalav, A. Rare-earth doped up-converting phoshors for an enhanced silicon solar cell response. Dissertation Thesis, University of New South Wales, 2006.

[79] Auzel, F. Chem. Rev., 2004, 104, 139.

[80] Strümpel, C; McCann, M; Beaucarne, G; Arkhipov, V; Slaoui, A; Švrček, V; Cañizo, C; Tobias, I. Solar Energy Materials \& Solar Cells, 2007, 91, 238.

[81] van der Ende, BM; Aarts, L; Meijerink, A. Phys. Chem. Chem. Phys., 2009, 11, 11081.

[82] Wild, J; Meijerink, A; Rath, JK; van Sark, WG; Schropp, REI. Energy Environ. Sci., 2011, 4, 4835.

[83] Shalav, A; Richards, BS; Trupke, T; Krämer, KW; Güdel, HU; Green, MA. Conference on Photovoltaic Energy Conversion, Osaka, Japan, 2003.

[84] Hehlen, M.; Krämer, K; Güdel, H; McFarlane, R; Schwartz, R. Phys. Rev. B, 1994, 49(18), 12475.

[85] Lahoz, F; et al. Optics Letters, 2008, 33(24), 2982.

[86] Boyd, RW. Nonlinear Optics, $3^{\text {rd }}$ Ed., Academic Press, San Diego, California, 2008.

[87] Ogusu, K; Yamasaki, J; Maeda, S; Kitao, M; Minakata, M. Opt. Lett., 2004, 29, 265.

[88] Hasegawa, T; Nagashima, T; Sugimoto, N. Opt. Commun., 2005, 250, 411.

[89] Mishra, SR; Rawat, HS; Mehendale, SC; Rustagi, KC; Sood, AK; Bandyopadhayay, R; Govindaraj A; Rao, CNR. Chem. Phys. Lett., 2000, 317, 510.

[90] Scalora, M; Dowling, JP; Bowden CM; Bloemer, MJ. Phys. Rev. Lett., 1994, 73, 1368.

[91] Michinel, H; Salgueiro, JR; Paz-Alonso, MJ. Phys. Rev. E., 2004, 70, 066605.

[92] Michinel, H; Campo-Taboas, J; García-Fernández, R; Salgueiro, JR; Quiroga-Teixeiro, ML. Phys. Rev. E., 2002, 65, 06604.

[93] Nóvoa, D; Tommasini, D; Michinel, H. EPL, 2012, 98, 44003.

[94] Kivshar, YS; Agrawal, GP. Optical Solitons: from fibers to photonic crystals, Academic Press: San Diego, California, 2003.

[95] Valore, A; Cariati, E; Righetto, S; Roberto, D; Tessore, F; et al. JACS, 2010, 132(13):4966.

[96] Agrawal, GP. Applications of Nonlinear Fiber Optics, Academic Press: San Diego, Calfornia, 2001.

[97] Andraud, C; Maury, O. Eur. J. Inorg. Chem., 2009, 29/30, 4357.

[98] Curry, RJ; Gillin, WP. Applied Physics Letters. 1999, 75(10), 1380.

[99] Wei, F; Li, YZ; Ran, GZ; Qin, GG. Opt. Express, 2010, 18(13), 13542. 
[100] Leuthold, J; Freude, W; Brosi, JM; Baets, R; Dumon, P; Biaggio, I; Scimeca, ML; Diederich, F; Frank, B; Koos, C. Proceedings of the IEEE, 2008, 97(7), 1304.

[101] Fraile-Peláez, FJ; Chamorro-Posada, P; Gómez-Alcalá, R. J. Nonlinear Opt. Phys. Mater., 2012, 21, 1250030.

[102] Eliseeva, SV; Bunzli, JCG. New J. Chem., 2011, 35, 1165.

[103] Dei, A; Gatteschi, D. Angew. Chem. Int. Ed., 2011, 50, 11852.

[104] (a) Sessoli, R; Hui, L; Schake, AR; Wang, S; Vincent, JB; Folting, K; Gatteschi, D; Christou, G. J. Am. Chem. Soc., 1993, 115, 1804. (b) Sessoli, R; Gatteschi, D; Caneschi, A; Novak, MA. Nature 1993, $365,141$.

[105] Gatteschi, D; Sessoli, R; Villain, J. Molecular Nanomagnets; Oxford University Press: Oxford, 2006.

[106] (a) Rinehart, JD; Fang, M; Evans, WJ; Long, JR. Nat. Chem., 2011, 3, 538; (b) Bogani, L; Wernsdorfer, W. Nat. Mater., 2008, 7, 179; (c) Mannini, M; Pineider, F; Danieli, C; Totti, F; Sorace, L; Sainctavit, P; Arrio, MA; Otero, E; Joly, L; Cezar, JC; Cornia, A; Sessoli, R. Nature, 2010, $468,417$.

[107] (a) Leuenberger, MN; Loss, D. Nature, 2001, 410, 789. (b) Ardavan, A; Rival, 0; Morton, JJL; Blundell, SJ; Tyryshkin, AM; Timco, GA; Winpenny, REP. Phys. Rev. Lett., 2007, 98, 057201.

[108] Urdampilleta, M; Nguyen, NV; Cleuziou, JP; Klyatskaya, S; Ruben, M; Wernsdorfer, W. Int. J. Mol. Sci., 2011, 12, 6656.

[109] (a) Gatteschi, D; Sessoli, R. Angew. Chem. Int. Ed., 2003, 42, 268; (b) Thomas, L; Lionti, F; Ballou, R; Gatteschi, D; Sessoli, R; Barbara, B. Nature, 1996, 383, 145; (c) Wernsdorfer, W; Sessoli, R. Science, 1999, 284, 133; (d) Wernsdorfer, W; Aliaga-Alcalde, N; Hendrickson, DN; Christou, G. Nature, 2002, 416, 406.

[110] Nakano, M; Oshio, H. Chem. Soc. Rev., 2011, 40, 3239.

[111] (a) Sessoli, R; Powell, AK. Coord. Chem. Rev., 2009, 253, 2328; (b) Osa, S; Kido, T; Matsumoto, N; Re, N; Pochaba, A; Mrozinski, J. J. Am. Chem. Soc., 2004, 126, 420.

[112] (a) Rinehart, JD; Long, JR. Chem. Sci., 2011, 2, 2078; (b) Sorace, L; Benelli, C; Gatteschi, D. Chem. Soc. Rev., 2011, 40, 3092; (c) Guo, YN; Xu, GF; Guo, Y; Tang, J. Dalton Trans., 2011, 40, 9953; (d) Wang, BW; Jiang, SD; Wang, XT; Gao, S. Sci. China, Ser. B: Chem., 2009, 52, 1739. (e) Brooker, S; Kitchen, JA. Dalton Trans., 2009, 7331. (f) Habib, F; Murugesu, M. Chem. Soc. Rev., 2013, 42(8), 3278.

[113] Woodruff, DN; Winpenny, REP; Layfield, RA. Lanthanide Single-Molecule Magnets, Chem. Rev., 2013, Article ASAP. DOI: 10.1021/cr400018q

[114] (a) Mishra, A; Wernsdorfer, W; Abboud, KA; Christou, G. J. Am. Chem. Soc., 2004, 126, 15648. (b) Papatriantafyllopoulou, C; Wernsdorfer, W; Abboud, KA; Christou, G. Inorg. Chem., 2011, 50, 421. (c) Karotsis, G; Kennedy, S; Teat, SJ; Beavers, CM; Fowler, DA; Morales, JJ; Evangelisti, M; Dalgarno, SJ; Brechin, EK. J. Am. Chem. Soc., 2010, 131, 12983.

[115] Pople, JA; Santry, DP; Segal, GA. J. Chem. Phys., 1965, 43, 129.

[116] Pople, JA; Beveridge, DL; Dobosh, PA. J. Chem. Phys., 1967, 47, 2026.

[117] Dewar, MJS; Thiel, W. J. Am. Chem. Soc., 1977, 99, 4907.

[118] Dewar, MJS; Thiel, W. J Am Chem Soc., 1977, 99, 4899.

[119] Dewar, MJS; Zoebisch, EG; Healy, EF; Stewart, JJP. J. Am. Chem. Soc., 1985, 107, 3902.

[120] Stewart, JJP. J. Comp. Chem., 1989, 10, 209.

[121] Stewart, JJP. J. Comp. Chem., 1989, 10, 221.

[122] Stewart, JJP. J. Mol. Model., 2007, 13, 1173.

[123] Rocha, GB; Freire, RO; Simas, AM; Stewart, JJP. J. Comp. Chem., 2006, 27, 1101.

[124] Stewart, JJP. J. Mol. Model., 2013, 19, 1.

[125] NIST (2012) NIST Chemistry WebBook: Species with data from [Stewart, JJP. J. Mol. Model., 2004, 10(1), 6]. http://webbook.nist.gov/cgi/cbook.cgi?Source=2004STE6-10\&Units=SI\&Mask=1

[126] Allen, FH. Acta Cryst. Cambr. Crystallogr. Data Centre., 2007, B58, 380.

[127] de Andrade, AVM; da Costa, NB; Simas, AM; de Sá, GF. Chem. Phys. Lett., 1994, 227, 349.

[128] Rocha, GB; Freire, RO; da Costa, NB; de Sá, GF; Simas, AM. Inorg. Chem., 2004, 43, 2346. 
[129] Freire, RO; Rocha, GB; Simas, AM. Inorg. Chem., 2005, 44, 3299.

[130] (a) Freire, RO; da Costa, NB; Rocha, GB; Simas, AM. J. Organomet. Chem., 2005, 690, 4099; (b) Freire, RO; Rocha, GB; Simas, AM. Chem. Phys. Lett., 2005, 411, 61; (c) Freire, R0; Rocha, GB; Simas, AM. J. Comput. Chem., 2005, 26, 1524; (d) da Costa, NB; Freire, RO; Rocha, GB; Simas, AM. Polyhedron, 2005, 24, 3046; (e) da Costa, NB; Freire, RO; Rocha, GB; Simas, AM. Inorg. Chem. Commun., 2005, 8, 831; (f) Freire, RO; da Costa, NB; Rocha, GB; Simas, AM. J. Chem. Theory Comput., 2006, 2, 64; (g) Bastos, CC; Freire, RO; Rocha, GB; Simas, AM. J. Photochem. Photobiol. A, 2006, 117, 225; (h) Freire, RO; Monte, EV; Rocha, G B; Simas, AM. J. Organomet. Chem., 2006, 691, 2584; (i) Freire, RO; da Costa, NB; Rocha, GB; Simas, AM. J. Phys. Chem. A, 2006, 110, 5897.

[131] (a) Freire, RO; Rocha, GB; Simas, AM. Chem. Phys. Lett., 2006, 425, 138; Freire, R0; Rocha, GB; Simas, AM. Chem. Phys. Lett., 2007, 441, 354; da Costa, NB; Freire, RO; Rocha, GB; Simas, AM. J. Phys. Chem. A, 2007, 111, 5015; Simas, AM; Freire, RO; Rocha, GB. Lect. Notes Comput. Sci., 2007, 4488, 312; Freire, RO; da Costa, NB; Rocha, GB; Simas, AM. J. Chem. Theory Comput., 2007, 3, 1588; Simas, AM; Freire, RO; Rocha, GB. J. Organomet. Chem., 2008, 693, 1952; Freire, RO; Rocha, GB; Simas, AM. J. Braz. Chem. Soc., 2009, 20, 1638.

[132] Freire, RO; Simas, AM. J. Chem. Theory Comput., 2010, 6, 2019.

[133] Dutra, JDL; Filho, MAM; Freire, RO; Rocha, GB; Simas, AM; Stewart, JJP. J. Chem. Theory Comput., 2013, Just Accepted Manuscript. DOI: 10.1021/ct301012h

[134] Stewart, JJP. Stewart Computational Chemistry, MOPAC2012 v.13.037L. http://OpenMOPAC.net

[135] Scott, AP; Radom, L. J. Phys. Chem., 1996, 100(41) 16502.

[136] Fekete, ZA; Hoffmannz, EA; Koertvelyesi, T; Penke, B. Mol. Phys., 2007, 105, 2597.

[137] (a) Neese, F; Wennmohs F; et al. ORCA v.2.9. Max-Planck-Institute for Bioinorganic Chemistry, Mülheim an der Ruhr, Germany. http://cec.mpg.de/forum/; (b) Petrenko, T; Neese, F. J. Chem. Phys., 2007, 127, 164319.

[138] Ridley, J; Zerner, M. Theor. Chim. Acta, 1973, 32, 111.

[139] Zerner, MC; Loew, GH; Kirchner, RF; Mueller-Westerhoff, UT. J. Am. Chem. Soc., 1980, 102, 589.

[140] de Andrade, AVM; Longo, RL; Simas, AM; de Sá, GF. J. Chem. Soc. Faraday Trans., 1996, 92, 1835.

[141] de Oliveira, CAF; da Silva, FF; Malvestiti, I; Rodrigues, V; Malta, S; Dutra, JDL; da Costa, NB; Freire, RO; Júnior, SA. J. Solid State Chem., 2013, 197, 7.

[142] Sheldrick, GM. SADABS. University of Göttingen, Germany, 1996.

[143] Sheldrick, GM. SHELXL97 and SHELXS97. University of Göttingen, Germany, 1997.

[144] Spek, AL. J. Appl. Crystallogr., 2003, 36, 7.

[145] Sabbah, R; Xu-wu, A; Chickos, JS; Planas Leitão, ML; Roux, MV; Torres, LA. Thermochim. Acta, 1999, 331(2), 93.

[146] Della Gatta, G; Richardson, MJ; Sarge, SM; Stolen, S. Pure Appl. Chem., 2006, 78(7), 1455.

[147] Sheik-Bahae, M; Said, AA; Stryland, EWV. Opt. Lett., 1989, 14, 955.

[148] Yoshino, F; Polyakov, S; Liu, M; Stegeman, G. Conference on Lasers and Electro-Optics 2000 (CLEO 2000), 2000, p. 150.

[149] Chappie, PB; Staromlynska, J; McDuff, RG. J. Opt. Soc. Am. B, 1994, 11, 975.

[150] Yin, M; Li, H; Tang, S; Ji, W. Appl. Phys. B, 2000, 70, 587.

[151] Paz-Alonso, MJ; Michinel, H; Bará, S. J. Opt. Soc. Am. B, 2003, 20, 2484.

[152] Zhao, F; Wang, C; Zhang, J; Zeng, Y. Opt. Express, 2012, 20, 26845.

[153] Sheik-Bahae, M; Said, A; Wei, TH; Hagan, D; Van Stryland, E. IEEE J. Quantum Elect., 1990, 26, 760.

[154] Ganeev, RA; Ryasnyansky, AI; Baba, M; Suzuki, M; Ishizawa, N; Turu, M; Sakakibara, S; Kuroda, H. Appl. Phys. B, 2004, 78, 433.

[155] Young, RA. The Rietveld Method, University Press: Oxford, 1993; Volume 1.

[156] Rodriguez-Carvajal, J. Physica B, 1993, 192, 55.

[157] Spek, AL. Acta Cryst., 2009, D65, 148.

[158] Fukuda, Y; Nakao A; Hayashi, K. J. Chem. Soc., Dalton Trans., 2002, 527. 
[159] Orpen, AG; Brammer, L; Allen, FH; Kennard, O; Watson, DG; Taylor, R. J. Chem. Soc., Dalton Trans. 1989, 1.

[160] Stewart, JJP. J. Mol. Model., 2008, 14, 499.

[161] Neelgund, GM; Shivashankar, SA; Narasimhamurthy, T; Rathore, RS. Acta Cryst. C, 2007, 63(2), 74.

[162] Feng, J; Yu, JB; Song, SY; Sun, LN; et al., Dalton Trans, 2009, 2406.

[163] Sun LN; Yu, JB; Zheng, GL; Zhang, HJ; et al. Eur. J. Inorg. Chem., 2006, 3962.

[164] Van Staveren, DR; et al. Inorg. Chim. Acta, 2001, 315, 163.

[165] Song, L; Liu, X; Zhen, Z; Cheng, C; Zhang, D. J. Mater. Chem., 2007, 17, 4586.

[166] Bellusci, A; Barberio, G; Crispini, A; Ghedini, M; La Deda, M; Pucci, D. Inorg. Chem., 2005, 44, 1818.

[167] Kononenko, LI; Melenteva, EV; Vitkun, RA; Poluektov, NS. Ukr. Khim. Zh., 1965, 31, 1031.

[168] Dzyubenko, NG. Candidate Dissertation in Chemical Sciences, Mosk. Gos. Univ., Moscow, 1984.

[169] Friedel, B; Keivanidis, PE; Brenner, TJK; Abrusci, A; et al. Macromolecules, 2009, 42(17), 6741.

[170] van Deun, R. Liquid Crystals with Lanthanides: A Thermal and Optical Investigation. PhD Thesis, Katholieke Universiteit Leuven, 2001.

[171] Knyazev, AA; Galyametdinov, IG; Goderis, B; Driesen, K; Goossens, K; Görller-Walrand, C; Binnemans, K; Cardinaels, T. Eur. J. Inorg. Chem., 2008, 756.

[172] Sherman, A. Chemical Vapor Deposition for Microelectronics, Noyes Publications: Park Ridge, NJ, 1987.

[173] Nikitenko, SI; et al. Ultrason. Sonochem., 2000, 7(4), 177.

[174] Pearce, CK; Grosse, DW; Hessel, W. Chem Eng Data, 1970, 15, 567.

[175] Deacon, GB; Phillips, RI. Coord Chem Rev., 1980, 33, 227.

[176] Nakamoto K. Infrared and Raman spectra of inorganic and coordination compounds. Wiley: New York, 1997.

[177] Vila Nova, SP; Batista, HJ; Alves, S; Mello Donegá, C; Longo, RL; de Sa, GF; Thompson, LC. J. Lumin., 2006, 118, 83.

[178] Hasegawa, Y; Tsuruoka, S; Yoshida, T; Kawai, H; Kawai, T. J. Phys. Chem. A, 2008, 112, 803.

[179] Bian, LJ; Xi, HA; Qian, XF; Yin, J; Zhu, ZK; Lu, QH. Mater. Res. Bull., 2002, 37, 2293.

[180] Nunn, S; Nishikida, K. Advanced ATR Correction Algorithm - Application Note 50581, Thermo Fisher Scientific, Madison, WI, USA, 2008

[181] Ryo, M; Shoko, S; Susumo, S. Rare Earths, 1999, 34, 26.

[182] Yu, J; Zhang, H; Fu, L; Deng, R; Zhou, L; Li, H; Liu, F; Fu, H. Inorg. Chem. Commun, 2003, 6(7), 852.

[183] Swavey, S; Krause, JA; Collins, D; D'Cunha, D; Fratini, A. Polyhedron, 2008, 27, 1061.

[184] Guo, J; Fu, L; Li, H; Zheng, Y; Meng, Q; Wang, S; Liu, F; Wang, J; Zhang, H. Mater. Lett., 2003, 57, 3899.

[185] Richards, G; Osterwyk, J; Flikkema, J; Cobb, K; Sullivan, M; Swavey, S. Inorg. Chem. Commun., 2008, 11, 1385.

[186] Qian, D; Chen, Z; Zhang, S. Polyhedron, 1993, 12(23) 2763.

[187] Baker, MH; Dorweiler, JD; Ley, AN; Pike, RD; Berry, SM. Polyhedron, 2009, 28, 188.

[188] Lahoz, F; Martín IR; Mendez-Ramos, J; Nunez, P. J. Chem. Phys., 2004, 120, 6180.

[189] Souza, RF; Alencar, MA; Meneghetti, MR; Hickmann, JM. Opt. Mater., 2009, 31, 1591.

[190] Nalwa, HS; Miyata, S. Nonlinear Optics of Organic Molecules and Polymers, CRC Press: Boca Raton, Florida, 1997.

[191] Jiang, SD; Liu, SS; Zhou, LN; Wang, BW; Wang, ZM; Gao, S. Inorg. Chem., 2012, 51 (5), 3079.

[192] Domingo, N; Luis, F; Nakano, M; Munto, M; Gomez, J; Chaboy, J; Ventosa, N; Campo, J; Veciana, J; Ruiz-Molina, D. Phys. Rev. B, 2009, 79, 214404.

[193] Ferreira, RAS; André, PS; Carlos, LD. Opt. Mater., 2010, 32, 1397.

[194] Le Quang, RH; Zyss, J; Ledoux, I; et al. Appl. Phys. Lett., 2006, 89, 141124.

[195] Chen, C; Zhang, D; Li, T; Zhang, D; Song, L; Zhen, Z. Appl. Phys. Lett., 2009, 94, 041119. 
[196] Kobayashi, T; Hogan, R. Applied Physics Letters, 2010, 97, 143303.

[197] Song, L; Wang, Q; Tang, D; Liu, X; Zhen, Z. New J. Chem., 2007, 31, 506.

[198] Song, L; Wang, Q; Tang, D; Liu, X; Zhen, Z. J. Non-Cryst. Solids, 2008, 354(28), 3375.

[199] Zhong, Q; Wang, H; Qian, G; Wang, Z; Zhang, J; Qiu, J; Wang, M. Inorg. Chem., 2006, 45, 4537.

[200] van Staveren, DR; Haasnoot, JG; Lanfredi, AMM; et al. Inorganica Chimica Acta, 2000, 307, 81.

[201] Roh, SG; Nah, MK; Oh, JB; et al. Polyhedron, 2005, 24, 137.

[202] Hong-Yan, L; Jing, W; Wei, H; et al. J. Photochem. Photobiol. A, 2009, 208, 110.

[203] Ilmi, R; Iftikhar, K. Inorg. Chem. Commun., 2010, 13, 1552.

[204] Irfanullah, M; Iftikhar, K. J. Photochem. Photobiol. A, 2011, 224, 91.

[205] Irfanullah, M; Iftikhar, K. J. Fluoresc., 2011, 21, 81.

[206] Irfanullah, M; Iftikhar, K. Inorg. Chem. Commun., 2010, 13, 694.

[207] Sofer, Z; Tresnakova, P; Spirkova, J; Rubas, S; Kalabova, M. J. Phys. Chem. Solids, 2007, 68(5-6), 1272.

[208] Tresnakova P; Spirkova, J; Rubas, S; Sofer, Z; Oswald, J. J Mater Sci: Mater Electron. 2007, 18, 379.

[209] Strek, W; Deren, PJ; Maruszewski, K; Pawlik, E; Wojcik, W; Malashkevich, GE; Gaishun, VI. J. Alloys Compd., 1998, 275/277, 420.

[210] Park, OH; Seo, S-Y; Jung, J-I; Bae, JY, Bae, BS. The $5^{\text {th }}$ International Meeting of Pacific Rim Ceramic Societies. Nagoya, Japan, 2003.

[211] Wang, H; Qian, G; Wang, Z; Zhang, J; Luo Y; Wang, M. J. Lumin., 2005, 113(3-4), 214.

[212] Reisfeld, R; Saraidarov, T; Pietraszkiewicz, M; Lis, S. Chem. Phys. Lett., 2001, 349, 266.

[213] Lenaerts, P; Storms, A; Mullens, J; D'Haen, J; Görller-Walrand, C; Binnemans K; Driesen, K. Chem. Mater., 2005, 17, 5194.

[214] Driesen, K. Spectroscopy of lanthanide ions in silica-based hybrid materials. PhD Thesis, Faculteit Wetenschappen, Katholieke Universiteit Leuven, 2003.

[215] Prajzler, V; Jeřábek, V; Lyutakov, O; Hüttel, I; Špirková, J; Machovič, V; Oswald, J; Chvostová D; Zavadil, J. Acta Polytechnica, 2008, 48(5), 14.

[216] Bortoluzzi, M; Paolucci, G; Gatto, M; Roppa, S; Enrichi, F; Ciorba, S; Richards, BS. J. Lumin, 2012, 132, 2378.

[217] Sun, LN; Zhang, HJ; Fu, LS; Liu, FY; Meng, QG; Peng, CY; Yu, JB. Adv. Funct. Mat., 2005, 15(6), 1041.

[218] Strek, W; Sokolnicki, J; Legendziewicz, J; Maruszewski, K; Reisfeld, R; Pavich, T. Opt. Mater., 1999, 13, 41.

[219] Curry, RJ; Gillin, WP; Knights, AP; Gwilliam, R. Opt. Mater., 2001, 17, 161.

[220] Artizzu, F; Mercuri, ML; Serpe, A; Deplano, P. Coord. Chem. Rev., 2011, 255, 2514.

[221] Zhao, WQ; Wang, PF; Ran, GZ; Ma, GL; Zhang, BR; Liu, WM; Wu, SK; Dai, L; Qin, GG. J. Phys. D, 2006, 39, 2711.

[222] Sun, RG; Wang, YZ; Zheng, QB; Zhang, H; Epstein, AJ. J. Appl. Phys., 2000, 87(10), 7589.

[223] O'Riordan, A; Van Deun, R; Mairiaux, E; Moynihan, S; Fias, P; Nockemann, P; Binnemans, K; Redmond, G. Thin Solid Films, 2008, 516, 5098.

[224] Zang, FX; Li, WL; Hong, ZR; Wei, HZ; Li, MT; Sun, XY; Lee, CS. Appl. Phys. Lett., 2004, 84, 5115.

[225] Hong, ZR; Liang, CJ; Li, RG; Zang, FX; Fan, D; Li, WL.; Hung, LS; Lee, ST. Appl. Phys. Lett., 2001, 79, 1942.

[226] Buckley, RV (Ed). Solid State Chemistry Research Trends. Nova Science Pub Inc.: New York, 2007. p. 236.

[227] Harrison, BS; Foley, TJ; Bouguettaya, M; Boncella, JM; et al. Appl. Phys. Lett., 2001, 79(23), 3770.

[228] Pizzoferrato, R; Lagonigro, L; Ziller, T; Di Carlo, A. Chem. Phys., 2004, 300, 217.

[229] Kang, TS; Harrison, BS; Foley, TJ; Knefely, AS; Boncella, JM; Reynolds, JR; Schanze, KS. Adv. Mater., 2003, 15, 1093.

[230] Schanze, KS; Reynolds, JR; Boncella, JM; Harrison, BS; et al. Synth. Met., 2003, 137, 1013. 
[231] Bulach, V; Sguerra, F; Hosseini, MW. Coord. Chem. Rev., 2012, 256(15), 1468.

[232] Kawamura, Y; Wada, Y; Yanagida, S. Jpn. J. Appl. Phys., 2001, 40, 350.

[233] Zang, FX; Hong, ZR; Li, WL; Li, MT; Sun, XY. Appl. Phys. Lett., 2004, 84(14), 2679.

[234] Zhang, D; Li, W; Chu, B; Li, X; Han, L; Zhu, J; Li, T; Bi, D; Yang, D; Yan, F; Liu, H; Wang, D. Appl. Phys. Lett., 2008, 92, 093501.

[235] Zang, FX; Sum, TC; Zhu, F; Hong, ZR; Sun, XY; Li, WL; Huan, CHA. J. Lightwave Technol., 2009, 27(11), 1522.

[236] Fan, WQ; Feng, J; Song, SY; Lei, YQ; Xing, Y; Deng, RP; Dang, S; Zhang, H. Eur. J. Inorg. Chem. 2008, 5513.

[237] Li, Z; Yu, J; Zhou, L; Zhang, H; Deng, R; Guo, Z. Org. Electron., 2008, 9, 487.

[238] Guo, X; Guo, H; Fu, L; Carlos, LD; Ferreira, RAS; Sun, L; Deng, R; Zhang, H. J. Phys. Chem. C., 2009, $113,12538$.

[239] Cheng, CH; Wang, J; Du, ZJ; Shi, SH; et al. J. Lumin., 2010, 130, 2293.

[240] Katkova, MA; Pushkarev, AP; Balashova, TV; Konev, AN; Fukin, GK; Ketkov, SY; Bochkarev, MN. J. Mater. Chem., 2011, 21, 16611.

[241] Sano, T; Fujita, M; Fuji, T; Hamada, Y; Shibata, K; Kuroki, K. Jpn. J. Appl. Phys., 1995. 34, 1883.

[242] Wang, KZ; Li, LJ; Liu, WM; Xue, ZQ; Huang, CH; Lin, JH. Mater. Res. Bull., 1997, 31, 993.

[243] Okada, K; Wang, YF; Chen, TM; Kitamura, M; Nakaya, T; Inoue, H. J. Mater. Chem., 1999, 9, 3023.

[244] (a) Liang, CJ; Zhao, D; Hong, ZR; Zhao, DX; Li, XY; Li, WL; Peng, JB; Yu, JQ; Lee, CS; Lee, ST. Appl. Phys. Lett., 2000, 76, 67; (b) Liang, CJ; Hong, ZR; Liu, XY; Zhao, DX; Zhao, D; Li, WL; Peng, JB; Yu, JQ; Lee, CS; Lee, ST. Thin Solid Films, 2000, 359, 14.

[245] Zheng, YX; Lin, J; Liang, YJ; Lin, Q; Yu, YN; Wang, SB; Guo, C; Zhang, HJ. Opt. Mater., 2002, 20, 273.

[246] Kido, J; Nagai, K; Okamoto, Y; Skotheim, T. Chem. Lett., 1991, 1267.

[247] McGehee, MD; Bergstedt, T; Zhang, C; Saab, AP; O’Regan, MB; Bazan, GC; Srdanov, VI; Heeger, AJ. Adv. Mater., 1999, 11, 1349.

[248] Yu, G; Liu, YQ; Wu, X; Zhu, DB; Li, HY; Jin, LP; Wang, MZ. Chem. Mater., 2000, 12, 2537.

[249] Kuriki, K; Koike, Y; Okamoto, Y. Chem. Rev., 2002, 102, 2347.

[250] Slooff, LH; van Blaaderen, A; Polman, A; Hebbink, GA; Klink, SI; van Veggel, FCJM; Reinhoudt, DN; Hofstraat, JW. J. Appl. Phys. 2002, 91, 3955.

[251] Werts, MHV; Verhoeven, J.W; Hofstraat, JW. J. Chem. Soc. Perkin Trans., 2000, 2, 433.

[252] Klink, SI; Hebbink, GA; Grave, L; Peters, FGA; van Veggel, FCJM; Reinhoudt, DN; Hofstraat, JW. Eur. J. Org. Chem., 2000, 1923.

[253] Klink, SI; Grave, L; Reinhoudt, DN; van Veggel, FCJM; Werts, MHV; Geurts, FAJ; Hofstraat, JW. J. Phys. Chem. A, 2000, 104, 5457.

[254] Ryo, M; Wada, Y; Okubo, T; Nakazawa, T; Hasegawa, Y; Yanagidoa, S. J. Mater. Chem., 2002, 12, 1748.

[255] Shavaleev, NM; Pope, SJA; Bell, ZR; Faulkner, S; Ward, MD. Dalton Trans., 2003, 808.

[256] Yang, X; Neher, D; Hertel, D; Daubler, TK. Adv. Mater, 2004, 16, 161.

[257] Yang, X; Muller, DC; Neher, D; Meerholz, K. Adv. Mater, 2006, 18, 948.

[258] Yang, XH; D. Neher, D. Appl. Phys. Lett., 2004, 84, 2476.

[259] Makinen, AJ; Hill, IG; Shashidhar, R; Nikolov, N; Kafafi, ZH. Appl. Phys. Lett., 2001, 79, 557.

[260] Phelan GD; Carlson B; Lawson R; et al. Proceedings of SPIE, 2004, 5214, 337.

[261] Choi, EY; Seo, JH; Kim, HM; et al. $3^{\text {rd }}$ International Nanoelectronics Conference (INEC), 2010, pp. 921-922, 3-8 Jan. 2010.

[262] Jiménez-Trillo, J; Alvarez, AL; Coya, C; Céspedes, E; Espinosa, A. Thin Solid Films, 2011, 520, 1318.

[263] Curry, RJ; Gillin, WP; Knights, AP; Gwilliam, R. Appl. Phys. Lett., 2000, 77(15), 2271.

[264] Álvarez, AL; Romero, B; Arredondo, B; Quintana, X; Mallavia, R; Oton, JM. Int. J. Numer. Model. Electron. Network Dev. Field., 2010, 23, 332.

[265] Coya, C; Álvarez, AL; Yoon, WS; Park, SY. J. Appl. Phys., 2011, 109, 094507. 
[266] Nicolai, HT; Wetzelaer, GAH; Kuik, M; Kronemeijer, AJ; de Boer, B; Blom, PWM. Appl. Phys. Lett., 2010, 96, 172107.

[267] Bässler, H. Phys. Status Solidi B., 1993, 175, 15.

[268] Tanasse, C; Meijer, EJ; Blom, PWM; de Leeuw, DM. Phys. Rev. Lett., 2003, 91, 216601.

[269] Murgatroyd, PN. J. Phys. D: Appl. Phys., 1970, 3, 151.

[270] Arkhipov, VI; Emelianova, EV; Bässler, H. Philos. Mag. B., 2001, 81, 985.

[271] Arkhipov, VI; Emelianova, EV; Tak, YH; Bässler, H. J. Appl. Phys., 1998, 84, 848.

[272] Kreouzis, T; Poplavskyy, D; Tuladhar, SM; Campoy-Quiles, M; Nelson, J; Campbell, AJ; Bradley, DDC. Phys. Rev. B., 2006, 73, 235201.

[273] Attridge, TH. Light and Plant Responses, Edward Arnold: London, 1990.

[274] Mohr, H; Schopfer, P; Quail, PH. Plant Physiology, Springer: Berlin, 1995.

[275] Fattori, V; Williams, JAG; Murphy, L; Cocchi, M; Kalinowski, J. Phot. Nano. Fund. Appl., 2008, 6, 225.

[276] Martins, JP; Ramos-Silva, M; Martín-Gil, J; Paixão, JA. Characterization of new europium complexes to be used as emissive layers in OLED. In $7^{\text {th }}$ International Meeting of Materials Materiais 2013, Coimbra, Portugal, 25-27 March 2013.

[277] McMahon, MJ; Kelly, JW; Decoteau, DR; Young, RE; Pollock, RK. J. Amer. Soc. Hort. Sci., 1991, $116(6), 950$.

[278] Rajapakse, NC; Pollock, RK; McMahon, MJ; Kelly, JW; Young, RE. HortScience, 1992, 27(11), 1208.

[279] Hori, K; Suzuki, J; Takamura, M; et al. Mitsubishi Heavy Industries Technical Review, 2012, 49(1), 47.

[280] Lee, CY; Lee, JY. Above 30\% External Quantum Efficiency in Blue Phosphorescent Organic LightEmitting Diodes Using Pyrido[2,3-b]indole Derivatives as Host Materials. Adv. Mater., 2013. DOI: 10.1002/adma.201301091 